This preliminary Technical Design Report (TDR) is the first document describing the full BTeV detector in detail. It describes the two arm version of the detector.

\title{
Proposal for an Experiment to Measure Mixing, CP Violation and Rare Decays in Charm and Beauty Particle Decays at the Fermilab Collider - BTeV $\dagger$
}

May 1999

A. Kulyavtsev, M. Procario, J. Russ, and J. You

Carnegie Mellon University, Pittsburgh, PA 15213, USA

J. Cumalat

University of Colorado, High Energy Physics, Campus Box 390,

Boulder, CO 80309, USA

J. A. Appel, C. N. Brown, J. Butler, H. Cheung, D. Christian, I. Gaines,

P. Garbincius, L. Garren, N. M. Gelfand, E. E. Gottschalk, G. Jackson,

P. Kasper, P. H. Kasper, R. Kutschke, S. W. Kwan, P. Lebrun, P. McBride,

L. Stutte, and J. Yarba

Fermilab, PO Box 500, Batavia, IL 60510, USA

P. Avery, and M. Lohner

University of Florida, Gainesville, FL 32611, USA

R. A. Burnstein, D. M. Kaplan, L. M. Lederman, H. A. Rubin, and C. White

Illinois Institute of Technology, Chicago, IL 60616, USA

M. Selen, and J. Wiss

University of Illinois at Urbana-Champaign, High Energy Physics,

441 Loomis Lab. of Physics, 1110 W. Green St., Urbana, IL 61801-3080, USA

R. W. Gardner and D. R. Rust

Physics Department, Indiana University

Bloomington, Indiana, 47405, USA

D. Menasce, L. Moroni, D. Pedrini, and S. Sala

INFN and University of Milano, Italy

G. Boca, G. Liguori, and P. Torre

Dipartimento di Fisica Nucleare e Teorica, Universita' di Pavia and INFN,

Sez. di Pavia, Italy

Y. Kubota, and R. Poling

University of Minnesota, High Energy Physics, Tate Lab of Physics,

116 Church St. S.E., Minneapolis, MN 55455, USA

T. Y. Chen

Nanjing University, Dept. of Physics, Nanjing 210008, China

V. Papavassiliou

New Mexico State University

\footnotetext{
${ }^{\dagger}$ Spokespersons: Joel Butler and Sheldon Stone
} 
K. Honscheid, and H. Kagan

Ohio State University,

HEP Group, Dept. of Experimental or Theoretical Physics,

Smith Lab, 174 W. 18th Ave., Columbus, OH 43210, USA

W. Selove

University of Pennsylvania, Philadelphia, PA 19104, USA

A. Lopez

University of Puerto Rico, Mayaguez, Puerto Rico

M. Alam, and S. Timm

State University of New York at Albany,

Dept. of Physics, Albany, NY 12222, USA

X. Q. Yu

University of Science and Technology of China, Dept. of Modern Physics,

Joint Institute for High Energy Physics, Hefei, Anhui 230027, China

M. He

Shandong University, High Energy Physics Group,

Jinan, Shandong 250100, China

S. Shapiro (emeritus)

Stanford Linear Accelerator Center, PO Box 4349, Stanford, CA 94309, USA

M. Artuso, M. Goldberg, T. Skwarnicki, S. Stone, and A. Wolf

Syracuse University, 201 Physics Bldg., Syracuse, NY 13244-1130, USA

T. Handler

University of Tennessee, Knoxville, TN 37996-1200, USA

A. Napier

Tufts University, High Energy Physics, Science \& Technology Center,

4 Colby St., Medford, MA 02155, USA

D. D. Koetke

Valparaiso University, Neils Science Center, Valparaiso, IN 46383, USA

P. Sheldon, and M. Webster

Vanderbilt University, Department of Physics and Astronomy,

Nashville, TN 37235, USA

M. Sheaff

University of Wisconsin, Phenomenology Inst., Dept. of Physics,

1150 University Ave., Madison, WI 53706, USA

J. Slaughter

Yale University, High Energy Physics, 219 Prospect St.,

554 JWG, Box 6666, New Haven, CT 06511, USA

S. Menary

York University, Dept. of Physics

4700 Keele St., Toronto, ON M3J 1P3, Canada 


\section{Contents}

I Physics Objectives of BTeV 1

1 Bottom Physics - Important Questions and Status of the Field in the year 20052

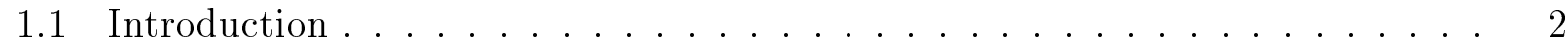

1.2 The CKM Matrix and CP Violation . . . . . . . . . . . . . . . . 2

1.3 CP Violation in Charged $B$ Decays . . . . . . . . . . . . . . . . 6

1.4 CP Violation Formalism in Neutral $B$ decays . . . . . . . . . . . . . . 7

1.5 Comment on Penguin Amplitude . . . . . . . . . . . . . . . . . . 12

1.6 Asymmetry Measurements . . . . . . . . . . . . . . . . . . . . . 12

1.7 Techniques for Determining $\beta \ldots \ldots \ldots \ldots$

1.7.1 Removal of two of the $\beta$ Ambguities . . . . . . . . . . . . . . 14

1.8 Techniques for Determining $\alpha \ldots \ldots \ldots \ldots$

1.9 Techniques for Determining $\gamma \ldots \ldots \ldots \ldots \ldots$

1.9.1 Measurement of $\gamma$ Using Time-Dependent CP violation in $B_{s}$ Decays . 19

1.9.2 Measurement of $\gamma$ Using Charged B Decay Rates . . . . . . . . . . . 20

1.9.3 Measurement of $\gamma$ Using $B \rightarrow K \pi$ and $B \rightarrow \pi \pi$ Decay Rates and

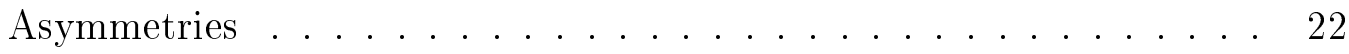

1.9.4 Measurement of $\gamma$ Using CP Asymmetries in $\boldsymbol{B}^{\boldsymbol{o}} \rightarrow \boldsymbol{\pi}^{+} \boldsymbol{\pi}^{-}$and

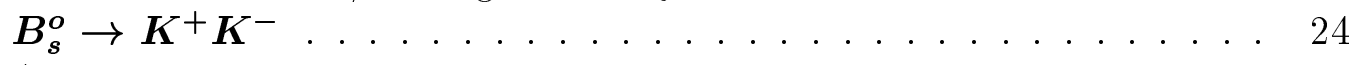

1.9.5 Additional Physics Opportunities with $B_{s}$ Mesons . . . . . . . . . 27

1.10 The Tests of the Standard Model . . . . . . . . . . . . . . . . . . . . 29

1.10.1 Better Measurements of the sides of the CKM triangle . . . . . . . 31

1.10 .2 Summary of CKM tests . . . . . . . . . . . . . . . . 32

1.10.3 Checks combining with measurements of magnitudes of CKM elements 33

1.11 Rare Decays as Probes beyond the Standard Model . . . . . . . . . . . . . . . 34

1.11.1 Standard Model Theory . . . . . . . . . . . . . . . . . . . . . . . . . . . 35

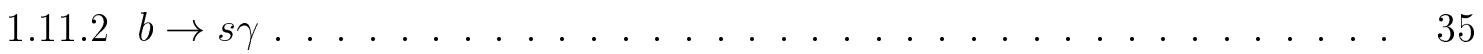

1.11 .3 The Exclusive Decays $K^{*} \gamma$ and $\rho \gamma \ldots \ldots \ldots \ldots$

$1.11 .4 b \rightarrow s \ell^{+} \ell^{-} \ldots \ldots \ldots \ldots \ldots \ldots \ldots \ldots$ 
2 Charm Physics - Important Questions and Status of the Field in the year 2005

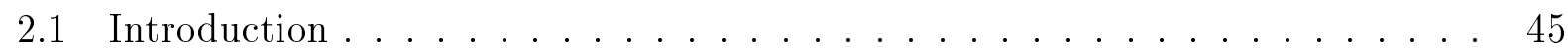

2.2 Important Questions in Charm Physics . . . . . . . . . . . . . . . . 45

2.2 .1 Standard Model Charm Physics . . . . . . . . . . . . . . . . 45

2.2.2 Charm as a Probe of New Physics . . . . . . . . . . . . . 48

2.2.3 Charm as a tool for Bottom Physics . . . . . . . . . . . . . . 54

2.3 Status of Charm Physics in the year $2005 \ldots \ldots \ldots \ldots$

2.4 Charm Physics in $\mathrm{BTeV} \ldots \ldots \ldots \ldots \ldots$. . . . . . . . . . . 57

3 The Main Physics Goals of BTeV 63

3.1 Physics Goals For $B$ 's . . . . . . . . . . . . . . . . . . 64

3.2 The Main Physics Goals for charm . . . . . . . . . . . . . . . . 65

3.3 Other Bottom and Charm Physics Goals . . . . . . . . . . . . . . . 65

$\begin{array}{lll}\text { II The BTeV Detector } & 66\end{array}$

4 The BTeV Baseline Detector $\quad \mathbf{6 7}$

4.1 Characteristics of Hadronic $\boldsymbol{b}$ Production . . . . . . . . . . . . 68

4.2 C0 Baseline Detector Description . . . . . . . . . . . . . . 73

4.2 .1 The C0 Experimental Area . . . . . . . . . . . . . . 75

4.2.2 The BTeV/C0 Spectrometer Magnet . . . . . . . . . . . . 78

4.2 .3 Tracking System . . . . . . . . . . . . . . . . . . 82

4.2 .4 Triggering System . . . . . . . . . . . . . . . . . . . . . . . 89

4.2 .5 Particle Identification . . . . . . . . . . . . . . . . . . 95

4.2 .6 Electromagnetic Calorimetry . . . . . . . . . . . . . . . . . 114

4.2 .7 Muon Detection . . . . . . . . . . . . . . . . . . . 136

4.3 Evolution of the $\mathrm{C} 0$ Program . . . . . . . . . . . . . . . . . 145

4.4 Completing the Simulation of the Baseline Detector . . . . . . . . . . 147

5 Pixel R\&D $\quad 150$

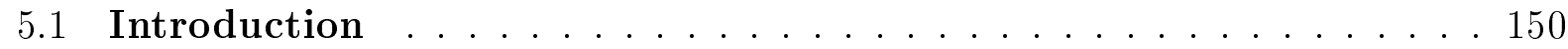

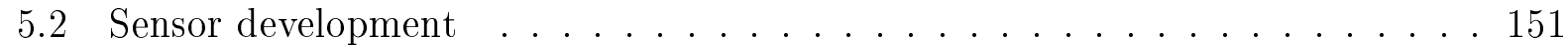

5.2 .1 Introduction . . . . . . . . . . . . . . . . . . . . 151

5.2 .2 Sensor simulation . . . . . . . . . . . . . . . . . . . . . 152

5.2 .3 Prototype sensor . . . . . . . . . . . . . . . . . . . . . . 174

5.2 .4 Collaborative effort with BNL/JHU . . . . . . . . . . . . 175

5.2 .5 Diamond option . . . . . . . . . . . . . . . . 176

5.2 .6 Goals of sensor development . . . . . . . . . . . . . . . . 176

5.3 Pixel readout chip . . . . . . . . . . . . . . . . . . . 176

5.3.1 Pixel readout chip development at Fermilab . . . . . . . . . . . 176

$5.3 .2 \quad$ FPIX0 . . . . . . . . . . . . . . . . . . . . . 177 


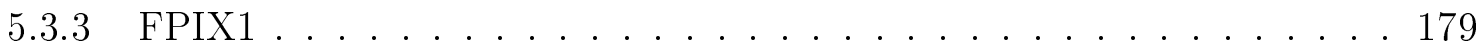

5.3 .4 Future plans . . . . . . . . . . . . . . . 183

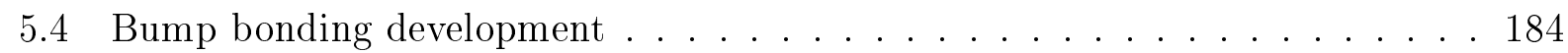

5.4 .1 Introduction . . . . . . . . . . . . . . 184

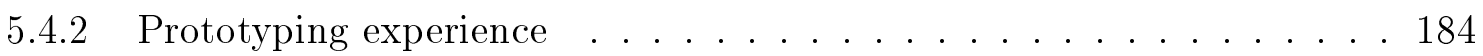

5.4 .3 Large scale study of bump bonding yield . . . . . . . . . . . . 186

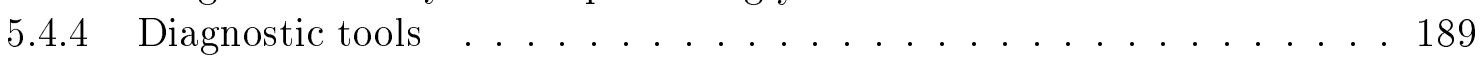

5.5 Data output and control . . . . . . . . . . . . . . . . . . . . . . . . . . . . . . 189

5.6 Pixel Readout and Quadrant Processors . . . . . . . . . . . . . 192

5.7 Assembly issues . . . . . . . . . . . . . . . . . . . . . . . . . . . . . . . . . . . . . . . . . . .

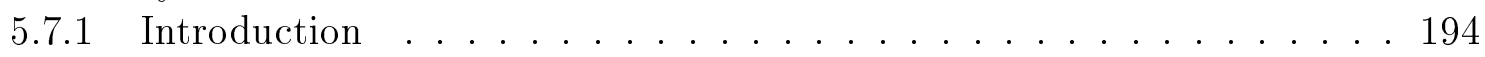

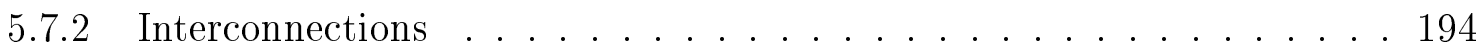

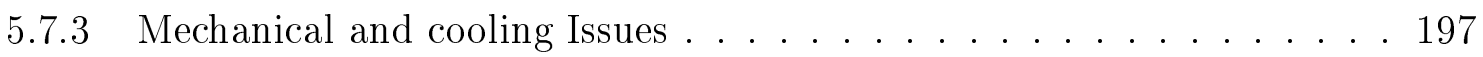

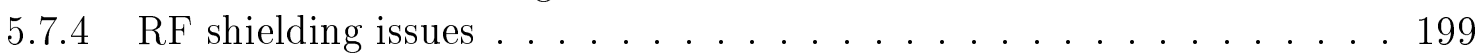

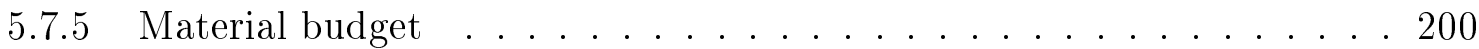

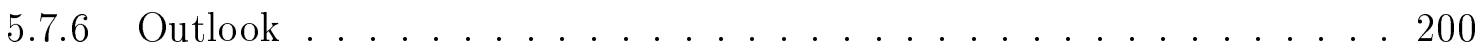

5.8 Pixel Beam Test . . . . . . . . . . . . . . . . . . . . . . . . . . . . . . . . 202

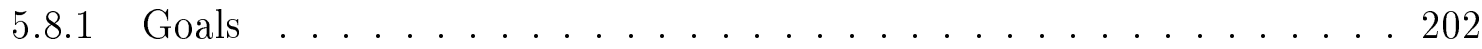

5.8 Test Stand . . . . . . . . . . . . . . . . . . . . . . . . . . . . . . . . . . . . . . . . 202

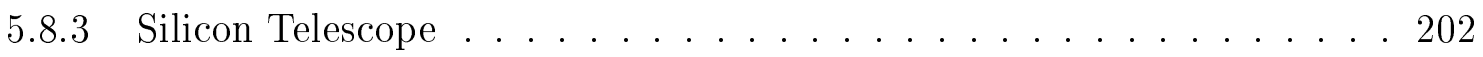

5.8.4 Pixel Readout Board . . . . . . . . . . . . . 203

6 Forward Tracking System 207

6.1 Introduction . . . . . . . . . . . . . . . 207

6.2 Detector Geometry . . . . . . . . . . . . . . . . . . . . . . . . . . . . . . . . . . .

6.2.1 Layout Considerations . . . . . . . . . . . . . 210

6.3 Performance Issues . . . . . . . . . . . . . . . . . . . . . . . . . . . . . . . . . . . . . . . . .

6.3.1 Straw Hit Occupancies . . . . . . . . . . . . . . 210

6.3.2 Momentum Resolution of the Forward Straw Tracker . . . . . . . . . . 211

6.4 Forward Straw Tracker . . . . . . . . . . . . . . . . . . 213

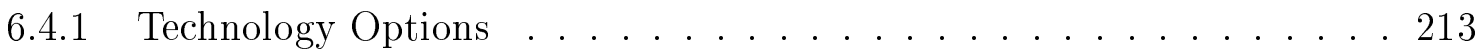

6.4.2 Straw Detecting Elements . . . . . . . . . . . . . . . 214

6.4.3 Construction of Straw Chamber Modules . . . . . . . . . . . . 219

6.4.4 Front-end Electronics . . . . . . . . . . . . . . 219

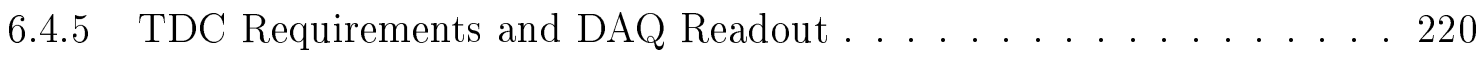

6.5 Forward Silicon Tracker . . . . . . . . . . . . . . . . . 221

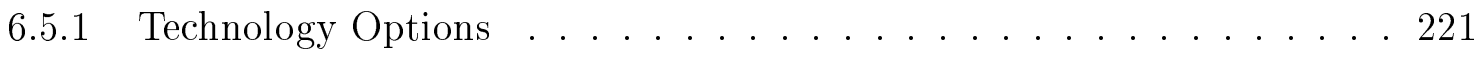

6.5.2 Detector Description and Layout . . . . . . . . . . . . 222

7 Design of the Muon Detector 224

7.1 Detector Design and Fabrication . . . . . . . . . . . . . . 224

7.1.1 Basic Building Block: Proportional Tube "Plank" . . . . . . . . . 224 
7.1.2 Design of a Detector Station . . . . . . . . . . . 225

7.1.3 Proportional Tube Details . . . . . . . . . . . . . 226

7.1.4 Miscellaneous Mechanical Details . . . . . . . . . . . 227

7.1.5 Summary of Mechanical Design . . . . . . . . . . . . . . 227

7.2 Front End Electronics . . . . . . . . . . . . . . . . 228

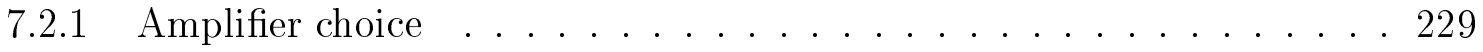

7.2.2 Post Front-end Considerations for the Muon System . . . . . . . . 231

7.3 Trigger Electronics . . . . . . . . . . . . . . . . . . . . . . . . . . . . . . . . . . . . . . . . . . . . .

7.4 Test Beam Plans and Goals . . . . . . . . . . . . . . . . 232

8 The BTeV Trigger Performance 235

8.1 Introduction . . . . . . . . . . . . . . . . . . . . . . . . . . . . . . . . . 355

8.2 Advanced Level 1 Trigger Studies . . . . . . . . . . . . . 235

8.2.1 Trigger Diagnostics and 'Software Instrumentation' . . . . . . . . . 236

8.2.2 Uncorrelated Noise Study . . . . . . . . . . . . . . . . 236

8.2.3 Correlated Noise Study . . . . . . . . . . . . . . . . . 237

8.2.4 Multiple Interactions per Crossing Study . . . . . . . . . . . 239

8.3 Efficiency and Rejection of the BTeV Level 1 Vertex Trigger for

Selected Physics States of Interest . . . . . . . . . . . . . . . . . 245

8.4 Other Level 1 triggers . . . . . . . . . . . . . . . . . 247

8.5 Status Report on Level 1 trigger R\&D and trigger prototype . . . . . . . . 247

8.6 Results on Baseline Level 1 Algorithm Timing on Digital Signal Processors . 249

8.7 Options to the Baseline Level 1 Vertex Trigger . . . . . . . . . . . . 252

8.7.1 An Alternative Using Two Plane Stations . . . . . . . . . . . . . . 252

8.7 .2 The 'Megapixel Trigger' . . . . . . . . . . . . . . . . . . . . . . . . . . 256

8.8 The Global Level 1 Trigger Framework . . . . . . . . . . . . . . . 260

8.9 The Level 2 and 3 Triggers . . . . . . . . . . . . . . . . 260

8.9.1 Preliminary studies of the Level 2 algorithm . . . . . . . . . . 261

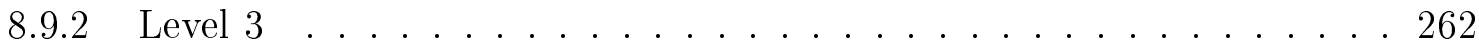

9 Trigger and Data Acquisition Architecture and Front End Electronics 268

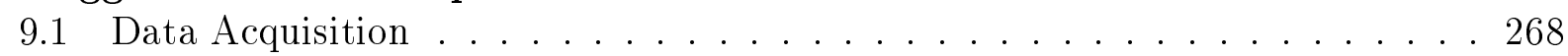

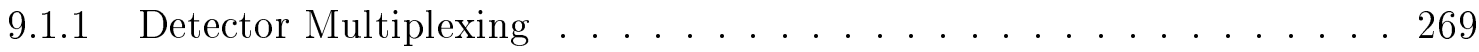

9.1 .2 Detector Data Links . . . . . . . . . . . . . 270

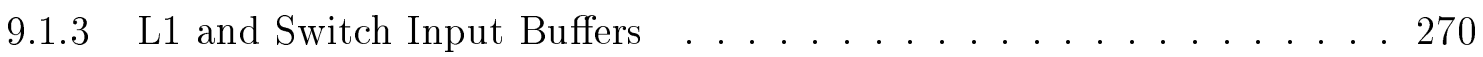

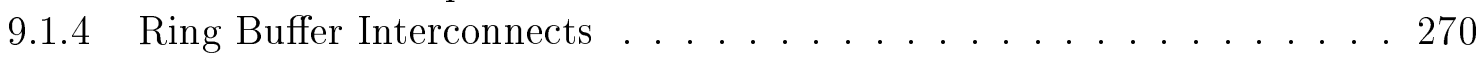

9.1.5 Staged Readout . . . . . . . . . . . . . . 271

9.1 .6 Data Switch . . . . . . . . . . . . . . . . . . . . . . . . . . . . . . 271

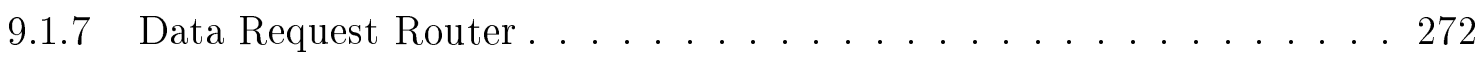

9.1 .8 Switch Output Buffers . . . . . . . . . . . . . 272

9.1 .9 Data Logging . . . . . . . . . . . . . . . . . . . . . . . . . . . . . . . . . . . . . . . . . .

9.1.10 Detector Control Links . . . . . . . . . . . . . 272 
9.1 .11 Diagnostics . . . . . . . . . . . . . . . . . . . . . . 272

9.1 .12 General Operation . . . . . . . . . . . . . . . . . . . 273

9.1 .13 Component Placement . . . . . . . . . . . . . . . . . . 273

9.2 Global Level 1 Trigger System . . . . . . . . . . . . . . . . . . . 273

9.3 Level $2 / 3$ Trigger . . . . . . . . . . . . . . . . . . . . . . . 276

9.4 Data Logging and Online Event Analysis . . . . . . . . . . . . . 277

9.5 Front End Electronics . . . . . . . . . . . . . . . . . . . . 278

9.5.1 Infrastructure and Other Support Systems . . . . . . . . . . . 278

9.5.2 Forward Tracker . . . . . . . . . . . . . . . . . . . . . . . . 283

9.5.3 Ring Imaging Cherenkov Counter . . . . . . . . . . . . . . . . . . . 284

9.5.4 Electromagnetic Calorimeter . . . . . . . . . . . . . . . . 285

9.5.5 Muon Detector . . . . . . . . . . . . . . . . . . . . 286

9.5.6 Software for Module and Subsystem Development and the Online Data Acquisition and Readout System . . . . . . . . . . . . 287

\section{Simulations Results: BTeV Physics Reach 288}

10 Status of the BTeV Simulation Effort $\quad 289$

10.1 Update on MCFast . . . . . . . . . . . . . . . . . . . . . . . . . . . . . . . . . . . . . . . . . . .

10.2 Kalman Filter . . . . . . . . . . . . . . . . . 290

10.3 Towards Realistic Pixel Resolution Functions . . . . . . . . . . . . . 291

10.4 Multiple interactions per crossing . . . . . . . . . . . . . . . . . . . 293

10.5 Simulation of Secondary Hadronic Interactions . . . . . . . . . . . . . 294

10.5.1 Inelastic Secondary Interactions . . . . . . . . . . . . . . 294

10.6 Elastic Interactions . . . . . . . . . . . . . . . . . . . . . . . . . . . . . . . . . . . . . . . . . . . . . . . .

10.7 Calorimeter Simulation . . . . . . . . . . . . . . . . . . . . . . . . . . . . . . . . . . . . . . . . . . . . .

10.7.1 Simulation Techniques . . . . . . . . . . . . . 295

10.7.2 The MCFast showering/calorimetry subpackage . . . . . . . . . . 295

10.8 Simulation of the BTeV Trigger . . . . . . . . . . . . . . . . . . . . . . . . . . . . . . . . . . . . . .

10.9 BTeV Geometry Description for MCFast . . . . . . . . . . . . 298

11 The Physics reach of BTeV 306

11.1 Measurement of the CKM angle $\gamma \ldots \ldots$. . . . . . . . . . . . . . . . . . . . . . . . .

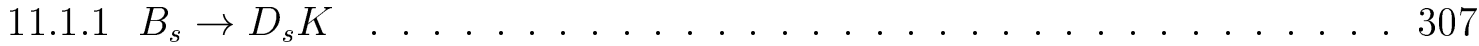

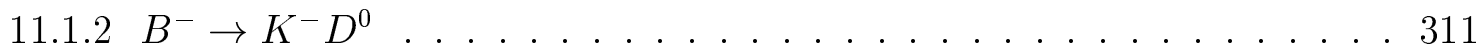

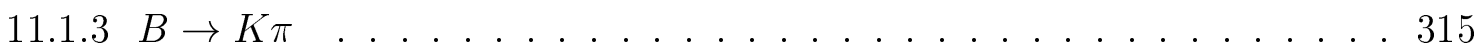

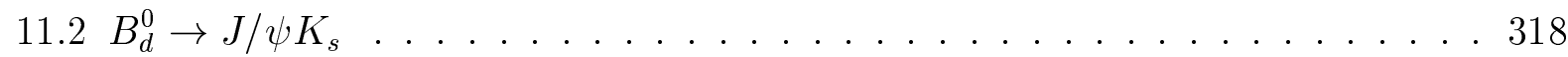

11.3 Flavor Tagging . . . . . . . . . . . . . . . . . . 322

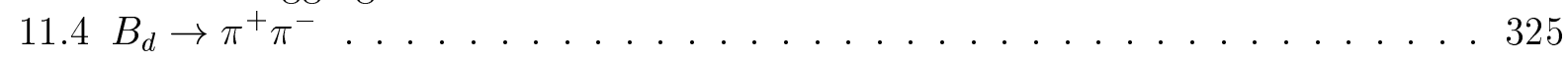

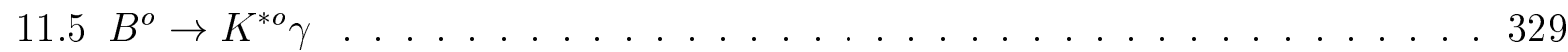

11.5.1 An Initial Shower Reconstruction Program . . . . . . . . . . . 330

11.5.2 Suppression of Combinatoric Backgrounds . . . . . . . . . . . 330 
11.5.3 $K^{* o} \gamma$ Analysis Cuts . . . . . . . . . . . . . . . . . 333

11.5.4 Signal Properties . . . . . . . . . . . . . . . . . . 333

11.5 .5 Backgrounds . . . . . . . . . . . . . . . . . . 335

11.5.6 Projections of Signal Size and Background . . . . . . . . . 336

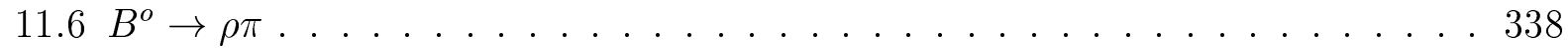

11.7 Flavor-changing $B$ meson decays $\ldots \ldots \ldots \ldots \ldots \ldots \ldots \ldots$

11.7.1 The Physics Significance of Rare B Meson Decays . . . . . . . . . . 340

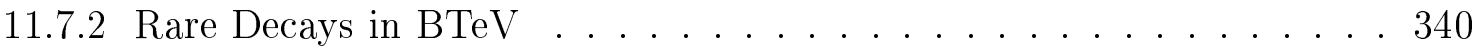

$11.8 \boldsymbol{B}_{\boldsymbol{s}}$ Mixing with $\boldsymbol{B}_{\boldsymbol{s}} \rightarrow \boldsymbol{D}_{\boldsymbol{s}}^{-} \boldsymbol{\pi}^{+}$and $\boldsymbol{B}_{\boldsymbol{s}} \rightarrow \boldsymbol{\psi} \overline{\boldsymbol{K}}^{* 0} \ldots \ldots \ldots \ldots \ldots \ldots \ldots$

11.8.1 Yields, Resolutions and Signal-to-Background Ratios . . . . . . . . 345

11.8.2 Computation of the $x_{s}$ Reach . . . . . . . . . . . . . 348

11.9 Charm Physics with the BTeV Detector . . . . . . . . . . . . . 356

11.9.1 Direct CP Violation in the Charm Sector . . . . . . . . . . 357

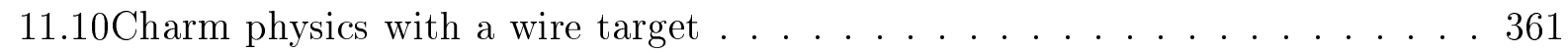

11.10.1 Vertex detector and trigger . . . . . . . . . . . . . 361

11.10 .2 Reconstruction . . . . . . . . . . . . . . . . . 362

11.10 .3 Physics reach . . . . . . . . . . . . . . . 362

12 Comparison of BTeV to Other Experiments 366

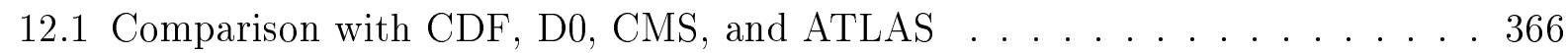

12.2 Comparison with $e^{+} e^{-} \boldsymbol{B}$ Factories $\ldots \ldots \ldots 367$

12.3 Comparison with LHC-B . . . . . . . . . . . . . . 367

$\begin{array}{lll}\text { IV Costs } & 371\end{array}$

13 Cost 372

13.1 Preliminary Cost Estimate . . . . . . . . . . . . . . . . . . 372

13.2 Support Required . . . . . . . . . . . . . . . . . . . . 373 


\section{Part I}

\section{Physics Objectives of BTeV}




\section{Chapter 1}

\section{Bottom Physics - Important Questions and Status of the Field in the year 2005}

\subsection{Introduction}

Here we will discuss CP violation and rare decays in the $b$ system as described by the Standard Model [1]. There are many important experimental measurements to be made. We will describe the reasons why these measurements are crucial. We will also point out the important tests that probe for physics beyond the Standard Model.

There are many other interesting and important physics topics concerning issues of heavy quark production, the phenomenology of weak decays, etc.., that we do not dicuss here. It should be kept in mind that other areas of interesting physics can be addressed by BTeV even though we don't explicitly discuss them here. The material at the beginning is pedagogical in nature. Experts may choose to skip to Section 1.7, below.

\subsection{The CKM Matrix and CP Violation}

The physical point-like states of nature that have both strong and electroweak interactions, the quarks, are mixtures of base states described by the Cabibbo-Kobayashi-Maskawa matrix $[2]$,

$$
\left(\begin{array}{c}
d^{\prime} \\
s^{\prime} \\
b^{\prime}
\end{array}\right)=\left(\begin{array}{lll}
V_{u d} & V_{u s} & V_{u b} \\
V_{c d} & V_{c s} & V_{c b} \\
V_{t d} & V_{t s} & V_{t b}
\end{array}\right)\left(\begin{array}{c}
d \\
s \\
b
\end{array}\right)
$$

The unprimed states are the mass eigenstates, while the primed states denote the weak eigenstates.

There are nine complex CKM elements. These 18 numbers can be reduced to four independent quantities by applying unitarity constraints and the fact that the phases of the quark wave functions are arbitrary. These four remaining numbers are fundamental constants of nature that need to be determined from experiment, like any other fundamental 
constant such as $\alpha$ or $G$. In the Wolfenstein approximation the matrix is written as [3]

$$
V_{C K M}=\left(\begin{array}{ccc}
1-\lambda^{2} / 2 & \lambda & A \lambda^{3}\left(\rho-i \eta\left(1-\lambda^{2} / 2\right)\right) \\
-\lambda & 1-\lambda^{2} / 2-i \eta A^{2} \lambda^{4} & A \lambda^{2}\left(1+i \eta \lambda^{2}\right) \\
A \lambda^{3}(1-\rho-i \eta) & -A \lambda^{2} & 1
\end{array}\right) .
$$

This expression is accurate to order $\lambda^{3}$ in the real part and $\lambda^{5}$ in the imaginary part. It is necessary to express the matrix to this order to have a complete formulation of the physics we wish to pursue. The constants $\lambda$ and $A$ have been measured using semileptonic $s$ and $b$ decays $[4] ; \lambda \approx 0.22$, and $A \approx 0.8$.

The phase $\eta$ allows for $\mathrm{CP}$ violation. $\mathrm{CP}$ violation thus far has only been seen in the neutral kaon system. If we can find CP violation in the $B$ system we could see if the CKM model works or perhaps discover new physics that goes beyond the model, if it does not.

The unitarity of the CKM matrix ${ }^{1}$ allows us to construct six relationships. These equations may be thought of triangles in the complex plane. They are shown in Fig. 1.1

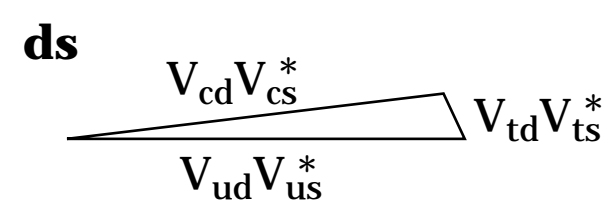

sb
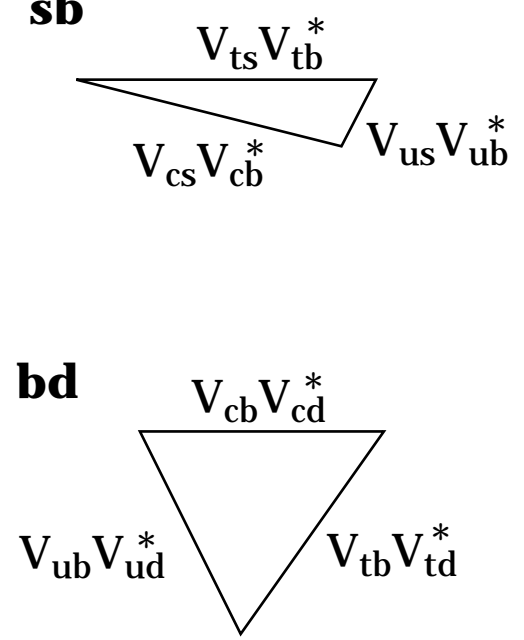
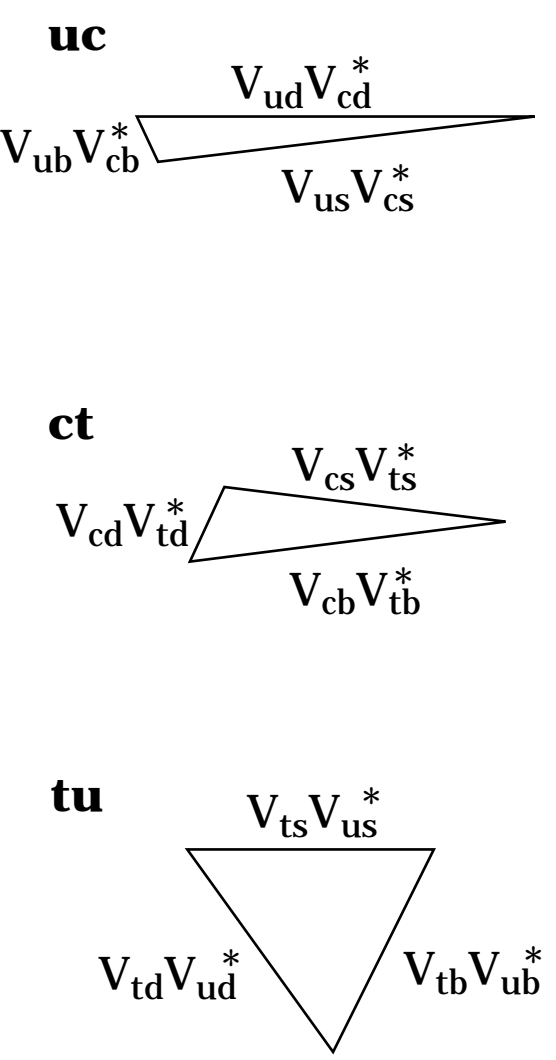

Figure 1.1: The six CKM triangles. The bold labels, i.e ds refer to the rows or columns used in the unitarity relationship.

\footnotetext{
${ }^{1}$ Unitarity implies that any pair of rows or columns are orthogonal.
} 
In the bd triangle, the one usually considered, the angles are all thought to be relatively large. It is described by:

$$
V_{u b} V_{u d}^{*}+V_{c b} V_{c d}^{*}+V_{t b} V_{t d}^{*}=0 .
$$

To a good approximation

$$
\left|V_{u d}^{*}\right| \approx\left|V_{t b}\right| \approx 1,
$$

then

$$
\frac{V_{u b}}{V_{c b}}+\frac{V_{t d}^{*}}{V_{c b}}+V_{c d}^{*}=0 .
$$

Since $V_{c d}^{*}=\lambda$, we can define a triangle with sides

$$
\begin{aligned}
\left|\frac{V_{t d}}{A \lambda^{3}}\right| & =\sqrt{(\rho-1)^{2}+\eta^{2}}=\frac{1}{\lambda}\left|\frac{V_{t d}}{V_{t s}}\right| \\
\left|\frac{V_{u b}}{A \lambda^{3}}\right| & =\sqrt{\rho^{2}+\eta^{2}}=\frac{1}{\lambda}\left|\frac{V_{u b}}{V_{c b}}\right| .
\end{aligned}
$$

This CKM triangle is depicted in Fig. 1.2.

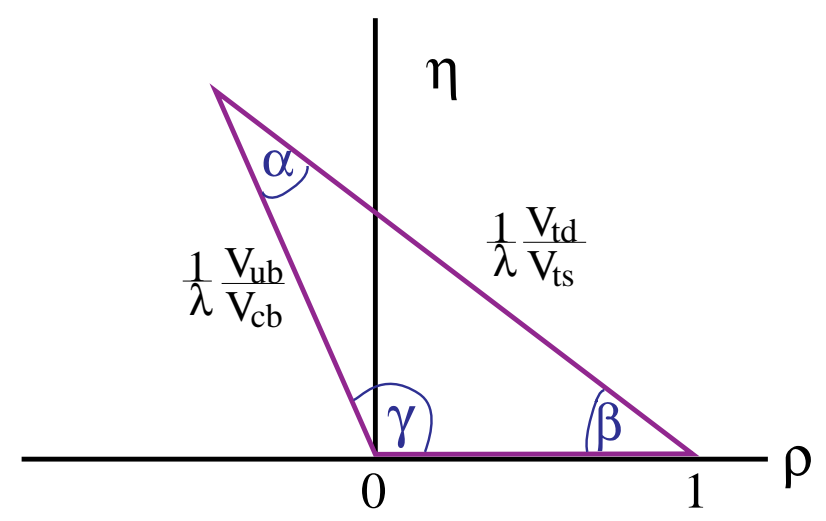

Figure 1.2: The CKM triangle shown in the $\rho-\eta$ plane. The left side is determined by $\left|V_{u b} / V_{c b}\right|$ and the right side can be determined using mixing in the neutral $B$ system. The angles can be found by making measurements of $\mathrm{CP}$ violation in $B$ decays.

We know two sides already: the base is defined as unity and the left side is determined within error by the measurements of $\left|V_{u b} / V_{c b}\right|$. The right side can be determined using mixing measurements in the neutral $B$ system. We will see, however, that there is a large error due to the uncertainty in $f_{B}$, the $B$-meson decay constant. Later we will discuss other measurements that can determine this side. The figure also shows the angles $\alpha, \beta$, and $\gamma$. These angles can be determined by measuring $\mathrm{CP}$ violation in the $B$ system.

Neutral $B$ mesons can transform to their anti-particles before they decay. The diagrams for $B_{d}$ mixing are shown in Fig. 1.3. (The diagrams for $B_{s}$ mixing are similar with $s$ quarks 
replacing $d$ quarks.) Although $u, c$ and $t$ quark exchanges are all shown, the $t$ quark plays a dominant role, mainly due to its mass, since the amplitude of this process grows with the mass of the exchanged fermion.

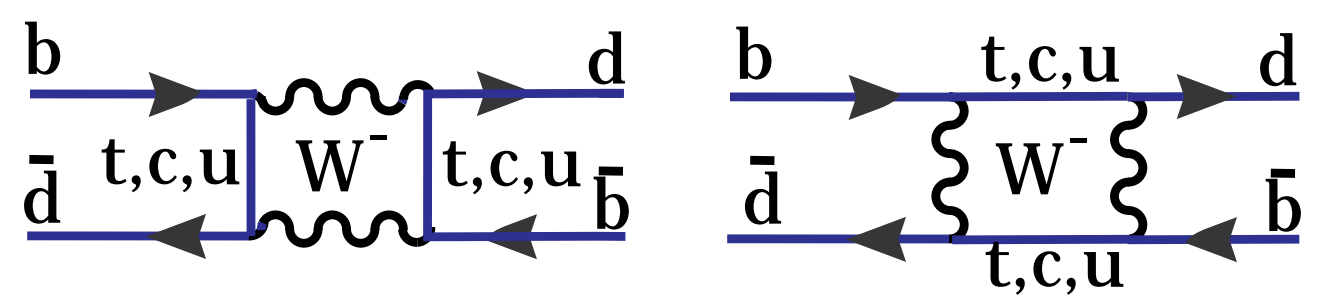

Figure 1.3: The two diagrams for $B_{d}$ mixing.

The probability of mixing is given by [5]

$$
x \equiv \frac{\Delta m}{\Gamma}=\frac{G_{F}^{2}}{6 \pi^{2}} B_{B} f_{B}^{2} m_{B} \tau_{B}\left|V_{t b}^{*} V_{t d}\right|^{2} m_{t}^{2} F\left(\frac{m_{t}^{2}}{M_{W}^{2}}\right) \eta_{Q C D}
$$

where $B_{B} f_{B}^{2}$ is related to the probability of the $d$ and $\bar{b}$ quarks forming a hadron and must be estimated theoretically; $F$ is a known function which increases approximately as $m_{t}^{2}$, and $\eta_{Q C D}$ is a QCD correction, with value about 0.8. By far the largest uncertainty arises from the unknown decay constant, $f_{B}$. This number gives the coupling between the $B$ and the $W^{-}$. It could in principle be determined by finding the decay rate of $B^{+} \rightarrow \mu^{+} \nu$ or to $\tau^{+} \nu$, both of which are very difficult to measure. Since

$$
\left|V_{t b}^{*} V_{t d}\right|^{2} \propto|(1-\rho-i \eta)|^{2}=(\rho-1)^{2}+\eta^{2}
$$

measuring mixing gives a circle centered at $(1,0)$ in the $\rho-\eta$ plane. The best recent mixing measurements have come from a variety sources [6], yielding a value for the $B_{d}$ of $\Delta m=$ $(0.464 \pm 0.018) \times 10^{12} \hbar s^{-1}$.

The fact that the CKM matrix is complex allows CP violation. So far CP violation has only been measured in decays of the $K_{L}^{o}$ meson [7]. In addition, CDF has seen an indication in $B^{o}$ decays [8].

There is a constraint on $\rho$ and $\eta$ given by the $K_{L}^{o} \mathrm{CP}$ violation measurement $(\epsilon)$, given by $[9]$

$$
\eta\left[(1-\rho) A^{2}(1.4 \pm 0.2)+0.35\right] A^{2} \frac{B_{K}}{0.75}=(0.30 \pm 0.06),
$$

where the errors arise from uncertainties on $m_{t}$ and $m_{c}$. The constraints on $\rho$ versus $\eta$ from the $V_{u b} / V_{c b}$ measurement, $\epsilon$ and $B$ mixing are shown in Fig. 1.4. The bands represent $1 \sigma$ errors, for the measurements and a $95 \%$ confidence level upper limit on $B_{s}$ mixing. The width of the $B_{d}$ mixing band is caused mainly by the uncertainty on $f_{B}$, taken here as $240>$ $f_{B}>160 \mathrm{MeV}$. Other parameters include $\left|V_{c b}\right|=0.0381 \pm 0.0021,\left|V_{u b} / V_{c b}\right|=0.087 \pm 0.012$ [10], limit on $\Delta m_{s}>12.4 \mathrm{ps}^{-1}$, and the ratio $f_{B_{s}} \sqrt{B_{B_{s}}} / f_{B_{d}} \sqrt{B_{B_{d}}} \leq 1.25$ [11]. 
The width of the $\epsilon$ band is caused by errors in $A, m_{t}, m_{c}$ and $B_{K}$. Here $B_{K}$ is taken as $0.75 \pm 0.15$ according to Buras [12]. The size of these error sources for $\epsilon$ is shown in Fig. 1.5. The largest error still comes from the measurement of $V_{c b}$, with the theoretical estimate of $B_{K}$ being a close second. The errors on $m_{t}$ and $m_{c}$ are less important.

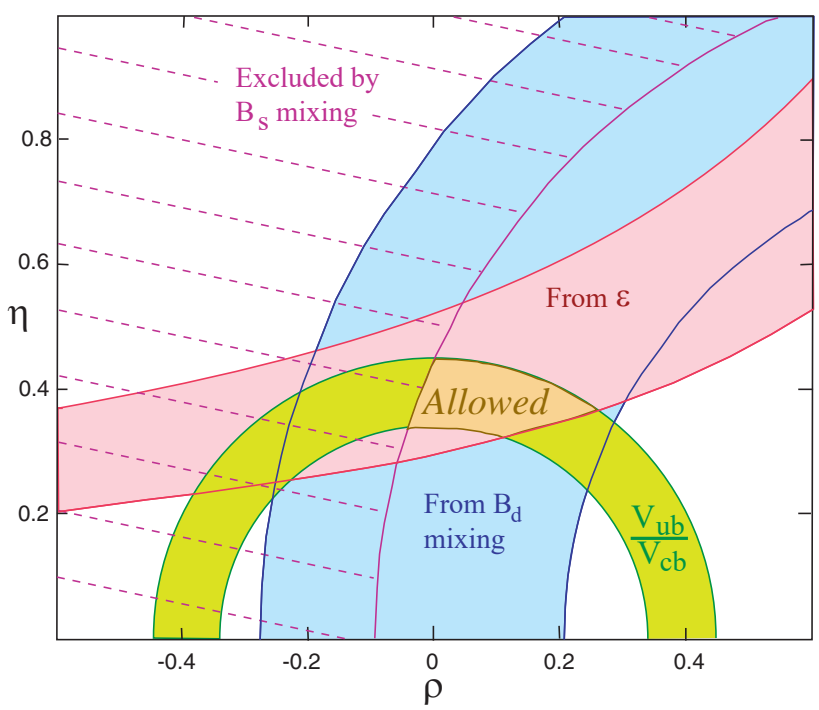

Figure 1.4: The regions in $\rho-\eta$ space (shaded) consistent with measurements of CP violation in $K_{L}^{o}$ decay $(\epsilon), V_{u b} / V_{c b}$ in semileptonic $B$ decay, $B_{d}^{o}$ mixing, and the excluded region from limits on $B_{s}^{o}$ mixing. The allowed region is defined by the overlap of the 3 permitted areas, and is where the apex of the CKM triangle sits.

This analysis is in good agreement with that of Rosner [11]. Recent measurements of $\epsilon^{\prime} / \epsilon$ determine $\eta$ directly [7]. However, the theoretical errors are so large that all that can be said is that the measurement is consistent with the allowed region. We caution the reader that this plot is only a guide, since the measured quantities all have large or even dominant errors due to theoretical models, and the bands are only $\pm 1 \sigma$ wide.

\subsection{CP Violation in Charged $B$ Decays}

The theoretical basis of the study of $\mathrm{CP}$ violation in $B$ decays was given in a series of papers by Carter and Sanda, and Bigi and Sanda [13]. We start with charged $B$ decays. Consider the final states $f^{ \pm}$which can be reached by two distinct weak processes with amplitudes $\mathcal{A}$ and $\mathcal{B}$, respectively.

$$
\mathcal{A}=a_{s} e^{i \theta_{s}} a_{w} e^{i \theta_{w}}, \quad \mathcal{B}=b_{s} e^{i \delta_{s}} b_{w} e^{i \delta_{w}} .
$$

The strong phases are denoted by the subscript $s$ and weak phases are denoted by the subscript $w$. Under the $\mathrm{CP}$ operation the strong phases are invariant but the weak phases change sign, so

$$
\overline{\mathcal{A}}=a_{s} e^{i \theta_{s}} a_{w} e^{-i \theta_{w}}, \overline{\mathcal{B}}=b_{s} e^{i \delta_{s}} b_{w} e^{-i \delta_{w}} .
$$




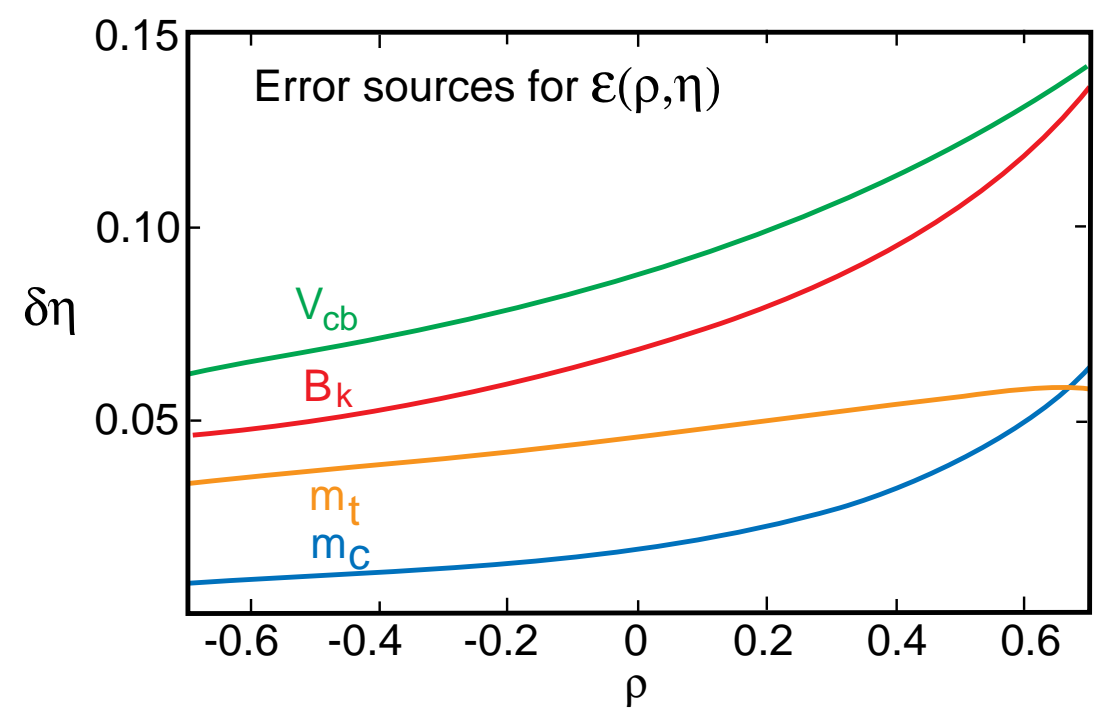

Figure 1.5: Error sources in units of $\delta \eta$ on the value of $\eta$ as a function of $\rho$ provided by the $\mathrm{CP}$ violation constraint in $K_{L}^{o}$ decay.

The rate difference is

$$
\begin{aligned}
\Gamma-\bar{\Gamma} & =|\mathcal{A}+\mathcal{B}|^{2}-|\overline{\mathcal{A}}+\overline{\mathcal{B}}|^{2} \\
& =2 a_{s} a_{w} b_{s} b_{w} \sin \left(\delta_{s}-\theta_{s}\right) \sin \left(\delta_{w}-\theta_{w}\right) .
\end{aligned}
$$

A weak phase difference is guaranteed in the appropriate decay mode (different CKM phases), but the strong phase difference is not; it is very difficult to predict the magnitude of strong phase differences.

As an example consider the possibility of observing $\mathrm{CP}$ violation by measuring a rate difference between $B^{-} \rightarrow K^{-} \pi^{o}$ and $B^{+} \rightarrow K^{+} \pi^{o}$. The $K^{-} \pi^{o}$ final state can be reached either by tree or penguin diagrams as shown in Fig. 1.6. The tree diagram has an imaginary part coming from the $V_{u b}$ coupling, while the penguin term does not, thus insuring a weak phase difference. This type of CP violation is called "direct." Note also that the process $B^{-} \rightarrow K^{o} \pi^{-}$can only be produced by the penguin diagram in Fig. 1.6(d). Therefore, in this simple example, we do not expect a rate difference between $B^{-} \rightarrow K^{o} \pi^{-}$and $B^{+} \rightarrow K^{o} \pi^{+}$. (There have been suggestions that rescattering effects may contribute here and produce a rate asymmetry, see section 1.9.)

\subsection{CP Violation Formalism in Neutral $B$ decays}

For neutral mesons we can construct the CP eigenstates

$$
\left|B_{1}^{o}\right\rangle=\frac{1}{\sqrt{2}}\left(\left|B^{o}\right\rangle+\left|\bar{B}^{o}\right\rangle\right)
$$



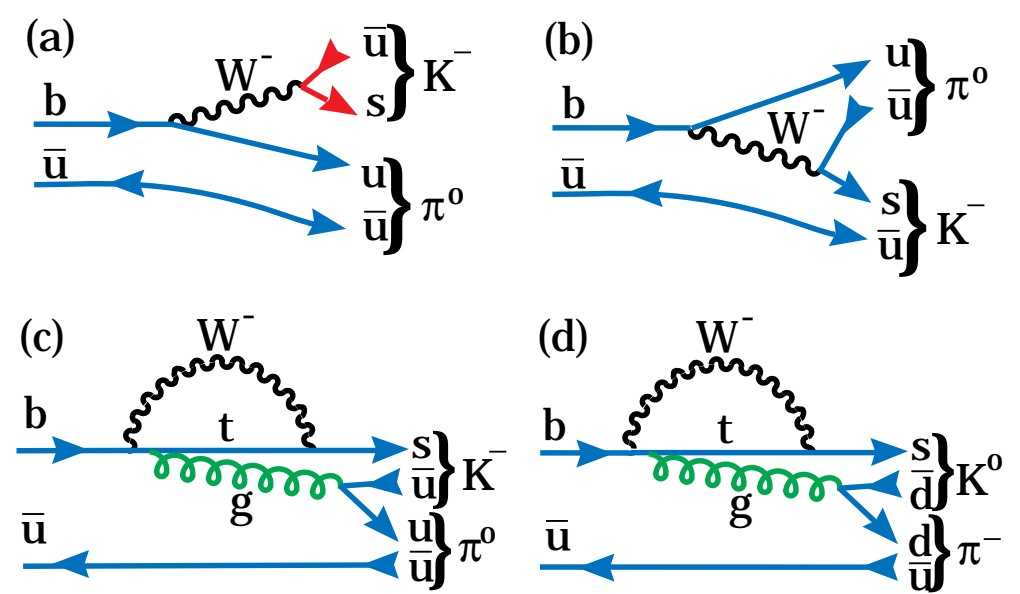

Figure 1.6: Diagrams for $B^{-} \rightarrow K^{-} \pi^{o}$, (a) and (b) are tree level diagrams where (b) is color suppressed; (c) is a penguin diagram. (d) shows $B^{-} \rightarrow K^{o} \pi^{-}$, which cannot be produced via a tree diagram.

$$
\left|B_{2}^{o}\right\rangle=\frac{1}{\sqrt{2}}\left(\left|B^{o}\right\rangle-\left|\bar{B}^{o}\right\rangle\right)
$$

where

$$
\begin{aligned}
& C P\left|B_{1}^{o}\right\rangle=\left|B_{1}^{o}\right\rangle, \\
& C P\left|B_{2}^{o}\right\rangle=-\left|B_{2}^{o}\right\rangle .
\end{aligned}
$$

Since $B^{o}$ and $\bar{B}^{o}$ can mix, the mass eigenstates are superpositions of $a\left|B^{o}\right\rangle+b\left|\bar{B}^{o}\right\rangle$ which obey the Schrodinger equation

$$
i \frac{d}{d t}\left(\begin{array}{c}
a \\
b
\end{array}\right)=H\left(\begin{array}{c}
a \\
b
\end{array}\right)=\left(M-\frac{i}{2} \Gamma\right)\left(\begin{array}{c}
a \\
b
\end{array}\right) .
$$

If $\mathrm{CP}$ is not conserved then the eigenvectors, the mass eigenstates $\left|B_{L}\right\rangle$ and $\left|B_{H}\right\rangle$, are not the CP eigenstates but are

$$
\left|B_{L}\right\rangle=p\left|B^{o}\right\rangle+q\left|\bar{B}^{o}\right\rangle, \quad\left|B_{H}\right\rangle=p\left|B^{o}\right\rangle-q\left|\bar{B}^{o}\right\rangle
$$

where

$$
p=\frac{1}{\sqrt{2}} \frac{1+\epsilon_{B}}{\sqrt{1+\left|\epsilon_{B}\right|^{2}}}, \quad q=\frac{1}{\sqrt{2}} \frac{1-\epsilon_{B}}{\sqrt{1+\left|\epsilon_{B}\right|^{2}}} .
$$

$\mathrm{CP}$ is violated if $\epsilon_{B} \neq 0$, which occurs if $|q / p| \neq 1$.

The time dependence of the mass eigenstates is

$$
\begin{aligned}
\left|B_{L}(t)\right\rangle & =e^{-\Gamma_{L} t / 2} e^{-i m_{L} t / 2}\left|B_{L}(0)\right\rangle \\
\left|B_{H}(t)\right\rangle & =e^{-\Gamma_{H} t / 2} e^{-i m_{H} t / 2}\left|B_{H}(0)\right\rangle,
\end{aligned}
$$


leading to the time evolution of the flavor eigenstates as

$$
\begin{aligned}
& \left|B^{o}(t)\right\rangle=e^{-\left(i m+\frac{\Gamma}{2}\right) t}\left(\cos \frac{\Delta m t}{2}\left|B^{o}(0)\right\rangle+i \frac{q}{p} \sin \frac{\Delta m t}{2}\left|\bar{B}^{o}(0)\right\rangle\right) \\
& \left|\bar{B}^{o}(t)\right\rangle=e^{-\left(i m+\frac{\Gamma}{2}\right) t}\left(i \frac{p}{q} \sin \frac{\Delta m t}{2}\left|B^{o}(0)\right\rangle+\cos \frac{\Delta m t}{2}\left|\bar{B}^{o}(0)\right\rangle\right)
\end{aligned}
$$

where $m=\left(m_{L}+m_{H}\right) / 2, \Delta m=m_{H}-m_{L}$ and $\Gamma=\Gamma_{L} \approx \Gamma_{H}$. Note, that the probability of a $B^{o}$ decay as a function of $t$ is given by $\left\langle B^{o}(t) \mid B^{o}(t)\right\rangle^{*}$, and is a pure exponential, $e^{-\Gamma t}$, in the absence of $\mathrm{CP}$ violation.

Indirect $C P$ violation in the neutral $B$ system

As in the case of $K_{L}$ decay, we can look for the rate asymmetry

$$
\begin{aligned}
a_{s l} & =\frac{\Gamma\left(\bar{B}^{o}(t) \rightarrow X \ell^{+} \nu\right)-\Gamma\left(B^{o}(t) \rightarrow X \ell^{-} \bar{\nu}\right)}{\Gamma\left(\bar{B}^{o}(t) \rightarrow X \ell^{+} \nu\right)+\Gamma\left(B^{o}(t) \rightarrow X \ell^{-} \bar{\nu}\right)} \\
& =\frac{1-\left|\frac{q}{p}\right|^{4}}{1+\left|\frac{q}{p}\right|^{4}} \approx O\left(10^{-2}\right) .
\end{aligned}
$$

These final states occur only through mixing as the direct decay occurs only as $B^{o} \rightarrow X \ell^{+} \nu$. To generate $\mathrm{CP}$ violation we need an interference between two diagrams. In this case the two diagrams are the mixing diagram with the $t$-quark and the mixing diagram with the $c$-quark. This is identical to what happens in the $K_{L}^{o}$ case. This type of CP violation is called "indirect." The small size of the expected asymmetry is caused by the off-diagonal elements of the $\Gamma$ matrix in equation (1.20) being very small compared to the off-diagonal elements of the mass matrix, i.e. $\left|\Gamma_{12} / M_{12}\right|<<1$ and $\operatorname{Im}\left(\Gamma_{12} / M_{12}\right) \neq 0$. This results from the nearly equal widths of the $B_{L}^{o}$ and $B_{H}^{o}[14]$. In the case of the $B_{s}^{o}$ there may be a significant enough difference between the widths of the light and heavy eigenstates that these equations would have to be modified [15].

$C P$ violation for $B$ via interference of mixing and decays

Here we choose a final state $f$ which is accessible to both $B^{o}$ and $\bar{B}^{o}$ decays. The second amplitude necessary for interference is provided by mixing. Fig. 1.7 shows the decay into $f$ either directly or indirectly via mixing. It is necessary only that $f$ be accessible from either state. However if $f$ is a CP eigenstate the situation is far simpler. For CP eigenstates

$$
C P\left|f_{C P}\right\rangle= \pm\left|f_{C P}\right\rangle
$$

It is useful to define the amplitudes

$$
A=\left\langle f_{C P}|\mathcal{H}| B^{o}\right\rangle, \quad \bar{A}=\left\langle f_{C P}|\mathcal{H}| \bar{B}^{o}\right\rangle .
$$




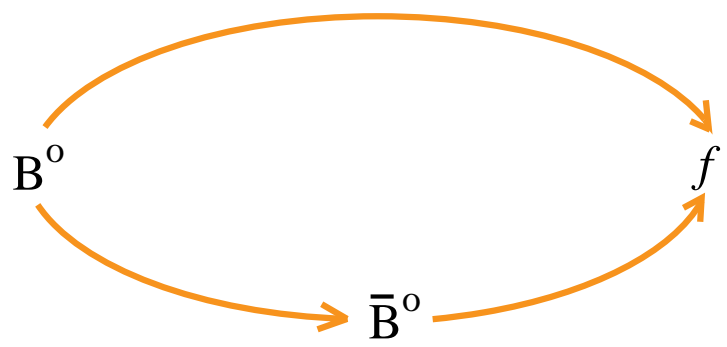

Figure 1.7: Two interfering ways for a $B^{o}$ to decay into a final state $f$.

If $\left|\frac{\bar{A}}{A}\right| \neq 1$, then we have "direct" CP violation in the decay amplitude, which was discussed above. Here CP can be violated by having

$$
\lambda=\frac{q}{p} \cdot \frac{\bar{A}}{A} \neq 1
$$

which requires only that $\lambda$ acquire a non-zero phase, i.e. $|\lambda|$ could be unity and $\mathrm{CP}$ violation can occur.

The asymmetry, in this case, is defined as

$$
a_{f_{C P}}=\frac{\Gamma\left(B^{o}(t) \rightarrow f_{C P}\right)-\Gamma\left(\bar{B}^{o}(t) \rightarrow f_{C P}\right)}{\Gamma\left(B^{o}(t) \rightarrow f_{C P}\right)+\Gamma\left(\bar{B}^{o}(t) \rightarrow f_{C P}\right)},
$$

which for $|q / p|=1$ gives

$$
a_{f_{C P}}=\frac{\left(1-|\lambda|^{2}\right) \cos (\Delta m t)-2 \operatorname{Im} \lambda \sin (\Delta m t)}{1+|\lambda|^{2}} .
$$

For the cases where there is only one decay amplitude $A,|\lambda|$ equals 1 , and we have

$$
a_{f_{C P}}=-\operatorname{Im} \lambda \sin (\Delta m t)
$$

Only the amplitude, $-\operatorname{Im} \lambda$ contains information about the level of $\mathrm{CP}$ violation, the sine term is determined only by $B^{o}$ mixing. In fact, the time integrated asymmetry is given by

$$
a_{f_{C P}}=-\frac{x}{1+x^{2}} \operatorname{Im} \lambda
$$

where $x=\frac{\Delta m}{\Gamma}$. For the case of the $B_{d}^{o} x /\left(1+x^{2}\right)=0.48$, which is quite lucky as the maximum size of the coefficient is -0.5 .

$\operatorname{Im} \lambda$ is related to the CKM parameters. Recall $\lambda=\frac{q}{p} \cdot \frac{\bar{A}}{A}$. The first term is the part that comes from mixing:

$$
\frac{q}{p}=\frac{\left(V_{t b}^{*} V_{t d}\right)^{2}}{\left|V_{t b} V_{t d}\right|^{2}}=\frac{(1-\rho-i \eta)^{2}}{(1-\rho+i \eta)(1-\rho-i \eta)}=e^{-2 i \beta} \text { and }
$$




$$
\operatorname{Im} \frac{q}{p}=-\frac{2(1-\rho) \eta}{(1-\rho)^{2}+\eta^{2}}=\sin (2 \beta) .
$$

To evaluate the decay part we need to consider specific final states. For example, consider $f \equiv \pi^{+} \pi^{-}$. The simple spectator decay diagram is shown in Fig. 1.8. For the moment we

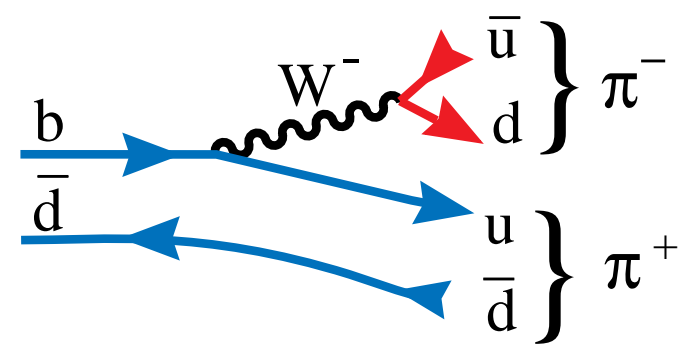

Figure 1.8: Decay diagram at the tree level for $B^{o} \rightarrow \pi^{+} \pi^{-}$.

will assume that this is the only diagram which contributes. Later we will show why this is not true. For this $b \rightarrow u \bar{u} d$ process we have

$$
\frac{\bar{A}}{A}=\frac{\left(V_{u d}^{*} V_{u b}\right)^{2}}{\left|V_{u d} V_{u b}\right|^{2}}=\frac{(\rho-i \eta)^{2}}{(\rho-i \eta)(\rho+i \eta)}=e^{-2 i \gamma},
$$

and

$$
\operatorname{Im}(\lambda)=\operatorname{Im}\left(e^{-2 i \beta} e^{-2 i \gamma}\right)=\operatorname{Im}\left(e^{2 i \alpha}\right)=\sin (2 \alpha) .
$$

The final state $J / \psi K_{s}$ plays an especially important role in the study of CP violation. It is a CP eigenstate and its decay is dominated by only one diagram, shown in Fig. 1.9. In this case we do not get a phase from the decay part because

$$
\frac{\bar{A}}{A}=\frac{\left(V_{c b} V_{c s}^{*}\right)^{2}}{\left|V_{c b} V_{c s}\right|^{2}}
$$

is real. In this case the final state is a state of negative $C P$, i.e. $C P\left|J / \psi K_{s}\right\rangle=-\left|J / \psi K_{s}\right\rangle$. This introduces an additional minus sign in the result for $\operatorname{Im} \lambda$. Before finishing discussion of this final state we need to consider in more detail the presence of the $K_{S}$ in the final state. Since neutral kaons can mix, we pick up another mixing phase. This term creates a phase given by

$$
\left(\frac{q}{p}\right)_{K}=\frac{\left(V_{c d}^{*} V_{c s}\right)^{2}}{\left|V_{c d} V_{c s}\right|^{2}}
$$

which is zero. It is necessary to include this term, however, since there are other formulations of the CKM matrix than Wolfenstein, which have the phase in a different location. It is important that the physics predictions not depend on the CKM convention. ${ }^{2}$

In summary, for the case of $f=J / \psi K_{s}, \operatorname{Im} \lambda=-\sin (2 \beta)$.

\footnotetext{
${ }^{2}$ Here we don't include CP violation in the neutral kaon since it is much smaller than what is expected in the $B$ decay.
} 


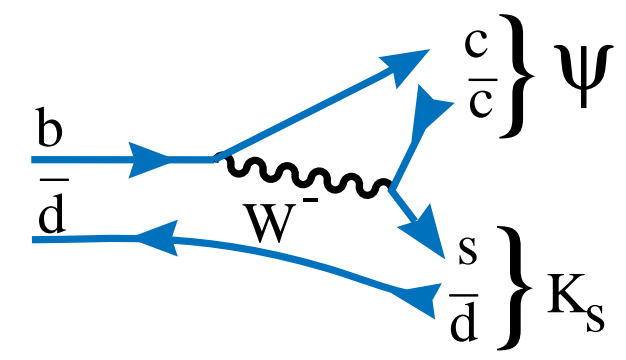

Figure 1.9: Decay diagram at the tree level for $B^{o} \rightarrow J / \psi K_{S}$.

\subsection{Comment on Penguin Amplitude}

Many processes can have penguin components. The diagram for $B^{o} \rightarrow \pi^{+} \pi^{-}$is shown in Fig. 1.10. The $\pi^{+} \pi^{-}$final state is expected to have a rather large penguin amplitude $\sim 20 \%$ of the tree amplitude. Then $|\lambda| \neq 1$ and $a_{\pi \pi}(t)$ develops a $\cos (\Delta m t)$ term. It turns out (see Gronau [16]), that $\sin (2 \alpha)$ can be extracted using isospin considerations and measurements of the branching ratios for $B^{+} \rightarrow \pi^{+} \pi^{o}$ and $B^{o} \rightarrow \pi^{o} \pi^{o}$. This method, though difficult, perhaps impossible, to apply, has almost no model dependence [19].

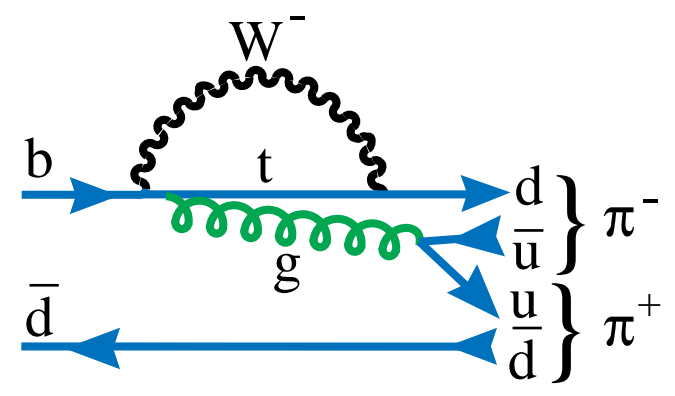

Figure 1.10: Penguin diagram for $B^{o} \rightarrow \pi^{+} \pi^{-}$.

In the $J / \psi K_{s}$ case, the penguin amplitude is expected to be small since a $c \bar{c}$ pair must be "popped" from the vacuum. Even if the penguin decay amplitude were of significant size, the decay phase, $\operatorname{Im}(\bar{A} / A)$ is the same as the tree level process, and quite small.

\subsection{Asymmetry Measurements}

In charged $B$ decays we only have to measure a branching ratio difference between $B^{+}$and $B^{-}$to see CP violation. For neutral $B$ decays we must find the flavor of the other $b$-quark produced in the event (this is called tagging), since we do not have any $B^{o}$ beams. We then 
measure a rate asymmetry that for $\mathrm{CP}$ eigenstates is given by

$$
a_{a s y}=\frac{\#\left(f, \ell^{+}\right)-\#\left(f, \ell^{-}\right)}{\#\left(f, \ell^{+}\right)+\#\left(f, \ell^{-}\right)}
$$

where $\ell^{ \pm}$indicates the charge of the lepton from the "other" $b$ and thus provides a flavor tag. In Fig. 1.11(a) the time dependence for the $B^{o}$ and $\bar{B}^{o}$ are shown as a function of $t$ in the $B$ rest frame for 500 simulated experiments of an average of 2000 events each with an input asymmetry of 0.3 . In Fig. 1.11(b) the fitted asymmetry is shown for 500 different "experiments."

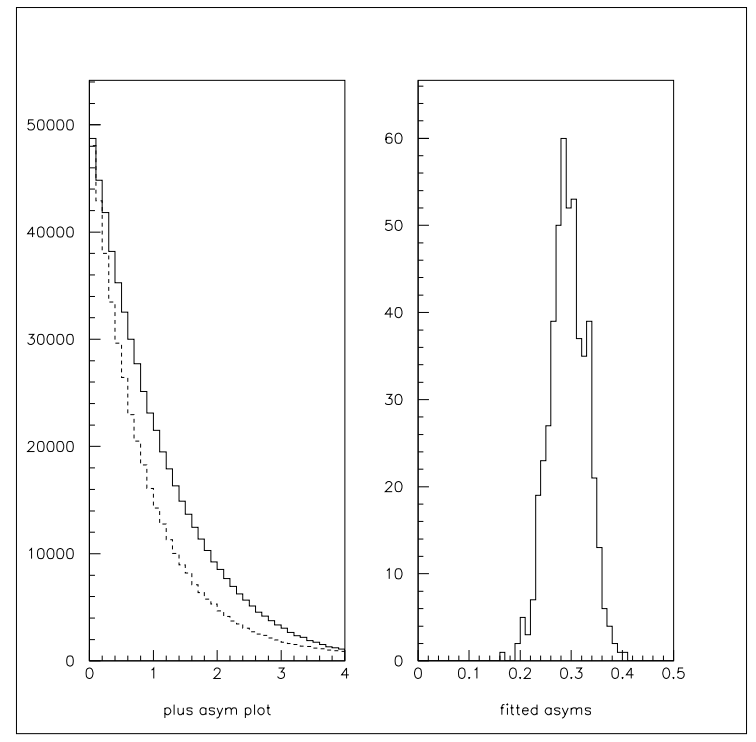

Figure 1.11: (a) Time dependence of $B^{o}$ and $\bar{B}^{o}$ decaying into a CP eigenstate, for an asymmetry of 0.3 for a total of 1 million events. The x-axis is proper time. In (b) the fitted asymmetry results are shown for 500 "experiments" of average of 2000 events each.

In $B_{s}^{o}$ decays the oscillations are far faster so the asymmetry must be measured as a function of time.

\subsection{Techniques for Determining $\beta$}

The decay $B^{o} \rightarrow J / \psi K_{s}$ is the primary source for measurements of $\sin (2 \beta)$. In the common phase convention, $\mathrm{CP}$ violation is expected to arise mostly from the mixing, driven by $\operatorname{Im}(q / p)$, while the decay amplitude, $\operatorname{Im}(\bar{A} / A)$, is expected to contribute only a small part (see Fig. 1.9). This decay is expected to have only a small Penguin contribution, but even if the Penguin contribution is significant it does not change the contribution of the mixing phase or the decay phase. ${ }^{3}$

\footnotetext{
${ }^{3}$ Actually the only phase that has physical meaning is the product of $q / p \cdot \bar{A} / A$.
} 
While we expect that $\sin (2 \beta)$ will have been measured before $\mathrm{BTeV}$, we do aim to improve significantly on the precision of the measurement. Furthermore, we intend to be able to remove "ambiguities." When we measure $\sin (2 \phi)$, where $\phi$ is any angle we have a four-fold ambiguity in $\phi$, namely $\phi, \pi / 2-\phi, \phi+\pi$ and $3 \pi / 2-\phi$. These ambiguities can mask the effects of new physics. Our task is to remove as many of the ambiguities as possible.

\subsubsection{Removal of two of the $\beta$ Ambguities}

The decay $B \rightarrow J / \psi K^{*}$, where $K^{*} \rightarrow K_{s} \pi^{o}$ can be used to get information about the sign of $\cos (2 \beta)$, which would remove two of the ambiguities [20]. This decay is described by three complex decay amplitudes. Following a suggestion of Dighe, Dunietz, and Fleischer [21, 22], we write the decay amplitudes $A_{0}=-\sqrt{1 / 3} S+\sqrt{2 / 3} D, A_{\|}=\sqrt{2 / 3} S+\sqrt{1 / 3} D$, and $A_{\perp}=P$, where $S, P$, and $D$ denote $\mathrm{S}, \mathrm{P}$, and $\mathrm{D}$ wave amplitudes, respectively. Normalizing the decay amplitudes to $\left|A_{0}\right|^{2}+\left|A_{\|}\right|^{2}+\left|A_{\perp}\right|^{2}=1$ and eliminating one overall phase leaves four independent parameters.

The full angular distribution of a $B$ meson decaying into two vector particles is specified by three angles. The helicity angle basis [23] has been used for angular analyses of $B \rightarrow J / \psi K^{*}$ decays. An alternative basis, called the transversity basis is more suitable for extracting parity information [22].

In this basis, the direction of the $K^{*}$ in the $J / \psi$ rest frame defines the x-axis of a righthanded coordinate system. The $K \pi$ plane fixes the y-axis with $p_{y}(K)>0$ and the normal to this plane defines the z-axis. The transversity angles $\theta_{\mathrm{tr}}$ and $\phi_{\mathrm{tr}}$ are then defined as polar and azimuth angles of the $l^{+}$in the $J / \psi$ rest frame. The third angle, the $K^{*}$ decay angle $\theta_{K^{*}}$, is defined as that of the $K$ in the $K^{*}$ rest frame relative to the negative of the $J / \psi$ direction in that frame. Using these definitions the full angular distribution of the $B \rightarrow J / \psi K^{*}$ decay is $[22]$ :

$$
\begin{aligned}
& \frac{1}{\Gamma} \frac{\mathrm{d}^{3} \Gamma}{\mathrm{d} \cos \theta_{\mathrm{tr}} \mathrm{d} \cos \theta_{K^{*}} \mathrm{~d} \phi_{\mathrm{tr}}} \\
& =\frac{9}{32 \pi}\left\{2\left|A_{0}\right|^{2} \cos ^{2} \theta_{K^{*}}\left(1-\sin ^{2} \theta_{\mathrm{tr}} \cos ^{2} \phi_{\mathrm{tr}}\right)\right. \\
& +\left|A_{\|}\right|^{2} \sin ^{2} \theta_{K^{*}}\left(1-\sin ^{2} \theta_{\mathrm{tr}} \sin ^{2} \phi_{\mathrm{tr}}\right) \\
& +\left|A_{\perp}\right|^{2} \sin ^{2} \theta_{K^{*}} \sin ^{2} \theta_{\mathrm{tr}} \sin ^{2} \phi_{\mathrm{tr}} \\
& -\operatorname{Im}\left(A_{\|}^{*} A_{\perp}\right) \sin ^{2} \theta_{K^{*}} \sin 2 \theta_{\mathrm{tr}} \sin \phi_{\mathrm{tr}} \\
& +\frac{1}{\sqrt{2}} \operatorname{Re}\left(A_{0}^{*} A_{\|}\right) \sin 2 \theta_{K^{*}} \sin ^{2} \theta_{\mathrm{tr}} \sin 2 \phi_{\mathrm{tr}} \\
& \left.+\frac{1}{\sqrt{2}} \operatorname{Im}\left(A_{0}^{*} A_{\perp}\right) \sin 2 \theta_{K^{*}} \sin 2 \theta_{\mathrm{tr}} \cos \phi_{\mathrm{tr}}\right\} \text {. }
\end{aligned}
$$

For $\bar{B}$ decays the interference terms containing $A_{\perp}$ switch sign while all other terms remain unchanged.

Results shown in Table 1.1 have been obtained from CLEO and CDF using the decay $\bar{K}^{* o} \rightarrow K^{-} \pi^{+}$. 


\begin{tabular}{ccc}
\hline \hline Parameter & CLEO [17] & CDF [18] \\
\hline$\left|A_{0}\right|^{2}=\Gamma_{\mathrm{L}} / \Gamma$ & $0.52 \pm 0.07 \pm 0.04$ & $0.59 \pm 0.06 \pm 0.02$ \\
$\left|A_{\perp}\right|^{2}=|P|^{2}$ & $0.16 \pm 0.08 \pm 0.04$ & $0.13_{-0.06}^{+0.12} \pm 0.03$ \\
\hline \hline
\end{tabular}

Table 1.1: Resulting decay amplitudes from the fit to the transversity angles. The first error is statistical and the second is the estimated systematic uncertainty.

The parity odd component is three standard deviations from zero in the average of the two experiments, and is $\approx 25 \%$ the rate of the parity even component. This is likely large enough to allow the determination of the sign of the interference terms in using the tagged $K^{* o} \rightarrow K_{s} \pi^{o}$ decays; that, in turn, allows a determination of the sign of the product of $\cos (2 \beta)$ with a strong phase-shift. The sign of this phase-shift can either be obtained from factorization, which is a dangerous procedure, or using the much weaker assumption of $\mathrm{SU}(3)$ symmetry, and analyzing the time-dependent oscillations in the decay $B_{s} \rightarrow J / \psi \phi$ [20], where the mixing phase is expected to be small.

Another independent method of removing two of the ambiguities is to measure the sign of the $\cos (2 \beta)$ term in the decay $B^{o} \rightarrow J / \psi K^{o}, K^{o} \rightarrow \pi^{ \pm} \ell^{\mp} \nu$. This idea developed by Kayser [24], works because of the interference between $K_{L}$ and $K_{s}$ in the decay, where the decay amplitudes are equal. The time evolution of the decay width can be expressed in terms of the $B^{o}$ decay time $\left(t_{B}\right)$ and the $K^{o}$ decay time $\left(t_{K}\right)$ as

$$
\begin{aligned}
& \Gamma\left(t_{B}, t_{K}\right) \propto \\
& e^{-\Gamma_{B} t_{B}}\left\{e^{-\gamma_{s} t_{K}}\left[1 \mp \sin (2 \beta) \sin \left(\Delta m_{B} t_{B}\right)\right]\right. \\
& +e^{-\gamma_{L} t_{K}}\left[1 \pm \sin (2 \beta) \sin \left(\Delta m_{B} t_{B}\right)\right] \\
& \pm(\mp) 2 e^{-\frac{1}{2}\left(\gamma_{s}+\gamma_{L}\right) t_{K}}\left[\cos \left(\Delta m_{B} t_{B}\right) \cos \left(\Delta m_{K} t_{K}\right)\right. \\
& \left.\left.+\cos (2 \beta) \sin \left(\Delta m_{B} t_{B}\right) \sin \left(\Delta m_{K} t_{K}\right)\right]\right\},
\end{aligned}
$$

where the top sign of each pair is for $B^{o}$, and the bottom for $\bar{B}^{o}$. The first pair of signs in the third line refers to the kaon decay mode $\pi^{-} \ell^{+} \nu(K)$, while the second pair is for $\pi^{+} \ell^{-} \bar{\nu}$ $(\bar{K})$.

To get an idea of the predicted asymmetries, we integrate this equation over $t_{B}$. There are four different rates that can be denoted as combinations of $B$ and $\bar{B}$ with $K$ and $\bar{K}$. In Fig. 1.12 we show the four rates as solid lines if $\cos (2 \beta)$ were positive and the four rates as dashed lines if $\cos (2 \beta)$ were negative. These were done for $\sin (2 \beta)=0.7$. If $\sin (2 \beta)$ were smaller the rate differences would be larger and vice-versa.

The differences are large over about five $K_{s}$ lifetimes. Since only the sign of the $\cos (2 \beta)$ term needs to be found, all other parameters, including $\sin (2 \beta)$ are specified. Unfortunately, the event rate is rather small, since $\mathcal{B}\left(K_{s} \rightarrow \pi \ell \nu\right)=1.4 \times 10^{-3}$ and although $\mathcal{B}\left(K_{L} \rightarrow \pi \ell \nu\right)=$ 


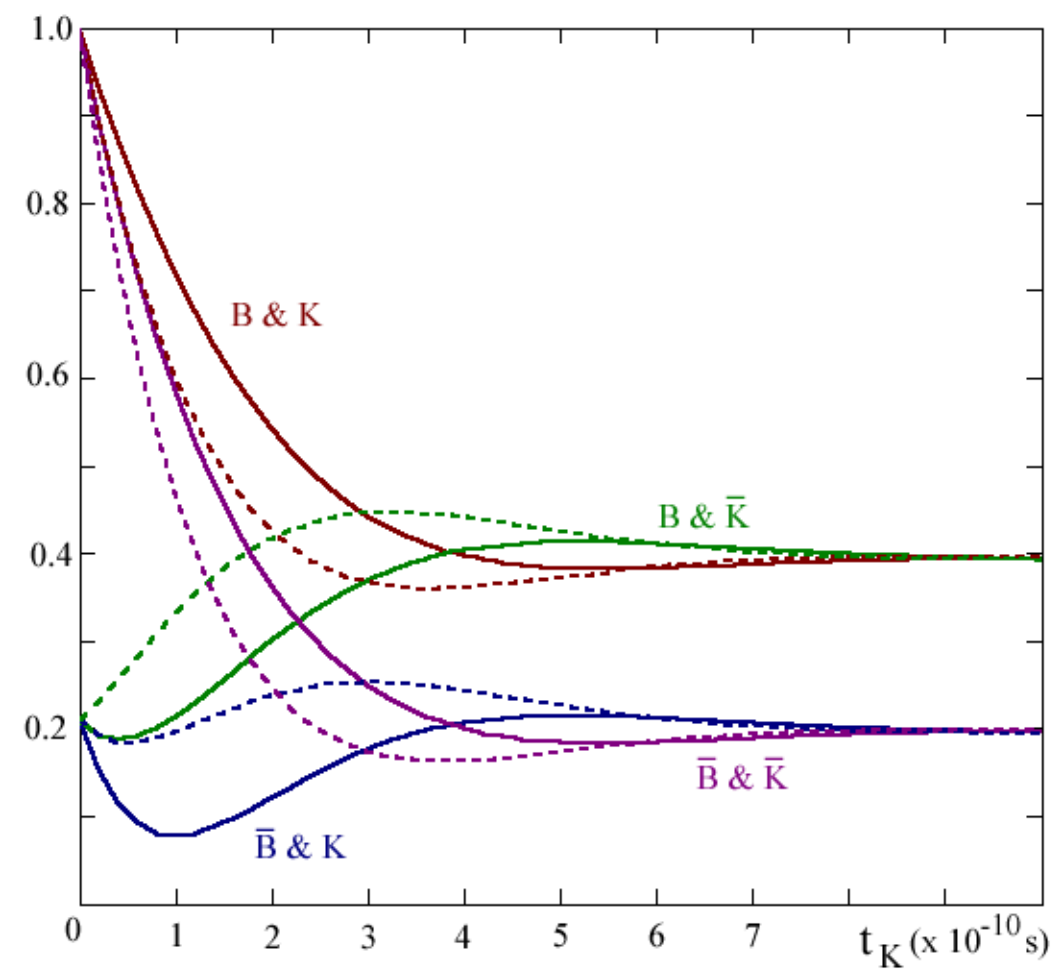

Figure 1.12: The decay rates for $B^{o} \rightarrow J / \psi K^{o}, K^{o} \rightarrow \pi \ell \nu$, as a function of $K^{o}$ decay time, integrated over the $B^{o}$ decay time. The solid lines have the sign of the $\cos (2 \beta)$ term as positive, while the corresponding dashed lines have negative values. The absolute normalization is arbitrary, and $\sin 2(\beta)$ was fixed at 0.7 .

0.66 , only $1 \%$ of the $K_{L}$ decay soon enough to be of use. Roughly, we have about 100 times fewer events than in $J / \psi K_{s}$. However, if the backgrounds are not too large, it will only take on the order of a hundred events to successfully determine the sign of $\cos (2 \beta)$ using this technique.

It is interesting to note that measuring this combination $B^{o}$ and $K^{o}$ decay modes can lead to measurements of CPT violation [25].

\subsection{Techniques for Determining $\alpha$}

Measuring $\alpha$ is more difficult than measuring $\beta$ in several respects. First of all, the decay amplitudes are modulated by $V_{u b}$ rather than $V_{c b}$, making the overall rates small. Secondly, the gluonic Penguin rates are of the same order causing well known difficulties in extracting the weak phase angle (see section 1.5 above). The Penguin diagrams add a third amplitude to the tree level and mixing amplitudes. It turns out, however, that this complication can be a blessing in disguise. The interference generates $\cos (2 \alpha)$ terms in the decay rate, that 
can be used to remove discrete ambiguities.

The decay $B^{o} \rightarrow \pi^{+} \pi^{-}$has oft been cited as a way to measure $\sin (2 \alpha)$. However, the Penguin pollution mentioned before, makes it difficult to extract the angle. Current data from CLEO gives $\mathcal{B}\left(B^{o} \rightarrow K^{\mp} \pi^{ \pm}\right)=(1.5 \pm 0.5 \pm 0.4) \times 10^{-5}$ and a $90 \%$ confidence upper limit on $\mathcal{B}\left(B^{o} \rightarrow \pi^{+} \pi^{-}\right)<0.84 \times 10^{-5}$ [34], showing a relatively large Penguin amplitude which cannot be ignored. Gronau and London [26] have shown that an isospin analysis using the additional decays $B^{-} \rightarrow \pi^{-} \pi^{o}$ and $B^{o} \rightarrow \pi^{o} \pi^{o}$ can be used to extract $\alpha$, but the $\pi^{o} \pi^{o}$ final state is extremely difficult to detect in any existing or proposed experiment. Other authors have suggested different methods, but they all have theoretical assumptions [27]. Thus, measurement of the CP asymmetry in $B^{o} \rightarrow \pi^{+} \pi^{-}$cannot, in our view, provide an accurate determination of $\sin (2 \alpha)$ unless some new breakthrough in theory occurs.

There is however, a theoretically clean method to determine $\alpha$. The interference between Tree and Penguin diagrams can be exploited by measuring the time dependent CP violating effects in the decays $B^{o} \rightarrow \rho \pi$ as shown by Snyder and Quinn [28]. There are three such neutral decay modes, listed in Table 1.2 with their respective Penguin and Tree amplitudes, denoted by $T^{i j}$, where $i$ lists charge of the $\rho$ and $j$ the charge of the $\pi$. For the $\rho^{o} \pi^{o}$ mode, isospin constraints are used to eliminate $T^{o o}$. The amplitudes for the charged decays are also given.

Table 1.2: $B^{o} \rightarrow \rho \pi$ Decay Modes

\begin{tabular}{ll}
\hline \hline & \\
Decay Model & Decay Amplitudes \\
\hline$\sqrt{2} A\left(B^{+} \rightarrow \rho^{+} \pi^{o}\right)$ & $=S_{1}=T^{+o}+2 P_{1}$ \\
$\sqrt{2} A\left(B^{+} \rightarrow \rho^{o} \pi^{+}\right)$ & $=S_{2}=T^{o+}-2 P_{1}$ \\
$A\left(B^{o} \rightarrow \rho^{+} \pi^{-}\right)$ & $=S_{3}=T^{+-}+P_{1}+P_{o}$ \\
$A\left(B^{o} \rightarrow \rho^{-} \pi^{+}\right)$ & $=S_{4}=T^{-+}-P_{1}+P_{o}$ \\
$2 A\left(B^{o} \rightarrow \rho^{o} \pi^{o}\right)$ & $=S_{5}=T^{+-}+T^{+-}-T^{+o}-T^{o+}-2 P_{o}$ \\
\hline \hline
\end{tabular}

For the $\rho \pi$ final state, the $\rho$ decay amplitude can be parameterized as

$$
f(m, \theta)=\frac{\cos (\theta) \Gamma_{\rho}}{2\left(m_{\rho}-m-i 0.5 \Gamma_{\rho}\right)},
$$

where $m_{\rho}$ is the $\rho$ mass of $0.77 \mathrm{GeV}$ and $\Gamma_{\rho}$, the width of $0.15 \mathrm{GeV}$. $\theta$ is the helicity decay angle and the $\cos (\theta)$ dependence arises because the $\rho$ must be fully polarized in this decay which starts with a spin-0 $B$ and ends up with a spin-1 $\rho$ and spin-0 $\pi$.

The full decay amplitude for $B^{o} \rightarrow \rho \pi \rightarrow \pi^{+} \pi^{-} \pi^{o}$ (and the corresponding $\bar{B}^{o}$ ) are given by

$$
\begin{aligned}
& A\left(B^{o}\right)=f^{+} S_{3}+f^{-} S_{4}+f^{o} S_{5} / 2 \\
& A\left(\bar{B}^{o}\right)=f^{+} \bar{S}_{3}+f^{-} \bar{S}_{4}+f^{o} \bar{S}_{5} / 2
\end{aligned}
$$


where the superscript on the $f$ indicates the charge of the $\rho$. The sum over the three neutral $B$ decay amplitudes involves only tree amplitudes; the Penguins vanish. The angle between this sum for $B^{o}$ decays $(\equiv T)$ and the sum for $\bar{B}^{o}(\equiv \bar{T})$ is precisely $\alpha$. Computing the amplitudes gives a series of terms which have both $\sin (\Delta m t)$ and $\cos (\Delta m t)$ time dependences and coefficients which depend on both $\sin (2 \alpha)$ and $\cos (2 \alpha)$.

To extract $\alpha$ only the neutral modes need be measured. Further constraints and information about Penguin phases can be extracted if the charged $B^{\prime}$ 's are also measured. But this is difficult because there are two $\pi^{o}$ 's in the $\rho^{+} \pi^{o}$ decay mode.

The $\rho \pi$ final state has many advantages. First of all it exists, the branching ratio for the $\rho^{o} \pi^{+}$final state was measured by CLEO as $(1.5 \pm 0.5 \pm 0.4) \times 10^{-5}[29]$. The rates for the neutral $B$ final state are expected to be similar, if not larger [30]. Secondly, since the $\rho$ is spin-1, the $\pi$ spin-0 and the initial $B$ also spinless, the $\rho$ is fully polarized in the $(1,0)$ configuration, so it decays as $\cos ^{2} \theta$, where $\theta$ is the angle of one of the $\rho$ decay products with the $\pi$ in the $\rho$ rest frame. This causes the periphery of the Dalitz plot to be heavily populated, especially the corners. A sample Dalitz plot is shown in Fig. 1.13. This kind of distribution is good for maximizing the interferences, which helps minimize the error. Furthermore, little information is lost by excluding the Dalitz plot interior, a good way to reduce backgrounds.

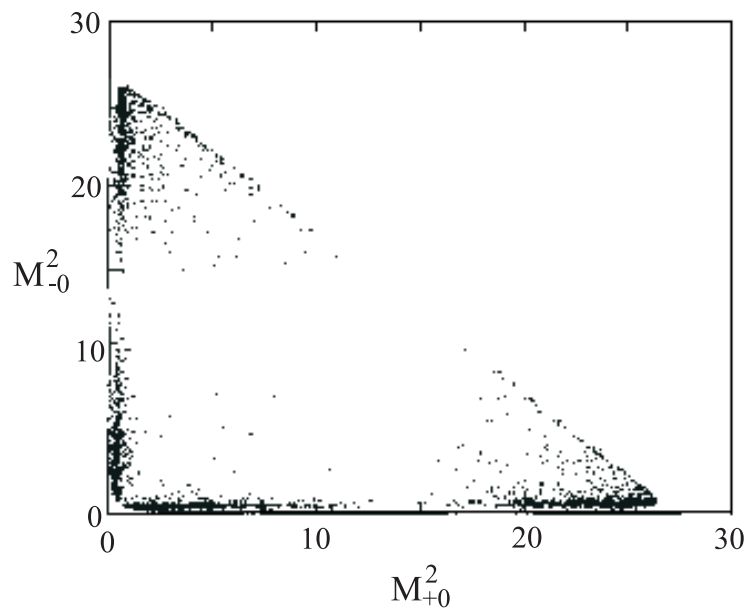

Figure 1.13: The Dalitz plot for $B^{o} \rightarrow \rho \pi \rightarrow \pi^{+} \pi^{-} \pi^{o}$ from Snyder and Quinn.

While we have not done our own Dalitz plot analysis yet, we refer to Snyder and Quinn who have. Their idealized analysis uses background free 1000 or 2000 flavor tagged event samples. The 1000 event sample usually yields good results for $\alpha$, but sometimes does not resolve the ambiguity. With the 2000 event sample, however, they always succeed.

The decay $B^{o} \rightarrow \pi^{+} \pi^{-}$can be used with some theoretical input to resolve the remaining ambiguity in $\sin (2 \alpha)$. The difference in CP asymmetries between $\pi \pi$ and $\rho \pi$ is given by

$$
a(\pi \pi)-a(\rho \pi)=-2\left(A_{P} / A_{T}\right) \cos \left(\delta_{P}-\delta_{T}\right)[\cos (2 \alpha) \sin (\alpha)]
$$


where $A_{P}$ and $A_{T}$, denote the Penguin and Tree amplitudes, respectively, and the $\delta$ 's represent their strong phase shifts. Factorization, can be used to get the sign of $A_{P} / A_{T}$ and the strong phase shifts are believed to be small enough that $\cos \left(\delta_{P}-\delta_{T}\right)$ is positive [59].

\subsection{Techniques for Determining $\gamma$}

The angle $\gamma$ could in principle be measured using a $\mathrm{CP}$ eigenstate of $B_{s}$ decay that was dominated by the $b \rightarrow u$ transition. One such decay that has been suggested is $B_{s} \rightarrow \rho^{o} K_{s}$. However, there are the same "Penguin pollution" problems as in $B^{\circ} \rightarrow \pi^{+} \pi^{-}$, but they are more difficult to resolve in the vector-pseudoscalar final state. (Note, the pseudoscalarpseudoscalar final state here is $\pi^{o} K_{s}$, which does not have a measurable decay vertex.)

Fortunately, there are other ways of measuring $\gamma$. CP eigenstates are not used, which introduces discrete ambiguities. However, combining several methods should remove these. We have studied three methods of measuring $\gamma$.

\subsubsection{Measurement of $\gamma$ Using Time-Dependent CP violation in $B_{s}$ Decays}

The first method uses the decays $B_{s} \rightarrow D_{s}^{ \pm} K^{\mp}$ where a time-dependent CP violation can result from the interference between the direct decay and the mixing induced decay [31]. Fig. 1.14 shows the two direct decay processes for $\bar{B}_{s}^{o}$.
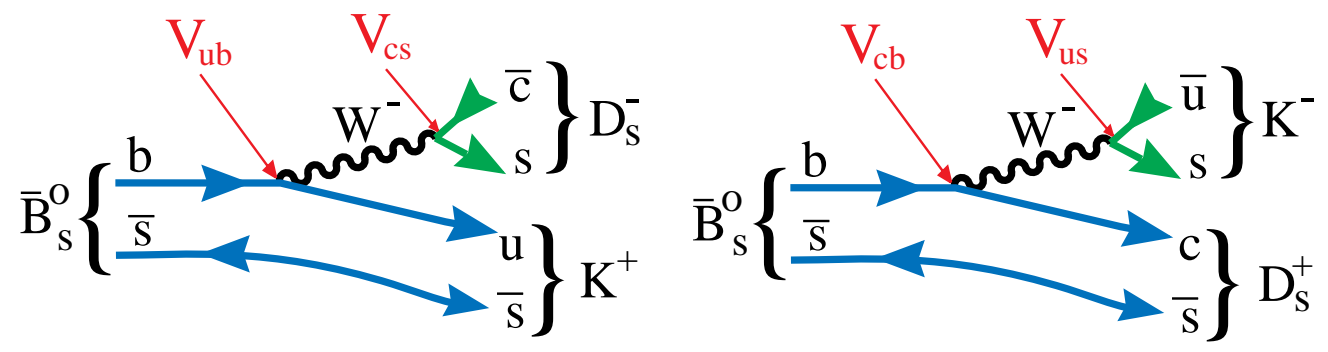

Figure 1.14: Two diagrams for $\bar{B}_{s}^{o} \rightarrow D_{s}^{ \pm} K^{\mp}$.

Consider the following time-dependent rates that can be separately measured using flavor tagging of the other $b$ :

$$
\begin{array}{r}
\Gamma\left(B_{s} \rightarrow f\right)=|M|^{2} e^{-t}\left\{\cos ^{2}(x t / 2)+\rho^{2} \sin ^{2}(x t / 2)-\rho \sin (\phi+\delta) \sin (x t)\right\} \\
\Gamma\left(\bar{B}_{s} \rightarrow \bar{f}\right)=|M|^{2} e^{-t}\left\{\cos ^{2}(x t / 2)+\rho^{2} \sin ^{2}(x t / 2)+\rho \sin (\phi-\delta) \sin (x t)\right\} \\
\Gamma\left(B_{s} \rightarrow \bar{f}\right)=|M|^{2} e^{-t}\left\{\rho^{2} \cos ^{2}(x t / 2)+\sin ^{2}(x t / 2)-\rho \sin (\phi-\delta) \sin (x t)\right\} \\
\Gamma\left(\bar{B}_{s} \rightarrow f\right)=|M|^{2} e^{-t}\left\{\rho^{2} \cos ^{2}(x t / 2)+\sin ^{2}(x t / 2)+\rho \sin (\phi+\delta) \sin (x t)\right\},
\end{array}
$$


where $M=\langle f \mid B\rangle, \rho=\frac{\langle f \mid \bar{B}\rangle}{\langle f \mid B\rangle}, \phi$ is the weak phase between the 2 amplitudes and $\delta$ is the strong phase between the 2 amplitudes. The three parameters $\rho, \sin (\phi+\delta), \sin (\phi-\delta)$ can be extracted from a time-dependent study. If $\rho=O(1)$ the fewest number of events are required.

In the case of $B_{s}$ decays where $f=D_{s}^{+} K^{-}$and $\bar{f}=D_{s}^{-} K^{+}$, the weak phase is $\gamma_{\cdot}{ }^{4}$ Using this technique $\sin (\gamma)$ is determined with a four-fold ambiguity.

\subsubsection{Measurement of $\gamma$ Using Charged $B$ Decay Rates}

Another method for extracting $\gamma$ has been proposed by Atwood, Dunietz and Soni [32], who refined a suggestion by Gronau and Wyler [33]. A large CP asymmetry can result from the interference of the decays $B^{-} \rightarrow K^{-} D^{o}, D^{o} \rightarrow f$ and $B^{-} \rightarrow K^{-} \bar{D}^{o}, \bar{D}^{o} \rightarrow f$, where $f$ is a doubly Cabibbo suppressed decay of the $D^{o}$ (for example $f=K^{+} \pi^{-}, K \pi \pi$, etc.). The overall amplitudes for the two decays are expected to be approximately equal in magnitude. (Note that $B^{-} \rightarrow K^{-} \bar{D}^{o}$ is color-suppressed and $B^{-} \rightarrow K^{-} D^{0}$ is color-allowed.) The weak phase difference between them is $\gamma$. To observe a CP asymmetry there must also be a nonzero strong phase between the two amplitudes. It is necessary to measure the branching ratio $\mathcal{B}\left(B^{-} \rightarrow K^{-} f\right)$ for at least 2 different states $f$ in order to determine $\gamma$ up to discrete ambiguities. Three-body $D^{o}$ decays, are not suggested since the strong $D$ decay phase shifts can vary over the Dalitz plot. Even in quasi-two body decays, such as $K^{*} \pi$ there may be residual interference effects which could lead to false results. Therefore, the modes that can best be used are $D^{o} \rightarrow K^{-} \pi^{+}$and $K^{+} K^{-}\left(\pi^{+} \pi^{-}\right)$final states.

We now discuss this method in more detail. Consider a two-body $B^{-}$decay into neutral charmed meson, either a $D^{o}$ or a $\bar{D}^{o}$ and a $K^{-}$. Let us further take the final state of the charmed meson to be a $K^{+} \pi^{-}$. There are two sequential decay processes that can lead to this situation, shown in Fig. 1.15. One is where the $B^{o}$ decays into a $D^{o}$, that decays in a doubly-Cabibbo suppressed process. The other is where the $B^{o}$ decays via a $b \rightarrow u$ transition to a $D^{o}$, that decays via a Cabibbo allowed process.

Remarkably, the decay rate for these two processes is quite similar leading to the possibility of large interference effects. Even if the interference effects are not large it is possible to use this method to determine $\gamma$, with some ambiguities. To see how this works, let us define the decay amplitudes and phases in Table 1.3 for two process, one as described above and the other where the $D^{o}$ or $\bar{D}^{o}$ decays into a CP eigenstate. (To be specific, we will take the $K^{+} K^{-}$final state.)

All quantities remain the same for the $B^{+}$decays, except that the phase $\gamma$ changes sign. The observed decay rates for the four processes can now be calculated by adding and squaring the amplitudes for the same final state. For example, the decay rate for $B^{-} \rightarrow\left[K^{+} \pi^{-}\right] K^{-}$ (where $\left[K^{+} \pi^{-}\right]$denotes a $K^{+} \pi^{-}$pair at the $D^{o}$ mass), is given by

$$
\Gamma\left(B^{-} \rightarrow\left[K^{+} \pi^{-}\right] K^{-}\right)=a c_{d}+b c+2 \sqrt{a c_{d} b c} \cos \left(\xi_{1}+\gamma\right)
$$

\footnotetext{
${ }^{4}$ This is an approximation. The phase is precisely $\gamma-2 \chi+\chi^{\prime}$, see section 1.10 .
} 

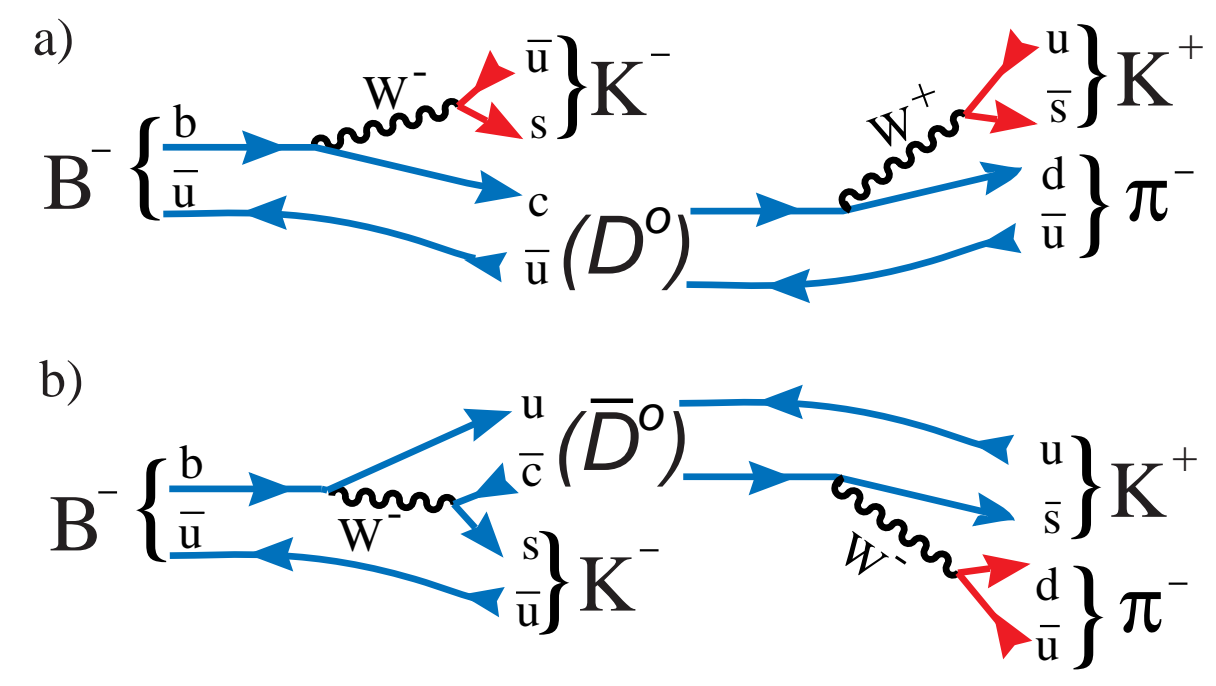

Figure 1.15: Diagrams for the two interfering processes, (a) $B^{-} \rightarrow D^{o} K^{-}$(color allowed) followed by $D^{o} \rightarrow K^{+} \pi^{-}$(doubly-Cabibbo suppressed), and (b) $B^{-} \rightarrow \bar{D}^{o} K^{-}$(color suppressed) followed by $D^{o} \rightarrow K^{-} \pi^{+}$(Cabibbo allowed).

Table 1.3: Amplitudes and Phases for $B^{-} \rightarrow D^{\circ} / \bar{D}^{o} K^{-}$

\begin{tabular}{lcccc}
\hline \hline Decay Mode & $B$ & $D$ & Strong & Weak \\
& Amplitude & Amplitude & Phase & Phase \\
\hline$B^{-} \rightarrow D^{o} K^{-}, D^{o} \rightarrow K^{+} \pi^{-}$ & $\sqrt{a}$ & $\sqrt{c_{d}}$ & $\delta_{B 1}+\delta_{C d}$ & 0 \\
$B^{-} \rightarrow \bar{D}^{o} K^{-}, D^{o} \rightarrow K^{-} \pi^{+}$ & $\sqrt{b}$ & $\sqrt{c}$ & $\delta_{B 2}+\delta_{C}$ & $\gamma$ \\
$B^{-} \rightarrow D^{o} K^{-}, D^{o} \rightarrow K^{+} K^{-}$ & $\sqrt{a}$ & $\sqrt{c_{C P}}$ & $\delta_{B 1}+\delta_{C P}$ & 0 \\
$B^{-} \rightarrow \bar{D}^{o} K^{-}, D^{o} \rightarrow K^{-} K+$ & $\sqrt{b}$ & $\sqrt{c_{C P}}$ & $\delta_{B 2}+\delta_{C P}$ & $\gamma$ \\
\hline \hline
\end{tabular}

where $\xi_{1}$ is a combination of $B$ and $D$ phase shifts, $\delta_{B 2}-\delta_{B 1}+\delta_{C}-\delta_{c}$ and is unknown. Similarly, the decay rates for the other processes are

$$
\begin{aligned}
\Gamma\left(B^{+} \rightarrow\left[K^{-} \pi^{+}\right] K^{+}\right) & =a c_{d}+b c+2 \sqrt{a c_{d} b c} \cos \left(\xi_{1}-\gamma\right) \\
\Gamma\left(B^{-} \rightarrow\left[K^{+} K^{-}\right] K^{-}\right) & =a c_{C P}+b c_{C P}+2 \sqrt{a b c_{C P}^{2}} \cos \left(\delta_{B}-\gamma\right) \\
\Gamma\left(B^{+} \rightarrow\left[K^{+} K^{-}\right] K^{+}\right) & =a c_{C P}+b c_{C P}+2 \sqrt{a b c_{C P}^{2}} \cos \left(\delta_{B}+\gamma\right)
\end{aligned}
$$

where $\delta_{B}=\delta_{B 1}-\delta_{B 2}$.

In these four equations, the quantities which are known, or will be precisely known before this measurement is attempted are the decay widths $a, c_{d}, c$ and $c_{C P}$. The unknowns are the decay width $b$, two strong phase shifts $\xi_{1}$ and $\delta_{B}$ and the weak phase shift $\gamma$. Thus the four 
equations may be solved for the four unknowns. We can find $\sin \gamma$ with a two-fold ambiguity. If more decay modes are added the ambiguity can be removed. The $B^{-}$decay mode can be changed from a $K^{-}$to a $K^{*-}$, which could change the strong $B$ decay phase shift, or a different $D^{o}$ decay mode can be used, such as $K^{-} \pi^{+} \pi^{+} \pi^{-}$, which would change the strong $D$ decay phase shift. In the latter case, we have to worry about differences in strong phase shifts between $D^{o}$ and $\bar{D}^{o}$ due to resonant structure, but use of this mode can shed some information on ambiguity removal.

Comparison of the solutions found here and using $B_{s} \rightarrow D_{s}^{ \pm} K^{\mp}$ as described in the previous section are likely to remove the ambiguities.

\subsubsection{Measurement of $\gamma$ Using $B \rightarrow K \pi$ and $B \rightarrow \pi \pi$ Decay Rates and Asymmetries}

CLEO has observed the pseudoscalar-pseudoscalar decays $B^{o} \rightarrow K^{\mp} \pi^{ \pm}, B^{-} \rightarrow K^{-} \pi^{o}, B^{-} \rightarrow$ $K^{o} \pi^{-}$, all with branching ratios around $1.5 \times 10^{-5}$ and set upper limits on the corresponding decays to di-pion final states [34]. CLEO has also measured the pseudoscalar-vector final state $\rho^{o} \pi^{-}$, with a similar branching ratio, showing that the dipion rates must be close to the upper limits. Therefore, the Penguin and Tree contributions for $B \rightarrow K \pi$ probably do not differ by more than a factor of five, so they can produce observable CP violating effects.

Proposals for extracting information on $\gamma$ have been made using the following experimental ratios:

$$
\begin{aligned}
R & =\frac{\tau\left(B^{+}\right)}{\tau\left(B^{0}\right)} \frac{\mathcal{B}\left(B^{0} \rightarrow \pi^{-} K^{+}\right)+\mathcal{B}\left(\bar{B}^{0} \rightarrow \pi^{+} K^{-}\right)}{\mathcal{B}\left(B^{+} \rightarrow \pi^{+} K^{0}\right)+\mathcal{B}\left(B^{-} \rightarrow \pi^{-} \bar{K}^{0}\right)} \\
R_{*} & =\frac{\mathcal{B}\left(B^{+} \rightarrow \pi^{+} K^{0}\right)+\mathcal{B}\left(B^{-} \rightarrow \pi^{-} \bar{K}^{0}\right)}{2\left[\mathcal{B}\left(B^{+} \rightarrow \pi^{0} K^{+}\right)+\mathcal{B}\left(B^{-} \rightarrow \pi^{0} K^{-}\right)\right]}
\end{aligned}
$$

The first, $R$, is by Fleischer and Mannel [35], and the second $R_{*}$, is by Neubert and Rosner [36], who updated an older suggestion of Gronau and Rosner [37]. The latter paper prompted much theoretical discussion about the effects of isospin conservation and rescattering [38][39][40][41]. A recent paper of Neubert [42] takes into account these criticisms and provides a framework to limit $\gamma$.

More information is obtainable if the CP averaged $\pi^{ \pm} \pi^{o}$ branching ratios are also measured, and a CP violating observable defined as

$$
\widetilde{A} \equiv \frac{A_{\mathrm{CP}}\left(\pi^{0} K^{+}\right)}{R_{*}}-A_{\mathrm{CP}}\left(\pi^{+} K^{0}\right),
$$

where for example

$$
A_{\mathrm{CP}}\left(\pi^{0} K^{+}\right)=\frac{\Gamma\left(B^{+} \rightarrow \pi^{o} K^{+}\right)-\Gamma\left(B^{-} \rightarrow \pi^{o} K^{-}\right)}{\Gamma\left(B^{+} \rightarrow \pi^{o} K^{+}\right)+\Gamma\left(B^{-} \rightarrow \pi^{o} K^{-}\right)} .
$$

To summarize Neubert's strategy for determining $\gamma$ : From measurements of the CPaveraged branching ratio for the decays $B^{ \pm} \rightarrow \pi^{ \pm} \pi^{0}, B^{ \pm} \rightarrow \pi^{ \pm} K^{0}$ and $B^{ \pm} \rightarrow \pi^{0} K^{ \pm}$, 
the ratio $R_{*}$ and a parameter $\bar{\varepsilon}_{3 / 2}$ are determined. Next, from measurements of the rate asymmetries in the decays $B^{ \pm} \rightarrow \pi^{ \pm} K^{0}$ and $B^{ \pm} \rightarrow \pi^{0} K^{ \pm}$the quantity $\widetilde{A}$ is determined.

In Fig. 1.16, we show the contour bands as given by Neubert in the $\phi-\gamma$ plane. Here $\phi$ is a strong interaction phase-shift. Assuming that $\sin \gamma>0$ as suggested by the global analysis of the unitarity triangle, the sign of $A$ determines the sign of $\sin \phi$. In the plot, we assume here that $0^{\circ} \leq \phi \leq 180^{\circ}$. For instance, if $R_{*}=0.7$ and $\widetilde{A}=0.2$, then the two solutions are $(\gamma, \phi) \approx\left(98^{\circ}, 25^{\circ}\right)$ and $(\gamma, \phi) \approx\left(153^{\circ}, 67^{\circ}\right)$, only the first of which is allowed by the upper bound $\gamma<105^{\circ}$ following from the global analysis of the unitarity triangle shown here (section 1.2 or in [11]). It is evident that the contours are rather insensitive to the rescattering effects. According to Neubert, the combined theoretical uncertainty is of order $\pm 10^{\circ}$ on the extracted value of $\gamma$.

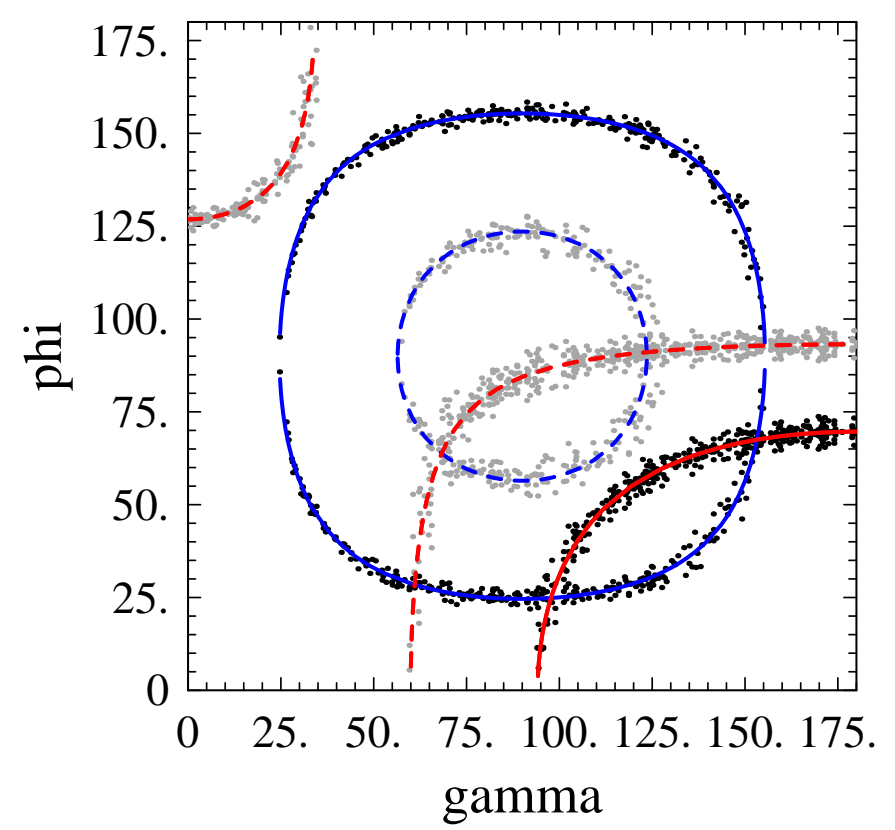

Figure 1.16: Contour plots from Neubert [42] for the quantities $R_{*}$ ("hyperbolas") and $\widetilde{A}$ ("circles"). The scatter plots show the results including rescattering effects, while the lines refer to $\varepsilon_{a}=0$. The solid curves correspond to the contours for $R_{*}=0.7$ and $\widetilde{A}=0.2$, the dashed ones to $R_{*}=0.9$ and $\widetilde{A}=0.4$.

From the contour plots for the quantities $R_{*}$ and $\widetilde{A}$ the phases $\gamma$ and $\phi$ can then be extracted up to discrete ambiguities. There are also errors in theoretical parameters that must be accounted for. 

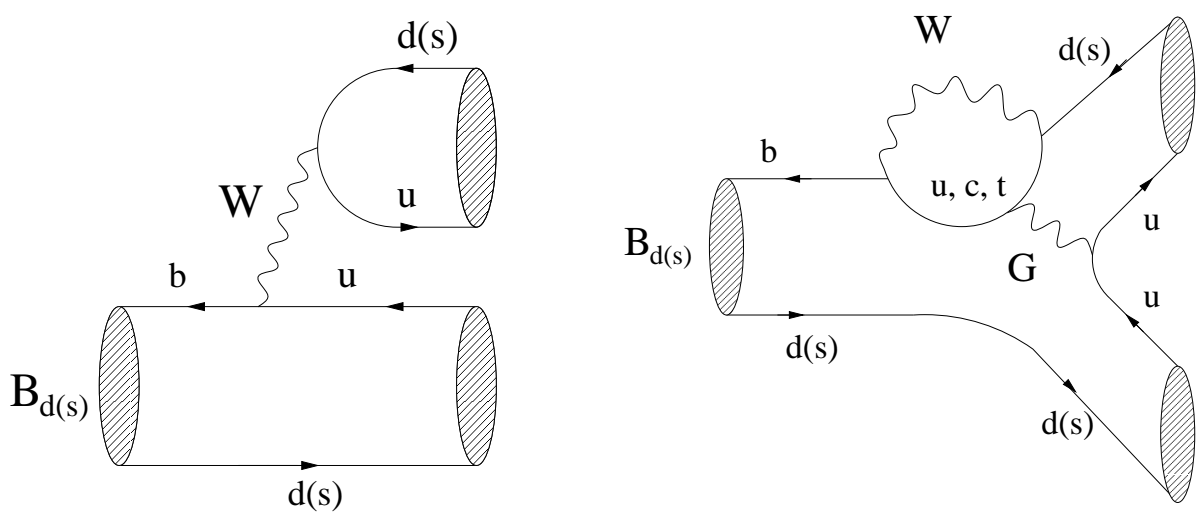

Figure 1.17: Feynman diagrams contributing to $B_{d} \rightarrow \pi^{+} \pi^{-}$and $B_{s} \rightarrow K^{+} K^{-}$(from Fleischer).

\subsubsection{Measurement of $\gamma$ Using CP Asymmetries in $B^{o} \rightarrow \pi^{+} \pi^{-}$and $B_{s}^{o} \rightarrow K^{+} K^{-}$}

Yet another interesting method for determining $\gamma$ has been suggested by Fleischer [43]. The decays $B^{o} \rightarrow \pi^{+} \pi^{-}$and $B_{s}^{o} \rightarrow K^{+} K^{-}$are related to each other by interchanging all down and strange quarks, which is called $U$-spin flavor symmetry [44]. Both channels can occur via Penguin or singly-Cabibbo-suppressed tree levels diagrams, shown in Fig. 1.17.

For $B^{o} \rightarrow \pi^{+} \pi^{-}$the transistion amplitude is given by

$$
A\left(B_{d}^{0} \rightarrow \pi^{+} \pi^{-}\right)=\lambda_{u}^{(d)}\left(A_{\mathrm{cc}}^{u}+A_{\mathrm{pen}}^{u}\right)+\lambda_{c}^{(d)} A_{\mathrm{pen}}^{c}+\lambda_{t}^{(d)} A_{\mathrm{pen}}^{t},
$$

where $A_{\mathrm{cc}}^{u}$ is due to the tree contributions, and the amplitudes $A_{\mathrm{pen}}^{j}$ describe penguin topologies with internal $j$ quarks $(j \in\{u, c, t\})$. These penguin amplitudes take into account both QCD and electroweak penguin contributions. The quantities

$$
\lambda_{j}^{(d)} \equiv V_{j d} V_{j b}^{*}
$$

are the usual CKM factors. If we make use of the unitarity of the CKM matrix and use the Wolfenstein parametrization, we have

$$
A\left(B_{d}^{0} \rightarrow \pi^{+} \pi^{-}\right)=e^{i \gamma}\left(1-\frac{\lambda^{2}}{2}\right) \mathcal{C}\left[1-d e^{i \theta} e^{-i \gamma}\right]
$$

where

$$
\mathcal{C} \equiv \lambda^{3} A R_{b}\left(A_{\text {cc }}^{u}+A_{\text {pen }}^{u t}\right)
$$

with $A_{\text {pen }}^{u t} \equiv A_{\text {pen }}^{u}-A_{\text {pen }}^{t}$, and

$$
d e^{i \theta} \equiv \frac{1}{\left(1-\lambda^{2} / 2\right) R_{b}}\left(\frac{A_{\mathrm{pen}}^{c t}}{A_{\mathrm{cc}}^{u}+A_{\mathrm{pen}}^{u t}}\right) .
$$


The quantity $A_{\text {pen }}^{c t}$ is defined in analogy to $A_{\text {pen }}^{u t}$, and the CKM factors are given by

$$
\lambda \equiv\left|V_{u s}\right|=0.22, \quad A \equiv \frac{1}{\lambda^{2}}\left|V_{c b}\right| \sim 0.8, \quad R_{b} \equiv \frac{1}{\lambda}\left|\frac{V_{u b}}{V_{c b}}\right| \sim 0.4, .
$$

For the following considerations, time-dependent CP asymmetries play a key role. In the case of a general $B_{d}$ decay into a final CP eigenstate $|f\rangle$, satisfying

$$
(\mathcal{C P})|f\rangle=\eta|f\rangle
$$

we have (see equation 1.33)

$$
\begin{aligned}
& a_{\mathrm{CP}}\left(B_{d}(t) \rightarrow f\right) \equiv \frac{\Gamma\left(B_{d}^{0}(t) \rightarrow f\right)-\Gamma\left(\overline{B_{d}^{0}}(t) \rightarrow f\right)}{\Gamma\left(B_{d}^{0}(t) \rightarrow f\right)+\Gamma\left(\overline{B_{d}^{0}}(t) \rightarrow f\right)} \\
& \quad=\mathcal{A}_{\mathrm{CP}}^{\operatorname{dir}}\left(B_{d} \rightarrow f\right) \cos \left(\Delta m_{d} t\right)+\mathcal{A}_{\mathrm{CP}}^{\operatorname{mix}}\left(B_{d} \rightarrow f\right) \sin \left(\Delta m_{d} t\right) .
\end{aligned}
$$

For the case of $B^{o} \rightarrow \pi^{+} \pi^{-}$, the decay amplitude takes the same form as (1.56), and we obtain the following expressions for the "direct" and "mixing-induced" CP-violating observables:

$$
\begin{aligned}
& \mathcal{A}_{\mathrm{CP}}^{\mathrm{dir}}\left(B_{d} \rightarrow f\right)=-\left[\frac{2 d \sin \theta \sin \gamma}{1-2 d \cos \theta \cos \gamma+d^{2}}\right] \\
& \mathcal{A}_{\mathrm{CP}}^{\mathrm{mix}}\left(B_{d} \rightarrow f\right)=\eta\left[\frac{\sin (2 \beta+2 \gamma)-2 d \cos \theta \sin (2 \beta+\gamma)+d^{2} \sin 2 \beta}{1-2 d \cos \theta \cos \gamma+d^{2}}\right],
\end{aligned}
$$

where $\eta$ is equal to +1 ; for negligible values of the "penguin parameter" $d$, we have $\mathcal{A}_{\mathrm{CP}}^{\text {mix }}\left(B_{d} \rightarrow\right.$ $\left.\pi^{+} \pi^{-}\right)=\sin (2 \beta+2 \gamma)=-\sin (2 \alpha)$. However, the penguin contributions are expected to play an important role.

Consider now the decay $B_{s}^{0} \rightarrow K^{+} K^{-}$. It originates from $\bar{b} \rightarrow \bar{u} u \bar{s}$ quark-level processes, as can be seen in Fig. 1.17. Using a notation similar to that in (1.56), we obtain

$$
A\left(B_{s}^{0} \rightarrow K^{+} K^{-}\right)=e^{i \gamma} \lambda \mathcal{C}^{\prime}\left[1+\left(\frac{1-\lambda^{2}}{\lambda^{2}}\right) d^{\prime} e^{i \theta^{\prime}} e^{-i \gamma}\right]
$$

where

$$
\mathcal{C}^{\prime} \equiv \lambda^{3} A R_{b}\left(A_{\mathrm{cc}}^{u^{\prime}}+A_{\mathrm{pen}}^{u t^{\prime}}\right)
$$

and

$$
d^{\prime} e^{i \theta^{\prime}} \equiv \frac{1}{\left(1-\lambda^{2} / 2\right) R_{b}}\left(\frac{A_{\mathrm{pen}}^{c t^{\prime}}}{A_{\mathrm{cc}}^{u^{\prime}}+A_{\mathrm{pen}}^{u t^{\prime}}}\right)
$$

correspond to (1.57) and (1.58), respectively. The primes remind us that we are dealing with a $\bar{b} \rightarrow \bar{s}$ transition. It should be emphasized that (1.56) and (1.64) are completely general parametrizations of the $B_{d}^{0} \rightarrow \pi^{+} \pi^{-}$and $B_{s}^{0} \rightarrow K^{+} K^{-}$decay amplitudes within 
the Standard Model, relying only on the unitarity of the CKM matrix. In particular, these expressions take into account also final-state interaction effects, which can be considered as long-distance penguin topologies with internal up- and charm-quark exchanges.

There may be a sizeable width difference $\Delta \Gamma_{s} \equiv \Gamma_{\mathrm{H}}^{(s)}-\Gamma_{\mathrm{L}}^{(s)}$ between the $B_{s}$ mass eigenstates [45], which may allow studies of CP violation with "untagged" $B_{s}$ data samples [46]. Such untagged rates take the following form:

$$
\Gamma\left(B_{s}^{0}(t) \rightarrow f\right)+\Gamma\left(\overline{B_{s}^{0}}(t) \rightarrow f\right) \propto R_{\mathrm{H}} e^{-\Gamma_{\mathrm{H}}^{(s)} t}+R_{\mathrm{L}} e^{-\Gamma_{\mathrm{L}}^{(s)} t},
$$

whereas the time-dependent $\mathrm{CP}$ asymmetry is given by

$$
\begin{aligned}
& a_{\mathrm{CP}}\left(B_{s}(t) \rightarrow f\right) \equiv \frac{\Gamma\left(B_{s}^{0}(t) \rightarrow f\right)-\Gamma\left(\overline{B_{s}^{0}}(t) \rightarrow f\right)}{\Gamma\left(B_{s}^{0}(t) \rightarrow f\right)+\Gamma\left(\overline{B_{s}^{0}}(t) \rightarrow f\right)} \\
& =2 e^{-\Gamma_{s} t}\left[\frac{\mathcal{A}_{\mathrm{CP}}^{\operatorname{dir}}\left(B_{s} \rightarrow f\right) \cos \left(\Delta m_{s} t\right)+\mathcal{A}_{\mathrm{CP}}^{\operatorname{mix}}\left(B_{s} \rightarrow f\right) \sin \left(\Delta m_{s} t\right)}{e^{-\Gamma_{\mathrm{H}}^{(s)} t}+e^{-\Gamma_{\mathrm{L}}^{(s)} t}+\mathcal{A}_{\Delta \Gamma}\left(B_{s} \rightarrow f\right)\left(e^{-\Gamma_{\mathrm{H}}^{(s)} t}-e^{-\Gamma_{\mathrm{L}}^{(s)} t}\right)}\right]
\end{aligned}
$$

with $\mathcal{A}_{\Delta \Gamma}\left(B_{s} \rightarrow f\right)=\left(R_{\mathrm{H}}-R_{\mathrm{L}}\right) /\left(R_{\mathrm{H}}+R_{\mathrm{L}}\right)$. If the $B_{s}^{0} \rightarrow f$ decay amplitude takes the same form as (1.64), we have

$$
\begin{gathered}
\mathcal{A}_{\mathrm{CP}}^{\mathrm{dir}}\left(B_{s} \rightarrow f\right)=+\left[\frac{2 \tilde{d}^{\prime} \sin \theta^{\prime} \sin \gamma}{1+2 \tilde{d}^{\prime} \cos \theta^{\prime} \cos \gamma+\tilde{d}^{\prime 2}}\right] \\
\mathcal{A}_{\mathrm{CP}}^{\operatorname{mix}}\left(B_{s} \rightarrow f\right)=+\eta\left[\frac{\sin (2 \chi+2 \gamma)+2 \tilde{d}^{\prime} \cos \theta^{\prime} \sin (2 \chi+\gamma)+\tilde{d}^{\prime 2} \sin 2 \chi}{1+2 \tilde{d}^{\prime} \cos \theta^{\prime} \cos \gamma+\tilde{d}^{\prime 2}}\right] \\
\mathcal{A}_{\Delta \Gamma}\left(B_{s} \rightarrow f\right)=-\eta\left[\frac{\cos (2 \chi+2 \gamma)+2 \tilde{d}^{\prime} \cos \theta^{\prime} \cos (2 \chi+\gamma)+\tilde{d}^{\prime 2} \cos 2 \chi}{1+2 \tilde{d}^{\prime} \cos \theta^{\prime} \cos \gamma+\tilde{d}^{\prime 2}}\right] .
\end{gathered}
$$

These observables are not independent quantities, and satisfy the relation

$$
\left[\mathcal{A}_{\mathrm{CP}}^{\mathrm{dir}}\left(B_{s} \rightarrow f\right)\right]^{2}+\left[\mathcal{A}_{\mathrm{CP}}^{\mathrm{mix}}\left(B_{s} \rightarrow f\right)\right]^{2}+\left[\mathcal{A}_{\Delta \Gamma}\left(B_{s} \rightarrow f\right)\right]^{2}=1
$$

In the general expressions (1.69)-(1.71), we have introduced the abbreviation

$$
\tilde{d}^{\prime} \equiv\left(\frac{1-\lambda^{2}}{\lambda^{2}}\right) d^{\prime},
$$

and $2 \chi=2 \arg \left(V_{t s}^{*} V_{t b}\right)$ denotes the $B_{s}^{o}-\overline{B_{s}^{o}}$ mixing phase. Within the Standard Model, we have $2 \chi \approx 0.03$ due to a Cabibbo suppression of $\mathcal{O}\left(\lambda^{2}\right)$, implying that $2 \chi$ is very small. This phase can be determined using $B_{s} \rightarrow J / \psi \eta^{\prime}$ decays (see section 1.10). 
Since the decays $B_{d} \rightarrow \pi^{+} \pi^{-}$and $B_{s} \rightarrow K^{+} K^{-}$are related to each other by interchanging all strange and down quarks, the $U$-spin flavour symmetry of strong interactions implies

$$
\begin{aligned}
& d^{\prime}=d \\
& \theta^{\prime}=\theta .
\end{aligned}
$$

In contrast to certain isospin relations, electroweak penguins do not lead to any problems in the $U$-spin relations (1.74) and (1.75), according to Fleischer.

In general we have five physics quantities of interest, $2 \chi, d, \theta, 2 \beta$ and $\gamma$. Let us now assume that $\sin (2 \beta)$ will be measured and $\sin (2 \chi)$ either measured or tightly limited. Only $d, \theta$ and $\gamma$ then need to be determined.

We have four possible measured quantities provided by the time-dependent $\mathrm{CP}$ asymmetries of the modes $B_{d} \rightarrow \pi^{+} \pi^{-}$and $B_{s} \rightarrow K^{+} K^{-}$. These four quantities are $\mathcal{A}_{\mathrm{CP}}^{\text {mix }}\left(B_{s} \rightarrow\right.$ $\left.K^{+} K^{-}\right), \mathcal{A}_{\mathrm{CP}}^{\text {mix }}\left(B_{d} \rightarrow \pi^{+} \pi^{-}\right), \mathcal{A}_{\mathrm{CP}}^{\mathrm{dir}}\left(B_{s} \rightarrow K^{+} K^{-}\right)$and $\mathcal{A}_{\mathrm{CP}}^{\mathrm{dir}}\left(B_{d} \rightarrow \pi^{+} \pi^{-}\right)$. To implement this plan we need measure only 3 of these four quantities, or combinations of them. For example, it may be difficult to independently determine $\mathcal{A}_{\mathrm{CP}}^{\text {mix }}\left(B_{d} \rightarrow \pi^{+} \pi^{-}\right)$and $\mathcal{A}_{\mathrm{CP}}^{\mathrm{dir}}\left(B_{d} \rightarrow \pi^{+} \pi^{-}\right)$, because of the small number of observable $B^{o}$ oscillations before the exponential decay reduces the number of events too much. However, the sum

$$
\begin{aligned}
a_{C P}^{\pi^{+} \pi^{-}} & =\int_{0}^{\infty} \mathcal{A}_{\mathrm{CP}}^{\mathrm{dir}} \cos \left(\Delta m_{d} t\right)+\mathcal{A}_{\mathrm{CP}}^{\mathrm{mix}} \sin \left(\Delta m_{d} t\right) \\
& =\frac{1}{1+x^{2}} \mathcal{A}_{\mathrm{CP}}^{\mathrm{dir}}+\frac{x}{1+x^{2}} \mathcal{A}_{\mathrm{CP}}^{\text {mix }}
\end{aligned}
$$

can be determined and used with the other two measurements from $B_{s}^{o} \rightarrow K^{+} K^{-}$. Clearly other scenarios are possible.

\subsubsection{Additional Physics Opportunities with $B_{s}$ Mesons}

A relatively large, $\approx 15 \%$ component of $B_{s}$ decays is predicted to end up as a $c \bar{c} s \bar{s}$ final state. Since $\bar{B}_{s}$ decay with the same rate into the same final state, it has been predicted [47] that there will be a substantial width difference $\Delta \Gamma=\Gamma_{H}-\Gamma_{L} \approx 15 \% \Gamma$, between $\mathrm{CP}+$ and CPeigenstates. $\mathrm{BTeV}$ can easily measure this lifetime difference by measuring the lifetime of a mixed CP state such as $D_{s}^{+} \pi^{-}$and comparing with the $\mathrm{CP}+$ state $J / \psi \eta^{\prime}$.

Measurement of $\Delta \Gamma$ can be used to estimate in an interesting but model dependent manner the value of $\Delta m_{s}$ and thus provides a redundant check on $B_{s}$ mixing measurements [46]

Should a large enough $\Delta \Gamma$ be determined there exist other possible ways to determine some of the interesting physics quantities discussed above. Some of these studies can be done without flavor tagging. In fact, the time evolution of untagged observables for a $B_{s}$ decay into a vector-vector final state is proportional to

$$
\left(e^{-\Gamma_{H} t}-e^{-\Gamma_{L} t}\right) \sin \phi_{C K M},
$$


where $\phi_{C K M}$ is a CP violating angle from the CKM matrix and depends on the specific decay mode.

In general the angular distribution for $B_{s} \rightarrow V V$ is expressed in terms of transversity in a manner similar to equation 1.43 , with the major difference being that the angular variables are time dependent. The time evolution of the decay $B_{s} \rightarrow J / \psi \phi$ is given in Table 1.4 [48].

\begin{tabular}{cc}
\hline \hline Observable & Time evolution \\
\hline$\left|A_{0}(t)\right|^{2}$ & $\left|A_{0}(0)\right|^{2}\left[e^{-\Gamma_{L} t}-e^{-\bar{\Gamma} t} \sin (\Delta m t) \sin (2 \chi)\right]$ \\
$\left|A_{\|}(t)\right|^{2}$ & $\left|A_{\|}(0)\right|^{2}\left[e^{-\Gamma_{L} t}-e^{-\bar{\Gamma} t} \sin (\Delta m t) \sin (2 \chi)\right]$ \\
$\left|A_{\perp}(t)\right|^{2}$ & $\left|A_{\perp}(0)\right|^{2}\left[e^{-\Gamma_{H} t}+e^{-\bar{\Gamma} t} \sin (\Delta m t) \sin (2 \chi)\right]$ \\
\hline $\operatorname{Re}\left(A_{0}^{*}(t) A_{\|}(t)\right)$ & $\left|A_{0}(0)\right|\left|A_{\|}(0)\right| \cos \left(\delta_{2}-\delta_{1}\right)\left[e^{-\Gamma_{L} t}-e^{-\bar{\Gamma} t} \sin (\Delta m t) \sin (2 \chi)\right]$ \\
$\operatorname{Im}\left(A_{\|}^{*}(t) A_{\perp}(t)\right)$ & $\left|A_{\|}(0)\right|\left|A_{\perp}(0)\right|\left[e^{-\bar{\Gamma} t} \sin \left(\delta_{1}-\Delta m t\right)\right.$ \\
& $\left.+\frac{1}{2}\left(e^{-\Gamma_{H} t}-e^{-\Gamma_{L} t}\right) \cos \left(\delta_{1}\right) \sin (2 \chi)\right]$ \\
$\operatorname{Im}\left(A_{0}^{*}(t) A_{\perp}(t)\right)$ & $\left|A_{0}(0)\right|\left|A_{\perp}(0)\right|\left[e^{-\bar{\Gamma} t} \sin \left(\delta_{2}-\Delta m t\right)\right.$ \\
& $\left.+\frac{1}{2}\left(e^{-\Gamma_{H} t}-e^{-\Gamma_{L} t}\right) \cos \left(\delta_{2}\right) \sin (2 \chi)\right]$ \\
\hline \hline
\end{tabular}

Table 1.4: Time evolution of the decay $B_{s} \rightarrow J / \psi\left(\rightarrow l^{+} l^{-}\right) \phi\left(\rightarrow K^{+} K^{-}\right)$of an initially (i.e. at $t=0)$ pure $B_{s}$ meson. $\delta_{1,2}$ are strong phase shifts. by

Combining with the decay of the $\bar{B}_{s}$ the time evolution of the untagged sample is given

$$
\begin{gathered}
\frac{d^{3} \Gamma\left(J / \psi\left(\rightarrow l^{+} l^{-}\right) \phi\left(\rightarrow K^{+} K^{-}\right)\right)}{d \cos \theta d \varphi d \cos \psi} \propto \frac{9}{16 \pi}\left[2\left|A_{0}(0)\right|^{2} e^{-\Gamma_{L} t} \cos ^{2} \psi\left(1-\sin ^{2} \theta \cos ^{2} \varphi\right)\right. \\
+\sin ^{2} \psi\left\{\left|A_{\|}(0)\right|^{2} e^{-\Gamma_{L} t}\left(1-\sin ^{2} \theta \sin ^{2} \varphi\right)+\left|A_{\perp}(0)\right|^{2} e^{-\Gamma_{H} t} \sin ^{2} \theta\right\} \\
+\frac{1}{\sqrt{2}} \sin 2 \psi\left\{\left|A_{0}(0)\right|\left|A_{\|}(0)\right| \cos \left(\delta_{2}-\delta_{1}\right) e^{-\Gamma_{L} t} \sin ^{2} \theta \sin 2 \varphi\right\} \\
+\left\{\frac{1}{\sqrt{2}}\left|A_{0}(0) \| A_{\perp}(0)\right| \cos \delta_{2} \sin 2 \psi \sin 2 \theta \cos \varphi\right. \\
\left.\left.-\left|A_{\|}(0) \| A_{\perp}(0)\right| \cos \delta_{1} \sin ^{2} \psi \sin 2 \theta \sin \varphi\right\} \frac{1}{2}\left(e^{-\Gamma_{H} t}-e^{-\Gamma_{L} t}\right) \delta \phi\right] .
\end{gathered}
$$

Thus a study of the time dependent angular distributions can lead to a measurement of $\sin (2 \chi)$, especially if $\Delta \Gamma$ is determined before hand. It is also possible to integrate over two of the angles if statistics is limited. The distribution in $J / \psi$ decay angle can be written as

$$
\frac{d \Gamma(t)}{d \cos \theta} \propto\left(\left|A_{0}(t)\right|^{2}+\left|A_{\|}(t)\right|^{2}\right) \frac{3}{8}\left(1+\cos ^{2} \theta\right)+\left|A_{\perp}(t)\right|^{2} \frac{3}{4} \sin ^{2} \theta
$$


where the $\mathrm{CP}$ violating angle originates from the imaginary parts of the interference terms in the $A$ 's.

Other final states have been suggested that provide a measurement of $\gamma$ using the above ideas. One particularly interesting set of decays is $B_{s} \rightarrow K^{*+} K^{*-}$ and $B_{s} \rightarrow K^{* o} \bar{K}^{* o}$ [49].

Finally, it is important to realize that determination of a non-zero $\Delta \Gamma$ allows the mea-

surement of $\operatorname{Re}\left(\frac{q}{p} \cdot \frac{\bar{A}}{A}\right)$, that in turn allows the removal of the ambiguities in the CKM angle of interest [46]. For the $B_{s}$ decays mentioned here this could be $\gamma$ or $\chi$.

\subsection{The Tests of the Standard Model}

There are several ways to view the problem of what the appropriate Standard Model tests are. We will discuss these. Another important consideration may be categorized as the "plague of ambiguities." In the phase measurements described above there are always ambiguities. For example, any determination of $\sin (2 \phi)$, where $\phi$ is any angle found using the interference of a single decay amplitude with a mixing amplitude has a four fold ambiguity, where $\phi$, $\pi / 2-\phi, \pi+\phi, 3 \pi / 2-\phi$ are all allowed solutions. One may take the point of view that we know that $\eta$ is a positive quantity and thus we can eliminate two of the four possibilities. However, this would be dangerous in that it could lead to our missing new physics. The only evidence that $\eta$ is positive arises from the measurements of $\epsilon$ and $\epsilon^{\prime}$ and the fact that theoretical calculations give $B_{K}>0$ for $\epsilon$. Even accepting that $K_{L}$ decays give $\eta>0$, it would be foolhardy to miss new physics just because we now assume that $\eta$ must be positive rather than insisting on a clean measurement of the angles that could show a contradiction.

Silva and Wolfenstein [50] following Kayser, Aleksan and London [51] argue that the best way to reveal the presence of new physics is to measure three independent CKM phases. In the six CKM triangles in Fig. 1.1, there are four and only four independent phases. These phases are taken as:

$$
\begin{array}{ll}
\beta=\arg \left(-\frac{V_{t b} V_{t d}^{*}}{V_{c b} V_{c d}^{*}}\right), & \gamma=\arg \left(-\frac{V_{u b}^{*} V_{u d}}{V_{c b}^{*} V_{c d}}\right), \\
\chi=\arg \left(-\frac{V_{c s}^{*} V_{c b}}{V_{t s}^{*} V_{t b}}\right), & \chi^{\prime}=\arg \left(-\frac{V_{u d}^{*} V_{u s}}{V_{c d}^{*} V_{c s}}\right),
\end{array}
$$

where we have changed the confusing notation of Silva and Wolfenstein from $\epsilon, \epsilon^{\prime}$ to $\chi$ and $\chi^{\prime}$.

Since the angles $\alpha, \beta$ and $\gamma$, when properly measured are expected to add to $180^{\circ}$, Silva and Wolfenstein take only two of them as independent. The angles $\beta$ and $\gamma$ are expected to be large in the Standard Model. $\chi$ is expected to be of the order $\lambda^{2} \eta \sim 0.02$ and $\chi^{\prime}$ of the order 0.003 .

The decay modes useful for measuring the first three of these angles, or alternatively, the angle $\alpha$ instead of $\gamma$ are shown in Table 1.5.

Other modes which also may turn out to be useful include $B^{o} \rightarrow D^{*+} \pi^{-}$and its charged 
Table 1.5: Primary modes useful for measuring CP asymmetries for different CKM angles

\begin{tabular}{lc}
\hline \hline Decay Model & Angle \\
\hline$B^{o} \rightarrow J / \psi K_{s}$ & $\sin (2 \beta)$ \\
$B^{o} \rightarrow \rho \pi \rightarrow \pi^{+} \pi^{-} \pi^{o}$ & $\sin (2 \alpha)$ \\
$B_{s}^{o} \rightarrow D_{s}^{\mp} K^{ \pm}$ & $\sin \gamma$ \\
$B^{-} \rightarrow \overline{D^{o}} K^{-}$, and c.c. & $\sin \gamma$ \\
$B_{s} \rightarrow J / \psi \eta^{\prime}$ & $\sin (2 \chi)$ \\
\hline \hline
\end{tabular}

conjugate [52], which measures $\sin (-2 \beta-\gamma)$ albeit with a small $\approx 1 \%$ predicted asymmetry, ${ }^{5}$ and $B \rightarrow K \pi$ modes which can be used to find $\gamma$ albeit with theoretical uncertainties.

There is no direct way of measuring the small angle $\chi^{\prime}$. However the angle $\chi$ can be measured by using $B_{s}$ decays. The most direct way is to measure the time dependent CP violation caused by the mixing of interference and decay in the modes $B_{s} \rightarrow J / \psi \eta, \eta \rightarrow \gamma \gamma$, or $B_{s} \rightarrow J / \psi \eta^{\prime}, \eta^{\prime} \rightarrow \rho^{o} \gamma$. The time resolution has to be excellent in order to resolve the fast $B_{s}$ oscillations. The all charged mode $B_{s} \rightarrow J / \psi \phi$ can also be used [48], but this requires a complicated angular analysis and consequently a great deal of data.

Let us try and get some idea of the sizes of the angles $\alpha, \beta$ and $\gamma$. The expected range of angles derived from Fig. 1.4 is shown in Fig. 1.18.

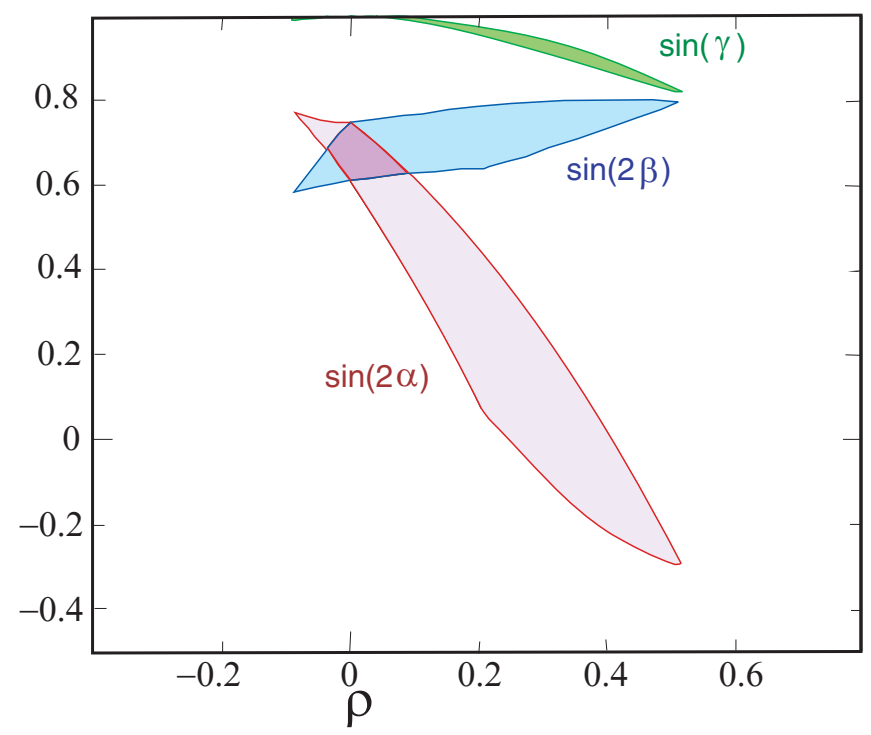

Figure 1.18: The "allowed" values for the three angles of the CKM triangle and derived from the allowed, i.e. $1 \sigma$ overlap region of Fig. 1.4.

\footnotetext{
${ }^{5}$ To measure a CP asymmetry this way requires using equations 1.48 , and extracting the strong phase, amplitude ratio, and a small asymmetry, a very difficult task.
} 
These plots show only the most likely values in the Standard Model. Recall that they are based on the overlap of $\pm 1 \sigma$ bands from constraints on $V_{u b} / V_{c b}, \epsilon$, and $B_{d}$ mixing. However, this gives us a good indication on where we should target our measuring potential. The angle $\chi$ as mentioned previously is expected to be small $\approx \lambda^{2} \eta$.

\subsubsection{Better Measurements of the sides of the CKM triangle}

One side of the triangle is determined by $\left|V_{u b} / V_{c b}\right|$. It appears that the best way to improve the values now is to measure the form-factors in the reactions $B \rightarrow \pi \ell \nu$ and $B \rightarrow \rho \ell \nu$. Input from the charm reactions $D \rightarrow \pi \ell \nu$ and $D \rightarrow \rho \ell \nu$ will also be helpful. This will decrease the model dependence error, still the dominant error, in the $V_{u b}$ determination. Lattice gauge model calculations are appearing and should be quite useful.

The other side of the triangle can be determined by measuring $B_{s}$ mixing, using the ratio

$$
\frac{\Delta m_{s}}{\Delta m_{d}}=\left(\frac{B_{s}}{B}\right)\left(\frac{f_{B_{s}}}{f_{B}}\right)^{2}\left(\frac{m_{B_{s}}}{m_{B}}\right)\left|\frac{V_{t s}}{V_{t d}}\right|^{2},
$$

where

$$
\left|\frac{V_{t d}}{V_{t s}}\right|^{2}=\lambda^{2}\left[(\rho-1)^{2}+\eta^{2}\right] .
$$

The large uncertainty in using the $B_{d}$ mixing measurement to constrain $\rho$ and $\eta$ is largely removed as the ratio of the first two factors in equation (1.81) is already known to $\pm 20 \%$.

As an alternative to measuring $x_{s}$, we can measure the ratio of the penguin decay rates

$$
\frac{\mathcal{B}(B \rightarrow \rho \gamma)}{\mathcal{B}\left(B \rightarrow K^{*} \gamma\right)}=\xi\left|\frac{V_{t d}}{V_{t s}}\right|^{2},
$$

where $\xi$ is a model dependent correction due to different form-factors. Arguments exist however, [53] that "long-distance" effects, basically other diagrams, spoil this simple relationship. If this occurs, however, then it is possible to find $\mathrm{CP}$ violation by looking for a difference in rate between $\rho^{+} \gamma$ and $\rho^{-} \gamma$.

The CLEO II data are already background limited. The limit quoted is [54]

$$
\frac{\mathcal{B}(B \rightarrow \rho \gamma)}{\mathcal{B}\left(B \rightarrow K^{*} \gamma\right)}<0.19
$$

at $90 \%$ confidence level.

An attractive alternative at a hadron collider is to measure $B_{s} \rightarrow \phi \gamma$ and $B_{s} \rightarrow K^{* o} \gamma$, instead of $K^{*} \gamma$ and $\rho \gamma$. The advantage is that the $\phi$ would not be the large background source to the $K^{* o}$ that the $K^{*}$ is for the $\rho$.

Another method of measuring $\left|V_{t d}\right|$ is to measure the branching ratio of $K^{+} \rightarrow \pi^{+} \nu \bar{\nu}$. A precise measurement would still be subject to theoretical uncertainties mostly arising from the uncertainty in the charmed quark mass and $\left|V_{c b}\right|$ [55]. A Brookhaven experiment, E787, has claimed a signal of one event[56], and hopes to obtain substantially more data. 


\subsubsection{Summary of CKM tests}

Our goal is to measure separately the four angles $\alpha, \beta, \gamma$ and $\chi$. We also want to measure as precisely as possible the side of the bd triangle (see Fig. 1.4) that requires a precise measurement of $B_{s}$ mixing. The other side, proportional to the magnitude of $V_{u b}$ will have been measured by $e^{+} e^{-} b$-factories and the precision will be limited by theoretical concerns if form-factors in the exclusive decays and $q^{2}$ distributions in the inclusive decays have been decisively measured.

After the three angles $\alpha, \beta$ and $\gamma$ have been measured, we need to check if they add up to $180^{\circ}$. A discrepancy here would show new physics. To be sure, this check is not complete if ambiguities have not been removed. (Even if the angles sum to $180^{\circ}$, new physics could hide.)

Each of the angles can be measured most easily via the decay modes shown in Table 1.5. New physics can add differently to the phases in different decay modes if it contributes differently to the relative decay amplitudes $A / \bar{A}$. Therefore it is interesting to measure $\mathrm{CP}$ violation in redundant modes. For example, the decay $B^{o} \rightarrow \phi K_{s}$ should also measure $\sin (2 \beta)$. If it is different than that obtained by $B^{o} \rightarrow J / \psi K_{s}$, that would be a strong indication of new physics [57]. We list in Table 1.6 other interesting modes to check $\sin (2 \beta)$. The branching ratios listed with errors have been measured [34] [58], while those without are theoretical estimates.

Table 1.6: Other modes useful for cross-checking $\sin (2 \beta)$

\begin{tabular}{lc}
\hline \hline & \\
Decay Model & Branching Ratio \\
\hline$B^{o} \rightarrow \phi K_{s}$ & $(0.04-1.5) \times 10^{-5}$ \\
$B^{o} \rightarrow D^{+} D^{-}$ & $\approx 10^{-3}$ \\
$B^{o} \rightarrow D^{*+} D^{-}$ & $\approx 10^{-3}$ \\
$B^{o} \rightarrow \eta^{\prime} K^{o}$, & $(5.9 \pm 1.9) \times 10^{-5}$ \\
$B^{o} \rightarrow J / \psi\left(\pi^{o}, \eta\right.$ or $\left.\eta^{\prime}\right)$ & $(3.4 \pm 1.6) \times 10^{-5}$ \\
\hline \hline
\end{tabular}

There are three alternative ways to measure $\gamma$, discussed in section 1.9, which serve both to remove ambiguities and perform checks. It will be much more difficult to find other modes to check $\alpha$, however. One approach is to measure the CP asymmetry in $B^{o} \rightarrow \pi^{+} \pi^{-}$and use theoretical models to estimate the effects of Penguin pollution. Minimally, a great deal would be learned about the models. It also turns out that the third ambiguity in $\alpha$ can be removed by comparing the CP violating asymmetry in $\pi^{+} \pi^{-}$with that found in $\rho \pi$ and using some mild theoretical assumptions [59]. 


\subsubsection{Checks combining with measurements of magnitudes of CKM elements}

We have taken the point of view that measurements of the magnitudes of CKM matrix elements all come with theoretical errors. Some of these are hard to estimate, we now try and view realistically how to combine $\mathrm{CP}$ violating phase measurements with the magnitude measurements to best test the Standard Model.

The best measured magnitude is that of $\left|V_{u s} / V_{u d}\right|=\lambda=0.2205 \pm 0.0018$, where the first error is experimental and the last theoretical. Silva and Wolfenstein [50], along with Aleksan, Kayser and London [51] show that the Standard Model can be checked in a profound manner by seeing if:

$$
\sin \chi=\left|\frac{V_{u s}}{V_{u d}}\right|^{2} \frac{\sin \beta \sin \gamma}{\sin (\beta+\gamma)} .
$$

Here the precision of the check is limited by the measurement of $\sin \chi$, not of $\lambda$. This check is likely to reveal that new physics is present, even if other checks have not shown new physics. The example given by Silva and Wolfenstein [50] is a new phase added to the standard model mixing phase, which is missed in the measurement of $\alpha$ plus $\beta$ since it cancels in the sum. Two other checks can be performed, which have similar structures.

$$
\begin{aligned}
& \sin \chi=\left|\frac{V_{u b}}{V_{c b}}\right|^{2} \frac{\sin \gamma \sin (\beta+\gamma)}{\sin \beta}, \\
& \sin \chi=\left|\frac{V_{t d}}{V_{t s}}\right|^{2} \frac{\sin \beta \sin (\beta+\gamma)}{\sin \gamma} .
\end{aligned}
$$

Note, that it is in principle possible to determine the magnitudes of $\left|V_{u b} / V_{c b}\right|$ and $\left|V_{t d} / V_{t s}\right|$, just by measuring the angles precisely enough. This is true because of the unitarity of the CKM matrix.

Before these precision tests can be carried out, it will be useful to check the consistency of the measurements of $\epsilon$ in $K_{L}$ decay, $\left|V_{u b} / V_{c b}\right|$ from semileptonic $b$ decay and the ratio of $x_{s} / x_{d}, B_{s} / B_{d}$ mixing parameters. The accuracy of the $\rho-\eta$ constraint from $\epsilon$ is currently determined mostly (see Fig. 1.5) by $V_{c b}$ and the parameter $B_{K}$, which can only be calculated not measured. While the determination of $V_{c b}$ will improve, in the next several years before it is limited by theoretical errors, the accuracy of $B_{K}$ will probably not improve, and it will remain difficult to assign a well defined error. Thus, the allowed band in $\epsilon$ will shrink somewhat, but it will remain relatively broad.

$B_{s}$ mixing may as yet be measured by LEP or CDF. If the oscillations prove to be fast enough, $\mathrm{BTeV}$ will make the first measurement. In either case, the ultimate utility of the measurement in restricting the region of the $\rho-\eta$ plane will be the inaccuracy of the calculation of ratio of $f_{B_{d}}^{2} B_{B_{d}} / f_{B_{s}}^{2} B_{B_{s}}$, after the measurement of $x_{s}$ becomes accurate enough. These parameters have been and are being calculated on the lattice. The accuracy in the above ratio is estimated not to be lower than about $10 \%$ [60]. 
The ultimate error on $\left|V_{u b}\right|$ will also be theoretical in origin. Experimental measurements from $e^{+} e^{-} B$ factories will perform extensive measurements of inclusive semileptonic $b \rightarrow u \ell \nu$ decays as well as exclusive decays to the $\pi \ell \nu$ and $\rho \ell \nu$ decays. The form-factors as well as the branching ratios will be measured and should help to restrict the models. However, at this time there isn't a compelling theoretical framework such as HQET for $b \rightarrow c \ell \nu$. Thus, we estimate the theoretical error to be currently on the order of $\pm 14 \%$, and hope for further improvements.

Thus the intersection of the mixing ratio band with the $V_{u b}$ band should provide a crossing point with reasonable errors. The measurement of each of the individual angles, $\alpha, \beta$ and $\gamma$ will need to be consistent within the error band, otherwise the standard model will be violated.

\subsection{Rare Decays as Probes beyond the Standard Model}

Rare decays have loops in the decay diagrams so they are sensitive to high mass gauge bosons and fermions. Thus, they are sensitive to new physics. However, it must be kept in mind that any new effect must be consistent with already measured phenomena such as $B_{d}^{o}$ mixing and $b \rightarrow s \gamma$.

These processes are often called "Penguin" processes, for unscientific reasons [61]. A Feynman loop diagram is shown in Fig. 1.19 that describes the transition of a $b$ quark into a charged $-1 / 3 s$ or $d$ quark, which is effectively a neutral current transition. The dominant charged current decays change the $b$ quark into a charged $+2 / 3$ quark, either $c$ or $u$.

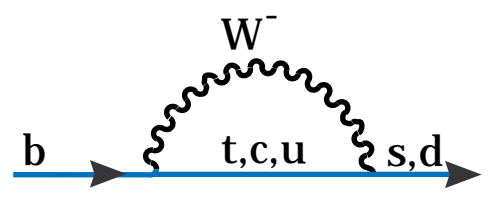

Figure 1.19: Loop or "Penguin" diagram for a $b \rightarrow s$ or $b \rightarrow d$ transition.

The intermediate quark inside the loop can be any charge $+2 / 3$ quark. The relative size of the different contributions arises from different quark masses and CKM elements. For $b \rightarrow s$, in terms of the Cabibbo angle $(\lambda=0.22)$, we have for $t: c: u-\lambda^{2}: \lambda^{2}: \lambda^{4}$. The mass dependence favors the $t$ loop, but the amplitude for $c$ processes can be quite large $\approx 30 \%$. Moreover, as pointed out by Bander, Silverman and Soni [62], interference can occur between $t, c$ and $u$ diagrams and lead to $\mathrm{CP}$ violation. In the standard model it is not expected to occur when $b \rightarrow s$, due to the lack of a CKM phase difference, but could occur when $b \rightarrow d$. In any case, it is always worth looking for this effect; all that needs to be done, for example, is to compare the number of $K^{*-} \gamma$ events with the number of $K^{*+} \gamma$ events.

There are other possibilities for physics beyond the standard model to appear. For example, the $W^{-}$in the loop can be replaced by some other charged object such as a Higgs; it is also possible for a new object to replace the $t$. 


\subsubsection{Standard Model Theory}

In the Standard Model the effective Hamiltonian for the intermediate $t$ quark is given by

$$
H_{e f f}=-\frac{4 G_{F}}{\sqrt{2}} V_{t b} V_{t s}^{*} \sum_{i=1}^{10} C_{i}(\mu) O_{i}(\mu) .
$$

Some of the operators are

$$
O_{2}=\bar{c}_{L}^{i} \gamma_{\mu} b_{L}^{i} \bar{s}_{L}^{j} \gamma^{\mu} c_{L}^{j}, \quad O_{7}=\frac{e}{16 \pi^{2}} m_{b} \bar{s}_{L}^{i} \sigma_{\mu \nu} b_{R}^{j} F^{\mu \nu} .
$$

The operators are obtained at the scale $\mu=M_{W}$ and then evolved to the $b$ mass scale using renormalization group equations, which mixes the operators:

$$
C_{i}(\mu)=\sum_{j} U_{i j}\left(\mu, M_{W}\right) C_{j}\left(M_{W}\right) .
$$

\subsection{2 $\quad b \rightarrow s \gamma$}

This process occurs when any of the charged particles in Fig. 1.19 emits a photon. The only operator which enters into the calculation is $C_{7}(\mu)$. CLEO first measured the inclusive rate [63] as well as the exclusive rate into $K^{*}(890) \gamma$ [64]. There is an updated CLEO measurement [65] using 1.5 times the original data sample and a new measurement from ALEPH [66].

To remove background CLEO used two techniques originally, one based on "event shapes" and the other on summing exclusively reconstructed $B$ samples. CLEO uses eight different shape variables [63], and defines a variable $r$ using a neural network to distinguish signal from background. The idea of the $B$ reconstruction analysis is to find the inclusive branching ratio by summing over exclusive modes. The allowed hadronic system is comprised of either a $K_{s} \rightarrow \pi^{+} \pi^{-}$candidate or a $K^{\mp}$ combined with 1-4 pions, only one of which can be neutral. The restriction on the number and kind of pions maximizes efficiency while minimizing background. It does however lead to a model dependent error. Then both analysis techniques are combined. Currently, most of the statistical power of the analysis $(\sim 80 \%)$ comes from summing over the exclusive modes.

Fig. 1.20 shows the photon energy spectrum of the inclusive signal, compared with the model of Ali and Greub [67]. A fit to the model over the photon energy range from 2.1 to 2.7 $\mathrm{GeV} / \mathrm{c}$ gives the branching ratio result shown in Table 1.7, where the first error is statistical and the second systematic.

ALEPH reduces the backgrounds by weighting candidate decay tracks in a $b \rightarrow s \gamma$ event by a combination of their momentum, impact parameter with respect to the main vertex and rapidity with respect to the b-hadron direction [66]. Their result is shown in Table 1.7, and the resulting photon energy spectrum in Fig. 1.21. The world average experimental value is also given, as well as the theoretical prediction. 


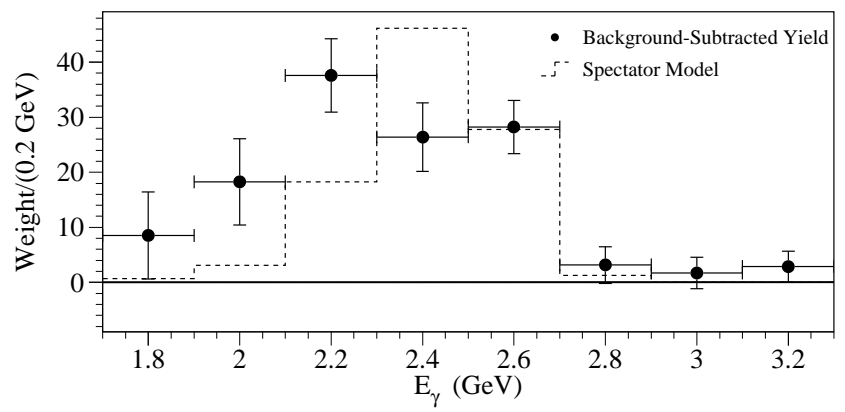

Figure 1.20: The background subtracted photon energy spectrum from CLEO. The dashed curve is a spectator model prediction from Ali and Greub.

Table 1.7: Experimental results for $b \rightarrow s \gamma$

\begin{tabular}{|lc|}
\hline Sample & branching ratio \\
\hline CLEO & $(3.15 \pm 0.35 \pm 0.41) \times 10^{-4}$ \\
ALEPH & $(3.11 \pm 0.80 \pm 0.72) \times 10^{-4}$ \\
Average & $(3.14 \pm 0.48) \times 10^{-4}$ \\
Theory[68] & $(3.28 \pm 0.30) \times 10^{-4}$ \\
\hline
\end{tabular}

The standard model prediction is in good agreement with the data. It is evaluated by including $u, c$ and $t$ loops. The $t$ loop has the CKM factors $V_{t b} V_{t s}^{*}$. The $c$ and $u$ can be combined, since by unitarity

$$
V_{c b} V_{c s}^{*}+V_{u b} V_{u s}^{*}=-V_{t b} V_{t s}^{*}
$$

The consistency with standard model expectation has ruled out many models. Hewett has given a good review of the many minimal supergravity models which are excluded by the data [69].

Triple gauge boson couplings are of great interest in checking the standard model. If there were an anomalous $W W \gamma$ coupling it would serve to change the standard model rate.

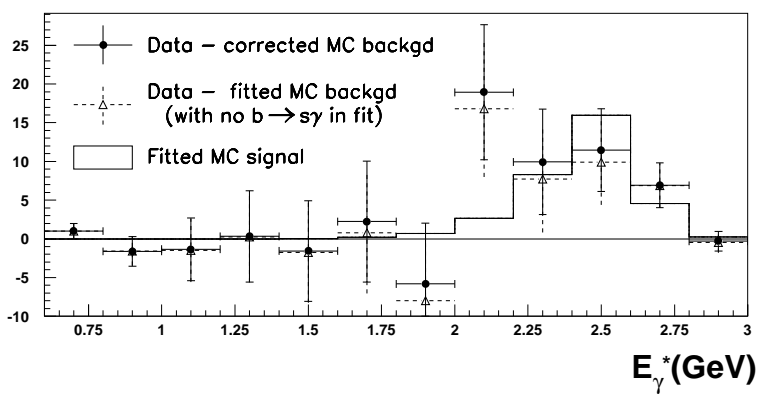

Figure 1.21: The average photon energy in the rest frame of the reconstructed $b$ jet from ALEPH. 
$p \bar{p}$ collider experiments have also published results limiting such couplings [70]. In a twodimensional space defined by $\Delta \kappa$ and $\lambda$, the D0 constraint appears as a tilted ellipse and the $b \rightarrow s \gamma$ as nearly vertical bands. In the standard model both parameters are zero.

\subsubsection{The Exclusive Decays $K^{*} \gamma$ and $\rho \gamma$}

The exclusive branching ratio is far more difficult to predict than the inclusive. CLEO measures $\mathcal{B}\left(B \rightarrow K^{*}(890) \gamma\right)=(4.2 \pm 0.8 \pm 0.6) \times 10^{-5}$, with this exclusive final state comprising $(18 \pm 7) \%$ of the total rate [54].

CLEO also limits $\mathcal{B}(B \rightarrow \rho \gamma)<1.2 \times 10^{-5}$ at $90 \%$ confidence level [54]. This leads to a model dependent limit on $\left|V_{t d} / V_{t s}\right|^{2}<0.45-0.56$, which is not very significant. It may be possible that improved measurements can find a meaningful limit, although that has been disputed [71].

\subsection{4 $b \rightarrow s \ell^{+} \ell^{-}$}

The diagrams that contribute to $b \rightarrow s \ell^{+} \ell^{-}$, where $\ell$ refers to either an electron or muon are shown in Fig. 1.22.

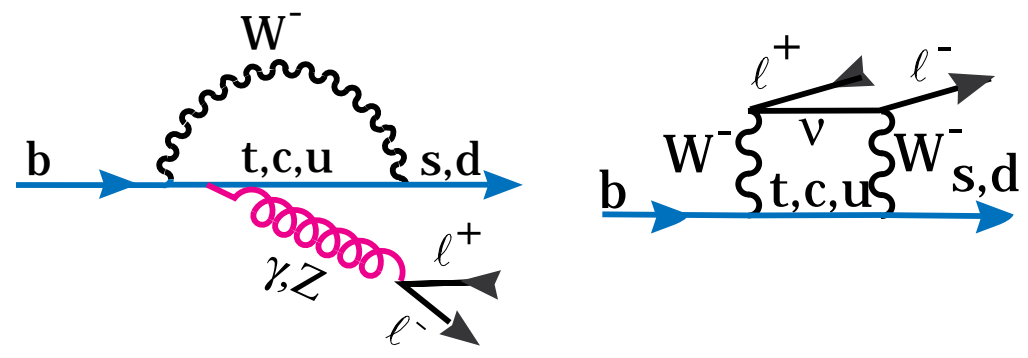

Figure 1.22: Loop or "Penguin" diagram for a $b \rightarrow s \ell^{+} \ell^{-}$transition.

The operator structure is more complicated as $C_{9}$ and $C_{10}$ contribute along with $C_{7}$. CP violation can be looked at in both the branching ratios and the polarization of the lepton pair [72]. No signals have been seen as yet in any inclusive or exclusive modes. When searching for such decays, care must be taken to eliminate the mass region in the vicinity of the $J / \psi$ or $\psi^{\prime}$ resonances, lest these more prolific processes, that are not rare decays, contaminate the sample. The results of searches are shown in Table 1.8.

$\mathrm{BTeV}$ has the ability to search for both exclusive and inclusive dilepton final states. The inclusive measurement can be done following the techniques used by CLEO to discover inclusive $b \rightarrow s \gamma$ and set upper limits on $b \rightarrow s \ell^{+} \ell^{-}$. CLEO doesn't have vertex information, so they choose track combinations assigning a kaon hypothesis to one track and pion hypotheses to the other charged tracks. They allow up to four pions, only one of which can be neutral and proceed to reconstruct each combination as if it were an exclusive decay mode. If any combination succeeds, they keep it. BTeV can improve on this procedure in two ways. First of all BTeV will have RICH $K \pi$ separation. Secondly we can insist that the charged particles 
Table 1.8: Searches for $b \rightarrow s \ell^{+} \ell^{-}$decays

\begin{tabular}{lclc}
\hline \hline$b$ decay mode & $90 \%$ c.l. upper limit & Group & Ali et al. Prediction [73] \\
\hline$s \mu^{+} \mu^{-}$ & $50 \times 10^{-6}$ & UA1 [74] & $(8 \pm 2) \times 10^{-6}$ \\
& $5.7 \times 10^{-6}$ & CLEO [75] & \\
$K^{* o} \mu^{+} \mu^{-}$ & $4.1 \times 10^{-6}$ & CDF [76] & $2.9 \times 10^{-6}$ \\
& $23 \times 10^{-6}$ & UA1 [74] & \\
& $9.5 \times 10^{-6}$ & CLEO [75] & \\
$K^{* o} e^{+} e^{-}$ & $13 \times 10^{-6}$ & CLEO [75] & $5.6 \times 10^{-6}$ \\
$K^{-} \mu^{+} \mu^{-}$ & $9.7 \times 10^{-6}$ & CLEO [75] & $0.6 \times 10^{-6}$ \\
$K^{-} e^{+} e^{-}$ & $5.4 \times 10^{-6}$ & CDF [76] & \\
\hline \hline
\end{tabular}

are consistent with coming from a $b$ decay vertex. Of course, we lose the power of the beam energy constraint that is so efficient at rejecting background at the $\Upsilon(4 S)$. However, it is a detailed question as to whether or not we more than make up the rejection power by using our advantages.

$B$ 's can also decay into dilepton final states. The Standard Model diagrams are shown in Fig. 1.23. In (a) the decay rate is proportional to $\left|V_{u b}\right|^{2} f_{B}^{2}$. The diagram in (b) is much larger for $B_{s}$ than $B_{d}$, again the factor of $\left|V_{t s} / V_{t d}\right|^{2}$. Results of searches are given in Table 1.9.
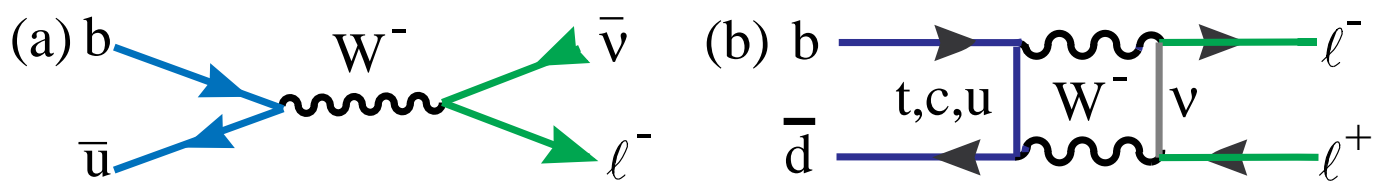

Figure 1.23: Decay diagrams resulting in dilepton final states. (a) is an annihilation diagram, and $(b)$ is a box diagram.

Table 1.9: Upper limits on $b \rightarrow$ dilepton decays (@90\% c.l.)

\begin{tabular}{lcccccc}
\hline \hline & \multicolumn{3}{c}{$\mathcal{B}\left(B^{o} \rightarrow \ell^{+} \ell^{-}\right)$} & $\mathcal{B}\left(B_{s} \rightarrow \ell^{+} \ell^{-}\right)$ & \multicolumn{3}{c}{$\mathcal{B}\left(B^{-} \rightarrow \ell^{-} \bar{\nu}\right)$} \\
& $e^{+} e^{-}$ & $\mu^{+} \mu^{-}$ & $\mu^{+} \mu^{-}$ & $e^{-} \bar{\nu}$ & $\mu^{-} \bar{\nu}$ & $\tau^{-} \bar{\nu}$ \\
\hline SM $^{\dagger}$ & $2 \times 10^{-15}$ & $8 \times 10^{-11}$ & $2 \times 10^{-9}$ & $10^{-15}$ & $10^{-8}$ & $10^{-5}$ \\
UA1 [74] & & $8.3 \times 10^{-6}$ & & & & \\
CLEO [77] & $5.9 \times 10^{-6}$ & $5.9 \times 10^{-6}$ & & $1.5 \times 10^{-5}$ & $2.1 \times 10^{-5}$ & $2.2 \times 10^{-3}$ \\
CDF [80] & & $2.0 \times 10^{-6}$ & $6.8 \times 10^{-6}$ & & & \\
ALEPH [78] & & & & & & $1.8 \times 10^{-3}$ \\
L3 [81] & & & & & $5.7 \times 10^{-4}$ \\
\hline \hline
\end{tabular}

${ }^{\dagger} \mathrm{SM}$ is the Standard Model prediction.[79]

Search for rare decays modes make up an important part of the BTeV physics program. 


\section{Bibliography}

[1] P. Langacker, "CP Violation and Cosmology," in CP Violation, ed. C. Jarlskog, World Scientific, Singapore p 552 (1989).

[2] N. Cabibbo, Phys. Rev. Lett. 10, 531 (1963); M. Kobayashi and K. Maskawa, Prog. Theor. Phys. 49, 652 (1973).

[3] L. Wolfenstein, Phys. Rev. Lett. 51, 1945 (1983).

[4] S. Stone, "Prospects For B-Physics In The Next Decade," in Techniques and Concepts of High-Energy Physics IX, ed. by T. Ferbel, NATO ASI Series, Plenum, NY (1996).

[5] M. Gaillard and B. Lee, Phys. Rev. D10, 897, (1974); J. Hagelin, Phys. Rev. D20, 2893, (1979); A. Ali and A. Aydin, Nucl. Phys. B148, 165 (1979); T. Brown and S. Pakvasa, Phys. Rev. D31, 1661, (1985); S. Pakvasa, Phys. Rev. D28, 2915, (1985); I. Bigi and A. Sanda, Phys. Rev. D29, 1393, (1984).

[6] Particle Data Group, C. Caso et al, The European Physical Journal C3 (1998) 1.

[7] Results for $\epsilon$ are from 1964. Recent results from KTeV are in agreement with earlier measurements from NA31 for a value of $\epsilon^{\prime} / \epsilon$ on the order of $2 \times 10^{-4}$.

[8] F. Bedeschi, presented at XIIIth Rencontres de Physique de la Vallee d'Aoste, La Thuile, Italy, Feb. 1999, to appear in proceedings.

[9] A. J. Buras, "Theoretical Review of B-physics," in BEAUTY '95 ed. N. Harnew and P. E. Schlein, Nucl. Instrum. Methods A368, 1 (1995).

[10] S. Stone, "Weak Decays, CKM and CP Violation," presented at The 17th Int. Workshop on Weak Interactions and Neutrinos (WIN 99) Cape Town, South Africa, January 1999, to appear in proceedings. See also, S. Stone hep-ex/9901001.

[11] J.L. Rosner, Preprint EFI-98-45 hep-ph/9809545, to appear in the Proceedings of the 16th International Symposium on Lattice Field Theory, Boulder, Colorado, 13-18 July 1998.

[12] Private communication from A. Buras. 
[13] The first papers explaining the phyiscs of mixing and CP violation in $B$ decays were A. Carter and A. I. Sanda, Phys. Rev. Lett. 45, 952 (1980); Phys. Rev. D23, 1567 (1981); I. I. Bigi and A. I. Sanda, Nucl. Phys. B193, 85 (1981); ibid B281, 41 (1987).

[14] I. Bigi, V. Khoze, N. Uraltsev, in CP Violation, ed. C. Jarlskog, World Scientific, Singapore 175 (1989).

[15] M. Beneke, G. Buchalla, I. Dunietz, Phys. Rev. D 54, 4419 (1996).

[16] M. Gronau, Phys. Rev. Lett. 63, 1451 (1989); M. Gronau and D. London, Phys. Rev. Lett. 65, 3381 (1990).

[17] C. P. Jessop, et al. (CLEO). Phys. Rev. Lett. 79, 4533 (1997).

[18] A. Ribon, "B-Physics at the Tevatron Collider," presented at Les Rencontres de Physique de la Valle d'Aoste, La Thuile, Italy, Feb. 28-March 6, 1999.

[19] The theoretical accuracy of this approach is limited by Electroweak penguins, that are expected to be rather small in this case. In principle, they can be taken into account, as pointed out by Buras and Fleischer in hep-ph/9810260, and also by Gronau, Pirjol and Yan in hep-ph/9810482.

[20] A. Dighe, I. Dunietz, and R. Fleischer, Phys. Lett. B433(1998) 147-149 (hep$\mathrm{ph} / 9804254)$.

[21] I. Dunietz, H. Quinn, A. Snyder, W. Toki, and H.J. Lipkin, Phys. Rev. D 43 (1991) 2193.

[22] A.S. Dighe, I. Dunietz, H.J. Lipkin and J.L. Rosner, Phys. Lett. B 369 (1996) 144.

[23] See, for example, M. Jacob and G.C. Wick, Ann. Phys. (N.Y.) 7 (1959) 404.

[24] B. Kayser, "Cascade Mixing and the CP-Violating Angle Beta," to appear in the Proceedings of the Moriond Workshop on Electroweak Interactions and Unified Theories, Les Arcs, France, March 1997, hep-ph/9709382. Previous work in this area was done by Y, Aimov, Phys. Rev. D42, 3705 (1990).

[25] D-S. Du and Z-T. Wei, "Test of CPT Symmetry in Cascade Decays," hep-ph/9904403 (1999).

[26] M. Gronau and D. London, Phys. Rev. Lett. 65, 3381 (1990).

[27] N. G. Deshpande, X. G. He, and S. Oh, Phys. Lett. B384 (1996) 283-287 (hep$\mathrm{ph} / 9604336)$, and references therein.

[28] A. E. Snyder and H. R. Quinn, Phys. Rev. D. 48, 2139 (1993). 
[29] Y. Gao, "Rare B Decays at CLEO (PV, s $\gamma, s \ell l, .$.$) , presented at Division of Particles$ and Fields Conference UCLA Jan., 1999. To appear in proceedings.

[30] A. Ali, G. Kramer, and C.D. Lu, Phys. Rev. D59 (1999) 014005 (hep-ph/9805403).

[31] D. Du, I. Dunietz and Dan-di Wu, Phys. Rev D34, 3414 (1986). R. Aleksan, I Dunietz, and B. Kayser, Z. Phys. C54, 653 (1992). R. Aleksan, A. Le Yaouanc, L. Oliver, O. Pène and J.-C. Raynal, Z. Phys. C67 (1995) 251 (hep-ph/9407406).

[32] D. Atwood, I. Dunietz and A. Soni, Phys. Rev. Lett. 78, 3257 (1997).

[33] M. Gronau and D. Wyler, Phys. Lett. B 265, 172 (1991).

[34] F. Wuerthwein (CLEO), "Penguins, Trees, and CP violation in B Decays," presented at DPF'99, to appear in proceedings.

[35] R. Fleischer, and T. Mannel, Phys. Rev. D57 (1998) 2752-2759 (hep-ph/9704423).

[36] M. Neubert and J. L. Rosner, Phys. Rev. Lett. 81 (1998) 5076-5079 (hep-ph/9809311).

[37] M. Gronau, and J. L. Rosner, Phys. Rev. D57 (1998) 6843-6850 (hep-ph/9711246); M. Gronau, and D. Pirjol, "A Critical Look at Rescattering Effects on $\gamma$ from $B^{+} \rightarrow K \pi$, hep-ph/9902482 (1999); M. Gronau and J. L. Rosner, "Combining CP Asymmetries in $B \rightarrow K \pi$ Decays, hep-ph/9809384 (1998).

[38] J.-M. Gerard and J. Weyers, "Isospin amplitudes and CP violation in $(B \rightarrow K \pi)$ decays," hep-ph/9711469 (1997).

[39] A. Falk, A. Kagan, Y. Nir and A. Petrov, JHU-TIPAC-97018 (December 1997).

[40] M. Neubert, "Rescattering Effects, Isospin Relations and Electroweak Penguins in $B \rightarrow$ $\pi K$ Decays," hep-ph/9712224 (1997).

[41] D. Atwood and A. Soni, "The Possibility of Large Direct CP Violation in $B \rightarrow K \pi$-Like Modes Due to Long Distance Rescattering Effects and Implications for the Angle $\gamma$," hep-ph/9712287 (1997).

[42] M. Neubert, "Model-Independent Analysis of $B \rightarrow \pi K$ Decays and Bounds on the Weak Phase $\gamma$, hep-ph/9812396 (1998).

[43] R. Fleischer, "New Strategies to Extract $\beta$ and $\gamma$ From $B_{d} \rightarrow \pi^{+} \pi^{-}$and $B_{s} \rightarrow K^{+} K^{-}$,", CERN-TH/99-79, hep-ph/9903456 (1999).

[44] I. Dunietz, "Extracting CKM Parameters from B Decays," in Proceedings of the Workshop on $B$ Physics at Hadron Accelerators, ed. P. McBride and S. Mishra, Snowmass, Co, June (1993), Fermilab-Conf-93/90-T. 
[45] For a recent calculation of $\Delta \Gamma_{s}$, see M. Beneke, G. Buchalla, C. Greub, A. Lenz and U. Nierste, preprint CERN-TH/98-261 (1998) [hep-ph/9808385].

[46] I. Dunietz, Phys. Rev. D52, 3048 (1995).

[47] A. S. Dighe, I. Dunietz, H. J. Lipkin, J. L. Rosner Phys. Lett. B369 144 (1996); R. Fleischer and I. Dunietz, Phys. Lett. B387 361 (1996); Y. Azimov and I. Dunietz Phys. Lett. B395 334 (1997);

[48] A. Dighe, I. Dunietz, and R. Fleischer, Eur. Phys. J. C6 (1999) 647-662 (hep$\mathrm{ph} / 9804253)$.

[49] R. Fleischer and I. Dunietz, Phys. Rev. D55 259 (1997); R. Fleischer, "Extracting CKM Phases from Angular Distributions of $B_{d, s}$ Decays into Admixtures of CP Eigenstates hep-ph/9903540 (1999).

[50] J. P. Silva, L. Wolfenstein, Phys. Rev. D55 (1997) 5331-5333 (hep-ph/9610208).

[51] R. Aleksan, B. Kayser and D. London, Phys. Rev. Lett. 73 (1994) 18 (hep-ph/9403341).

[52] I. Dunietz, Phys. Lett. B427 (1998) 179-182 (hep-ph/97124).

[53] D. Atwood, B. Blok and A. Soni, Int. J. Mod. Phys. A11, 3743 (1996); see also N. Deshpande, X. He \& J. Trampetic, Preprint OITS-564-REV (1994); see also J. M. Soares, Phys. Rev. D53, 241 (1996). G. Eilam, A. Ioannissian R. R. Mendel and P. Singer, Phys. Rev. D53, 3629 (1996).

[54] R. Ammar et al, "Radiative Penguin Decays of the B Meson," CLEO-CONF 96-6 (1996).

[55] G. Buchalla, A. J. Buras, and M. E. Lautenbacher, Rev. Mod. Phys., 68, 1125 (1996) (hep-ph/9512380).

[56] S. Adler, et al(E787), Phys. Rev. Lett 79, 2204 (1997).

[57] Y. Grossman, G. Isidori, M. Worah, Phys. Rev. D58 057504 (1998).

[58] S. Kopp, "Studies of $B^{\circ}$ Decays for Measuring $\sin (2 \beta)$ " presented at DPF'99, to appear in proceedings.

[59] Y. Grossman and H. R. Quinn, "Removing Discrete Ambiguities in CP Asymmetry Measurments," hep-ph/9705356 (1997).

[60] Private communication from J. Rosner, A. Buras, C. Bernard and others.

[61] K. Lingel, T. Skwarnicki and J. G. Smith, "Penguin Decays of the $b$ Quark," hepex/9804015, submitted to Annual Reviews of Nuclear and Particle Physics (1998).

[62] M. Bander, D. Silverman and A. Soni, Phys. Rev. Lett. 43, 242 (1979). 
[63] M. S. Alam et al (CLEO), Phys. Rev. Lett. 74, 2885 (1995).

[64] R. Ammar et al (CLEO), Phys. Rev. Lett. 71, 674 (1993).

[65] S. Glenn et al (CLEO), "Improved Measurement of $\mathcal{B}(b \rightarrow s \gamma)$," submitted to XXIX Int. Conf. on High Energy Physics, Vancouver, Canada, July 1998 paper ICHEP98 1011 (1998).

[66] B. Barate et al (ALEPH), "A Measurement of the Inclusive $b \rightarrow s \gamma$, Branching Ratio," CERN-EP/98-044 (1998).

[67] A. Ali and C. Greub, Phys. Lett. B 259, 182 (1991). The parameters for this fit are $<m_{b}>=4.88 \mathrm{GeV}$ and $P_{F}=250 \mathrm{MeV} / \mathrm{c}$.

[68] A. Czarnecki and W. J. Marciano, "Electroweak Radiative Corrections to $b \rightarrow s \gamma$," submitted to XXIX Int. Conf. on High Energy Physics, Vancouver, Canada, July 1998 paper ICHEP98 714 (1998); ibid Phys. Rev. Lett. 81, 277 (1998); see also see also M. Neubert, "Theoretical Status of $b \rightarrow X_{s} \gamma$ Decays," hep-ph/9809377 (1998); A. Ali, "Theory of Rare B Decays," hep-ph/9709507 DESY 97-192 (1997); N. G. Deshpande, "Theory of Penguins in B Decays," in B Decays Revised 2nd Edition, ed. by S. Stone, World Scientific, Singapore, (1994).

[69] J. L. Hewett, "B Physics Beyond the Standard Model," hep-ph/9803370 (1998).

[70] S. Abachi et al (D0), Phys. Rev. D 56, 6742 (1997); F. Abe et al (CDF), Phys. Rev. Lett. 78, 4536 (1997).

[71] D. Atwood, B. Blok \& A. Soni, Int. J. Mod. Phys. A11, 3743 (1994) and Nuovo Cimento 109A, 873 (1994); N. Deshpande, X. He \& J. Trampetic, Phys. Lett. B 362, 1996 (;) see also J. M. Soares, Phys. Rev. D 53, 241 (1996); G. Eilam, A. Ioannissian \& R. R. Mendel, Z. Phys. C71, 95 (1995).

[72] S. Fukae, C.S. Kim , T. Morozumi, and T. Yoshikawa, "A Model Independent Analysis of the Rare $B$ Decay $B \rightarrow X_{s} \ell^{+} \ell^{-}$, KEK-TH578, hep-ph/9807254 (1998), and references cited therein.

[73] A. Ali, C. Greub and T. Mannel, "Rare B Decays in the Standard Model," in Hamburg 1992, Proceedings, ECFA Workshop on a European B-meson Factory, Eds. R. Aleksan and A. Ali, p155 (1993).

[74] C. Albajar et al, Phys. Lett. B262, 163 (1991).

[75] R. Godang et al, "Search for Electroweak Penguin Decays $B \rightarrow K \ell^{+} \ell^{-}$and $B \rightarrow K^{*} \ell^{+} \ell^{-}$ at CLEO," CLEO-CONF 98-22 (1998).

[76] F. Abe et al(CDF), Phys. Rev. Lett. 76, 2015 (1996), and updates on the CDF bottom physics web page. 
[77] R. Ammar et al, Phys. Rev. D49, 5701 (1994); M. Artuso, et al, Phys. Rev. Lett. 75, 785 (1995).

[78] D. Buskulic et al, Phys. Lett. B343, 444 (1995).

[79] A. Ali and T. Mannel, Phys. Lett. B264, 447 (1991). Erratum, ibid, B274, 526 (1992).

[80] F. Abe et al (CDF), Phys. Rev. D 57, R3811 (1998).

[81] M. Acciarri et al. (L3), Phys. Lett. B 396 (1997) 327-337. 


\section{Chapter 2}

\section{Charm Physics - Important Questions and Status of the Field in the year 2005}

\section{$2.1 \quad$ Introduction}

Although there will still be many open questions in charm physics in the year 2005, one should distinguish the more important ones from those that are more mundane. The first section of this chapter outlines what we think are the most interesting issues in charm physics in the year 2005 .

The second section of this chapter addresses what charm physics would already have been done by fixed-target experiments (FOCUS, HERA-B, COMPASS) and at $e^{+} e^{-}$colliders (CLEO, BaBar, KEKB, BES) and outlines where BTeV can make important contributions.

\subsection{Important Questions in Charm Physics}

Charm decays are theoretically complex because the relatively low mass of the charm quark allows large non-perturbative strong interaction effects. Therefore, there will likely to be many open questions in charm physics, we concentrate on the most important of these, (those likely to be of general and enduring interest). Note, that we do not include issues that we think will be resolved by data collected before the year 2005 .

We divide our discussion of charm physics into three parts. The first deals with Standard Model charm physics and the second with opportunities to discover physics beyond the Standard Model. The last section deals with topics that have a connection to bottom physics.

\subsubsection{Standard Model Charm Physics}

\subsubsection{CP Violation}

Observation of Standard Model CP violation in charm decays is very unlikely prior to the year 2005. This can become a realistic possibility for BTeV. Although CP violation due to $D^{0}-\overline{D^{0}}$ 
mixing within the Standard Model is expected to be unobservably small, and direct CP violation in Cabibbo-favored and doubly-Cabibbo-suppressed decays are negligible, direct $\mathrm{CP}$ violation in some singly-Cabibbo-suppressed decay modes could be observable. For example Buccella et al. [1] have calculated the CP asymmetry for the decay $D^{+} \rightarrow \bar{K}^{* 0} K^{+}$to be $a_{C P}=2.8 \times 10^{-3}$, and the CP asymmetry for the decay $D_{s}^{+} \rightarrow K^{*+} \eta^{\prime}$ to be $a_{C P}=-8.1 \times 10^{-3}$, where the asymmetry is defined by the asymmetry in the rates:

$$
a_{C P}=\frac{\Gamma(D \rightarrow f)-\Gamma(\bar{D} \rightarrow \bar{f})}{\Gamma(D \rightarrow f)+\Gamma(\bar{D} \rightarrow \bar{f})} .
$$

These CP asymmetries would be observable if one can achieve an error of less than $1 \times 10^{-3}$ for these rate asymmetries.

Although difficult, such sensitivities are achievable in $\mathrm{BTeV}$ if the trigger is sufficiently efficient for the relevant charm decay modes. The more important issue is the reliability of these Standard Model CP asymmetry calculations. Currently they are viewed as very uncertain due to our poor knowledge of the hadronic matrix elements and strong interaction phases. This means that it would be difficult to use any measurements of $a_{C P}$ to constrain parameters of the Standard Model. However, even the observation of Standard Model CP violation in charm would be interesting in itself. There is justified hope that our knowledge of hadronic physics will improve by the year 2005 (or later) so that measurements of Standard Model CP violation in charm will provide another useful handle in our quest to understand $\mathrm{CP}$ violation and its role in nature.

\subsubsection{Strong Interaction Studies}

Another dogma that may fall with time is the notion that one cannot precisely test QCD using charm decays. Lattice QCD calculations of the bottom and charm decay constants are much better understood now than 10 years ago. For example the uncertainty in the calculations of $f_{B}$ have been reduced substantially in the last ten years [2]. By the year 2005 one can expect that the quenched approximation will no longer be needed and the Lattice QCD calculations of the charm decay constant could reach uncertainties below $\pm 5 \%$ [3]. However purely leptonic decays of charm mesons will be difficult to observe cleanly in a hadron collider experiment, so the more interesting tests of QCD will be done with form factors measured using semileptonic decays of charm. One can expect that by the year 2005 these form factors can be calculated using Lattice QCD with uncertainties approaching the \pm few $\%$ level, at least for the zero recoil point, i.e. at maximum $q^{2}$. (The 4-momentum of the lepton pair in the semileptonic decay is denoted by $q^{2}$ ). Although the uncertainty in the calculations will increase at lower $q^{2}$ due to the finite lattice spacing, one expects no obstacles for future calculations to reach the same level of uncertainty as experimental measurements.

If one assumes unitarity for the three generation CKM matrix, the coupling $\left|V_{c d}\right|$ and $\left|V_{c s}\right|$ are known very well within the Standard Model. This means that experimental measurements of semileptonic charm decays can give precise determinations of the form factors in the decay and thus can be compared to Lattice QCD calculations to test QCD. One expects 
this to be an important field of study by the latter part of the next decade, and one where more precise data would be required.

Alternatively, one can use precise measurements of semileptonic decay rates to make precise direct measurements of $\left|V_{c s}\right|$ and $\left|V_{c d}\right|$ to test unitarity of the 3 generation CKM matrix. This assumes that, in the future, theoretical uncertainties in the form factors can be controlled at the $1 \%$ level. A $1 \%$ uncertainty in the decay rate corresponds to a $0.5 \%$ uncertainty in $\left|V_{c q}\right|$ when theoretical errors in the form factors are not included. Figure 2.1(a) shows the current values of the direct measurements on these CKM parameters together with the unitarity constraint coming from the direct measurement of $\left|V_{c b}\right|$ [13]. Figure 2.1(b) shows possible $0.5 \%$ measurements which could reveal violations in the unitarity of the Standard Model 3 generation CKM matrix. The unitarity constraint gives the CKM values given by the small box in the figure.
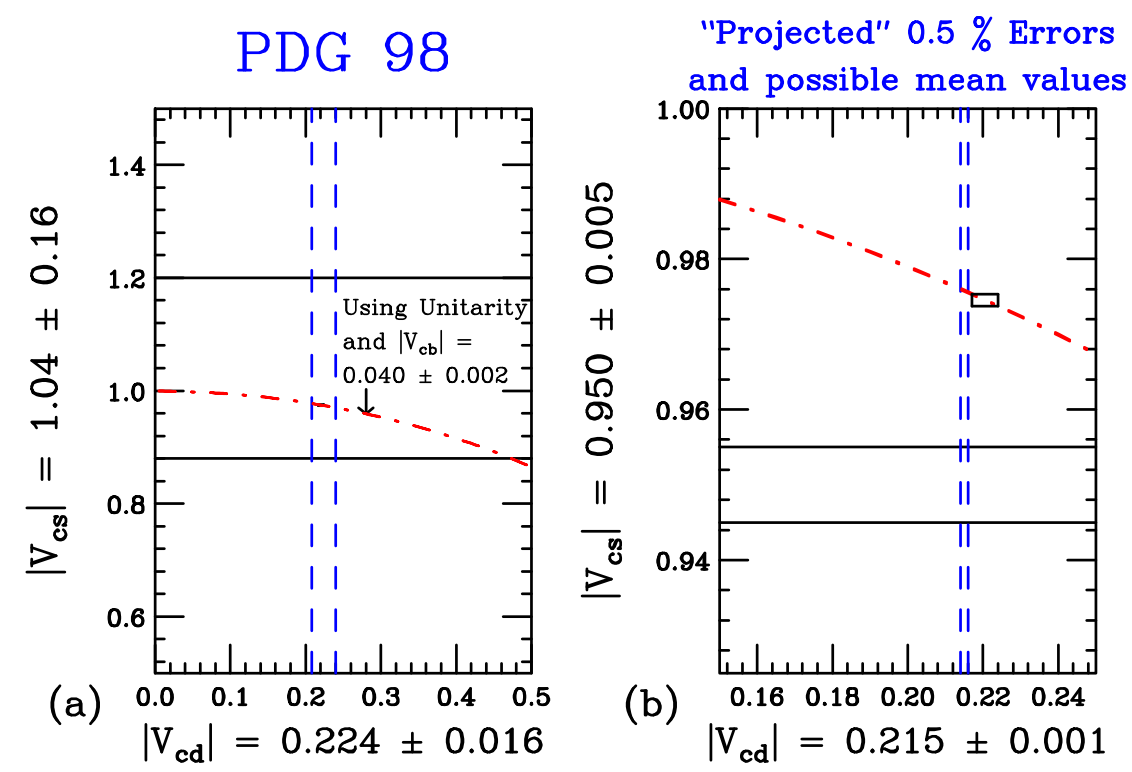

Figure 2.1: (a) Current direct measurements of $\left|V_{c s}\right|$ and $\left|V_{c d}\right|$ together with the unitarity constraint using $\left|V_{c b}\right|=0.040 \pm 0.002$ from PDG [13]. (b) Projected possible measurements for $\left|V_{c s}\right|$ and $\left|V_{c d}\right|$ with $1 \%$ errors in the semileptonic decay rates. (No theory errors are included). The small box gives the current $90 \%$ C.L. limits for $\left|V_{c s}\right|$ and $\left|V_{c d}\right|$ assuming unitarity and the circular band is just the $1 \sigma$ band using $\left|V_{c b}\right|$ alone.

Another area of enduring interest is the observation of particles expected in the Standard Model, but not yet observed. In this case we mean baryons containing two or three charm quarks. For example, baryons with the quark contents ccq, cbq or ccc. These baryons are expected within the quark model. Their observation and measurements of their properties and comparison with expectations would be very interesting. 


\subsubsection{Charm as a Probe of New Physics}

One virtue of charm decays that has been growing in recognition is their potential for discovery of physics beyond the Standard Model. The areas of interest that have been discussed quite extensively are $D^{0}-\overline{D^{0}}$ mixing, $\mathrm{CP}$ violation, and decays that are rare or forbidden in the Standard Model. The virtue which makes these phenomena interesting is that they are predicted to be negligibly small within the Standard Model. As a result there is a large discovery window, as absence of Standard Model "background" means that enhancements due to new physics do not have to be large to be observable.

The usefulness of each phenomenon depends on how reliably the Standard Model rates for each can be calculated, and thereby how large a window of opportunity there exists for discovery of new physics. Also any enhancements due to new physics have to be compared to all relevant existing experimental constraints to ensure compatability.

\subsubsection{1 $\quad D^{0}-\overline{D^{0}}$ Mixing}

Probably the most theoretically studied aspect of charm physics as a tool for the discovery of new physics is $D^{0}-\overline{D^{0}}$ mixing. Standard Model "short-distance" (1-loop) contributions to $D^{0}-\overline{D^{0}}$ mixing have been known to be small for a long time now. The Standard Model "long-distance" contributions (due to final state hadronic effects as opposed to quark degrees of freedom) are not well known since they are inherently nonperturbative. Calculations of the long-distance effects used to range from very small to very large [4], but it appears there is now a consensus on the size of these long-distance effects. Although they are larger than the short-distance effects, they are still small enough that a sizable window of opportunity exists for the discovery of new physics in $D^{0}-\overline{D^{0}}$ mixing $[5,6]$.

Experimental analyses of $D^{0}-\overline{D^{0}}$ mixing study the ratio of the wrong-sign to right-sign decay rates:

$$
r_{D}=\frac{\Gamma\left(D^{0} \rightarrow \ell^{-} X\right)}{\Gamma\left(D^{0} \rightarrow \ell^{+} X\right)} \approx \frac{1}{2}\left[\left(\frac{\Delta m_{D}}{\Gamma}\right)^{2}+\left(\frac{\Delta \Gamma_{D}}{2 \Gamma}\right)^{2}\right] .
$$

Where the relationship to $\Delta m_{D} / \Gamma$ and $\Delta \Gamma_{D} / \Gamma$ assumes that $\Delta m_{D} / \Gamma$ and $\Delta \Gamma_{D} / \Gamma \ll 1$. The current best published experimental limit from semileptonic decays is $r_{D}<5 \times 10^{-3}$ [7]. If we assume that $\Delta \Gamma_{D} / \Gamma$ can be neglected this translates into an upper limit for the mass difference

$$
\begin{gathered}
\frac{\Delta m_{D}}{\Gamma}<0.1 \\
\Delta m_{D}<1.6 \times 10^{-13} \mathrm{GeV} / \mathrm{c}^{2}
\end{gathered}
$$

The Standard Model short-distance contribution comes from W box diagrams with internal $d, s$ and $b$ quarks. Its value is given by

$$
\Delta m_{D}^{S D} \approx 2.5 \times 10^{-17} \mathrm{GeV}\left(\frac{m_{s}}{0.3 \mathrm{GeV}}\right)^{4}\left(\frac{f_{D}}{f_{\pi}}\right)^{2}
$$


So that $\Delta m_{D}<2 \times 10^{-16} \mathrm{GeV}$, or $\Delta m_{D} / \Gamma<1 \times 10^{-4}$. Again assuming that $\Delta \Gamma_{D} / \Gamma$ is negligible, that translates into $r_{D}<10^{-8}-10^{-10}$ or even smaller.

The Standard Model long-distance effects have been computed via two different methods: a dispersive approach (using current data on intermediate hadronic particle states that mediate the transition) and an approach that uses Heavy Quark Effective Theory (HQET). The dispersive approach using current experimental data yields [5]

$$
\frac{\Delta m_{D}}{\Gamma} \approx 8 \times 10^{-4} \times(1.46-\sqrt{b})
$$

where $b$ is defined by

$$
\frac{\Gamma\left(D^{0} \rightarrow K^{+} \pi^{-}\right)}{\Gamma\left(D^{0} \rightarrow K^{-} \pi^{+}\right)}=b \times \tan ^{4} \theta_{C}
$$

The current value for Doubly Cabibbo Suppressed decay gives $\Delta m_{D}^{L D} / \Gamma \approx 2 \times 10^{-4}$, i.e. not much larger than the short-distance contribution. The calculation using the dispersive approach is not complete in that not enough data is available on all the two particle decay modes. The calculation uses the branching fractions of the $D^{0}$ into two particle intermediate states that can bring about the transition from $D^{0}$ to $\overline{D^{0}}$ and vice versa. However only enough information exists for the pseudoscalar-pseudoscalar two particle states $(K K, \pi \pi$ and $K \pi)$. The sizes of contributions from pseudoscalar-vector and vector-vector two particle states are uncertain but could be more kinematically suppressed than for PP. The sign of these contributions is also uncertain and can cause cancellations. Although one cannot be $100 \%$ sure that these PV and VV contributions are not in fact large without more data, a good estimate is to assume similar sizes for the contributions from these PV and VV states. In this case one gets $r_{D} \approx 10^{-8}$.

The second method of calculating the long-distance contribution uses HQET and gives $[5]$

$$
\frac{\Delta m_{D}}{\Gamma} \approx(1-2) \times 10^{-5}
$$

which is about an order of magnitude smaller than that given by the dispersive method. This may indicate that there are cancellations in the sum of the dispersive contributions that are not yet accounted for.

We conclude that the Standard Model contribution to $D^{0}-\overline{D^{0}}$ mixing is given by $r_{D} \approx$ $10^{-8}$ (and $\Delta m \approx 2 \times 10^{-17} \mathrm{GeV}$ ). This means there is a window of about 5 orders of magnitude in which to search for physics beyond the Standard Model.

The contributions to $D^{0}-\overline{D^{0}}$ mixing from extensions to the Standard Model have been studied and summarized by Burdman [5], Hewett [8], and Pakvasa [9, 10]. New physics scenarios that can give rise to large observable, above Standard Model $D^{0}-\overline{D^{0}}$ mixing are summarized below (note that this list is by no means complete).

1. Two Higgs Doublet Models - In this extension of the Standard Model there are two models of how the quark masses arise; these are called Model I and Model II in the literature $[11,12]$. Model I can only modify the Standard Model result for $\Delta m_{D}$ for 
very small values of $\tan \beta$, and this is already excluded by existing data on $b \rightarrow s \gamma$ and $B_{d}^{0}-\overline{B_{d}^{0}}$ mixing. In Model II the extra contribution due to box diagrams with $W^{ \pm}$ replaced by charged Higgs can produce large $\Delta m_{D}$ for large values of $\tan \beta$, and possibly observable effects for charged Higgs masses below about $250 \mathrm{GeV} / \mathrm{c}^{2}$. See Figure 2.2(b) taken from reference [8]. This is an area where charm physics is complementary to bottom physics in that different regions of $\tan \beta$ are tested.

2. Heavy $Q=-1 / 3$ Quark - This could be an extension of the Standard Model involving four generations with heavy neutrinos or just a singlet $Q=-1 / 3$ quark. This heavy quark would participate in the box diagrams and enhance $\Delta m_{D}$ as long as the product of the couplings $\left|V_{c b^{\prime}} V_{u b^{\prime}}^{*}\right|$ is large enough. Direct searches have placed a limit $m_{b^{\prime}}>128$ $\mathrm{GeV} / \mathrm{c}^{2}[13]$ and for the case of a $b^{\prime}$ belonging to a fourth generation, current data on the CKM matrix gives the upper limits

$$
\left|V_{u b^{\prime}}\right|<0.08,\left|V_{c b^{\prime}}\right|<0.6
$$

Values of $\Delta m_{D}>10^{-15}$ up to the experimental limit are possible for $m_{b^{\prime}} \approx 100-200$ $\mathrm{GeV} / \mathrm{c}^{2}$ and $\left|V_{c b^{\prime}} V_{u b^{\prime}}^{*}\right|>0.001$. See Figure 2.2(a) taken from reference [8].

3. Tree-Level Flavor Changing Neutral Currents - Any tree-level FCNC must satisfy the constraints imposed by experimental measurements of $K^{0}$ and $B^{0}$ mixing and rare $K$ and $B$ decays. One model which can do this and still produce large values of $\Delta m_{D}$ is one with a Flavor Changing Neutral Higgs. Values of $\Delta m_{D}$ as large as $10^{-13} \mathrm{GeV}$ are possible [8]. Topcolor assisted technicolor models [14] which generate top-pions that couple to the up-type quarks can produce values of $\Delta m_{D}$ as large as $8 \times 10^{-14} \mathrm{GeV}$ [15], and composite technicolor schemes can produce $\Delta m_{D}$ as large $4 \times 10^{-15} \mathrm{GeV}$ [16].

4. Supersymmetry - Although in the Minimal Supersymmetric Standard Model the additional contributions to $\Delta m_{D}$ from squark and gluinos box diagrams are very small $\approx 10^{-18}$ [17], there are alternative ways proposed to link the squark mass matrix with the quark mass matrix which can produce values of $\Delta m_{D}$ as large as the experimental limit [18]. Different approaches to handle FCNC in supersymmetry can also produce sizable values of $\Delta m_{D}[19]$.

5. Leptoquarks - Contributions to $\Delta m_{D}$ via box diagrams involving scalar leptoquark bosons with charged leptons or neutrinos are possible [20]. Values of $\Delta m_{D}$ up to the current experimental limit are theoretically possible, though in fact the limit on $\Delta m_{D}$ has been used to place constraints on the allowed leptoquark coupling and mass [8].

6. Family Symmetry - A gauged family symmetry proposed by Volkov, Monich and Struminski [21] can produce interesting effects in charm while still satisfying the constraints from experimental measurements of kaon physics. Values as large as $10^{-13} \mathrm{GeV}$ are possible for $\Delta m_{D}[9]$. 
So far we have completely neglected $\Delta \Gamma$ even though it contributes to $r_{D}$ as can be seen from equation (2.2). Within the Standard Model $\Delta \Gamma$ is expected to be small and of the same order of magnitude as $\Delta m[4,22]$. The $\Delta \Gamma$ contribution is just the absorptive part of the long-distance diagrams that determine $\Delta m$ which were already discussed earlier [6, 23]. This means that the $\Delta \Gamma$ contribution may be neglected when we consider the approximate size of the Standard model contribution to $r_{D}$. The new physics contributions we discussed contribute to $\Delta m$ and are generally assumed to not contribute to $\Delta \Gamma$. If we wish to constrain $\Delta m$ in order to reduce the parameter space of new physics scenarios one needs to make measurements of $\Delta \Gamma$ or set a limit on its size. This can easily be done if one measures the lifetime difference between a CP-even eigenstate like $D^{0} \rightarrow K^{+} K^{-}, \pi^{+} \pi^{-}$and a CP-odd eigenstate like $D^{0} \rightarrow K_{s}^{0} \rho, K_{s}^{0} \phi$. An alternative way is to measure the lifetime difference for a mixed state like $D^{0} \rightarrow K^{-} \pi^{+}$and compare this to say the lifetime of a CP-even eigenstate. Under certain assumptions the mixed state $D^{0} \rightarrow K^{-} \pi^{+}$is composed of known relative sizes of the two CP-even and CP-odd eigenstates [24], thus $\Delta \Gamma$ may also be extracted from these lifetime difference measurements [25]. The relationship of these measured lifetime differences to $\Delta \Gamma$ becomes more complicated if sizable $\mathrm{CP}$ violation exists [26].

Current mixing limits without using knowledge of $\Delta \Gamma$ produce an allowed region in the parameter space of $\Delta m / \Gamma$ versus $\Delta \Gamma / 2 \Gamma$. For current limits even a few $\%$ measurement of $\Delta \Gamma / 2 \Gamma$ can reduce this allowed parameter region, e.g. see Figure 2.3.

\subsubsection{CP Violation}

While Standard Model predictions for CP asymmetries can be as high as few $\times 10^{-3}$ in some cases, this is still very small compared to the current experimental limits of the order of 0.05-0.10 [13]. Less theoretical work has been done in predicting CP asymmetries in charm decays within the Standard Model or due to new physics scenarios. This may change by the year 2005, but at present a search for $\mathrm{CP}$ violation is a less clean way in general to search for new physics than a search in $D^{0}-\overline{D^{0}}$ mixing.

It is useful to split the discussion into the types of $\mathrm{CP}$ violation that could occur.

- Direct $C P$ Violation - This is a non-zero value of $a_{C P}$ (defined in equation (2.1)). For this to occur, there has to be at least two amplitudes that give rise to the same final state. There must be two different relative phases associated with these amplitudes, one $(\Delta \alpha)$ that does not change sign under CP (such as due to final state interactions) and a weak phase difference $(\Delta \phi)$ that does [28]:

$$
\begin{aligned}
& A(D \rightarrow f)=\left|M_{1}\right|+e^{i \Delta \alpha^{\text {strong }}} e^{i \Delta \phi^{\text {weak }}}\left|M_{2}\right| \\
& A(\bar{D} \rightarrow \bar{f})=\left|M_{1}\right|+e^{i \Delta \alpha^{\text {strong }}} e^{-i \Delta \phi^{\text {weak }}}\left|M_{2}\right|
\end{aligned}
$$

then $a_{C P}$ is proportional to $\sin \left(\alpha_{1}-\alpha_{2}\right) \sin \left(\phi_{1}-\phi_{2}\right)$ :

$$
a_{C P}=\frac{2 \rho \sin \Delta \alpha^{\text {strong }} \sin \Delta \phi^{\text {weak }}}{1+\rho^{2}+2 \rho \cos \Delta \alpha^{\text {strong }} \cos \Delta \phi^{\text {weak }}}
$$


where $\rho=\left|M_{2} / M_{1}\right|$. To lowest order within the Standard Model, this condition can only occur for Cabibbo suppressed decays and not for Cabibbo-favored or doublyCabibbo-suppressed decays [5, 10]. This means that a particularly clean window for searches for new physics is to observe CP asymmetries in Cabibbo-allowed decays. For example Left-Right Symmetric models can give sizable values of $a_{C P} \sim 10^{-2}$ in Cabibbo-allowed decays [10, 29]. Note that small $\left(\sim 10^{-4}\right) \mathrm{CP}$ asymmetries are possible in certain specific Cabibbo-favored decay modes in the Standard Model, due to an interference with the doubly-Cabibbo-suppressed decay mode, for example in $D \rightarrow$ $K_{s}^{0} \pi,\left(K_{s}^{0} \pi\right)_{K^{*}} \pi$ decays [30].

In the Standard Model weak phases enter through the CKM matrix so relative weak phases can occur in Cabibbo suppressed decays via the interference of spectator and penguin amplitudes [5]. The difficulty is in evaluating the relative strong phase between the spectator and penguin amplitudes. Specific model calculations for $D \rightarrow K K, \pi \pi, K^{*} K$ yield $a_{C P} \leq 10^{-3}$ though higher values in some cases are possible [1]. Despite the uncertainties in evaluating the relative strong phases, an observation of $\mathrm{CP}$ asymmetries of $\geq 10^{-2}$ would indicate new physics in most cases. By the year 2005 , one can hope that the theoretical picture will be clearer and that this window of discovery would thus be cleaner. The way that new physics scenarios could produce large CP asymmetries is, for example, through extra phases, maybe large phases in the penguin diagram. Although it seems that $\mathrm{CP}$ asymmetries of $\geq 10^{-2}$ are possible in new physics scenarios, there are not yet clear theoretical calculations.

- Indirect $C P$ Violation - This involves $D^{0}-\overline{D^{0}}$ mixing and thus can only occur in $D^{0}$ decay modes. In the Standard Model, CP violation in mixing is very small $a_{C P} \sim 10^{-4}$ [10]. New physics can increase $\Delta m_{D}$ to produce larger mixing which can generate CP asymmetries of the order $\Delta m_{D} / \Gamma[5]$. This means that indirect CP asymmetries of the order of $10^{-2}-10^{-1}$ could be possible in new physics scenarios.

\subsubsection{Rare Decays and Forbidden Decays}

What makes rare decays interesting is not just that these decay rates are small as this does not by itself constitute a good window for the search for new physics. Rather, it is their sensitivity to new physics. The rare decays of interest are ones which transform $c \rightarrow u$ in some form, i.e. contain Flavor Changing Neutral Currents. The new physics scenarios that enhance FCNC in charm leading to large observable $D^{0}-\overline{D^{0}}$ mixing may also increase specific rare FCNC charm decays. Unfortunately, although the Standard Model short-distance contributions to these rare decays gives very small decay rates, Standard Model long-distance effects can be very large and are likely to overwhelm any increase in the rates caused by modifications of the short-distance 1-loop contributions by new physics. As usual, the long-distance contributions are not known with much certainty, however there has been much recent theoretical work trying to evaluate the size of the long-distance contributions and, at least, put upper limits on their size. In this way, some rare decays have been identified as more interesting than others in the search for new physics. 
The rare decays of interest are $c \rightarrow u \gamma$ and $c \rightarrow u \ell^{+} \ell^{-}$, the latter occurring either as $D^{0} \rightarrow \ell^{+} \ell^{-}$or $c \rightarrow u \ell^{+} \ell^{-}$. There are other decays of interest that are forbidden within the Standard Model, Lepton Family Number Violating decays $\left(D \rightarrow X \ell_{1}^{+} \ell_{2}^{-}\right)$and Lepton Number Violating decays $\left(D \rightarrow X \ell^{+} \ell^{+}\right)$.

1. $c \rightarrow u \gamma$ - Unlike $b \rightarrow s \gamma$, the short-distance contributions to $c \rightarrow u \gamma$ are very small within the Standard Model due to the absence of a very heavy down-type quark in the 1-loop contribution and to the fact that the b-quark CKM couplings $\left(\left|V_{c b}\right|\right.$ and $\left.\left|V_{u b}\right|\right)$ are small. Before perturbative QCD corrections the bare electroweak penguin for $c \rightarrow u \gamma$ yields a tiny branching ratio of $10^{-17}$, which is enhanced by perturbative QCD corrections to $10^{-12}[31,32]$. The huge QCD corrections for $c \rightarrow u \gamma$ suggest that there may be even larger contributions from long-distance effects. In fact, many exclusive $D \rightarrow X \gamma$ modes have been calculated based on consideration of long distance effects to have branching ratios in the $10^{-4}-10^{-6}$ range $[31,32]$. This means that any contribution of new physics to $c \rightarrow u \gamma$ would have to be extremely large in order to dominate the long-distance effects. For example a heavy $Q=-1 / 3$ quark could increase the $c \rightarrow u \gamma$ branching ratio to as high as $10^{-8}$ for sufficently large CKM factors [8], but this is still tiny compared to long-distance effects. An interesting alternative method for searching for new physics in $c \rightarrow u \gamma$ decays is not to compare a single measured rate to the Standard Model theory, but to compare two particular decays, e.g. $D \rightarrow \rho \gamma$ and $D \rightarrow K^{*} \gamma$ [33]. Observing $B(D \rightarrow \rho \gamma) / B\left(D \rightarrow K^{*} \gamma\right)$ significantly different from $\tan ^{2} \theta_{C}$ may indicate new physics that contributes to $c \rightarrow u \gamma$ and thus to $D \rightarrow \rho \gamma$ but not to $D \rightarrow K^{*} \gamma$, e.g. in non-minimal SUSY [34]. However this may be spoilt by long-distance effects which will produce uncertainties in this ratio.

2. $D^{0} \rightarrow \ell^{+} \ell^{-}-$The short-distance contribution to the $D^{0} \rightarrow \mu^{+} \mu^{-}$branching ratio is $10^{-19}$ while long distance contributions increase this to as high as $10^{-15}[5,9]$. New physics, for example an additional Higgs doublet or a heavy $Q=-1 / 3$ quark, could enhance this rate to the $10^{-10}$ level [9]. Thus any observation at a large rate compared to $10^{-15}$ would signify new physics.

3. $c \rightarrow u \ell^{+} \ell^{-}-$Since $D^{0} \rightarrow \ell^{+} \ell^{-}$is helicity suppressed, $10^{-3}$ for muons and $10^{-7}$ for electrons, it is thought that any new physics that contributes to $c \rightarrow u$ might produce larger observable rates in $c \rightarrow u \ell^{+} \ell^{-}$modes which do not have this suppression. The Standard Model short-distance contribution to $B\left(c \rightarrow u \ell^{+} \ell^{-}\right)$is $10^{-8}$ while longdistance contributions gives $10^{-6}$ [35], e.g. $D \rightarrow \pi \ell^{+} \ell^{-}$is calculated to be $10^{-6}$ [36]. The long-distance effects are thus still rather large and most new physics scenarios are unlikely to produce large enough rates. The current experimental limits for these decay modes leave little room for new physics discoveries, (see Table 2.1).

4. $c \rightarrow u \nu \bar{\nu}$ - The Standard Model prediction for this process is very small, the shortdistance contribution to the inclusive process is a $\mathrm{few} \times 10^{-15}$ while the long-distance contributions to specific exclusive modes is also very small: $B\left(D^{0} \rightarrow \pi^{0} \nu \bar{\nu}\right) \sim 6 \times 10^{-16}$ 
and $B\left(D^{+} \rightarrow \pi^{+} \nu \bar{\nu}\right) \sim 8 \times 10^{-16}[9]$. However these decays are extremely difficult to observe experimentally.

5. $D^{0} \rightarrow \gamma \gamma-$ This is another mode that is experimentally difficult to observe. The shortdistance contribution to this branching ratio is $10^{-16}$ while long-distance contributions increase this to as high as $10^{-9}[9]$.

6. $L F N V$ and $L N V$ Decays - These forbidden decays may offer an opportunity to discover new physics. There is little theoretical work on their expected size in charm in new physics scenarios. The LFNV decay $D^{0} \rightarrow \mu^{ \pm} e^{\mp}$ is forbidden in the Standard Model with zero mass neutrinos. A fourth generation model with massive neutrinos and non-zero neutrino mixing could in theory mediate $D^{0} \rightarrow \mu^{ \pm} e^{\mp}$ in box diagrams with internal massive neutrinos. However, to satisfy constraints from current experimental limits, this branching ratio must be smaller than $10^{-22}$ [9]. New physics that could produce larger branching fractions than this could be tested, e.g. leptoquarks [8]. A gauged family symmetry could generate a branching ratio for $D^{0} \rightarrow \mu^{ \pm} e^{\mp}$ of $10^{-13}$. It is unclear what new physics scenarios could increase the rates of these decays so that they can be at an observable level.

\subsubsection{Charm as a tool for Bottom Physics}

Since bottom quarks decay predominantly to charm quarks, some knowledge of charm physics is sometimes required to extract interesting physics from measurements of bottom particle decays. In some cases the uncertainty in our knowledge of charm physics may become the limiting factor in our understanding of bottom decays.

The most obvious quantities that are needed are the absolute branching fractions of the various charm particles. The absolute branching fractions of the $D^{0}$ and $D^{+}$are measured fairly well and by the year 2005 they should be measured with sufficient accuracy for any physics we would be interested in at that time. On the other hand measurements of the $D_{s}$ and $\Lambda_{c}$ absolute branching fractions are not known accurately. Their determination from experimental measurements are model dependent. This should improve with time. On the other hand the absolute branching fractions of the other charm baryons are not currently known at all.

There are other quantities of interest that are less obvious. For example, although $c \rightarrow u \gamma$ and $c \rightarrow u \ell^{+} \ell^{-}$decays may not end up being particularly good places to look for new physics, it is a good place to try to understand Standard Model long-distance effects. Similar longdistance effects are present in $B \rightarrow \rho \gamma$ and these are comparable in size to the penguin contribution in this decay. They must be corrected for before an accurate extraction of $\left|V_{t d}\right| /\left|V_{t s}\right|$ can be made from a measurement of $B(B \rightarrow \rho \gamma) / B\left(B \rightarrow K^{*} \gamma\right)$. Long-distance effects can also be studied in $D^{0} \rightarrow \bar{K}^{* 0} \mu^{+} \mu^{-}$and $D_{s}^{+} \rightarrow \rho^{+} \mu^{+} \mu^{-}$which are predicted to have large branching ratios $\left(2 \times 10^{-6}\right.$ and $3 \times 10^{-5}$ respectively) [38] and should be observable soon. 
Table 2.1: Experimental limits and Standard Model expectations for rare decays. The experimental limits come from [13] except for $D^{0} \rightarrow \rho^{0} \gamma, \phi \gamma, \bar{K}^{* 0} \gamma$ [37]. The Standard Model predictions (dominated by long-distance effects) come from references [8, 31, 32, 36, 38].

\begin{tabular}{ccc}
\hline \hline Decay & Exp. Limit & SM expectation \\
\hline \hline FCNC & & \\
\hline$D^{0} \rightarrow \mu^{+} \mu^{-}$ & $4.1 \times 10^{-6}$ & $<3 \times 10^{-15}$ \\
$D^{0} \rightarrow \pi^{0} e^{+} e^{-}$ & $4.5 \times 10^{-5}$ & \\
$D^{0} \rightarrow \pi^{0} \mu^{+} \mu^{-}$ & $1.8 \times 10^{-4}$ & \\
$D^{0} \rightarrow \bar{K}^{0} e^{+} e^{-}$ & $1.1 \times 10^{-4}$ & $<2 \times 10^{-15}$ \\
$D^{0} \rightarrow \bar{K}^{0} \mu^{+} \mu^{-}$ & $2.6 \times 10^{-4}$ & $<2 \times 10^{-15}$ \\
$D^{+} \rightarrow \pi^{+} e^{+} e^{-}$ & $6.6 \times 10^{-5}$ & $\sim 1 \times 10^{-6}$ \\
$D^{+} \rightarrow \pi^{+} \mu^{+} \mu^{-}$ & $1.8 \times 10^{-5}$ & $\sim 1 \times 10^{-6}$ \\
$D^{+} \rightarrow K^{+} e^{+} e^{-}$ & $2.0 \times 10^{-4}$ & $<10^{-15}$ \\
$D^{+} \rightarrow K^{+} \mu^{+} \mu^{-}$ & $9.7 \times 10^{-5}$ & $<10^{-15}$ \\
$D^{0} \rightarrow \rho^{0} \gamma$ & $2.4 \times 10^{-4}$ & $(0.1-6.0) \times 10^{-5}$ \\
$D^{0} \rightarrow \phi \gamma$ & $1.9 \times 10^{-4}$ & $(0.1-3.4) \times 10^{-5}$ \\
$D^{0} \rightarrow \bar{K}^{*} \gamma$ & $7.6 \times 10^{-4}$ & $(0.7-8.0) \times 10^{-4}$ \\
$D^{+} \rightarrow \rho^{+} \gamma$ & & $(0.4-6.3) \times 10^{-5}$ \\
$D_{s}^{+} \rightarrow \rho^{+} \gamma$ & & $(0.6-3.8) \times 10^{-4}$ \\
$D^{0} \rightarrow \rho^{0} \mu^{+} \mu^{-}$ & $2.3 \times 10^{-4}$ & $(3.5-4.7) \times 10^{-7}$ \\
$D^{0} \rightarrow \bar{K}^{* 0} \mu^{+} \mu^{-}$ & $1.2 \times 10^{-3}$ & $(1.6-1.9) \times 10^{-6}$ \\
$D^{+} \rightarrow \rho^{+} \mu^{+} \mu^{-}$ & $5.6 \times 10^{-4}$ & $(1.5-1.8) \times 10^{-6}$ \\
$D_{s}^{+} \rightarrow \rho^{+} \mu^{+} \mu^{-}$ & & 0 \\
\hline $\mathrm{LF}$ or LN Viol. $\mathrm{LN}^{ \pm}$ & $\sim 3 \times 10^{-5}$ \\
\hline$D^{0} \rightarrow \mu^{ \pm} e^{\mp}$ & $1.9 \times 10^{-5}$ & 0 \\
$D^{+} \rightarrow \pi^{+} \mu^{ \pm} e^{\mp}$ & $1.3 \times 10^{-4}$ & 0 \\
$D^{+} \rightarrow K^{+} \mu^{ \pm} e^{\mp}$ & $1.3 \times 10^{-4}$ & 0 \\
$D^{+} \rightarrow \pi^{-} \mu^{+} \mu^{+}$ & $8.7 \times 10^{-5}$ & 0 \\
$D^{+} \rightarrow K^{-} \mu^{+} \mu^{+}$ & $1.2 \times 10^{-4}$ & \\
$D^{+} \rightarrow \rho^{-} \mu^{+} \mu^{+}$ & $5.6 \times 10^{-4}$ & \\
\hline \hline
\end{tabular}


The branching ratio for the purely leptonic decay $B^{-} \rightarrow \ell^{-} \nu_{\ell}$ is expected to be small $\left(10^{-5}\right)$ and probably not observable in the near future for a direct extraction of the value of $f_{B}$. However the uncertainty in $f_{B}$ is a major source of uncertainty in the determination of $\rho$ (in the usual CKM triangle) from fits to the CKM parameters. Also, the main uncertainty in the estimate of $x_{s} \equiv(\Delta m / \Gamma)_{B_{s}}$ is associated with $f_{B_{s}}$ [39]. A better value of $f_{B}$ and $f_{B_{s}}$ may be derived from measurements of $f_{D}$ and $f_{D_{s}}$ since their ratios can be more reliably calculated than the individual decay constants [40]. However, precision measurements of $f_{D}$ and $f_{D_{s}}$ is also difficult, especially in a hadron collider. Precise tests of form factors may give confidence in the theoretical methods used to calculate $f_{D}$ and $f_{D_{s}}$.

\subsection{Status of Charm Physics in the year 2005}

By the year 2005 there could be many experiments with very large samples of charm decays. The photoproduction fixed target experiment FOCUS has already collected data and has about $10^{6}$ fully reconstructed $D^{0}$ and $D^{+}$decays [41]. The future COMPASS hadroproduction experiment is expected to collect enough data to reconstruct over $5 \times 10^{6}$ charm hadrons [42]. There will be several $e^{+} e^{-}$collider experiments with large samples of charm. CLEO II.5 (with a silicon vertex detector) has collected about $5 \mathrm{fb}^{-1}$ or about $5 \times 10^{6}$ produced $c \bar{c}$ events, this could increase to about a $f e w \times 10^{7}$ produced $c \bar{c}$ events together with CLEO III running. The b-factories BaBar and KEKB could collect about $1 \times 10^{8}$ produced $c \bar{c}$ by the year 2005. There is of course a reduction in numbers of events when one considers fully reconstructed charm decays which pass the trigger. It is not clear at this point if there will be a $\tau$-charm factory producing competitive results at that time. Although the charm cross section is relatively huge in $p \bar{p}$ collisions at $\sim 2 \mathrm{TeV},(\approx 1 \mathrm{mb}), p \bar{p}$ collider experiments have so far not made competitive measurements of charm decay physics except as regards its connection with bottom physics, e.g. the observation of the $B_{c}$ meson [43]. Since charm decays are normally a significant background to bottom physics in hadron collider experiments, one has to take care in order not to eliminate most of the interesting charm decays in the experimental trigger. Since the charm particle lifetimes are significantly shorter than the bottom particle lifetimes one also needs sufficient vertex resolution at the trigger level to get efficient charm detection while eliminating light quark backgrounds. Even with a trigger bandwidth devoted mainly to high $p_{T}$ and bottom physics, it may still be possible for CDF and D0 to make some competitive charm physics results in very specific channels, e.g. $D^{0}-\overline{D^{0}}$ mixing with semileptonic decays or heavier mass particles like those containing two or more charm quarks.

We expect that by the year 2005 there will be many new precise measurements of charm hadronic and semileptonic decays. There should also be measurements of purely leptonic decays. Charm particle lifetimes and absolute branching ratios will be precisely measured. Many hadronic decay amplitudes and phases will be precisely measured leading to more complete final state interaction estimates. Theory for charm decays should be better understood and more reliable calculations of non-perturbative effects should be available. Semileptonic decay form factors will be precisely measured, however a comparison with Lattice QCD or 
other theoretical calculations may be hindered by a lack of statistics or by poor $q^{2}$ resolution at the maximum $q^{2}$ where the theoretical uncertainties are the smallest. Searches for $D^{0}-\overline{D^{0}}$ mixing should have reached the $1 \times 10^{-4}$ level and the sensitivity for searches of direct $\mathrm{CP}$ violation in Cabibbo-suppressed decays should be at the $1 \times 10^{-2}$ level by the year 2005 . Both of these searches will thus begin to constrain the parameters of non-Standard Model physics scenarios. The search for rare decays should have reached a low enough sensitivity that Standard Model $c \rightarrow u X$ decays via long-distance effects would be observable. Thus beginning to test the long-distance calculations of $c \rightarrow u \gamma$ and $c \rightarrow u \ell^{+} \ell^{-}$rates.

\subsection{Charm Physics in BTeV}

One year of data taking at $2 \times 10^{32}$ will produce about $2 \times 10^{12} c \bar{c}$ pairs. This is an enormous sample if one can achieve sufficiently high trigger efficiency. Without modification a typical vertex trigger for b-quark events might be a factor of ten less efficient for c-quark decays than for b-quarks. With a $1 \%$ trigger efficiency and a $10 \%$ reconstruction efficiency one could expect about $1 \times 10^{9}$ reconstructed charm particle decays.

Probably the most promising area to see non-Standard Model physics is to observe $D^{0}-\overline{D^{0}}$ mixing. Sensitivities of a $\mathrm{few} \times 10^{-5}$ for $r_{D}$ should be achievable in BTeV using semileptonic decays. Searches for $D^{0}-\overline{D^{0}}$ mixing in hadronic decays are complicated by possible interference with Doubly-Cabibbo-suppressed decays and possible sizable CP violation in the decays $[26,44]$. However similar sensitivities using hadronic decays should be achievable. In addition, it may be possible for $\mathrm{BTeV}$ to contribute to better limits for $\Delta \Gamma$ though by the year 2005 these limits may be limited by systematic uncertainties rather than by the statistical sample of $\mathrm{CP}$ even and $\mathrm{CP}$ odd charm states. These $r_{D}$ and $\Delta \Gamma$ measurements should constrain large areas of parameter space for new physics scenarios if no $D^{0}-\overline{D^{0}}$ mixing is observed. Actual achievable sensitivities require careful study using the BTeV Monte Carlo.

Searches for direct $\mathrm{CP}$ violation in $\mathrm{BTeV}$ should reach the few $\times 10^{-3}$ level for Cabibbosuppressed modes and about $1 \times 10^{-3}$ for Cabibbo-favored decay modes. It would be interesting to see $\mathrm{CP}$ violation in charm decays. To reach uncertainties of less than $1 \times 10^{-3}$ in $a_{C P}$ (for Cabibbo-suppressed decays) requires more than $10^{6}$ background free decays. This is probably not possible in a short running time in BTeV without implementing charm specific changes in the trigger. Probably a trigger efficiency of about $10 \%$ would be necessary to achieve the desired sensitivity. The potential and likelihood of achieving this level of sensitivity will be studied carefully.

Due to the large number of produced $c \bar{c}$ pairs, searches for the rare decays of interest should reach very small sensitivities maybe as low as $10^{-7}-10^{-8}$. Searches for decay modes with two leptons are helped by the fact that the vertexing constraint in the trigger may be relaxed for events with two leptons in the secondary vertex. This could make rare decays an especially good place to find new physics in BTeV. 

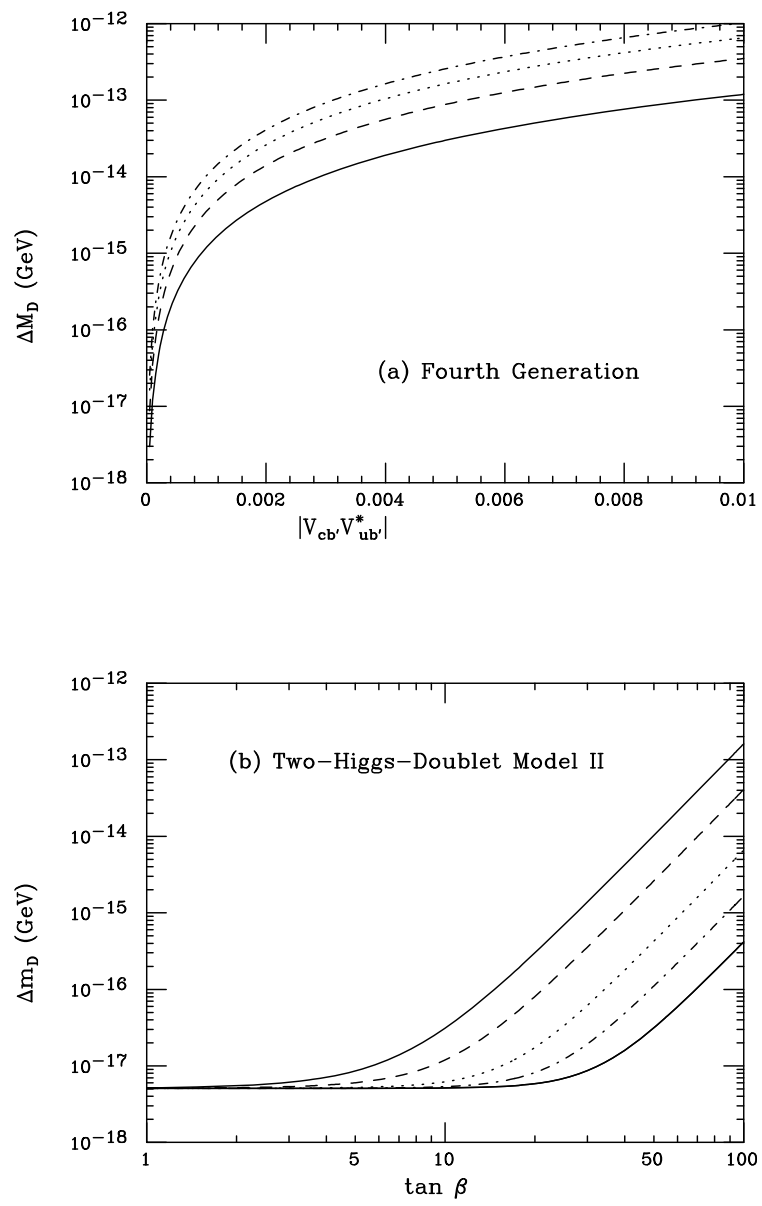

Figure 2.2: Figures taken from reference [8]. (a) $\Delta m$ in the four generation Standard Model as a function of the CKM mixing factor, with the solid, dashed, dotted, dash-dotted curves corresponding to $m_{b^{\prime}}=100,200,300,400 \mathrm{GeV}$ respectively. (b) $\Delta m$ in two-Higgs-doublet model II as a function of $\tan \beta$ with, from top to bottom, curves representing $m_{H^{ \pm}}=50$, $100,250,500,1000 \mathrm{GeV}$. 


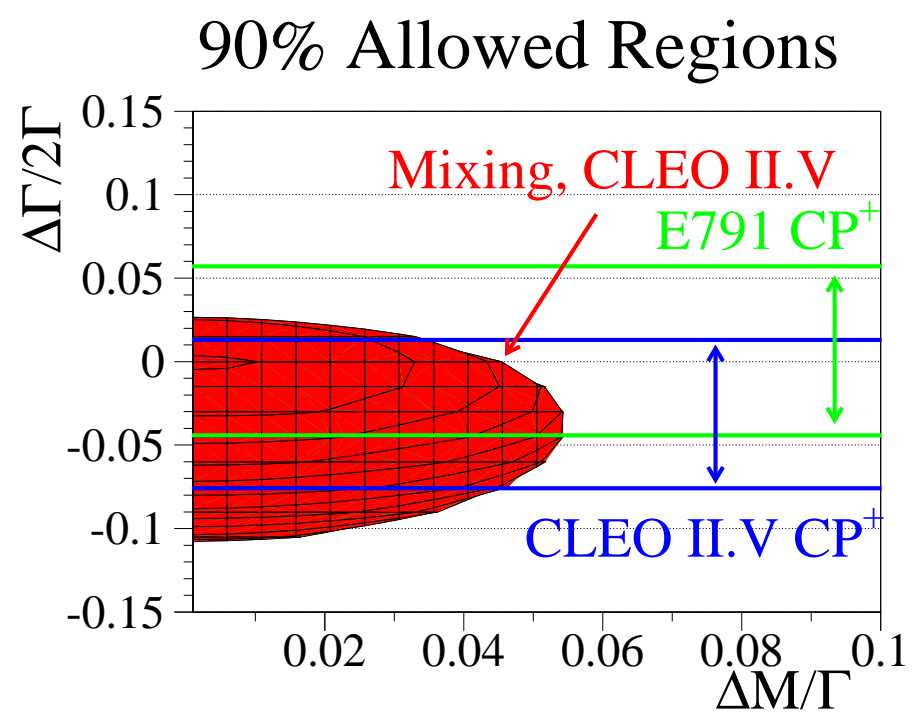

Figure 2.3: Plot taken from reference [27] showing the E791 result for $\Delta \Gamma$ [25] together with the preliminary results from CLEO on $\Delta \Gamma$ and $r_{D}$. The $90 \%$ C.L. allowed region in the $x=\Delta m / \Gamma$ versus $y=\Delta \Gamma / 2 \Gamma$ space is shown and one can see that the $\Delta \Gamma$ measurements cut out some of the region allowed by the $r_{D}$ measurement (shaded section). 


\section{Bibliography}

[1] F. Buccella et al., Phys. Lett. B 302, 319 (1993); F. Buccella, M. Lusignoli and A. Pugliese, Phys. Lett. B 379, 249 (1996).

[2] A. S. Kronfeld, "Lattice QCD Calculations of Leptonic and Semileptonic Decays," in Proceedings of the 1998 Workshop on Heavy Quarks at Fixed Target, Fermilab, Illinois, Oct. 10-12, 1998, pp. 355-365.

[3] A. S. Kronfeld, Private Communication.

[4] L. Wolfenstein, Phys. Lett. B 164, 170 (1985); J. Donoghue, E. Golowich, B. R. Holstein and J. Trampetic, Phys. Rev. D 33, 179 (1986).

[5] G. Burdman, "Potential for Discoveries in Charm Meson Physics," talk presented at the Workshop on the Tau/Charm Factory, Argonne, Illinois, Jun. 21-23, 1995. FERMILABConf-95/281-T. hep-ph/9508349.

[6] H. Georgi, Phys. Lett. B 297, 353 (1992); T. Ohl, G. Riccardi and E. H. Simmons, Nucl. Phys. B 403, 603 (1993).

[7] E. M. Aitala, et. al., Phys. Rev. Lett 77,2384 (1996).

[8] J. L. Hewett, "Searching for New Physics with Charm," talk presented at the Lafex International School on High Energy Physics (LISHEP95), Rio De Janeiro, Brazil, Feb. 6-22, 1995. SLAC-PUB-95-6821. hep-ph/9505246.

[9] S. Pakvasa, "Flavor Changing Neutral Currents in Charm Sector," in Proceedings of the FCNC 97, Santa Monica, CA, Feb. 19-21, 1997. UH-511-871-97. hep-ph/9705397.

[10] S. Pakvasa, "Charm as Probe of New Physics", in Proceedings of the CHARM2000 Workshop, Fermilab, Jun. 7-9, 1994, pp. 85-93.

[11] L. F. Abbott, P. Sikivie and M. Wise, Phys. Rev. D 21, 179 (1980).

[12] V. Barger, J. Hewett and T. Rizzo, Phys. Rev. D 41, 3421 (1990).

[13] PDG, C. Caso et al., Eur. Phys. J. C 3, 1 (1998); 
[14] C. T. Hill, Phys. Lett. B 345, 483 (1995).

[15] E. Golowich and S. Pakvasa, Phys. Rev. D 51, 1215 (1995).

[16] C. D. Carone and R. T. Hamilton, Phys. Lett. B 301, 196 (1993).

[17] J. S. Hagelin, S. Kelley and T. Tanaka, Nucl Phys. B 415, 293 (1994); Mod. Phys. Lett. A 8, 2737 (1993).

[18] Y. Nir and N. Seiberg, Phys. Lett. B 309, 337 (1993).

[19] A. G. Cohen, D. B. Kaplan, F. Lepeintre and A. Nelson, Phys. Rev. Lett. 78, 2300 (1997); Phys. Lett. B 388, 588 (1996); A. Nelson in FCNC97?

[20] S. Davidson, D. Bailey and B. A. Campbell, Z. Phys. C 61, 613 (1994).

[21] G. Volkov, V. A. Monich and B. V. Struminski, Yad. Fiz. 34, 435 (1981)

[22] G. Burdman, "Charm Mixing and CP Violation in the Standard Model", in Proceedings of the CHARM2000 Workshop, Fermilab, Jun. 7-9, 1994, pp. 75-93.

[23] E. Golowich and A. .A. Petrov, Phys. Lett. 427, 172 (1998).

[24] I. Dunietz, Phys. Rev. D 52, 3048 (1995); W. F. Palmer and Y. L. Wu, Phys. Lett. B 350, 245 (1995).

[25] E. M. Aitala, et. al., "Measurement of Lifetimes and a Limit on the Lifetime Difference in the Neutral D-Meson System", Fermilab preprint FERMILAB-Pub-99/036-E.

[26] G. Blaylock, A. Seiden and Y. Nir, Phys. Lett. B 355, 555 (1995); L. Wolfenstein, Phys. Rev. Lett. 75, 2460 (1995);

[27] M. Selen, "Charm Physics from CLEO", talk presented at the APS Centennial Meeting, Atlanta, Mar. 21-26, 1999.

(http://www.lns.cornell.edu/public/CLEO/analysis/results/D-mixing).

[28] T. Brown, S. F. Tuan and S. Pakvasa, Phys. Rev. Lett. 51, 1823 (1983); O. E. Overseth and S. Pakvasa, Phys. Rev. 184, 1663 (1969).

[29] A. Le Yaouanc, L. Oliver and J. C. Raynal, Phys. Lett. B 292, 353 (1992); M. Gronau and S. Wakaizumi, Phys. Rev. Lett. 68, 1814 (1992).

[30] I. I. Bigi and H. Yamamoto, Phys. Lett. B 349, 363 (1995).

[31] G. Burdman, E. Golowich, J. L. Hewett and S. Pakvasa, Phys. Rev. D 52, 6383 (1995).

[32] S. Fajfer, S. Prelovšek and P. Singer, Eur. Phys. J. C 6, 471 (1999); S. Fajfer and P. Singer, Phys. Rev. D 56, 4302 (1997). 
[33] I. I. Bigi, "Open Questions in Charm Decays Deserving an Answer", in Proceedings of the CHARM2000 Workshop, Fermilab, Jun. 7-9, 1994, pp. 323-333.

[34] I. I. Bigi, F. Gabbiani and A. Masiero, Z. Phys. C 48, 633 (1990).

[35] G. Burdman, "Theoretical Issues in Rare $K, D$ and $B$ Decays", in Proceedings of the 1998 Workshop on Heavy Quarks at Fixed Target, Fermilab, Illinois, Oct. 10-12, 1998, pp. 461-471.

[36] P. Singer and D. Zhang, Phys. Rev. D 55, R1127 (1997).

[37] D. M. Asner et al., Phys. Rev. D 58, 092001-1 (1998).

[38] S. Fajfer and S. Prelovšek, Phys. Rev. D 58, 094038-1 (1998).

[39] J. Rosner, "What Charm Can Tell Us About Beauty", in Proceedings of the CHARM2000 Workshop, Fermilab, Jun. 7-9, 1994, pp. 297-311.

[40] B. Grinstein, Phys. Rev. Lett. 71, 3067 (1993).

[41] J. M. Link, "The FOCUS Spectrometer and Hadronic Decays in FOCUS", in Proceedings of the 1998 Workshop on Heavy Quarks at Fixed Target, Fermilab, Illinois, Oct. 10-12, 1998, pp. 261-269.

[42] A. Bravar, "The COMPASS Experiment at CERN", in Proceedings of the 1998 Workshop on Heavy Quarks at Fixed Target, Fermilab, Illinois, Oct. 10-12, 1998, pp. 390-398.

[43] F. Abe et al., Phys. Rev. D 58, 112004-1, (1998); Phys. Rev. Lett. 81, 2432 (1998).

[44] T. E. Browder and S. Pakvasa, Phys. Lett. B 383, 475 (1996) 


\section{Chapter 3}

\section{The Main Physics Goals of BTeV}

Below, we review the important questions in the physics of bottom and charm that must be addressed by experiments. (The physics case is laid out in much more detail in Chapter's 1 and 2.) Some key measurements, for example $\sin 2 \beta \mathrm{CP}$ violation in some rare decays, and $B_{s}$ mixing may well be measured before $\mathrm{BTeV}$ goes into operation. Most of the program, however, will not have been done, and even where initial measurements have been made, more precise measurements will probably be justified.

The strength of $\mathrm{BTeV}$ rests on several pillars:

- Excellent time resolution provided by a pixel tracking system inside the main beampipe and thus close to the beam. This time resolution is critical for detached vertex triggering in the first level trigger and for background rejection in the final data analysis.

- A highly efficient and redundant trigger algorithm. We trigger at the first level on $n$-tracks which miss the primary vertex each by $m-\sigma$. We can prescale different values of $n$ and $m$ creating redundancy. In certain cases redundancy is provided by a di-lepton trigger.

- Excellent particle identification over virtually the entire momentum range provided by a Ring Imaging Cherenkov (RICH) detector.

- A high resolution Electromagnetic Calorimeter using lead-tungstate crystals. This gives us the capability to successfully measure final states with single photons, $\pi^{o}$ 's, $\eta$ 's and $\eta^{\prime \prime}$ s that is absolutely crucial in finishing the physics program discussed in Chapter 1 and outlined below.

Below, we list some of the measurements that we believe that BTeV can make as well if not better than any other bottom or charm experiment that will be running during this period. We want to emphasize that $\mathrm{BTeV}$, with its powerful and general trigger and complete array of detection elements will be capable of pursuing a wide variety of final states and can be optimized to undertake whatever interesting measurements need to be made as we learn 
more in this area in the next few years. Furthermore BTeV, because of its powerful trigger and vertexing capability, is a better charm experiment than any current or planned. Since it is important to try to find phenomena which are not expected within the Standard Model, it is essential that searches be undertaken for CP violation, mixing, and rare decays of charm, where the Standard Model predicts very low rates and where a signal from new physics may therefore stand out clearly.

\subsection{Physics Goals For B's}

Here we briefly list the main physics goals for studies of the $b$ quark. BTeV will be capable of measuring standard model parameters including CKM matrix elements, and performing all of the necessary tests outlined in Chapter 1 to either confirm the standard model or find firm evidence that the standard model is violated and provide a view as to how it is violated.

- Measurement of the "CP violating" angle $\gamma$. We measure $\gamma$ using several different methods including resolving time dependent $B_{s}$ oscillations in the final states $B_{s} \rightarrow D_{s}^{\mp} K^{ \pm}$. Measurement of $B^{+} \rightarrow D^{o} K^{+}, B^{+} \rightarrow \bar{D}^{o} K^{+}$, where the $D^{o}$ can decay directly or via a doubly Cabibbo suppressed decay mode. We also need to measure the conjugate $B^{-}$ decay modes. Furthermore, we will measure the reactions $B^{o} \rightarrow K^{ \pm} \pi^{\mp}, B^{ \pm} \rightarrow K^{ \pm} \pi^{o}$ and $B^{ \pm} \rightarrow K_{s} \pi^{\mp}$, to make model dependent determinations.

- Measurement of the CP violating angle $\alpha$ using the neutral decays $B^{o} \rightarrow \rho \pi$ to measure both $\sin (2 \alpha)$ and $\cos (2 \alpha)$ to remove two of the possible ambiguities in the extraction of $\alpha$ from $\sin (2 \alpha)$

- Measurement of the small CP asymmetry in $B_{s} \rightarrow J / \psi \eta^{\prime}$ or $J / \psi \eta$.

- Search for rare final states such as $K \mu^{+} \mu^{-}, K^{*} \mu^{+} \mu^{-}, \pi \mu^{+} \mu^{-}, \rho \mu^{+} \mu^{-}$, and similar states with electrons, which could result from new high mass particles coupling to $b$ quarks.

- We assume that the CP violating function $\sin (2 \beta)$ will have already been measured using $B^{o} \rightarrow J / \psi K_{s}$, but we will be able to significantly reduce the error. Furthermore, we will be able to remove two of the ambiguities in the determination of $\beta$ using either the reaction $B^{o} \rightarrow J / \psi K^{* o}, K^{* o} \rightarrow K_{s} \pi^{o}$, by getting the strong phase shift from the analogous reaction $B_{s} \rightarrow J / \psi \phi$, or by the reactions $B^{o} \rightarrow J / \psi K^{o}, K^{o} \rightarrow \pi^{ \pm} \ell^{\mp} \nu$. We will also search for new physics by checking the measurement of $\sin (2 \beta)$ using several other decay modes, such as $\phi K_{s}$.

- Search for unexpectedly high asymmetries in places where the Standard Model predicts small CP asymmetries. The decay $B_{s} \rightarrow J / \psi \phi$ is an example.

- Precision measurements of $B_{s}$ mixing, both the time evolution $x_{s}$ and the lifetime difference, $\Delta \Gamma$, between the positive $\mathrm{CP}$ and negative $\mathrm{CP}$ final states. 
- Measurement of the CP asymmetries in the simple pseudoscalar-pseudoscalar decay modes $B^{o} \rightarrow \pi^{+} \pi^{-}$and $B_{s}^{o} \rightarrow K^{+} K^{-}$depend on interferences between tree, mixing and Penguin amplitudes. These measurements can lead to an independent measurement of the angle $\gamma$ with some theoretical input or reveal new physics.

\subsection{The Main Physics Goals for charm}

According to the Standard Model, charm mixing and CP violating effects should be "small." Thus charm provides an excellent place for non-Standard Model effects to appear. Specific goals are listed below.

- Search for mixing in $D^{o}$ decay, by looking for both the rate of wrong sign decay, $r_{D}$ and the width difference between positive $\mathrm{CP}$ and negative $\mathrm{CP}$ eigenstate decays, $\Delta \Gamma$.

- Search for CP violation in $D^{o}$. Here we have the advantage over $b$ decays in that there is a large $D^{*+}$ signal which tags the initial flavor of the $D^{o}$ through the decay $D^{*+} \rightarrow$ $\pi^{+} D^{o}$. Similarly $D^{*-}$ decays tag the flavor of inital $\bar{D}^{o}$. The current experimental upper limits on $\mathrm{CP}$ violating asymmetries are on the order of $10 \%$, while the standard model prediction is about $0.1 \%$.

- Search for direct CP violation in charm using $D^{+}$and $D_{s}^{+}$decays.

- Search for rare decays of charm, which if found would signal new physics.

\subsection{Other Bottom and Charm Physics Goals}

There are many other physics topics that can be addressed by BTeV. A short list is given here.

- Measurement of the $b \bar{b}$ production cross-section and correlations between the $b$ and the $\bar{b}$ in the forward direction.

- Measurement of the $B_{c}$ production cross-section and decays.

- The spectroscopy of $b$ baryons.

- Measurement of the $c \bar{c}$ production cross-section and correlations between the $c$ and the $\bar{c}$ in the forward direction.

- Precision measurement of $V_{c d}$ and the form-factors in the decays $D \rightarrow \pi \ell^{+} \nu$ and $D \rightarrow$ $\rho \ell^{+} \nu$.

- Precision measurement of $V_{c s}$ and the form-factors in the decay $D \rightarrow K \ell^{+} \nu$ and $D \rightarrow$ $K^{*} \ell^{+} \nu$. 


\section{Part II}

\section{The BTeV Detector}




\section{Chapter 4}

\section{The BTeV Baseline Detector}

In this chapter, we describe the BTeV detector baseline design. We begin with a review of the important characteristics of hadronic $B$ production and the properties of the Tevatron which influence the design. We then give a description of the C0 Collision Hall and IR. We discuss each major component of the detector in turn, including the key requirements that drive the design, the choice of the baseline technology, the expected performance, and the options which are still under consideration. More details on each major component are presented in later chapters. 


\subsection{Characteristics of Hadronic $b$ Production}

The kinematics of hadronic $B$ and charm production play a major role in the design of $\mathrm{BTeV}$. We review the most important features here.

It is customary to characterize heavy quark production in hadron collisions with the two variables $p_{t}$ and $\eta$. The latter variable was first invented by those who studied high energy cosmic rays and is assigned the value

$$
\eta=-\ln (\tan (\theta / 2)),
$$

where $\theta$ is the angle of the particle with respect to the beam direction.

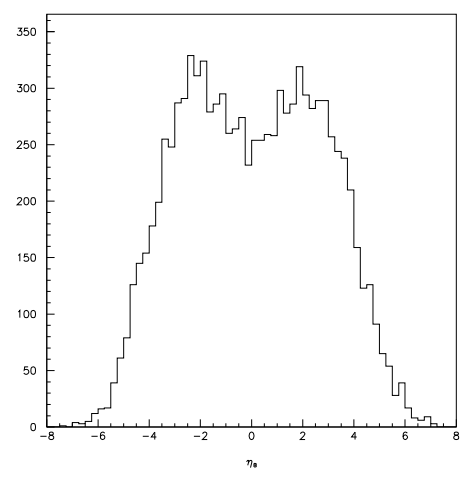

Figure 4.1: The $B$ yield versus $\eta$.

According to QCD calculations of $b$ quark production, the b's are produced "uniformly" in $\eta$ and have a truncated transverse momentum, $p_{t}$, spectrum, characterized by a mean value approximately equal to the $B$ mass [1]. The distribution in $\eta$ is shown in Fig. 4.1.

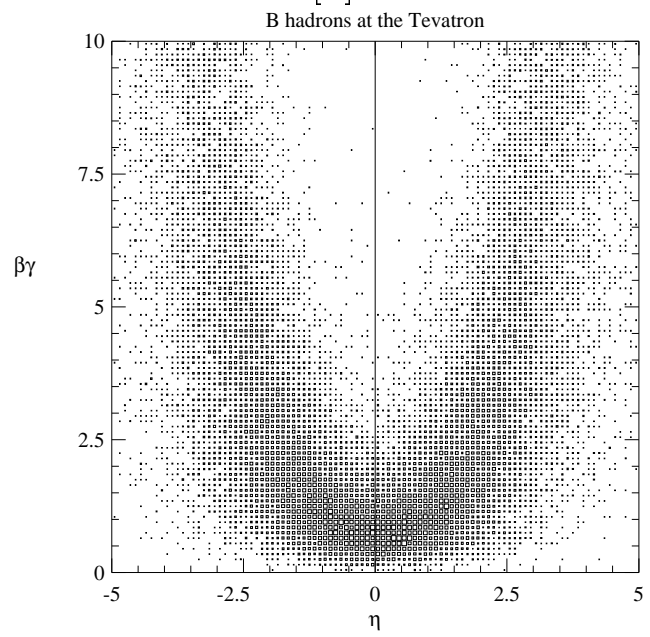

Figure 4.2: $\beta \gamma$ of the $B$ versus $\eta$.

There is a strong correlation between the $B$ momentum and $\eta$. Shown in Fig. 4.2 is the $\beta \gamma$ of the $B$ hadron versus $\eta$ from Pythia at $\sqrt{s}=2 \mathrm{TeV}$. It can clearly be seen that near $\eta$ of 
zero, $\beta \gamma \approx 1$, while at larger values of $|\eta|, \beta \gamma$ can easily reach values of 6 . This is important because the observed decay length varies with $\beta \gamma$ and furthermore the absolute momenta of the decay products are larger allowing for a suppression of the multiple scattering error.

Since the detector design is somewhat dependent on the Monte Carlo generated $b$ production distributions, it is important to check that the correlations between the $b$ and the $\bar{b}$ are adequately reproduced. Fig. 4.3 shows the azimuthal opening angle distribution between a muon from a $b$ quark decay and the $\bar{b}$ jet as measured by CDF [2] and compares it with the MNR predictions [3].

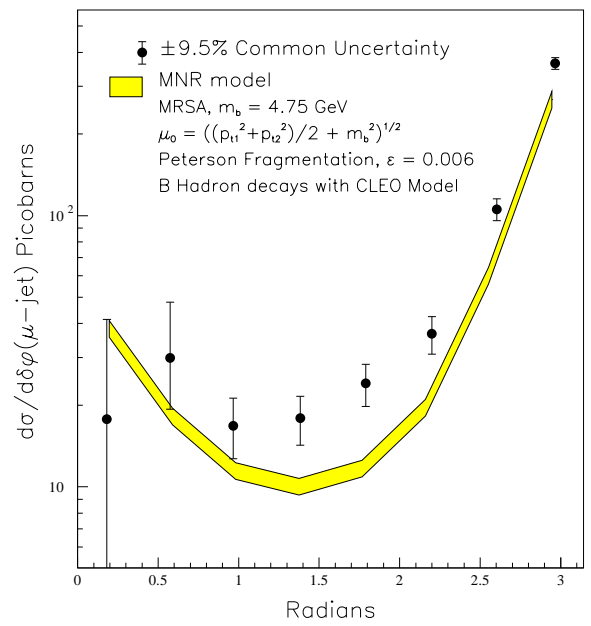

Figure 4.3: The differential $\delta \phi$ cross-sections for $p_{T}^{\mu}>9 \mathrm{GeV} / \mathrm{c},\left|\eta^{\mu}\right|<0.6, \mathrm{E}_{T}^{\bar{b}}>10 \mathrm{GeV}$, $\left|\eta^{\bar{b}}\right|<1.5$ compared with theoretical predictions. The data points have a common systematic uncertainty of $\pm 9.5 \%$. The uncertainty in the theory curve arises from the error on the muonic branching ratio and the uncertainty in the fragmentation model.

The model does a good job representing the shape, which shows a strong back-to-back correlation. The normalization is about a factor of two higher in the data than the theory, which is generally true of CDF $b$ cross-section measurements [4]. In hadron colliders all $B$ species, $B^{o}, B^{+}, B_{s}^{o}$, b-baryons, and even $B_{c}$ mesons, are produced at the same time.

The "flat" $\eta$ distribution hides an important correlation of $b \bar{b}$ production at hadronic colliders. In Fig. 4.4 the production angle of the hadron containing the $b$ quark is plotted versus the production angle of the hadron containing the $\bar{b}$ quark. Here zero degrees represents the direction of the incident proton and 180 degrees, the incident anti-proton. There is a very strong correlation in the proton or the anti-proton directions: when the $B$ is forward the $\bar{B}$ is also forward. (We call both the proton and anti-proton directions forward.) This correlation between $B$ and $\bar{B}$ production is not present in the central region (near 90 degrees). By instrumenting a relatively small region of angular phase space, a large number of $b \bar{b}$ pairs can be detected. Furthermore, the $B$ 's populating the forward regions have large values of $\beta \gamma$ and this permits more accurate separation of the primary and secondary vertices and better overall detection efficiency for $b$ hadrons. In addition, the techniques for particle identification in this kinematic range are very well developed. Powerful particle identification is a key 
to high sensitivity bottom and charm experiments. For these reasons, we have designed a detector with 'forward coverage'.

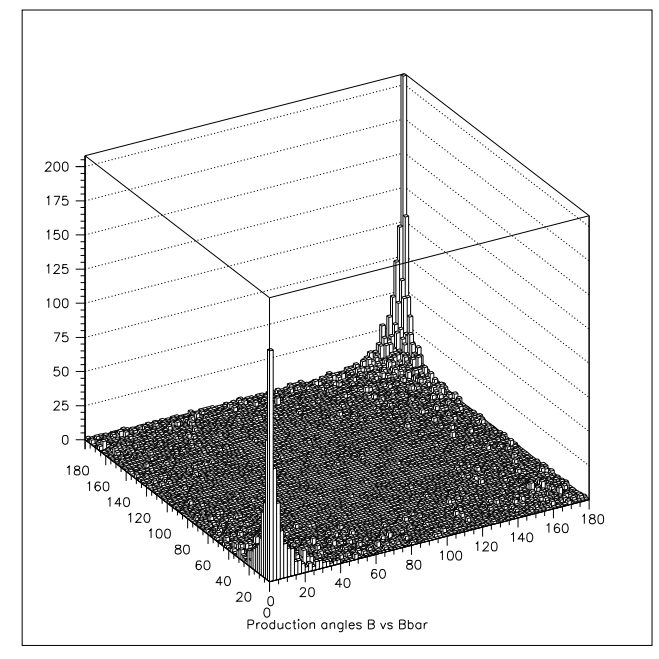

Figure 4.4: The production angle (in degrees) for the hadron containing a $b$ quark plotted versus the production angle for a hadron containing $\bar{b}$ quark, from the Phythia Monte Carlo generator.

Charm production is similar to $b$ production but has a much larger cross section. Current theoretical estimates are that charm is $1-2 \%$ of the total $p \bar{p}$ cross-section. The cross section is even more strongly peaked in the forward directions because the average transverse momentum is of the order of only $1.5 \mathrm{GeV} / \mathrm{c}$. The charm cross section has never been measured because texperiments with good acceptance in the central region have very low efficiency for triggering and reconstructing charm. The favorable kinematics in the forward direction gives $\mathrm{BTeV}$ a very high efficiency for reconstructing charm.

Table 4.1 gives the relevant Tevatron parameters. We expect to start serious data taking in Fermilab Run II with a luminosity of about $5 \times 10^{31} \mathrm{~cm}^{-2} \mathrm{~s}^{-1}$; our ultimate luminosity goal, to be obtained in Run III, is $2 \times 10^{32} \mathrm{~cm}^{-2} \mathrm{~s}^{-1}$.

While BTeV is designed to study $p \bar{p}$ collisions at $2 \mathrm{TeV}$, it can also run in 'Fixed Target' mode. In that mode, a thin wire is inserted into the halo of the circulating beam and the detector studies the collisions of $800 \mathrm{GeV} / \mathrm{c}$ protons (or anti-protons) with the nuclei of the wire. For this reason, Fixed Target mode is also referred to as 'wire mode'.

In this mode, particles diffusing laterally out of the beam eventually reach the wire target where they can interact. Since the diffusion is very slow, each particle will traverse the wire many times until it either interacts or multiple scattering blows its orbit up so badly that it escapes from the machine aperture altogether. We are learning from accelerator physicists what overall interaction rate we can expect.

The cross section for fixed target charm production on a proton at $800 \mathrm{GeV} / \mathrm{c}$ is about $30 \mu \mathrm{b}$. While this is much lower than in the collider, there are some advantages: first, it 
Table 4.1: The Tevatron as a $b$ and $c$ source for BTeV

\begin{tabular}{|l|c|}
\hline Luminosity (BTeV design) & $2 \times 10^{32} \mathrm{~cm}^{-2} \mathrm{~s}^{-1}$ \\
$b$ cross-section & $100 \mu \mathrm{b}$ \\
$\#$ of $b$ 's per $10^{7}$ sec (Run II) & $4 \times 10^{11}$ \\
$\frac{\sigma_{b-\bar{b}}}{\sigma_{\text {inel }}}$ & $0.2 \%$ \\
$c$ cross-section & $>500 \mu \mathrm{b}$ \\
Bunch spacing & $132 \mathrm{~ns}$ \\
Luminous region length & $\sigma_{z}=30 \mathrm{~cm}$ \\
Luminous region length & $\sigma_{x} \sigma_{y}=\approx 5 \mu \mathrm{m}$ \\
Interactions/crossing & $<2.0>$ \\
\hline
\end{tabular}

may not require low- $\beta$ quadrupoles to be installed so it might be available to us earlier than collider mode; second, it is not expected to affect the luminosity at B0 or D0, which is not the case when the beams collide at $\mathrm{C} 0$ and this will translate into longer running times; the region along the beam that has to be instrumented with precision vertex detectors is much smaller which means that a small scale vertex detector prototype is all that is needed for initial experiments; and the fact that the center of mass is boosted towards the spectrometer increases the acceptance. However, this mode is not useful to us for $B$-physics studies because the cross section is of order $10 \mathrm{nb}$. While we believe that the most sensitive charm experiment will be using the collider, this mode does give us the opportunity to do physics early with a partially instrumented detector. 


\section{Bibliography}

[1] M. Artuso, "Experimental Facilities for b-Quark Physics," in B Decays revised 2nd Edition, Ed. S. Stone, World Scientific, Singapore (1994).

[2] F. Abe et al, Phys. Rev. D 53, 1051 (1996).

[3] M. Mangano, P. Nason and G. Ridolfi, Nucl. Phys. B 373, 295 (1992).

[4] F. Abe et al, Phys. Rev. Lett. 75, 1451 (1995). Previous UA1 measurements agreed with the theoretical predictions, see C. Albajar et al, Phys. Lett. B 256, 121 (1991). Recent D0 measurements agree with both the CDF measurements and the high side of the theoretically allowed range. See S. Abachi et al, Phys. Rev. Lett. 74, 3548 (1995). 


\subsection{C0 Baseline Detector Description}

We describe here the baseline detector for BTeV, which can achieve our physics goals, and is designed to have the capability to perform new measurements on new interesting final states that may turn out to be recognized as important in the future.

The challenges for any future hadron collider $B$ experiment are to achieve high efficiency and low background in the hostile and complex environment of a hadron collider. This requires the effective and aggressive use of the most modern technologies. For BTeV this represents a significant step beyond what will be achieved by the upcoming generation of hadron collider $B$ experiments and $e^{+} e^{-}$collider experiments running on the $\Upsilon(4 \mathrm{~S})$, and their possible extensions and upgrades. Furthermore, we must be able to compete successfully with the LHC-b experiment, when it starts running.

$\mathrm{BTeV}$ has begun to take shape based on an understanding of what needs to be done to meet these challenges. These are the key design features of BTeV:

- a dipole located on the IR, which gives BTeV an effective 'two arm' acceptance;

- a precision vertex detector based on planar pixel arrays;

- a vertex trigger at Level 1 which makes BTeV very efficient for many final states, even if they have no leptons in them; and

- excellent particle identification. Especially important is the requirement of very good charged hadron identification. Many of the states that will be of interest in this phase of bottom and charm physics will only be separable from other states if this capability exists. Also, it will allow for the possibility of kaon tagging. Muon and electron identification are important for tagging and for studies of decay modes involving leptons, too.

- A high quality $\mathrm{PbWO}_{4}$ electromagnetic calorimeter capable of detecting final states with single photons, $\pi^{o}$ 's, $\eta$ 's or $\eta^{\prime}$ 's.

The parameters of the Tevatron that must be taken into account in creating a design for meeting these challenges are given in Table 4.1. A schematic of the experiment is given in Fig. 4.5. We now discuss the key elements of the baseline design. 

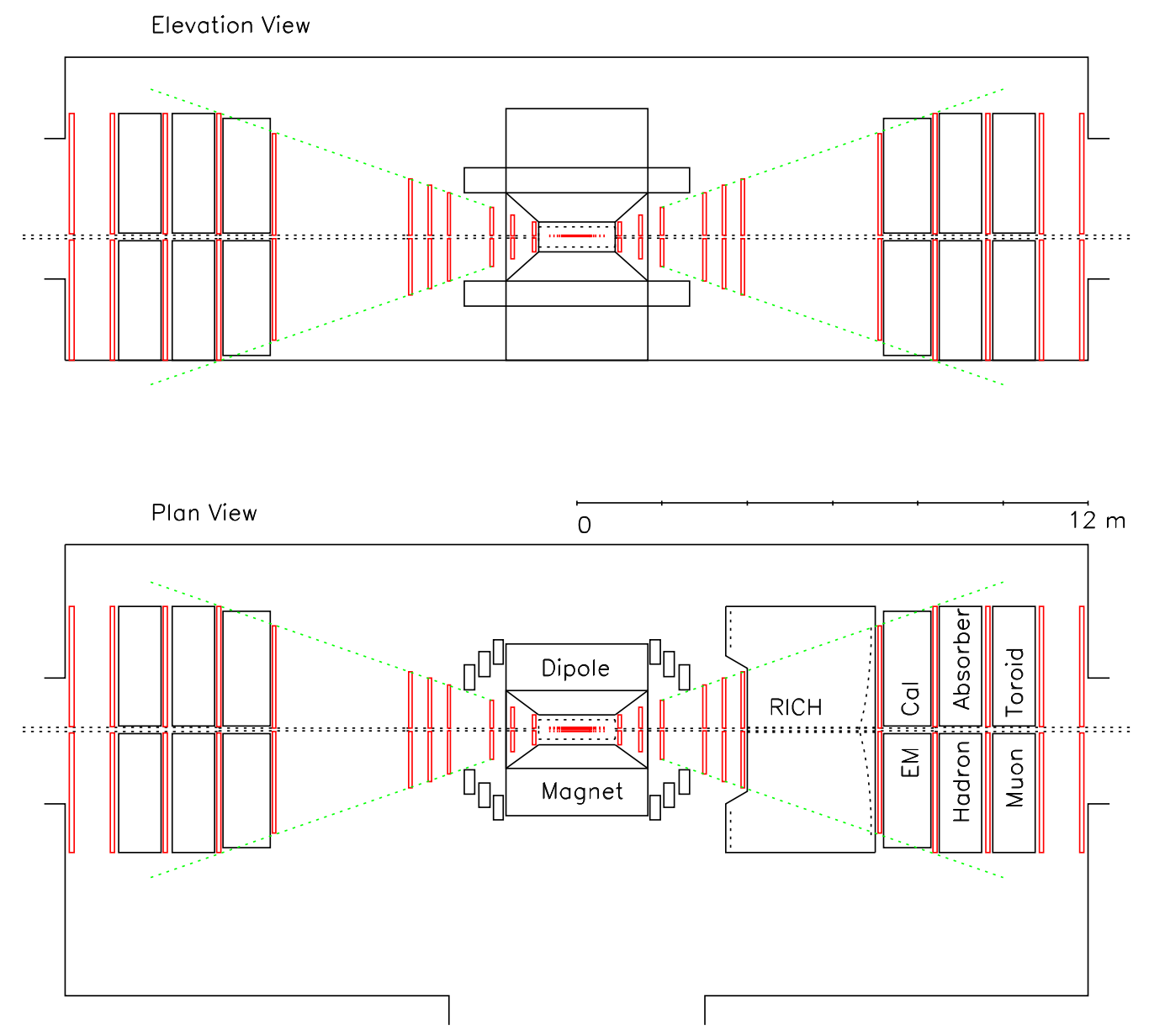

Figure 4.5: Layout of BTeV/C0 Spectrometer 


\subsubsection{The Co Experimental Area}

The experiment will be carried out in the newly constructed $\mathrm{C} 0$ test hall, see Figure 4.6. The test hall is a $216 \mathrm{~m}^{2}$ (9m wide by $24 \mathrm{~m}$ long) enclosure centered along the $\mathrm{C} 0$ straight section of the Tevatron. (In plan view, the hall is asymmetric transversely, extending $3.5 \mathrm{~m}$ from the beam on the west side and $5.5 \mathrm{~m}$ from the beam on the east side.) The Tevatron beam is $2.5 \mathrm{~m}$ above the floor slab and $4.25 \mathrm{~m}$ below the roof of the hall. There are flared transition enclosures both upstream and downstream of the test hall. The test hall specifications and dimensions are compatible with the detector described in this document.

To the east of the test hall is the C0 assembly building (see Figure 4.7) a steel framed, industrial type, structure containing a $150 \mathrm{~m}^{2}$ assembly hall at the test hall elevation. Detector components will be constructed in the assembly hall and moved into the test hall through a $6 \mathrm{~m} \times 6 \mathrm{~m}$ 'shield door'. The $\mathrm{C} 0$ assembly building also has space on various levels for the electrical, water and air handling systems needed for the BTeV detector. Figure 4.8 shows a layout of the assembly building and test hall with the magnet and muon toroids of the proposed BTeV detector superimposed.

Detector elements are brought into the area at a ground level loading dock and lowered to the assembly floor using the 30 ton crane which covers the loading dock and assembly hall. There is no crane in the beam enclosure; all detector elements must be designed so they can be constructed or staged in the assembly area and moved through the shielding door into the enclosure. During operation the shielding door is sealed with concrete blocks for radiation protection. The blocks are stored in the south end of the assembly area in an alcove when the door is open.

There are cable ducts from the experiment enclosure to a $150 \mathrm{~m}^{2}$ equipment room at grade level on the north end of the assembly building. Preliminary engineering has been done on converting this equipment room into a two or three level electronics/counting room and office area for experimenters. Electric power, air handling, other utilities, as well as an elevator, lavatories, etc., although not yet installed, have been designed to accommodate $\mathrm{BTeV}$ needs in these areas. 
Figure 4.6: C0 Test Hall

Figure 4.7: Tevatron berm and C0 Assembly Building 
Figure 4.8: Layout of C-0 Test Area 


\subsubsection{The BTeV/C0 Spectrometer Magnet}

We have identified a magnet that satisfies the specifications for the $\mathrm{BTeV} / \mathrm{C} 0$ spectrometer. The SM3 magnet, currently part of the decommissioned Fermilab MEast spectrometer, is shown on the proposed layout, Fig. 4.5, of the $\mathrm{BTeV} / \mathrm{C} 0$ spectrometer. This magnet was assembled in 1981 from soft iron blocks recovered from the decommissioned Nevis Cyclotron. The coils for the magnet were built of $5 \mathrm{~cm}$ square aluminum conductor by the Sumitomo Corporation under the aegis of the US-Japan Agreement on High Energy Physics. The magnet operated in MEast from 1982 until 1997, at a central field of about 0.8 Tesla, serving experiments E605, E772, E789, and E866.

The SM3 magnet was assembled by welding together, in place, various blocks of steel. It has a total weight of 500 metric tons. The construction and assembly drawings for this magnet have been located. Engineering preparations are now underway for disassembling the magnet. The modified magnet could then be reassembled in the $\mathrm{C} 0$ assembly hall and rolled into the $\mathrm{C} 0$ collision hall, as shown in Fig. 4.6.

The central field specified for the $\mathrm{BTeV} / \mathrm{C} 0$ spectrometer is much higher than the past operating excitation of the SM3 magnet. Preliminary studies with magnetostatic modeling programs (POISSON and OPERA) have led to a design for a new pole-piece for SM3. This pole-piece, indicated in Fig. 4.9, yields a central field of 1.6 Tesla, and an integrated dipole field of $5.2 \mathrm{~T}-\mathrm{m}$.

The design has an integrated sextupole field component on the symmetry axis of 0.002 $\mathrm{T} / \mathrm{m}$. This is small compared to the natural sextupole moment of the Tevatron dipoles and is acceptable for insertion into the Tevatron lattice. In this design the magnet would draw $650 \mathrm{~kW}$ of power at 4200 amps, similar to its past operating power level (it was powered by two $500 \mathrm{~kW}$ Transrex power supplies). Note that the magnet will be oriented so that charged particles are deflected in the vertical plane. The properties of the magnet, with the pole faces shimmed to the BTeV requirements, are shown in Table 4.2.

Table 4.2: BTeV/C0 Vertex Dipole Properties

\begin{tabular}{|l|l|l|}
\hline Property & Value & Comment \\
\hline $\int B \times d l$ & $5.2 \mathrm{~T}-\mathrm{m}$ & $\begin{array}{l}2.6 \mathrm{~T} \text {-m on each } \\
\text { side of center of IR }\end{array}$ \\
Central Field & 1.6 Tesla & \\
Steel Length & $3.2 \mathrm{~m}$ & \\
Overall length & $5.3 \mathrm{~m}$ & \\
Magnet Vert. aperture & $\pm 0.3 \mathrm{rad}$ & \\
Magnet Horz. aperture & $\pm 0.3 \mathrm{rad}$ & \\
\hline
\end{tabular}

The magnet is centered on the interaction region in $\mathrm{Z}$ thus creating both an 'upstream' and 'downstream' spectrometer. In quark-antiquark production at $2 \mathrm{TeV}$, the bottom quark 


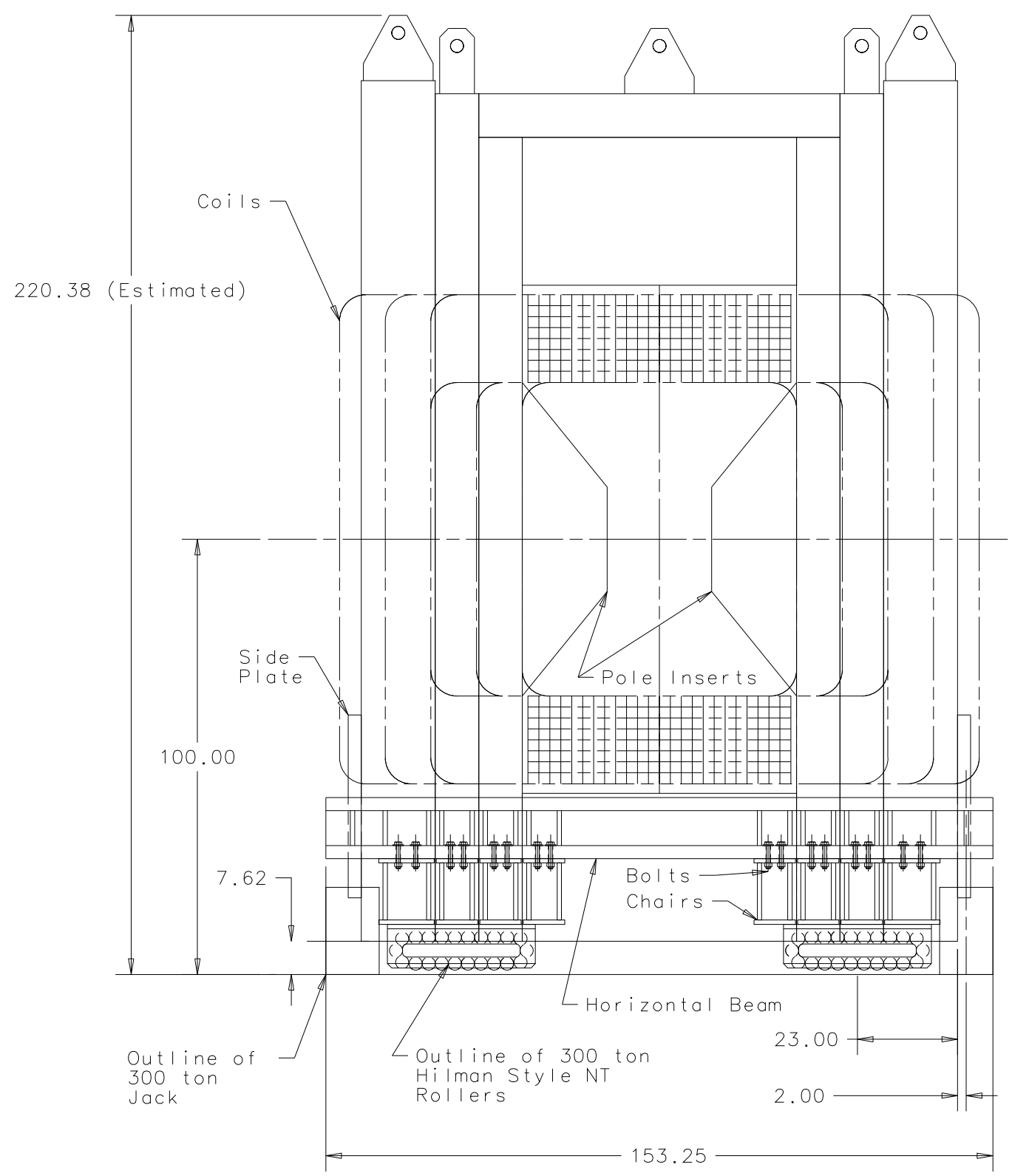

Figure 4.9: Cross section of the modified SM3 dipole with rollers and pole piece inserts. All dimensions are in inches. 


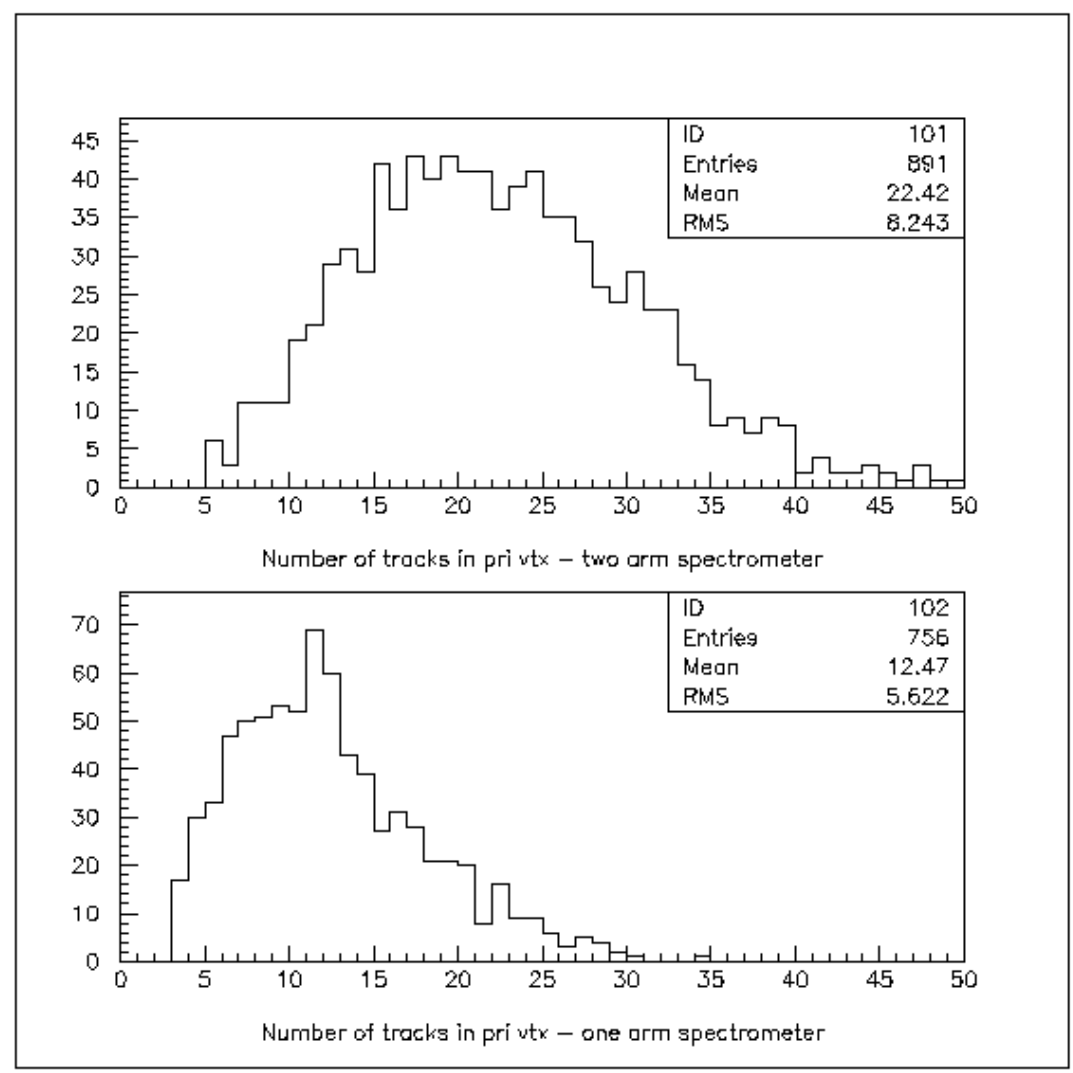

Figure 4.10: Fraction of events with $\mathrm{N}$ primary tracks above $1 \mathrm{GeV} / \mathrm{c}$ momentum for a) a dipole centered on the IR giving an effective two-arm acceptance and b) a single forward spectrometer arm.

and antiquark are usually either both boosted upstream or both boosted downstream. Thus having two spectrometers doubles the acceptance of the experiment for tagged decays. Having two spectrometers further improves the experiment because the extended coverage increases the number of tracks from the underlying event that can be used to determine the primary vertex. Fig. 4.10 shows the number of high energy primary tracks (low multiple scattering) that contribute to locating the primary vertex for single arm coverage and for two arm coverage. If we require three such tracks to determine the primary vertex, then 2.2 times more events survive with two arms than with one, and the primary vertex resolution is enhanced.

In this central dipole geometry, there is a strong magnetic field at the vertex detector. Because of the excellent spatial resolution of the vertex detector, it is possible to get a crude measurement of the track momentum using the vertex detector alone. This measurement 
can be used to reject tracks at the trigger level that are at such low momentum that multiple coulomb scattering errors make their assignment to a vertex uncertain.

The second spectrometer arm will also be useful during the R\&D and early data-taking phases of the experiment. It is possible, given the lab's budget, that only one arm will be fully instrumented initially. The other side can be used to test new detector concepts or to test prototypes of production components under actual beam conditions until it becomes possible to instrument it fully. 


\subsubsection{Tracking System}

The tracking system is designed to reconstruct tracks and measure their angles and momenta over the full aperture $( \pm 300 \mathrm{mr} \times \pm 300 \mathrm{mr}$ ) and momentum acceptance (from $1 \mathrm{GeV} / \mathrm{c}$ to more than $100 \mathrm{GeV} / \mathrm{c}$ ) of the experiment. The main requirements that have to be satisfied by the system are:

- Provide adequate resolution on track positions, at least $9 \mu \mathrm{m}$ or better, and angles, at least $0.1 \mathrm{mr}$, to separate with high efficiency and robustness the secondary vertices of bottom and charm decays from the primary interaction vertex

- Provide sufficient momentum resolution, about $1 \%$ at $100 \mathrm{GeV} / \mathrm{c}$, to permit the reconstruction of the invariant mass of multibody bottom and charm decays with good precision

- Deliver its information to a Level 1 trigger processor which implements a trigger based on detached vertices or tracks with large impact parameters

- Handle the rates and tolerate the radiation doses expected in the experiment

To satisfy these requirements, the experiment will employ two tracking subsystems which work together to provide efficient track reconstruction, excellent vertex resolution, and excellent momentum and mass resolution. These are

- The vertex detector which provides the precise vertex measurement and also has momentum measurement capability. The signals from the vertex detector provide the main input to the Level 1 vertex trigger.

- The forward tracker, which provides, along with the vertex detector, precision momen-

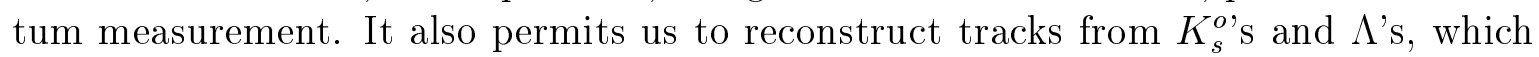
often decay downstream of the vertex detector.

In the following paragraphs, we discuss the design of each of these systems.

\subsubsection{Vertex Detector}

The vertex pixel detector provides the high resolution tracking near the interaction which is required to associate tracks with their proper vertices - primary and secondaries. In designing a vertex tracking system, one must consider the long interaction region (IR) of the Tevatron, which has a $\sigma_{z}$ of $30 \mathrm{~cm}$. This forces one to have a rather long vertex detector. In addition, the detector must be placed very close to the interaction region in order to achieve good resolution and acceptance; so radiation damage is an issue. In this experiment, the vertex detector will be used in the Level 1 trigger to select events with secondary vertices. This places special requirements on the detector and its readout.

The baseline BTeV vertex tracking system uses planar arrays of silicon pixel detectors, placed at intervals along the IR and upstream and downstream of it (to catch tracks coming from the end of the IR). Silicon pixel detectors are chosen because of their: 
- Superior signal to noise (expected to be better than 100 to 1 for $300 \mu \mathrm{m}$ thick detectors) as compared to other solutions such as silicon strips

- Excellent spatial resolution. This includes the ability to improve the spatial resolution by measuring the charge sharing between adjacent pixels

- Low occupancy due to the large number of channels

- Intrinsically better pattern recognition capabilities compared to strip detectors

- Speed of response

- Radiation hardness

It is especially important for the trigger that the number of spurious noise hits be as low as possible. Also, the vertex tracking system must not produce pattern recognition ambiguities or ghost tracks which would take extra time to sort out at the trigger level. The two dimensional nature of the pixels is an enormous help in this regard.

The baseline vertex detector, shown in Fig. 4.11, has rectangular $50 \mu \mathrm{m}$ by $400 \mu \mathrm{m}$ pixel detectors. It has triplets of planes distributed along the IR separated by $3.2 \mathrm{~cm}$. The individual planes cover $\pm 5 \mathrm{~cm}$ in both transverse dimensions. Acceptance studies show that this is an adequate size for these detectors. They are mounted above and below the beam, and are arranged so that a small square hole of $\pm 6 \mathrm{~mm} \times \pm 6 \mathrm{~mm}$ is left for the beams to pass through.

Each triplet consists of one plane that measures the bend view, one that measures the non-bend view, and a third that measures the bend view again. The reason for this particular arrangement of pixel orientations is related to the trigger and is discussed below in the trigger section. A crude momentum measurement can be made using only information from three or four triplets. If the pulse height is read out and made available to the trigger, charge sharing can be used to improve the momentum resolution (the point resolution is expected to be between $5 \mu \mathrm{m}$ and $9 \mu \mathrm{m}$ ). The momentum information can be used to reject very soft tracks that would adversely affect the trigger algorithm, and to properly assign multiple coulomb scattering weights to tracks in the vertex fits.

The implementation of this system will be very challenging. Because pixel information must be made available to the first-level trigger, a custom electronic design is necessary. There are also significant mechanical (e.g. alignment and support) and thermal issues that must be addressed. The detector must be shielded electronically from the circulating beam which is a significant $\mathrm{rf}$ source. It will be enclosed in a secondary vacuum, isolated from the main accelerator vacuum, by a thin aluminum vacuum container. The silicon should be as close to the beam as possible. A hole of $\pm 6 \mathrm{~mm}$ on a side will result in the innermost edge of the detector receiving a fluence of close to $2.0 \times 10^{14}$ per year $\left(10^{7} \mathrm{sec}\right)$ at a luminosity of $2 \times 10^{32} \mathrm{~cm}^{-2} \mathrm{sec}^{-1}$. The detector needs to be retractable to a distance of $2 \mathrm{~cm}$ from the beam while the collider is being filled and until acceleration is complete. Table 4.3 summarizes the properties of the pixel detector. 
While pixel detectors have been successfully operated in HEP experiments, no pixel detector of the proposed size which operates at the high rates we will experience in BTeV, and which can provide signals to the first-level trigger has yet been built. A substantial $\mathrm{R} \& \mathrm{D}$ effort is required to meet the BTeV specifications. Although the BTeV pixel detector is unique, pixel detectors are necessary for several other experiments, and we are taking advantage of work being done all over the world. A status report on the R\&D to develop this detector is given in Chapter 5 .

We recognize that the design presented here is very challenging. We are studying ways of reducing the technical requirements while incurring only minimal loss of physics reach. We are also particularly interested in reducing the amount of material in the pixel detector in order to reduce the number of conversions of photons from $B$ decays. This is discussed in the chapter on the electromagnetic calorimeter below.

\subsubsection{Forward tracking system}

The purpose of the forward tracking system is to:

- Provide high precision momentum measurements for charged tracks. The vertex detector will provide precise position and direction measurements, but it is too short and contains too much material to provide precise momentum measurements.

- Provide the only trajectory measurements for important classes of tracks which do not pass through the vertex detector. The most important such class consists of $K_{s}$ 's and $\Lambda$ 's which decay downstream of the pixel planes. $K_{s}$ 's are daughter particles in some of the most important bottom and charm decays.

- Confirm and locate tracks at the entrance (and exit) of the RICH, the EM calorimeter, and the muon system. In particular, this system allows one to flag charged tracks that interacted or decayed within the spectrometer volume.

The baseline technology for most of the forward tracker is planes of $4 \mathrm{~mm}$ diameter straw tubes. Seven stations of tubes are distributed along each arm of the spectrometer. Three of these stations are located in the fringe field of the dipole magnet. Three more are placed between the end of the magnet coils and the RICH entrance window (these establish the direction of tracks entering the RICH). The final plane is located between the RICH and the EM calorimeter. A single station has three sets of straws directed along the three views: $\mathrm{Y}$, $\mathrm{U}$, and $\mathrm{V}$ ( $\mathrm{U}$ and $\mathrm{V}$ are at $\pm 45^{\circ}$ from $\mathrm{Y}$ ). Each set in a station is made up of three layers of parallel straws.

The straw tube chambers are expected to provide a spatial resolution of $\sim 150 \mu \mathrm{m}$ per plane. Three parallel straw tubes per station will yield a resolution of better than $100 \mu \mathrm{m}$ in each of the three views. This resolution is sufficient to provide a momentum resolution between $0.5 \%$ and $1 \%$ for momenta between $1 \mathrm{GeV} / \mathrm{c}$ and $100 \mathrm{GeV} / \mathrm{c}$.

Recent simulations have demonstrated that at a luminosity of $2 \times 10^{32} \mathrm{~cm}^{-2} \mathrm{sec}^{-1}, 4 \mathrm{~mm}$ diameter straw tubes do not provide adequate segmentation near the beamline in the first 
three measuring stations. As is discussed in Chapter 6, we have modified the baseline design to include silicon strip detectors to cover the central portion of these stations.

Tables 4.4 and 4.5 summarize the baseline design specifications for the straw tube and silicon strip subsystems of the forward tracker.

\subsubsection{Diamond Pixel Option}

Diamond pixel detectors could have significant advantages over silicon pixel detectors but are in an earlier stage of development. The recombination length of diamond has improved steadily in the last couple of years and is now around $150 \mu \mathrm{m}$. The resulting signal is about a factor of three lower than that of silicon. Research on improving the signal collection continues. Properties of diamond that make it superior to silicon if sufficient pulse height can be obtained are:

- lower radiation length leading to lower multiple scattering;

- better radiation hardness; and

- better thermal conductivity which leads to simpler cooling schemes which can result in less material in the detector. 


\section{The BTeV Baseline Pixel Detector}

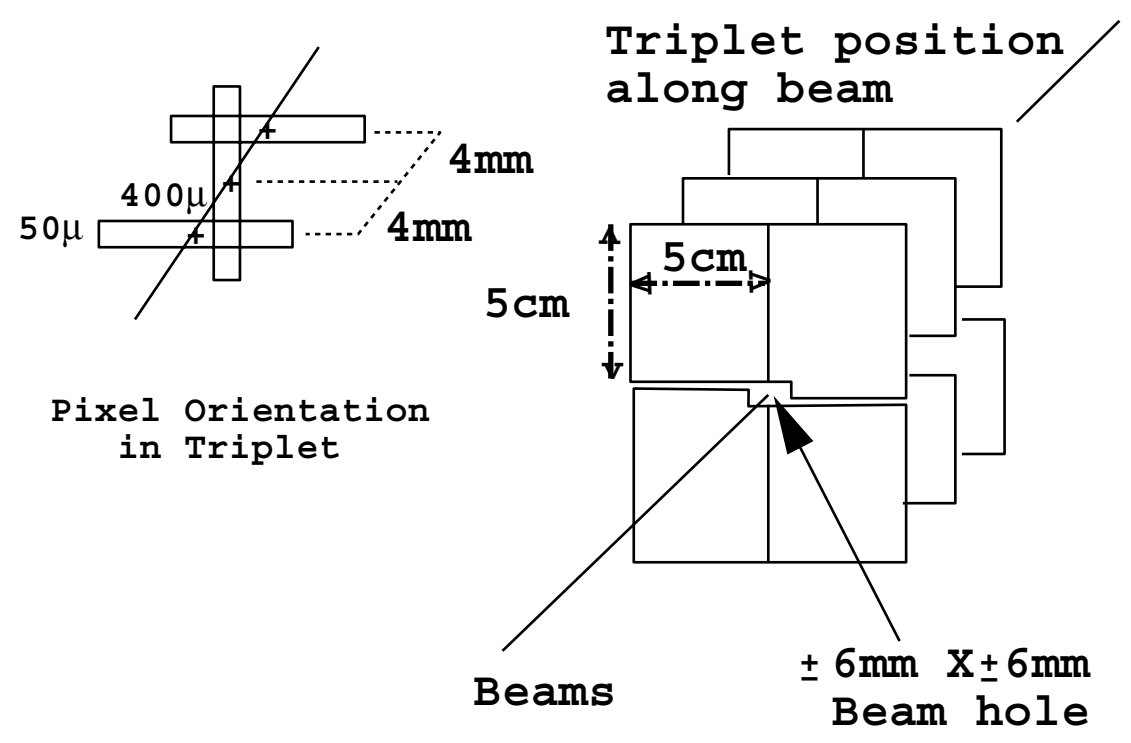

Elevation View

10 of 31 Triplet Stations

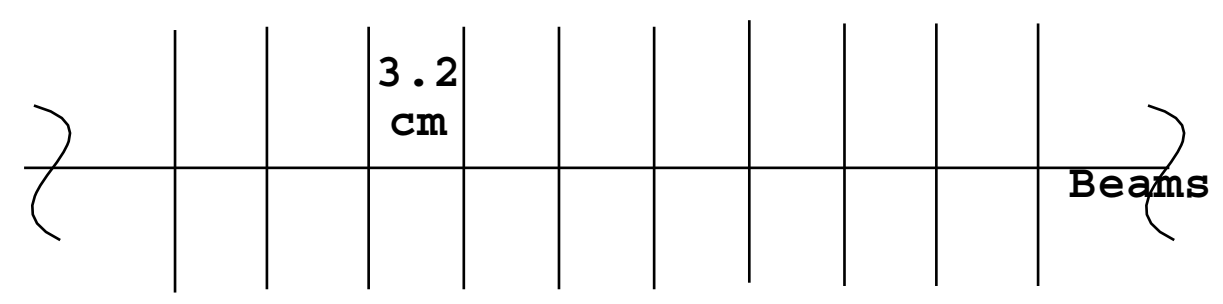

Figure 4.11: Baseline pixel vertex detector 
Table 4.3: Properties of Baseline Pixel Vertex Detector

\begin{tabular}{|l|l|}
\hline Property & Value \\
\hline Pixel size & rectangular: $50 \mu \mathrm{m} \times 400 \mu \mathrm{m}$ \\
Plane Dimensions- upper half & $10 \mathrm{~cm} \times 5 \mathrm{~cm}$ \\
Plane Dimensions- lower half & $10 \mathrm{~cm} \times 5 \mathrm{~cm}$ \\
Central square hole (adjustable) & nominal setting: $12 \mathrm{x} 12 \mathrm{~mm}$ \\
Total Planes & 93 \\
Total Stations & 31 , each consisting of \\
& three planes \\
Pixel orientations & the outer two with narrow \\
& pixel dimension vertical \\
& the middle one with narrow \\
Separation of Stations & pixel dimension horizontal \\
Plane to plane separation & $3.2 \mathrm{~cm}$ \\
(within station) & 4 mm \\
Total Plane Thickness & $1000 \mu \mathrm{m}$ Si equivalent \\
(incl cooling, supports) & $3200 \mu \mathrm{m} \mathrm{Si} \mathrm{equivalent}$ \\
Station Thickness & \\
(incl. Al shielding skin) & $4.2 \times 10^{7}$ \\
Total Pixels & $\approx 1 \mathrm{~m}^{2}$ \\
Total Silicon Area & analog readout (no. of bits to be decided) \\
Readout & signals are used in Level 1 trigger \\
Trigger & beam crossing rate is 132 ns. \\
Rate requirements & $<3 \%$ at $2 \times 10^{32} \mathrm{~cm}^{-2} \mathrm{~s}^{-1}$ \\
Deadtime & desired: $<10^{-6}$ per channel per crossing \\
Noise requirement & required: $<10^{-5}$ per channel per crossing \\
& $9 \mu \mathrm{m}$ or better \\
Resolution & $5 \mu \mathrm{m}$ or better \\
Alignment accuracy & $\approx 6 \times 10^{14}$ particles $/ \mathrm{cm}^{2}$ \\
Radiation tolerance & $<40 \mu$ Watt \\
Power per pixel &
\end{tabular}


Table 4.4: Properties of the baseline forward straw tracker

\begin{tabular}{|l|l|}
\hline \hline Property & Value \\
\hline Straw size & $4 \mathrm{~mm}$ diameter \\
Central hole & $5 \mathrm{~cm} \times 5 \mathrm{~cm}$ to $24 \mathrm{~cm} \times 24 \mathrm{~cm}$ \\
Total Stations & $14(7$ per arm $)$ \\
$Z$ positions $(\mathrm{cm})$ & $96,146,196,296,341,386,706$ \\
Half size $(\mathrm{cm})$ & $30,45,60,90,105,118,210$ \\
Views per station & 3 \\
Layers per view & 3 \\
Total number of straws & 78132 \\
Total station thickness $\left(<L>/ X_{0}\right)$ & $0.6 \%$ \\
Readout & ASD + timing chip $(6$ bits $)$, sparsified \\
Total channels & 118,464 \\
Rate requirements & beam crossing rate is $132 \mathrm{ns.}$ \\
\hline \hline
\end{tabular}

Table 4.5: Properties of the proposed baseline forward silicon tracker

\begin{tabular}{|l|l|}
\hline \hline Property & Value \\
\hline Wafers & $4 \times 6 \mathrm{~cm}^{2} / 2.5 \times 4.75 \mathrm{~cm}^{2}$ \\
Pitch & $100 \mu \mathrm{m}$ \\
Thickness & $300 \mu \mathrm{m}$ \\
Ladder modules & 12 per station \\
Coverage & $24 \mathrm{~cm} \times 24 \mathrm{~cm}$ \\
Central hole & $5 \mathrm{~cm} \times 5 \mathrm{~cm}$ \\
Total stations & $6(3 \mathrm{per}$ arm $)$ \\
Z positions $(\mathrm{cm})$ & $100,150,200$ \\
Measurement views & $4(\mathrm{X}, \mathrm{Y}, \mathrm{U}, \mathrm{V})$ \\
Channels per view & 2400 \\
Total channels & 57600 \\
Readout & double-sided, sparsified binary \\
\hline \hline
\end{tabular}




\subsubsection{Triggering System}

The proposed broad charm and beauty physics program of $\mathrm{BTeV} / \mathrm{C} 0$ calls for a trigger whose efficiency

- is large for heavy-quark decay events which can be found off-line and

- is relatively independent of decay mode.

Because the program has many goals and will undergo several phases of development, the trigger needs to be flexible. We plan to implement a multi-level trigger with several "branches" that can be independently prescaled and then combined into a global trigger. These will include muon and dimuon triggers, general interaction triggers, and possibly electron and dielectron triggers. However, the demands of a collider $B$ experiment are difficult to meet with simple, conventional approaches to triggering. In particular, the second criterion above is not satisfied by simple triggers commonly used in hadron collider experiments, e.g. lepton triggers. BTeV's main $B$-physics trigger focuses on the key difference between heavy quark events and typical hadronic events - the presence of detached vertices - at the first trigger level, Level 1. It is very efficient for the $B$ decays that can be successfully reconstructed by the spectrometer. It also avoids the need to focus at Level 1 on specific final states, which can limit the accessible physics. Its implementation requires a very sophisticated trigger architecture which also provides a more than adequate foundation for any of the other triggers we are considering.

In this section, we focus on the Level 1 detached vertex trigger, for which we are carrying out a program of research and development, based on extensive simulation and design studies. Details some of the other triggers - the muon trigger and the electron trigger - are given in the sections on the muon detector and the electromagnetic calorimeter.

\subsubsection{Level 1 Detached Vertex Trigger}

The trigger algorithm has the goal to reconstruct tracks and find vertices in every interaction up to an interaction rate of order $15 \mathrm{MHz}$ (luminosity of $2 \times 10^{32} \mathrm{~cm}^{-2} \mathrm{~s}^{-1}$ at $\sqrt{s}=2 \mathrm{TeV}$ ). This entails an enormous data rate coming from the detector $(\sim 100 \mathrm{~GB} / \mathrm{s})$, thus a careful organization of the data-flow is crucial. This trigger must be able to operate both in collider and fixed-target modes. It must be capable of reducing the trigger rate by a factor of at least 100 .

The key ingredients for such a trigger are

1. a vertex detector with excellent spatial resolution, fast readout, and low occupancy;

2. a heavily pipelined and parallel processing architecture well suited to tracking and vertex finding;

3. inexpensive processing nodes, optimized for specific tasks within the overall architecture; 
4. sufficient memory to buffer the event data while the calculations are carried out; and

5. a switching and control network to orchestrate the data movement through the system.

The pixel detector described above is the first ingredient. Use of pixels for the tracker will produce very high spatial resolution for the trigger (and the final analysis) and, because of their 2-dimensional nature and excellent signal-to-noise, will reduce the level of potential confusion during the crucial track-finding phase of the trigger. Since the pixel detector is located in a magnetic field, some momentum information can be derived from the bending in the pixel detector alone without needing to use the downstream tracking system. If, in addition, the pixels have pulse height readout, they can achieve a spatial resolution of less than $12 \mu \mathrm{m}$, which provides rather good momentum information to the Level 1 trigger.

To provide the second ingredient, we employ parallelism both at the subevent level and at the event level. The detector layout described above has "stations" consisting of three planes. Processors organize the hits in each triplet of planes into "station hits". Still at the subevent level, individual processors work on the station hits from a slice of the detector, as will be described below. At the event level, track candidates from the individual slices are brought together in a common processing node, one member of a large farm, for vertex finding.

The third ingredient is provided by using large numbers of inexpensive, commercially available CPU chips. New processors are constantly appearing on the market, often optimized for very specific tasks, and prices are dropping rapidly. Research is in progress to determine the characteristics of the processors needed at each stage.

The fourth ingredient is also commercially available. The time allowed to process the data is limited by the number of events which can be stored while the trigger decision is being made. An event is expected to be significantly less than 200,000 bytes. The memory required to buffer 256 crossings even for 200,000 byte events is only $50 \mathrm{MB}$ and is adequate for a decision time of $33 \mu \mathrm{s}$. This system can be off the detector (i.e. not in a strong radiation environment) so commercial (PC) memories are adequate and the costs are small on the scale of the full trigger system. Memory costs do not limit the decision time which could be much longer if necessary.

The final ingredient is switching networks which appear at the input of each section of the Level I trigger and between the Level 1 trigger and next level of triggering. The design of this switching hardware is under study.

The reference trigger scheme was originally developed by the Penn Group - Selove, Sterner, and Isik [4]. While it has undergone major revisions and is still being updated, it has been an excellent starting point for studying this kind of trigger.

The proposed algorithm has four steps:

1. In the first step, hits from each pixel plane are assigned to detector subunits. This can be done very rapidly for all planes in parallel using field-programmable gate arrays (FPGAs). It is desirable to subdivide the area of the detectors in a way which minimizes the number of tracks crossing from one subunit into the next and assures uniform and 
low occupancy $(<1$ hit/event) in all subunits. We therefore divide each plane into 32 azimuthal sectors (" $\phi$-slices"). Simulation shows that at $2 \mathrm{TeV}$, on average each $\phi$-slice contains 0.3 hits per inelastic interaction, approximately independent of the plane's $z$ coordinate.

2. In the second step, "station hits" are formed in each triplet of closely-spaced planes using hits from each $\phi$-slice. Since the pixels under consideration have a rectangular shape (e.g. $50 \mu \mathrm{m}$ by $400 \mu \mathrm{m}$ ), the two outer planes in each station are oriented for best resolution in the bend view, and the inner plane for the non-bend view. There is a "hit processor" for each $\phi$-slice of each station. The hit processor finds triplets of overlapping pixels, and sends to the next stage a single "minivector" consisting of $x, y, \frac{d x}{d z}$ and $\frac{d y}{d z}$. Given the good position resolution of each measurement, a detector station determines a space point accurate to $5-9 \mu \mathrm{m}$ in $x$ and $y$, and $y$ and $x$ slopes to $\frac{d y}{d z} \approx 1 \mathrm{mr}$ (bend-view) and $\frac{d x}{d z} \approx 10 \mathrm{mr}$ (non-bend). The use of these minivectors in the track-finding stage substantially reduces combinatorics.

3. In the third step, minivectors in each $\phi$-slice from the full set of stations are passed via a sorting switch to a farm of "track processors." The sign of the bend-view slope is used to distinguish forward-going and backward-going minivectors and send them to separate farms. To handle the few percent of tracks crossing segment boundaries, hits near a boundary between two $\phi$-slices can be sent to the processors for both slices.

Each track processor links minivectors into tracks, proceeding along $z$ from station to station. For each pair of minivectors in adjacent stations, the processor averages the $y$-slopes in the two stations and uses this average slope (which represents the slope of the chord of the magnetically deflected trajectory) to project from the first station into the second. It then checks whether the $y$-value of the minivector in the downstream station agrees with the projection within an acceptance window. If three or more hits satisfy this requirement, a fast fitting algorithm finds the momentum of the track candidate.

4. In the fourth step, the tracks are passed to a farm of "vertex processors" and used to form vertices. The vertex with $\sum p_{z}$ closest to zero is designated as the primary vertex. Tracks which have a large impact parameter with respect to this vertex are taken as evidence for heavy quark production in an event. To reduce the effect of multiple scattering on vertex resolution, tracks below an adjustable bend-view transverse-momentum $\left(p_{y}\right)$ threshold are excluded from the vertex finding.

It is desirable to have pulse-height information included with each hit so that interpolation can be used to improve the point resolution beyond the $50 \mu \mathrm{m} / \sqrt{12}$ otherwise available. Digital (1 bit) readout seems to be adequate for the $p_{y}$ cut, but the improved momentum resolution with analog information would allow the processor to calculate the mass of the charged particles at a vertex, which may be a useful variable on which to cut if additional rejection is needed. It also can improve the position accuracy for tracks incident at large angles, which cross over rows or columns of pixels. 


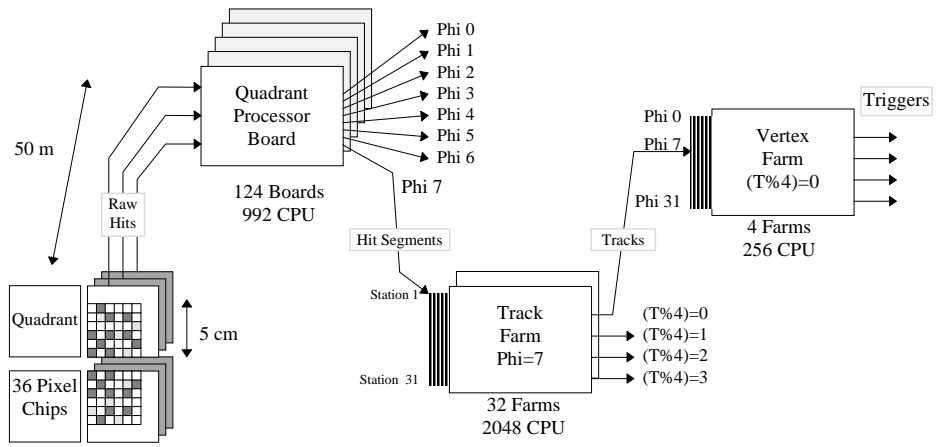

Figure 4.12: Schematic of Proposed Level 1 vertex trigger

The Level 1 rejection factor for events containing only light quarks is required to be approximately $10^{-2}$ in order to reduce the data rate into Level 2 to sufficiently low levels. The overall acceptance for $B \rightarrow \pi^{+} \pi^{-}$events is expected to be $10-20 \%$ for the proposed $\mathrm{BTeV}$ Level 1 vertex trigger. The expected trigger efficiency for $B \rightarrow \pi^{+} \pi^{-}$events that will have both $B$ daughter $\pi$ s reconstructed should be much higher - about $50 \%$. Results of simulation studies with this trigger will be described below in Chapter 8 .

D. Husby and W. Selove have developed a detailed proposal for this trigger system. In this design there are about 1000 hit processors, 2000 track processors, and 128 vertex processors. Since the architecture is scalable, there is flexibility for these numbers to be optimized to meet unanticipated conditions. The design uses a combination of digital signal processors (DSPs), FPGAs and commercial CPUs. Fig. 4.12 shows a schematic diagram of the full system. Details of their current design can be found in Ref. [5]. A hardware design has been worked out for a small-scale prototype; see the trigger research and development section for details.

\subsubsection{Development of other Level 1 triggers and the global trigger framework}

While we have emphasized the "vertex/impact parameter" trigger because it is our most crucial and most ambitious trigger, we will also have other triggers at Level 1. These will include muon and electron triggers and perhaps some triggers derived from the same hardware that is used for the vertex trigger but optimized for different physics. At the output of the first level trigger there will be a 'global Level 1 trigger' subsystem that will allow us to combine the various individual trigger elements into final triggers (for example, an event with only weak evidence for a secondary vertex but with an indication of a high $p_{t}$ single lepton might be accepted). It will also have the ability to apply a programmable prescale to the various triggers before the are 'or'ed together to form the final trigger. We will probably also include the ability to dynamically change the prescales during the course of the store. 


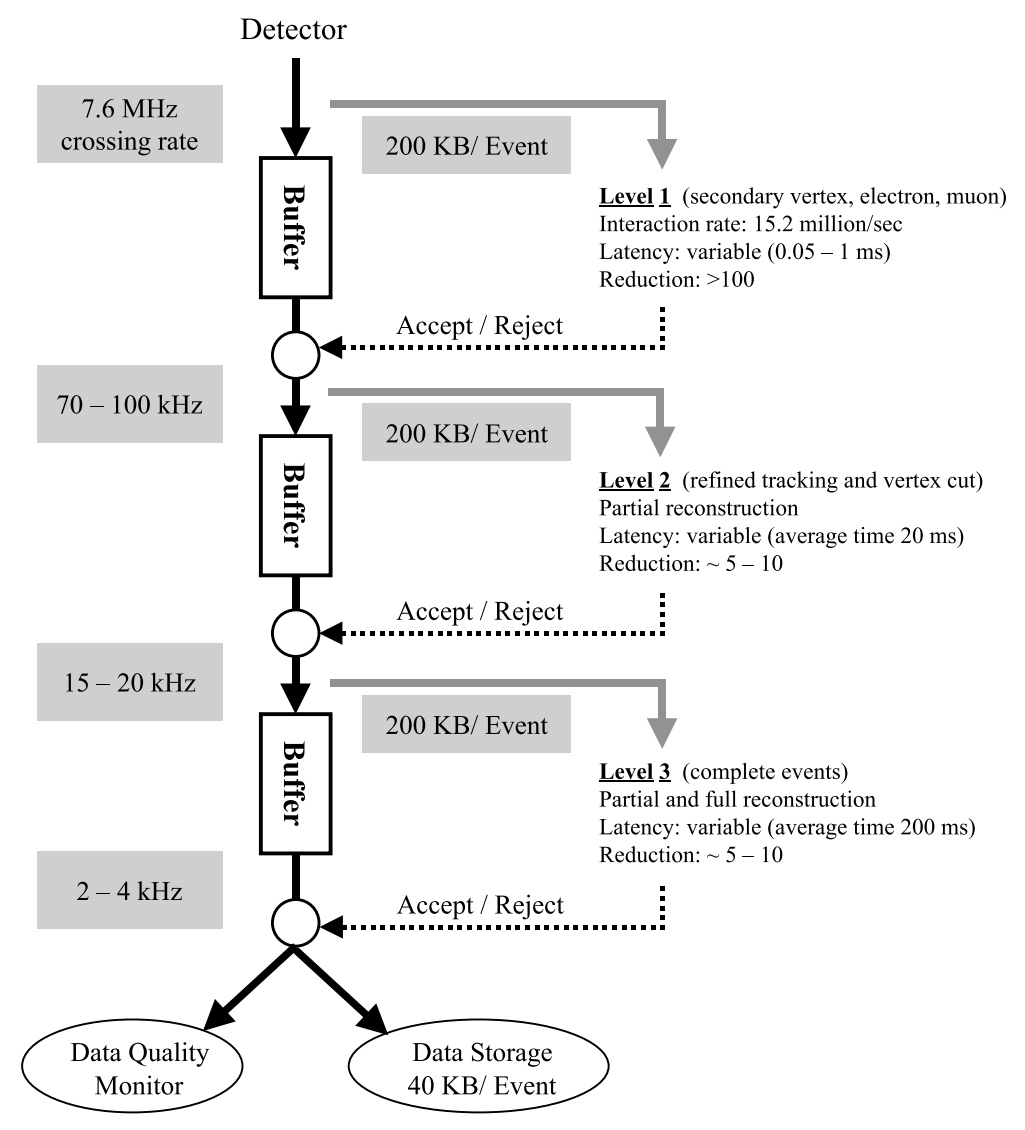

Figure 4.13: The BTeV data flow diagram. The numbers are for the nominal luminosity of $2 \times 10^{32} \mathrm{~cm}^{-2} \mathrm{~s}^{-1}$.

\subsubsection{Beyond the Level 1 Trigger}

Modern experiments in high energy physics implement hierarchical trigger systems and BTeV is no exception. Many details of the full data acquisition system have to be worked out as the design of the detector components and trigger algorithms become more mature. At the design luminosity of $2 \times 10^{32} \mathrm{~cm}^{-2} \mathrm{~s}^{-1}$ and with a total inelastic cross section of $\sigma_{\text {inel }} \approx 60 \mathrm{mb}$ almost every crossing will have an interaction, so events will have to be examined at the crossing rate of $7.5 \mathrm{MHz}$. Fig. 4.13 gives the outline of the expected data flow at the nominal design luminosity.

At our current level of understanding, the needed level 1 rejection factor seems to be achievable even if the only test made is for a detached vertex in vacuum. The event rate is then reduced to $\approx 100 \mathrm{kHz}$ in the worst case during the highest luminosity running. It is still possible to move all this data, amounting to a maximum rate of $10 \mathrm{~GB} / \mathrm{s}$, to the Level 2 trigger.

Events that pass the first trigger level are forwarded to compute nodes to be further analyzed. First, information from other detectors besides the pixel detector is used to get 
better values of the momentum of the tracks. The vertex detachment requirement is then imposed with a better knowledge of the error matrices of the tracks and improved vertex assignments are computed. Events remaining after this step are then fully assembled in a switch-based event builder and passed to a processor farm. Even so, it will probably be impractical to write several $\mathrm{kHz}$ of events to archival storage.

The final trigger level will combine information from different sub-detectors to further suppress background events. Particle identification will be available at this stage and could be used to obtain a very clean direct charm signal for specific final states. We intend to fully reconstruct the events at this level before the data are sent to a storage system and made available for off-line processing.

Because the event rate surviving this last level may still be close a few $\mathrm{kHz}$, the software will probably have to reduce the amount of data per event to archival storage by writing out an event summary which eliminates much of the raw data. The event summary would be around $50 \mathrm{~KB}$ so that the output rate could still be as high as $80 \mathrm{MB} / \mathrm{s}$. This results in a dataset size of $800 \mathrm{~TB} / \mathrm{yr}$, somewhat more than is expected from CDF or D0 in Run II. However, initial phases of the experiment will run at much lower rates and will be comparable in dataset size to a current fixed target experiment.

\subsubsection{Additional Work}

Although simulation results are very encouraging, much additional simulation work and a vigorous $R \& D$ program are needed to implement such a triggering system. The simulation work involves studying the performance of these proposed Level 1 and Level 2 algorithms under realistic experimental conditions, which include detector inefficiencies, photon conversions, delta ray production, hadronic interactions in the silicon, low energy loopers, multiple interactions, electronic noise, machine backgrounds, and beam-induced backgrounds (e.g. scraping in the support structures and/or beam pipe). Some of these studies have already been done and are presented in Chapter 8 . 


\subsubsection{Particle Identification}

Excellent charged hadron particle identification is a critical component of a heavy quark experiment. Even for a spectrometer with the excellent mass resolution of BTeV, there are kinematic regions where signals from one final state will overlap those of another final state. For example, $B_{d}^{o} \rightarrow \pi^{+} \pi^{-}, B_{d}^{o} \rightarrow K^{+} \pi^{-}$, and $B_{s}^{o} \rightarrow K^{+} K^{-}$all overlap to some degree leading to ambiguous classification of the final state. These ambiguities can be eliminated almost entirely by an effective particle identifier. In addition, many physics investigations involving neutral $B$-mesons require 'tagging' of the flavor of the signal particle by examining the properties of the 'away-side' particle. Our studies show that kaon tagging is a very effective means of doing this.

\subsubsection{Requirements}

In the design of any particle identification system, the dominant consideration is the momentum range over which efficient separation of the various charged hadron types $-\pi, K$, and $p$ - must be provided. In BTeV, the physics goal which sets the upper end of the momentum requirement is the desire to cleanly separate $B_{d}^{o} \rightarrow \pi^{+} \pi^{-}$from $B_{d}^{o} \rightarrow K^{+} \pi^{-}$and $B_{s}^{o} \rightarrow K^{+} K^{-}$. These two-body decays produce reasonably high momentum pions and kaons. Fig. 4.14 shows the momentum distribution of pions from the decay $B_{d}^{o} \rightarrow \pi^{+} \pi^{-}$mentioned above for the case where the two particles are within the spectrometer's acceptance. The low momentum requirement is defined by having high efficiency for 'tagging' kaons from generic B decays. Since these kaons come mainly from daughter $D$-mesons in multibody final state $B$-decays, they typically have much lower momentum than the particles in two body decays. Fig. 4.15 shows the momentum distribution of 'tagging' kaons for the case where the signal particles are within the geometric acceptance of the spectrometer. About $1 / 5$ of the tagging kaons never exit the end of the spectrometer dipole. Almost all kaons exiting the dipole have momenta above $3 \mathrm{GeV}$. Based on these plots, we have set the momentum range requirement for the particle identification system to be

$$
3 \mathrm{GeV} / \mathrm{c}<P_{\text {particle id }}<\sim 70 \mathrm{GeV} / \mathrm{c}
$$

Finally, kaons and pions from directly produced charm decays have momenta which are not very different from the kaons from $B$-decays. Fig. 4.16 shows the momentum spectra of kaons from accepted $D^{o} \rightarrow K^{-} \pi^{+}, D^{o} \rightarrow K^{-} \pi^{+} \pi^{-} \pi^{+}$, and $D_{s}^{+} \rightarrow K^{+} K^{-} \pi^{+}$in both collider and fixed target mode. The range set by the $B$-physics requirements is a reasonable, if not optimal, choice also for charm physics.

\subsubsection{RICH radiators}

Because of the large momentum range and limited longitudinal space available for a particle identification system in the $\mathrm{C} 0$ enclosure, there is really only one choice of detector technology - a gaseous ring-imaging Cherenkov counter. Pions and kaons can be separated in this momentum region with a single gas radiator like $C_{4} F_{10}(n=1.00138$ in visible range). Some 

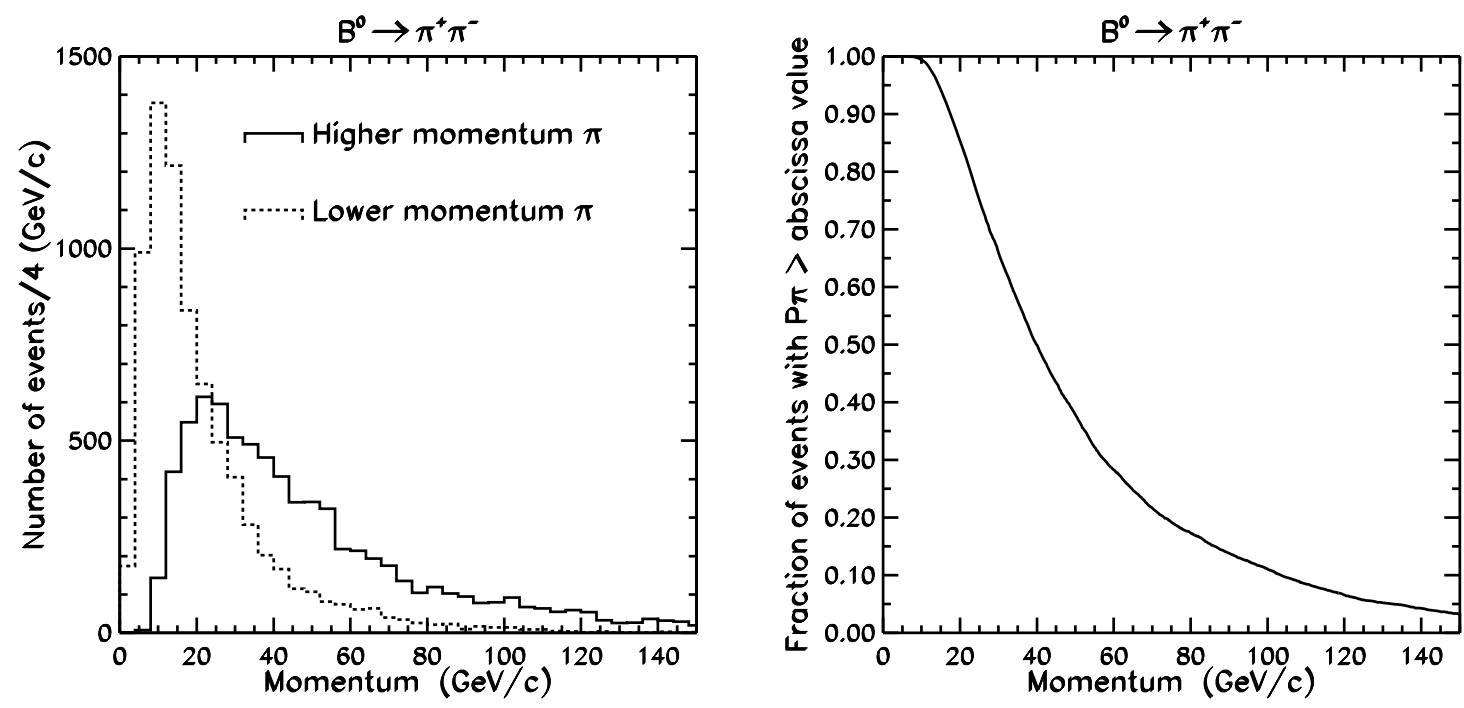

Figure 4.14: The momentum distribution of pions in $B_{d} \rightarrow \pi^{+} \pi^{-}$decays. The left plot shows distributions for the lower (dashed line) and higher (solid line) momentum pion in this decay. The right plot presents the latter distribution in integral form, which gives loss of efficiency as a function of the high momentum cut-off of the particle ID device.

\begin{tabular}{||c|c|c||}
\hline \hline Parameter & $C_{4} F_{10}$ & aerogel \\
\hline $\mathrm{n}$ & 1.00138 & 1.03 \\
$\Theta_{c}(\beta=1)$ & $52.5 \mathrm{mrad}$ & $243 \mathrm{mrad}$ \\
$\pi$ threshold & $2.5 \mathrm{GeV} / \mathrm{c}$ & $0.6 \mathrm{GeV} / \mathrm{c}$ \\
$K$ threshold & $9.0 \mathrm{GeV} / \mathrm{c}$ & $2.0 \mathrm{GeV} / \mathrm{c}$ \\
$p$ threshold & $17.1 \mathrm{GeV} / \mathrm{c}$ & $3.8 \mathrm{GeV} / \mathrm{c}$ \\
thickness & $283 \mathrm{~cm}$ & $5 \mathrm{~cm}$ \\
\hline \hline
\end{tabular}

Table 4.6: Several parameters for the proposed gaseous and solid radiators. 

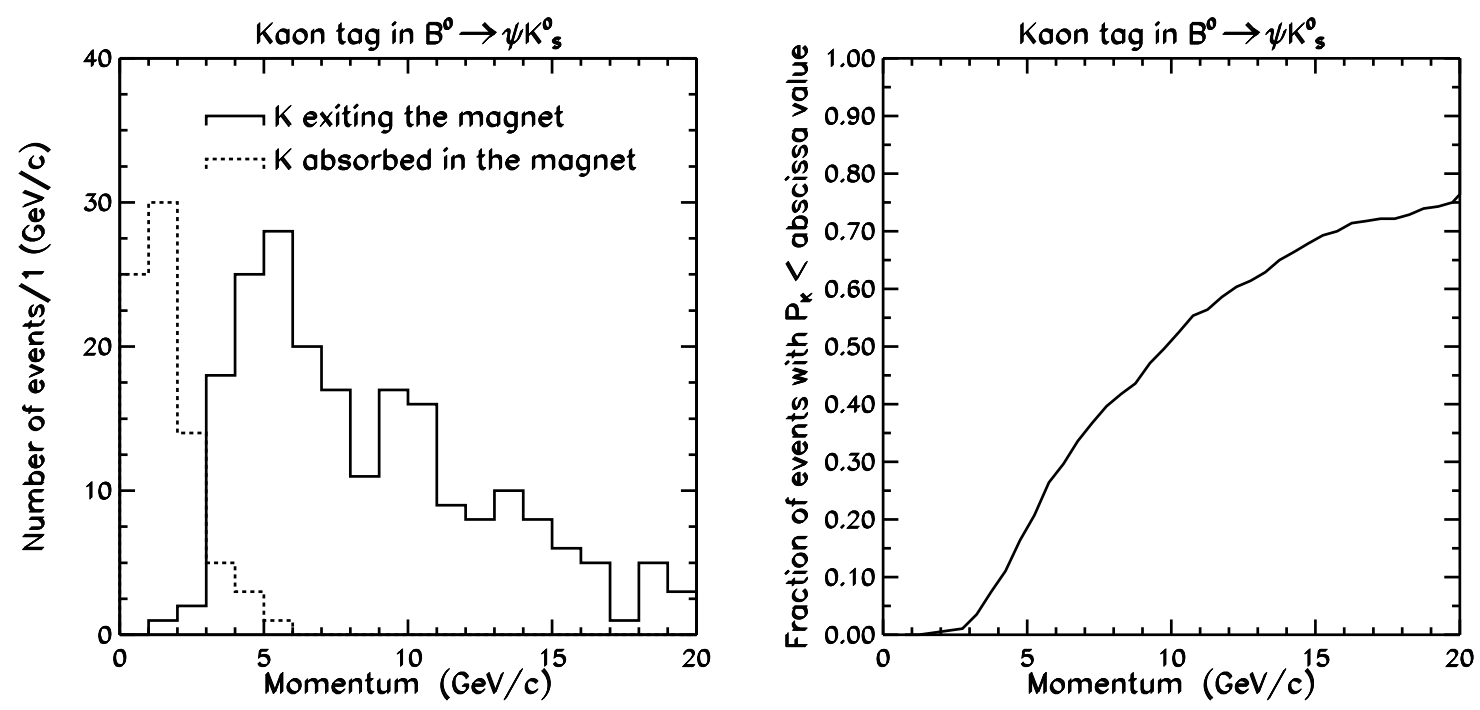

Figure 4.15: The momentum distribution of 'tagging' kaons for the case where the signal particles $\left(\psi K_{S}^{0}\right)$ are within the geometric acceptance of the spectrometer. The left plot shows distributions for kaons absorbed in (dashed line) and exiting from (solid line) the magnet. The right plot presents the latter distribution in integral form, which gives loss of efficiency as a function of the low momentum cut-off of the particle ID device.

parameters of this gas are given in Table 4.6. Among other experiments, $C_{4} F_{10}$ is used in DELPHI (endcap) [1], HERA-B [2] and HERMES RICH detectors [3]. It was also chosen for one of the LHC-B RICH detectors [4].

Note that below about $9 \mathrm{GeV}$, no gas can provide $K / p$ separation and that, since kaons are below threshold, the RICH operates in a threshold mode (except that it has much better noise discrimination than a normal threshold counter because it still measures a Cherenkov ring for pions). In order to improve identification of low momentum particles, one interesting possibility is to insert a thin $(\sim 5 \mathrm{~cm})$ piece of aerogel at the entrance to the gas RICH as proposed by LHC-B [5] and already implemented by HERMES [3]. For example, aerogel with a refractive index of $n=1.03$ would lower the $\pi, K, p$ momentum thresholds to $0.6,2.0$, $3.8 \mathrm{GeV} / \mathrm{c}$ respectively as illustrated in Fig. 4.17. Shorter wavelength Cherenkov photons undergo Raleigh scattering inside the aerogel itself. They are absorbed in the radiator or exit at random angles. A thin window between the aerogel and the gas radiator passes photons only in the visible range, diminishing the scattered component.

The same photo-detection system can detect Cherenkov rings produced in both the gaseous and the aerogel radiators if it operates in visible wavelengths.

\subsubsection{RICH dimensions}

The RICH detector can be located behind the tracking chambers just outside the central dipole magnet, about 4 meters away from the interaction point. The length of the RICH 


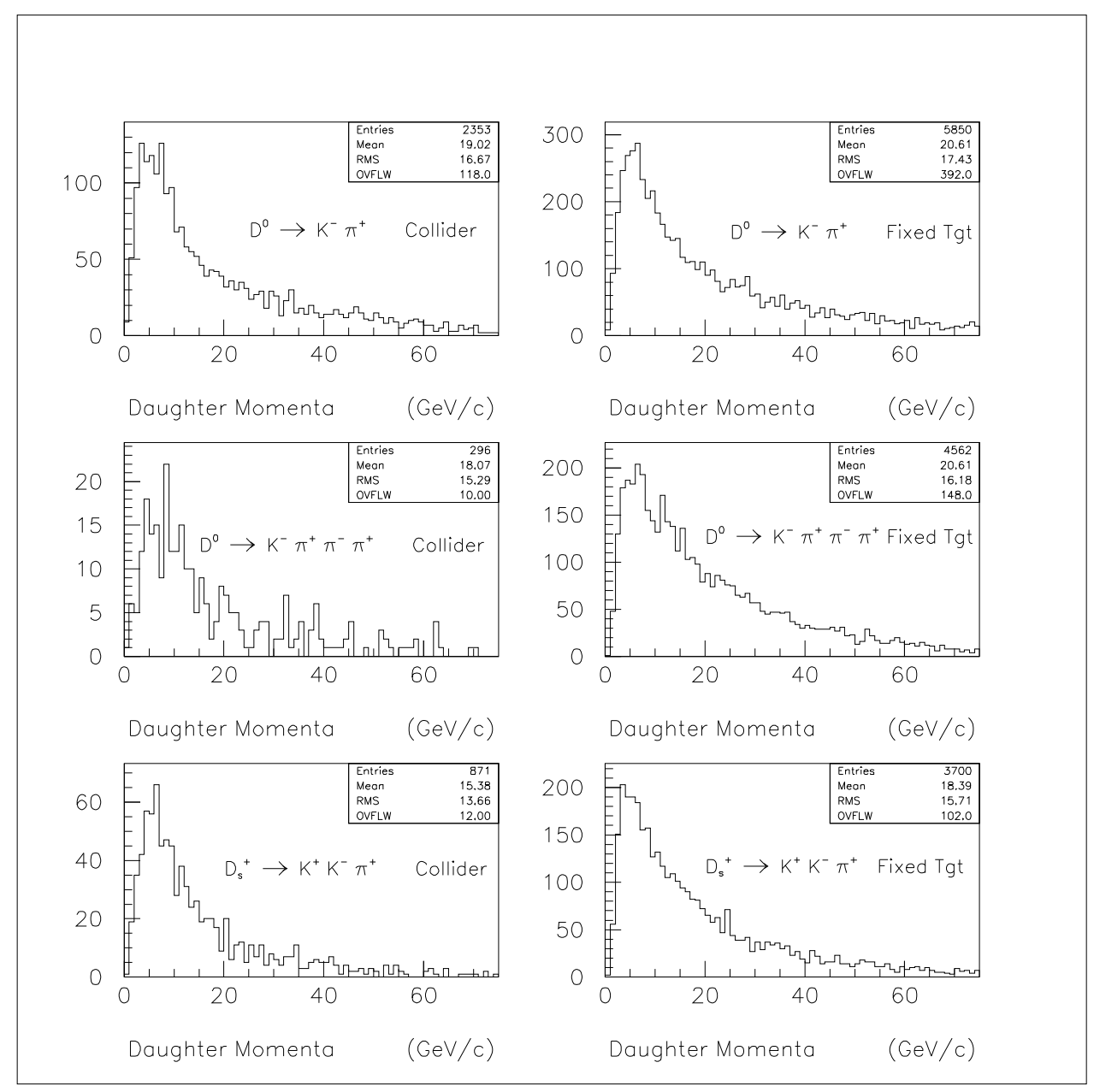

Figure 4.16: The momentum spectra of kaons from accepted $D^{o} \rightarrow K^{-} \pi^{+}, D^{o} \rightarrow$ $K^{-} \pi^{+} \pi^{-} \pi^{+}$, and $D_{s}^{+} \rightarrow K^{+} K^{-} \pi^{+}$in both collider and fixed target modes. 


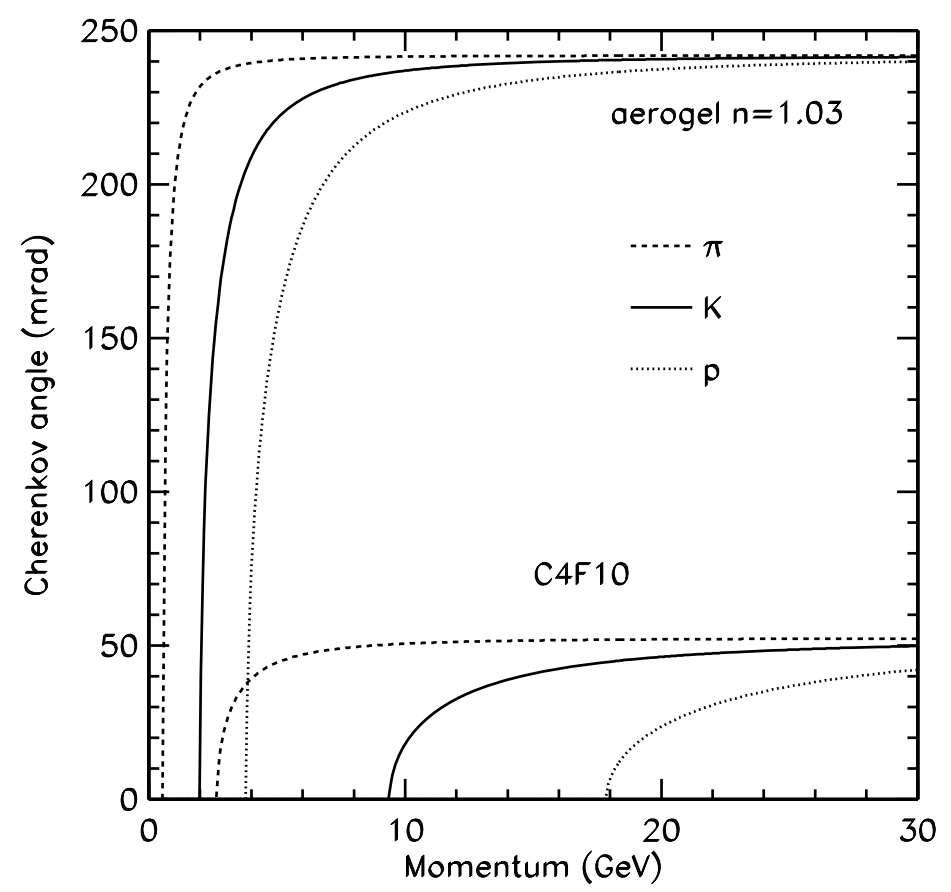

Figure 4.17: Cherenkov angles for various particle species as a function of particle momentum for $C_{4} F_{10}$ and aerogel $(n=1.03)$ radiators.

detector must be less than 3 meters to allow sufficient space for the EM calorimeter and the muon system. The aerogel radiator with thickness of about $5 \mathrm{~cm}$ will be mounted at the entrance to the RICH vessel. The gaseous $C_{4} F_{10}$ radiator will fill the rest of the RICH volume. The transverse dimensions of the RICH entrance window are set by the aperture of the dipole magnet to be about $1.35 \mathrm{~m} \times 1.35 \mathrm{~m}$.

Spherical mirrors at the end of the gas volume reflect Cherenkov photons back and focus them into rings at the photo-detection surface. The photo-detectors should be located away from the flux of particles exiting the magnet, therefore the mirrors are tilted. In order to minimize geometric aberrations from an off-axis mirror configuration, the mirrors will be split along the mid-line of the detector, reflecting photons to photo-detectors located on each side of the vessel in the non-bend view ( $x-z$ plane). The longer the RICH detector the smaller the tilt angle. Since the geometric aberrations due to the mirror tilt are significant for the gas radiator, we plan on the longest RICH detector we can accommodate within the space limitations. This also maximizes the photon yield from the gaseous radiator, again improving the resolution of the device. Thus, the gas radiator will be approximately 3 meters long and the mirror radius will be 7 meters. The mirror tilt angle will be about 240 mrads. Note that the mirror tilt angle cannot be further reduced by use of additional flat mirrors at the other end of the detector, like in the designs of HERA-B and downstream LHC-B RICH detectors, since the front of the gas volume must stay transparent to the aerogel photons. The transverse size of the mirror will be about $4 m \times 4 m$ A possible configuration for the mirrors is to make them from an array of individual hexagons as shown in Fig. 4.18. Each 
mirror half would consist of 18 full hexagons ( $76.2 \mathrm{~cm}$ tip-to-tip) and 6 half hexagons. A reflectance efficiency over $85 \%$ at all relevant wavelengths has been achieved in HERA-B detector with $7 \mathrm{~mm}$ thick pyrex coated with $200 \mathrm{~nm} \mathrm{Al}$ and $30 \mathrm{~nm} \mathrm{MgF}_{2}$.

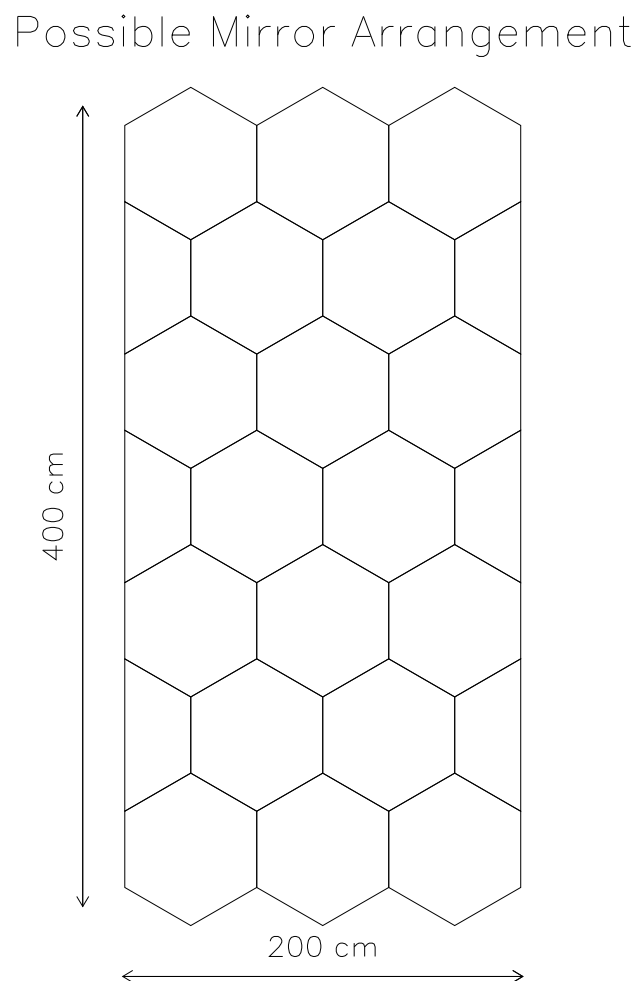

Figure 4.18: Possible arrangement for each half of the mirror using hexagonal mirrors of size $76.2 \mathrm{~cm}$ tip-to-tip.

To find the size and optimal position and orientation of the photo-detection surface, we have used a ray tracing Monte Carlo. Even though the true focal plane of a spherical mirror is not planar, non-planar surfaces do not improve resolution significantly and are difficult to realize in practice. Thus, we have assumed that the Cherenkov rings are focused on a plane. We then minimized emission point error for the gaseous radiator with respect to the location of the plane and its orientation using a sample of $B_{d} \rightarrow \pi^{+} \pi^{-}$events. The untilted orientation of the photo-detectors is very close to the optimum. The corresponding contribution to the Cherenkov angle resolution for the gaseous radiator is 0.4 mrad per photon, compared to $0.2 \mathrm{mrad}$ for untilted mirror. As expected for spherical mirrors the photo-detectors must be located about half way between the interaction point and the mirror, which puts them slightly behind the RICH entrance window.

Geometry of the proposed RICH detector is shown in Fig. 4.19. 

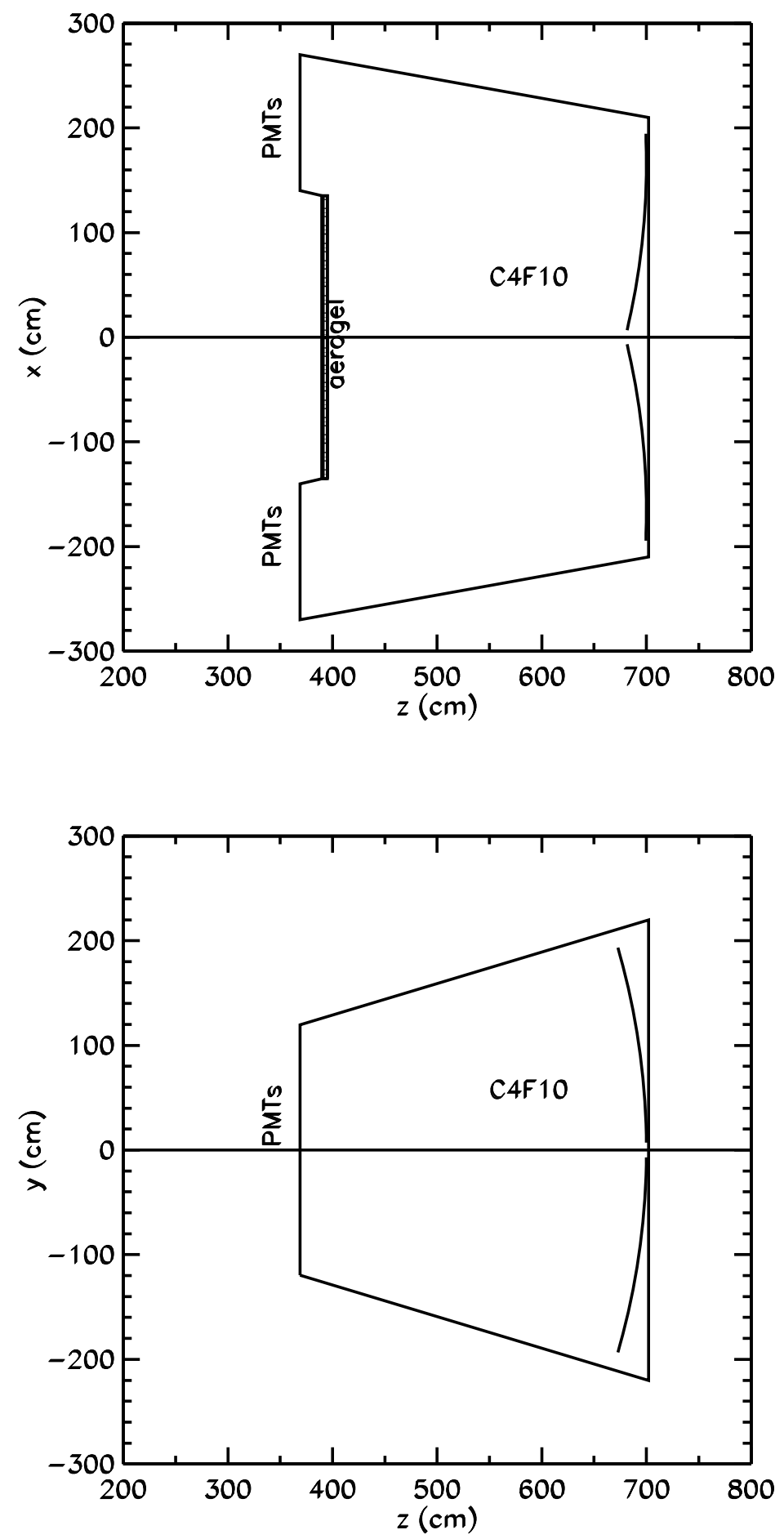

Figure 4.19: BTeV RICH geometry outline. 


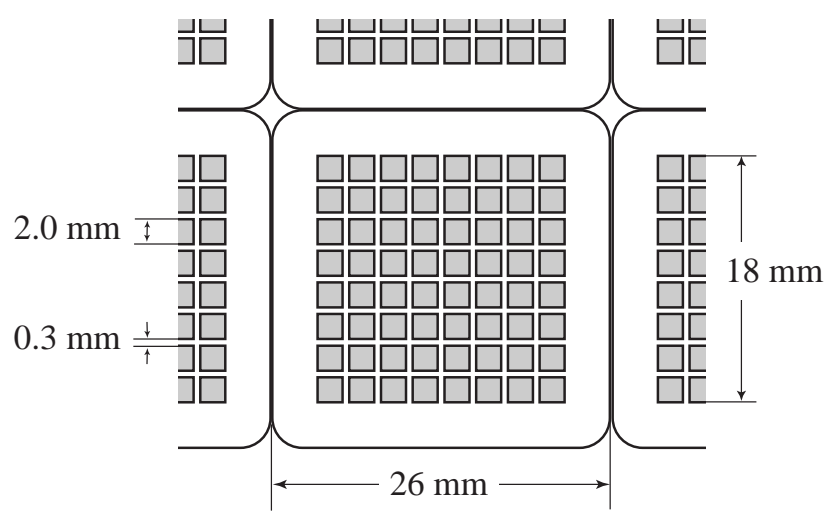

Figure 4.20: Closely packed array of Hamamatsu R5900-M64 tubes as seen from the front. The anode cells are shaded. The picture taken from Ref.[6].

\subsubsection{Photo-detectors}

The photo-detectors must be sensitive to visible wavelengths in order to detect Cherenkov rings from the aerogel. Because of the open geometry of the forward spectrometer and the absence of magnetic field around the RICH detector, arrays of photo-multipliers can be used. Detection of the Cherenkov spectrum in visible wavelengths allows the use of inexpensive window materials, standard photo-cathodes and a simple gas system. The photo-multiplier solution offers durability of the device. Large intrinsic gain and fast response provide for noise resistance and a straightforward read-out system. Hamamatsu has recently developed R5900 multi-anode photo-tubes (MAPMT) which are about 1 square inch in cross-section and are segmented into four, sixteen or sixty-four separate anodes. Thus, anode size can be selected to match other contributions to Cherenkov angle resolution. With the largest segmentation, the anode cell size is $2 \times 2 \mathrm{~mm}$. A closely packed array of R5900-M64 tubes is illustrated in Fig. 4.20. The multi-anode photo-tubes R5900-M16 were adopted for the HERA-B RICH detector [2]. The active photo-cathode area of R5900 tubes constitutes only about $50 \%$ of the total area of the tube (not including additional $0.3 \mathrm{~mm}$ wide dead spaces in between the anode cells).

To increase the geometrical efficiency, a light focusing system right in front of the PM array is needed. Some losses of light in the light collection system are unavoidable. HERA-B uses a two lens system to concentrate light on the active area of a MAPMT. The magnification of the HERA-B system is 0.5 , thus the effective cell size is doubled relative to the true anode cell size and a single photo-tube is serving an area which is larger than its own dimensions (i.e. the tubes are not closely packed). UVT plexiglass lenses have a combined transmission 
efficiency of about $70 \%$ at larger wavelengths (including reflective losses) [2]. LHC-B has proposed a single lens system [6]. An array of convex-plano lenses is in direct optical contact with MAPMT windows (see Fig. 4.21). The MAPMT tubes are closely packed. To recover the inactive area of MAPMTs the magnification of the system is 0.7. Such a design provides almost full coverage of the photo-detection plane with the minimal effective anode cell size $(2.8 \times 2.8 \mathrm{~mm}$ for R5900-M64 tube). There is just one refractive surface to pass, thus the reflective losses are minimized. LHC-B is also studying a light collection system based on light guides concentrating light into active parts of the anodes [8]. Such a system could even avoid small dead areas in between the anode cells which add up to $22 \%$ inefficiency for the R5900-M64 tube. Similar solutions will work for our RICH as well. 

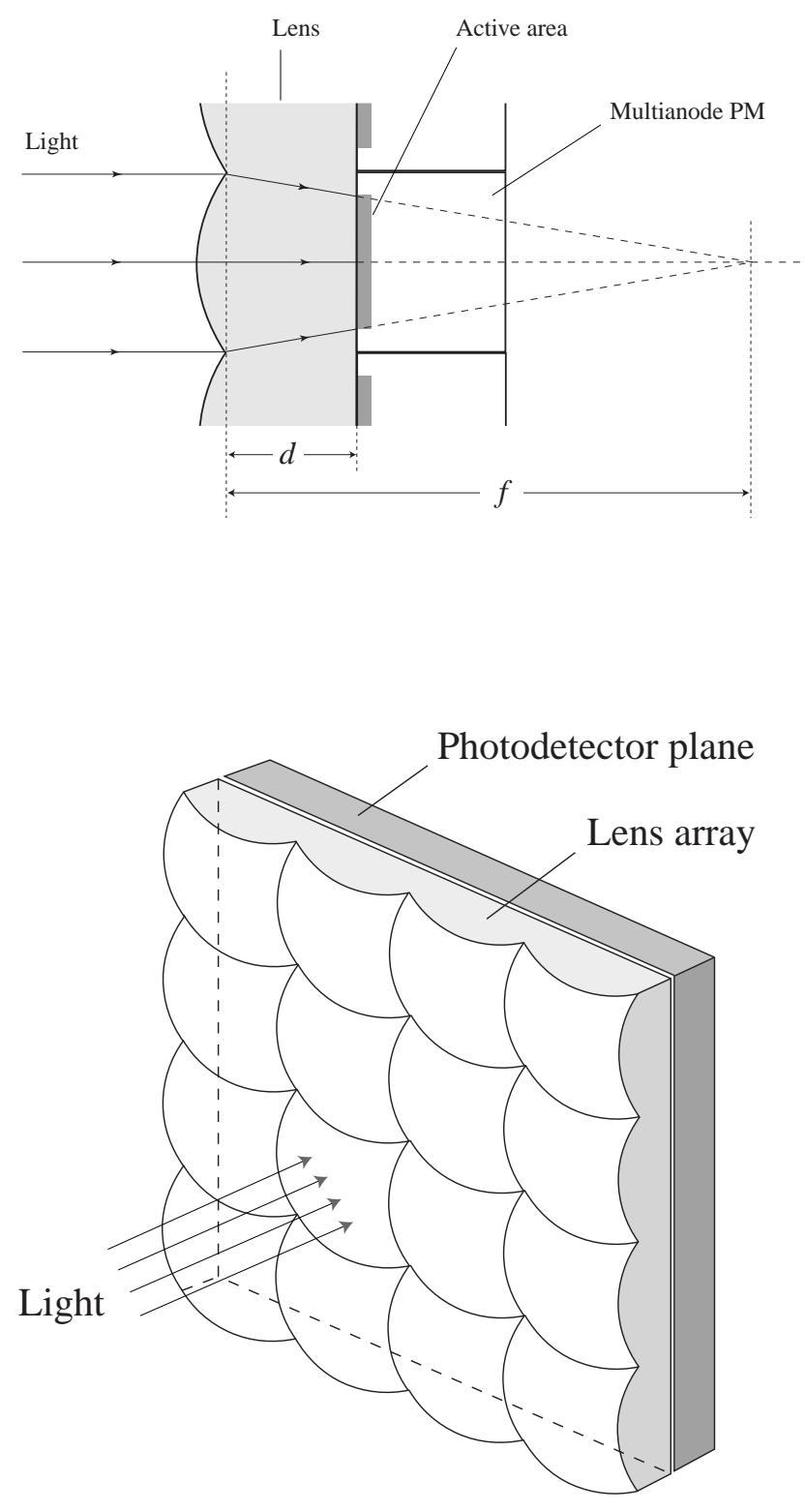

Figure 4.21: Two views of the lens system proposed by R. Forty for use with closely packed arrays of Hamamatsu R5900 MAPMTs. The pictures are taken from Ref.[6]. 
Hybrid Photo-Diodes offer an alternative photo-detection scheme. In an HPD, a photoelectron emitted by the photo-cathode is accelerated onto a silicon pixel detector by a very high voltage. Commercially available HPDs from DEP in the Netherlands have 61 closely packed hexagonal pixels, each with an area of $3.5 \mathrm{~mm}^{2}$, enclosed in a single cylindrical tube (see Fig. 4.22). The active area of the tube is small, $\approx 30 \%$ of the total area. These HPDs were successfully used to detect Cherenkov photons in a beam-test setup by the LHC-B group [9]. To recover geometrical inefficiency, a light focusing system with a magnification of $<0.5$ could be designed. An aggressive R\&D program has been pursued at CERN to develop HPDs with a higher active area for LHC-B RICH detectors. This is mostly achieved by increasing the overall size of the vacuum tube to $80-127 \mathrm{~mm}$ in diameter, with a much larger number of pixels inside (1024-2048). To maximize the active area of the front face of the tube, photoelectrons are electrostatically focused onto a smaller diameter silicon pixel array inside, while being accelerated at the same time. Two different focusing schemes are under development. For such a large number of channels per tube it is impractical to have frontend electronic chips situated outside the tube. Thus, the read-out chips are being integrated into the vacuum tubes of HPDs. One of the new HPDs is being developed in collaboration with DEP. Even if suitable HPDs are available in the relevant time scale for BTeV, HPD technology has some drawbacks. Lower intrinsic gain ( $5 \cdot 10^{3}$ vs $10^{6}$ for MAPMT) requires more sophisticated read-out electronics. Also much higher voltages are required ( $20 \mathrm{kV}$ vs $1 \mathrm{kV}$ for MAPMTs). HPDs are more sensitive to fringe magnetic fields than MAPMTs. On the other hand, if the CERN R\&D program succeeds, HPDs with a larger fraction of the active area may be available, perhaps eliminating the need for a light collection system in front of HPD arrays. The overall price for a full size detector is estimated, at present, to be comparable between the MAPMTs and HPDs.

We choose the MAPMT solution for the baseline design, however we intend to continue to evaluate HPD option as well.

\subsubsection{Expected resolution for gaseous radiator}

The emission point error magnified by the mirror tilt is about $0.4 \mathrm{mrad}$ per photon, slightly dependent on the the exact charged track trajectory.

The chromatic error depends on the choice of photo-cathode, window and lens materials. Extending the transparency of the optical elements towards shorter wavelengths increases the chromatic error per photon. At the same time, the number of detected $C_{4} F_{10}$ photons per track is also increased, making the Cherenkov angle resolution per track almost invariant. It should be noted that the increased wavelength sensitivity has no effect on photon yield and chromatic error for the aerogel radiator. Since materials with good UV transparency (e.g. quartz glass) are expensive, we assume standard borosilicate glass windows on MAPMTs. The dependence of quantum efficiency on photon energy used in the simulations is shown in Fig. 4.23. With this wavelength acceptance, the chromatic error per photon is expected to be $0.39 \mathrm{mrad}$, well matched to the emission point error.

The third significant contribution to the single photon resolution will come from a finite 


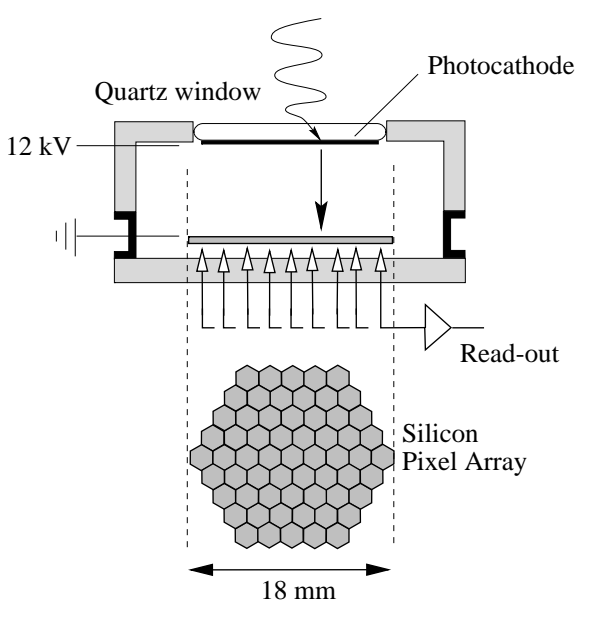

61-Pixel HPD

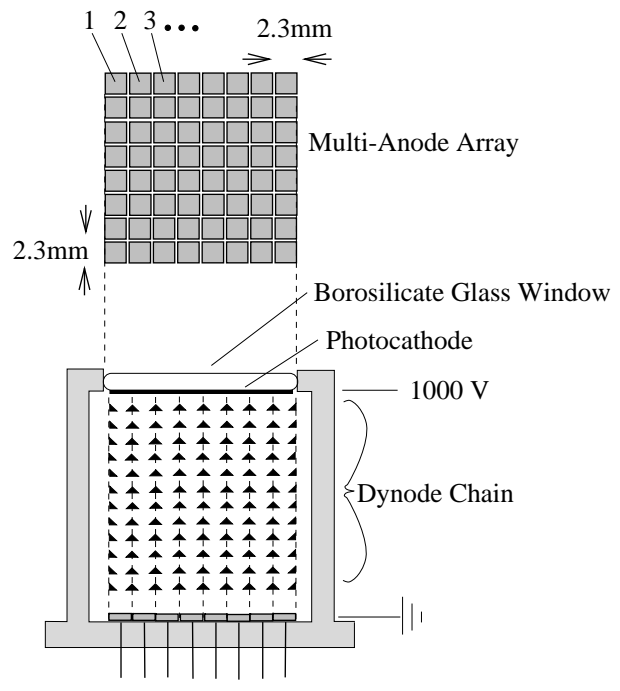

64-Channel MAPMT

Figure 4.22: 61-Pixel HPD from DEP and R5900-M64 MAPMT from Hamamatsu. The pictures are taken from Ref.[7].

effective cell size of the photo-detector. For $d \times d$ cell size, this contribution is expected to be $\sigma_{\theta}=d / \sqrt{12} \times 2 / R$. Closely packed R5900-M64 photo-tubes with the single lens light collection system described previously would result in a detector granularity error of 0.24 mrad, below the contributions from the emission point and chromatic errors. Covering the entire photo-detection area with the finest segmentation escalates the number of electronic channels and therefore, the cost of the detector. The best possible Cherenkov angle resolution is needed for high momentum tracks radiating photons in the the gaseous radiator. The higher momentum tracks tend to be closer to the beam pipe and illuminate a smaller region of the photo-detection plane than slower tracks. This region also has the highest occupancy of photons since the track multiplicity increases in the beam direction. For these two reasons, the inner region of the photo-detection plane must be populated with the finest segmentation. The outer region of the photo-detection plane is populated by $C_{4} F_{10}$ photons from slower tracks. All aerogel photons tend to fall into the outer region too, because of the much larger value of the Cherenkov angle. This is fortunate, since low intensity aerogel rings would have been heavily contaminated by high intensity gaseous rings in the inner region. The Cherenkov angle resolution becomes less critical for slower tracks. Since occupancy is lower in the outer regions, it will be possible to populate these areas with coarser segmentation without a significant impact on the performance of the device. We intend to use R5900-M16 tubes here. In this region, the photo-detector segmentation error will be around $0.51 \mathrm{mrad}$ and will dominate the $C_{4} F_{10}$ Cherenkov angle resolution. To determine the boundaries of 


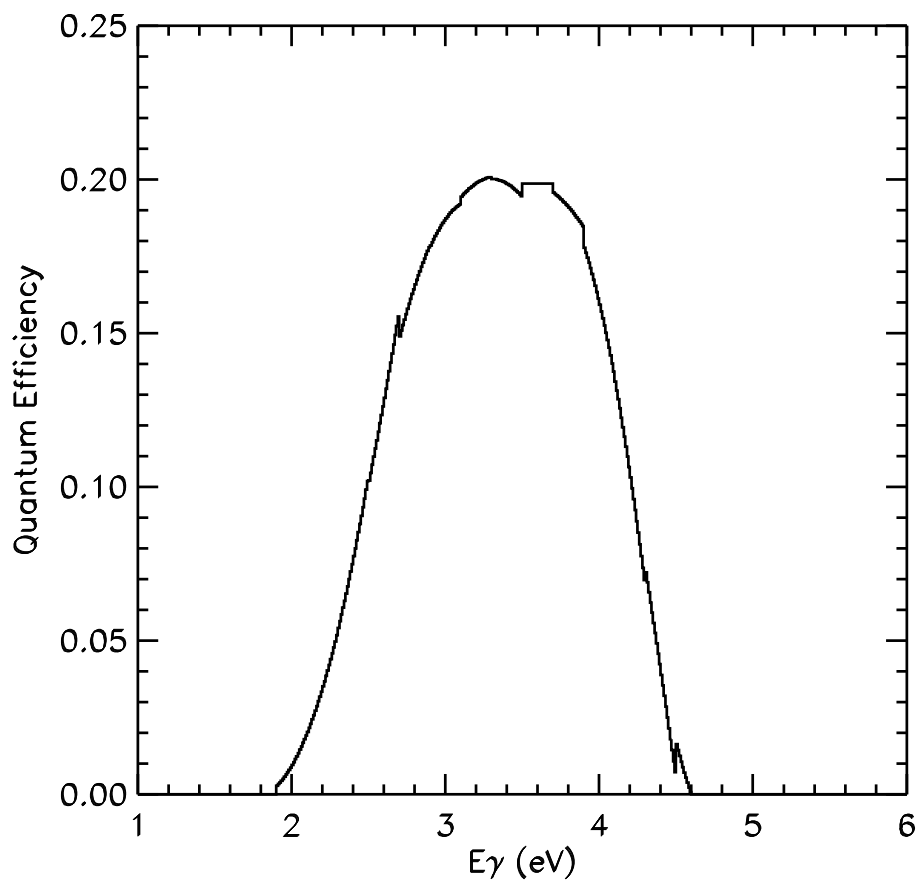

Figure 4.23: Quantum Efficiency of MAPMTs with borosilicate glass windows (as used in HERA-B RICH) used in our simulations.

the regions with fine and coarse segmentation of MAPMTs, we studied the occupancy of the photo-detection plane by various sources of Cherenkov photons. The distribution of photons produced in $C_{4} F_{10}$ by the faster track in the reconstructed $B^{0} \rightarrow \pi^{+} \pi^{-}$events is shown in Fig. 4.24. This should be compared to the similar distribution of $C_{4} F_{10}$ photons produced by all tracks from generic $b \bar{b}$ events shown in Fig. 4.25. The distribution of aerogel photons from generic $b \bar{b}$ events is also shown in Fig. 4.25. The selected regions of the fine and coarse MAPMT segmentation are indicated on these plots. In total, we intend to cover $3.1 \mathrm{~m}^{2}$ of area in each of the two identical RICH detectors. The inner $\left(1.2 \mathrm{~m}^{2}\right)$ part of this area will be finely segmented. Table 4.7 summarizes the number of tubes and electronic channels. The coarser segmentation in the outer photo-detection region has almost no effect on Cherenkov angle resolution for the faster track in the $B^{0} \rightarrow \pi^{+} \pi^{-}$events ( $2 \%$ effect). The resolution for the aerogel radiator is also not effected, since the chromatic error is large (see the next section). To match this error we could use R5900-M4 tubes in the outer region and further reduce number of electronics channels. However, such a scheme would deteriorate the $C_{4} F_{10}$ high momentum resolution by 10\%. Perhaps, another intermediate area with R5900-M16 tubes could fix the problem. Further optimization studies are needed.

Overall Cherenkov angle resolution per photon for the $C_{4} F_{10}$ radiator is expected to be 


\begin{tabular}{||c|c|c|c||}
\hline \hline PMT Tube type & Area & Number of tubes & Number of channels \\
\hline R5900-M64 & $1.22 \mathrm{~m}^{2}$ & 1800 & 115,200 \\
R5900-M16 & $1.89 \mathrm{~m}^{2}$ & 2800 & 44,800 \\
\hline Together & $3.11 \mathrm{~m}^{2}$ & 4600 & 160,000 \\
\hline \hline Two RICH detectors & $6.22 \mathrm{~m}^{2}$ & 9200 & 320,000 \\
\hline \hline
\end{tabular}

Table 4.7: Number of Tubes and channels per detector arm and total in two arms.

$0.61 \mathrm{mrad}$. Per track resolution depends on the observed photo-electron yield.

In addition to quantum efficiency losses, photo-electron yield is reduced by imperfect mirror reflectivity, absorption in light collection and PMT window materials and reflective losses on refractive boundaries. The MAPMT tubes also have a small inactive area in between anode cells. This effect has been recently studied for R5900-M64 tubes by the Oxford group [10]. Their measurement ( $23 \%$ light loss) comes close to the naive expectation from the geometrical dimensions of the anode cells and the dead area in between. We simulate this effect explicitly in a geometrical way. Loss of light yield due to finite cell size (photon overlaps) is about $5.5 \%$ for saturated rings, and is also modeled explicitly. For mirror reflectivity we assume $87 \%$. In the HERA-B light collection system, there are five refractive boundaries resulting in $15 \%$ light loss due to reflections on these surfaces [2]. There is only one refractive boundary in the light focusing scheme described above, thus we assume only $4 \%$ loss. Finally, we assume $20 \%$ loss for absorption in the light collection system compared to $15 \%$ estimated for the HERA-B lens system. After all these corrections the average photoelectron yield from $C_{4} F_{10}$ for $\beta=1$ particles is expected to be close to 50. LHC-B expects to achieve a similar photo-electron yield with a 3 times shorter $C_{4} F_{10}$ radiator. This is mostly (factor of 2.3) due the quantum efficiency assumed by LHC-B, which extends towards much shorter wavelengths. The rest can be accounted for by a more optimistic assumption about light collection efficiency. Even though the extended wavelength coverage increases photoelectron statistics, chromatic error is also increased producing almost no gain in Cherenkov angle resolution per track. To maintain wide wavelength coverage expensive window and light collection materials would have to be used. Therefore, we prefer to work in a narrower range of wavelengths. Our estimated photo-electron yield is larger than projected for the HERA-B RICH (32-35 photo-electrons)[2]. This is partially due to a different light collection scheme assumed here, and the lack of a second mirror in our design.

Our simulations predict the average Cherenkov angle resolution per track from the $C_{4} F_{10}$ radiator to be $0.0935 \mathrm{mrad}$. The resulting $K / \pi$ separation at higher momenta is shown in Fig. 4.26. The projected performance satisfies our physics goals well. Various contributions to Cherenkov angle resolution are summarized in Table 4.8. 
Table 4.8: Expected performance of BTeV RICH system.

\begin{tabular}{||l|l|l||}
\hline \hline & $C_{4} F_{10}$ & Aerogel $n=1.03$ \\
\hline emission point error & $0.4 \mathrm{mrad}$ & $0.1 \mathrm{mrad}$ \\
chromatic error & $0.4 \mathrm{mrad}$ & $1.9 \mathrm{mrad}$ \\
segmentation & $0.2 \mathrm{mrad}$ & $0.5 \mathrm{mrad}$ \\
\hline total error per photon & $0.6 \mathrm{mrad}$ & $2.0 \mathrm{mrad}$ \\
\hline number of photons & 50 & 9 \\
\hline total error per track & $0.094 \mathrm{mrad}$ & $0.7 \mathrm{mrad}$ \\
\hline \hline
\end{tabular}

\subsubsection{Expected resolution for aerogel radiator}

The chromatic error for aerogel photons is expected to be about $1.9 \mathrm{mrad}$. This is likely to be an overestimate since we did not simulate attenuation of shorter wavelengths in the radiator itself. The effect of the lucite filter between the aerogel and gas radiators was simulated as a sharp cut-off at $300 \mathrm{~nm}$.

HERMES reports [3] additional 0.7 mrad error, mostly due to aerogel density variation. The emission point error is expected to be very small $(0.09 \mathrm{mrad})$ because the radiator in only $5 \mathrm{~cm}$ thick. The detector granularity error is below $0.51 \mathrm{mrad}$. Putting all these factors together we predict the Cherenkov resolution per photon to be around 2 mrad.

We assume 9 detected photo-electrons per track according to the recent reports from HERMES. This photon yield cannot be increased by using a thicker radiator because of light scattering and absorption in aerogel itself. Further improvements in aerogel transparency would increase the number of photons per Cherenkov ring.

The expected Cherenkov resolution per track is about $0.7 \mathrm{mrad}$. The resulting $K / p$ separation at lower momenta is shown in Fig. 4.27. The required $K / p$ separation up to the proton Cherenkov radiation threshold in $C_{4} F_{10}$ should be easily achieved. Various contributions to Cherenkov angle resolution are again summarized in Table 4.8. 


\section{$\mathbf{Y}(\mathbf{c m})$}

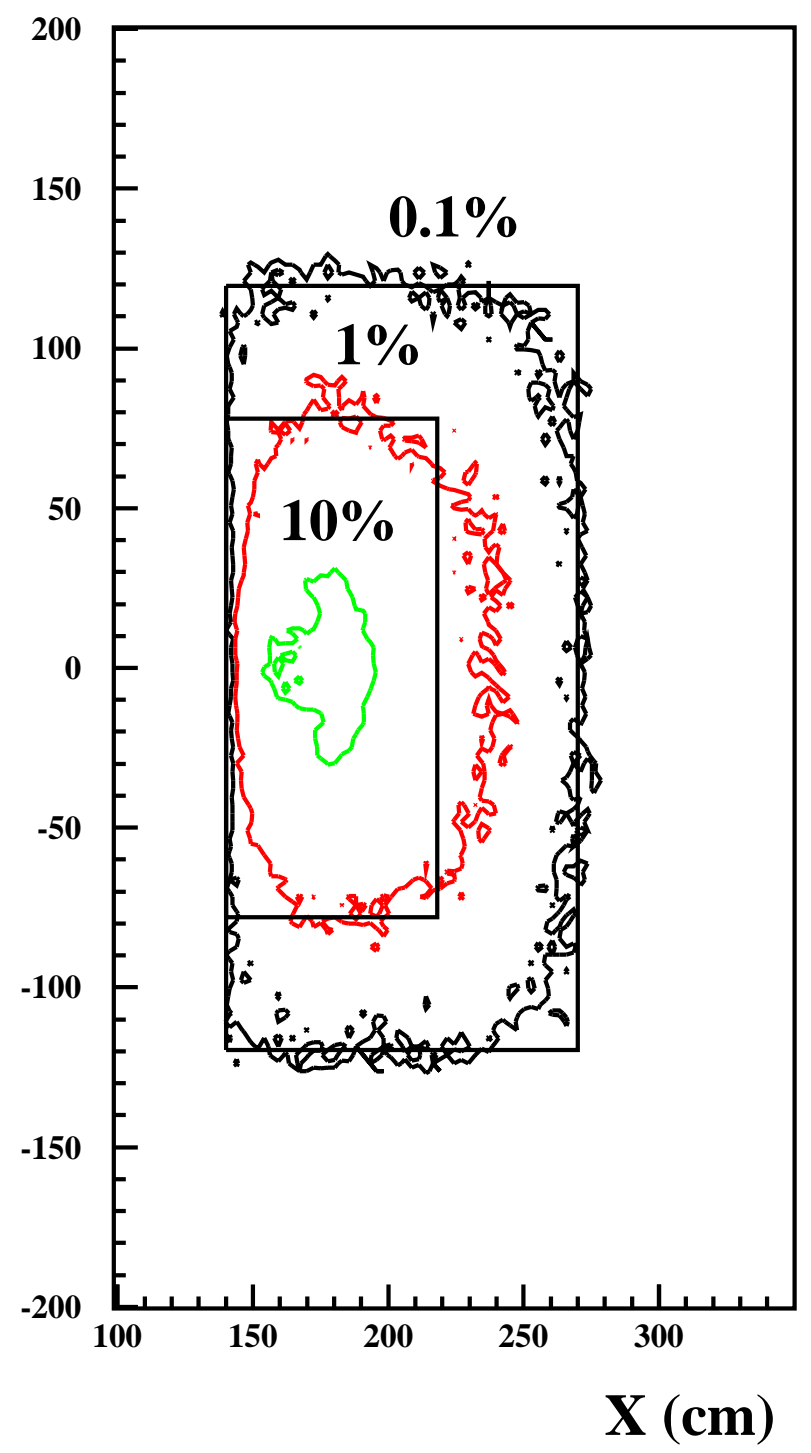

Figure 4.24: Contours of the number of Cherenkov photons per one MAPMT $(2.6 \times 2.6$ $\mathrm{cm}^{2}$ ) per event produced in $C_{4} F_{10}$ by the faster track in reconstructed $B^{0} \rightarrow \pi^{+} \pi^{-}$decays. Rectangular boxes illustrate areas to be covered with MAPMTs. The inner box corresponds to the region of finer segmentation. 


\section{Y (cm)}

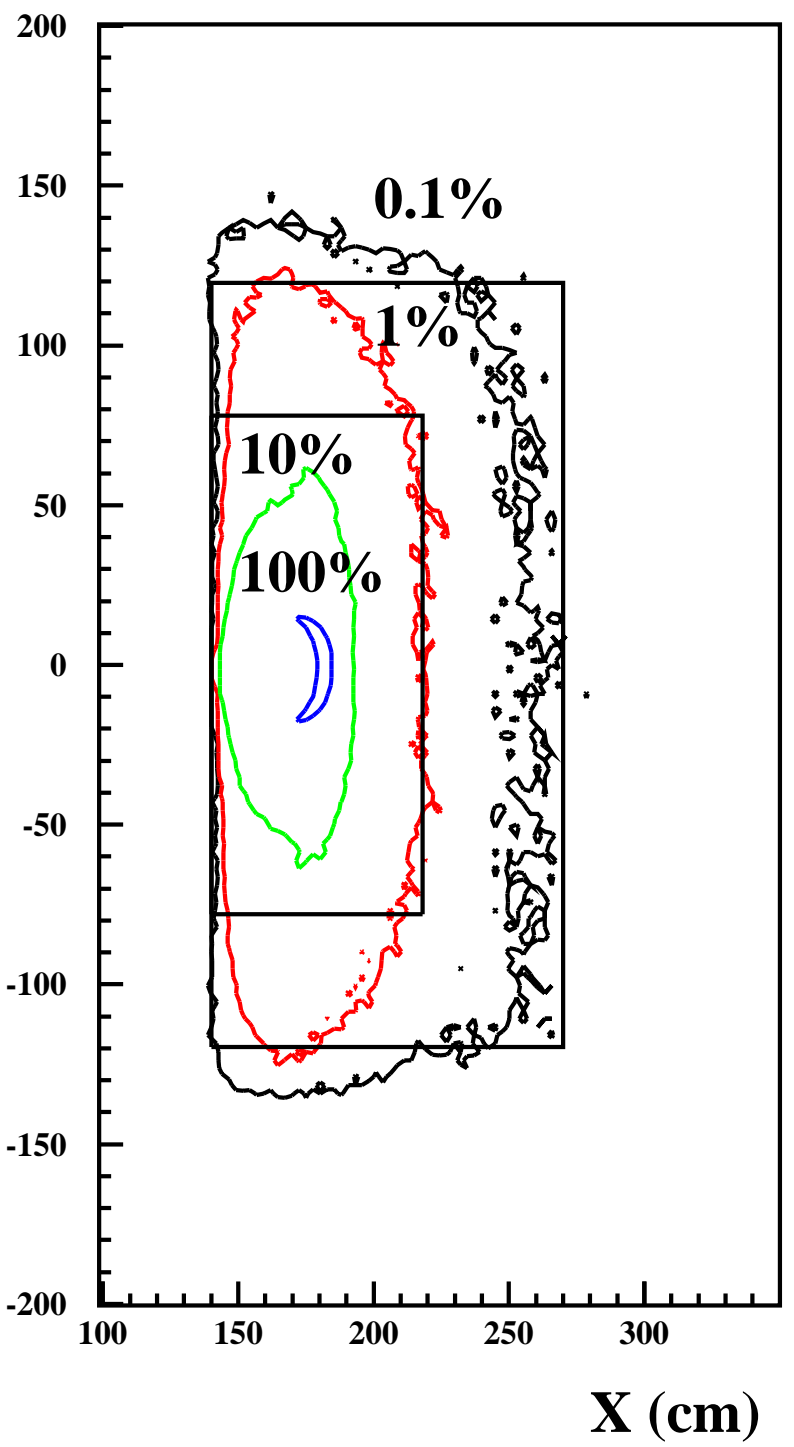

Y (cm)

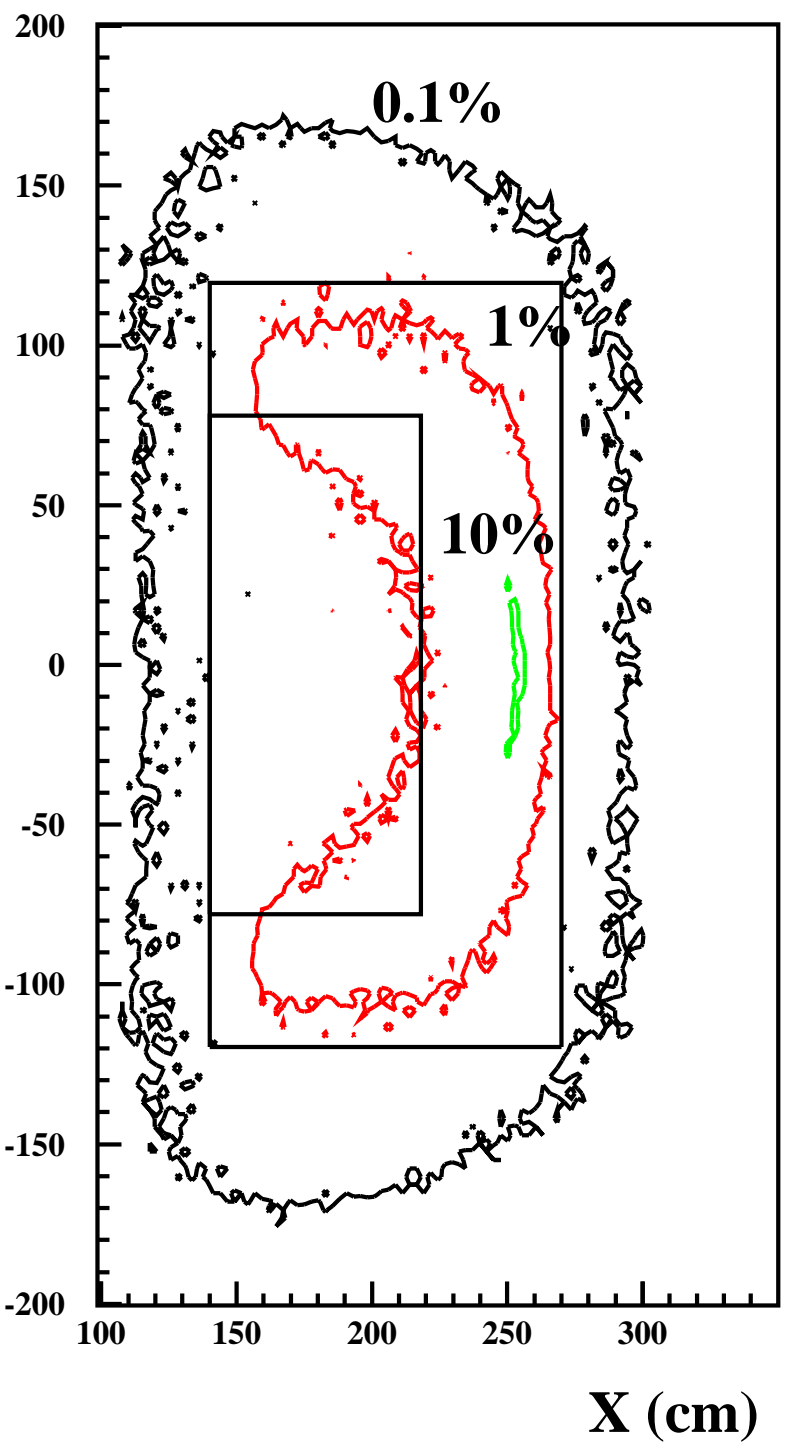

Figure 4.25: Contours of number of Cherenkov photons per one MAPMT $\left(2.6 \times 2.6 \mathrm{~cm}^{2}\right)$ per event produced by all tracks in generic $b \bar{b}$ event originating from $C_{4} F_{10}$ (left) and aerogel (right) radiators. Rectangular boxes illustrate areas to be covered with MAPMTs. The inner box corresponds to the region of finer segmentation. 


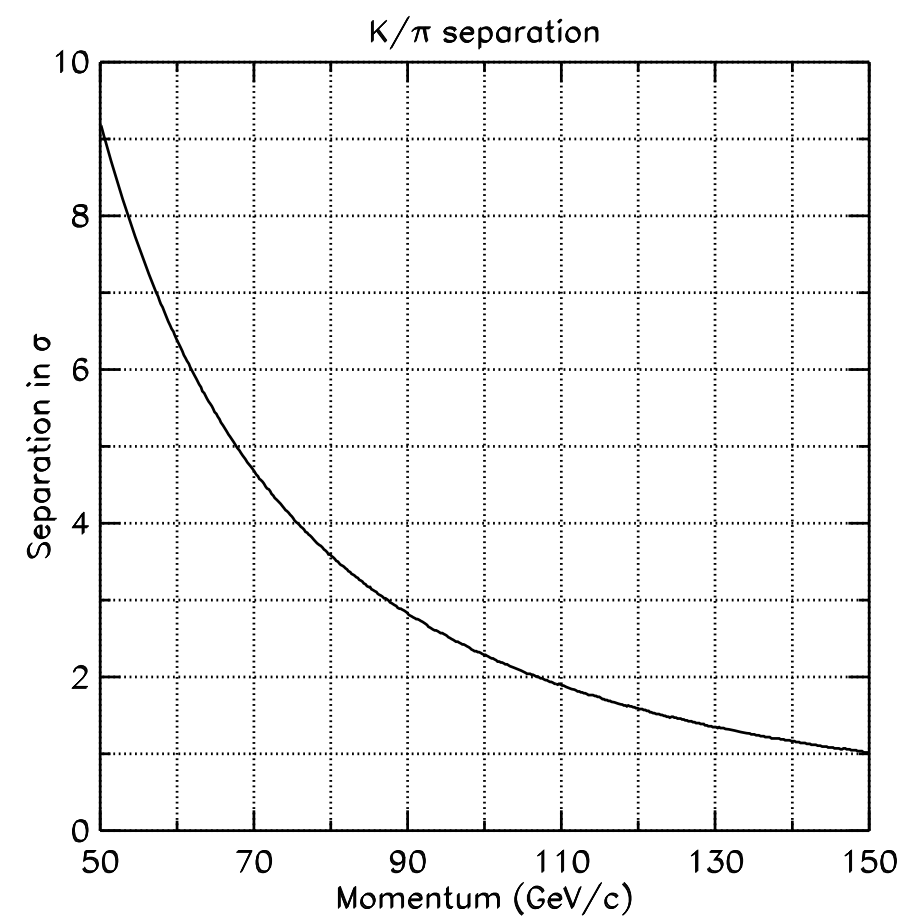

Figure 4.26: Expected $K / \pi$ separation from $C_{4} F_{10}$ radiator.

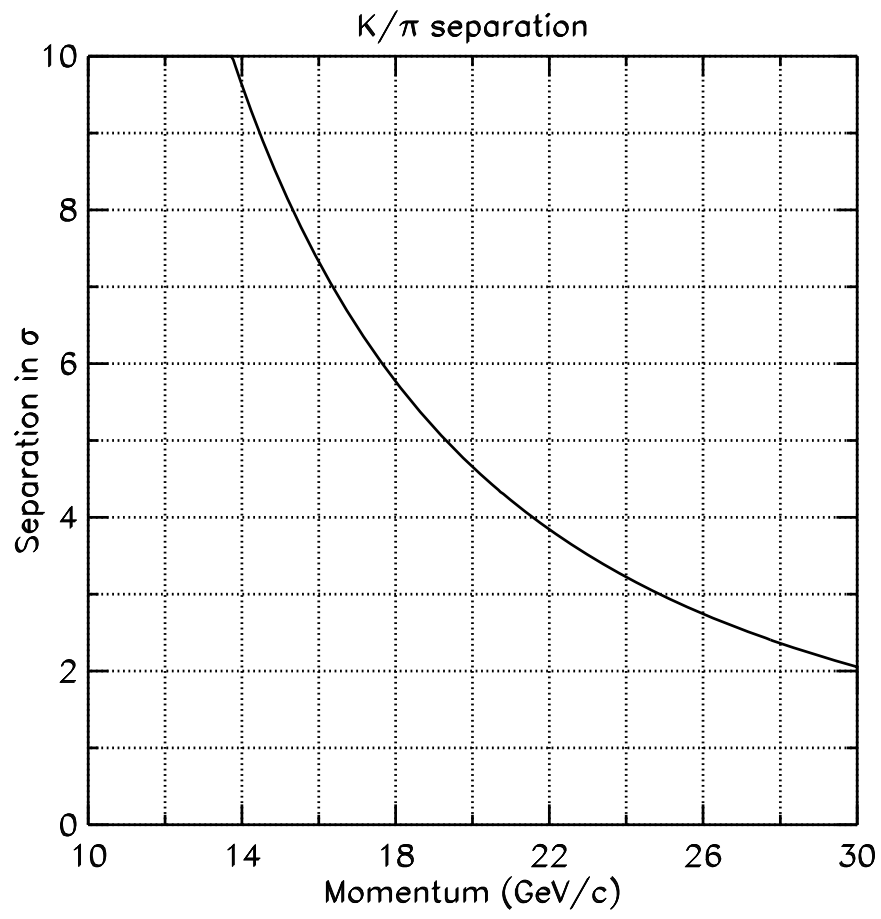

Figure 4.27: Expected $K / p$ separation from aerogel radiator. 


\section{Bibliography}

[1] W. Adam et al., Nucl. Inst. \& Meth. A343, 60 (1994).

[2] J.L. Rosen, Proceedings of The Fifth International Workshop on $B$-Physics at Hadron Machines, Los Angeles, USA, Oct. 13-17, 1997, published in Nucl. Inst. and Meth. A408 (1998) 191; S. Kopar, Proceedings of the The 3rd International Workshop on Ring Imaging Cherenkov Detectors Weizmann Institute of Science Ein-Gedi, Dead-Sea, Israel, Nov. 15-20, 1998.

[3] P. Carter, Proceedings of the The 3rd International Workshop on Ring Imaging Cherenkov Detectors Weizmann Institute of Science Ein-Gedi, Dead-Sea, Israel, Nov. 15-20, 1998. "Proposal for Dual Radiator RICH for HERMES", E. Cisbani et al., Hermes Internal Note 97-005.

[4] LHC-B, Technical Proposal, CERN LHCC 98-4, LHCC/P4, 1998.

[5] R. Forty, CERN-PPE/96-176, Sept. 1996 published in Proc. of the $4^{\text {th }}$ Int. Workshop on $B$-physics at Hadron Machines, Rome, Italy, June 1996, F. Ferroni, P. Schlein (Eds.), North-Holland, 1996.

[6] R. Forty, "Use of lenses to increase the RICH photo-detector coverage", LHCb Internal Note LHCB/98-038.

[7] A. Duane et al., LHCb Internal Note LHCB/98-039.

[8] V. Grecco, A. Petrolini, "Study of a possible light collector system for multi-anode photo-multiplier tubes", LHCb Internal Note LHCB/99-005.

[9] S. Easo, Proceedings of the The 3rd International Workshop on Ring Imaging Cherenkov Detectors Weizmann Institute of Science Ein-Gedi, Dead-Sea, Israel, Nov. 15-20, 1998.

[10] N. Smale et al., "Evaluation of the multi-anode photo-multipliers for the LHCb RICH detectors", LHCb Internal Note LHCB/98-066. 


\subsubsection{Electromagnetic Calorimetry}

\subsubsection{Introduction}

A thorough investigation of $B$ decays requires the ability to study decay modes containing single photons, $\pi^{o}$ s, and $\eta$ 's. Some of the important decay modes mentioned in chapter 1 are:

- $B^{o} \rightarrow(\rho \pi)^{o} \rightarrow \pi^{+} \pi^{-} \pi^{o}$

- $B^{o} \rightarrow K^{* o} \gamma$ and $\rho^{o} \gamma$

- $B_{s}^{o} \rightarrow \psi \pi^{o}, \psi \eta$, and $\psi \eta^{\prime}$;

- $B^{o} \rightarrow K^{* o} \pi^{o}$; and

- $B^{ \pm} \rightarrow K^{ \pm} \pi^{o}$ and $\pi^{ \pm} \pi^{o}$.

We have begun an intensive study of the problems associated with reconstructing electromagnetic showers in the environment of a hadron collider and using them to obtain signals for $B$ mesons containing photons in the final state. While this study is still in progress, we are sufficiently encouraged by our initial results to develop a baseline design for an electromagnetic calorimeter which is capable of reconstructing photons. The electromagnetic calorimeter will also be used to identify electrons which can be used to tag the flavor of the associated $B$ for $\mathrm{CP}$ studies or to isolate high mass electron-positron pairs that form exclusive final states. Examples are $B \rightarrow D^{(*)} e \nu$ (for electron tagging) and $B \rightarrow J / \psi K_{\mathrm{s}}$, $J / \psi \rightarrow e^{+} e^{-}, K_{\mathrm{s}} \rightarrow \pi^{+} \pi^{-}$.

Below in chapter 11, we discuss the challenges of isolating the small signals of the most interesting decay modes from the large backgrounds that are encountered in hadronic interactions. Here, we present the key requirements on energy and position resolution and on radiation hardness that the calorimeter must satisfy. We then present the BTeV baseline electromagnetic calorimeter design. In chapter 11, we show simulations of key signals and in chapter 14 we present a preliminary cost estimate for the proposed baseline device.

\subsubsection{Key Requirements and Issues}

To determine the appropriate size, segmentation, and technology for the calorimeter requires an understanding of the kinematic properties of the photons in signals, of potential sources of backgrounds, and of the radiation environment.

The area available to the calorimeter is located about 7.0 meters from the nominal interaction point and extends in $\mathrm{Z}$ for 1 meter. The distance from the beam to the floor is $2.5 \mathrm{~m}$. The transverse dimension which matches the acceptance of the spectrometer is a $4 \mathrm{x}$ 4 meter box centered on the Tevatron beam line.

We have begun very detailed Monte Carlo studies of the following two benchmark processes to understand the signals we hope to measure: 
- $B^{o} \rightarrow K^{* o} \gamma$ and

- $B^{o} \rightarrow \rho^{+} \pi^{-}, \rho^{-} \pi^{+}$, and $\rho^{o} \pi^{o}$.

The results of the full simulation are presented in chapter 11. Here, we present only the 'generator level' study for $K^{*} \gamma$ and for $B^{o} \rightarrow \rho^{+} \pi^{-}$. In the first study, we simply require all tracks of the final state of interest to be within the angular acceptance of the $\mathrm{BTeV}$ spectrometer and to satisfy a minimum energy requirement. We chose these two modes because the photon in the $K^{*} \gamma$ decay has rather high momentum and $P_{t}$, while those from the $\pi^{o}$ decay of the $\rho^{+}$are much softer. The results of these studies are shown in Fig. 4.28 and Fig. 4.29. Based on these studies, the energy range of interest is extends from $1 \mathrm{GeV}$ to $60 \mathrm{GeV}$ and the angular range of interest extends out to 200-300 milliradians.

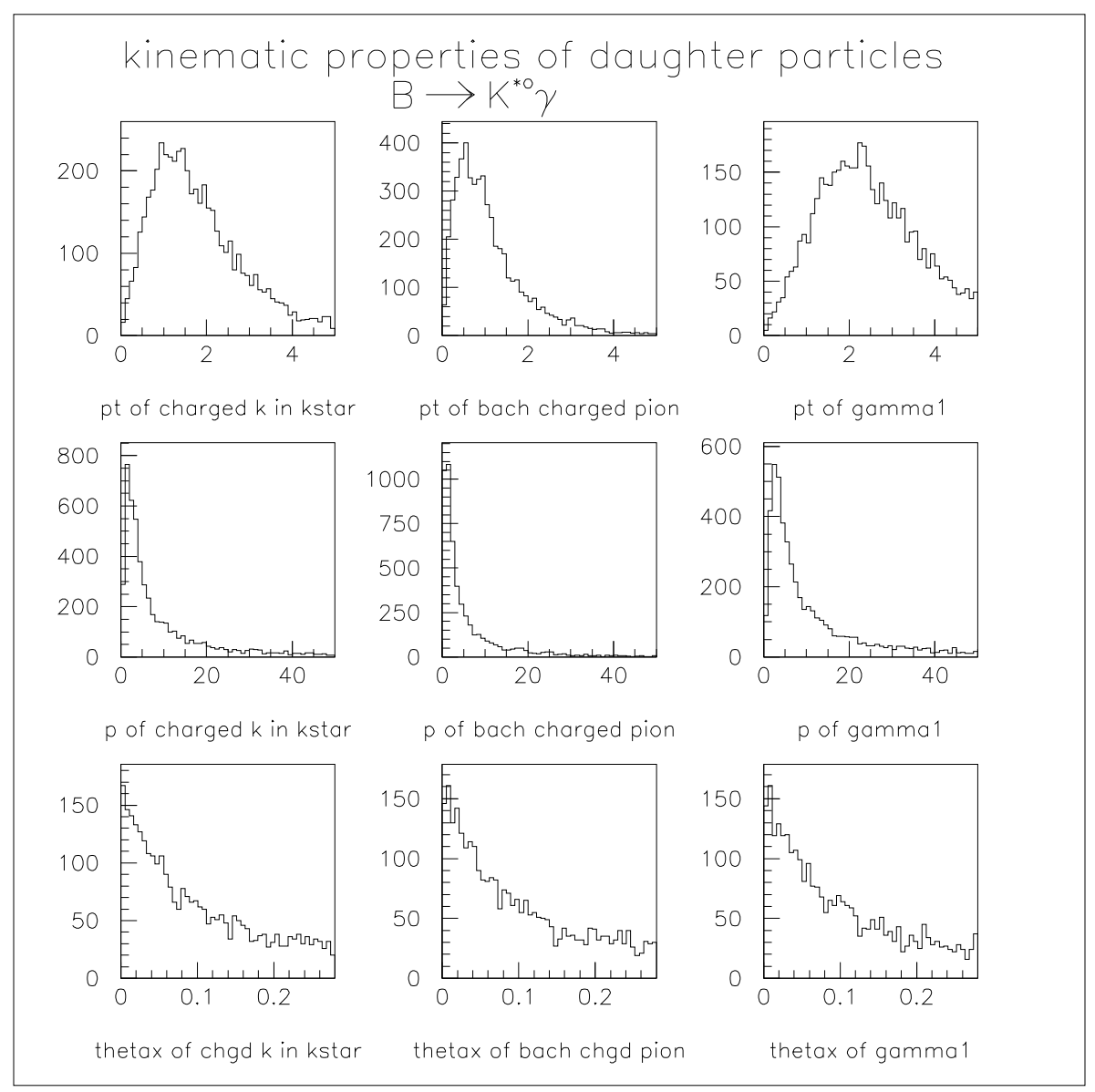

Figure 4.28: Kinematic properties of $B \rightarrow K^{*} \gamma$ where all generated tracks satisfy rudimentary acceptance requirements

To understand the requirements on energy and angular resolution, we have done a more sophisticated simulation. In this simulation, we carried out a full Monte Carlo generation and offline tracking of the charged tracks. For the photons, we smeared the momentum vectors 


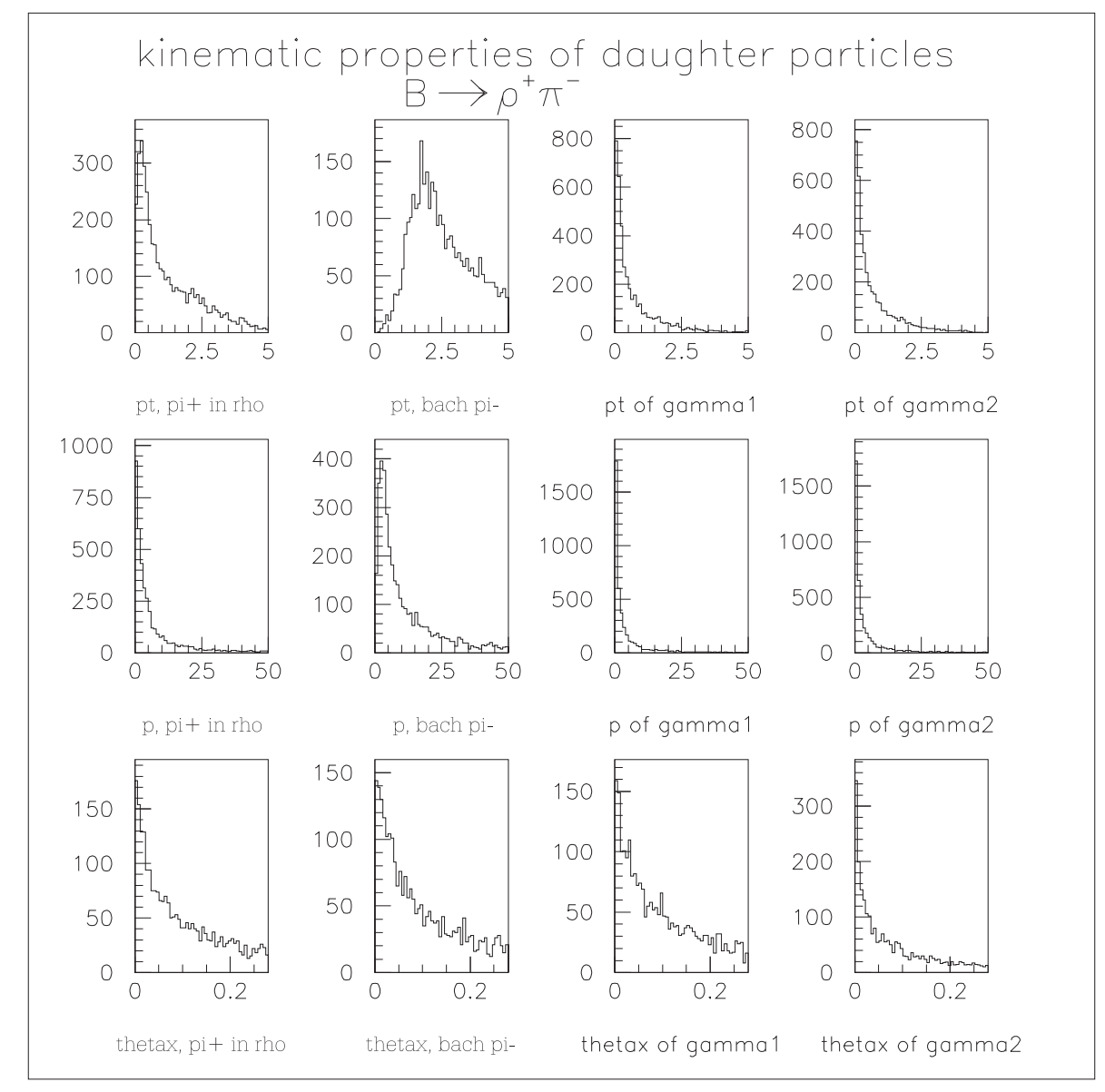

Figure 4.29: Kinematic properties of $B^{o} \rightarrow \rho^{+} \pi^{-}$where all generated tracks satisfy rudimentary acceptance requirements

of the generated photons in energy and angle and used the smeared momentum vectors in mass calculations. The energy is smeared according to

$$
\frac{\sigma_{E}}{E}=\sqrt{\frac{a^{2}}{E}+b^{2}}=\frac{a}{\sqrt{E}} \oplus b,
$$

with $E$ in units of $\mathrm{GeV}$. We refer to the parameters $a$ and $b$ as the stochastic term and the constant term, respectively. The angles were smeared by projecting the generated $\gamma$ to the surface of the calorimeter, smearing the position there using a Gaussian of width $\Delta X / \sqrt{12}$ and $\Delta Y / \sqrt{12}$, where $\Delta X$ and $\Delta Y$ are the transverse dimensions of the calorimeter elements. The angles were recalculated (using the primary vertex for the upstream point). Finally, a smeared $\gamma$ momentum vector and its correctly propagated covariance matrix were calculated. These are used together with the charged tracks found in the offline analysis to reconstruct the $B$ mass. The parameters $a, b, \Delta X$, and $\Delta Y$ can be varied and their effect on the mass resolution and other quantities can be obtained. Some results from these studies are given 
below in Table 4.9. The results agree well with the results from the full MCFAST simulation and shower reconstruction shown later.

\subsubsection{Backgrounds}

There are two major issues concerning backgrounds which influence the requirements on the calorimeter. The first has to do with problems in the reconstruction of objects $-\gamma$ 's, $\pi^{o}$ 's, electrons, $\eta$ 's, etc. - which can result in poor efficiency, the reconstruction of 'false' objects, and the reconstruction of objects with seriously degraded resolution. The second has to do with combinatoric backgrounds - that is, not knowing which of the many neutral objects appearing in a typical event to associate with the decaying $B$. We have learned that the backgrounds due to problems in object reconstruction place the most demanding requirements on the detector, except for the energy resolution which determines the overall mass resolution of the final state. Good mass resolution is a final defense against combinatoric backgrounds. In this section, we discuss the requirements that help reduce the object reconstruction backgrounds. Methods for defeating combinatoric backgrounds are discussed in chapter 11 below.

The dominant processes which mimic a single photon are:

- High momentum $\pi^{o}$ s whose two photons are emitted within a very small angle. Because of the finite size of the calorimeter elements and of the electromagnetic shower, these can appear to merge or 'coalesce' into a single shower. Since there is an energy dependent minimum opening angle between the photons in $\pi^{o}$ decay, this problem affects mainly the highest energy photons of interest to BTeV. A high degree of segmentation can reduce this background and an analysis which uses transverse shower shape variables can be effective at detecting coalesced photons up to quite high energies. Below we show that coalesced photons can be identified up to over $90 \mathrm{GeV}$ with the proposed baseline calorimeter.

- $\pi^{o}$ decays where the less energetic of the two photons is below the sensitivity of the calorimeter or falls outside the calorimeter's geometric acceptance. A $\pi^{o}$ can decay so one photon takes most of the energy while the other photon has very little. If the ability exists to detect the low energy photon, it can be paired with the higher energy single photon candidates to see if they form a $\pi^{o}$ and if they do, those high energy photons can be rejected as single photon candidates. However, since the $\mathrm{BTeV}$ calorimeter is far from hermetic, this approach is not very efficient. Instead, one has to understand which states containing $\pi^{o}$ decays are potential sources of fake single photon signals and demonstrate that they have not polluted the single photon signal. For example, the decay $B^{o} \rightarrow K^{* o} \pi^{o}$ can mimic $B^{o} \rightarrow K^{* o} \gamma$ if the $\pi^{o}$ decays very asymmetrically.

The dominant source of background to $\pi^{o}$ identification is combinatoric background. All pairs of photon candidates (some of which will be false) must be considered in reconstructing $\pi^{o}$ 's. Random pairings make a rather smooth background in invariant mass. Since the mean number of photons recorded in the calorimeter in a typical $B$ event is 30 , the total number of 
combinations is very large. Many of the photons are unpaired - that is they come from a $\pi^{o}$, $\eta^{\circ}$ or other parent but one of the daughter photons is outside the calorimeter's acceptance or below its energy sensitivity. Coalesced $\pi^{o}$ s also mimic individual photons and participate in incorrect pairings. Shower overlaps both with other photons and hadronic showers in the calorimeter can distort the energy and angles of the photons and push the $\pi^{o}$ masses to an incorrect value. Converted photons produce $e^{+} e^{-}$pairs which can bremsstrahlung, producing other photons that create background. The signal to background ratio of the $\pi^{o}$ signal depends on the energy resolution since the narrower the $\pi^{o}$ mass peak, the smaller is the integrated background under it.

Electrons will be identified by matching their energy with the momentum measured for the track by the spectrometer $\left(\frac{E}{p}\right)$ and by looking at their transverse profiles. The dominant background which results in the false identification of objects as electrons is expected to be photon conversions due to interactions in the beam pipe, in the silicon near the primary vertex, or in the material of the RICH or forward tracker elements. Other backgrounds will be misidentified charged pions which charge exchange early in the calorimeter, and other rare processes which can mimic the behavior of an electron.

The ability to reject backgrounds will depend on the choice of the calorimeter technology, the segmentation, and to some extent the performance of the other detector elements. These processes are being evaluated with Monte Carlo tools (such as GEANT and MCFAST) and perhaps eventually with test beam studies.

We have identified combinatoric background as a serious problem. A final defense against the combinatoric background is mass resolution. The calorimeter is the only means of measuring the energy and angles of neutral objects in the event. The energy resolution of the calorimeter is a more significant source of mass smearing than its angular resolution. For the range of calorimeter technologies satisfying other BTeV requirements, the energy measurement of the photons will dominate the total mass resolution of the state. Table 4.9 shows the mass resolution for $B^{o} \rightarrow K^{* o} \gamma$, using an energy resolution parameterized as above with stochastic terms of $3.0 \%, 2.5 \%, 2.0 \%$, and $1.5 \%$ and constant terms of $1.0 \%, 0.75 \%$, and $0.55 \%$, and $0.25 \%$. Both the constant and stochastic terms are important contributors to the resolution because of the large energy range of the photons. 
Table 4.9: Mass Resolution for Different Calorimeter Energy Resolutions (statistical accuracy is $2-3 \mathrm{MeV} / \mathrm{c}^{2}$ )

\begin{tabular}{|l|c|c|c|}
\hline State & $a$ & $b$ & Mass Resolution \\
$\%$ & $\%$ & $\mathrm{MeV} / \mathrm{c}^{2}$ \\
\hline$B^{o} \rightarrow K^{* o} \gamma$ & 3.0 & 1.0 & 54 \\
& 2.5 & 1.0 & 48 \\
& 2.0 & 1.0 & 49 \\
& 1.5 & 1.0 & 49 \\
& 3.0 & 0.75 & 46 \\
& 2.5 & 0.75 & 50 \\
& 2.0 & 0.75 & 42 \\
& 1.5 & 0.75 & 39 \\
& 3.0 & 0.55 & 45 \\
& 2.5 & 0.55 & 41 \\
& 2.0 & 0.55 & 44 \\
& 1.5 & 0.55 & 40 \\
& 3.0 & 0.25 & 41 \\
& 2.5 & 0.25 & 45 \\
& 2.0 & 0.25 & 40 \\
& 1.5 & 0.25 & 36 \\
\hline \hline
\end{tabular}

\subsubsection{Radiation Hardness Requirements}

A full calculation of the radiation environment takes into account radiation deposited in the calorimeter from the direct products of collisions, from the interactions of those products with the material of the spectrometer, from beam halo, and from backscatter and residual radiation from other detectors. We are in the process of carrying out such a calculation, but it is not yet complete. Here, to get an idea of the radiation environment the detector must tolerate, we show, Fig. 4.30 the energy dose in the calorimeter from photons, electrons, interacting hadrons, and non-interacting charged particles per (minimum bias) interaction. We set the radiation requirement based on tripling the deposition from these events to account for sources not included in this calculation. The calorimeter begins at $\pm 9.88 \mathrm{~cm}$ in both $\mathrm{X}$ and $\mathrm{Y}$. The annual radiation dose is 60 KGrays at a radius of about $14 \mathrm{~cm}(20 \mathrm{mr})$. The dose falls off like a power of the radius to the 2.3 power from $20 \mathrm{~cm}$ on out. The detector needs to be radiation hard towards the middle and radiation tolerant on the outside. At 20 $\mathrm{mr}$, the detectors nearest the beam will receive doses that are a quarter of those expected at the LHC (at greater radius but much higher luminosity and energy). We plan to use a solid state chip (the QIE) to digitize the pulse in or near the photomultiplier tube base. The radiation levels at the downstream end of the detector are therefore also of concern. 


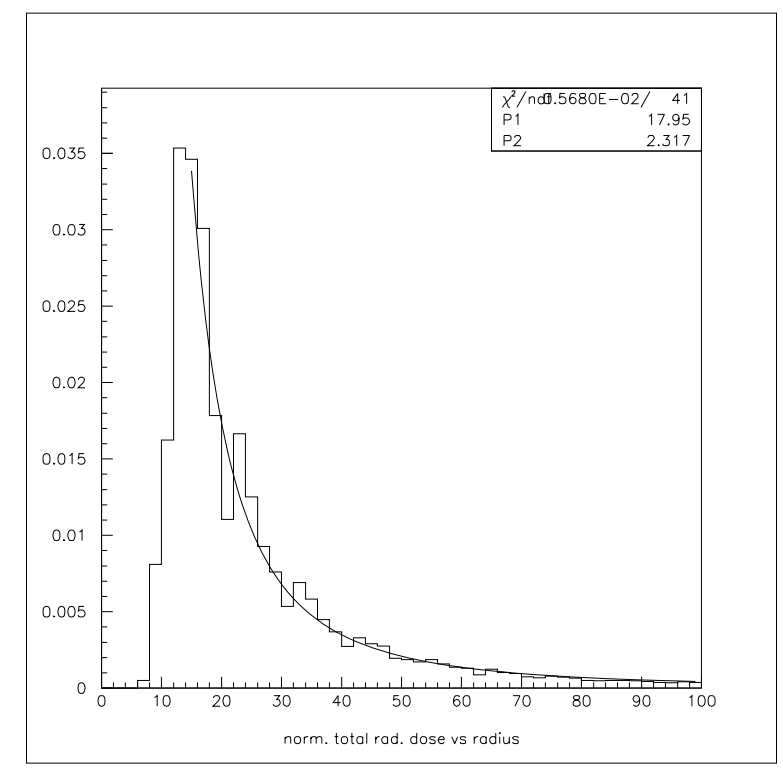

Figure 4.30: Total energy deposition in $\mathrm{GeV}$ per $\mathrm{cm}^{2}$ per interaction (minimum bias) vs radius $(\mathrm{cm})$ due to photons, electrons, interacting hadrons, and non-interacting (minimum ionizing) particles. The curve is the result of a fit to a power law, $A \times R^{n}$. The best fit gives $n=-2.3$. A rough conversion gives $20 \mathrm{KGrays} /$ year for each $0.01 \mathrm{GeV}$ per $\mathrm{cm}^{2}$ per interaction.

\subsubsection{Time resolution}

The spacing of beam crossings at the Tevatron will be 132 ns. As long as the occupancy is low and there is some way of tagging which beam crossing each hit goes with, it is not necessary to have a signal which is so fast that it can be collected between crossings. However, it is certainly a convenience if this can be accomplished and may also reduce machine backgrounds as well.

\subsubsection{Summary of Requirements}

The above considerations lead to a range of requirements on various characteristics which are shown in Table 4.10. These requirements are not uniquely determined by our studies. They also reflect our current understanding of available technologies and their limitations. So, they have certain compromises already built in to them.

\subsubsection{Baseline Technology}

Several technologies were considered (from the point of view of performance, cost, and availability), including:

- A sampling calorimeter with lead and either scintillator or liquid argon 
Table 4.10: Requirements of Baseline Electromagnetic Calorimeter

\begin{tabular}{|l|l|}
\hline Property & Value \\
\hline $\begin{array}{l}\text { Maximum length } \\
\text { Angular Resolution } \\
\text { Energy resolution: }\end{array}$ & $\begin{array}{l}1 \text { meter } \\
\text { Stochastic Term }\end{array}$ \\
Constant Term & $1-3 \%$ \\
Minimum angle & $0.55-0.75 \%$ \\
Maximum angle & $10-20 \mathrm{mr}$ at $700 \mathrm{~cm}$ \\
Length & $200-300 \mathrm{mr}$ \\
Pulse pair resolution & $\begin{array}{l}25 \text { radiation lengths } \\
\text { must be able to associate } \\
\text { pulse height to individual beam crossings } \\
\text { with low pile up }\end{array}$ \\
\hline \hline
\end{tabular}

- A total ionizing calorimeter based on either CsI or liquid krypton

- A calorimeter based on $\mathrm{PbWO}_{4}$ crystals.

Of these, lead and scintillator was rejected because of poor resolution. Fermilab is reluctant to deal with the numerous safety issues related to operating liquid argon or liquid krypton in the $\mathrm{C} 0$ enclosure. These devices also have long charge collection times which can, however, be overcome by careful pulse shaping. Liquid krypton, in particular, looked like an attractive possibility. Liquid detectors are radiation hard. However, the constant term in the energy resolution may be considerably larger due to the relatively large amount of material before the active sampling material. CsI (undoped) was attractive from many points of view. However, it is not radiation hard enough to be used close to the beam. Furthermore, it is not quite fast enough to completely produce all its charge in under one crossing but it this problem is easily overcome with electronics that samples two or three crossings. Lead glass has been rejected because it is not likely to survive the yearly radiation dose.

After study, we have selected for our baseline a calorimeter made of $\mathrm{PbWO}_{4}$ crystals. The $\mathrm{BTeV} \mathrm{PbWO}_{4}$ choice for an Electromagnetic Calorimeter (EMC) is largely based on the work of CMS. Our choice is based on several considerations, listed roughly in order of priority:

- It satisfies our requirements on energy and spatial resolution. Blocks of size roughly $2.5 \mathrm{~cm}$ can be made. CMS expects to achieve a stochastic term of $2.7 \%$ and a constant term of $0.55 \%$ using Avalanche PhotoDiodes (APDs) for readout. We plan to use photomultiplier tubes (PMTs) and expect to do better.

- This material is very resistant to radiation damage, especially when doped with either $\mathrm{Nb}$ or La. CMS requires the crystals to survive a dose of $50 \mathrm{kGy}$. This should be sufficient for $\mathrm{BTeV}$. 
- They are fast - they deliver $99 \%$ of their light output within $100 \mathrm{~ns}$.

The properties of $\mathrm{PbWO}_{4}$ which are important for the calorimeter are given in Table 4.11.

We describe some properties of the CMS calorimeter for purposes of comparison with the device we are proposing. CMS has chosen $\mathrm{PbWO}_{4}$ for both barrel and endcaps. The crystals are tapered to point at the interaction region. The sizes differ, the barrel crystals are $230 \mathrm{~mm}$ long, while the endcap crystals, which follow a 5 radiation length preradiator, are $220 \mathrm{~mm}$ long. The endcaps are $24.7 \mathrm{~mm}$ square in the front, while the barrel counters are smaller at $21.5 \mathrm{~mm}$ square. BTeV will use untapered blocks of very similar dimensions. Many details about the $\mathrm{PbWO}_{4}$ calorimeter can be found in the CMS writeup [1].

CMS will use Avalanche Photodiodes (APD) to read out the barrel and Vacuum Phototriodes to read out the endcap. These devices are necessary because of the high magnetic field in which the crystals are positioned. In BTeV we are far from any magnetic fields, so we can use photomultiplier tubes. This provides less noise, better resolution as will be described below, in a well understood and tested technology. In fact, BTeV could use the same tubes that KTeV used for their inner blocks, the Hamamatsu R5364, provided that a radiation hard glass such as C96-1 is used.

As part of its acceptance criteria, CMS requires that all of their crystals emit more than 10 photoelectrons per MeV into a XP2262B photomultiplier tube covering the back face of the crystal. Using a smaller phototube, we expect to collect in excess of 7 photoelectrons per $\mathrm{MeV}$. CMS actually collects $\approx 4$ photoelectrons per MeV into two $25 \mathrm{~mm}^{2}$ APD's on each crystal.

Table 4.11: Properties of $\mathrm{PbWO}_{4}$

\begin{tabular}{|l|l|}
\hline Property & Value \\
\hline Density $\left(\mathrm{gm} / \mathrm{cm}^{3}\right)$ & 8.28 \\
Radiation Length $(\mathrm{cm})$ & 0.89 \\
Interaction Length (cm) & 22.4 \\
Light Decay Time (ns): & $5(39 \%)$ \\
& $15(60 \%)$ \\
& $100(1 \%)$ \\
Refractive Index & 2.30 \\
Maximum of emission $(\mathrm{nm})$ & 440 \\
Temperature Coefficient $\left(\% /{ }^{\circ} \mathrm{C}\right)$ & -2 \\
Light output/NaI(Tl) $(\%)$ & 1.3 \\
Light output (pe/MeV into a 2" PMT) & 10 \\
\hline \hline
\end{tabular}

Despite all the work that has been done by CMS, there are significant open issues and risks in this approach. The main problem is growing crystals with sufficient quality and reproducibilty (yield), to lead to acceptable costs. There are only four producers working with CMS which currently have the ability to grow these crystals, and they will be kept busy for some time producing the crystals for CMS. These companies are in the Czech Republic, Russia and two in China. We will attempt to look for new sources, however. 
It may be possible to use another less radiation hard crystal at the periphery of the detector and we are evaluating such alternatives. However, in this document, we describe a system made totally from $\mathrm{PbWO}_{4}$ crystals.

\subsubsection{GEANT Studies}

The EMC geometry was defined as a rectangular array of crystals $24.7 \times 24.7 \mathrm{~mm}^{2}$. The length of the crystals was varied. A full simulation of the material properties was done with photons normally incident on the array, but smeared over the front face of the crystals.

\section{i. Crystal length}

We first explored the desired crystal lengths. In Figs. 4.31 and 4.32, we show the total energy deposited in the crystal for $2.5 \mathrm{GeV} / \mathrm{c}$ and $80 \mathrm{GeV} / \mathrm{c}$ incident photons, representing a low energy and an energy near the upper range of interest.
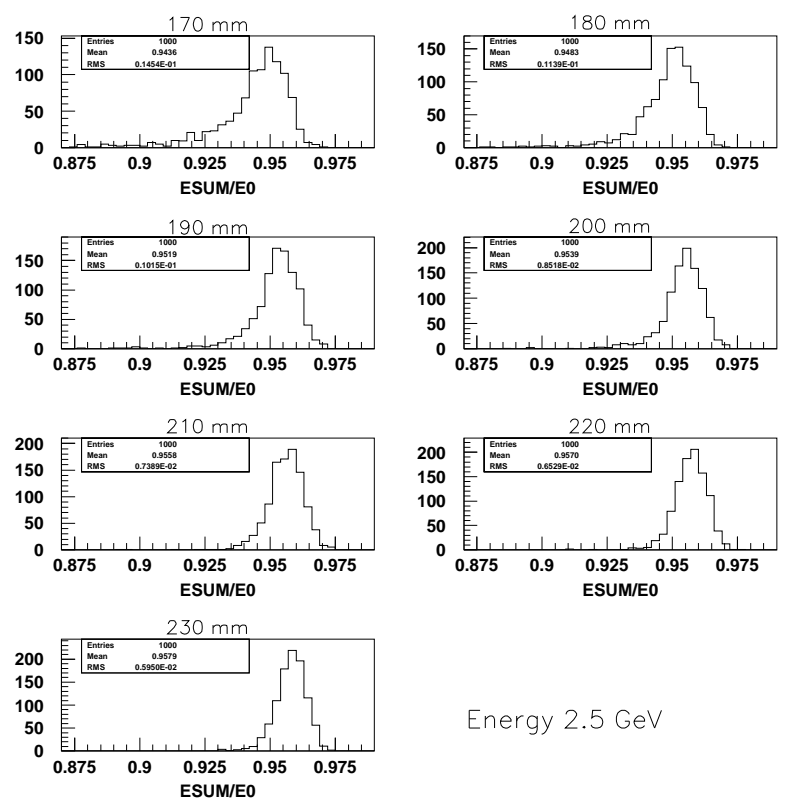

Energy $2.5 \mathrm{GeV}$

Figure 4.31: Energy deposited for $2.5 \mathrm{GeV} / \mathrm{c}$ photons normally incident on $\mathrm{PbWO}_{4}$ crystals of the labeled lengths.

It appears that 220 or $230 \mathrm{~mm}$ lengths are required. In order to make this study more quantitative, we clustered the energy in a $5 \times 5$ array of crystals centered on the crystal with the largest energy deposit. Then we fit the distributions with a function that reasonably describes the central part of the distribution as a Gaussian with an asymmetric low energy tail. This function has been called the "Crystal Ball lineshape," and was named after a former experiment based on NaI crystals [2]. The function form is 

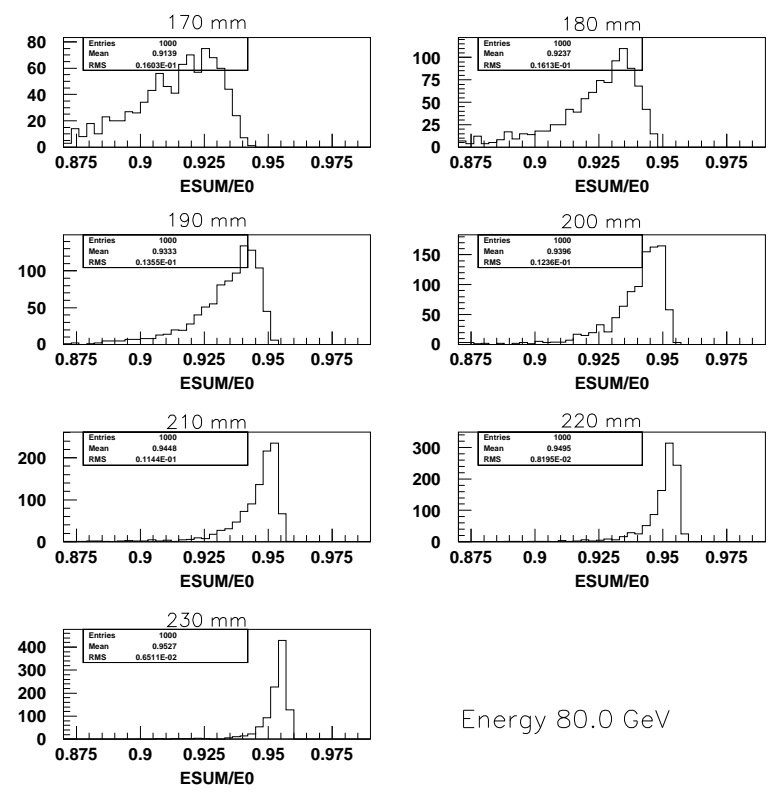

Energy $80.0 \mathrm{GeV}$

Figure 4.32: Energy deposited for $80 \mathrm{GeV} / \mathrm{c}$ photons normally incident on $\mathrm{PbWO}_{4}$ crystals of the labeled lengths.

$$
f\left(E \mid E^{*}, \sigma_{E^{*}}, \alpha, n\right)=\left(\begin{array}{lc}
A \cdot \exp \left[-\frac{1}{2}\left(\frac{E^{*}-E}{\sigma_{E^{*}}}\right)^{2}\right] & \text { for } E>E^{*}-\alpha \cdot \sigma_{E^{*}} \\
A \cdot \frac{\left(\frac{n}{\alpha}\right)^{n} e^{-\frac{1}{2} \alpha^{2}}}{\left(\frac{E^{*}-E}{\sigma_{E^{*}}}+\frac{n}{\alpha}-\alpha\right)^{n}} & \text { for } E<E^{*}-\alpha \cdot \sigma_{E^{*}} \\
\text { here } A^{-1} \equiv \sigma_{E^{*}} \cdot\left[\frac{n}{\alpha} \cdot \frac{1}{n-1} e^{-\frac{1}{2} \alpha^{2}}+\sqrt{\frac{\pi}{2}}\left(1+\operatorname{erf}\left(\frac{\alpha}{\sqrt{2}}\right)\right)\right]
\end{array}\right.
$$

Here $E$ is the measured energy, $E^{*}$ is the "true" (or most likely) energy and $\sigma_{E^{*}}$ is the energy resolution. To use this formula, the parameter $n$ is fixed to value of about 5 .

GEANT events for different energy photons and lengths were fit with this function for different energies and lengths varying from 210 to $230 \mathrm{~mm}$. An example of such a fit is shown in Fig. 4.33.

The result of this study is summarized on Fig. 4.34, where the $\sigma$ from each of the the fits is plotted. We see that the energy resolution determined by the crystal length and transverse size is hardly affected by using crystals of $220 \mathrm{~mm}$, but there is a degradation going to 210 $\mathrm{mm}$. This study uses energy deposited in the $5 \mathrm{x} 5$ array of blocks without the smearing due to photon statistics, so the resolutions are unreasonably good.

The energy resolution determined without photon statistics or electronic noise for 220 


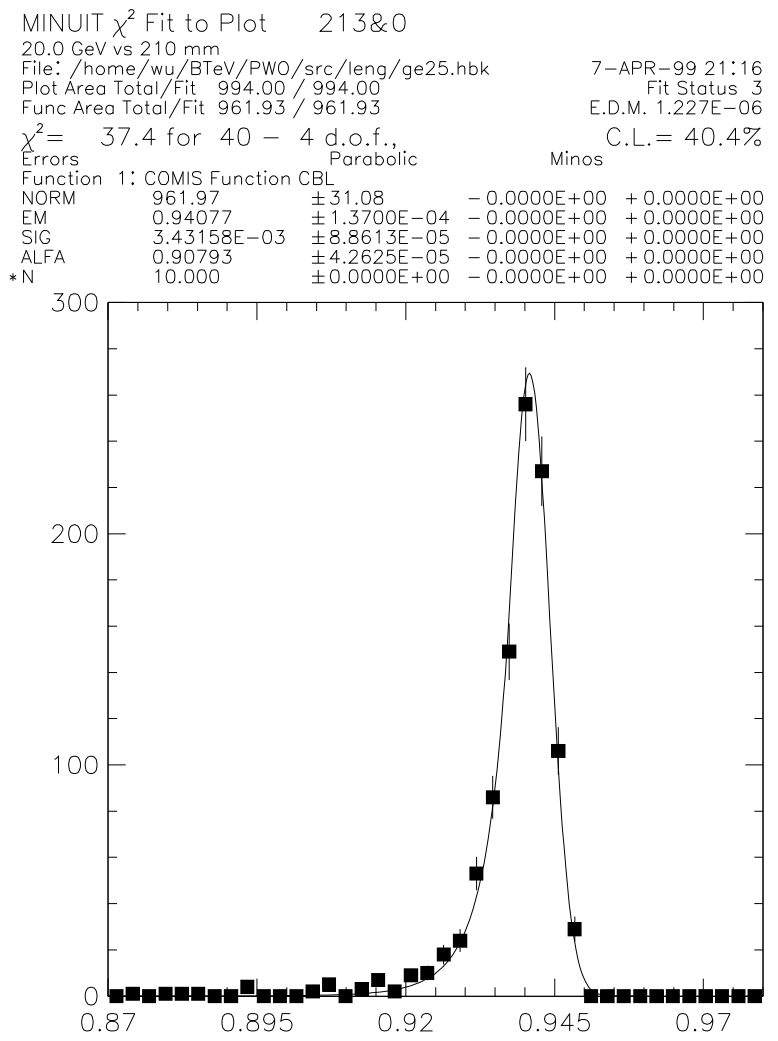

Figure 4.33: Fit of clustered energy deposited for $20 \mathrm{GeV}$ photons normally incident on $\mathrm{PbWO}_{4}$ crystals of $210 \mathrm{~mm}$ in length using the Crystal Ball lineshape. The x-axis is normalized to one at $20 \mathrm{GeV}$.

mm long crystals is shown on Fig. 4.35 .

$$
\sigma_{E}=\sqrt{\frac{a^{2}}{E}+b^{2}} \equiv \frac{a}{\sqrt{E}} \oplus b,
$$

with $a=1.08 \%$ and $b=0.2 \%$, and $E$ in units of GeV.

We can determine the component purely due to transverse leakage by fitting the distributions for total energy deposit and subtracting in quadrature the $\sigma$ 's from these fits and those with the $5 \times 5$ clustering. The transverse leakage component is shown in Fig. 4.36. We see the effect of transverse leakage can be characterized as

$$
\sigma_{E}^{\text {trans }}=\frac{0.7 \%}{\sqrt{E}} .
$$

The transverse leakage component is sufficiently small that it is not necessary to attempt to add in more blocks to the energy sum

ii. Expected Energy Resolution

The final resolution in energy that we can expect from a $\mathrm{PbWO}_{4}$ system will depend on several factors. One of these, is the transverse and longitudinal leakage. Another is 


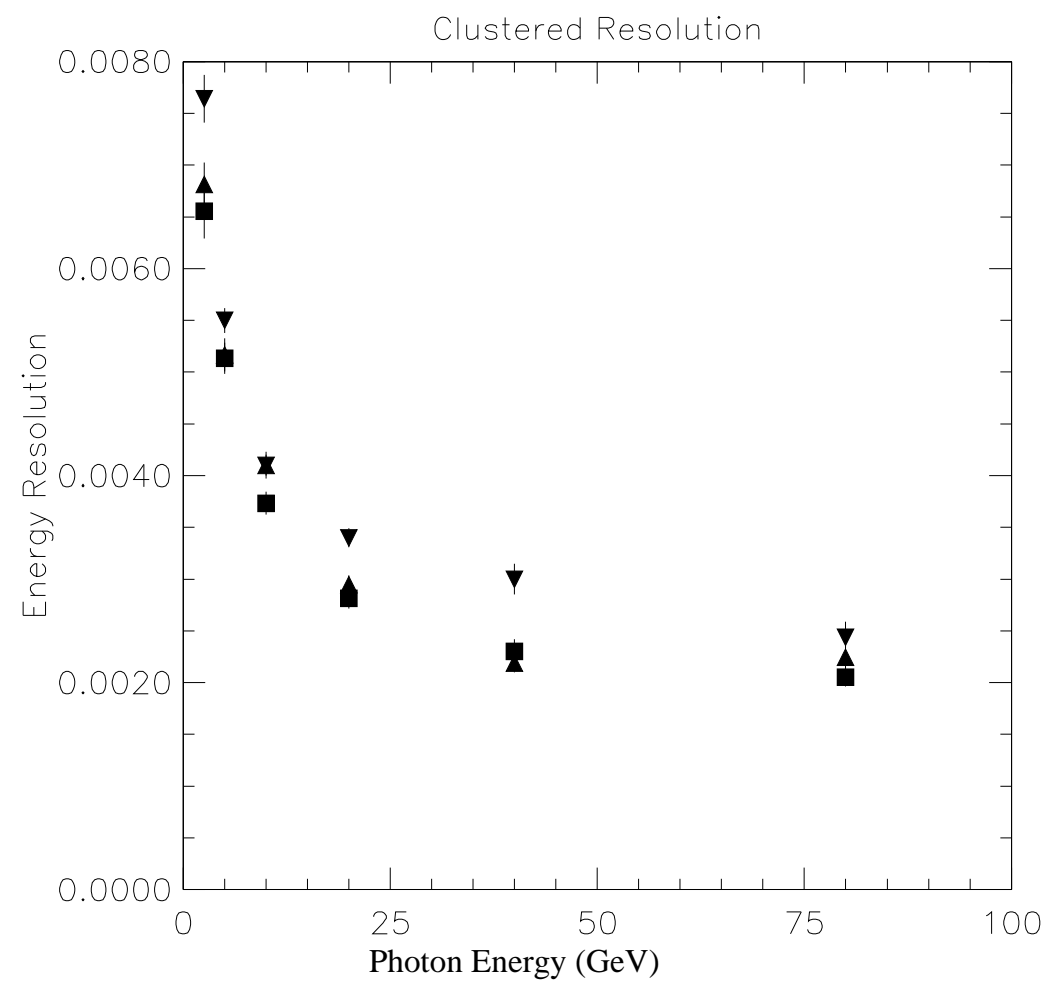

Figure 4.34: Energy resolutions as a function of incident photon energy for different crystal lengths using $5 \times 5$ clustering. The squares are for $230 \mathrm{~mm}$, the triangles for $220 \mathrm{~mm}$ and the inverted triangles for $210 \mathrm{~mm}$.

photoelectron statistics. These contribute to the so called "stochastic" term which has a $1 / \sqrt{E}$ dependence. We will evaluate this term below. Other effects that could contribute include electronics noise, negligibly small for phototubes, variations in light output along the crystal length, errors in the relative calibration between crystals, and possible other yet to be identified sources. These latter effects contribute to the "constant" term.

We can use the energy resolution expected by CMS to guide our expectations. They base their study on 4 photoelectrons/MeV into two APD's. There is an excess noise factor in APD's due to correlated gain in the device. This gives them effectively a factor of two fewer photons. Thus in CMS, photostatistics gives a contribution of $2.3 \% / \sqrt{E}$. The term for lateral containment is based on $21.5 \mathrm{~mm}^{2}$ crystals and a $5 \times 5$ clustering algorithm. They find a term $1.5 \% / \sqrt{E}$. Adding these together in quadrature gives $2.7 \% / \sqrt{E}$. CMS estimates a constant term of $0.55 \%$. They also include extra noise due to leakage currents in the photodiodes, and the effect of summing over $\approx 25$ interactions per crossing each with 7 times more energy than the Tevatron. We do not have these later effects at a significant level.

Additional guidance can be found by viewing the performance of the KTeV CsI calorime- 


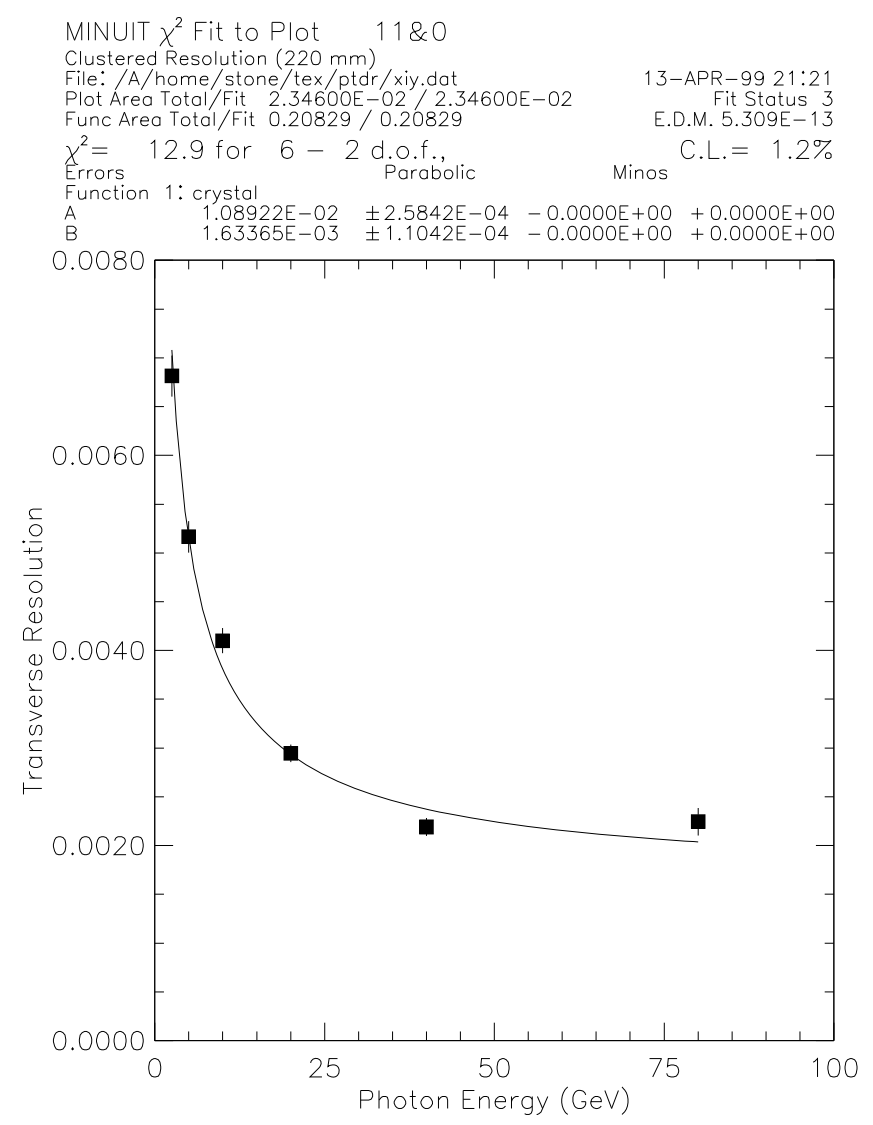

Figure 4.35: Energy resolutions as a function of incident photon energy for $220 \mathrm{~mm}$ long crystals using $5 \times 5$ clustering.

ter. They have achieved [3]

$$
\sigma_{E}=\frac{2 \%}{\sqrt{E}} \oplus 0.45 \% .
$$

The dominant cause of the constant term seems to be a differing crystal response depending on where on the face of the crystal the incident photon strikes. This effect was not observed in the CLEO CsI calorimeter [4].

The photon statistics term for BTeV, taking 7 photoelectrons $/ \mathrm{MeV}$ is expected to be $1.2 \% / \sqrt{E}$. We have already shown that the effect of transverse and longitudinal leakage in $220 \mathrm{~mm}$ long crystals is $1.1 \% / \sqrt{E}$. Adding these two non-correlated components in quadrature gives $1.6 \% / \sqrt{E}$. We have less knowledge of the constant term so we take the CMS estimated value of $0.55 \%$. The expected energy resolution in $\mathrm{BTeV}$ then is

$$
\sigma_{E}=\frac{1.6 \%}{\sqrt{E}} \oplus 0.55 \%
$$

To be conservative the physics simulations have been done using $2.5 \%$ rather than $1.6 \%$. iii. $\gamma-\pi^{o}$ Overlap 


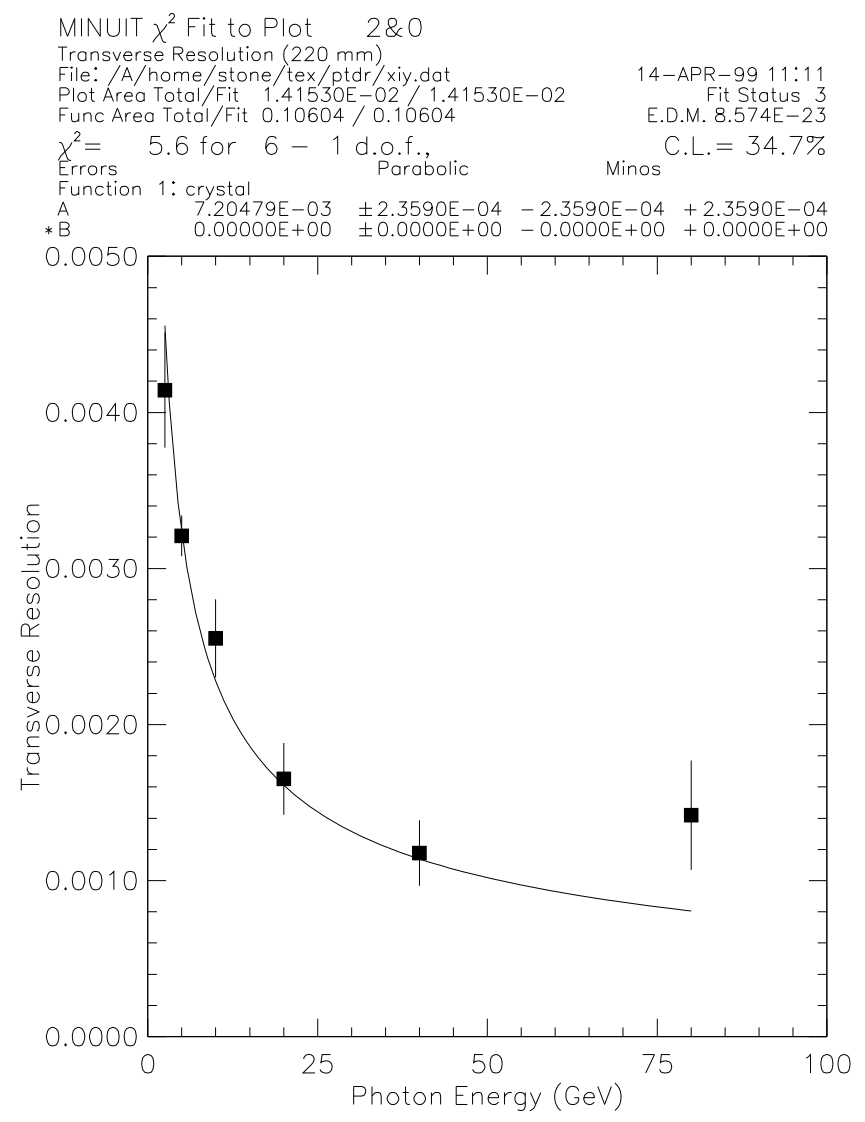

Figure 4.36: The contribution to the energy resolution from transverse leakage using a 5x5 array of crystals of $220 \mathrm{~mm}$ length and $24.7 \mathrm{~mm}^{2}$ in cross-section.

It is clearly quite important for BTeV to be able to distinguish photons from $\pi^{o}$ 's. Many of the $\pi^{o}$ s will be fast and could in principle appear as single photons. To study how well we could separate these two components with the proposed transverse segmentation, we generated both of them at fixed energies letting the $\pi^{o} \rightarrow \gamma \gamma$. We assumed only 4 photoelectrons $/ \mathrm{MeV}$, and a constant term of $0.55 \%$ thereby forcing a poorer energy resolution than we may achieve.

To study the probability of overlaps quantitatively, we calculate the second-moment of the energy distribution, $S_{2}$, defined as

$$
S_{2}=\frac{\sum_{i}\left(\vec{R}_{i}-\vec{R}_{0}\right)^{2} \cdot E_{i}}{\sum_{i} E_{i}}
$$

where $\vec{R}_{i}$ is the position vector to the center of each individual crystal in the cluster, $\vec{R}_{0}$ is the energy weighted position vector of the entire cluster, and $E_{i}$ is the energy deposited in each crystal. The $S_{2}$ distribution for both photons and $\pi^{o}$ 's of fixed energies is compared in Fig. 4.37. 

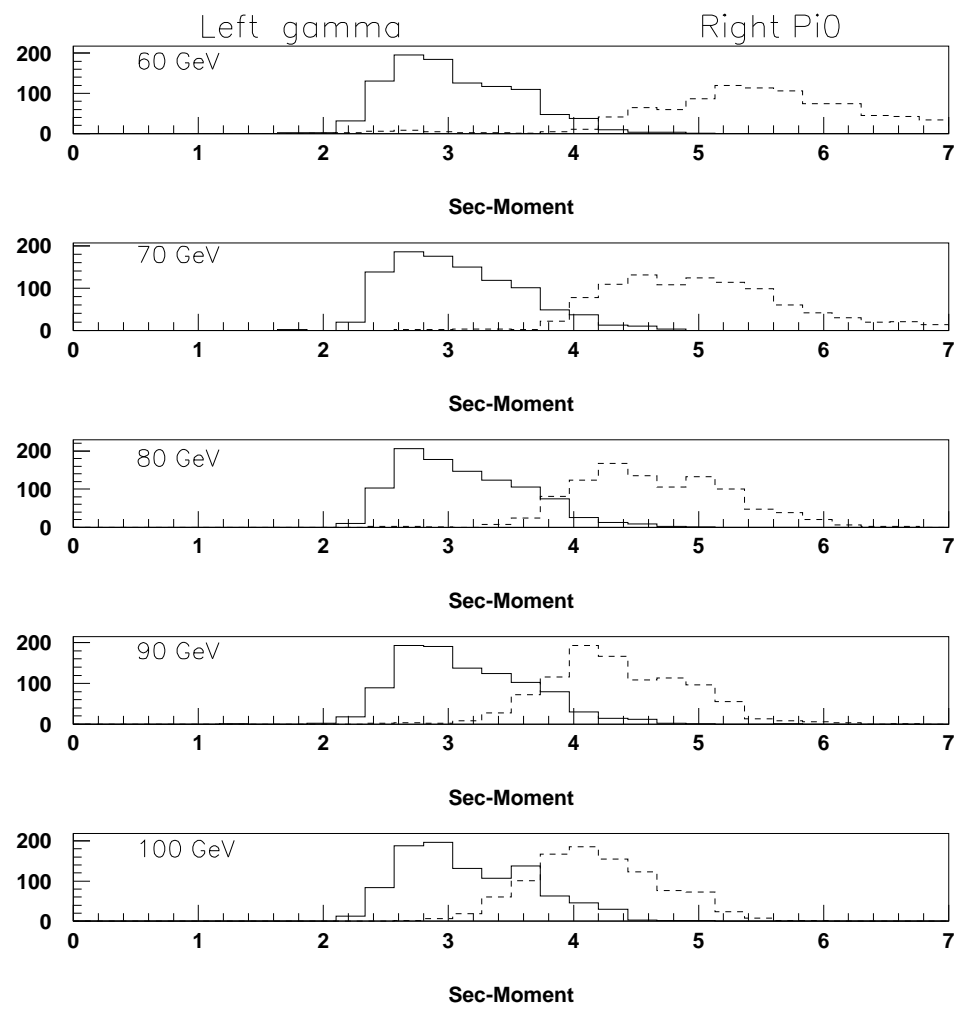

Figure 4.37: Second-moment energy distribution, $S_{2}$, for $\gamma^{\prime}$ s and $\pi^{o}$ s, as derived using GEANT and 4 photoelectrons/MeV.

In Table 4.12 we show the mis-identification rates for $\pi^{o} \rightarrow \gamma$ and $\gamma \rightarrow \pi^{o}$, both for $90 \%$ efficiency.

As most of the photons and neutral pions of interest in $\mathrm{BTeV}$ are below $60 \mathrm{GeV}$, the performance of the calorimeter in separating them is excellent.

iv. Position Resolution

The electromagnetic calorimeter is 7 meters from the center of the magnet. The resolution on projected angle is given in first order by the error in transverse position divided by 7 meters. The angular resolution enters directly in the mass resolution of fully reconstructed $b$ and charm final states.

We again used GEANT to simulate the energy deposit in $220 \mathrm{~mm}$ long crystals taking 7 photoelectrons/MeV. Positions were then determined in both directions transverse to the crystal axes. As we have not seen any difference in the two directions, an advantage of square cross-section crystals, we show results only for one direction, called $x$. The position of the 
Table 4.12: Neutral Particle Fake Rates for 90\% Efficiency

\begin{tabular}{ccc}
\hline \hline & & \\
Energy $(\mathrm{GeV})$ & $\pi^{o}$ to $\gamma$ fake rate $(\%)$ & $\gamma$ to $\pi^{o}$ fake rate $(\%)$ \\
\hline 60 & 2.9 & 0.8 \\
70 & 1.3 & 2.9 \\
80 & 4.2 & 5.9 \\
90 & 13.9 & 16.0 \\
100 & 38.5 & 30.7 \\
\hline \hline
\end{tabular}

reconstructed photon is determined from the energy deposit by using

$$
X c l=\frac{\sum_{i=1}^{5} x_{i} \cdot E_{i}}{\sum_{i=1}^{5} E_{i}},
$$

where $E_{i}$ is the energy reconstructed in each crystal, $x_{i}$ is the center of each crystal and the sum extends two crystals in the direction of $-x$ from the highest energy crystal to two crystals in the $+x$ direction.

In Fig. $4.38 \mathrm{Xcl}$ is shown versus the known position of the photon, $\mathrm{X} 0$ for $30 \mathrm{GeV}$ incident photons. The characteristic "S-curve" shape is apparent. To evaluate the position resolution, the overall shape is removed by a fit and the remaining width of the curve gives the position uncertainty. In Fig. 4.39 the central value of the S-curve has been removed and the distribution in $d x=X c l-X 0$ fit with a Gaussian. The width of the distribution then is the r.m.s. position resolution.

The results of performing this study over a range of energies is shown in Fig. 4.40, where we show the r.m.s. position resoluton as a function of energy. The fit gives

$$
\sigma_{x}=3526 \mu \mathrm{m} / \sqrt{E} \oplus 217 \mu \mathrm{m} .
$$

The projected position resolution then is much better than that assumed in the simulations, $\sigma_{x}=247 \mathrm{~mm} / \sqrt{12}=7100 \mu \mathrm{m}$. These position resolutions give excellent angular resolution. At $6 \mathrm{GeV}$, the projected r.m.s. angular resolution is $0.2 \mathrm{mr}$.

\subsubsection{Baseline Design}

The baseline $\mathrm{BTeV}$ electromagnetic calorimeter consists of two wheels of $2.47 \mathrm{~cm} \mathrm{X} 2.47 \mathrm{~cm}$ blocks of $\mathrm{PbWO}_{4}$ crystals - one in each arm of the spectrometer. Each wheel is split down the middle to accommodate the beam pipe and to allow it to be moved away from the IR for servicing if necessary. The block length is chosen to be 25 radiation lengths $(22 \mathrm{~cm})$. We consider two options. They differ only in outer radius. The inner radius for both options is currently chosen to be $7 \mathrm{~cm}$, which corresponds to $\pm 10 \mathrm{mr}$. For the first option the outer radius is chosen to be $140 \mathrm{~cm}$, which corresponds to $200 \mathrm{mr}$. The radius is less than we would like, but costs less. The total number of blocks is 10,000 per arm or 20,000 total. In size and 


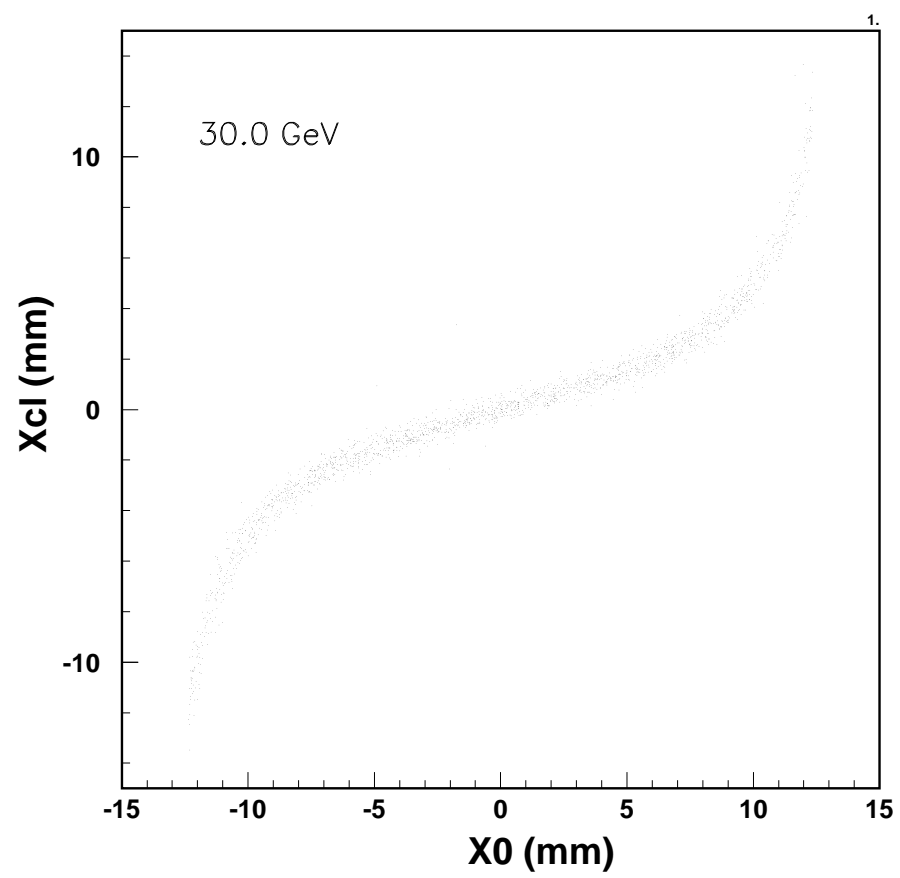

Figure 4.38: Measured photon position $X c l$ versus known photon position $X 0$ for $30 \mathrm{GeV}$ photons.

number of blocks, the system is only a little smaller than the CMS endcap calorimeter. It is this option that we call the baseline and have used in the simulations. The second option extends the calorimeter out to $300 \mathrm{mr}$. This is not only useful in getting more photon rate, but also extends the electron coverage. It does double the number of blocks, however, to 20,000 per arm.

At present, our blocks are rectangular solids. CMS has tapered its blocks so that the crystals almost point to the interaction point. We may consider doing this if further study shows it to be advantageous. The cross-section is chosen to be the same as the maximum size of the CMS end-cap blocks and the length is the same as the CMS endcap crystals.

There are detectors in each arm of the spectrometer. Each detector is housed in a temperature and humidity controlled hut. Temperature stabilization or some kind of correction is necessary because of the thermal coefficient of the $\mathrm{PbWO}_{4}$. CMS plans to control the temperature to $0.1^{\circ} \mathrm{C}$. We will also employ temperature stabilization but have not yet designed the system. In addition to temperature stabilization, the gains are monitored with a laser pulsing system.

Detectors are read out with PMTs. The light emitted by the crystal peaks at $440 \mathrm{~nm}$. The output of the PMT will go to a modified QIE chip located in or near the photomultiplier base. This chip provides a pulse height for each beam crossing. The expected light output is 


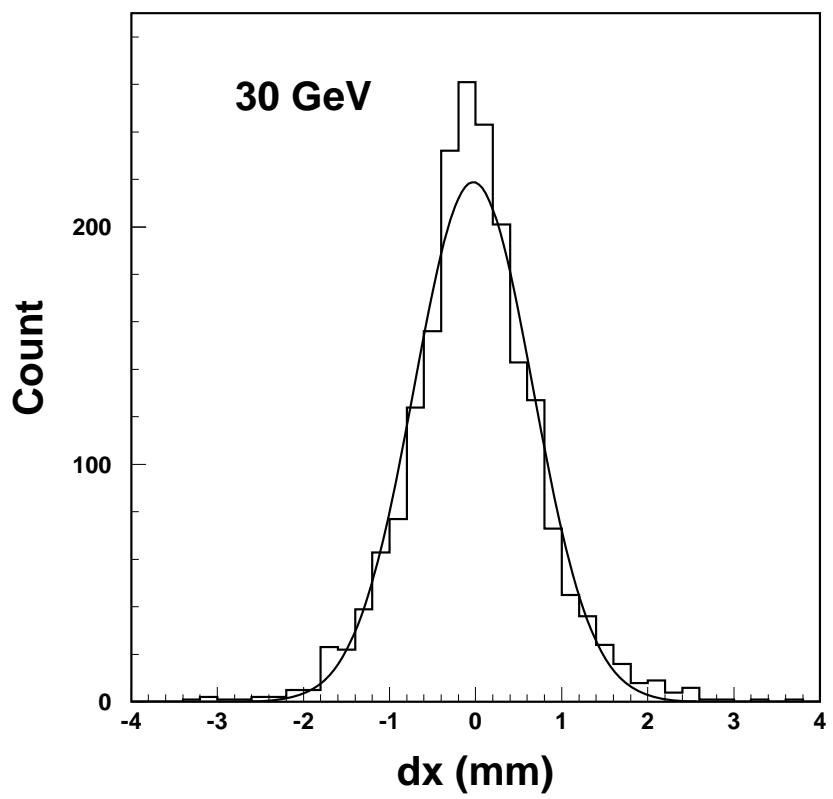

Figure 4.39: Distribution of $d x=X c l-X 0$ for $30 \mathrm{GeV}$ photons. The fit is a Gaussian with mean consistent with zero and $\sigma=0.7 \mathrm{~mm}$.

7000 photoelectrons at $1 \mathrm{GeV}$. The minimum sensitivity of the system is set so that a muon transversing the system, which corresponds to an energy deposition of $300 \mathrm{MeV}$, will have peak 50 counts above pedestal.

The detector is far enough away from the BTeV dipole so that there should be no need for special magnetic shielding. It is possible that we will magnetize the iron of the muon filter just downstream of the detector. In that case, we will put an iron shield plate between the calorimeter's PMTs and the muon filter.

Based on our extrapolation of information from CMS on the energy resolution, and our own GEANT studies, we expect a stochastic term of about $1.6 \%$. The constant term is expected to be about $0.55 \%$. The spatial resolution in both directions transverse to the Tevatron is expected to be about $3500 \mu \mathrm{m} / \sqrt{E}+200 \mu \mathrm{m}, E$ in units of $\mathrm{GeV}$.

Tests by CMS have indicated that these blocks can sustain integrated doses of 200,000 Grays. The only blocks that receive doses close to this number are the blocks right near the beam.

We have not yet started engineering on the support, but our detector is similar in size and shape to the CMS endcap electromagnetic calorimeter. A solution similar to theirs should work for BTeV. Because our detector is not embedded in a large device, and because at least at present we do not plan to use tapered blocks, our supports will be simpler and less expensive than those for CMS.

The properties of the baseline detector are summarized in Table 4.13. The GEANT studies described above demonstrate that the proposed device will satisfy the requirements of Table 4.9. 


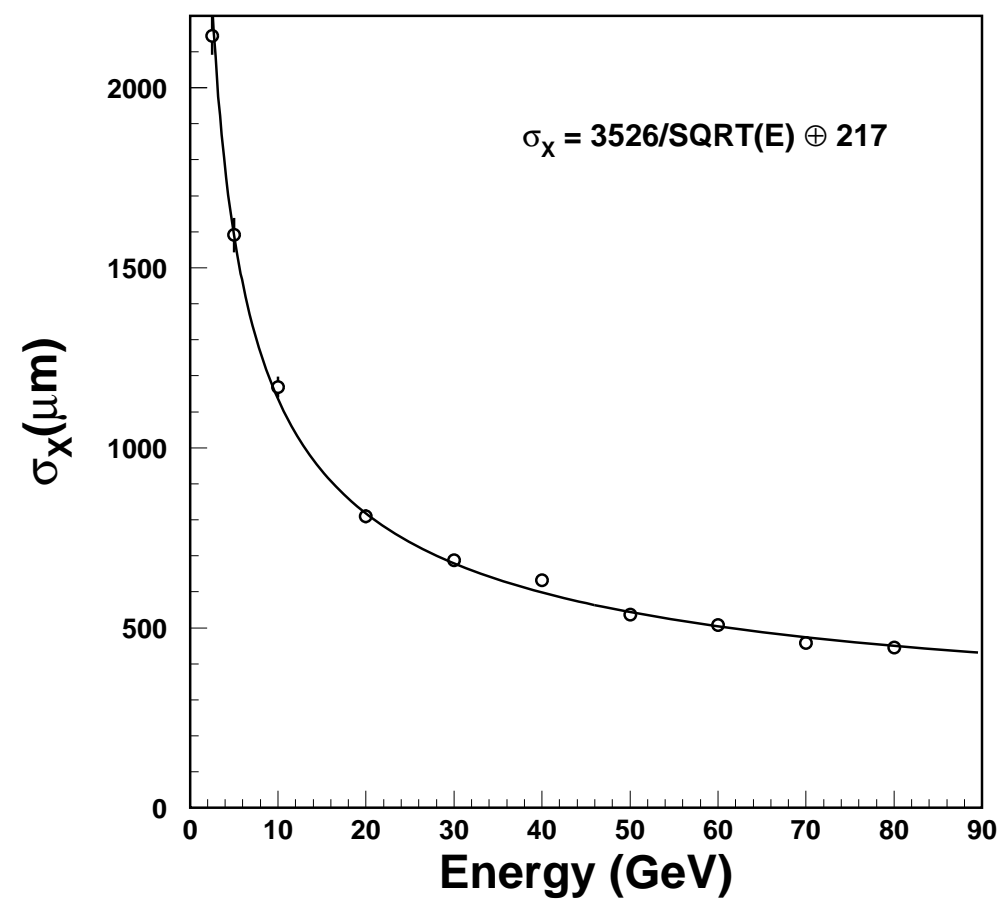

Figure 4.40: The expected r.m.s. position resolution for photons as a function of energy.

\subsubsection{BTeV Electromagnetic Calorimeter R\&D Program}

At the time of our original R\&D proposal, we did not have a plan for the Electromagnetic Calorimeter. We just listed several options, each of which had problems. Many of the problems we were worried about have been resolved by the development by CMS of $\mathrm{PbWO}_{4}$ crystals as a viable calorimeter technology. We are taking full advantage of the work that CMS has done. We do have to carry out some R\&D because we want to use photomultipliers rather than avalanche photodiodes. We will also be using a modified version of the QIE chip to read the data out. We want to obtain a few crystals and to try them with different types of PMTs and with QIE readout. We will have to explore cost issues with phototube manufacturers. Eventually we will want to expose a 5X5 (or better 7X7) stack of crystals to a low energy electron beam to study the resolution, leakage, temperature sensitivity, etc.

It is also critical that we begin to understand the issues related to producing crystals for $\mathrm{BTeV}$. We will need to visit the existing production sites as well as to explore the possiblity of producing crystals with other manufacturers. 
Table 4.13: Properties of the BTeV electromagnetic Calorimeter

\begin{tabular}{|l|l|}
\hline Property & Value \\
\hline transverse block size & $2.47 \mathrm{~cm} \mathrm{X} 2.47 \mathrm{~cm}$ \\
Block length & $22 \mathrm{~cm}$ \\
Radiation Length $(\mathrm{cm})$ & 25 \\
Front end electronics & PMT \\
Digitization/readout & QIE (FNAL) \\
Inner Dimension & $\pm 9.88 \mathrm{~cm} \times \pm 9.88 \mathrm{~cm}$ \\
Outer Radius (baseline) & $140.0 \mathrm{~cm}$ \\
Total blocks per arm (baseline) & 10000 \\
Total blocks per arm (option) & 20000 \\
Outer Radius (option) & $215.0 \mathrm{~cm}$ \\
\hline \hline
\end{tabular}




\section{Bibliography}

[1] CMS, "The Electromagnetic Calorimeter Project Technical Design Report," CERN/LHCC 97-33, CMS TDR 4 (1997).

[2] T. Skwarnicki, "A Study of the Radiative Cascade Transitions Between the UpsilonPrime and Upsilon Resonances," DESY F31-86-02 (thesis, unpublished) (1986).

[3] Private communication from Ed Blucher.

[4] E. Blucher, B. Gittelman, B. K. Heltsley, J. Kandaswamy, R. Kowalewski, Y. Kubota, N. Mistry, A. Bean, and S. Stone, "Tests of Cesium Iodide Crystals for an Electromagnetic Calorimeter," Nuclear Instruments \& Methods A249, 201 (1986). 


\subsubsection{Muon Detection}

The BTeV muon system has two primary functions:

- $J / \psi$ and Prompt Muon Trigger: Besides providing interesting physics, this trigger performs an important service role by selecting (bottom) events on which the more aggressive and technically challenging triggers (such as the vertex trigger) can be debugged and evaluated.

- Muon Identification: Many of the experiment's physics goals (rare decay searches, CP violation studies which require tagging, studies of beauty mixing, searches for charm mixing, etc.) rely on efficient muon identification with excellent background rejection.

We have selected a magnetic (toroidal) design combined with fine-grained tracking elements (described in detail in a subsequent chapter). This design permits "stand-alone" momentum/mass triggering: i.e. a muon trigger based solely on information from the muon detector. In addition, improved background rejection is possible via comparisons of this stand-alone measurement with momentum and tracking information from the rest of the spectrometer. The design of the system, including the fine-grained detector segmentation, has been chosen to reduce and uniformly distribute occupancies and to minimize confusion in pattern recognition (both at the trigger level and in offline reconstruction).

In order to provide a viable trigger, the system must get a rejection at Level 1 of a few $10^{-2}$. The goal for muon misidentification is $10^{-3}$.

Given the objective of a "stand-alone" trigger and the size limitations set by the experimental hall, one can make fairly general calculations that place specific (and restrictive) constraints on the design of the system. We will first describe these general calculations and use them to motivate the overall design of the muon system. We will then describe trigger efficiency and rejection studies we have performed for $B^{0} \rightarrow J / \psi K_{s}^{0}$, which illustrate the performance of the design for a specific (and important) physics mode.

\subsubsection{General Design Considerations}

In the absence of any special momentum dependent coordinate weighting designed to reduce the effects of multiple Coulomb scattering, the fractional momentum resolution in a magnetic spectrometer can be parameterized by the expression:

$$
\frac{\sigma_{p}}{p}=\sqrt{a^{2}+(b p)^{2}}
$$

In Equation 4.12, the $a$ term depends on the bending power and multiple scattering environment of the detectors, while the $b$ term depends on the bending power and and the detector layout and spatial resolution. Fig.4.41 shows that the potential rejection provided by a trigger for low momentum muons significantly degrades once the low momentum fractional resolution exceeds $25 \%$. An $a=25 \%$ value means that the trigger rejects very soft muons at roughly the $4 \sigma$ level. At higher momentum, where multiple scattering is less important, 


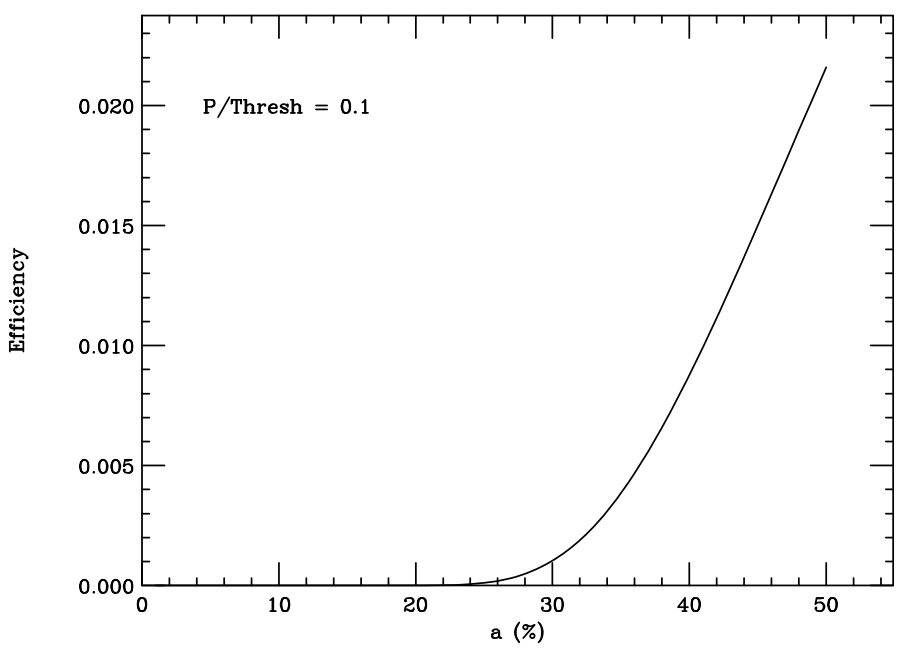

Figure 4.41: The efficiency, calculated in a simple Gaussian model, for a muon with 1/10 the threshold momentum to pass a momentum threshold as a function of $a$, the MCS term in the resolution formula. We say that a muon at the threshold momentum should fire the trigger $50 \%$ of the time. Note the dramatic rise in sensitivity above $a=25 \%$.

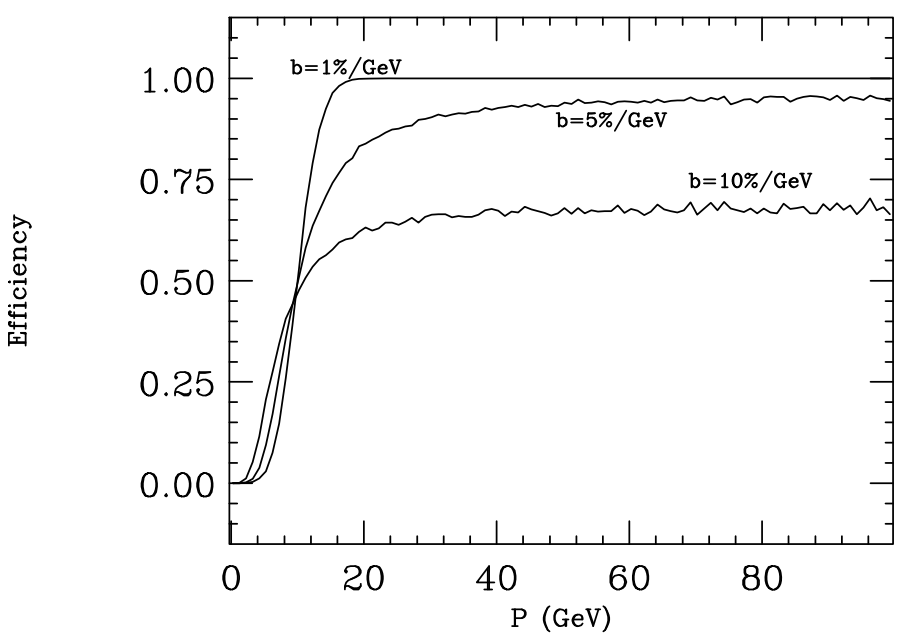

Figure 4.42: Simulated trigger efficiency as a function of muon momentum for a trigger designed to fire with a $50 \%$ efficiency at $10 \mathrm{GeV}$. The multiple scattering dominated term is set to $a=25 \%$ while the measurement dominated term varies from $1 \% / G e V<b<$ $10 \% / \mathrm{GeV}$.

one becomes sensitive to the $b$ term of Eq. 4.12. The high momentum resolution influences how sharp a momentum threshold one can make in a stand-alone muon trigger. Figure 4.42 illustrates this point by showing the trigger efficiency as a function of momentum for several $b$ values. 


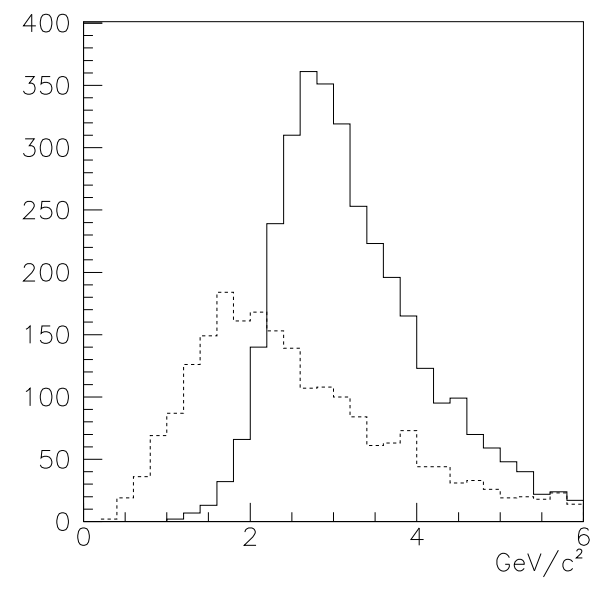

Figure 4.43: Reconstructed $J / \psi \rightarrow \mu^{+} \mu^{-}$mass resolution for $b=1 \% / \mathrm{GeV}$ (solid) and $b=5 \% / G e V$ (dashed), where $b$ is defined in Eq. 4.12. Equal numbers of $J / \psi$ candidates were generated for each case.

These considerations suggest minimum performance criteria of $a<25 \%$ and $b \leq$ $1 \% / \mathrm{GeV}$. As a further illustration, Fig. 4.43 shows the mass resolution obtainable at the trigger level for $J / \psi \rightarrow \mu^{+} \mu^{-}$events for two values of $b$.

\subsubsection{Possible Design Layouts}

Several possible measurement and shielding layouts were studied before we reached the baseline design. In assessing possible layouts, we compute the momentum resolution using an error matrix which incorporates Gaussian models for both the detector resolution and multiple Coulomb scattering. The beam is modeled as a Gaussian beam spot with $\sigma_{x}=$ $\sigma_{y}=1 \mathrm{~mm}$ and $\sigma_{z}=30 \mathrm{~cm}$. In cases where there is redundant coordinate information, the error matrix assumes that the fit weighted the space points according to momentum in order to get the best possible momentum measurement.

Our early calculations showed that it is not possible to get a good momentum measurement using just the dispersion provided by the central dipole (and a constraint that muon system tracks point back to the interaction point). The extrapolation distance is too large given the level of multiple scattering up to the muon planes, making it infeasible to point the muon track segment back to the interaction region. A magnetic toroid with magnetic fields circulating in the $\hat{\phi}$ direction is the only (simple) option which allows us to trigger effectively using tracking information from only the muon system.

The first arrangement we considered is illustrated in Figure 4.44. In this design, one determines the momentum of a muon by measuring its slope in $\hat{\theta}$ before and after a toroid. The entering and exiting slopes of the muon are measured using two pairs of measuring stations $\left(\mu_{1}-\mu_{4}\right)$ separated by one meter lever arms. At low momentum, where the multiple scattering dominates resolution, a single 1.5 Tesla, 1 meter thick toroid achieves the required 


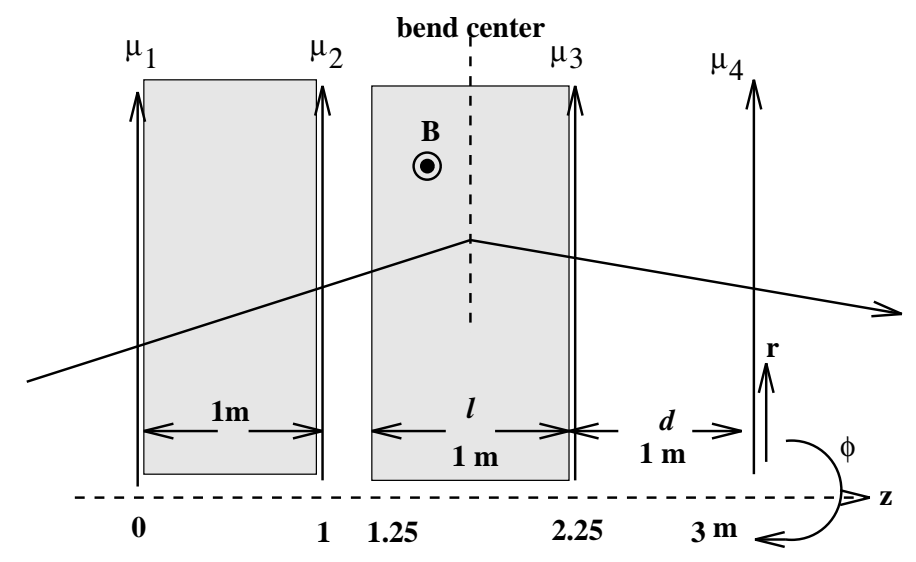

Figure 4.44: Layout of one arm of the muon detector in $2 \oplus 2$ geometry.

fractional momentum resolution of $a=25 \%$. In this arrangement the steel between the measurement stations of the upstream doublet $\left(\mu_{1}-\mu_{2}\right)$ primarily serves to shield the muon system from hadrons and need not be magnetized. The requirement of good momentum resolution $(b \approx 1 \% / \mathrm{GeV})$ dictates a spatial resolution of $\sigma=2.5 \mathrm{~mm}$. We will call this the " $2 \oplus 2$ " geometry.

Note that the intrinsic rms spatial resolution $(\sigma=2.5 \mathrm{~mm})$ required by this (and subsequent) geometries is consistent with what we require from timing considerations alone. Timing information is useful for rejecting background hits such as those associated with accelerator backgrounds (these hits come 60-70 ns before tracks from the interaction point). Assuming a fast gas drift velocity of roughly $9 \mathrm{~cm} / \mu \mathrm{s}$, a drift tube system with a maximum drift time of $30 \mathrm{~ns}$ has a maximum drift length of $2.5 \mathrm{~mm}$ (leaving $30 \mathrm{~ns}$ for charge integration). For a double layer of $1 \mathrm{~cm}$ diameter round proportional tubes, offset by half a tube in a "picket fence" design (to avoid dead regions between the tubes), the maximum drift time for the first signal from a track is 30 ns. This arrangement yields an rms resolution of $\sigma=1.44 \mathrm{~mm}$.

The major problem with the $2 \oplus 2$ design is that the most upstream of the four detector stations is unshielded against hadronic showers originating in the electromagnetic calorimeter and is thus likely to be too confused for use in a first level trigger. One could, in principle, measure momentum using the downstream doublet $\mu_{3}$ and $\mu_{4}$ and a single upstream point $\mu_{2}$. This geometry gives essentially the same $a$ parameter, but for the same spatial resolution the $b$ parameter is degraded by a factor of 2.8 because the effective upstream lever arm has been reduced to just the distance between the bend center and $\mu_{2}$.

This led us to the revised design, our "baseline" geometry, illustrated in Fig. 4.45 where the upstream muon trajectory is measured using information from the nominal beam constraint. The effectiveness of this approach is illustrated in Fig. 4.46. The upper black curves give the fractional momentum resolution as a function of muon momentum for the case where only the downstream steel is magnetized and only information from $\mu_{3}, \mu_{4}$ and the nominal beam center is used. The three curves are for muons emerging at three different azimuthal 


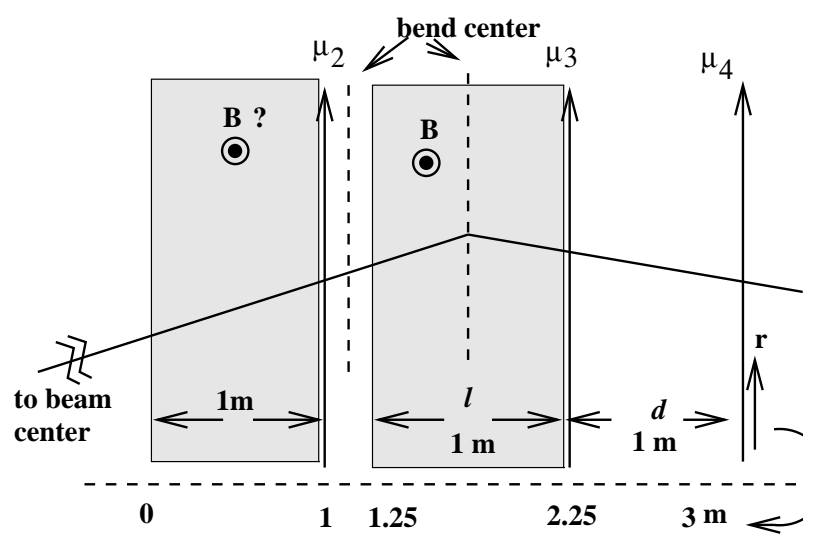

Figure 4.45: Layout showing the baseline geometry. The downstream trajectory is measured by $\mu_{3}$ and $\mu_{4}$. The upstream trajectory is measured using the nominal beam center with possible help from $\mu_{2}$. In order to get sufficient bending power, both steel sections will need to be magnetized.

$(\phi)$ angles. The pronounced azimuthal variation in fractional momentum resolution arises because of constructive and destructive interference between the dipole and toroid fields. Figure 4.46 shows that at destructive $\phi$ angles, the $a$ term exceeds our criteria of $a<25 \%$.

The incorporation of information from $\mu_{2}$ significantly helps the fractional momentum resolution for the case of a single toroid at low momenta as shown in the three blue curves of Fig. 4.46. Essentially the fit used to obtain the "blue" resolution curves ignores the beam constraint at low momentum while exploiting it at high momentum through momentum varying weights. Such a resolution is obtainable off-line, but would be difficult to achieve in a first level trigger.

If one instead magnetizes both the upstream and downstream steel, the bending power of the toroid overwhelms the power of the dipole and a much simpler triggering scenario emerges which is illustrated by the lower seven curves of Fig. 4.46. The case where only a beam constraint and $\mu_{3}-\mu_{4}$ are used in the trigger is illustrated by the dashed red curves. The green curve shows the resolution one would obtain using a beam constraint and the downstream $\mu_{3}-\mu_{4}$ doublet if the central dipole were turned off. The green curve shows that the dipole provides very little additional bending power in the ultimate system. When information from $\mu_{2}$ is included one gets the solid magenta curves. Again, curves are shown for three different azimuths. Each of these curves exhibit good performance relative to our criteria on $a$ and $b$ (over the complete azimuth).

Interestingly enough, Fig. 4.46 tells us that in a two magnet, toroid geometry, $\mu_{2}$ contributes very little to the momentum resolution. In the absence of noise or confusion, one can design a very simple but effective trigger by correlating information from just the two $\hat{\theta}$ views of the downstream doublet consisting of $\mu_{3}$ and $\mu_{4}$ and the nominal beam center. Since this is the minimal amount of information required to obtain a momentum estimate, no momentum dependent weighting of detector information is used. We also have the benefit of deriving this trigger from the most shielded chambers of the muon system, minimizing 


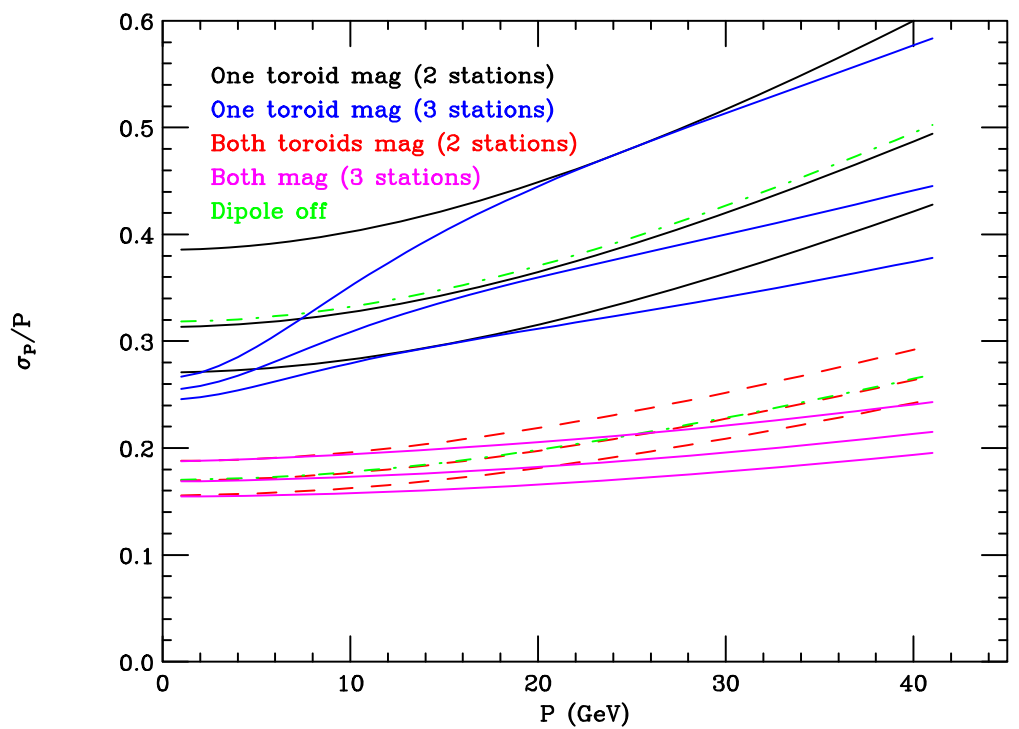

Figure 4.46: Plots of the fractional momentum resolution as a function of momentum for various readout scenario's of the BTeV muon system. All plots assume chambers with $2.5 \mathrm{~mm}$ rms spatial resolution. The plots appear in groups of 3 corresponding to $\phi=270^{\circ}$ (best) $0^{\circ}$ and $90^{\circ}$ (worst). The $\phi$ dependence of the resolution illustrates interference between the central dipole and muon toroid(s). The black and red families of curves illustrate the case where only the two stations after the second toroid are used in the trigger. In this case the trajectory upstream of the toroid comes entirely from the beam constraint. The blue and magenta curves illustrate the case where the station between the two toroids is used to help determine the trajectory prior to the toroids. The green curves assume only the final two stations are used, and that the dipole is turned off .

hadronic punch-through effects.

Although $\mu_{2}$ is not required to measure muon information in our two toroid scenario, it is likely to be of substantial use in eliminating false triggers due to noise and confusion. Figure 4.47 illustrates just one example by showing how $\mu_{2}$ can be used at the trigger level to eliminate false triggers from soft muons with an accompanying $\delta$-ray.

\subsubsection{Baseline Design}

Based on the above, our baseline muon system consists of two $1 \mathrm{~m}$ thick iron toroids and three measurement stations with spatial resolutions of $\sigma=1.5 \mathrm{~mm}$. Each of the measurement stations is separated by approximately 1 meter. The spatial resolution is set by both timing considerations and momentum resolution requirements. 


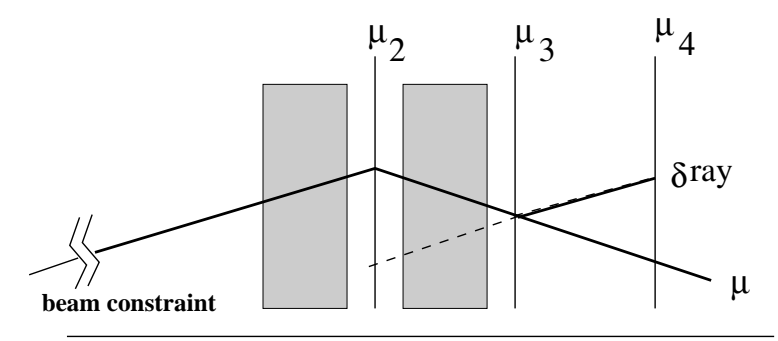

Figure 4.47: This figure illustrates the usefulness of $\mu_{2}$ in reducing false triggers due to confusion from $\delta$-rays. We illustrate a trajectory for a low momentum $\mu$ which is severely bent by the two toroids and therefore extrapolates far from the nominal beam center. As this muon emerges from $\mu_{3}$ it emits a wide angle $\delta$-ray which leaves an additional hit in $\mu_{4}$. In the case illustrated, the two hits in stations $\mu_{3}$ and $\mu_{4}$ from the $\delta$-ray point back to the nominal beam center and mimic a high momentum muon. However, the $\delta$-ray trajectory extrapolates very far from the muons hit in $\mu_{2}$ and thus fails to satisfy a trigger which requires a confirming hit in $\mu_{2}$.

\subsubsection{Trigger Studies for $B^{0} \rightarrow J / \psi K_{s}^{0}$}

We have studied the triggering performance of our baseline muon system using MCFAST. We used minimum bias events to study rejection ratios and $B^{0} \rightarrow J / \psi K_{s}^{0}$, where $J / \psi \rightarrow \mu \mu$, to investigate trigger efficiency. The minimum bias events were generated with Pythia and include elastic scattering, single and double diffractive, low $p_{T}$ scattering and semi-hard QCD $2 \rightarrow 2$ processes. Events are overlapped to simulate multiple events per crossing. The number of events per crossing is generated from a Poisson distribution with average two. In generating the $B^{0} \rightarrow J / \psi K_{s}^{0}$ events, minimum bias events are also added so that the total number of interactions per crossing is also Poisson with an average of two. For the studies presented below, we only consider "real" muons, which can be prompt or can come from decays in flight. In minimum bias events, the muons come almost entirely from pion $(70 \%)$ and kaon (30\%) decays. "Fake" muons from punch through, delta rays, and noise hits in the chamber are not yet simulated.

In the calculations below, efficiency is the ratio of accepted events to generated events. This includes geometrical acceptance and the selection cuts. For our purposes, a signal $\left(B^{0} \rightarrow J / \psi K_{s}^{0}\right)$ event is considered to be in the geometrical acceptance if both tracks from the $J / \psi$ muons are tracked by the silicon and make it through all stations of the muon system. The muon system is assumed to have an outer radius of $2.4 \mathrm{~m}$ and the inner radius is varied between $10 \mathrm{~cm}$ and $20 \mathrm{~cm}(10 \mathrm{~cm}$ is the smallest possible inner radius given the inner radius of the toroids, while $15-20 \mathrm{~cm}$ is the smallest inner radius that can probably be achieved with the baseline design). At that point, both minimum bias and signal events are treated identically. They are accepted if muon(s) in the event pass cuts on momentum, transverse momentum, and/or a minimum invariant mass cut on muon pairs. In all cases where two muons are required, they must have opposite sign and be in different octants in $\phi$ (each detector plane in the baseline muon system is divided into eight pie shaped octants). 
The pair of muons must also be in the same arm of the detector.

The momentum and transverse momentum of each muon in the event is obtained by smearing its generated momentum using the appropriate resolution (see Fig. 4.46) and smearing function. We then vary the cuts on momentum, transverse momentum, and invariant mass of pairs of muons and make two dimensional plots showing rejection of minimum bias events on one axis and $J / \psi$ efficiency on the other. An example plot is shown in Fig. 4.48. We have made similar plots for differing inner radii, one versus two toroids, and so on. Based

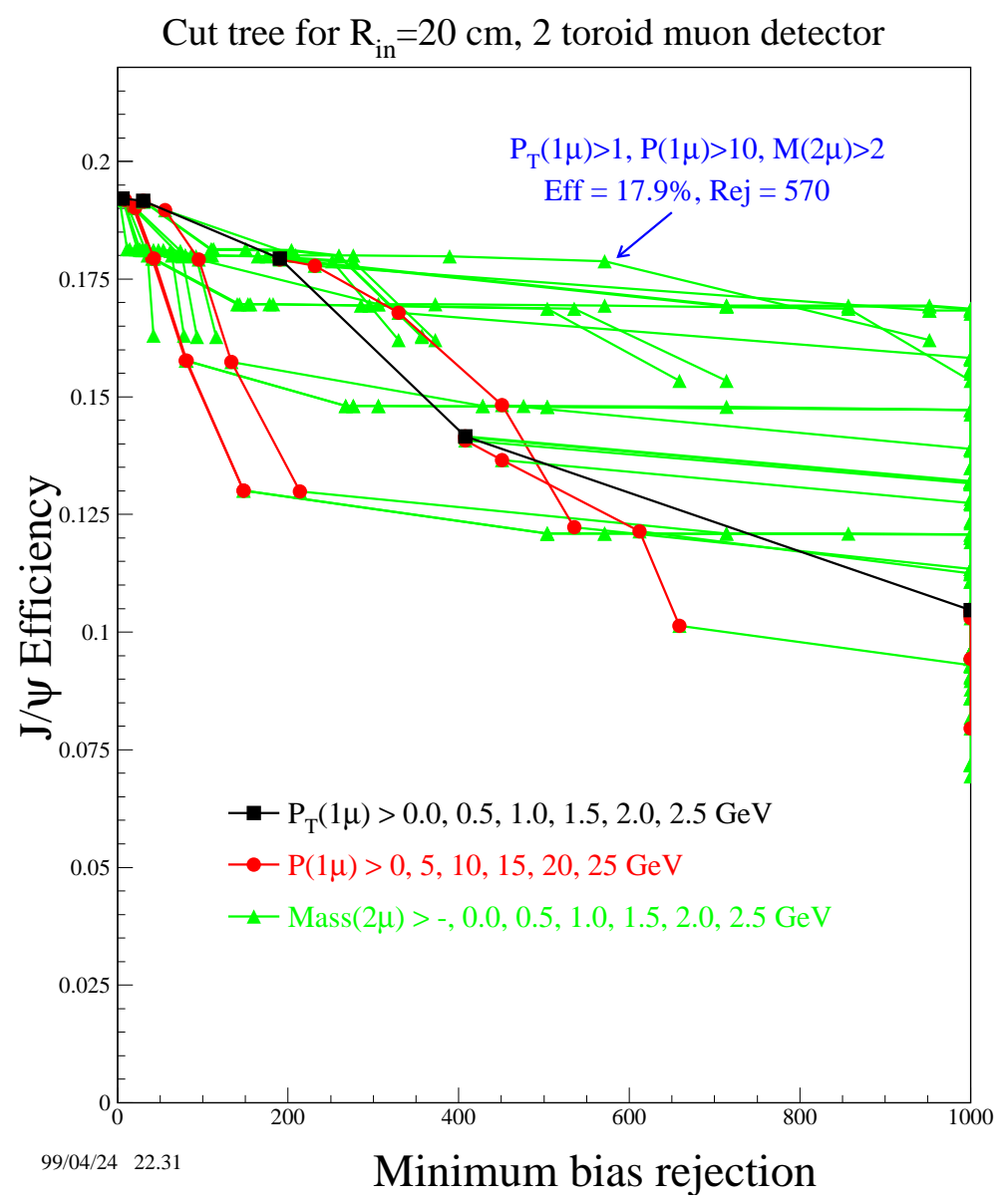

Figure 4.48: Absolute $J / \psi$ efficiency versus minimum bias rejection for cuts on the minimum muon $p_{T}$, the minimum muon momentum and the minimum invariant mass. Black lines connect the squares which show the variation as the minimum muon $p_{T}$ is increased. From each black square a red line connecting the circles shows the change as the minimum muon momentum is increased. From each red circle a green line connecting the triangles shows the variation as the minimum invariant mass requirement is increased. A cut of "-" indicates no cut. Results are for a two-toroid muon detector with inner radius of $20 \mathrm{~cm}$.

on these studies, we find typical rejection ratios of 500-600 with efficiencies of roughly $18 \%$. Note that the geometric acceptance (no momentum or mass cuts) of a $10 \mathrm{~cm}$ inner radius 
system is $22 \%$. Based on these studies, we have found:

1. Most of the rejection power comes from requiring minimum $p$ and $p_{T}$ for two muons in the event. An invariant mass cut is beneficial, but is not required to obtain large rejections with good efficiency. If it turns out that an invariant mass cut is difficult to implement in the first level trigger, we will not be at a severe disadvantage.

2. An inner radius of $15-20 \mathrm{~cm}$ is acceptable. If we insist on a rejection ratio of 500 or more, the efficiency for signal events is $18.8 \%$ for an inner radius of $10 \mathrm{~cm}$ and $17.9 \%$ for an inner radius of $20 \mathrm{~cm}$ (20 cm is worst case).

3. Two toroids are needed. If we require a rejection ratio of 500-600, the efficiency for signal events drops significantly (from $\sim 18 \%$ to $\sim 14 \%$ ) in going from two toroids to one. In fact, it appears that the only hope for a single muon trigger is the two toroid case: acceptable rejections are not possible with only one toroid.

\subsubsection{Summary of Detector Requirements}

To summarize, the BTeV muon system should have the following attributes:

1. Robust: It will be very difficult to access the detectors once they are installed.

2. High Rate Capability: Rates of a few $\mathrm{kHz} / \mathrm{cm}^{2}$ are possible in parts of the system (based on calculations of accelerator backgrounds).

3. Good Time Resolution: Timing information on hits with a resolution of 50-60 ns will allow rejection of hits due to accelerator backgrounds.

4. Magnetic: Two toroids of 1.5 Tesla field and roughly $1 \mathrm{~m}$ thickness (in each arm).

5. Fine-Grained Spatial Resolution: Tracking resolutions of 2-3 mm are required.

6. Longitudinal Compactness: Given the size constraints, the detectors should be of minimal thickness.

7. Minimal Neutron Susceptibility: We will avoid the use of hydrogenic detector elements.

8. Uniform Occupancy: A geometry designed to minimize pattern recognition confusion and minimize occupancy at smaller radii.

The details of the baseline muon system design are discussed in a subsequent chapter. 


\subsection{Evolution of the C0 Program}

We want to congratulate Fermilab on the completion of a new experimental area at $\mathrm{C} 0$ which is capable of supporting a state of the art bottom and charm decay experiment. We have carefully designed BTeV to be a good match to this new enclosure. We propose to develop the experiment in stages. Some of these stages involve R\&D into key technologies required for the experiment. Others involve a program of physics and background measurements. Only by doing tests under actual running conditions does one truly confront all the problems of efficiency, backgrounds, and systematic errors in the design.

The rate at which the full experiment can be mounted depends on many things. One is when the low $\beta$ quadrupoles will be available, since this determines the earliest time when we can have collisions at high luminosity. Another is when the R\&D for each component is completed. A third is, of course, when manpower for design work and money to build detectors and buy the electronics and computing become available. Finally, all the equipment must be installed, integrated, and made to work.

We assume that the running schedule around which we plan our activities is: each year there will be about 9 months of collider running; that there will be a three month shutdown for maintenance during which installation can take place; and that at some point after a few years of running, the 3 month shutdowns will be arranged back-to-back so that there can be a reasonable period for envisioned upgrades.

Our first goal is to get the 'large components' installed in $\mathrm{C} 0$ during the shutdown for Run II installation. At the minimum, this means the SM3 analysis magnet. If we can locate the steel for one or both of the muon detectors, we would like to get that installed too. Once these components are in, all other installation activities are similar to installing components in a fixed target experiment. Individual modules of the detector can be moved into the enclosure and installed in short shutdowns of a few days.

BTeV R\&D is proceeding well. Pixel R\&D is well along and we would like to complete it in 2002. Two different readout chips, bump-bonded to detectors will be tested in the 1999 fixed target run. Trigger R\&D is also proceeding well and will be completed in 2001 . The design of the forward tracking system, muon chambers, particle identification system, and electromagnetic calorimetry can be completed in 2001. Prototype modules of the forward tracker and the muon chambers will be tested in the 1999 fixed target run.

Starting at the beginning of Run II we will put counters into C0 to measure backgrounds near the IR and in the region of the muon detector and RICH; to set up the prototype modules of the muon detector and the forward tracker and operate them in the $\mathrm{C} 0$ enclosure; to install a wire target and study the interaction rate and backgrounds, and establish the effect on the other collision regions.

In 2000, we also plan to install three pixel planes inside the SM3 magnet and several forward tracking planes, along with trigger scintillators. We will use this arrangement to study the initial full size pixel detector chips in a prototype housing inside the beam pipe. Interactions on a wire target will be used. We are also proposing to establish the ability to test electronics near the beam by placing them in existing Roman Pots. 
We plan to have our first $p \bar{p}$ collisions in 2002 using an intermediate $\beta *$ IR constructed from existing quadrupole magnets. This arrangement will not permit us to run simultaneously with both CDF and D0 since separators will need to be turned off to bring the beams into collision at $\mathrm{C} 0$. We envisage intermittent running perhaps using the ends of stores. We will install $10 \%$ of the final pixel system, a serious full scale system test of both the pixels and the trigger. We will also measure the backgrounds in the collider mode. We will interleave wire mode and collider running and test as much of the vertex tracker and vertex trigger as possible.

In 2003 we plan to start installing the rest of the BTeV spectrometers, and have some collider running after each installation period.

We look forward to full runs in collider mode building up to full luminosity in 2004 and 2005.

With this plan, we can steadily build our understanding of the detector over a period of a few years and can be ready to do physics with a tested analysis program in place when the full detector is complete. It also allows the lab to spread its resources, people and money, over several years. Following this plan, Fermilab will have a serious forward collider $B$ experiment well in progress before the LHC turns on. 


\subsection{Completing the Simulation of the Baseline Detector}

The simulation work on all-charged states is quite far along and has dominated the requirements for the baseline detector. The study of states with photons and $\pi^{o}$ 's has just begun. More physics channels will be investigated and background calculations for states with photons will be pursued aggressively even though they require us to run a very large number of events. The current baseline detector has been modeled and extensively studied and we are now confronting very detailed problems which involve more global optimizations - those requiring an understanding of the impact of various choices on the whole ensemble of physics topics. For example, the thickness of the pixel detector is a problem not so much for its effect on charged particle tracking but for its impact on photon reconstruction because so many photons undergo conversion to $e^{+} e^{-}$pairs.

We have also made much progress in improving the detail of the simulation and bringing it closer to reality. Significant design work is occuring on nearly all the detectors. As the design of the detectors begins to firm up, we are feeding information into the simulations to make them more realisitic with respect to cell sizes, resolutions, and material budget. Much of the work in the next year will be in modeling the details of the detector elements and understanding more complex issues and detector dependent issues such as pattern recognition and trigger rates to make sure that the proposed design can actually do the measurements we are interested in.

The trigger simulation includes pattern recognition in tracking the charged particles through the silicon detector. More detailed hit generation and digitization have been implemented so that we can more fully understand the efficiency and rejection of the trigger. We have begun to investigate other trigger algorithms as part of the trigger $R \& D$ effort. We are pursuing schemes with fewer planes to reduce the amount of material in the vertex detector and to reduce its complexity. Further simulations will be required to fully understand the effects of multiple interactions on the tracking and vertexing efficiencies although much work has already been done in this area. Serious work has been carried out on the Level 2 and Level 3 trigger algorithms and the overall trigger and data acquisition architecture.

Detailed simulations of the expected background rates in the $\mathrm{C} 0$ hall have not yet been performed. We will study backgrounds from all sources including those generated in the machine.

There are tools available to study the beam backgrounds [1]. Proton-antiproton collisions are typically simulated with DTUJET93. Beam halo interactions with scrapers and particle tracking through the Tevatron are modeled with STRUCT. Particle loss induced hadronic and electromagnetic showers, and secondary particle transport in accelerator and detector components, including shielding, are simulated using the MARS simulation package. We expect to carry out studies of the beam related backgrounds in cooperation with the Beams Division as the design of the detector and interaction region move ahead. We also are planning to undertake a series of measurements of the environment in the $\mathrm{C} 0$ Collision Hall as soon as collider operation resumes.

The simulation tools are now in place to study all the physics and detector issues that 
need to be addressed to make the final refinement of the $\mathrm{BTeV}$ detector. Over the next year, we expect to investigate a wide variety of physics topics and detector issues connected with bottom and charm physics and improve the background studies and to be in a position to make a final "global optimization" that will result in a final design that will give BTeV the greatest possible physics reach. 


\section{Bibliography}

[1] From the C0 Workshop, Fermilab, Dec 4-6, 1996. See http://fnphyxwww.fnal.gov/conferences/czero/simulations_summary.html 


\section{Chapter 5}

\section{Pixel R\&D}

\subsection{Introduction}

The $\mathrm{BTeV}$ pixel detector is central to the capability of the BTeV experiment. At the same time, it stretches the technical frontier beyond what has so far been achieved in other experiments. In these circumstances, the plan has been to gain experience in the various required technologies and to demonstrate the necessary component capabilities that will be needed for the final device. In following this plan, as much as possible we have collaborated with industry and with groups working on LHC experiments developing similar detectors.

The major components for the pixel detector system are the sensor, readout chip, sensorto-readout-chip connection, mechanical support and cooling, high-density interconnection between the readout chips and the control chips on the detector plane peripheries, and the multiplexing and data transmission to the first-level trigger. Details on the current state of $R \& D$ on these areas from various groups and industry can be found in [1]. For some components, we have demonstrated capability beyond that required for the baseline $\mathrm{BTeV}$ detector (e.g., $30 \mu \mathrm{m}$-pitch bump bonding for sensor-to-readout-chip connection, while the baseline calls for $50 \mu \mathrm{m}$ pitch). For others we have demonstrated capability which meets the tough $\mathrm{BTeV}$ specifications (e.g., front-end amplifier of the FPIXi designs). For yet other components, we can make straightforward extensions to devices already tested which, when modified, will meet the BTeV needs (e.g., readout architecture). Finally, there are areas where additional work will be needed to get to the final Technical Design Report level (e.g., higher-level integration of components and radiation-hard implementation of the ASIC design). One choice that we will have to make in implementing a radiation-hard front-end is between deep sub-micron CMOS processes and more traditional radiation-hard technologies. We are just beginning to gain experience with yields for our readout chip designs, and have started a program to test yields at the component level too (e.g., for bump bonding).

On the road to gaining local experience and demonstrating necessary components, we have an active program of work in the MT test beam. We are ready to test two versions of Fermilab readout ASICs bump bonded to sensors (with pixel size of $50 \mu \mathrm{m}$ by $400 \mu \mathrm{m}$ ). By the 
time of the TDR, we should have gained experience in assembling and operating multichip modules which will be the basic building block of the final BTeV pixel detector. We also have a physical simulator of the electrical noise from the Tevatron beam for testing electronic components and their shielding inside the beam vacuum-pipe. We are also preparing to place devices into the Tevatron in borrowed Roman Pots as a test of the designs which grow out of the simulator experience.

Finally, we have begun to develop industrial contacts and joint efforts with other experiments on the remaining areas. We have ordered test components for high-density interconnects (HDIs), fiber-optic data transmission, support structures, and cooling channel prototypes. Test stands for these are in various stages of construction, and making rapid progress.

\subsection{Sensor development}

\subsubsection{Introduction}

The sensor unit cell size is closely related to the achievable spatial resolution and to the occupancy in the system. The sensor thickness affects the signal-to-noise ratio achievable in the course of the detector lifetime, the overall material budget and the power dissipation of the system, in particular after large irradiation doses. The overall material budget is determined not only by the active elements (sensor and readout electronics), but also by our ability to design a low mass mechanical support and cooling system.

One of the key elements driving our design is the necessity to put these detectors as close to the beam line as possible, in order to optimize the vertex resolution. In our baseline geometry, the inner detector edge is located $6 \mathrm{~mm}$ from the beam line. As a consequence, the inner portion of our detector will be exposed to radiation levels comparable to the ones expected for the LHC experiments. One of the effects produced by high-energy particles on semiconductor devices is displacements. These are dislocations of atoms from their normal sites in the lattice, producing defects and trapping sites. Displacements determine the degradation of the bulk material. In detectors, they cause changes of the internal electric field due to the modified doping concentration. For very-high-dosage irradiation, this will eventually lead to inversion of the conduction type of the bulk material (type-inversion), increases in leakage current and depletion voltage, changes in capacitance and resistivity, and charge collection losses. The key to ensure that the sensor could still provide useful signal after high radiation dosage is to be able to operate the detector at high voltage. In order to do this, the breakdown point must be increased and controlled. A common solution to this problem is to use multiple guard rings [2].

In order to increase the useful operating time of the silicon sensors, running with partial depletion has to be considered. This is more suitable for n-type pixel readout, since the depleted region will grow from the $n^{+}$side of the junction after type inversion. Thus, the sensor technology preferred is the so-called " $\mathrm{n}^{+} / \mathrm{n} / \mathrm{p}^{+}$" adopted by both ATLAS and CMS

pixel systems $[3,4]$, where the collection electrodes are $\mathrm{n}^{+}$pixels and the $\mathrm{p}^{+}$implant biases 
the back of the detector.

Results from PSI for CMS showed that for a fluence of $6 \times 10^{14} \pi / \mathrm{cm}^{2}$, a depletion depth of $150 \mu \mathrm{m}$ is obtained for 300 volts. At high bias voltages, charge trapping in the depleted region is below the $10 \%$ level. [4].

For an $n^{+} / \mathrm{n}$ pixel detector, the charge-collecting pixels are defined by $n^{+}$implants. Due to the oxide-charge-induced electron accumulation layer between the silicon and silicon oxide, neighboring $n^{+}$implants will be shorted together unless they are isolated from one another. There are two techniques to implement the isolation: p-stop and p-spray. In the p-stop isolation, a high dose p-implant surrounds the $n^{+}$implant. In the p-spray isolation, a medium dose p-implant covers the whole $n$-side and the collecting $n^{+}$pixels are done by overcompensation with the high dose of $n^{+}$implant [5]. The ideal design for the p-stop (single, common or multiple, width and spacing of the p-implants) needs to be determined. It will have a significant impact on the minimum pixel pitch, the noise, charge collection, and capacitance of the sensor. Recent studies by both ATLAS and CMS also showed that in order to keep the field low for non-connected pixels (failed bump bonding) so as to avoid stochastic discharges, the p-stop has to be designed carefully to create a high resistivity accumulation path between neighboring pixels. The advantages of the p-spray technique is that one or two mask steps and the risk of overlapping implants will be eliminated. A finer pitch will also be possible. A further advantage of the p-sray is that the electric field in the region between the p-spray and the $n^{+}$implant decreases with increasing irradiation, minimizing the risk of micro-discharge.

The use of $p^{+} / \mathrm{n}$ detectors in a high radiation environment also needs to be studied. One interesting option in this case is to go to thinner detectors (say 200 to $250 \mu \mathrm{m}$ ) and lower resistivity silicon(say around $1 \mathrm{~K}$ ohms-cm). Simulations show that such detectors can be fully depleted at a voltage no higher than $300 \mathrm{~V}$ up to a fluence of a few times $10^{14}$ particles $/ \mathrm{cm}^{2}$.

There is a new development on radiation hardened silicon reported by the CERN RD48 (Rose) collaboration. Radiation hardness of silicon detectors can be significantly improved by using oxygen-diffused silicon. Various silicon detector manufacturers, working in collaboration with RD48, have started on a program of optimization of the oxygen diffusion process. We plan to get some pixel detectors made from oxygenated silicon wafers.

\subsubsection{Sensor simulation}

A detailed understanding of the factors affecting the sensor performance is crucial to its design. We have studied a number of issues through simulation.

In a $n^{+} / \mathrm{n}$ detector the charge carriers collected at the pixel electrode are electrons. We have studied these $n^{+} / \mathrm{n}$ sensors , as well as the conventional choice of $\mathrm{p}^{+} / \mathrm{n}$ pixel devices, which have holes as the collected charge. In addition, we have considered radiation damage effects. These include the deterioration of the noise performance due to the increased leakage current and the change in detector response induced by the change in the effective donor concentration. 
Other factors that affect the ultimate resolution achievable in this system are related more closely to the design approach and the performance of the readout electronics. In particular, the electronic noise, and the threshold that determines the minimal charge deposition that will be recorded as a signal hit, are important. The sensitivity to these parameters has been studied, as well as the tradeoff between analog and digital readout.

In order to understand these effects, we have developed a stand-alone simulation, based on a two-dimensional model of the signal formation in silicon. This program has been interfaced with the Monte Carlo software used to study our physics reach. This integration allows us to have a more realistic model of the detector occupancy, crucial in trigger simulations, and also provides a more realistic implementation of the hit resolution achievable for different track angles of incidence.

While this simulation program has lead us to a much better understanding of the interplay between hit resolution, occupancy and physics reach of our experiment, an additional effort is under way to develop a more accurate model of the static and dynamic properties of the pixel sensors, taking into account the true three-dimensional nature of the electric field distribution and signal charge-flow to the collection electrode. These studies will be crucial to determine our final sensor choice.

\subsubsection{Spatial resolution studies}

We have developed a program to evaluate the spatial resolution achievable with different sensor and readout electronics specifications[6]. Our approach is similar to several other studies performed for silicon microstrip detectors [7], [8]. We have investigated the interdependence of charge diffusion, magnetic field effects, electronic noise, discriminator threshold and number of bits in the back-end electronics.

The ability to achieve a spatial resolution better than the nominal 'digital' resolution $(\sigma=$ width $/ \sqrt{12}$ ) is closely related to how accurately we can interpolate between the location of the centers of individual pixels, using the information of the reconstructed charge collected at each sensor in a cluster.

We have modeled the charge produced by the minimum ionizing track traversing silicon using a Landau probability distribution. Electrons and holes produced by the energy deposited by the track drift along the electric field lines $(\vec{E})$ in the detector. The equations describing this drift motion are:

$$
\begin{aligned}
\vec{J}_{n} & =q \rho_{n} \mu_{n} \vec{E} \\
\overrightarrow{J_{h}} & =q \rho_{h} \mu_{h} \vec{E}
\end{aligned}
$$

where $q$ is the electron charge, $\mu_{n}$ and $\mu_{h}$ are the mobility of electrons and holes respectively, $\rho_{n}$ is the number of free electrons per unit volume, and $\rho_{h}$ is number of free holes per unit volume.

In parallel, the charge cloud spreads laterally due to diffusion. The parameters characterizing the drift in the electric field $\left(\mu_{h}, \mu_{e}\right)$ are related to the parameter describing the 
diffusion of the charge cloud $\left(D_{h}, D_{e}\right)$ by the Einstein equation:

$$
D_{h(e)}=\frac{k T}{q} \mu_{h(e)}
$$

where $D_{h}$ and $D_{e}$ are the diffusion coefficients, $q$ is the electron charge and $k T$ is the product of the Boltzmann constant and the absolute temperature of silicon. The average square deviation with respect of the trajectory of the collected charge without diffusion is $\left.<\Delta r^{2}\right\rangle=$ $2 D \Delta t$. In our study we have used $\mu_{h}=400 \mathrm{~cm}^{2} / V s, \mu_{e}=1450 \mathrm{~cm}^{2} / V s$.

When the silicon detector is located inside a magnetic field, there is an additional source of charge spreading: the lateral motion induced on electrons and holes by the magnetic field. This effect shifts the centroid of the detection point of the charged cloud and also widens it, because the amount of drift is proportional to the path length of the detected electrons (holes). This so called " $E \times B$ effect" depends on a parameter called the Hall mobility $\mu_{H}$, proportional to the charge carrier mobility discussed above. In order to take into account the effect of the magnetic field in the bend plane, the following values of the Hall mobility have been taken from experimental data quoted in [9]:

$$
\begin{gathered}
\mu_{H, h}=0.72 \mu_{h}, \\
\mu_{H, e}=1.15 \mu_{e} .
\end{gathered}
$$

The charge-cloud image appears on more pixels when the incident track crosses the detector at an angle, as the generation point of the electron-hole pair spreads out along the track path length. These various effects are illustrated in Fig. 5.1, which shows the charge broadening due to diffusion, the magnetic field and the combination of the these two effects together with track inclination with respect to the normal to the detector.

An analog readout improves the spatial accuracy by charge weighting. A low intrinsic noise is obviously a key element in this approach, together with a low and uniform threshold. These features are important, not only to maintain the advantages of an analog readout, but also to minimize problems in data flow related to excessive data transfer. Finally, the achievable digitization resolution and the knowledge of the gain of the analog front-end are additional issues that should not be overlooked. We will address the interdependence among these parameters.

Two assumptions are common to all the configurations discussed in this study. We have considered a $300 \mu \mathrm{m}$ thick Si detector biased in over-depleted mode (200 V for a detector that is fully depleted at $120 \mathrm{~V}$ ). In this first stage we have assumed full charge collection and we have assumed negligible capacitive cross talk between adjacent pixels. These assumptions will be relaxed in subsequent studies.

\subsubsection{Factors affecting the spatial resolution}

We have carefully examined the different factors affecting the hit resolution in the small pixel direction, both in the bend plane and in the direction orthogonal to it, with reference to a pixel geometry of $50 \mu \mathrm{m} \times 300 \mu \mathrm{m}$, using several track orientations. 


\section{Charge Distribution}

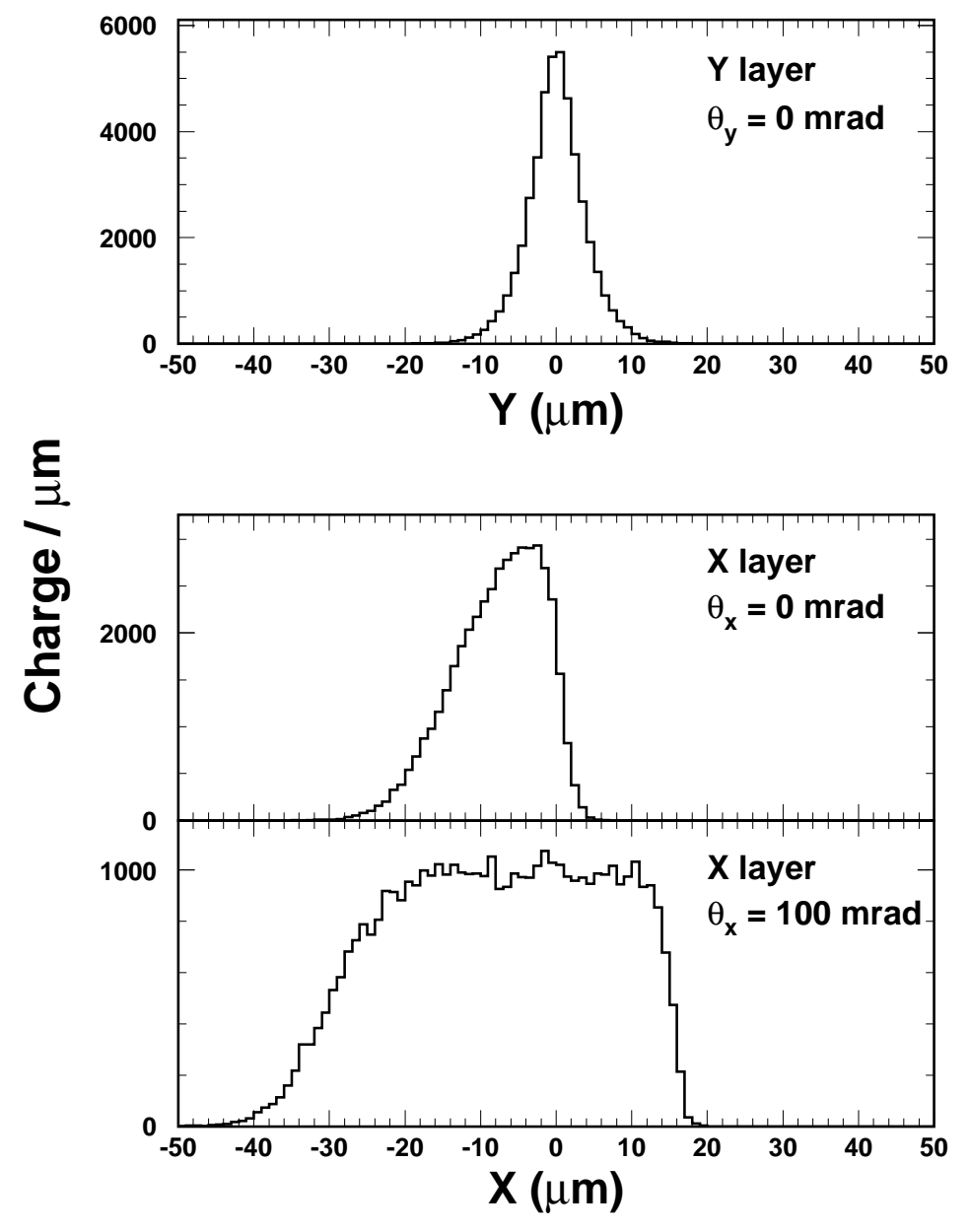

Figure 5.1: Collected charge spreading in a $300 \mu \mathrm{m}$ silicon detector a) produced by diffusion, b) produced by the interplay of diffusion and the magnetic field for $\mathrm{B}=1.6 \mathrm{~T}$, and c) produced by diffusion and magnetic field effects when the charged track is incident at an angle of 100 $\mathrm{mr}$ in the bend plane. 
Fig. 5.2 shows the relationship between the position of incidence at the pixel plane and the reconstructed coordinate for tracks at normal incidence on the pixel detector, whereas Fig. 5.3 assumes a track inclined at an angle $\theta_{x}=300 \mathrm{mr}$. We have started our study assuming a readout scheme with the intrinsic electronic noise switched off, and we consider both a binary and an analog readout (with 6-bit ADC resolution). We can see that if the electronics noise is negligible, a digital readout actually can achieve a better resolution than the naive expectation of the width of the cell divided by $\sqrt{12}$. This is due to the fact that when the charge is shared between two or more pixels it is possible to do a digital charge weighting algorithm that provides some interpolation between the position of individual pixels. This improvement is constant, on the average, for different angles.

Let us start our discussion on analog readout with tracks at normal incidence. Fig. 5.2 shows that, in presence of the magnetic field, the charge is actually 'focused' inside the pixel instead of spread out by virtue of the magnetic field. This makes the analog and digital readout equivalent, because there is not a second pixel helping to interpolate the track position using charge sharing. On the other hand, tracks incident on the pixel region where the magnetic field spreads the charge further with respect to diffusion, are helped significantly by analog readout. In order to take full advantage of the analog information, it is necessary to adopt an optimal choice of the charge weighting algorithm that reflects the expected distribution of the charge profile. In particular, the center-of-gravity linear weighting algorithm is suitable only for relatively large angle tracks, where enough charge sharing is produced by the track inclination. For tracks at normal incidence, it is necessary to perform a non-linear weighting [8].

An additional important constraint in the achievable resolution is the discriminator threshold. In this study we have assumed that only the pixels having a signal above threshold are read out, and we have varied the threshold for analog and binary readout. Fig. 5.4.a shows the effect of increasing the threshold for an incident angle $\Theta_{x}=300 \mathrm{mr}$, for analog and digital readout respectively. Fig. 5.4.b shows the corresponding pixel occupancy. Each curve shows the fraction of events having $N$ pixels hit for a given threshold. For instance, for a threshold of 1000 electrons, less than $5 \%$ of the events have 4 or more pixels hit, $45 \%$ have 3 pixels hit, and about $50 \%$ have two pixels hit. The resolution achievable for binary readout shows a characteristic oscillatory behavior as we change the threshold. The worst resolution is always at the thresholds for which most of the events have clusters with the same size, corresponding to less interpolation power. In the analog readout case, the higher the threshold is, the coarser the charge sharing process is. In several of the results presented below, we have assumed a threshold of 1000 electrons, a somewhat optimistic assumption, supported by promising initial test results on the FPIX0 pixel readout chip [10]. The slope of the threshold dependence of the resolution is not very high below 3000 electrons, as illustrated by Fig. 5.4, so the results are largely unaffected if we use less restrictive conditions.

Each readout circuit features some intrinsic noise, most commonly the series noise associated with the input transistor of the charge-sensitive preamplifier integrating the charge collected from the pixel sensor. In addition, at very high radiation damage, a contribution from the parallel noise produced by the excess leakage current may become non-negligible 


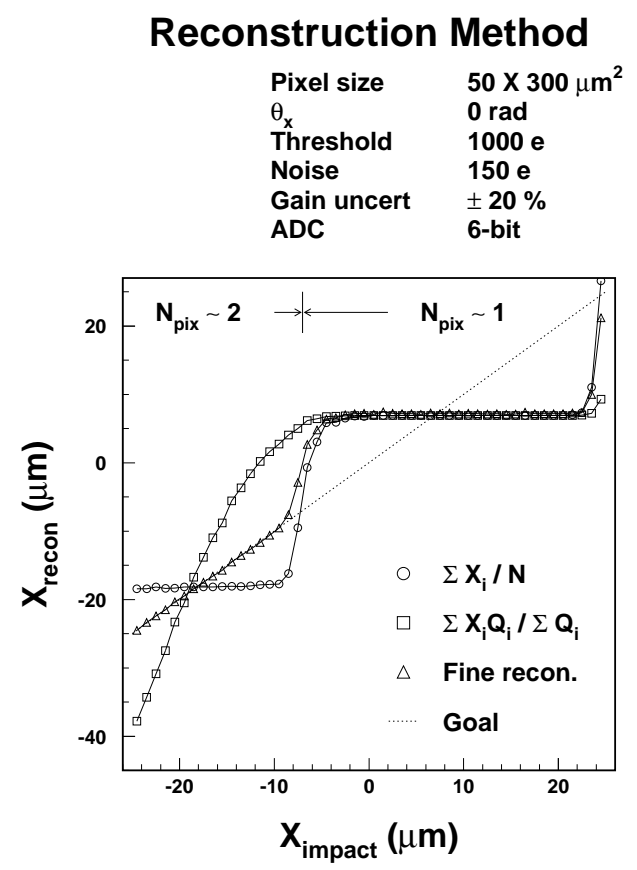

Figure 5.2: The reconstructed $\mathrm{x}$ coordinate as a function of the charged track impact point on the pixel cell for a pixel size of $50 \mathrm{x} 300 \mu \mathrm{m}^{2}$ and normal incidence. 


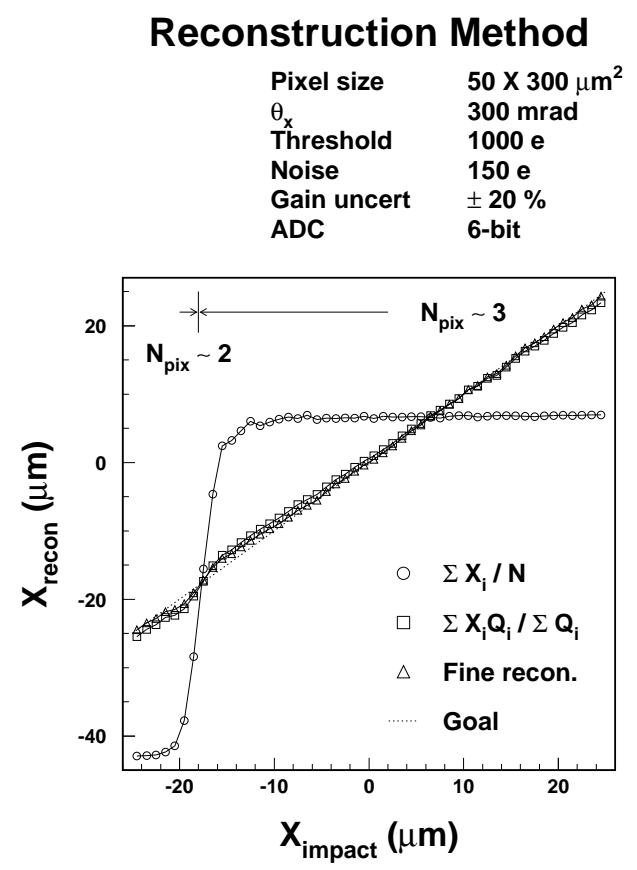

Figure 5.3: The reconstructed $\mathrm{x}$ coordinate as a function of the charged track impact point on the pixel cell for a pixel size of 50x $300 \mu \mathrm{m}^{2}$ and incidence angle in the bend plane $\theta_{X}=300$ $\mathrm{mr}$. 


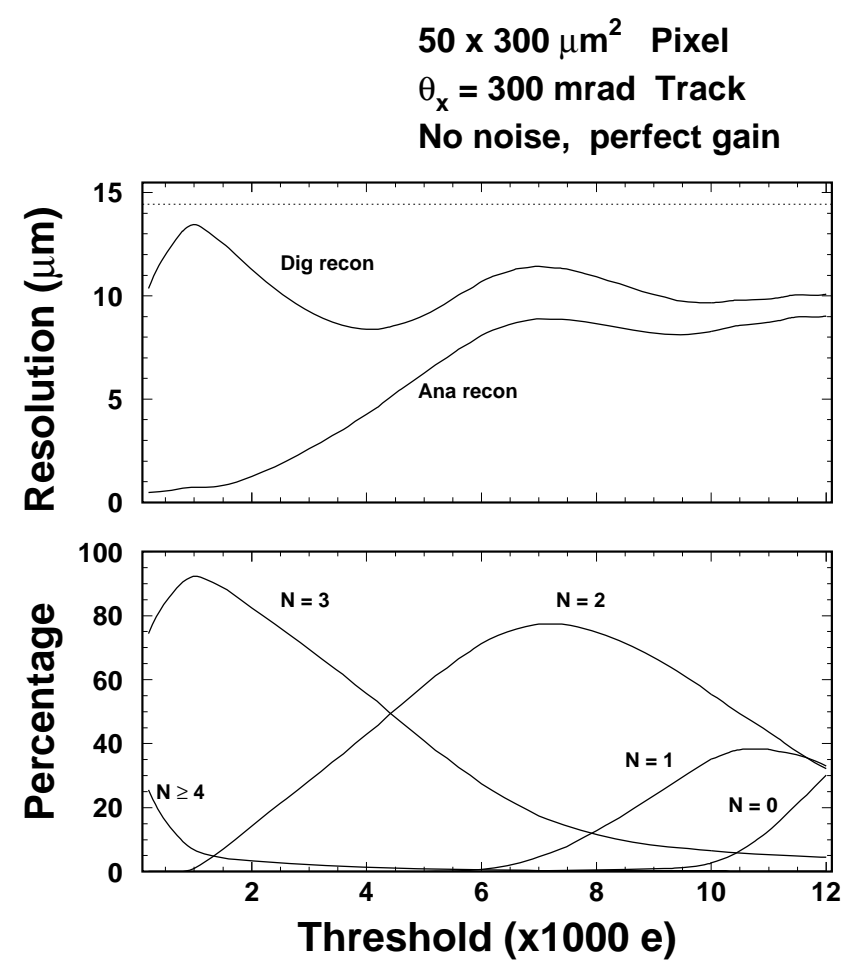

Figure 5.4: a) Spatial resolution in the reconstructed $\mathrm{x}$ coordinate as a function of the threshold for a pixel size of $50 \times 300 \mu \mathrm{m}^{2}$ and incidence angle in the bend plane $\theta_{X}=300$ mr. b) percentage of events having $\mathrm{N}$ pixels hit as a function of the threshold for the same configuration. 
or even the dominant effect. A pixel detector has the potential for being a very low noise system, as the capacitive load at the input of the charge sensitive preamplifier is quite negligible. The recent results achieved at Fermilab in the prototype development of the front-end electronics for our pixel system are quite promising, and lead us to assume that an equivalent noise charge of about 150 electrons is an achievable goal. However to take full advantage of the low noise, the minimum threshold spread among channels needs to be comparable, otherwise either our assumption on the threshold needs to be modified or the effective noise is higher than assumed.

Fig. 5.5 shows the number of pixels hit as a function of the track angle for electron and hole collection. Fig. 5.6 shows the resolution as a function of the angle in the bending plane $\left(\theta_{x}\right)$, and in the non-bend plane $\left(\theta_{y}\right)$ expected with digital readout and with a 4 bit ADC respectively. At angles above $\pm 50 \mathrm{mr}$ the resolution achieved with analog readout is significantly better.

One of the questions that we have addressed in this study is the interplay between maximum dynamic range and ADC resolution. The known shape of the Landau distribution, with a long tail above the most probable energy loss for a minimum ionizing particle in $300 \mu \mathrm{m}$ silicon (24,000 electrons), suggests a dynamic range at least of 48,000 electrons. However, the goal of measuring the charge collected in the pixel with the maximum pulse height more accurately obviously conflicts with the accuracy of the charge measurement in the neighboring pixels at any given bit resolution. It is interesting to see the interplay of these two factors. We have studied this issue at two different angles in the non-bend plane: $\theta_{x}=100 \mathrm{mrad}$ and $\theta_{x}=200 \mathrm{mrad}$. Fig. 5.7 shows the study at $\theta=100 \mathrm{mrad}$ and Fig. 5.8 the corresponding results at $\theta=200 \mathrm{mrad}$. In both cases the solid line defines binary readout, quite insensitive to the dynamic range of the front-end electronics. However it can be seen that for the quite limited resolution provided by a 2 bit ADC, there is actually an optimum in the dynamic range at about 25,000 electrons. For higher resolution, this optimum appears to have a threshold, above which there is not much improvement in resolution achieved by increasing the dynamic range. At a larger angle $(200 \mathrm{mrad})$, where the ratio between the maximum pixel charge signal and the signal in its neighbors becomes progressively lower, the results are consistent, but are even more favorable to the lower dynamic range.

Another effect that we have studied is the sensitivity of the hit resolution to the digitization accuracy of the ADC chosen. Fig. 5.9 shows a comparison of the performance of different ADC's for a threshold choice of 2000 electrons. There is a definite difference between the region within $100 \mathrm{mr}$ of normal incidence and the rest of the solid angle. The angle $\theta_{x}$ is the track angle in the non-bend plane and the angle $\theta_{y}$ is the angle in the bend plane. In the former region the resolution improves smoothly from 10 to about $5 \mu \mathrm{m}$ as the angle increases. The higher number of bits is helpful at larger angles, where a resolution of about $3 \mu \mathrm{m}$ is achievable with a 4 bit ADC. Finally, a non linear response flattens the resolution achievable in the region of larger angles. Fig. 5.10 shows the effects of adding 450 electron rms threshold spread between different channels and of raising the threshold to 3000 electrons. We can see that it is quite valuable to be able to maintain a low threshold and try to achieve a 4 bit resolution in the ADC. 


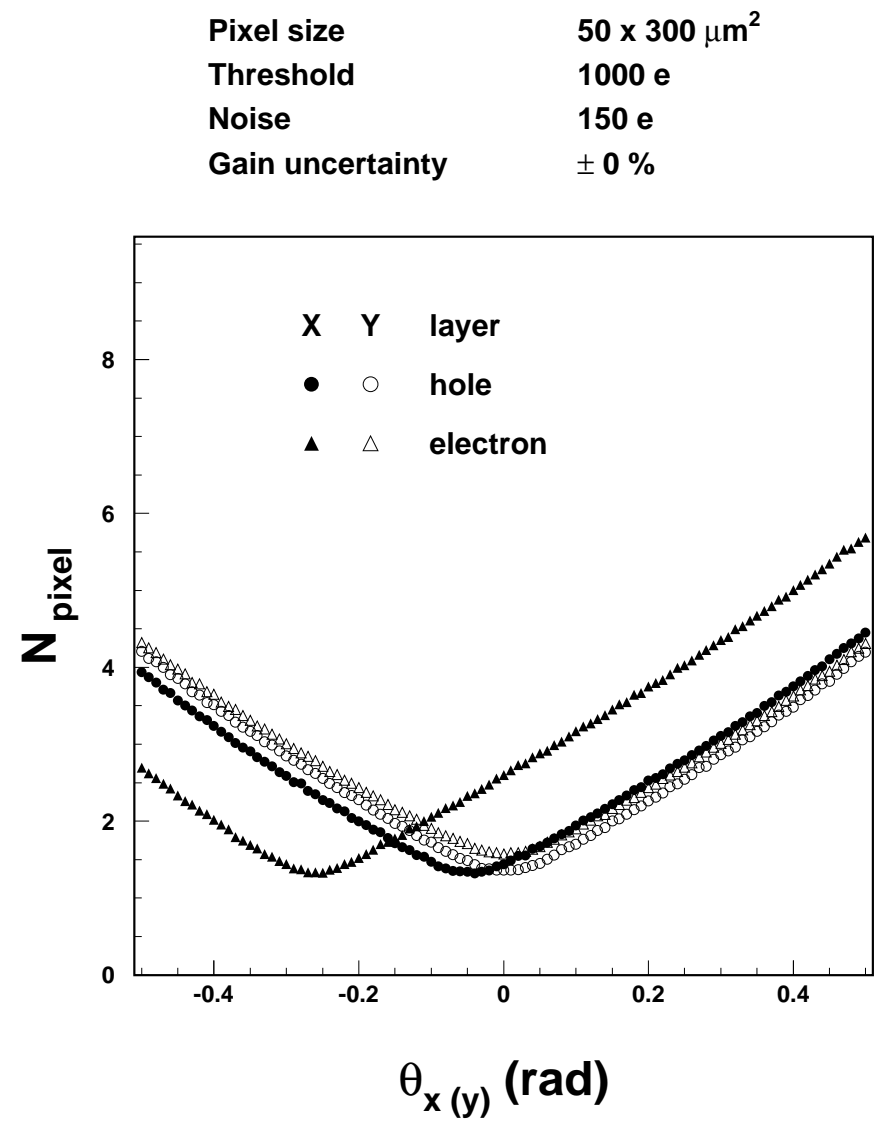

Figure 5.5: Number of pixels hit for a $50 \mu \mathrm{m}$ pixel small dimension as a function of the track orientation. 


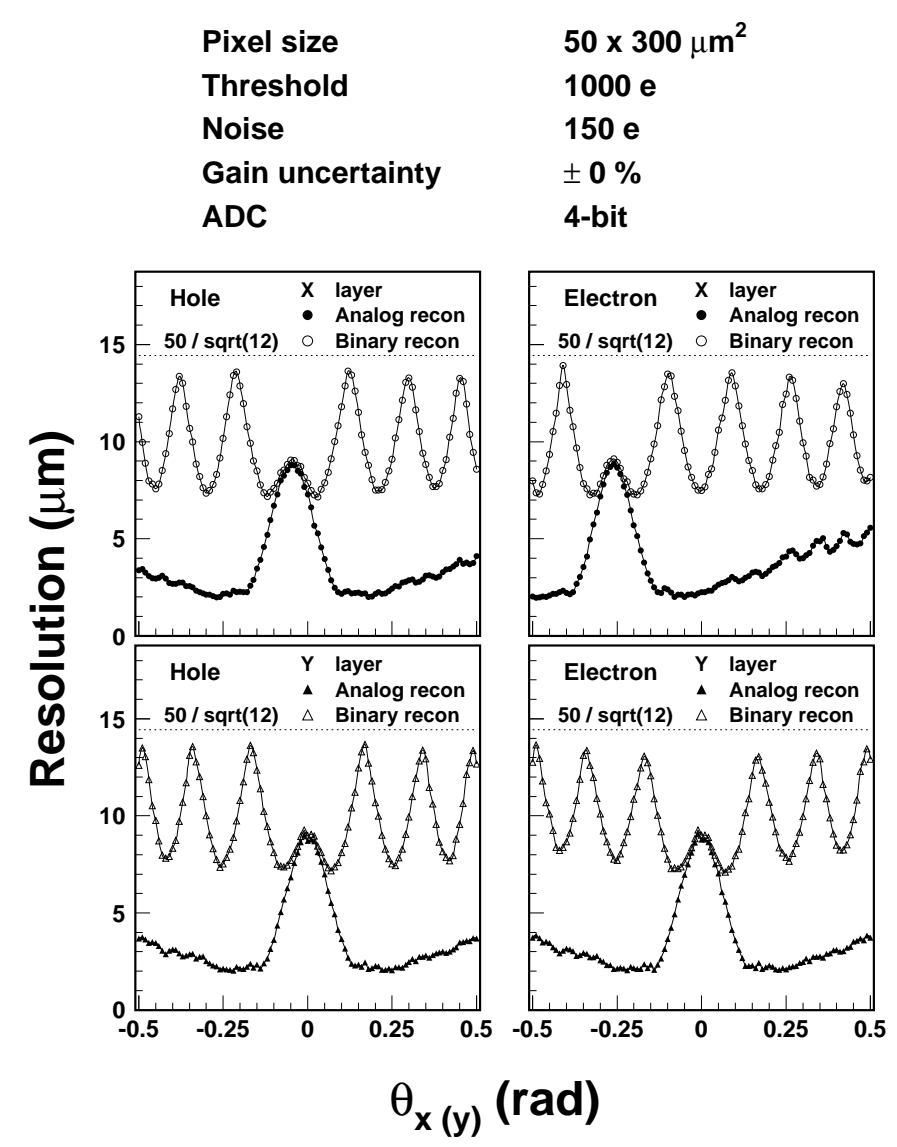

Figure 5.6: Resolution for binary readout and 4-bit ADC readout for $50 \mu \mathrm{m}$ pixel small dimension. 


\section{Effect of ADC Dynamic region Non-bending direction \\ $\theta=100 \mathrm{mrad}$}

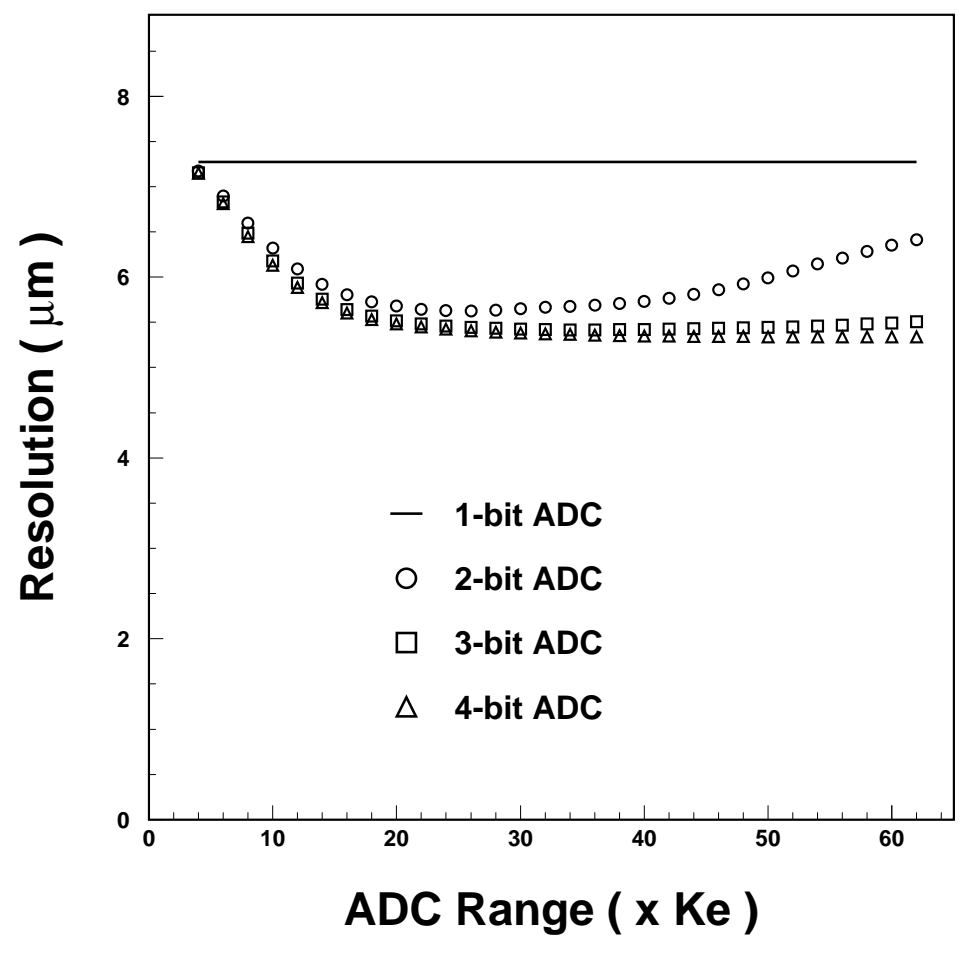

Figure 5.7: Effect of dynamic range of the ADC for different number of bits, for tracks incident at $100 \mathrm{mrad}$. 


\section{Effect of ADC Dynamic region Non-bending direction $\theta=200 \mathrm{mrad}$}

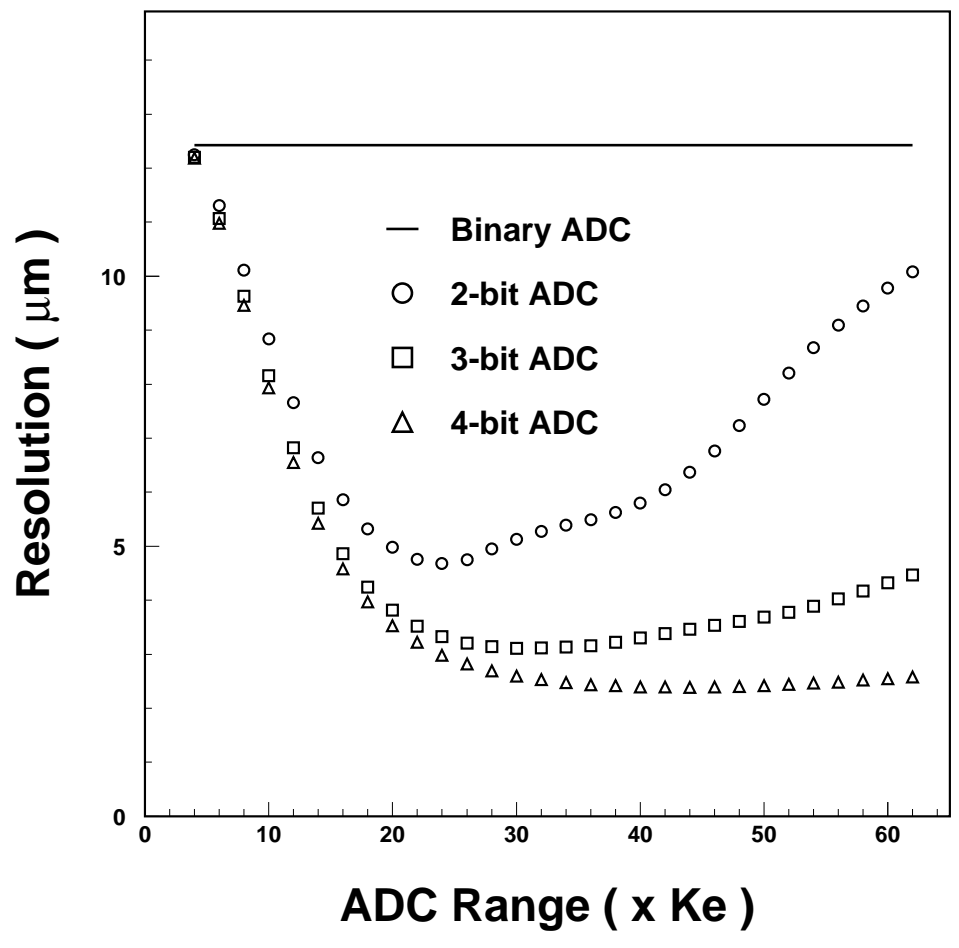

Figure 5.8: Effect of dynamic range of the ADC for different number of bits, for tracks incident at $200 \mathrm{mrad}$. 


\section{Different type of ADC}

$\begin{array}{lrlr}\text { threshold } & 2,000 \mathrm{e} & \text { noise } & 150 \mathrm{e} \\ \text { threshold rms } & 0 & \text { gain uncert } & 0 \\ \text { adc range } & 24,000 \mathrm{e} & \text { cross talk } & 0\end{array}$

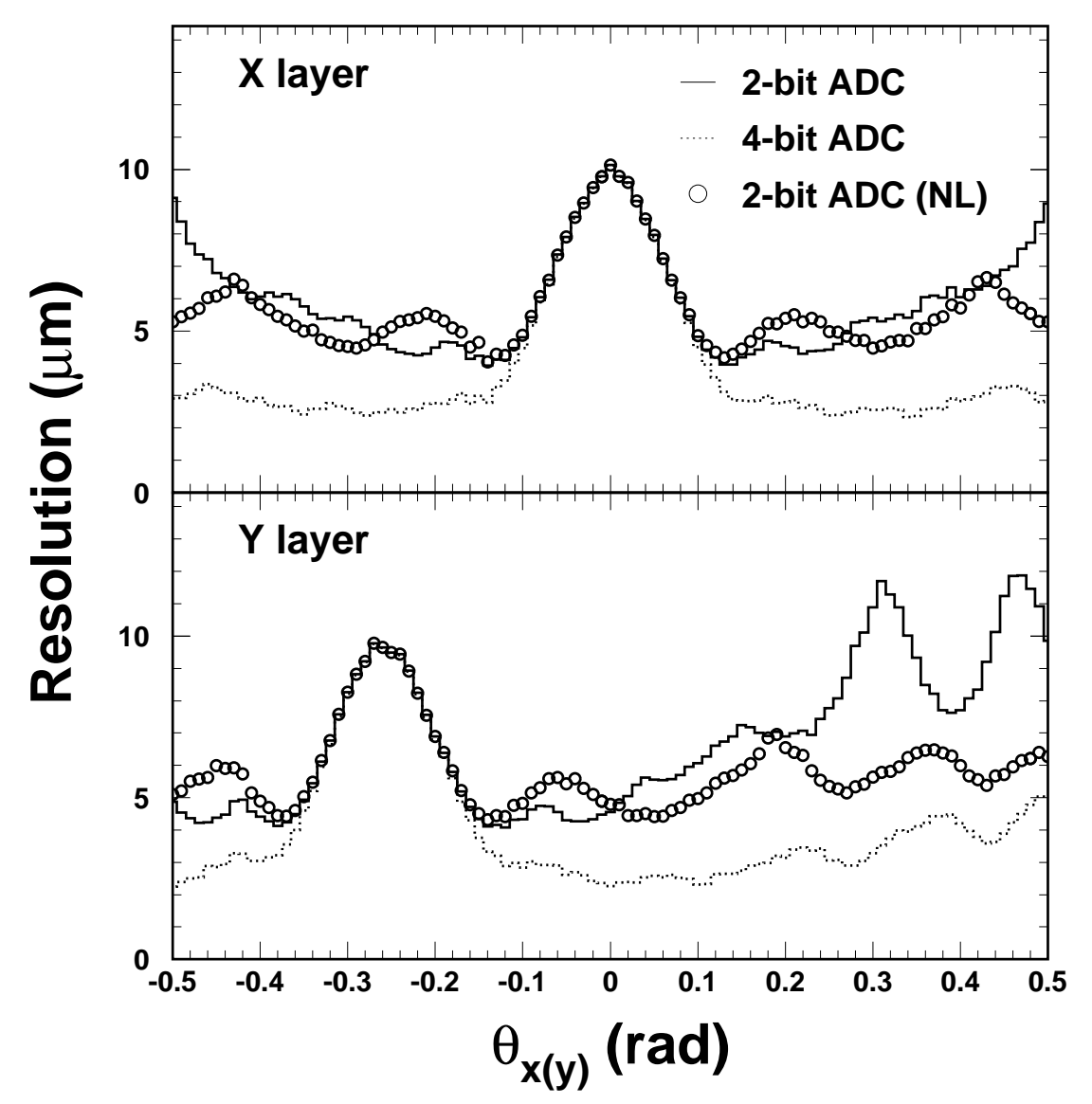

Figure 5.9: Comparison between different ADC resolution, with a nominal threshold of 2000 electrons and no threshold spread. 


\section{Different ADC threshold}

$\begin{array}{lrlr}\text { ADC type } & \text { linear } & \text { noise } & 150 \mathrm{e} \\ \text { threshold rms } & 450 \mathrm{e} & \text { gain uncert } & 0 \\ \text { adc range } & 24,000 \mathrm{e} & \text { cross talk } & 0\end{array}$

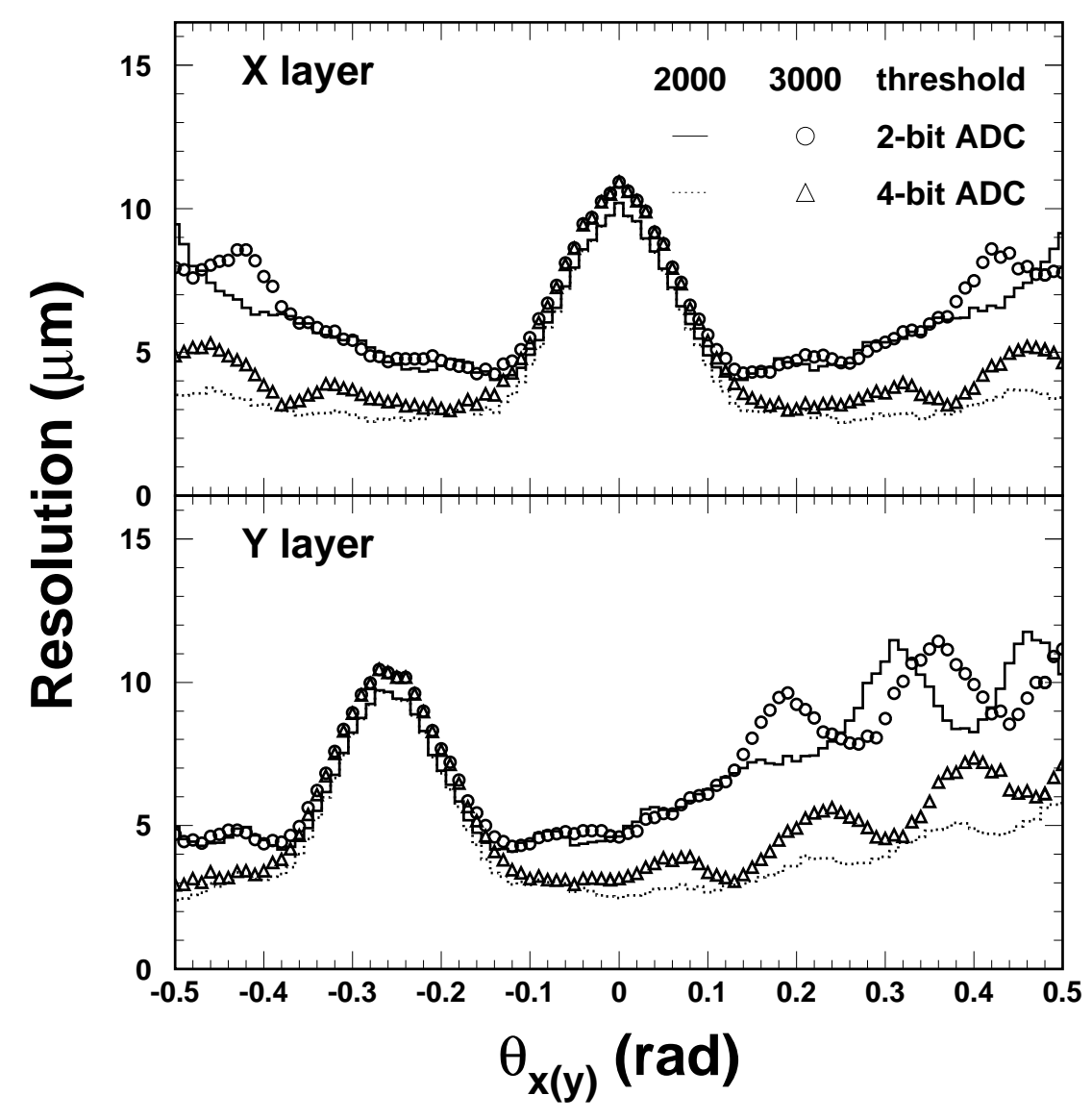

Figure 5.10: Comparison between different ADC resolution, with nominal thresholds of 2000 and 3000 electrons and a threshold spread of 450 electrons. 
Table 5.1: Pixel occupancy for electron collection for different cell geometries and event type. One interaction per crossing is assumed.

\begin{tabular}{|l|c|c|c|c|}
\hline & \multicolumn{2}{|c|}{$(30 \mu \mathrm{m} \times 500 \mu \mathrm{m})$} & \multicolumn{2}{c|}{$(50 \mu \mathrm{m} \times 300 \mu \mathrm{m})$} \\
\cline { 2 - 5 } & Light Quark Evt & B evt & Light Quark Evt & B Evt \\
\hline npix/chip & 0.87 & 1.76 & 0.65 & 1.30 \\
ntrk/chip & 0.26 & 0.51 & 0.25 & 0.52 \\
ncol/chip & 0.25 & 0.50 & 0.26 & 0.53 \\
npix/trk & 3.5 & 3.55 & 2.65 & 2.59 \\
ncol/trk & 1.03 & 1.03 & 1.06 & 1.06 \\
\hline
\end{tabular}

Table 5.2: Pixel occupancy for hole collection for different cell geometries and event type. One interaction per crossing is assumed.

\begin{tabular}{|l|c|c|c|c|}
\hline & \multicolumn{2}{|c|}{$(30 \mu \mathrm{m} \times 500 \mu \mathrm{m})$} & \multicolumn{2}{c|}{$(50 \mu \mathrm{m} \times 300 \mu \mathrm{m})$} \\
\cline { 2 - 5 } & Light Quark Evt & B evt & Light Quark Evt & B Evt \\
\hline npix/chip & 0.52 & 1.11 & 0.40 & 0.90 \\
ntrk/chip & 0.25 & 0.51 & 0.25 & 0.52 \\
ncol/chip & 0.24 & 0.50 & 0.25 & 0.53 \\
npix/trk & 2.16 & 2.24 & 1.69 & 1.79 \\
ncol/trk & 1.03 & 1.04 & 1.05 & 1.06 \\
\hline
\end{tabular}

\subsubsection{Study of the correlation between hit resolution and $B$ decay time reso- lution for different detector configurations}

The studies discussed above have allowed us to map the achievable hit resolution for any given geometry as a function of the track incidence angle. They have also provided us with more accurate information on the hit multiplicity associated with a given track angle. We have used this more realistic information to achieve a better understanding of several key features of our detector performance.

We have investigated[11] the occupancy in the inner portion of our detector for different event classes and different assumptions on the interaction rate. This study has been a crucial input for the engineering group responsible for the design of the pixel readout chip and to test the readout architecture and identify possible bottlenecks in the data throughput. The pixel occupancy has been studied for a chip located with its inner edge $6 \mathrm{~mm}$ from the beam pipe. The chip has been modeled as an $8 \times 8 \mathrm{~mm}^{2}$ square. Two different cell geometries have been studied, $30 \times 500 \mu \mathrm{m}^{2}$ and $50 \times 300 \mu \mathrm{m}^{2}$ : they correspond to two different choices of the pixel small dimension with the same unit cell area. The results of this study are summarized in Tables 5.1 and 5.2 for electron and hole collection respectively.

In light quark events, it can be seen that the average number of tracks per chip in this "hot" region is only 0.25 : thus $75 \%$ of the events do not produce any tracks crossing any given chip located near the beam. This study also provides data samples for the readout architecture simulation and the trigger processor generator. Initial studies have shown that, 
assuming a pixel size of $50 \mu \mathrm{m} \times 400 \mu \mathrm{m}$ and 2 or 3 bit flash ADC's digitizing the pixel cell signal, the proposed column-based data-driven architecture should be capable of achieving the hit readout rate required by the $\mathrm{BTeV}$ experiment [12].

The crucial parameter to determine the physics reach of $\mathrm{BTeV}$ is the vertex resolution, or equivalently, the resolution in the proper decay time. The time resolution is affected not only by the hit resolution in the pixel sensor and extrapolation distances, but also by the material in the pixel system, including the sensor, the readout electronics and the material used for mechanical support and cooling. The study of the effect of increasing the material budget in the detector system is currently under way [13]. The importance of keeping the material budget to a minimum is illustrated by a comparison of the time resolution achieved for the decay $B_{s} \rightarrow D_{s} K$ under two assumptions shown in Fig. 5.11. The top plot shows the resolution achieved with a pixel small dimension of $50 \mu \mathrm{m}$ and pixel planes organized in stations containing 3 detectors whose material budget was described by three layers of thickness $0.6,0.5$ and $0.6 \mathrm{~mm}$ of silicon equivalent respectively. The bottom plot corresponds to the same geometry but with an increased equivalent Si thickness (1.1, 1.0, $1.1 \mathrm{~mm})$. The time resolution degrades from 42 to 50 fs. This study illustrates the importance of keeping the material budget to a minimum.

The simulation included in the original EOI was based on an assumed hit resolution that was uniform throughout the sensor. However we have shown that the resolution improves substantially for angled tracks due to the increased charge sharing that allows a better interpolation. This more realistic mapping of the effective hit resolution has been introduced in our simulation and has shown that this improvement is quite important and compensates the adverse effects of having taken a more conservative model of the material included in our detector.

\subsubsection{Non-Gaussian Resolution Functions}

All previous discussions of the physics reach of BTeV, contained the approximation that the measurements produced by the pixel detectors had a Gaussian shape. With the simulation tools now available it is possible to remove this approximation and to demonstrate that a realistic shape for the resolution function does not significantly degrade the physics reach.

The information in the preceding sections was used to implement several different models of the pixel resolution in the MCFast. These models are discussed in detail in section 10.3. The third of these models, the fast clustering model, is the one of interest; it involves the generation of digitized pulse heights and the extraction of a measured coordinate from the pulse height distribution. The shapes of the resolution functions for this model can be seen in Fig. 10.2 parts c) and d). Both of these have significant non-Gaussian components. For reference, two other models will be considered, both of which have Gaussian resolution functions. In the first model the Gaussian has a constant $\sigma=\operatorname{pitch} / \sqrt{12}$, while in the second model $\sigma$ varies with angle of incidence. The resolution functions for the variable $\sigma$ model can be see in Fig. 10.1; over the entire parameter space the resolution for the short dimension is

better than pitch divided by $\sqrt{12}$. Also, careful inspection will show that resolution function 


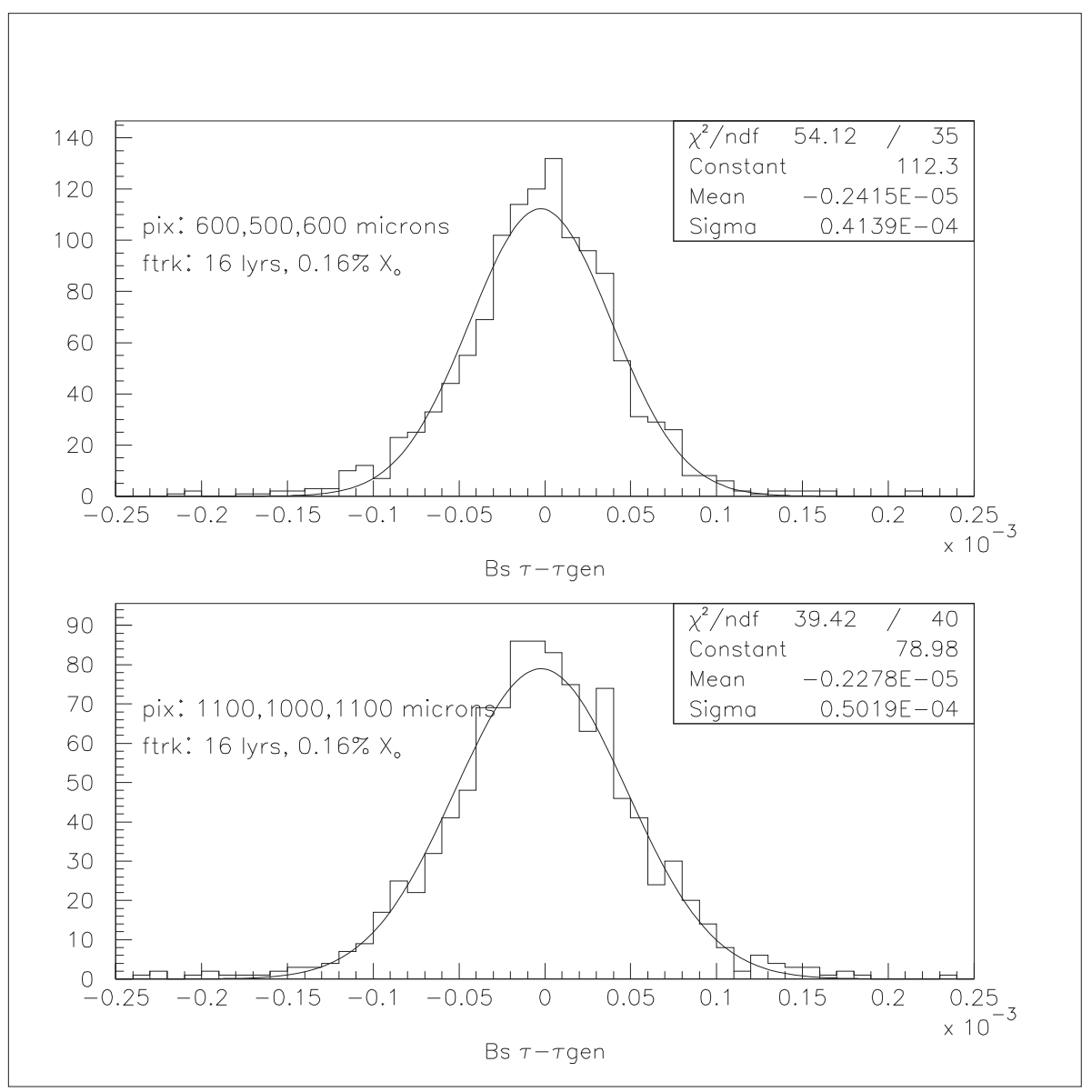

Figure 5.11: Comparison between the time resolution for two different assumptions of the material budget in a triplet station. The decay mode considered is $B_{s} \rightarrow D_{s} K$. 
for the fast cluster model is slightly poorer than the resolution function for the variable $\sigma$ model; the reasons are discussed in section 10.3.

In order to investigate the physics consequences of these different spatial resolution functions, the decay chain $B_{s} \rightarrow \psi \bar{K}^{* 0}, \psi \rightarrow \mu^{+} \mu^{-}, \bar{K}^{* 0} \rightarrow K^{-} \pi^{+}$was considered. After all analysis cuts and background suppression cuts, the resolution on the proper decay time of each $B_{s}$ candidate was computed. This quantity, in this decay mode, makes one of the most severe demands on the spatial resolution of the pixel detectors. The histograms in Fig. 5.12 show the distribution of this resolution over many reconstructed $B_{s}$ candidates. In part a), the distribution is shown for the constant $\sigma$ model of the pixel spatial resolution. In part b) the distribution is shown for the variable $\sigma$ model and, in part c) the distribution is shown for the fast clustering model. The most prominent feature in these plots is that there is a large improvement in resolution going from constant $\sigma$ to variable $\sigma$. This is expected because the variable $\sigma$ model, as discussed above, has better resolution than the constant $\sigma$ model. There is also an increase in yield, which comes from the improvement in detachment and isolation cuts which derives from improved spatial resolution. A third observation is that, when going from the variable $\sigma$ model to the fast clustering model, only minor changes are seen, consistent with the small differences between the two models. The conclusion of this study is that the expected resolution is not significantly degraded when using a model with explicit pulse height generation.

Part a) of Fig. 5.13 shows the distribution of the quantity $\left(t_{\text {measured }}-t_{\text {generated }}\right) / \sigma_{t}$ for the variable $\sigma$ model of the pixel spatial resolution. Here $t$ is the proper decay time of a $B_{s}$ candidate and $\sigma_{t}$ is its error, computed on a candidate by candidate basis. Notice that the distribution is very close to a unit Gaussian and that there are few outliers. This is expected because the underlying resolution model is Gaussian; the outliers come from the small number of cases in which fit seeds are outside the radius of convergence of the fitter and from the few cases in which the first order error propagation formulae do not work. All entries in these plots are for true signal combinations. Now compare this to part b), which contains the same plot for the fast clustering model, in which the underlying resolution functions are not Gaussian. The core of this plot remains consistent with a unit Gaussian but the number of outliers has clearly increased. The increased number of outliers are attributable to the tails of the pixel spatial resolution functions. The clear conclusion is that the increased number of outliers is small enough to be of no concern.

Parts c) and d) of Fig. 5.13 compare the confidence level of the fit of the $\mu^{+} \mu^{-} K^{-} \pi^{+}$ system to a common vertex. Again all entries are for true signal combinations. Part c) is for the variable $\sigma$ model, in which the pixel resolution function is explicitly Gaussian; in this case the confidence level distribution is flat, as expected. Part d) shows the distribution for the fast clustering model which contains two sorts of non-Gaussian contributions, a box-like component in the core and long tails. The combined effect of these non-Gaussian components is the spike in the few bins near a confidence level of zero and a slight downwards slope in the body of the plot. The area in the spike is about $4 \%$ of the total area of the plot. Again the clear conclusion is that the introduction of non-Gaussian resolution functions does not raise significant concerns about the physics reach of $\mathrm{BTeV}$. 

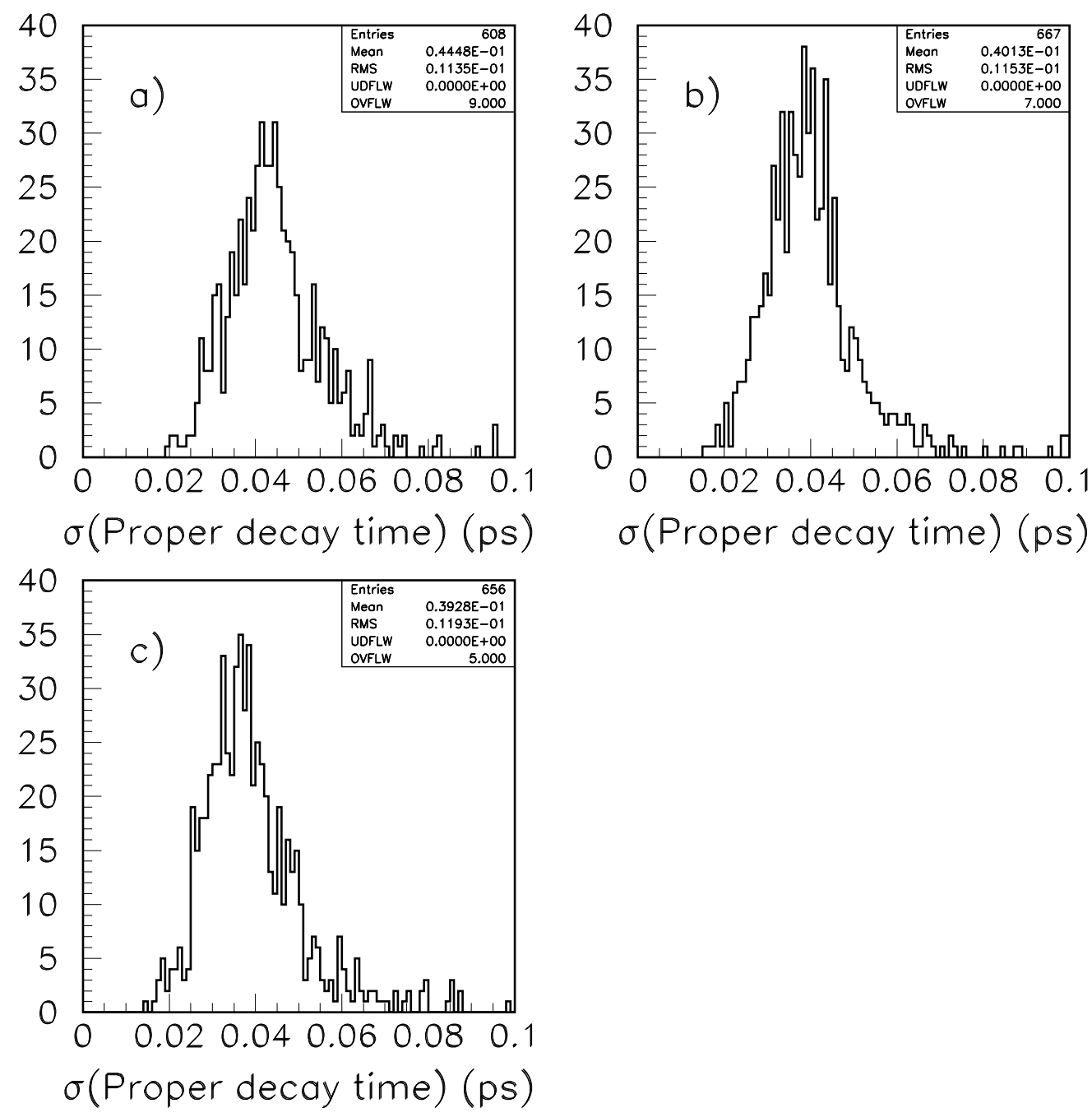

Figure 5.12: The expected resolution on the proper decay time for the decay chain $B_{s} \rightarrow$ $\psi \bar{K}^{* 0}, \psi \rightarrow \mu^{+} \mu^{-}, \bar{K}^{* 0} \rightarrow K^{-} \pi^{+}$: a) for the constant $\sigma$ model of the pixel spatial resolution; b) for the variable $\sigma$ model; and c) for the fast clustering model. All entries are for true signal combinations. These models are described in section 10.3. 

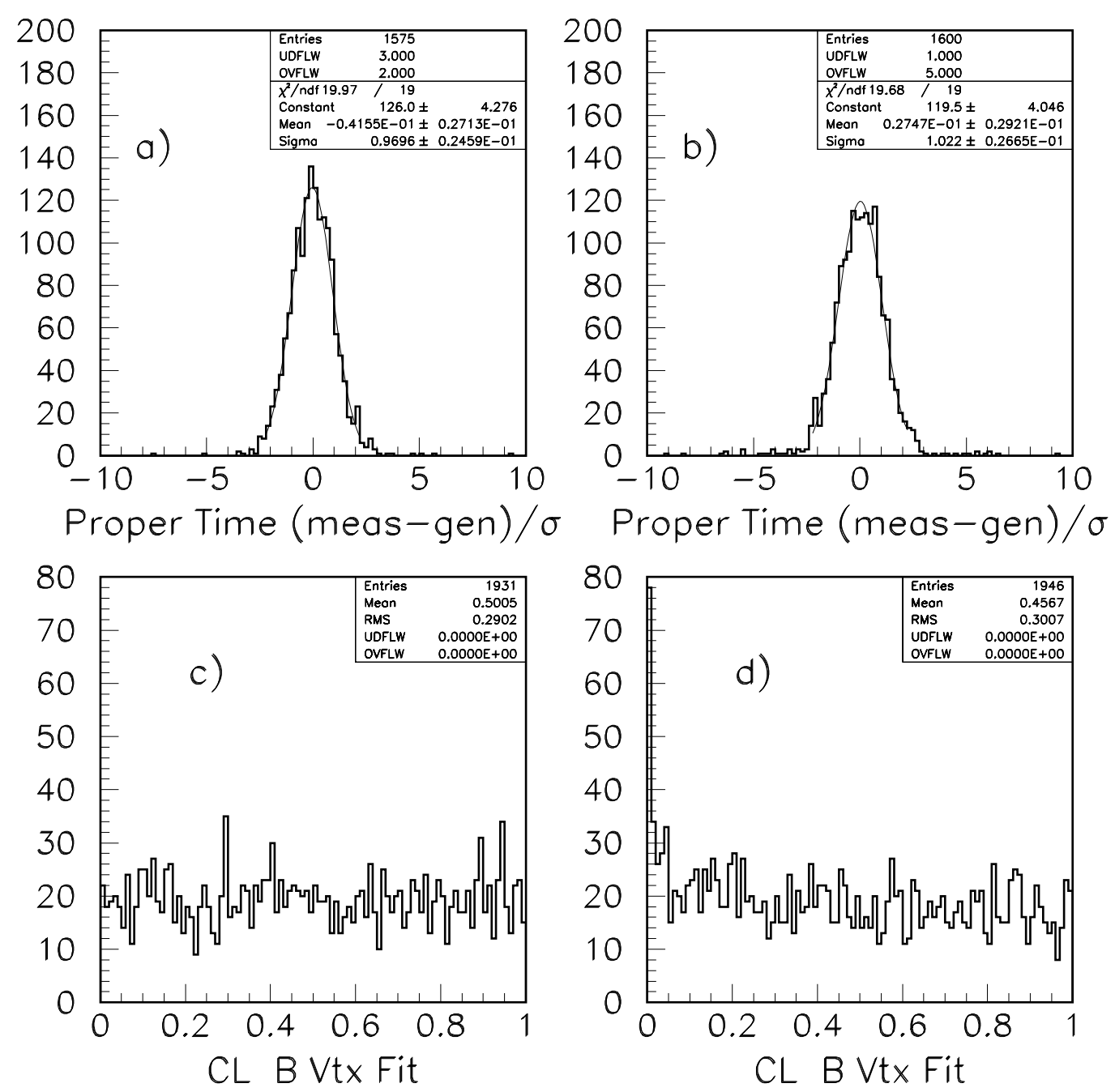

Figure 5.13: Parts a) and c) show quantities computed using the variable $\sigma$ model of the spatial resolution of the pixels, in which the resolution function is explicitly Gaussian. Part a) shows the normalized residual on the proper decay time of a $B_{s}$ candidate, while part c) shows the confidence level of the fit four tracks to a common vertex. All entries are for true signal combinations. Part a) is expected to be a unit Gaussian while part c) is expected to be flat. The reasons for the few outliers in a) are discussed in the text. Parts b) and d) show the same quantities for the fast clustering model, which does have non-Gaussian contributions; in these cases the deviations from the ideal behavior present but are small enough not to raise significant concerns. 


\subsubsection{Future sensor simulations}

There are several other issues affecting the sensor performance that we would like to investigate. Some of them determine the optimal performance throughout the lifetime of the sensor while radiation damage effects accumulate. Other studies are dynamical in nature and will provide a more realistic model of the charge sharing between adjacent electrodes.

Our baseline sensor has a collection electrode on the ohmic side of the sensor $\left(\mathrm{n}^{+}\right)$. This requires a more complex geometry on this side in order to provide electrical insulation between the individual pixels, typically with p-stop or p-spray implants [14], [15]. These structures introduce additional complexity into our modeling of the sensor, by producing a lateral component in the electric field that sometimes has quite dramatic effects in the detector performance. It can introduce local regions of very high field that may determine the break-down voltage. In addition, it can produce modulation of the charge sharing between pixel cells introducing some lateral component to the drift motion that can increase charge sharing when the track crosses the detector at normal incidence [16]. It is intuitive to see that this electric field profile is of true tridimensional nature and thus requires 3 -D simulation tools. We have chosen to perform our studies using a very sophisticated simulation program, DaVinci [17]. It has been used successfully by teams at CERN[9] and the University of Hawaii[18] to simulate their pixel sensors. The CERN group has even added an interface to study the charge collection profile in the presence of a magnetic field that we need to implement eventually if we want to model the charge collection in our experiment.

The issues that we are planning to study are:

1- a mapping of the electric field throughout the whole sensor (both inside the active volume and at the periphery for different guard ring configurations). This study is crucial to understand the sensor breakdown voltage and to design sensors that ultimately will withstand high reverse bias. The sensor is likely to be operated at high voltage during some stages of its lifetime. In addition, a detailed mapping of the electric field profile in these regions is an essential input to the the p-isolation design.

2- a study of the profile of the charge pulses in a cluster of neighboring pixels while we change the track impact parameter inside a central pixel. This study is crucial to understand the actual charge sharing between neighboring pixels and the expected pulse height with different sensors and at different irradiation doses. The radiation effects will be modeled with a different space charge density $N_{\text {eff }}$ and with a degraded carrier lifetime due to the excess of trapping centers induced by radiation effects. In addition we are planning to introduce a realistic simulation of transport properties in the magnetic field.

These studies will be complemented by the extensive measurement program detailed in other sections of this chapter. In particular, the test beam data and extensive electrical characterization of sensors before and after irradiation will provide crucial tests of our model. 


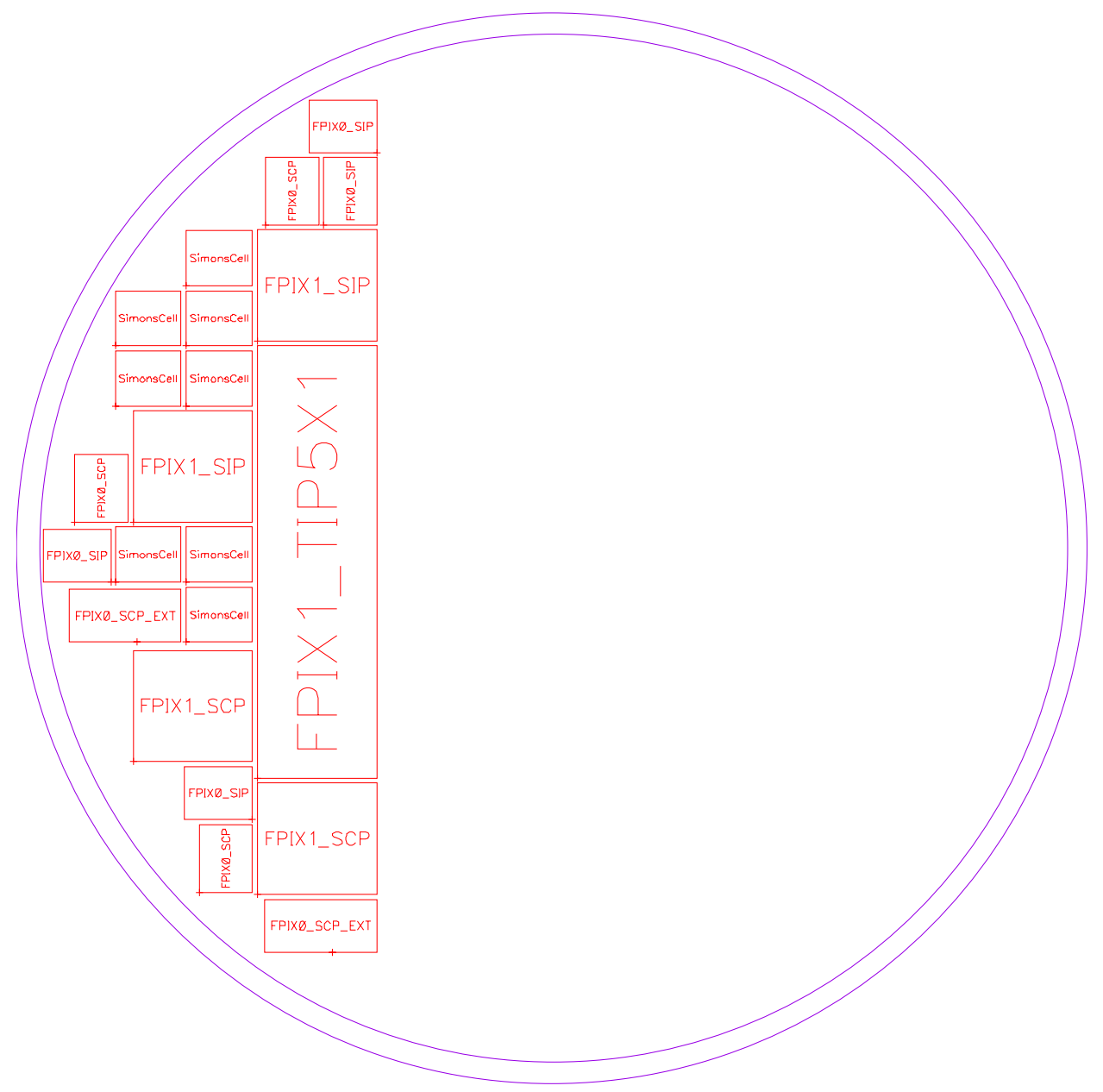

Figure 5.14: Wafer map

\subsubsection{Prototype sensor}

We have recently sent out for bids to a number of vendors for prototype sensor wafers, jointly with the US-CMS collaboration. The sensors will be $n^{+}$on $n$ with p-stop isolation between $n^{+}$implants on the n-side of the wafer. Fig. 5.14 shows the wafer layout of the BTeV portion.

Our sensor designs are very similar to the p-stop designs of the ATLAS "First Pixel Prototype" [19]. We also borrowed heavily from their experience with the guard-ring design.

The BTeV portion of the wafer contains the following structures:

- FPIX1-SIP: A sensor with individual ("atoll") p-stops - designed to mate to a single FPIX1 readout chip (2 per wafer).

- FPIX1-SCP: A sensor with a single common p-stop - designed to mate to a single FPIX1 readout chip (2 per wafer).

- FPIX0-SIP: A sensor with individual ("atoll") p-stops - designed to mate to a single FPIX0 readout chip (4 per wafer). 
- FPIX0-SCP: A sensor with a single (floating) common p-stop - designed to mate to a single FPIX0 readout chip (3 per wafer).

- FPIX0-SCP-EXT: A sensor with a single common p-stop which is extended to one side so that it may be biased - designed to mate to a single FPIX0 readout chip (2 per wafer).

- FPIX1-TIP5X1: A sensor with individual ("atoll") p-stops - designed to mate to 5 FPIX1 readout chips (1 per wafer).

- SimonsCell: test structures(both diodes and pixels) with a variety of guard ring configurations (9 per wafer). Three different guard ring designs will be tested. These are the ATLAS design, the CMS-strip design [20], and a new design from BNL and Johns Hopkins University (JHU) [21].

Additional test structures will also be present on the wafer to monitor flat band voltages, layer thickness, implant resistivity, aluminum sheet resistance, etching uniformity and alignment. Our plan for this submission is to have enough sensors to be mated to the FPIX0 and FPIX1 chips and to gain experience on multichip-module assembly. We will also use the devices to do systematic studies on guard ring designs, p-stop designs, as well as effects such as surface damage, stability and variation from wafer to wafer and vendor to vendor. Irradiation studies will also be carried out so that dependence of the breakdown voltage, depletion voltage, and leakage current on the fluence can be studied.

A number of wafers will be made from oxygen-diffused silicon.

This round of submissions is focussed on p-stop designs. However, recent tests reported by the ATLAS collaboration show that the use of p-spray will significantly improve the performance of detectors after a radiation dosage exceeding $2 \times 10^{14} \mathrm{p} / \mathrm{cm}^{2}$. We plan to have a separate submission to vendors to study this technology. We will collaborate with the ATLAS collaboration on the submission and study of p-spray detectors.

\subsubsection{Collaborative effort with BNL/JHU}

Because of the commonalities of the problems faced by BTeV and LHC experiments, a close collaboration amongst various institutes is called for. We have collaborated with BNL and JHU on sensor development. BNL possesses facilities to fabricate high quality prototype detectors. The advantage of this path is cost-effectiveness and fast turn-around. As a result of our collaborative effort, a large number of detectors of various configurations have been fabricated and tested under different processing conditions [22, 23]. BNL can now produce good detectors reliably. Various guard-ring designs have been studied and improved upon. So far, we have demonstrated that $n^{+} / n$ devices can be operated at $500 \mathrm{v}$ without breaking down, both before and after irradiation. Single pixel devices with pixel pitch of 60 and $120 \mu \mathrm{m}$ with common or atoll p-stops have also been studied. One of the pixel cells in these devices is connected to a large probing pad on the periphery. All the other pixels in the devices are ganged together to another probing pad. During measurement, the ganged 
pixels were grounded together, and I-V measurements were taken on the single pixel cell. Our results showed that breakdown occured more frequently for devices with atoll p-stop than with the common p-stop. This is even more so for devices with small pitch. We will modify the separation between the $n^{+}$implant and the p-stop in a future fabrication.

\subsubsection{Diamond option}

In addition to the baseline silicon-sensor plan, some members of $\mathrm{BTeV}$ are participating in R\&D into diamond pixel detectors as an option for BTeV. Test results on diamond from the CERN RD42 collaboration indicate that diamond detectors have a useful range of operation up to fluences exceeding $10^{15} \mathrm{n} / \mathrm{cm}^{2}$. Diamond has excellent thermal conductivity and mechanical properties. These, coupled with the fact that diamond detectors have no leakage current, make diamond detectors a very attractive option for the innermost region where the radiation dosage is very high. On the other hand, this is a technology still under development, and has never been used in a real high-energy physics experiment. There are concerns about the quality of the diamonds, collection efficiencies, thickness of the diamond, large scale production, and cost.

Our simulation shows that there are significant gains in resolution by moving the detectors close to the beam. In our baseline design, we have chosen to place the pixel detector no closer than $6 \mathrm{~mm}$ from the beam because of radiation damage to silicon. The use of diamond pixel detectors would enable us to operate the vertex detectors closer to the beam.

We plan to test diamond pixel detectors using the FPIX0 readout chip in the MT test beam during the fixed target run at Fermilab.

\subsubsection{Goals of sensor development}

Our goal is to have a final specification for the sensors by May 2000. To achieve this goal, we have to do studies of guard rings, p-stop designs, and p-spray devices before and after irradiation. Electrical characteristics of devices before and after irradiation also will be simulated using computer models.

At the same time, we will keep in close contact with the CERN RD42 collaboration to investigate the possibility of using diamond detectors in the innermost area of our pixel system.

\subsection{Pixel readout chip}

\subsubsection{Pixel readout chip development at Fermilab}

One of the defining characteristics of $\mathrm{BTeV}$ is the plan to use a vertex trigger as the primary trigger for the experiment. This means that the pixel readout ASIC must be capable of reading out all hit information from every $p \bar{p}$ interaction. MCFAST simulations have shown that an $8 \mathrm{~mm} \times 7.2 \mathrm{~mm}$ pixel sensor located $6 \mathrm{~mm}$ from the beamline (corresponding to the 
innermost pixel readout chip, given the baseline BTeV design and the current readout chip size) will be hit by one or more tracks in approximately one bunch crossing out of four at a luminosity of $2 \times 10^{32} \mathrm{~cm}^{-2} \mathrm{sec}^{-1}$. The number of pixel cells hit by a single track depends on the pixel size and the track angle with respect to the pixel sensor. Given a pixel size of $50 \mu \mathrm{m} \times 400 \mu \mathrm{m}$ and a sensor thickness of $300 \mu \mathrm{m}$, our simulations indicate that an average of five pixels will be hit in the innermost readout chip in those crossings in which one or more tracks hit the chip. Therefore, this pixel chip has to sustain an average readout rate of 1.25 pixels per BCO (beam crossing). To account for statistical fluctuations and allow for effects not included in the simulations, the chip must be capable of even higher data transfer rates, and must be able to buffer hit information which can not be immediately read out.

The BTeV pixel readout chip should be optimized for the 132 ns time between crossings planned for the Tevatron collider. It must be radiation hard so that it can be used close to the beamline. As described above, the baseline BTeV design calls for $\mathrm{n}^{+}$on $\mathrm{n}$ silicon sensors with appropriate guard ring structures for high voltage operation. These sensors provide adequate signals after significant radiation exposure, but also have rather large radiationdamage-induced leakage current. The BTeV pixel readout chip must be able to tolerate this leakage current at least up to $25-50 \mathrm{nA}$ per pixel.

An R\&D program has begun at Fermilab whose goal is the design of a pixel readout ASIC for BTeV[24][25]. The program envisions a series of prototype pixel readout chips, each with specific engineering goals. The current expectation is that the final design will be implemented using the radiation hard Honeywell 0.5 $\mu \mathrm{m}$ CMOS Silicon-On-Insulator (SOI) process. For this reason, the first prototype devices have been made using relatively inexpensive radiation soft CMOS processes using only features (number of metal and polysilicon layers, for example) shared by the Honeywell SOI process.

Significant progress has been made in the development of a pixel readout ASIC. Two prototype circuits have been fabricated and are being tested. The first chip, FPIX0, was designed in early 1997, and received at Fermilab in September, 1997. It has been extensively tested, both alone and bonded to a sensor [26]. FPIX0 chips bonded to pixel sensors will be tested in the Meson test beam, as described below. The second prototype pixel readout chip, FPIX1, was designed during 1998, and received in December, 1998. This circuit, which contains $\sim 970000$ transistors, is the largest ASIC ever designed at Fermilab. It is currently being bench tested. Beam tests are planned for late summer, 1999.

\subsubsection{FPIX0}

FPIX0 has achieved both of its primary design goals. These were: 1) To establish a frontend design appropriate for use at the Tevatron collider; and 2) To verify that deadtimeless operation is possible. Deadtimeless operation requires that the analog and digital sections of the chip be isolated from one another, and that readout signals be isolated from the sensor so they are not coupled back to the amplifier inputs. In the FPIX designs, the metal 3 layer is used to isolate the sensor from the readout chip.

The FPIX0 amplifier consists of two folded cascode stages, AC coupled to one another. 
The first stage is a charge amplifier that uses a current controlled feedback circuit [27]. The second stage provides additional gain and is DC coupled to the discriminator. The FPIX0 amplifier is a continuous time amplifier which does not require front-end reset signals. The feedback current controls the return-to-baseline time and also compensates for sensor leakage current. The circuit was designed to completely compensate for leakage current up to $\sim 25$ $\mathrm{nA}$. Tests of the FPIX0 demonstrate that the circuit works as designed, and in fact currents of $100 \mathrm{nA}$ or more can be tolerated with only minor signal degradation. This is essential for operation in $\mathrm{BTeV}$ close to the Tevatron beam, where radiation damage will result in large sensor leakage current (pixel sensors can not be AC coupled to the readout electronics because the required capacitor would be larger than the pixel).

One of the primary goals of our test beam activity is to verify the simulation results on resolution as a function of the number of ADC bits. FPIX0 chips (which include an analog output) bonded to ATLAS sensors will be used for this purpose.

\subsubsection{Test results}

Fluorescence x-rays from a number of metal foils have been used to provide an absolute calibration of an FPIX0 bonded to an ATLAS (Seiko ST1) pixel sensor. Figure 5.15 shows the pulse height spectrum collected using a single FPIX0 pixel amplifier while the sensor was being illuminated with terbium x-rays. The $K_{\alpha 1}$ peak $(44.5 \mathrm{keV})$ is easily identified at $407 \mathrm{mV}$. Since it is known that a $44.5 \mathrm{keV}$ x-ray creates 12300 mobile electron-hole pairs in fully depleted silicon, the scale for this channel is 30.2 input electrons per $\mathrm{mV}$ peak output signal. We have verified the linearity of the FPIX0 amplifier response to small input signals using a variety of characteristic x-rays.

Measurements of amplifier noise and discriminator threshold dispersion have been made for a number of FPIX0 chips, and also for a smaller test chip (Pre-FPIX1) which contains a variety of FPIX0-like amplifiers. Two conclusions have been drawn from these measurements. First, there is a trade-off between dynamic range on the one hand, and low noise and small discriminator threshold dispersion on the other. Second, the cells which are bonded to sensor pixels are only slightly noisier than identical unbonded cells. If all of this noise difference is attributed to the difference in input capacitance, then a SPICE simulation indicates that the total input capacitance of the (Seiko ST1) sensor pixel and bump bond is approximately $180 \mathrm{fF}$, which is very close to the expected value.

No pickup of the digital activity associated with readout has been detected. With the metal 3 shield grounded, there is no significant coupling through the detector. The capacitance between the metal 3 shield and a sensor pixel has been inferred to be approximately 1.8 $\mathrm{aF} / \mu^{2}$. This capacitance was inferred by disconnecting the shield from its ground, injecting a pulse on the shield, and measuring the output voltage from a single pixel unit cell. Finally, we have measured the pixel-to-pixel cross talk to be less than $2 \%$. 


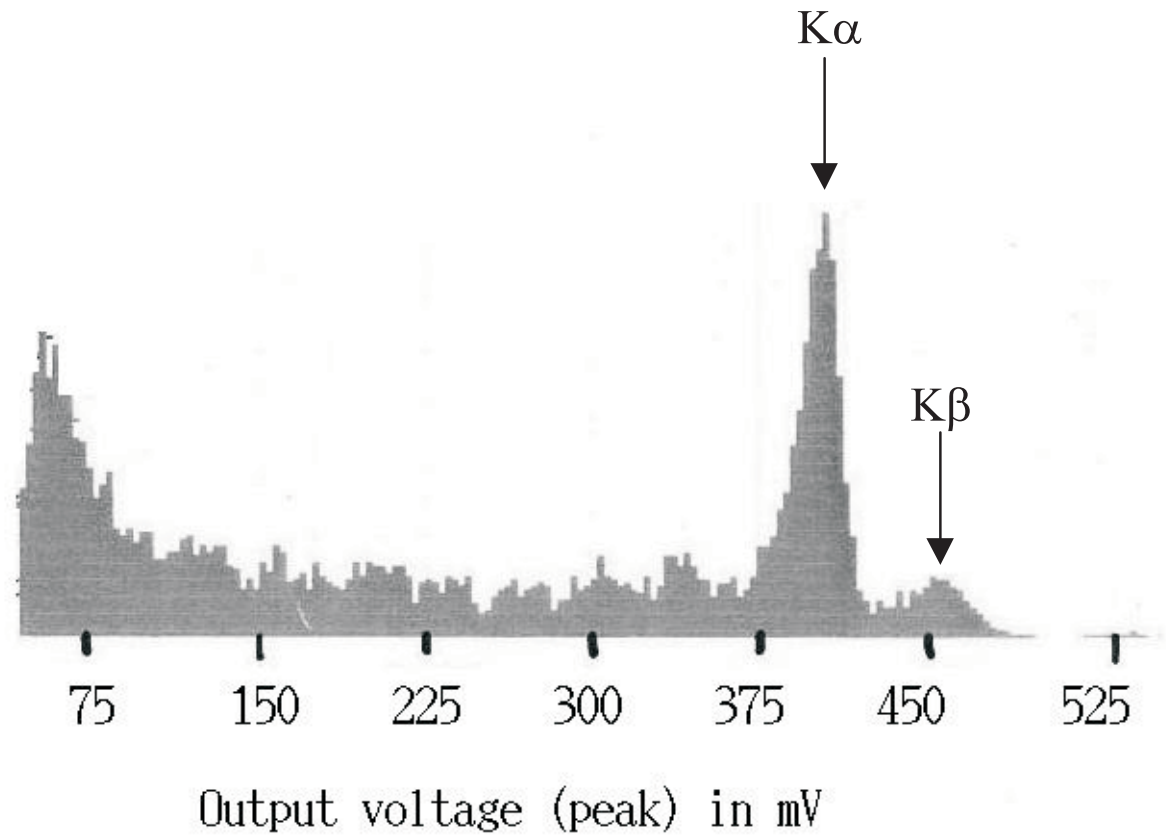

Figure 5.15: Terbium x-ray spectrum recorded by a single FPIX0 channel.

\subsubsection{FPIX1}

\subsubsection{Design features and architecture}

The FPIX1 readout chip is the first implementation of a new column-based pixel architecture designed to meet the requirements of $\mathrm{BTeV}$. The most stringent requirement is that all pixel hit information from every Tevatron crossing must be digitized and read out so that it may be used to form the primary trigger for the experiment. Simulations indicate that, with a 26.5 MHz readout clock, FPIX1 is capable of reading out an average of more than three pixels per BCO. Relatively straightforward extensions of the FPIX1 architecture should increase the readout bandwidth by a factor of four or more.

In FPIX1, each pixel hit is associated with a six-bit time stamp (the beam crossing number) and the hit information is held in the pixel unit cell until readout. In normal operation for $\mathrm{BTeV}$, the chip itself generates a readout trigger for every beam crossing. The time stamp associated with a hit pixel is held in a register located at the end of the column of pixels containing the hit. All pixels in a given column which are hit in the same $132 \mathrm{~ns}$ beam crossing are associated with the same end-of-column timestamp register. Each column in FPIX1 has four timestamp registers.

Figure 5.16 is a photo-micrograph of a single FPIX1 chip. Figure 5.17 is a schematic diagram of the layout. FPIX1 is composed of three building blocks: the pixel unit cell, the end of column logic, and the chip control logic. The active area consists of an array of 160 rows by 18 columns of pixel cells, each $50 \mu \mathrm{m} \times 400 \mu \mathrm{m}$. All "normal" chip I/O is through a double row of pads (shown along the bottom in Figures 5.16 and 5.17). The opposite edge of 
the chip is used to provide direct access to amplifier and discriminator outputs from one row of pixel cells. These are for diagnostic use during the prototype phase of chip development, and will not exist in the production version of the chip. In addition, one column of pixels is designed to be read out under external control using pads on this "top" edge of the chip.

A block diagram of the FPIX1 pixel unit cell is given in Figure 5.18. The FPIX1 frontend is an optimized version of the FPIX0 front-end described above. The dynamic range of the FPIX1 amplifier is approximately $32000 \mathrm{e}^{-}$. FPIX1 was designed with a two bit flash ADC in every pixel cell.

The digital section of the FPIX1 pixel unit cell consists of a command interpreter and a bus controller. Four pairs of lines carry commands from the end-of-column-logic to the pixel unit cells. Each pair of command lines is associated with a particular timestamp register in the end-of-column logic. The commands which control the action of the pixel cells are: "idle," "reset," "output," and "look-for-data." Commands are presented by the end-ofcolumn logic simultaneously to all pixel cell command interpreters in a column. When a pixel discriminator fires, then if the "look-for-data" command is present on one of the four pairs of command lines, the command interpreter in the hit cell associates the hit with the timestamp register corresponding to the "look- for-data" command. Simultaneously, it alerts the end-of-column logic to the presence of a hit. After the association of a hit pixel with a timestamp register is made, the command interpreter in the hit pixel cell waits for an "output" or "reset" command on the line corresponding to the associated timestamp register, and ignores the other command lines.

In normal operation, the FPIX1 chip control logic generates a readout trigger for every beam crossing after waiting for two crossings to allow hit information to settle. A token passes quickly through columns containing no data associated with the requested crossing number, and stops at the first hit column. Another token is generated by the end of column logic in this hit column, and this token drops down the column through unhit cells until it reaches the first hit pixel. At the next rising readout clock edge, the hit pixel cell loads its data onto the column bus and drives it to the end-of-column logic for one clock cycle. In parallel, the column token is transmitted to the next hit pixel. The time required for the token to pass through unhit columns, and unhit pixel unit cells in a hit column, determines the maximum allowable readout clock frequency. A good deal of effort was spent in the design of a high speed token-passing circuit for FPIX1. The time required to skip an unhit

pixel cell has been measured to be $\sim 190 \mathrm{ps}$ (greater than $5 \mathrm{GHz}$ skip rate). This allows a 26.5 $\mathrm{MHz}$ readout clock to be used with a comfortable safety margin.

\subsubsection{Preliminary test results}

The first measurements of FPIX1 performance are very encouraging. The front-end performance is approximately as expected. Both the amplifier noise and the discriminator threshold rms are $\sim 40 \%$ smaller than in FPIX0, and acceptable for use in BTeV. The discriminator overdrive required to latch a hit in the proper beam crossing is only $\sim 250 e^{-}$. The two-bit ADCs operate as designed, and all of the readout logic works. An IR drop on the line which 


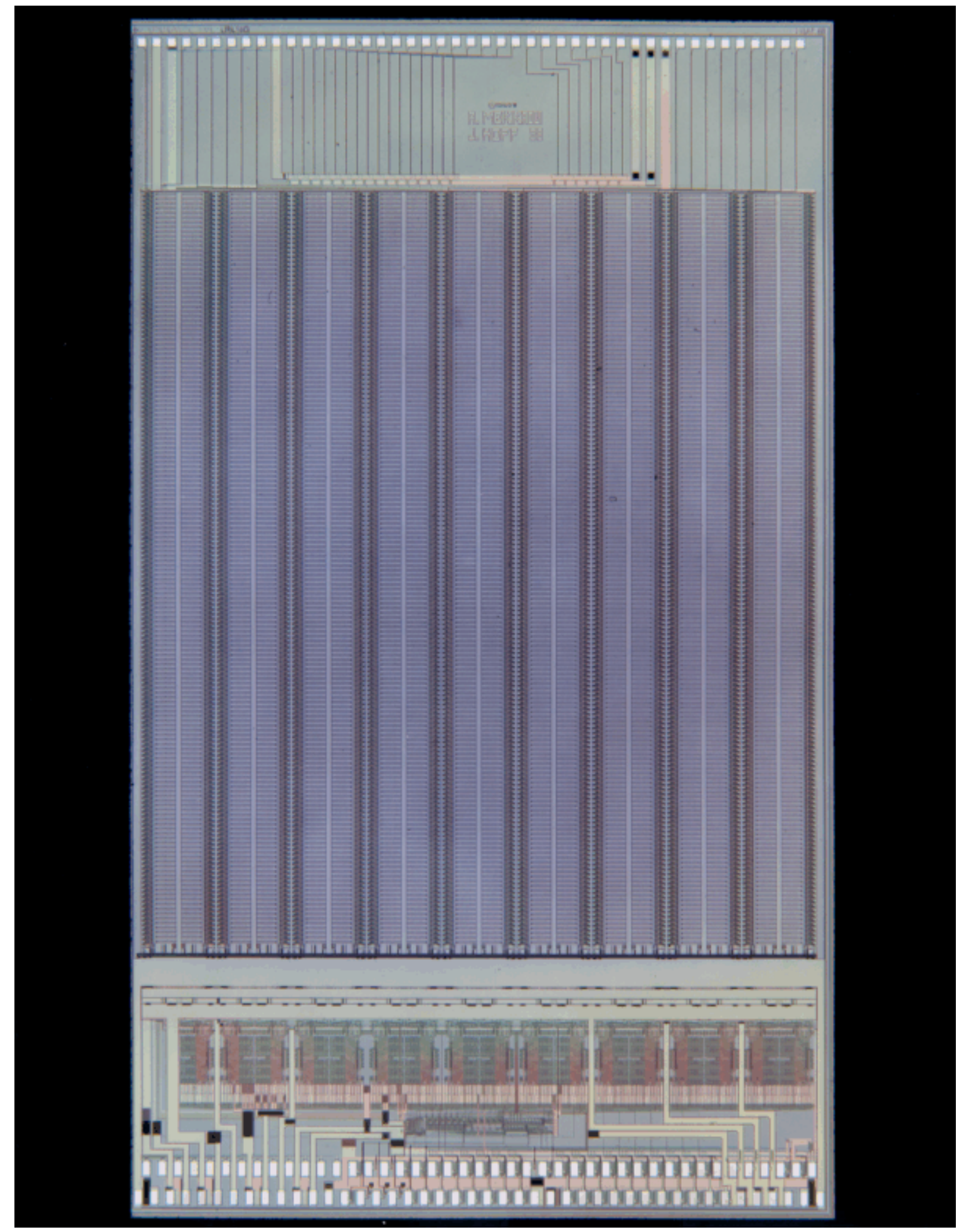

Figure 5.16: Photomicrograph of FPIX1. 


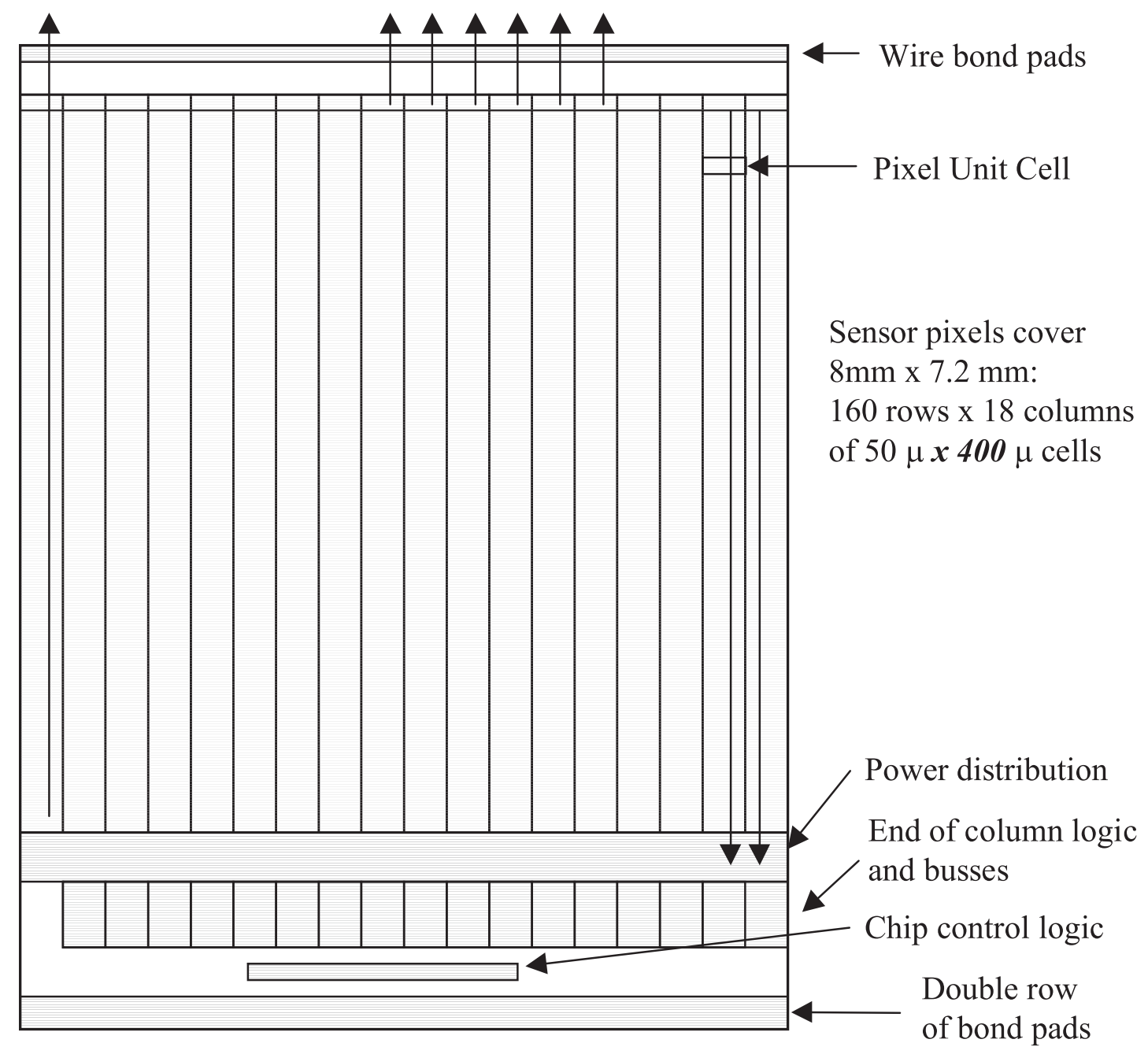

Figure 5.17: FPIX1 Floor Plan. 


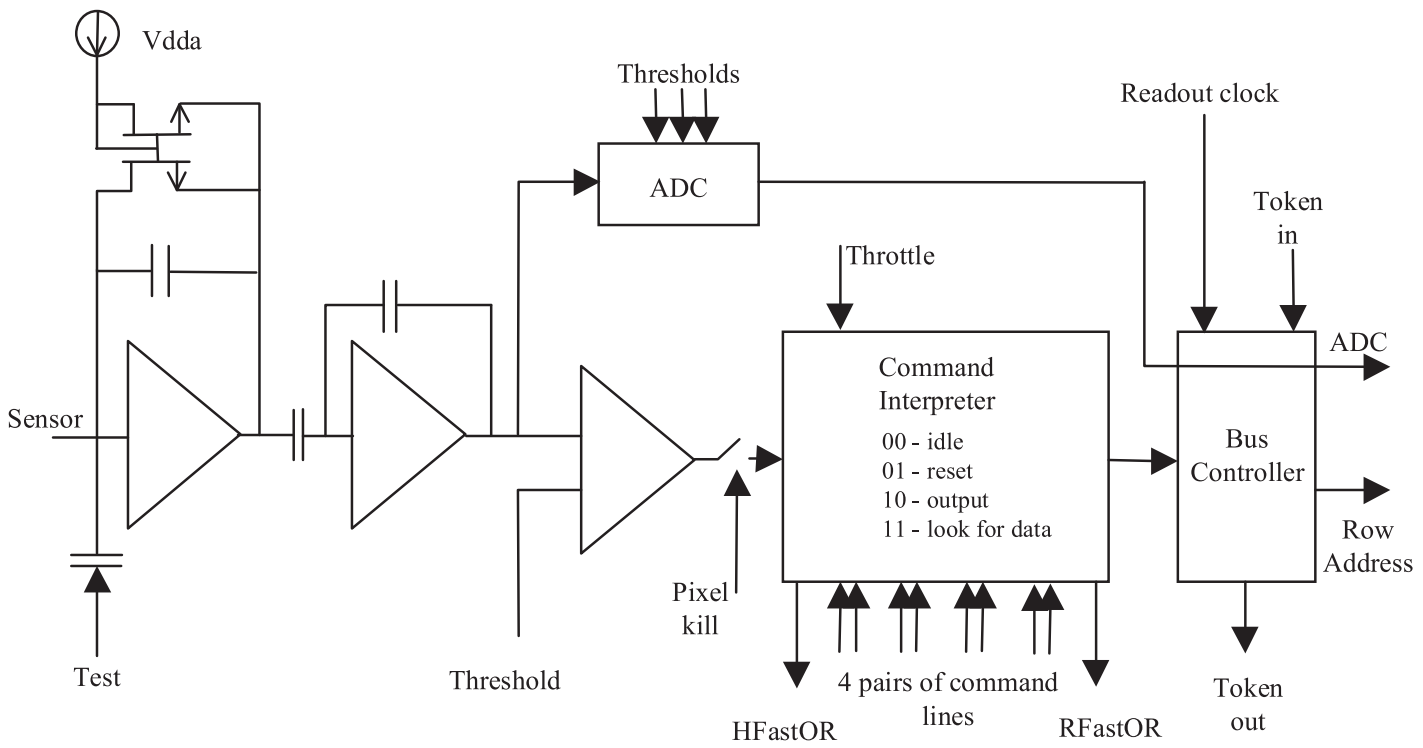

Figure 5.18: The FPIX1 Pixel Unit Cell.

alerts the end-of-column logic to the presence of a hit in the column may mean that only $\sim 125 / 160$ pixels in each column can be used in the beam test. This error is embarrassing, but completely understood, and trivial to correct in the next FPIX design.

As FPIX1 was designed, simulations were performed using both SPICE and Verilog. SPICE simulations were used in the analog design and also to provide parameters (e.g. time delays) for the Verilog simulations. Layout versus schematic checking was performed both at the cell level and at the full-chip level. The behavior of the entire chip was simulated using Verilog. We are continuing these simulations using pixel hits generated by MCFAST and Verilog models updated with measured FPIX1 parameters. We will rely heavily on Verilog simulations as we decide what features of FPIX1 to modify in the FPIX2 design.

\subsubsection{Future plans}

FPIX1 was fabricated using the HP $0.5 \mu \mathrm{m}$ CMOS process. This choice was made in order to facilitate the production of a final $\mathrm{BTeV}$ pixel readout chip using the radiation hard Honeywell 0.5 $\mu \mathrm{m}$ SOI CMOS process. However, recent results from RD-49 at CERN indicate that standard commercial "deep submicron" $(0.25 \mu \mathrm{m}$ and below) CMOS processes may be even more radiation hard than military processes such as the Honeywell $0.5 \mu \mathrm{m}$ SOI, provided only that a set of special design rules is followed [29]. An ASIC is currently being designed at Fermilab which contains amplifiers, comparators, and other circuits similar to those required for the front-end of the pixel readout. This chip will be fabricated using a standard commercial $0.25 \mu \mathrm{m}$ CMOS process. Its performance before, during, and after exposure to radiation will be evaluated. Based on this evaluation, we will decide whether to implement the next FPIX chip in HP $0.5 \mu \mathrm{m}$, or to redirect our efforts towards a deep 
submicron implementation.

\subsection{Bump bonding development}

\subsubsection{Introduction}

The BTeV baseline pixel detector, like all other pixel systems, is based on a design relying on a hybrid approach. With this approach, the readout chip and the sensor array are developed separately, and the detector is constructed by flip-chip mating of the two. This method offers maximum flexibility in the development process, choice of fabrication technologies, and the choice of sensor materials. However, it requires the availability of a highly reliable, reasonably low cost, fine-pitch flip-chip attachment technology.

There are several bonding technologies which may be suitable for this. These include indium bumps, Pb-Sn solder bumps, fluxless solder bumps, and anisotropic conductive films (ACF) or tapes. The technology has to be able to fulfill the following requirements:

- small bump - the typical bump diameter and height for our pixel detector is between $10-12 \mu \mathrm{m}$.

- fine pitch $(50 \mu \mathrm{m})$

- high yield - a defect rate of better than $10^{-4}$ is required.

\subsubsection{Prototyping experience}

Given the fact that we are mostly dealing with dies (for the readout chip) so far, and that our pitch is extremely fine, we chose to go with indium bumps which possess many attractive features. We have successfully deposited indium bumps on 30 micron pitch for an earlier tests of four hybridized arrays. The low melting point of pure indium, $156^{\circ} \mathrm{C}$, allows for cold pressure welding, instead of solder reflow, and low forces (2-3 gram/bump) are used during the bonding process. For small prototype runs, there are only a handful of companies that can provide such services to outside customers. Because of the technical challenges (small die, large aspect ratio, fine pitch), we decided to go to two different vendors. These are Advanced Interconnect Technology (AIT) in Hong Kong, and Boeing North America Inc. in Anaheim. Our preliminary results showed that the failure rate in bump deposition from AIT was better than $2 \times 10^{-4}$ at $90 \%$ C.L. There were a couple of dies with poor bump deposition(bridges or shorts, missing bumps) from the BOEING batch. Ignoring those two bad ones, the failure rate from BOEING was comparable to that of AIT. However, since the beginning of last year, BOEING no longer provides bump-bonding service to outside customers. We did, however, manage to get four pixel detectors using the FPIX0 chip bumped and mated at BOEING before they decided to stop their service. So far, we have fully tested one detector and found out that all the channels have been successfully connected. Fig. 5.19 shows the hit-map of a 

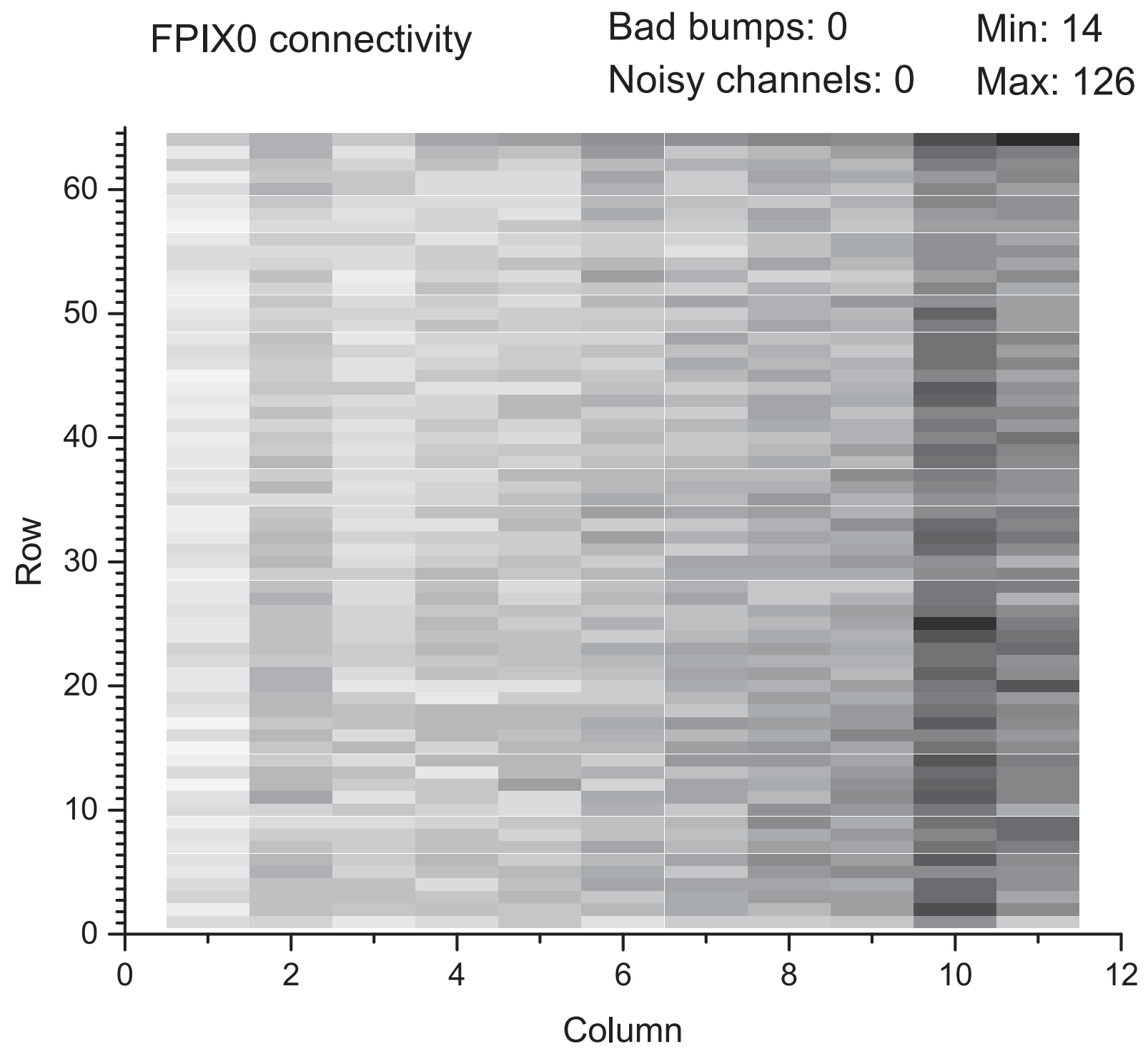

Figure 5.19: Hit map of a bump-bonded FPIX0 pixel detector showing $100 \%$ connectivity. 
prototype pixel detector using a $S r^{90}$ source. All the channels were found to work, showing that all the bump bonds were good.

We have also received from AIT four thinned silicon wafers with bumps put on. The wafers were thinned at BNL. Two of the wafers were thinned to $250 \mu \mathrm{m}$ and two to $200 \mu \mathrm{m}$. They were then sent to AIT where indium bumps were deposited. The pitch in the narrow dimension for the pixels on these wafers was $30 \mu \mathrm{m}$. Visual inspection under a microscope showed that apart from a few bad or missing bumps on the edge of the wafers, all the bumps were good and fairly uniform. Based on these limited statistics, the failure rate in bump deposition on thin wafers is about the same as for $300 \mu \mathrm{m}$ thick wafers. However, we have not done any tests on flip-chip mating with thinned wafers.

There are lots of companies which can provide flip-chip mating services for large pitch (typically $200 \mu \mathrm{m}$ pitch and $100 \mu \mathrm{m}$ diameter bumps). Our requirements are at the very high end of the technology due to the small bumps, fine pitch, and relative low volume by industrial standards. These rule out high volume companies such as Flip-Chip Technology, Abpac, and Delco.

\subsubsection{Large scale study of bump bonding yield}

The goals of the large scale bump bonding study are to find a suitable vendor to do reliable and cost-effective bump bonding and to establish a quality control procedure so as to minimize the failure rate. We have established good working relations with industry and institutes working on LHC related projects. We have, and will continue to benefit from, sharing experience and information with LBNL.

Because of our fine pitch and small bumps requirement and that we have been dealing with small readout dies, we have opted to go for indium bumps. However, our recent contacts showed that $\mathrm{Pb} / \mathrm{Sn}$ solder has been made to work at various places(e.g., IZM in Germany, SII in Japan, and AIT in Hong Kong) for a pitch as fine as $50 \mu \mathrm{m}$. Solder bumping is a self-aligning process and is a bit cheaper than indium bumps. However, the process typically involves the use of flux which needs to be removed after flip-chip mating. With small bumps, this may not be easy. Apart from environmental considerations, flux residue will lead to corrosion of the joint. The use of flux-less solder bump is now being pursued by industry. MCNC has a new fluxless, no-clean process called PADS (Plasma Assisted Fluxless Soldering) which has shown considerable success with flip-chip assemblies [34]. This relies on a pretreatment which enables the subsequent solder reflow in inert ambients. The use of this process, however, will pose stringent requirements on the thickness and material of the passivation on the readout and sensor wafers. This needs to be studied. Another concern is that the heavy $\mathrm{Pb}$ component in the solder bump may lead to a substantial increase in the electronics single-event upset rate. We plan to study this carefully together with the US-CMS collaboration.

Both pure In and $\mathrm{Pb} / \mathrm{Sn}$ solder appear to meet our requirements for providing adequate electrical and mechanical bonds at $50 \mu \mathrm{m}$ pitch. Our task is to start qualifying vendors

with facilities to provide such services to outside customers and demonstrate the quality and 
yield of their bump bonding services by providing bumping and bonding of a large number of dummy pixel detectors. Long term effects on the bump bonding will also be studied. These include mechanical stability, effect of thermal cycles, irradiation, and handling the parts during assembly. The study will be done on different pitches and both technologies (Indium and $\mathrm{Pb} / \mathrm{Sn}$ ) will be tested.

For this purpose, we have designed a dummy assembly module consisting of an upper and a lower array. As much as possible, the two arrays will be close to the size of the real sensor and readout chip that we will have. Each array will have just metallization (Aluminum or gold) which will be covered with passivation with openings for the bump-bond pads and probe pads. The wafers will be made with two pitches, $30 \mu \mathrm{m}$ and $50 \mu \mathrm{m}$. The upper array mimics the sensor chip and consists of pairs of bump-bond pads connected together. The lower array mimics the readout chip and consists of a large number of bump-bond pads ganged together with traces leading to probing pads on the periphery (Figure 5.20). When the two are mated, one can use an automatic probe station, with the appropriate probe card to test connectivity, and hence determine the presense of bad connections. The probing pads on the periphery are large enough so that they can also be used for wire-bonding. Assembly using these dummy modules can be practiced.

To characterize a vendor, we will send them a number of these dummy wafers. The wafers will be $300 \mu \mathrm{m}$ thick. Depending on the success of these tests, we will send them real sensor wafers and FPIX1 readout wafers so that we can do a real evaluation. We will also do tests on thin wafers. Table 5.3 lists the vendors that we have contacted and have expressed some interests in working with us. Both TRONICS and Rockwell can only handle 4" silicon wafers. This may be a concern for future production runs.

\begin{tabular}{|c|c|c|c|}
\hline Vendor & Technology & Location & Status \\
\hline AIT & $\begin{array}{c}\text { Indium } \\
\text { solder }\end{array}$ & $\begin{array}{c}\text { Hong Kong } \\
\text { Hong Kong/Singapore }\end{array}$ & $\begin{array}{c}\text { contract } \\
\text { pending }\end{array}$ \\
\hline IZM & Solder & Germany & SOWsent \\
\hline LMCO & Indium & Massachusett & SOWsent \\
\hline MCNC/Unitef & $\begin{array}{c}\text { solder } \\
\text { fluxless solder }\end{array}$ & $\begin{array}{c}\text { North Carolina } \\
\text { North Carolina }\end{array}$ & $\begin{array}{c}\text { proposal rec'd } \\
\text { proposal rec'd }\end{array}$ \\
\hline TRONICS & solder & France & proposal rec'd \\
\hline Rockwell & Indium & California & on hold \\
\hline
\end{tabular}

Table 5.3: A list of potential vendors that can provide bump bonding services. SOW stands for Statement of Work. Proposal received means that they have already sent in their proposal to our SOW.

Our plan is to select up to four vendors from the list and work together with them. A lot of interaction between us and the companies is needed to understand the possible causes of failures, to rectify them, and to establish a detailed quality control procedure. 


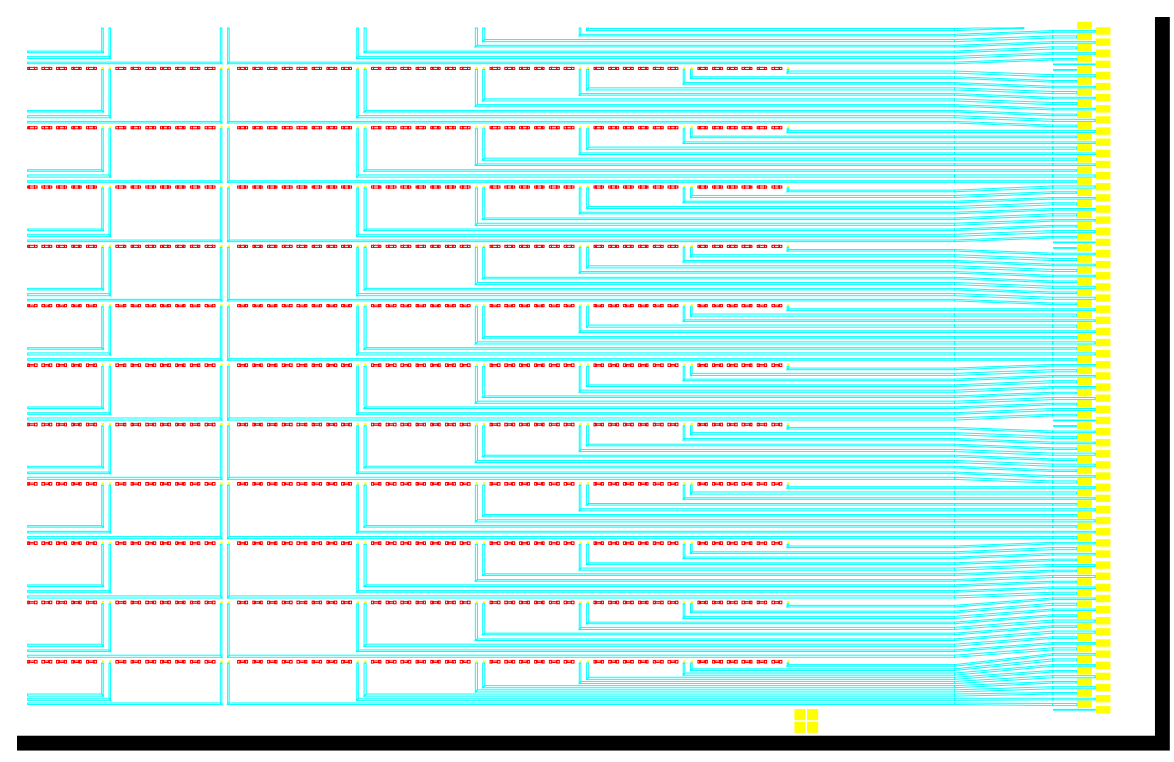

Figure 5.20: Layout of the lower array in the dummy wafer that will be used for large scale bump bonding studies. 


\subsubsection{Diagnostic tools}

To characterize the various vendors and to understand the yield of the process, we need some diagnostic tools. Some of these will also be valuable during the production phase as a means of quality control. Possible tools include:

- optical microscope - Coupled with a precision $\mathrm{x}-\mathrm{y}$ table, this is a simple and cheap way of detecting defects such as missing bumps, bridges, misplaced bumps, and irregular bump deposition.

- scanning electron microscope - This is useful to measure precisely the bump diameter, height, and placement precision. It also shows the under-bump-metallization.

- wafer profiler system - As a defect inspection tool, the profiler automatically detects bridges, under and oversized bumps, missing and extra bumps, and bump position. Furthermore, the system calculates bump height, diameter, volume, as well as coplanarity.

- acoustic imaging system - Using high frequency ultrasound, high resolution images can be produced to specific levels within a flip-chip mated device.

- X-ray imager - Using a high resolution micro-focus X-ray tube, a highly magnified $\mathrm{X}$-ray image of the flip-chip mated device will show the presence of poorly connected or missing joints between the sensor and the readout chip.

We are working together with local industries and the bump bonding companies to determine which tool will provide the most useful information.

\subsection{Data output and control}

The input and output links to the pixel planes can be categorized based on the data bandwidth. The highest bandwidth corresponds to the pixel hits generated during the normal operation of the pixel detectors and collected by the pixel detector amplifiers and discriminator chips. However, these chips must be initialized, controlled and monitored by the DAQ system. This latter information will travel to and from the pixel detector planes at a considerably slower speed.

The electronics dedicated to the functions of receiving and transmitting data will be located on the corners of each pixel detector plane. This circuitry must be in charge of adapting electrical protocols, data formats, and data speeds to interface with the pixel detector chips. This electronics, called Data In and Out (DIO) is severely constrained by factors such us physical space, power dissipation, radiation dosage, EMI noise, mass, and outgassing.

The expected occupancy of the pixel detector planes, at the maximum luminosity BTeV intends to run $\left(2 \times 10^{32} \mathrm{~cm}^{-2} \mathrm{sec}^{-1}\right)$, can generate as much as $16 \mathrm{~Gb} / \mathrm{s}$ of digitized data. The 
initialization, control and supervision data will not exceed $160 \mathrm{Mb} / \mathrm{s}$ for each half-plane of the pixel detector system.

Figure 5.21 shows a block diagram of the proposed scheme. The two links on the upper left corner receive the $53 \mathrm{MHz}$ clock and the initialization and control data for the pixel readout chips. The links on the bottom right of the block diagram transmit the pixel hit data to the DAQ and Trigger Processor. These links will also be used to read out the configuration and status information of the pixel readout chip for supervision purposes.

The main building blocks of the DIO electronics are the optical receiver, the dataconcentrator and the optical transmitter chips. The optical receiver is an interface chip between the serial optical channels and the pixel readout chips. At the physical level, it must detect the current generated by the pixel detector and generate the CMOS and LVDS signals to interface with the pixel readout chips. Furthermore, it must provide a readout clock with very low jitter $(<150 \mathrm{ps})$. At the logical level, it must decode serial information into initialization and control commands for the pixel readout chips, and decode the BCO clock.

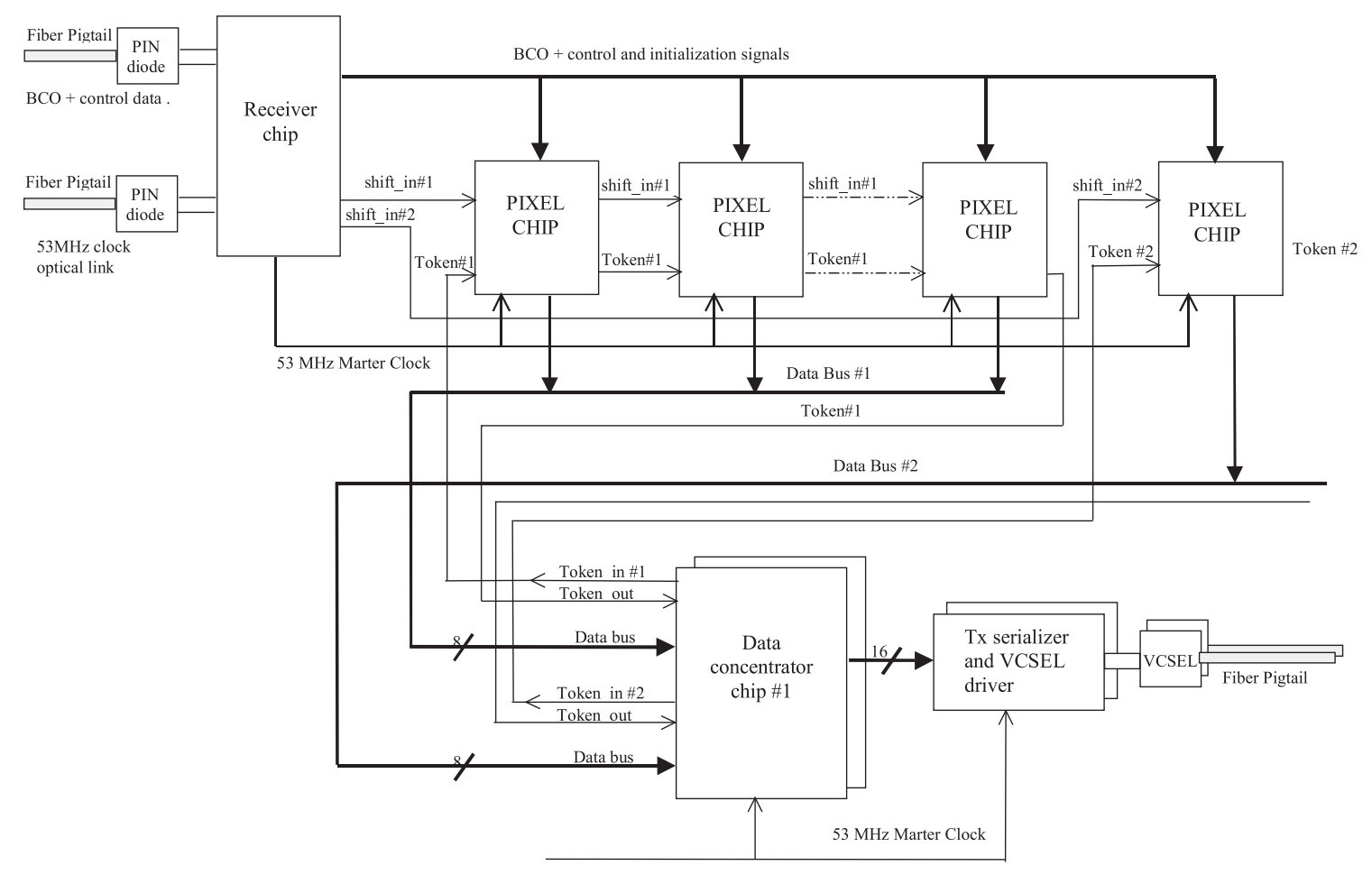

Figure 5.21: Block diagram of the proposed data I/O scheme.

The data-concentrator chip will group the data output of pixel readout chips. Its main function is to control the data flow from a pixel module, manage the token bit passing, equalize the data stream from the pixel chips into a serializer and optical transmitter. The data equalization is needed due to the fact that the hit and data rate is different in every pixel chip according with its proximity to the beam. The pixel chips closest to the beam will 
generate as much as $400 \mathrm{Mb} / \mathrm{s}$ of data and may need a dedicated link to the data-concentrator chip. Other chips, which do not generate as much data, will share a common bus and will be readout following a token passing algorithm. The data-concentrator must keep the token passing algorithm consistency and interface to the serializer and optical transmitter.

The optical receiver and the data-concentrator chips will be designed at Fermilab. The data $\mathrm{I} / \mathrm{O}$ of the pixel detector planes will be carried through fiber-optic cables. Current studies show that these cables present the best solution to meet the requirements of mass, EMI noise, radiation, power consumption, and physical space. Since other experiments in the HEP community are also designing optical links for pixel detectors and calorimeters, we will benefit by collaborating with other groups working in this area. The following new technologies are currently under study:

- Vertical Cavity Surface Emission Lasers (VCSELs): Allow gigabits of data to be transmitted several hundreds of meters with few $\mathrm{mW}$ of optical power. VCSELs have been proven to be very radiation tolerant [30].

- Step Index Pure Silica Fibers: Accept high levels of radiation without increasing attenuation more than a small percentage [31]. Other graded index fibers are also under study. They are not as tolerant as the pure silica core, but they are less costly. Radiation tests will be performed to determine the level of acceptability.

- Custom ceramic and silicon packages: Very small custom designed packages for VCSEL and PIN diodes, and fiber-optic pigtails may allow optimizing for size, along with meeting the requirements of mass budget and radiation tolerance [32].

The fast data output links must deliver up to $16 \mathrm{~Gb} / \mathrm{s}$ in every half-plane. We are researching the convenience of fast serial links, about $1 \mathrm{~Gb} / \mathrm{s}$, versus slower multifiber parallel links. Fast serial links are harder to design, but will reduce the number of fiber channels. No commercial devices exist which can accomplish the task of interfacing to the pixel chips, serializing the data, and transmitting over a fiber optic channel, and meet the pixel detector requirements. Most of the commercial devices are inappropriate for radiation environments. Furthermore, none of the commercial gigabit-speed data serializers meet the power budget. Among the possible alternatives, one of the most attractive is a CHFET-GaAs serializer being designed for the CMS experiment at CERN. We have already started to collaborate with the chip designer to meet BTeV specifications and to support testing at Fermilab. The protocol of this chip will be compatible with the so-called G-Link receiver at a frequency of $1.2 \mathrm{~Gb} / \mathrm{s}$, and its power consumption is less than $100 \mathrm{~mW}$ [33].

A second possibility is a prototype chip being designed by an R\&D section of Honeywell. It codifies four channels of 16 bit words into 3 fiber links. Each link has two fibers for data and a third for clock. The chip is being implemented in a $0.25 \mu \mathrm{m}$ CMOS process. This is not a radiation hard process by design. However, some $0.25 \mu \mathrm{m}$ CMOS processes have proven to be very radiation resistant [29], which may possibly exceed the required levels. Some research groups at the RD47 collaboration at CERN are currently looking into this problem. 
The optical components will require a special package. The package must be of low material, non-magnetic, must not outgas and be as small as possible. Furthermore, it must couple the VCSEL or PIN efficiently to the fiber. The fiber must be radiation tolerant as well. Figure 5.22 shows a possible solution where a ceramic substrate is used to host the VCSELs, PINs and fiber pigtails. This kind of substrate has ceramic or silicon alignment blocks, strain reliefs, and wire bond pad to interface to the transmiitter and receiver chips. The fiber pigtails can be ribbonized some distance outside the detector plane and connected to a standard fiber ribbon cable. This connection can be performed either by splicing or through a small-form-factor fiber ribbon connector.

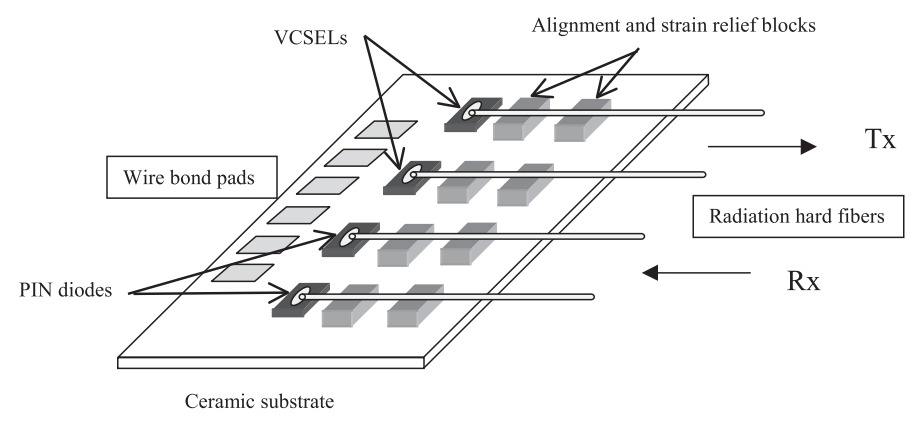

Figure 5.22: Sketch of a possible arrangement of the optical components.

\subsection{Pixel Readout and Quadrant Processors}

The data read out from the pixel detectors are transmitted through fiber-optic cables to Quadrant Processors. From the Quadrant Processors the data will be delivered to the pixel trigger system and the DAQ system.

There are several factors that impact the number of fiber optic links needed to transmit the data from the pixel detector to the Quadrant Processors: pixel hit and noise rate, forms of data compression of the pixel data, maximum optical-link data rate, and safety margin on the data transmission bandwidth.

The pixel detector will be used for the lowest level trigger, and all the data it generates, whether noise or actual hits, has to be transmitted to the Quadrant Processor. For this reason, it is important to include additional bandwidth.

A simple form of data compression can be achieved directly by the pixel chip, thereby decreasing the data rate. Data alignment by timestamp implemented in FPIX1 already 
achieves an initial degree of data compression. During readout, the timestamp has to be transmitted just once for all the hits that occurred simultaneously. In the next FPIX generation, we hope to implement what we have named "group" reading. The concept is that instead of reading a column by individually reading each pixel cell, we will read groups of consecutive pixel cells. So, we will not have pixel row address, but actually pixel group address, and the row position of several pixels can be uniquely identified with just one row address. Simulations have shown a data compression by a factor of 2.36 with respect to a pixel by pixel readout if we use a group size composed of four pixels.

To estimate the data that a pixel plane generates, we used the data compression scheme which includes alignment by timestamp and group reading formed by the data of four pixel cells. The readout data is composed of time stamp, group row and column address and two more bytes of pulse heights, each byte with two pulse heights. The pixel size is $50 \mathrm{x} 400 \mu \mathrm{m}^{2}$. Other assumptions used in the simulation were:

1 . There are no noise hits

2 . Charge carriers: electrons

3 . Threshold: 2000 electrons

4. Magnetic field of $1.6 \mathrm{~T}$

5 . Minimum bias events with two interactions per $132 \mathrm{~ns}$ bunch crossing.

With these assumptions, we obtained a data rate for a pixel plane half of $2.5 \mathrm{GBits} / \mathrm{s}$. We will now assume that the data rate for the fiber optic cables is $1.06 \mathrm{GBits} / \mathrm{s}$, and we can now estimate the number of fiber-optic cables required per half plane. Assuming a safety margin of four, we obtain ten fiber-optic data links per half, or five per quadrant.

The Quadrant Processors will have inputs from three groups of five fiber-optic cables coming from the quadrant. These quadrants are the three adjacent quadrants that form a triplet. There will be a total of 124 Quadrant Processors. The main functions of the Quadrant Processors are:

- Sort the data from the pixel detector by time stamp

- Store the data from the pixel detector in its internal buffers until a decision from the Global Trigger

- Find the center of mass of the pixel hits

- Calculate the minivectors from the triplet and send the data to the Track processor

- After a decision from the Global Trigger, if the event was accepted, deliver the data to the DAQ. Otherwise, just discard the event and clear the event buffer. 


\subsection{Assembly issues}

\subsubsection{Introduction}

The assembly design for the BTeV pixel detector system is dominated by the need to keep the amount of material to a minimum. This requirement is motivated by the adverse effects of multiple coulomb scattering on the achievable spatial and mass resolution. The former parameter is the most critical, since it influences many elements in the final capability of BTeV: separation of decay vertices from interaction vertices, trigger efficiency and enrichment, signal to background levels, proper time resolution, and sensitivity to multiple interactions per crossing. The mass resolution is also important, but mostly influences just the signal to background quality of BTeV data.

Counter to the need to keep materials at a minimum are the need for reproducible, stable position-determining supports, the need to remove significant amounts of heat directly from the active sensor areas, the need to move the detectors back from the interaction region during injection and machine-study periods, and the need to reposition the detectors reliably and accurately for physics data-taking.

In addition to these design criteria, we have to take into account that the pixel readout chips are limited in size, and the final detector assembly must be made as a matrix of communicating, overlapping pieces. The deadspace and/or gaps between materials also need to be minimized to maintain good efficiency for multiparticle charm and, especially, beauty decay modes of interest.

\subsubsection{Interconnections}

In principle, each half of a pixel plane could be made from a monolithic sensor with a single large readout device bump bonded to it for signal processing. In practice, this concept cannot be used for many reasons. The size of readout chips cannot be arbitrarily large due to the reticle sizes which are repeated to pattern a full wafer. In any event, yields on sensors and readout devices are such that the cost of making pieces so large would be more than prohibitive. Furthermore, the radiation dose varies dramatically from the nearbeam region to the edge of each plane (approximately as the distance to the power 1.7 according to experience at CDF). Thus, various regions of sensor need different bias voltages to achieve depletion (as required for sensitivity), and the local breakdown voltages may also vary with radiation dose. Thus, we are necessarily drawn to a matrix of devices and their interconnections.

Current FPIX1 prototypes have sensitive areas of up to 7.4 by $8.0 \mathrm{~mm}^{2}$. There is additional area on one edge for control and I/O circuitry, and for wire-bond pads. We may be able to increase the readout chip size somewhat, but the basic final element is not going to be much different from what we have now. We do plan to make many of the readout chips double size (back-to-back) and use larger sensors with multiple readout chips in the final detector. This can reduce the complexity of the interconnection scheme to a certain extent. 
Nevertheless, each chip will have a full set of pads for interconnection, and the density of control and readout lines remains very high.

In order to gain experience on the technical issues, we are making a five-readout-chip sub-assembly with flexible cable interconnections. This will test industrial capability and allow us to get some design experience in this area.

Fallback options include the more ambitious, and technically challenging solution using traces on the sensor with bump bonds in place of wire bonds for readout-chip-to-control and output-line connection. This technology, referred to as MCM-D technology, is being pursued as an option by the ATLAS collaboration [35].

\subsubsection{Pixel Plane High Density Circuitry Interconnect}

The pixel detector design is severely bounded by several constraints which reflects on the choices of high density cables and pixel plane circuitry interconnect. Some of these constraints are mass, outgassing, distribution of the high voltage for detector bias, radiation exposure, reliability, and cost.

In a pixel detector, the readout chips are interconnected and the output signals are routed to the edges of the pixel planes. There, the output signals are wire bonded to the fiber optics data serializer integrated circuit for data readout.

In this application, the circuit density of the pixel module circuit interconnect is highly associated with the readout speed of the pixel chip. All data generated inside the pixel chip has to be readout for the lowest level trigger decision. In order to accommodate reasonable readout throughput, several readout buses will have to routed from the pixel chips to the data serializers. Based on the space available for routing it is easy to recognize that very high density circuits need to be employed.

We are presently researching the usage of low-mass flex-circuit interconnect. A preliminary analysis and consulting with vendors strongly suggest that this approach should effectively meet the constraints already outlined. For example, Kapton, one of the polyimide dielectrics used for flex-circuit manufacturing supports as much as $118 \mathrm{KV} / \mathrm{mm}$, which is very good for the high voltage distribution of the detector bias. Other advantages include low mass (the radiation length $X_{o}$ of Kapton is $286 \mathrm{~mm}$ ), reasonable cost, radiation tolerance, etc.

We have researched a number of flex-circuit manufacturers and decided to work in conjunction with Fujitsu Computer Package Technologies, a division of Fujitsu, on the design, characterization and production of very high density flex-circuits for the pixel-plane interconnect. Fujitsu's nominal design rules include $25 \mu \mathrm{m}$ hole diameter and $20 \mu \mathrm{m}$ line width and spacing in four or more metal layers which represents the state of the art in this technology.

The present proposal for the interconnect is to glue flex-circuits on the top of the pixelplane substrate, possibly a carbon-fiber plate, which will hold the detector and associated electronics. A similar approach is being pursued by D0 for the silicon detector hybrids and in the implementation of the majority of the pixel plane interconnect for ATLAS [36]. We estimate that the following four layers of flex-circuit would suffice: 
- two layers for signal interconnects,

- one more layer for power and other signals and

- one layer for the ground plane.

These layers are quite thin and can be kept within $5 \mu \mathrm{m}$ of copper thickness. However, substantial mass can be saved if we can implement a three layer circuit, two with signals and power and the third, a ground plane made of aluminum. We will investigate the possibility of depositing a thin aluminum layer on the top of the carbon fiber substrate, use this layer as the ground plane, and laminate and interconnect the flex-circuit directly to this substrate. We are also researching vendors that could manufacture aluminum-on-polyimide flex-circuits.

As our first attempt to build a pixel module, we are presently laying out a five-chip pixel module using the FPIX1 readout chip. Figure 5.23 shows the progress on the layout. The wire bonding pads of three FPIX1 chips are already interconnected in this layout. These chips will be bump bonded to a detector and wired bonded to a high density flex-circuit interconnect. Both the chips and the circuit will be glued to a G10 board. The characteristics of this circuit interconnect will be the following:

- Metal layers: $5 \mu \mathrm{m}$ thick $\mathrm{Cu}$, with $20 \mu \mathrm{m}$ line and space design rules. The first metal layer is capped with $2 \mu \mathrm{m}$ of $\mathrm{Ni}$, and $1 \mu \mathrm{m}$ for wire-bondable gold.

- Film: $50 \mu \mathrm{m}$ thick Upilex SGA (which is comparable to Kapton)

- Film via: $25 \mu \mathrm{m}$ via on $108 \mu \mathrm{m}$ capture pad.

- Lamination: $50 \mu \mathrm{m}$ epoxy

- Z-vias: $200 \mu \mathrm{m}$ via on $350 \mu \mathrm{m}$ capture pad. Via filled with $S n S b_{5}$ solder.

- Size: $27 \mathrm{~mm}$ x $54 \mathrm{~mm} \times 0.2 \mathrm{~mm}$

We intend to test this pixel module for several important performance characteristics: noise, threshold dispersion and crosstalk. Specifically for the flex-circuit itself, we intend to measure the electrical and mechanical performance $[36,37]$ and the effect of radiation, thermal cycle, and outgassing in vaccum.

Future research for the pixel plane interconnect will include technologies like multi-chipmodule interconnect type D (MCM-D) and silicon (or possibly diamond) module hybrid to allow greater use of bump bonding and reduce the number of wire bonds. However, important issues such as high voltage distribution need to be carefully considered. 


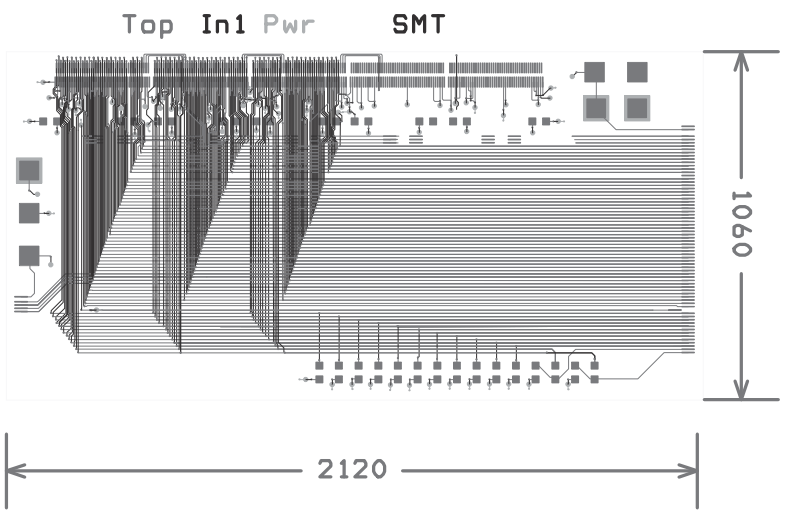

Figure 5.23: Schematic layout of the High Density interconnect prototype. Units are given in mils (0.001 inch).

\subsubsection{Mechanical and cooling Issues}

The mechanical design is driven by the goal of minimizing the material needed to achieve a satisfactory solution. Carbon-carbon, beryllium and CVD diamond structures appear most suited. We are focusing on plates attached to both sides of cooling pipes. The plates would only be required in the region where they support the pixel devices, with enough periphery to support control chips and pixel-plane-output cables. Rigid support to the outside world can, thus, be limited to a minimum, say the corners of the half-planes.

We will operate with a thermal budget of 40 microwatts per pixel, and doing the engineering as if all the sensors would be placed on the two sides of the above sandwich structure. Furthermore, the sensors will have to be operated at around $-10^{\circ} \mathrm{C}$ and the temperature for each half-plane have to be maintained uniformly to within $\pm 1^{\circ} \mathrm{C}$. We plan to hire Energy Science Laboratories Inc. (ESLI), San Diego, to perform part of the research work for the development of the mechanics and cooling for a pixel detector plane. This contract will be divided in two phases. The first phase is the theoretical study and simulation of different options for the pixel plane mechanics and cooling, while the second phase is the actual implementation of a prototype of the most promising proposal.

Probably the most important item in this interaction will be the communication between ELSI and Fermilab. Since this is an investigative project and it may have unexpected results, we need to keep evaluating and assessing other promising solutions.

This investigation should answer questions about the ability of different substrates and cooling structures to remove heat from integrated circuits attached to their surface. Also, the mechanical stability of the structure is important for the performance. Two basic approaches are to be considered during this design study: 
a) a plate that conducts the heat from the integrated circuits to one or more edges that are attached to a cold sink,

b) a plate that has the cooling channels embedded.

Several different schemes can be envisioned for the second option, such as fluid in pipes between plates, spacers with fluid flow "everywhere" inside, and "fuzzy" carbon spacer with fluid everywhere. Figure 5.24 shows the assembly of pixel plane with pipes between plates.

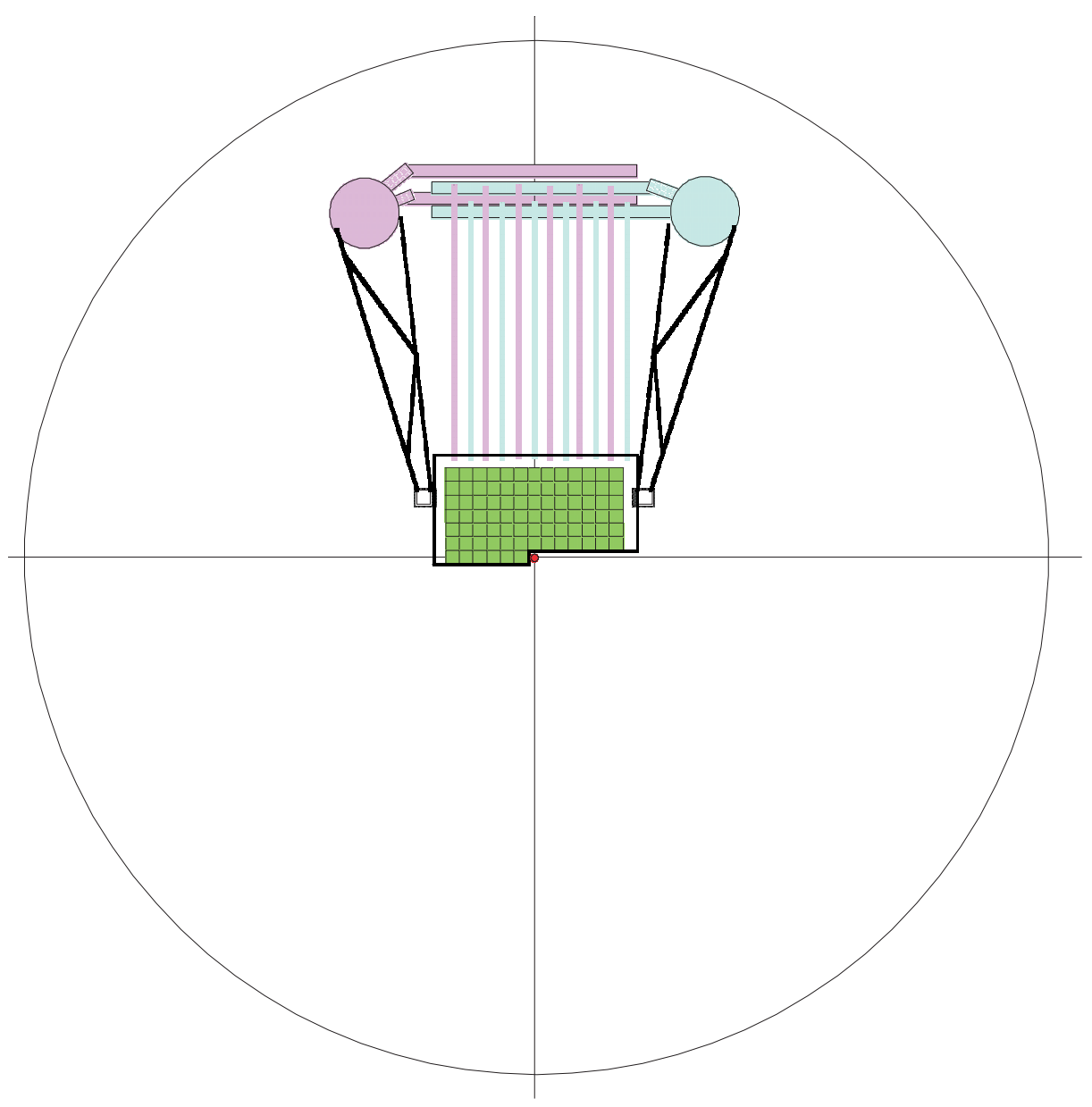

Figure 5.24: Schematic drawing of the pixel plane with cooling channels between the plates

A report from ESLI for the first phase will include the estimated performance of cooling systems and design recommendations, judgements on the feasibility of the economical construction of each option, estimates of the pressure drop of the cooling fluid, heat transfer properties, stiffness, vibration modes, reliability of piping, and temperature profiles. From these, the best solution will be picked.

In the second phase, a prototype of the best solution will be constructed. This model does not need to be a full working unit. However, it will contain enough of the features of the proposed design that it can be tested and compared to simulations. 


\subsubsection{RF shielding issues}

The pixel detector will be installed inside the beam pipe enclosure in the $\mathrm{C} 0$ interaction region. The inside of the beam pipe is, in several respects, an aggressive environment for operation of a large, sophisticated detector. We would like to carry out a series of tests both in the laboratory as well as in the Tevatron to study the rf pickup and the shielding that will be required. Our objective with these tests is to investigate the electro- and magnetic (EM) interaction of the Tevatron beam with pixel detectors and associated electronics. When the beam passes close to the pixel detectors and electronics, it generates the equivalent of an electromagnetic pulse which can induce noise and cause other problems in the electronics. Shielding is required to protect the electronics from this pickup. This shielding has traditionally been a metallic skin and adds to the material budget. We are pursuing two kinds of tests, one in a simulator of the Tevatron beam in a laboratory set-up, the other inside the Tevatron itself. We feel that these tests will give us very important insights and will help us to generate guidelines for the design and shielding of the pixel system.

\subsubsection{Beam simulator}

An apparatus has been built to simulate the rf environment of the Tevatron. The basic structure of the test stand is a 5' long cylindrical coaxial pipe made out of stainless steel. It will be used to simulate the electromagnetic field from a bunch of beam particles. The electronics to be tested will be inserted through a slot in the outer conductor. An rf amplifier is used to generate the required current, estimated to be about 8.4Amp. Along with the beam simulator, we have constructed a computer driven test circuit which will feed a controlled bit pattern across a variety of signal paths inside the simulator apparatus. We also anticipate testing various shielding techniques. Our goal is to determine the level of rf pickup in the test circuit and to determine which signal path designs and shielding configurations minimize noise while keeping material use at a minimum. The results of these tests will then be applied to produce a prototype rf shield (if needed) to be tested in the $\mathrm{C} 0$ interaction region.

\subsubsection{Roman Pot Tests for BTeV}

We plan to install an existing Roman Pot and castle from E811 into the Tevatron. The pot will make it easy for us to insert various test devices into the region of the beam and make the desired measurements. We will make measurements with pixel detectors and chips placed closed to the beam, and study methods to decrease the EM interference of the beam on the electronics. Our current plans are for a two-year investigation. This will start with simple devices to make EM measurements. Each test will build on the previous tests. The goal is to construct sophisticated electronic test devices with active circuitry similar to whar we could use in the real detector. We hope to learn how to construct a small test pixel detector and take data from it.

The following topics will be investigated:

- layout issues and line terminations; 
- rf shielding: its shape, thickness, material, grounding, and support;

- power supplies: their design, location and distribution

Theoretical analysis of the susceptibility of layout traces and transmission lines will be conducted as a first stage of design, to evaluate electromagnetic compatibility rules between the detector and the surrounding media. In such an analysis, it is very difficult to consider all the variables affecting the detector design. Physical prototypes of both general and critical parts of the detector will help to understand the final EM susceptibility of the detector.

We envision two options for the location of the power supplies: outside and inside the enclosure. The first option simplifies the design and procurement of power supplies, but imposes restriction on the distribution cables. The cables can act as EM pick-up elements conducting noise into the detector or generating conductive paths between the different layers of the detector. In these tests we will be able to analyze the performance of this topology, the level of filtering and shielding, and the input-output isolation of the power supplies. Note that the addition of filtering and shielding may require too much mass for the experiment.

The second option imposes more challenges due to the need of locating DC-DC converters near the detector to break conductive paths and decrease the EM pick-up. The pixel detector will be subject to strong magnetic field, which does not allow any magnetic material in the design of such converters. Research will be conducted to evaluate the option of using DC-DC converters based on piezoelectric ceramic transformers [38, 39] and the use of high frequency air core transformers.

\subsubsection{Material budget}

Table 5.4 summarizes our current understanding of the material budget for one single pixel plane. Each pixel plane will consist of a number of modules. Each module may consist of 4 to 7 readout chips. Figure 5.25 illustrates the way we envisage each plane will be assembled. To account for non-sensitive area in each pixel sensor required for guard rings and scribe edge, the table assumes a $25 \%$ overlap of modules on opposite sides of the substrate (this overlap is not shown in the figure). Our baseline design calls for a $5 \mathrm{~mm}$ thick Be substrate with integrated cooling channels.

\subsubsection{Outlook}

Between the time of this PTDR and a full TDR, we anticipate having results from the various prototypes and the engineering studies described above. These will be used to develop engineering calculations and assembly drawings of an actual BTeV pixel detector - optimized for minimum material and stability of positioning. We do anticipate final alignment using charged particles from real physics events for each run (each repositioning of the pixel halfplanes), including whatever is needed to make the trigger work effectively. 


\begin{tabular}{|c||c|c|c|}
\hline Item & Thickness $(\mathrm{mm})$ & $X_{0}(\mathrm{~mm})$ & $X / X_{0}(\%)$ \\
\hline Sensor & 0.25 & 93.6 & 0.30 \\
\hline Readout chip & 0.15 & 93.6 & 0.20 \\
\hline Bumps and wire bond & 0.02 & 10.0 & 0.004 \\
\hline HDI & 0.224 & 284 & 0.08 \\
\hline Components on HDI & & & 0.04 \\
\hline Glue & & & 0.02 \\
\hline Coolant(water/alcohol) & 0.35 & 40 & 0.02 \\
\hline Substrate $($ Be $)$ & 0.5 & 353 & 0.14 \\
\hline Total & & & 0.80 \\
\hline
\end{tabular}

Table 5.4: Material budget of a BTeV Pixel plane. This assumes that we can build a pixel plane using only one substrate. Within a plane, we assume that there is a $25 \%$ overlap in sensor or readout chip, that the HDI has $100 \%$ coverage and that the cooling fluid and cooling channel covers $20 \%$ of the substrate. Each HDI has 4 layers with each layer consisting of $50 \mu \mathrm{m}$ kapton and $6 \mu \mathrm{m}$ of $\mathrm{Cu}$.

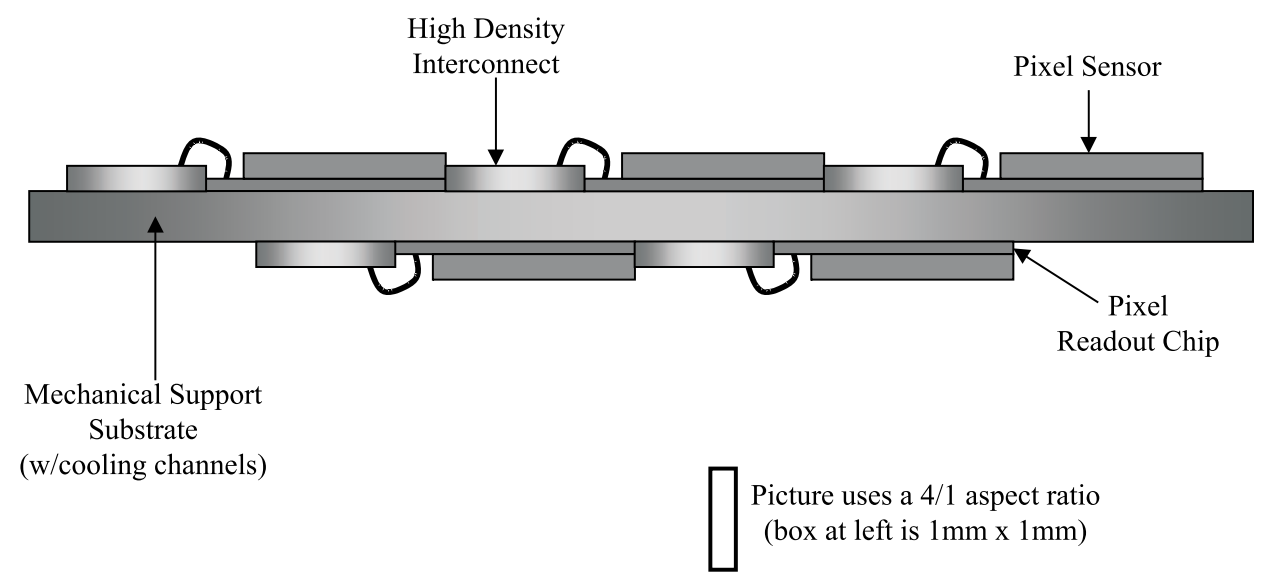

Figure 5.25: Schematic of the assembly of a pixel plane. 


\subsection{Pixel Beam Test}

Beam tests of prototype pixel detectors will take place during the 1999 fixed target run. During this six month period it is planned to test silicon sensors bonded to both FPIX0 and FPIX1 readout chips and a diamond sensor bonded to an FPIX0 chip.

\subsubsection{Goals}

\section{FPIX0}

- To measure the efficiency, charge sharing and resolution as a function angle of incidence, number of ADC bits, and readout threshold. The effect of magnetic field will also be studied.

\section{FPIX1}

- To test the fast readout in a high intensity beam.

- To study the resolution as a function angle of incidence, threshold, and different sensor designs.

\section{Diamond Sensor}

- To determine whether diamond pixel detectors bonded to FPIX0 chips provide good efficiency, resolution, uniformity, and signal to noise.

\subsubsection{Test Stand}

The test stand is shown in Fig. 5.26. The central aluminum box at the bottom of the figure has slots for mounting pixel test boards at several different angles. Two triplets of silicon strip detectors form a telescope for tracking the beam particles.

\subsubsection{Silicon Telescope}

There are six planes of $20 \mu \mathrm{m}$ pitch silicon strip detectors which are read out with SVX2b chips. A VME based data acquisition system has been adapted from a CDF test stand. It consists of an SGI workstation, a VME CPU, a Silicon Test Acquisition and Readout board (STAR), a Test Fiber Interface board (TFIB), and a Test Port Card (TPC). The STAR

has 3 data buffers which can accept data from the SVX2b chips via the TPC. Simulations indicate that we should be able to project track locations at the pixel detector with $4-5 \mu \mathrm{m}$ precision. 


\subsubsection{Pixel Readout Board}

The pixel readout has been designed to be integrated with the existing SVX2b readout. An RS485 VME interface board is used to send control signals to the pixel readout chip via a pixel readout board. The pixel readout board is physically separated into two boards - an inner board on which is mounted the pixel sensor bonded to the readout chip, an 8-bit flash $\mathrm{ADC}$ and power supplies, and an outer board which has all the readout control components. The outer board can accept a trigger either via a NIM or ECL input or through the RS485 cable. After receiving a trigger, the data is read out into one of the STAR buffers. The same outer board can be used for either FPIX0 or an FPIX1 chip. The inner board will be different for each readout chip. For example, the FPIX0 board has a flash ADC whereas the FPIX1 chip itself has a 2-bit ADC in each pixel.

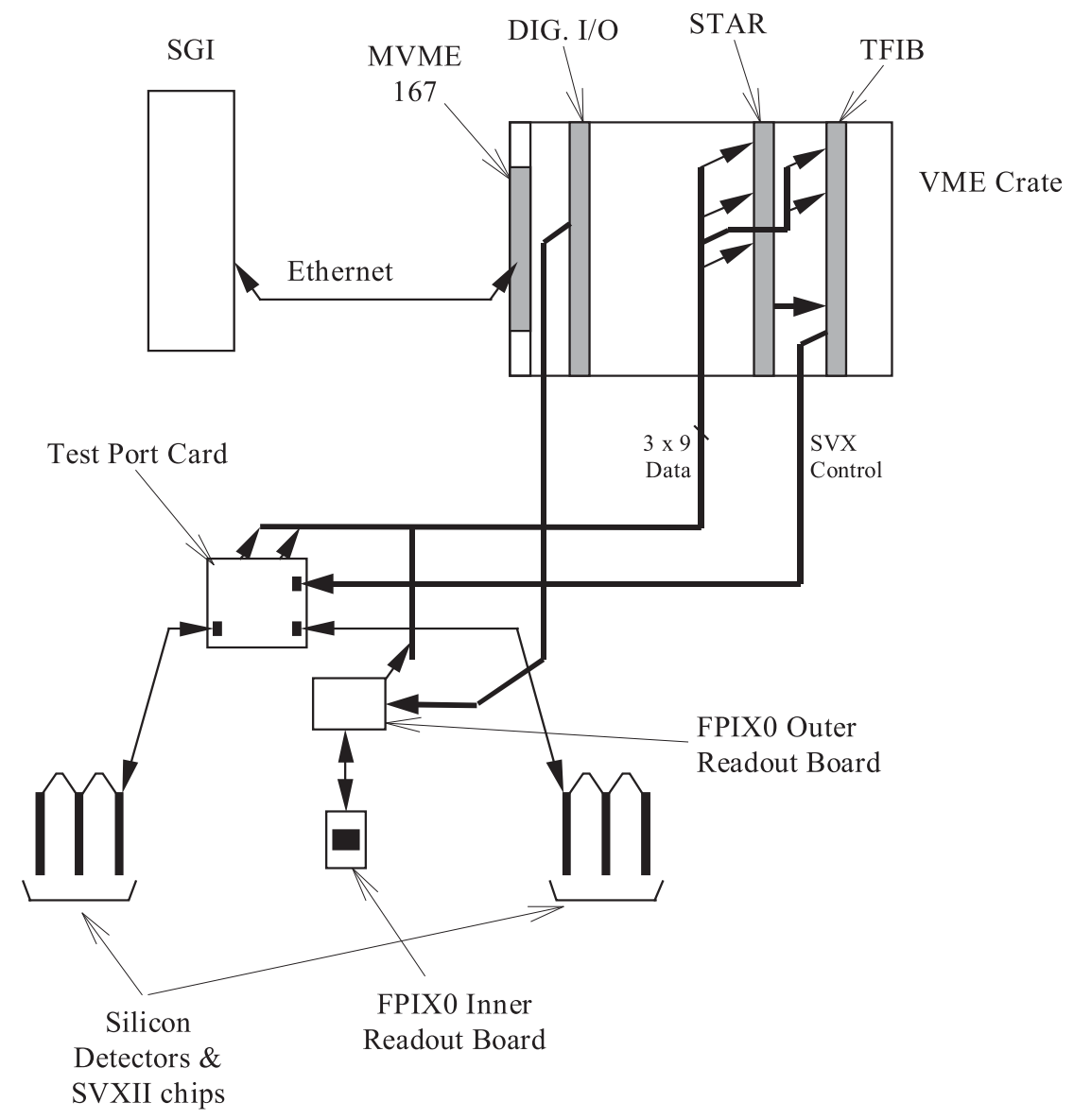

Figure 5.26: Test Stand for Pixel Beam Test. 


\section{Bibliography}

[1] D. Anderson and S. Kwan, Proc. of Pixel98 - International Pixel Detector Workshop, May 7-9 1998, Fermilab-CONF-98/196.

[2] B.S. Avset and L. Evensen, Nucl. Instrum. Meth. A377 (1996), 397.

[3] The ATLAS Pixel Detector Technical Design Report, CERN/LHCC-98-13.

[4] The CMS Tracker Project Technical Design Report, CERN/LHCC-98-6.

[5] R.H. Richter, Nucl. Instrum. Meth. A377 (1996), 412.

[6] Marina Artuso and Janchun Wang, BTeV Internal Report BTeV-Int-97-18, February 1998

[7] E. Belau et al, Nucl. Instr. and Meth. 124, (1983) 253.

[8] R. Turchetta, Nucl. Instr. and Meth. A 335, (1993) 44.

[9] L.H.H. Scharfetter (RD19), Active Pixel Detectors for Large Hadron Colliders, CERN Thesis, 1997.

[10] A. Mekkaoui, Talk given at the 3rd International Meeting on front-end Electronics for High Resolution Detectors, Taos, NM (1997).

[11] Penny Kasper, BTeV Internal Report BTeV-int-98/3, March 1998.

[12] G. Cancelo et al., BTeV Internal Report BTeV-int-98/16, September 1998.

[13] Penny Kasper, BTeV Internal Report BTeV-int-99/4, March 1999.

[14] G. Lutz et al., Talk Presented at the Workshop on Vertex Detectors: State of the Art and Perspectives, Erice, Sicily, (1986).

[15] P. Fisher et al., ATLAS Internal Note INDET-NO-086 (1994).

[16] M. Artuso, Presented at 29th International Conference on High-Energy Physics (ICHEP 98), Vancouver, Canada, 23-29 Jul 1998, Syracuse Un. Preprint HEPSY-98-2, (1998). 
[17] Technology Modeling Associates, Inc., Sunnyvale, CA

[18] S. Parker, talk given at Fermilab (1998).

[19] S. Seidel, "Pixel Sensors for ATLAS", in Proc. if Pixel98 Workshop, Fermilab, Batavia, IL, May 1998, ed. D. Anderson and S. Kwan, Fermilab-CONF-98/196, 263.

[20] M. Da Rold et al., IEEE Trans. Nucl. Sci. 44 (1997), 721.

[21] C-Y. Chien, private communication.

[22] Z. Li et al., IEEE Trans. Nucl. Sci. 45 (1998) 348.

[23] Z. Li et al., Nucl. Instrum. Meth. A408 (1998).

[24] D.C. Christian, et al., "Development of a Pixel Readout Chip for BTeV",FERMILABCONF-98-354, 1998. To be published in the proceedings of the $7^{\text {th }}$ International Workshop on Vertex Detectors (VERTEX'98).

[25] S. Zimmermann, et al.,"High Readout Speed Pixel Chip Development at Fermilab", FERMILAB-CONF-98-278, 1998. Published in Rome, 1998, Electronics for LHC Experiments, 528.

[26] A. Mekkaoui, et al., "Results from an FPIX0 Chip Bump Bonded to an ATLAS Pixel Detector", FERMILAB-CONF-98-278, 1998. Published in Rome, 1998, Electronics for LHC Experiments, 599.

[27] L. Blanquart, et al., Nucl. Instrum. Meth. A395 (1997), 313.

[28] M. Artuso and J. Wang, "Some Further Studies on Factors Affecting the Pixel Resolution", BTeV-int-98/13, BTeV Internal note.

[29] W. Snoeys, "Radiation tolerance beyond 10 Mrad for a pixel readout chip in standard submicron CMOS", Proc. of the 4th Workshop on Electronics for LHC Experiments, Rome, September, CERN/LHCC/98-36, 114.

[30] R. Gomez, M. Johnson, and M. Baert, "Preliminary studies of the use of Vertical Cavity Surface-emitting lasers for data transmission in High Energy Physics", Fermilab-Pub$97 / 098$.

[31] I. Bhandal, "Optical Fiber for telecommuncations", presented at the 2nd Workshop on Optical Readout Technologies for ATLAS, Oxford University, England, Jan. 7-8, 1999.

[32] J. Hall, "Packaging for a high-radiation environment",presented at the 2nd Workshop on Optical Readout Technologies for ATLAS, Oxford University, England, Jan. 7-8, 1999. 
[33] P. Denes, "Radiation hard gigabit systems", presented at the 2nd Workshop on Optical Readout Technologies for ATLAS, Oxford University, England, Jan. 7-8, 1999.

[34] N. Koopman et al., "Fluxless no-clean assembly of solder bumped flip chips", Presented at 46th ECTC 1996, Orlando, Florida, May 28-31, 1996.

[35] P. Gerlach, "MCM-D Technology for Pixel Detector Modules", in Proc. of the Pixel98 Workshop, Fermilab, Batavia, IL, May 1998, ed. D. Anderson and S. Kwan, FermilabCONF-98/196, 497.

[36] P. Skubic et al., "Flex Circuits for the ATLAS Pixel Detector", in Proc. of the Pixel98 Workshop, Fermilab, Batavia, IL, May 1998, ed. D. Anderson and S. Kwan, FermilabCONF-98/196, 519.

[37] K. Neriyanuri, "ATLAS Pixel Detector High Density Inerconnect design and testing of ASIC analog multiplexer", Master Thesis (unpublished), 1997.

[38] M. Imori et al., "A High voltage Supply Using a Piezo Ceramic Transformer", IEEE Nuclear Science Symposium, 1995.

[39] Chih-yi Lin, "Development of a piezoelectric transformer converter", Virginia Power Electronics Center, Current Magazine, Spring 1993. 


\section{Chapter 6}

\section{Forward Tracking System}

\subsection{Introduction}

The forward charged particle tracking system will play a central role in nearly every physics analysis in $\mathrm{BTeV}$. The major functions of the system are to measure momenta for tracks found in the pixel system, to measure all parameters for tracks which do not pass through the vertex detector (such as $K_{s}$ and $\Lambda^{0}$ daughter tracks), and to project tracks into the RICH systems and EM calorimeters. Measurements from the forward tracking system can also be used at Level 2 of the trigger to greatly improve the momentum estimate for muon tracks found in the muon spectrometer at Level 1. Level 2 triggers can use forward tracking information to link these tracks to the pixel system and associate them with vertices.

The requirements of the forward tracking system are:

1. Provide tracking coverage in each arm in the angular range $\theta= \pm 10 \mathrm{mrad}$ to $\theta= \pm 300$ mrad over a single arm distance of $7 \mathrm{~m}$.

2. Achieve a bend-view resolution of $\leq 100 \mu \mathrm{m}$ (per station). This corresponds to a momentum resolution of $\sim 1 \%$ for $100 \mathrm{GeV}$ tracks reaching the last measurement station just before the EM calorimeter.

3. Provide robust pattern recognition for charged particles, in conjunction with the pixel system.

4. Materials in the sensitive detector region should be minimized in order to reduce the effects of multiple Coulomb scattering, ionization energy losses, and hadronic secondary interactions. A design goal is to introduce less than $1 \% \mathrm{X}_{0}$ per measurement station.

5. Separate information from different beam crossings. This means that the maximum drift time must be less than the time between bunch crossings (132 ns).

6. The detectors should be robust mechanically and electrostatically but should be serviceable during accelerator down times. This implies an ability to remove chambers without removing the beam pipe. 
7. Cost effective, proven technologies should be used to the extent possible.

Meeting these requirements in a forward hadron collider environment will be challenging. One reason is that the particle flux varies by three orders of magnitude over the tracking volume. Sensitive detector elements must be kept short in the high occupancy regions. Since the tracking volume is quite large (the most downstream chamber covers an area $4.2 \mathrm{~m}$ by $4.2 \mathrm{~m}$ ), we require large area, low mass detectors such as drift chambers, straw tube detectors, or honeycomb drift chambers in the forward region. One finds that maintaining short sense wire lengths in the high occupancy region while minimizing the amount of material associated with the necessary readout and detector services (electronics connections, gas manifolds, module/wire supports) becomes especially difficult. We considered straw tube configurations which placed shorter straws in the high occupancy region, growing longer with radial distance from the beam pipe. These solutions however always resulted in unacceptable amounts of material in the sensitive detector volume. Our conclusion was that a divided geometry which employs high granularity detectors with low mass readout in the inner region and large area tracking devices for the outer region was necessary to achieve acceptable occupancy levels while maintaining good momentum resolution.

\subsection{Detector Geometry}

The baseline BTeV design consists of 14 tracking stations (7 in each arm) employing straw drift tube detectors and silicon microstrip detectors. The straw tracker provides large area outer coverage and is comprised of planar straw tubes arranged in three measurement views: $Y$ (vertical, bend view), and two stereo views $U$ and $V$ oriented at \pm 45 degrees to $X$ (these stereo angles are still under study). Each measurement view consists of three physical layers of $4 \mathrm{~mm}$ diameter straw tubes in a close-packed configuration. Two such layers are required to resolve the left-right ambiguity in the drift-time coordinate measurement. The third layer provides redundancy and increases the efficiency of the coordinate measurement. The first three measuring stations nearest the interaction region cover only the outer tracking region while the downstream stations have an inner square aperture with dimensions corresponding to the $\pm 10 \mathrm{mrad}$ acceptance limit. The details of the forward straw tracker are summarized in Table 6.1.

In the first three tracking stations on either side of the dipole, silicon microstrip detectors cover the high occupancy region near the beamline. Design work for the silicon portion of the forward tracker has only recently begun. We have performed studies both with three planes of single-sided detectors, and with two planes of double-sided detectors. We simulated singlesided detectors with 300 micron pitch (resulting in a factor of 10 reduction in hit occupancy with respect to straw tubes) and covering an area of $24 \mathrm{~cm}$ by $24 \mathrm{~cm}$ arranged as three views: $X, Y$ and a 45 degree stereo view. While single-sided detectors have an advantage in being simpler in design, the main disadvantage is the additional material required to make the same number of position measurements. Since keeping material to a minimum is one of our primary concerns, our baseline proposal is to employ two planes of $300 \mu \mathrm{m}$ thick 
Table 6.1: Properties of the baseline forward straw tracker

\begin{tabular}{|l|l|}
\hline \hline Property & Value \\
\hline Straw size & $4 \mathrm{~mm}$ diameter \\
Central hole & $5 \mathrm{~cm} \times 5 \mathrm{~cm}$ to $24 \mathrm{~cm} \times 24 \mathrm{~cm}$ \\
Total Stations & $14(7$ per arm $)$ \\
$Z$ positions $(\mathrm{cm})$ & $96,146,196,296,341,386,706$ \\
Half size $(\mathrm{cm})$ & $30,45,60,90,105,118,210$ \\
Views per station & 3 \\
Layers per view & 3 \\
Total number of straws & 78132 \\
Total station thickness $\left(<L>/ X_{0}\right)$ & $0.6 \%$ \\
Readout & ASD + timing chip $(6$ bits $)$, sparsified \\
Total channels & 118,464 \\
Rate requirements & beam crossing rate is $132 \mathrm{~ns}$. \\
\hline \hline
\end{tabular}

double-sided silicon microstrip detectors with $100 \mu \mathrm{m}$ pitch. Standard sized silicon wafer modules are mounted on low mass carbon fiber support pads and are arranged in a ladder readout configuration. Four views are provided: $X, Y, U, V$ where the $U$ and $V$ stereo angles are matched to the corresponding straw tracker stereo views. The properties of the proposed baseline forward silicon microstrip tracker are summarized in Table 6.2.

The forward tracking system provides up to 63 measurement points for tracks within the $\pm 300 \mathrm{mrad}$ acceptance.

Table 6.2: Properties of the proposed baseline forward silicon tracker

\begin{tabular}{|l|l|}
\hline \hline Property & Value \\
\hline Wafers & $4 \times 6 \mathrm{~cm}^{2} / 2.5 \times 4.75 \mathrm{~cm}^{2}$ \\
Pitch & $100 \mu \mathrm{m}$ \\
Thickness & $300 \mu \mathrm{m}$ \\
Ladder modules & 12 per station \\
Coverage & $24 \mathrm{~cm} \times 24 \mathrm{~cm}$ \\
Central hole & $5 \mathrm{~cm} \times 5 \mathrm{~cm}$ \\
Total stations & $6(3 \mathrm{per}$ arm $)$ \\
Z positions $(\mathrm{cm})$ & $100,150,200$ \\
Measurement views & $4(\mathrm{X}, \mathrm{Y}, \mathrm{U}, \mathrm{V})$ \\
Channels per view & 2400 \\
Total channels & 57600 \\
Readout & double-sided, sparsified binary \\
\hline \hline
\end{tabular}




\subsubsection{Layout Considerations}

The important considerations for the forward tracking layout are:

1. Material should be kept to a minimum, to minimize both multiple Coulomb scattering and photon conversions.

2. Coordinate views should be chosen to emphasize position measurement resolution in the bend (vertical) direction.

3. Stereo coordinate views should be chosen to maximize pattern recognition capability without adversely complicating the detector module construction or significantly increasing its cost.

4. Sensitive lengths of straw tubes close to the beam pipe should be kept as short as possible in order to minimize the hit occupancy in a given straw detector.

5. The number of measurement layers per view should be chosen to maintain good efficiency while minimizing the material in the decay volume.

Our baseline layout places three stations in the fringe field region of the dipole magnet, three stations in the field free region just upstream of the RICH, and one station just downstream of the RICH.

\subsection{Performance Issues}

\subsubsection{Straw Hit Occupancies}

We anticipate large occupancies in the inner forward tracking region detectors, and have concluded that detectors with higher granularity than straws are necessary to provide adequate performance. To study the occupancies in the forward straw tracker we simulate generic $b \bar{b}$ events using the PYTHIA event generator and the MCFAST detector simulation package. Minimum bias inelastic events are added to the $b \bar{b}$ event to yield a Poisson event multiplicity distribution with a mean of two. This simulates running conditions at a luminosity of $\mathcal{L}=2 \times 10^{32} \mathrm{~cm}^{-2} \mathrm{~s}^{-1}$. Particles from hadronic secondary interactions, photon conversions, and decays in flight are included in the event sample. The occupancies for the baseline geometry are shown in Figs. 6.1-6.3 for the $Y$ layer in four of the seven forward straw tracking stations. Here we define the occupancy as the average number of charged particles traversing a straw cell in an event. The average occupancy is plotted as a function of the straw number offset from the beam center. For comparison, we show the occupancy resulting from the $b \bar{b}$ event itself.

Forward stations which are closest to the interaction region have occupancies approaching $8 \%$ for straw tubes nearest the beam pipe. If a $5 \mathrm{~cm}$ beam aperture is used, the occupancy rises to $\approx 20 \%$, which is an extremely high value. The notch pattern where the occupancy 


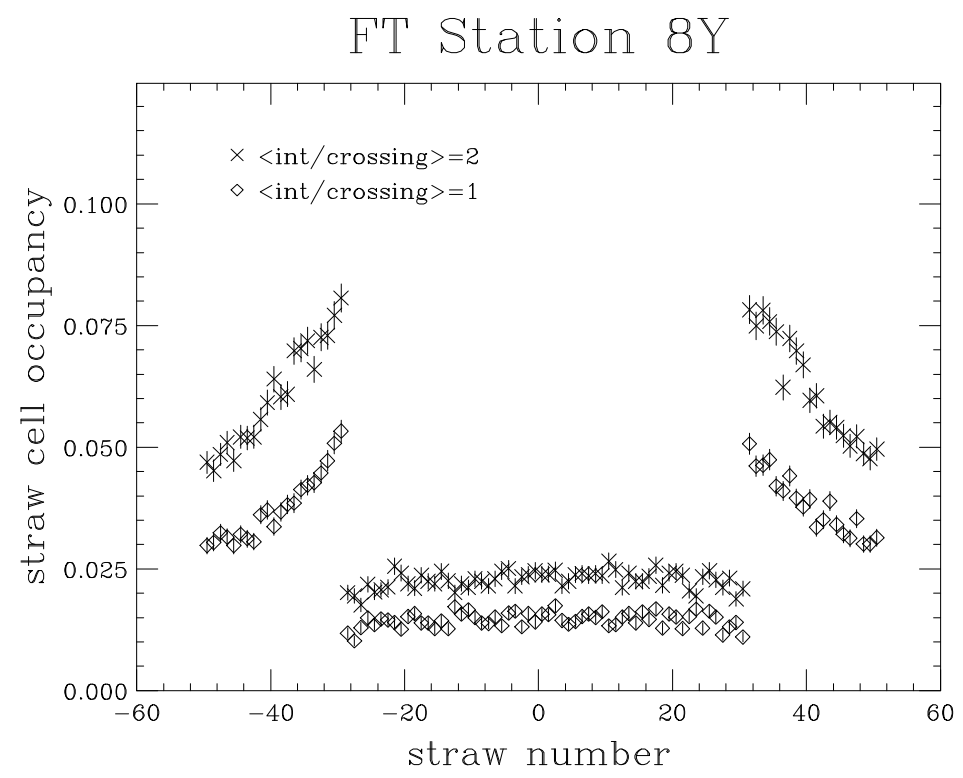

Figure 6.1: Hit occupancy for the forward straw tracking station closest to the interaction region. The occupancy is the average number of particles passing through a given tube in an event.

is reduced to about $2 \%$ results from shortening the tubes so that they end $12 \mathrm{~cm}$ from the beam. Forward silicon microstrip detectors provide coverage for this $\pm 12 \mathrm{~cm}$ region.

Figs. 6.2-6.3 show the occupancy for stations located immediately upstream and downstream of the RICH. These stations have beam pipe apertures of $\pm 3.5 \mathrm{~cm}$ and $\pm 7.0 \mathrm{~cm}$ respectively, so their straw tubes extend into the high rate region. The occupancy in the station just upstream of the RICH is close to $10 \%$, an uncomfortably large value. The track separation due to the increased distance from the interaction point results in lower occupancies for the station downstream of the RICH.

In Fig. 6.4 we plot the fraction of tracks which are shadowed as a result of the high occupancy in the station upstream of the RICH. Tracks closest to the beam pipe will be shadowed by other tracks in the event about $0.6 \%$ of the time. This can be reduced by enlarging the beam gap in the chamber, with a corresponding loss in acceptance.

It should be noted that the occupancies and shadow fractions shown are for a single layer and not for the resolved 3-layer hits. We plan to investigate ways in which the drift time information from all three layers can be exploited to recover track information from these doubly hit cells.

\subsubsection{Momentum Resolution of the Forward Straw Tracker}

In this section we study the momentum resolution of the proposed baseline forward straw tracking system. For the time being we limit our attention to multiple scattering and the 


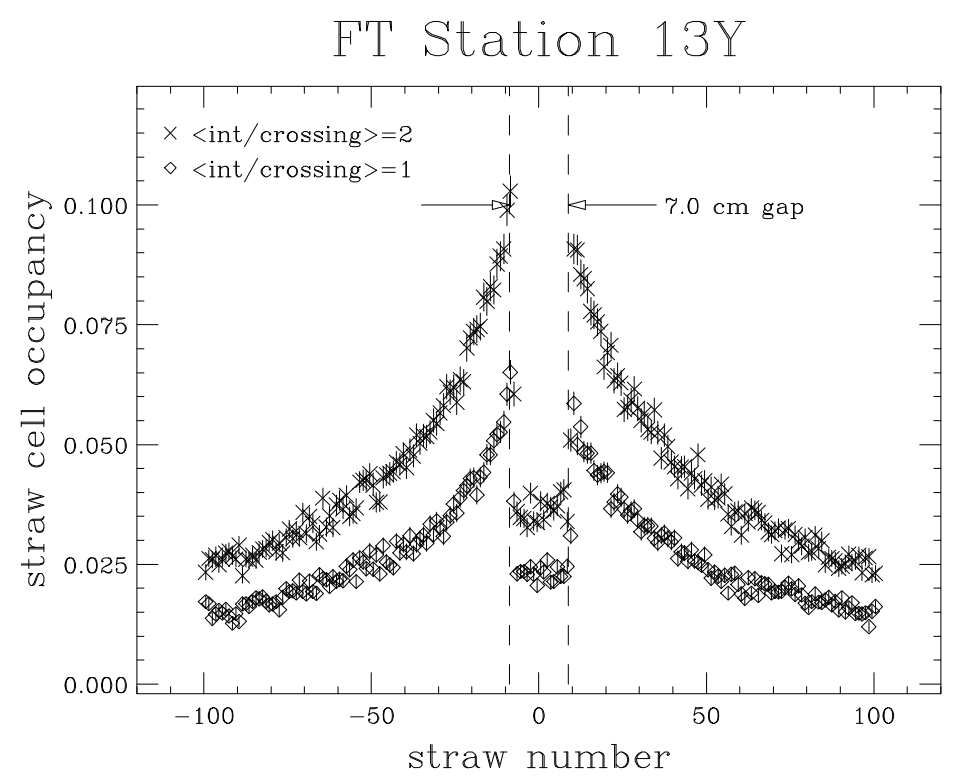

Figure 6.2: Hit occupancy for the forward straw tracking station immediately upstream of the RICH. The occupancy is the average number of particles passing through a given tube in an event.

intrinsic resolution of the straw detectors, since these are the parameters most directly affected by technology choices for the forward tracking system. Additional contributions to the resolution, to be considered later, will come from module misalignments, non-uniformities in the manufacture of detector elements, errors in the dipole field mapping, and non-linearities caused by hit adjacencies. To allow for these errors, we have assumed a conservative intrinsic straw resolution of $150 \mu \mathrm{m}$ per measurement layer. Drift-time measurement accuracy of 100-120 $\mu \mathrm{m}$ or better has been achieved by the ATLAS TRT group [9] for 4mm diameter straw cells for certain electronic threshold and signal peaking times.

Multiple scattering causes random changes in a particle's direction, producing scattering angles that vary inversely to the particle momentum. This results in a fractional momentum resolution which is roughly constant. The intrinsic position resolution of the detector produces a linear dependence of $\sigma(p) / p$ on particle momentum. These two effects contribute to the behavior seen in the following figures.

In Fig. 6.5 the fractional momentum resolution $\sigma(p) / p$ for the baseline geometry is plotted as a function of $p$ and the polar angle $\theta$ of the track at its production vertex. In order to study the performance of the straw tracking system itself, we consider only tracks which pass through the outer straw tracking planes (no forward silicon hit information is used). Furthermore, we select only those tracks which pass through at least two pixel stations, which insures an accurate track vector in the magnetic field region. We also require that tracks pass through at least three forward straw tracking stations. In Fig. 6.5 we show the effects arising from the straw materials and position resolution. 


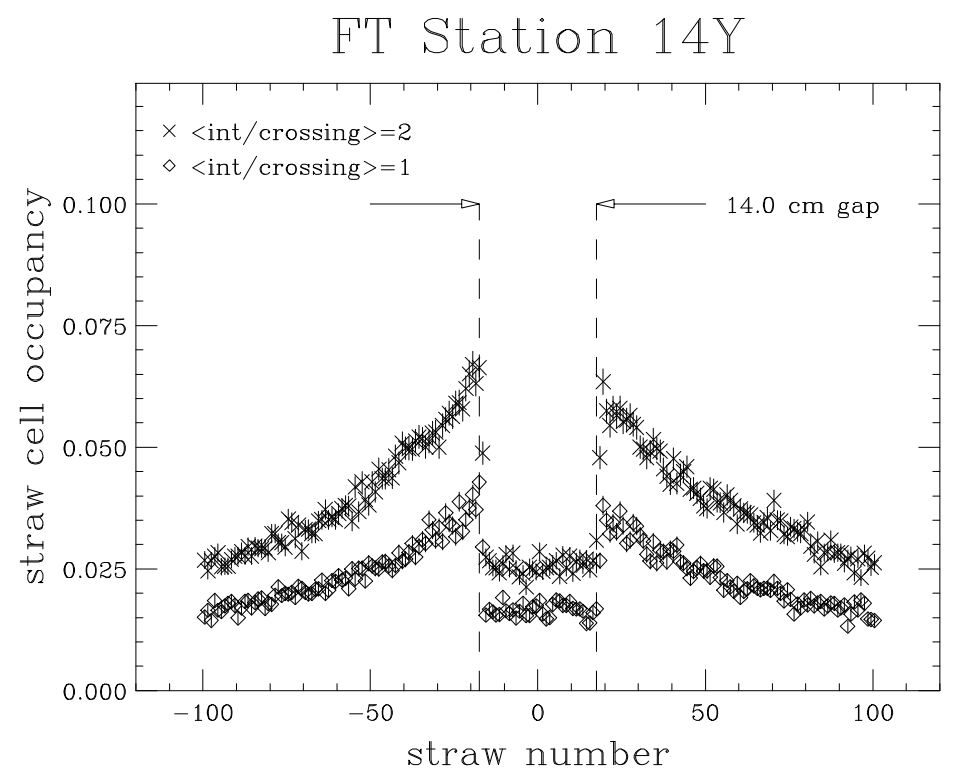

Figure 6.3: Hit occupancy for the forward straw tracking station immediately downstream of the RICH, just before the EM calorimeter. The occupancy is the average number of particles passing through a given tube in an event.

For the baseline system, the fractional momentum resolution is roughly constant (approximately $0.5 \%$ ) up to approximately $16 \mathrm{GeV} / \mathrm{c}$ at which point measurement errors begin to contribute. At approximately $35 \mathrm{GeV} / \mathrm{c}$ the contributions from multiple scattering and position resolution are about equal. The curve which plots the resolution for a straw system with zero intrinsic resolution does not approach a constant value at low momentum, since in these plots no constraint on the number of measurement points has been applied.

The bottom plot in Fig. 6.5 indicates that multiple scattering clearly dominates tracks emerging at large angles, since these are mostly low momentum tracks.

\subsection{Forward Straw Tracker}

\subsubsection{Technology Options}

There are several technology options for the outer forward tracking system. The large tracking volume $\left(420 \times 420 \mathrm{~cm}^{2}\right.$ for the most downstream station) suggests use of conventional drift chambers which offer a low mass, high precision tracking solution for designs with small drift distances (eg. $\sim 2 \mathrm{~mm}$ ). However, supporting the wire tensions over considerable lengths will lead to mechanical difficulties, and most likely will require additional support frame material in the decay region of the detector. There are additional problems: small drift distances over a long length can lead to electrostatic instabilities; space charge effects occur at very high rates; and broken wires are difficult to replace or retrieve and can disable 


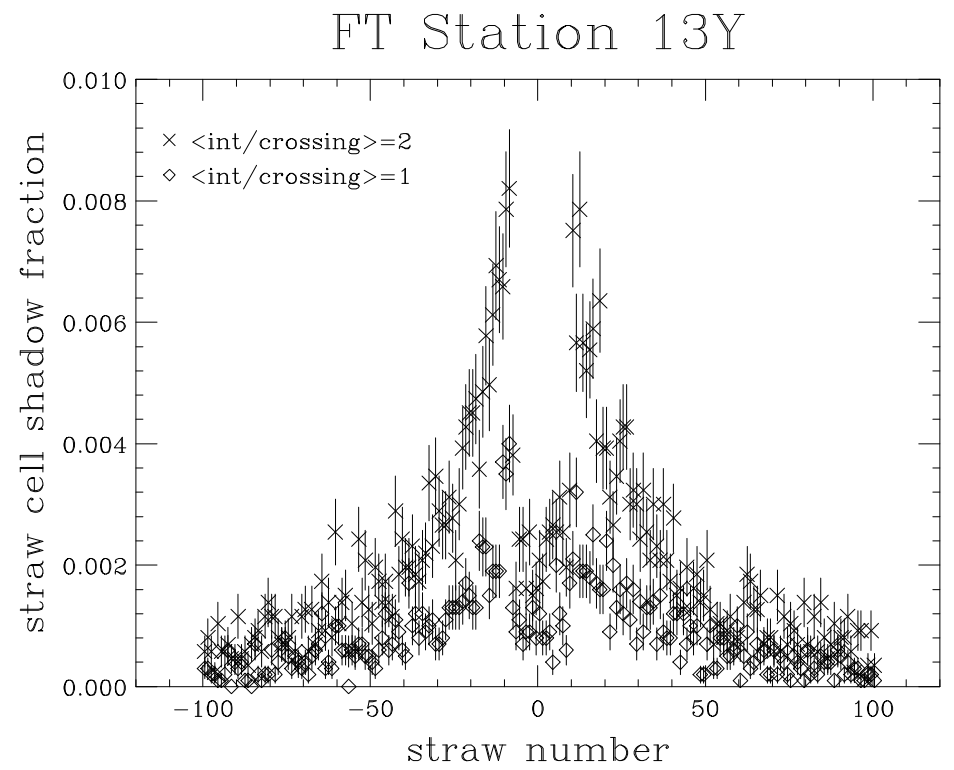

Figure 6.4: Straw cell shadow fraction for the forward straw tracking station immediately upstream of the RICH. A hit is shadowed when another track in the event passes through the same detector.

an entire chamber. These and other drawbacks of the conventional drift chamber design for high luminosity hadron collider experiments were considered in detail in Ref. [1].

Honeycomb drift chambers (HDCs) [5] can be used as a low-cost, wide area tracking detector. This technology option is being pursued by HERA-B for their outer tracking system [4]. Layers of an HDC are fabricated by folding three Kapton foils into a hexagonal structure. The result is a planar array of staggered drift cells which are mechanically stable and selfsupporting. The construction methods for HDCs are considered to be simpler and more efficient compared to straw chambers, an important consideration for very large tracking systems. However, HDCs have not been thoroughly tested in high rate environments and their long term operation has yet to be proven.

Over the past several years there has been extensive development of straw tube trackers for use in high luminosity hadron collider environments [6, 7, 8, 9]. Straw tube detectors offer the following advantages: electrical and mechanical isolation between cells, ease of wire support, the ability to operate in high rate environments, overall low cost per unit volume, ease of serviceability, and construction of lightweight, low-Z materials. These features make it a suitable baseline technology choice for BTeV.

\subsubsection{Straw Detecting Elements}

Since we believe straw tracking chambers are the most promising technology choice for the forward tracking system, we have begun a program of research and development to determine 

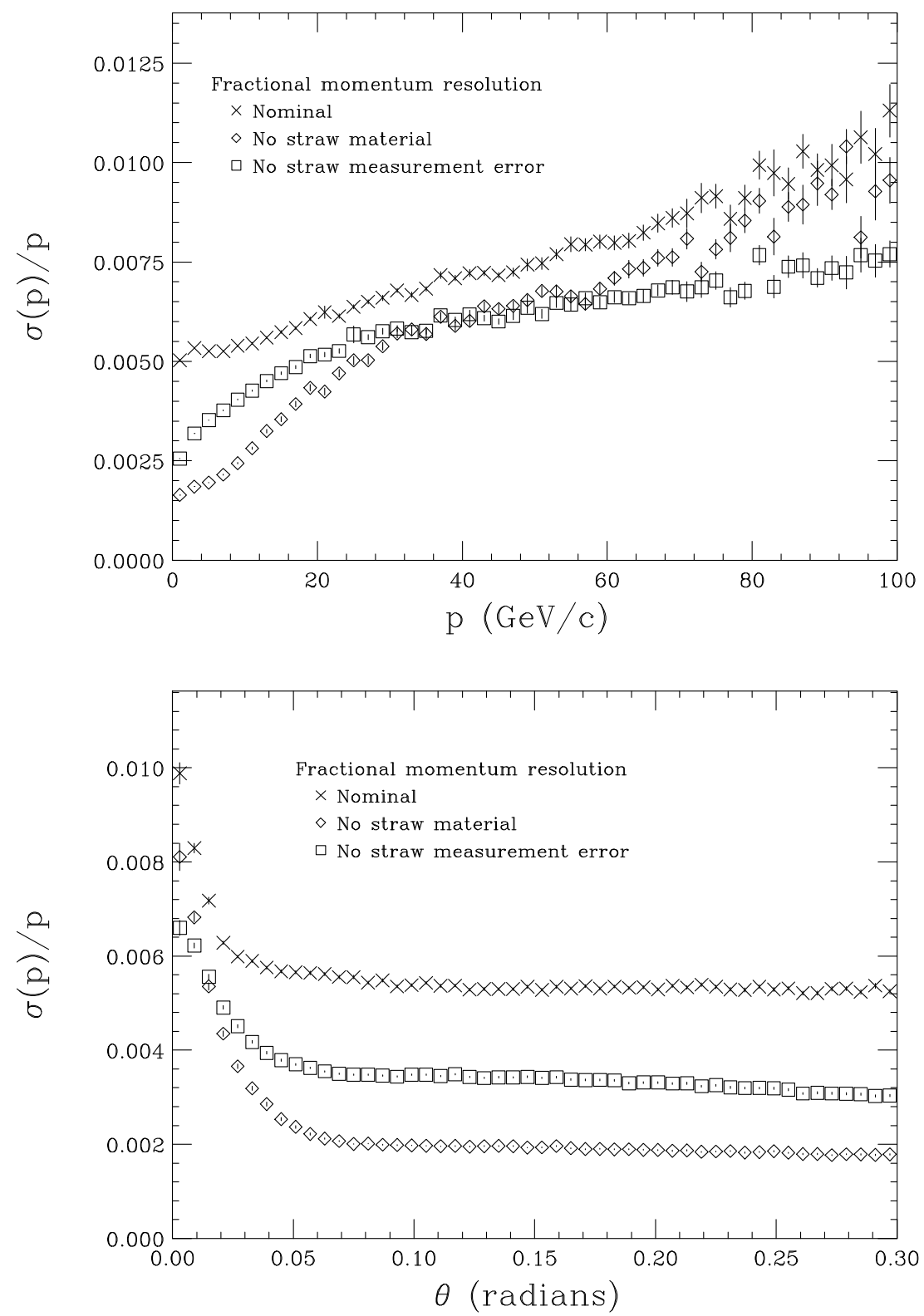

Figure 6.5: Fractional momentum resolution as a function of momentum and angle $\theta$ for tracks passing through at least two pixel stations and through the outer straw tracker (averaged over all tracks in $b \bar{b}$ events). 
the basic straw drift cell properties required for stable operation in the high rate environment anticipated for BTeV. We plan to develop straw cells capable of achieving high drift time measurement efficiency and drift time accuracy while maintaining low noise counting rates. Where possible we will build on the significant body of research carried out for the SDC straw drift tubes [6] and the ATLAS TRT straw tubes [9].

\subsubsection{Straw Tube Design}

There are several design choices which will need to be specified for BTeV straws. The first is the choice of straw tube diameter and materials. The SDC and ATLAS straw trackers both employ $4 \mathrm{~mm}$ diameter tubes. Smaller tube diameters reduce the occupancy but there is a limit to practical operation at very small diameters. The material will most likely be a Kapton film with copper or aluminum conduction layers, and possibly protective carbonpolyimide layers. The manufacturing process is rather involved depending on the rigidity requirements and choice of reinforcement scheme. The SDC straws were manufactured by an industrial firm[10] which also applied the conduction layers (copper in this case). The straw manufacture process for the ATLAS TRT is more involved, as carbon fiber threads are applied at CERN for additional mechanical support. This reflects a difference in design philosophy: the ATLAS straw tubes, to some extent, provide some of the tension relief (in addition to the carbon fiber reinforced plastic shell and alignment planes) while for the SDC straw tracker the shell and endplates carried the entire wire tension and the close-packed straws were subject to no external mechanical stresses.

\subsubsection{Materials}

The choice of specific straw materials and accompanying support structure materials has significant implications for possible background counting rates in the straw tubes. The neutron flux in particular needs to be estimated for the chosen BTeV forward system layout.

We are developing a prototype straw tube which places an aluminum conduction layer between two Kapton films, the inner one next to the gas volume being a carbon loaded, low resistivity film (see Fig. 6.6). The idea is that the Kapton forms a protective barrier, similar to the graphite layers deposited on the inner surface of the ATLAS TRT straws. We measured the surface resistivity of the aluminum coated, carbon loaded Kapton film of our prototype to be $6.5 \pm 1.0 \Omega$ /square, which is comparable to the specified value for the TRT straw tube. The details of the prototype straw material are listed in Table 6.3. The 1 mil thickness of each film is chosen as a compromise between the 0.5 mil thick films used for SDC straw tubes (which had very little mechanical rigidity) and the thicker and reinforced TRT straws. Our final design will likely have the straws supporting some of their own weight. If this turns out to not to be the case, we may use instead 0.5 mil Kapton films which have been shown to work and would provide a reduction of material in the detector volume. 


\section{Straw Wall}

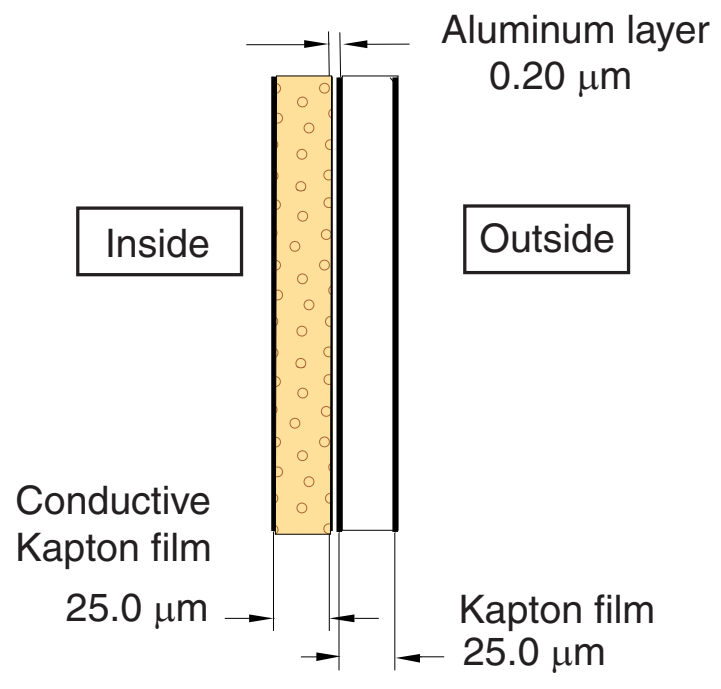

Figure 6.6: Cross section sketch of the straw wall for the prototype BTeV straw tube. The straw is manufactured by winding around a mandrel two $1 \mathrm{~cm}$ wide Kapton strips offset by $5 \mathrm{~mm}$.

\subsubsection{Gas Composition}

We are considering several possibilities for the optimal BTeV gas mixture. The SDC straw design used $80 \% \mathrm{CF}_{4}$ and $20 \%$ isobutane, a mixture chosen for its fast drift velocity $(\approx 100 \mu \mathrm{m} / \mathrm{ns})$ and well known operating characteristics in high radiation rate applications. This very fast drift velocity was targeted for the $16 \mathrm{~ns}$ SSC bunch crossing time. The ATLAS TRT gas mixture $\left(70 \% \mathrm{Xe}+20 \% \mathrm{CF}_{4}+10 \% \mathrm{CO}_{2}\right)$ contains xenon, which is a good x-ray absorber (required for a transition radiation detector), $\mathrm{CF}_{4}$, to insure high drift velocity, and $\mathrm{CO}_{2}$ as a quencher. For BTeV straw cells we plan to determine the gas mixture which optimizes drift time resolution, efficiency, and stability of operation. One possibility is to use a TRT-like mixture in which Xe is replaced by Argon: $70 \% \mathrm{Ar}+20 \% \mathrm{CF}_{4}+10 \% \mathrm{CO}_{2}$. We have observed the analog signal properties of this mixture and found them very similar to the xenon based mixture. Another candidate is Argon-Ethane- $\mathrm{CF}_{4}, 50: 35: 15$, used for the Central Outer Tracker (COT) by CDF. This gas has a fast drift time of $88 \mu \mathrm{m} / \mathrm{ns}$ when their chamber is operated at $2.5 \mathrm{kV} / \mathrm{cm}$. This configuration gives the COT a $100 \mathrm{~ns}$ maximum drift time, suitable for $132 \mathrm{~ns}$ bunch crossings. Since our cell size is much smaller than that of the COT, we are not forced to use a fast gas. Mixtures with little or no $\mathrm{CF}_{4}$ will also be considered.

Closely associated with the choice of gas mixture will be the determination of optimal gas gain at which to operate the detector. Higher values of gas gain are known to introduce space-charge effects which can lead to significant non-linearities in the straw response. The probability that self-limiting streamer discharges occur rises quickly with gas gain. This 


\begin{tabular}{|l|l|}
\hline Description & BTeV Straw Prototype \\
\hline \hline Kapton film & Inner: Polyimide type XC \\
& $25 \pm 2.5 \mu m$ thickness \\
& Outer: Polyimide type $100 \mathrm{VN}$ \\
& $25 \pm 2.5 \mu m$ thickness \\
\hline Density & $1.42 \mathrm{~g} / \mathrm{cm}^{3}$ \\
\hline Aluminum layer & $(0.2 \pm 0.08) \mu m$ thickness \\
\hline Resistivity of inner Kapton layer & $6.5 \pm 1.0 \Omega /$ square \\
\hline \hline
\end{tabular}

Table 6.3: Summary of straw tube material specifications for the BTeV prototype straw tube

in turn leads to an overloading of the front-end electronics and introduces deadtime in the amplifier/shaper/discriminator circuitry.

Perhaps the most important factor for robust operation is mechanical integrity of the straw cell itself. The quality of the anode and cathode surfaces must be high and the position of the anode wire within the tube must be held to strict tolerances. Thus an important part of our program will be to develop reliable construction techniques and quality assurance procedures.

The high voltage range at which the straws may operate without inducing large amounts of dark current will be measured for the selected straw design. At high voltages the streamer discharge rate increases until most of the signal from the straw is from such streamers. Corona discharges occur even without significant wire offsets. This effect is compounded for straw tubes with large wire offsets. Experience from the ATLAS TRT straw tubes shows these offsets must be kept below $300 \mu \mathrm{m}$ to prevent such breakdowns if one expects to be able to vary the voltage over a reasonable range of operating gains. These types of studies will need to be performed for $\mathrm{BTeV}$ straws and candidate gas mixtures.

\subsubsection{Signal Wire, Joints and Supports}

The sense wire for the straw cells is 30 micron diameter gold plated tungsten held under 50 grams of tension. In addition, following the technique used for the ATLAS TRT, most of the sense wires will be divided electrically using a small glass capillary bead [9]. This cuts the occupancy rates in half.

The sense wires in straws which span more than $80 \mathrm{~cm}$ will require additional support. Fig. 6.7 shows the helical design developed for ATLAS TRT which can be adopted for BTeV straws. 


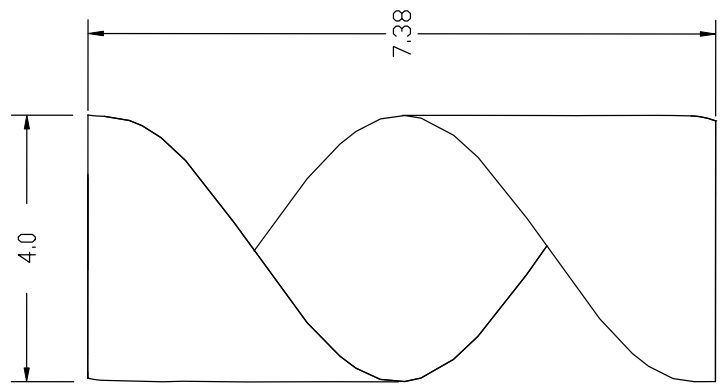

Figure 6.7: Twister wire support used in ATLAS TRT [9]. Dimensions shown are mm.

\subsubsection{Construction of Straw Chamber Modules}

\subsubsection{Mechanical Support Considerations}

The module concept for straw drift tube arrays was developed for the SDC straw tracker. Rather than constructing large monolithic arrays of several thousand drift tubes, one assembles groups of layers in packages consisting of a few hundred tubes. This method lends itself well to mass production techniques, quality assurance, and ease of repairability. Fig. 6.8 shows a sketch for one of six modules comprising the station nearest the interaction region. The module positions 3 layers of close packed straw tubes via an outer aluminum frame, which provides the gas manifold and wire tension support. Two such modules fit together to comprise a measurement view. The straw tube services are provided at the two ends by the frame, which forms a gas manifold, holds signal wires, interfaces with the front-end boards, and is the attachment and alignment fixture for the module. Fig. 6.9 shows how these modules may be combined to form a tracking station.

Additional services may be required to remove the heat generated in the straws as positive ions flow to the cathode. Straws near the beam pipe will have high counting rates and thus may have significant heat to dissipate. Temperature variations will lead to non-uniformity of the straw tube response and so must be kept to a minimum (yet to be specified). Calculations of the power dissipation rates as a function of straw position will need to be made once the overall design is specified.

\subsubsection{Front-end Electronics}

The straw tubes require a relatively high gain amplifier, to allow high efficiency operation at moderate gas gain. Current is induced in a proportional chamber anode wire by the motion of positive ions away from the wire. The fall time of this signal is quite slow (relative to the Tevatron crossing time of $132 \mathrm{~ns}$ ) and must be shaped using a network designed with the appropriate ion velocity in mind. The University of Pennsylvania ASD series of integrated circuits [11] was developed initially for the SDC straw chambers, and more recently for the ATLAS TRT. These ASICs include high gain preamplifiers, pole-zero networks for pulse 


\section{Station 7,8 Module}

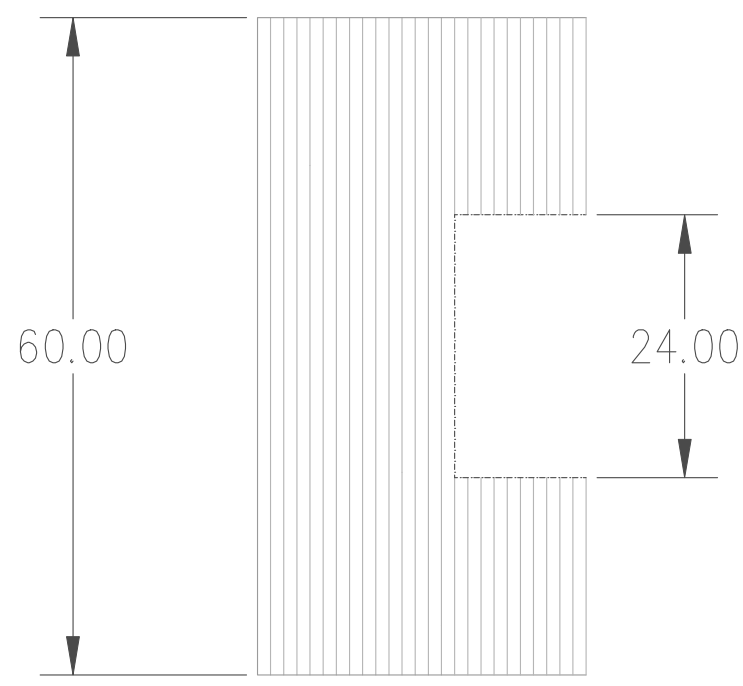

Figure 6.8: View of a straw module for the forward tracking station nearest the interaction region. Dimensions shown in $\mathrm{cm}$.

shaping and ion-tail cancellation, and leading edge discriminators. These ASICs are radiation hard, and are almost exactly suited to our use. We expect to use one of the ASD ASICs for the BTeV straw chamber system.

\subsubsection{TDC Requirements and DAQ Readout}

The baseline design requires a position resolution less than $100 \mu \mathrm{m}$ per measurement station ( $~ 150 \mu \mathrm{m}$ per plane). The error introduced by the granularity of the TDC bins should be small compared to the intrinsic straw tube resolution. This intrinsic error is a function of drift distance as well as other factors. An RMS error of $150 \mu \mathrm{m}$ is achieved by having a much larger error for tracks passing very close to the wire, and a significantly smaller error $(\sim 100 \mu \mathrm{m})$ for tracks passing at an optimal distance from the wire. The TDC binning error should thus be much less than $100 \mu \mathrm{m}$. If we operate the straw tube with a gas mixture and high voltage setting which results in a $100 \mu \mathrm{m} / \mathrm{ns}$ drift velocity, then the TDC bin width of $1.5 \mathrm{~ns}$ (corresponding to a binning error of $44 \mu \mathrm{m}$ ) would suffice. This is not a difficult specification. We have begun discussions with the Fermilab PPD Electrical Support group about the design of a purely digital TDC to meet our needs. 


\section{Stations 7,8}

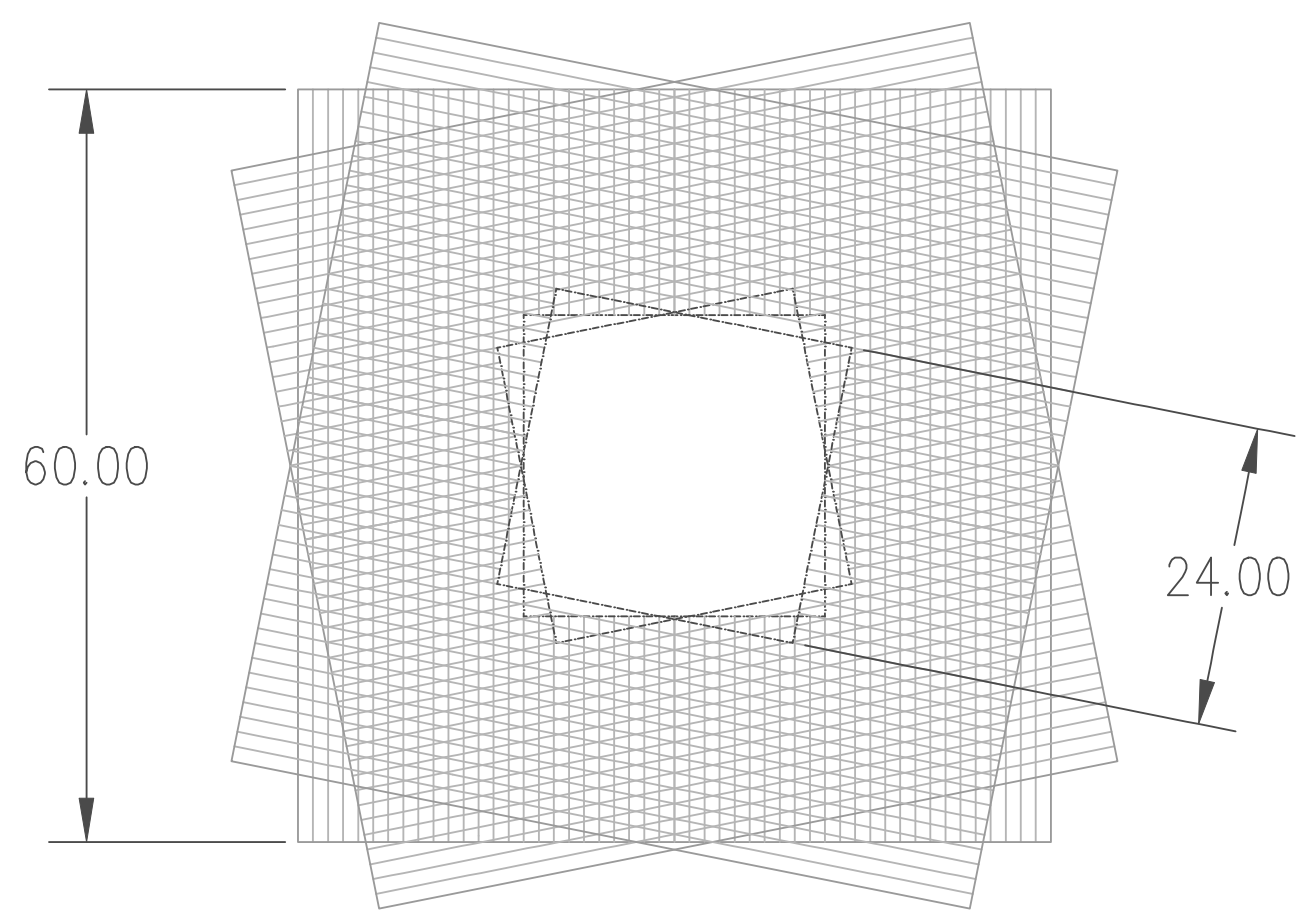

Figure 6.9: Front view of the forward straw tracking station nearest the interaction region. Dimensions shown in $\mathrm{cm}$. The station consists of three measurement views built from six independently built and serviced modules. The station shown, which is a proposed change to the baseline design, consists of an $X$ view and two \pm 11.3 degree stereo views. Note that the stereo views mostly measure the $Y$, bend view coordinate.

\subsection{Forward Silicon Tracker}

\subsubsection{Technology Options}

Microstrip Gas Chambers[2] (MSGC's) are being pursued by the LHC-B[3] and HERA-B[4] Collaborations for their inner region tracking detectors. These devices offer good granularity, low occupancy, resolutions better than $80 \mu \mathrm{m}$, and high rate capability. However the boro-silicate glass substrates, on which the MSGC's are based, introduce considerably more radiation length per measurement plane than either conventional drift chambers or straw tube cells. Cost is a major factor for large area coverage as well. These considerations make MSGC's an unattractive option for the outer regions of the forward tracking system where large areas need to be instrumented while keeping the radiation lengths to a minimum to maintain good resolution for wide angle, low momentum tracks. MSGC's can be considered for the the inner region in close proximity to the beam pipe where the occupancy rate is 
high and the tracks tend to be of higher momenta. However, MSGC's are not yet a proven technology.

Silicon microstrip detectors are a well-proven technology. They offer excellent resolution and low occupancy. Additionally they can be operated in high radiation dose environments. The thickness can be kept to a minimum using double-sided silicon sensors. A ladder arrangement of silicon detector modules can provide relatively large area coverage.

\subsubsection{Detector Description and Layout}

The baseline proposal consists of two planes of $300 \mu \mathrm{m}$ thick double-sided silicon microstrip detectors with $100 \mu \mathrm{m}$ pitch. Standard sized silicon wafer modules are mounted on low mass carbon fiber support pads and are arranged in a daisy-chained ladder readout configuration as shown in Fig. 6.10. Four views are provided: $X, Y, U, V$ where the $U$ and $V$ stereo angles are matched to the corresponding straw tracker stereo views. Each layer consists of 16 silicon strip modules measuring $6 \mathrm{~cm}$ by $4 \mathrm{~cm}$ and four measuring $2.5 \mathrm{~cm}$ by $4.75 \mathrm{~cm}$. The configuration shown here is similar to that proposed by LHC-B [13].

Each view consists of 2400 channels and the whole system has 57,600 channels. With $100 \mu \mathrm{m}$ pitch we need only readout the hits since the hit alone provides $\sigma=100 \mu \mathrm{m} / \sqrt{12}=$ $29 \mu \mathrm{m}$ resolution. A new binary readout ASIC, designed for high speed readout, will be required.

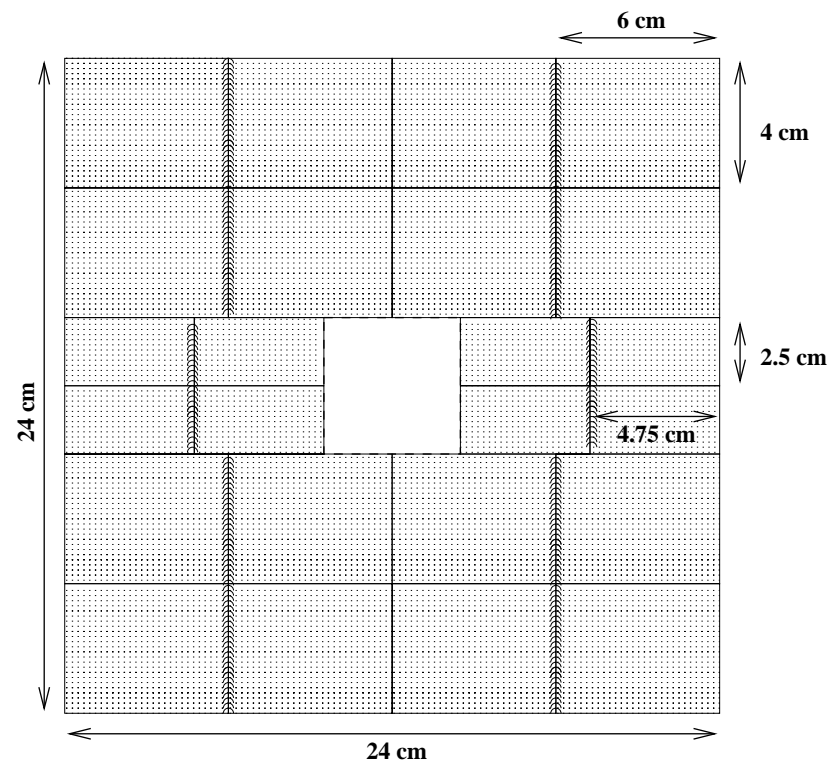

Figure 6.10: Proposed schematic of a forward silicon tracking layer. 


\section{Bibliography}

[1] D.G. Cassel, G.G. Hanson et al., "Report of the Central Tracking Group", Proceedings of the 1986 Summer Study of the Physics of the Superconducting Supercollider, eds. R. Donaldson and J. Marx, Snowmass, CO (1986) 377.

[2] A. Oed, Nucl. Instrum. Meth. A 263 (1988) 351.

[3] LHC-B Letter of Intent, CERN/LHCC 95-5, August 1995.

[4] HERA-B Design Report, DESY-PRC 95/01, January 1995.

[5] H. van der Graaf, et al., Nucl. Instrum. Meth. A 307 (1991) 220.

[6] Y. Arai, et al., "A modular straw drift tube tracking system for the Solenoidal Detector Collaboration experiment, Part I: Design"; Nucl. Instrum. Meth. A 381 (1996) 355.

[7] Y. Arai, et al., "A modular straw drift tube tracking system for the Solenoidal Detector Collaboration experiment, Part II: Performance"; Nucl. Instrum. Meth. A 381 (1996) 372.

[8] RD6 Collaboration, "The ATLAS TRT straw proportional tubes: performance at very high counting rate", Nucl. Instrum. Meth. A 367 (1995) 143.

[9] ATLAS Inner Detector Technical Design Report, CERN/LHCC/97-16,17.

[10] Lamina Dielectrics Ltd., Billingshurst, W. Sussex, UK.

[11] F.M. Newcomer, R. Van Berg, J. Van der Spiegel and H.H. Williams, Nucl. Instrum. Meth. A 283 (1989) 806.

[12] Y. Arai and M. Ikeno, IEEE J. of Solid-State Circuits SSC-27 (1992) 359.

[13] LHC-B Technical Proposal. 


\section{Chapter 7}

\section{Design of the Muon Detector}

As described earlier, the $\mathrm{BTeV}$ muon system should have the following attributes:

1. Robust: It will be very difficult to access the detectors once they are installed.

2. High Rate Capability: Rates of a few $\mathrm{kHz} / \mathrm{cm}^{2}$ are possible in parts of the system (based on calculations of accelerator backgrounds).

3. Good Time Resolution: Timing information on hits with a resolution of 50-60 ns will allow rejection of hits due to accelerator backgrounds.

4. Magnetic: Two toroids of 1.5 Tesla field and roughly $1 \mathrm{~m}$ thickness (in each arm).

5. Fine-Grained Spatial Resolution: Tracking resolutions of 2-3 mm are required.

6. Longitudinal Compactness: Given the size constraints, the detectors should be of minimal thickness.

7. Minimal Neutron Susceptibility: We will avoid the use of hydrogenic detector elements.

8. Uniform Occupancy: A geometry designed to minimize pattern recognition confusion and minimize occupancy at smaller radii.

\subsection{Detector Design and Fabrication}

In this section we describe the detector system designed to satisfy these criteria.

\subsubsection{Basic Building Block: Proportional Tube "Plank"}

The basic building block in the construction of a detector station is a "plank" of $3 / 8$ " stainless steel proportional tubes (see Fig. 7.1). Thirty-two tubes are arranged in a double layer with an offset of half a tube ("picket fence" geometry) and are glued together at two or three points by aluminum ribs ("Fred Ribs" in the figure). This should provide an extremely 


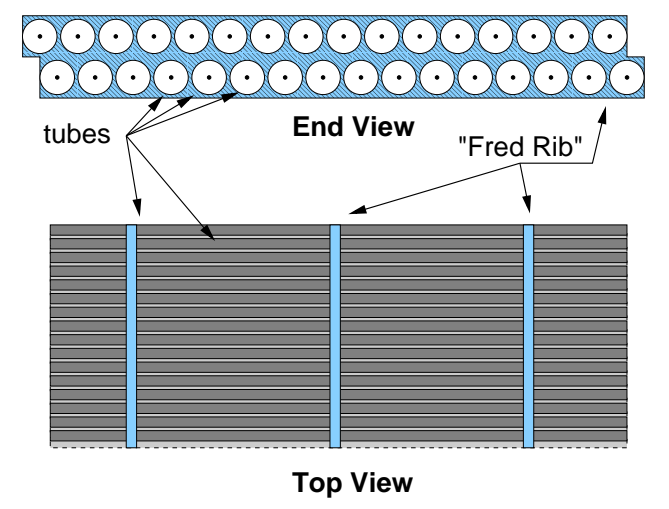

Figure 7.1: End and top views of one "plank" of proportional tubes. The plank is the basic building block of the muon system.

sturdy, self-supporting building block. Proportional tubes have been selected as the detector technology because they are robust and have the necessary rate capability. We intend to use a fast gas (such as $88 \% \mathrm{Ar}, 10 \% \mathrm{CF}_{4}, 2 \% \mathrm{CO}_{2}$, which has a drift velocity of roughly $9 \mathrm{~cm} / \mu \mathrm{s}$ [1]) so the maximum collection time (drift plus charge integration) for a signal should be less than 60 ns. We intend to use thin walled (0.01") stainless steel tubes. This is a proven technology - it has been used successfully by the CDF collaboration where similar tubes [2] had a long lifetime with low failure rate.

The $0.5 \mathrm{~cm}$ wire spacing of this design has an effective spatial resolution of at most $1.5 \mathrm{~mm}$, with no dead regions between tubes.

\subsubsection{Design of a Detector Station}

To minimize occupancy at small radii and to minimize pattern recognition confusion, each detector station will consist of eight overlapping pie shaped "octants," as shown in Fig. 7.2. Each octant is a single unit, which will be hung from a hanging frame. There are three views $(r, u$, and $v)$ in each octant as shown in Fig. 7.3. The views stack on top of each other, and are built from the planks described above. We are considering adding a thin layer of aluminum between each view to act as a delta ray "shield." While the shield itself will generate delta rays, the idea is to regenerate them near the tubes so that they will tend to fire the same tubes as their parent muons. There will be 15 planks (butted up against each other) in each view of an octant (more than shown in Fig. 7.3).

Currently the angle of the $u$ and $v$ views with respect to the $r$ view is $\pm 22.5^{\circ}$. The three views provide redundancy and allow studies to be made in which two views are beat against the third (such as in efficiency studies). The angle of $22.5^{\circ}$ is not yet set in stone, further Monte Carlo studies need to be performed. This geometry gives excellent resolution and redundancy in the bend direction $(\hat{\theta})$ of the toroids, and the resolution in the $\hat{\phi}$ direction is reasonable $\left(\sigma_{\phi}=2.8 \mathrm{~mm}\right.$, which is approximately twice the single plank resolution).

This octant geometry has several advantages. In all three views the planks are short near 


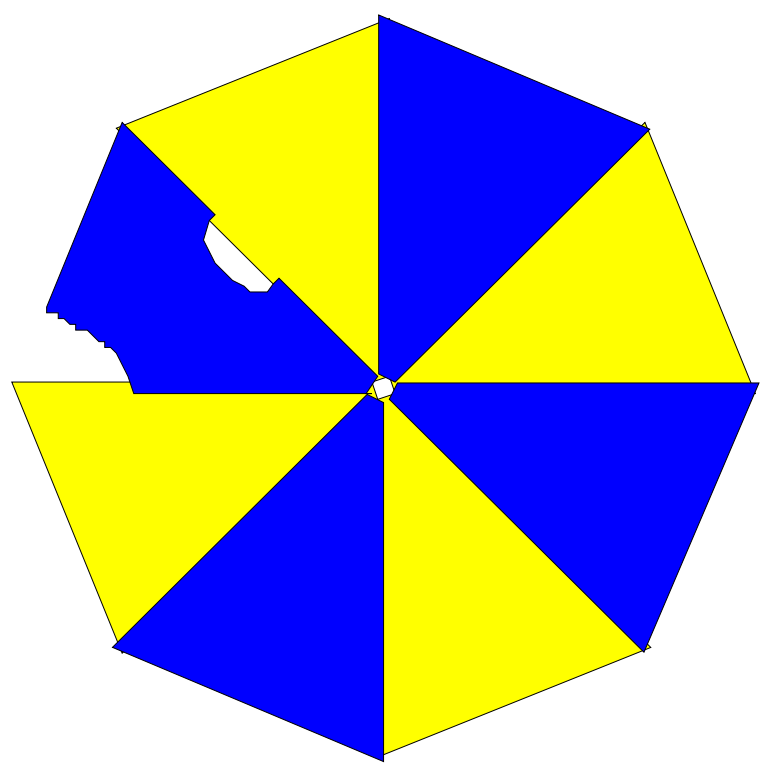

Figure 7.2: Beams-eye view of one muon detector station, which consists of eight overlapping octants arranged in two layers. One octant is cut away in places to show the overlap between adjacent octants.
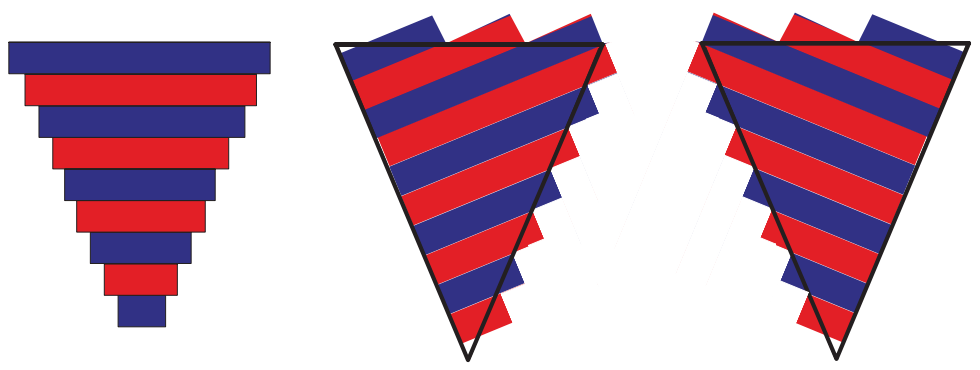

Figure 7.3: Arrangement of planks to form each of the three views in an octant. There will be 15 planks per octant (more than shown).

the beam pipe, and get longer at larger radii. This should result in approximately uniform occupancy as a function of $r$. This geometry should also help minimize pattern recognition confusion.

With 15 planks per view per octant, and 32 channels in a plank, the channel count for each octant will be 1440 and for each detector station will be 11,520, for a total of 34,560 channels in each arm and 69,120 channels in both arms.

\subsubsection{Proportional Tube Details}

Each of the 32 tubes in a plank will share a common gas manifold at each end. The wires will be held at positive high voltage and read out through a blocking capacitor by front-end 
electronics which will attach at one end. High voltage will be fed to the plank at the same end as the electronics. We intend to use a "fast" gas mixture of $88 \% \mathrm{Ar}, 10 \% \mathrm{CF}_{4}, 2 \% \mathrm{CO}_{2}$ or something similar. For more details on the front-end electronics, expected signals, etc., see the section on front-end electronics below.

The longest tubes in the system will be approximately 6 feet in length, and we are considering several different wire diameters (20-50 microns). We will decide on a wire diameter based on the results of beam tests to be performed this summer (1999, see description below). In any case, our calculations indicate that no wire supports are necessary. Again, we hope to get some feedback on that this summer.

The tubes will be strung individually and the wires held in place via a crimp pin. The arrangement is very similar to that used in the CDF system [2]. The crimp pins will extend out of the gas manifold and will attach to a circuit board which contains the blocking capacitors and an edge connector for the front-end cards.

\subsubsection{Miscellaneous Mechanical Details}

Because of the overlapping octant geometry of each detector station, there is plenty of room for cabling and front-end electronics everywhere except near the beam pipe. It is contention for space at small radii that limits the inner radius of the system to between 15 and $20 \mathrm{~cm}$.

Weight is a potential concern because we are using stainless tubes. Using thin walled tubes reduces this concern significantly. We estimate the weight of the stainless tubes in one octant (all three views) to be $200 \mathrm{lbs}$, the same as if we were to use 1/32" thick aluminum tubes. However, the hanging frame used to support the octants and the delta ray shields will probably double the weight of each octant, so that each detector station will weigh 1.5-2 tons.

We plan on building at least 5 spare octants ( $10 \%$ of those in the total system), so that if one develops problems (more than a handful of broken wires, etc.) we can swap a new octant in and work on the problem octant "offline." We do not plan on restringing bad tubes - we will simply make a new plank.

The octants will be fabricated at Illinois (50\%) and Vanderbilt (50\%). We estimate the cost of the stainless tubing to be $\$ 500,000$. Wire will cost $\$ 250,000$. Machined parts such as the gas manifolds, gas connectors, wire crimp pins and feedthroughs will cost $\$ 500,000$. Including the hanging frame and contingency, we estimate the total cost of materials for the detector planes to be $\$ 1.5$ million, not including electronics.

\subsubsection{Summary of Mechanical Design}

A summary of the baseline BTeV Muon System is given in Table 7.1. 
Table 7.1: Parameters of the baseline BTeV Muon System.

\begin{tabular}{|l|c|}
\hline \hline Transverse size & $\pm 200 \mathrm{mrads}$ \\
Total Length & $3.3 \mathrm{~m}$ \\
Rate Capability Req'd. & few $\mathrm{kHz} / \mathrm{cm}^{2}$ \\
Steel Length & $2 \mathrm{~m}$ \\
Toroidal Field & $1.5 \mathrm{~T}$ \\
No. of Longitudinal segments (steel) & 2 \\
Plane size: & $20 \mathrm{~m}^{2}$ \\
Tube cell size & $1 \mathrm{~cm}($ diameter $)$ \\
Wire spacing: & $0.5 \mathrm{~cm}$ \\
Spatial resolution & $1.5 \mathrm{~mm}$ \\
No. of channels/octant (3 views) & 1440 \\
Octants per station & 8 \\
Total channels & $35,000 \mathrm{per}$ arm \\
Momentum resolution & $\sigma_{p} / p=19 \% \oplus 0.6 \% \times p$ \\
Total thickness/station & $20 \mathrm{~cm}$ \\
\hline \hline
\end{tabular}

\subsection{Front End Electronics}

In order to capture all the charge coming from a typical proportional tube for a single incident ionizing particle, one must integrate the charge due to the positive ion drift over a long period of time relative to the expected beam crossing for the BTeV experiment. The time needed to collect a portion of the total charge is a function of the gas used in the proportional tube, the geometry of the tube, and the high voltage bias of the center wire, relative to the outer conductor:

$$
\frac{Q}{Q_{o}}=\frac{\ln \left(1+t / t_{o}\right)}{2 \ln (b / a)}[3],[4]
$$

where $Q$ is the charge collected in an amount of time, $t, Q_{o}$ is the total charge from the ionization (depends on the gain of the tube), $b$ and $a$ are the outer and inner radius, respectively, of the conductors in the proportional tube, and:

$$
t_{o} \sim \frac{a}{2 v_{d}}[4]
$$

where $v_{d}$ is the drift velocity of the gas for the chosen value of the gain in the tube [1]. Alternatively, for a very fast signal, one could try to just capture the fast signal coming from the electron amplification, but this is only $1-2 \%$ of $Q_{o}$.

In $\mathrm{BTeV}$ we intend to use the muon proportional tubes in the Level 1 Trigger. The natural cutoff for integrating a signal then is on the order of a beam crossing, or 100 ns. However, we would like to reduce backgrounds associated with the collider beams, which pass through about 60-70 ns before interaction products arrive. For a fast gas like $\mathrm{ArCO}_{2} \mathrm{CH}_{4}[1]$, 
and a wire-to-wire spacing of $0.5 \mathrm{~cm}$, the maximum drift time before the first signals from a track arrive is about 30 ns. This means that if we would like to be able to separate the various "crossings", our electronics should be able to resolve 2 hits occurring 30 ns apart (minimum).

\subsubsection{Amplifier choice}

Fortunately, electronics to amplify and digitize the analog signal from a chamber hit in a high rate environment were developed for the SSC [3], and continue to be refined for use in detectors for the LHC [5] and at Fermilab [6]. We will take full advantage of these developments.

We intend to mimic the setup used for the CDF COT [6]. There will be a PC board to deliver High Voltage to each Proportional Tube, and a PC board with electronics to amplify and digitize the tube signal. Both boards will be located directly at the end of a group of 16-32 proportional tubes.

We plan to utilize the ASD family of integrated circuits developed at the University of Pennsylvania to amplify and digitize the signals coming from the proportional tubes. These

chips (and applications) are described in detail elsewhere [3],[6], but we briefly mention some more important features here.

The ASD8B, which is being used for evaluation and beam tests, amplifies the first $\sim 6$ ns of the the chamber signal and outputs an LVDS (equivalent) differential logic signal. This chip, when mounted on a PC board, has a low effective threshold of about $\sim 1 \mathrm{fC}$. This is the threshold as quoted in the ASD8B reference and verified in tests that we performed on an evaluation board supplied by the University of Pennsylvania in December of 1998. The chip also features a double pulse resolution of $\sim 20 \mathrm{~ns}$. This chip seems to be an ideal choice for the BTeV muon system, and it is important to determine if we have sufficient signal to utilize the ASD8B (or other ASD chip!).

\subsubsection{Practical Considerations}

To calculate the signal we expect in the proportional tube, we will use the data presented in reference [1]. For the same gain as quoted in the $\operatorname{paper}\left(\sim 1.8 \times 10^{5}\right)$, we can use the quoted results to determine our operating parameters for different tube geometries and gas mixtures. In Table 7.2.1.1, we summarize the results for different gases and inner conductor radii.

In the table, we have made some assumptions. We've assumed that the most important factor in calculating the gain is sensitivity to a single ionization cluster of a single electronion pair; we haven't taken into account the effect of adding a termination resistor (see below); we haven't taken into account the effect of a magnetic field; and we've neglected aging effects in the tube. We've also taken for granted that the electronics will be located at the end of the proportional tubes: lowering the electronic noise and increasing the amount of signal we get in the small integration time available. Even so, we can learn a great deal from looking at the table. 
Table 7.2: Operating parameters for different gases and inner wire radii (a). The values of $t_{d r i f t}^{M}$ assume a wire space of $0.5 \mathrm{~cm}$.

\begin{tabular}{|l|llllll|}
\hline \hline Gas & $\left.E_{\text {field }}\right|_{a}$ & $v_{d}$ & $V_{\text {bias }}$ & $Q_{6 \mathrm{~ns}}$ & $t_{\text {drift }}^{M}$ & $\mathrm{a}$ \\
\hline$\frac{\mathrm{ArC}_{2} \mathrm{H}_{6}}{50 / 50}$ & $150\left(\frac{\mathrm{kV}}{\mathrm{cm}}\right)$ & $4.8\left(\frac{\mathrm{cm}}{\mu \mathrm{s}}\right)$ & $3545(\mathrm{~V})$ & $7.8(\mathrm{fC})$ & $50(\mathrm{~ns})$ & $50(\mu \mathrm{m})$ \\
$\frac{\mathrm{ArCO}}{80 / 20}$ & 164 & 2.4 & 3768 & 5.9 & 100 & 50 \\
$\frac{\mathrm{ArCO} \mathrm{CF}_{4}}{88 / 2 / 10}$ & 150 & 8.7 & 3454 & 9.5 & 30 & 50 \\
$\frac{\mathrm{ArC}_{2} \mathrm{H}_{6}}{50 / 50}$ & 150 & 4.8 & 1656 & 8.8 & 50 & 20 \\
$\frac{\mathrm{ArCO}}{80 / 20}$ & 164 & 2.4 & 1806 & 7.1 & 100 & 20 \\
$\frac{\mathrm{ArCO}}{88 / 2 / 10}$ & 150 & 8.7 & 1656 & 10.4 & 30 & 20 \\
\hline \hline
\end{tabular}

\subsubsection{Amplifier Observations}

A smaller wire size is desirable from an electronics standpoint: the operating voltage $\left(V_{\text {bias }}\right)$ is lower and the integrated charge collected $\left(Q_{6 \mathrm{~ns}}\right)$ is a little higher. The lower operating voltage gives us more flexibility in the choice of a decoupling capacitor, allowing the option of surface mount components in the high voltage system. The higher integrated charge means we can safely raise the threshold of the electronics without losing signal, allowing greater flexibility in our noise budget. Additionally, it is expected that the gain of a tube has less sensitivity to the operating voltage with a smaller wire [1].

Also evident is the advantage of having a fast gas. Not only can one integrate more charge in a smaller period of time, one has less sensitivity to beam "crossings". We can achieve our goal, stated previously, of the first signal to arrive after a maximum time of about $t_{\mathrm{drift}}^{M}=30 \mathrm{~ns}$.

Another way to look at the table is to reason that all the combinations calculated will produce a working detector, and that using the ASD chip allows us a lot of flexibility in our choice of wire diameter, gas, and operating voltage.

At most, a termination resistor will raise our noise by $1 \mathrm{fC}$ and lower our gain by a factor of 2. This is to be offset by the expectation that about 10 primary ion-pair clusters will be produced, on average, for a MIP passing through $1 / 3 \mathrm{~cm}$ of gas volume.

One final consideration in evaluating the ASD8B for $\mathrm{BTeV}$ is that the process used to make the chip is obsolete and the chip will not be available in production quantities [7]. We will have to use an improved version (ASDQ) at a slightly higher cost. It integrates for a slightly longer period, has the same double pulse resolution, and has a higher noise level of $2-3 \mathrm{fC}$.

\subsubsection{Front-end Readout}

As mentioned previously, the ASD chips produce differential logic signals (LVDS) suitable for driving long $(10 \mathrm{~m})$ cable lengths. The LVDS signal consists of 1 bit/channel signifying whether or not a channel is on or off. If we choose to do any logic on the ASD board, we 
must first convert the LVDS, perform the required function, and finally redrive the signal. This option, though decreasing cable cost, will require more power to be delivered to the front-end, more power to be dissipated in a small area near the detector, and will increase the amount of noise on the board. This option was not used on CDF and is not recommended by the designer of the ASD8B chip [7].

Finally, reading out the raw hits provides for maximum flexibility in designing, prototyping or even upgrading and good utility in re-using these electronics or interchanging electronics with other BTeV detectors. Cards designed with a similar philosophy have been in continual use for 25 years in high energy physics experiments. For the reasons given, we intend to read out the raw hits from the front-end electronics.

\subsubsection{Feasibility}

By utilizing existing electronics packages and packaging for the front-end of the BTeV muon system we will save considerable time and developmental costs. For instance, the major modification needed to utilize the 24 channel CDF COT front-end card for BTeV, is to remove one of the chips from the CDF COT circuit board to accommodate the smallest muon drift tube channel count footprint of 16 channels.

Additionally, such a scheme is easily utilized by other detectors, such as the straw tracker, and we can benefit from standardization. To use this scheme and front-end board in other BTeV gas tracking systems, one has to consider what information is needed for tracking, and build a suitable post front-end circuit board to suit those needs. The front-end can be used right away.

\subsubsection{Post Front-end Considerations for the Muon System}

As stated, we intend to do no post processing on the front-end amplifier board. We still need to read the signals out, and we would like to keep costs to a minimum.

In order for the front-end to operate correctly, we will need to provide a mechanism to modify the threshold for a channel. Rather than include a digital to analog converter on the front-end card, we would rather provide a single analog threshold for each chip (8 channels) and provide a digital mask for channels that fire continuously.

To reduce cable cost and logistics for long cable runs, we will convert the parallel bitmap coming from the front-end to a serial signal that can be transmitted on a single fiber optic cable. A single optical cable will read out 96 channels and do so in the $132 \mathrm{~ns}$ between beam crossings. (The hard limit is 132 bits for a 1 Gbit/s optical link.)

Converting the data from parallel to serial destroys the fine timing information of the front-end signal but allows us to apply a gate to the signals coming from the front-end. By applying this gate we provide both the means to compress the bitmap information in time for the conversion and the ability to filter out-of-time hits from the information sent to the trigger and the DAQ.

All these actions are to be done on a circuit board that intercepts 96 channels of input 


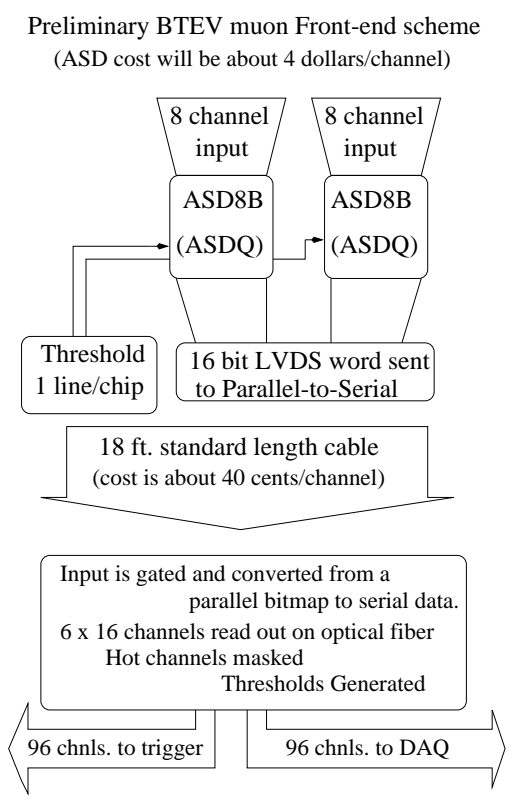

Figure 7.4: Schematic diagram of the front-end and post front-end circuit boards for the BTeV Muon Proportional Tube system. Some highly preliminary cost estimates are included in the figure.

from 6 frond-end cards, and converts the signals for later use. Schematically, the front-end and post front-end card are shown in Fig. 7.4.

\subsection{Trigger Electronics}

\subsection{Test Beam Plans and Goals}

During the 1999 test beam running we will investigate the stability, reliability and operating characteristics of our proportional tubes with various tube lengths (1,3,6 feet) and with various wire diameters $(20,30,50$ micron). We will analyze the response of the system at different incident beam angles and will record rate capability, noise, efficiency and resolution. We also will test the performance of prototypes of the front-end electronics and a prototype trigger processor.

We will construct a tracking system consisting of ten planks of proportional tubes. The planks are identical in design to the ones described above: 32 tubes arranged in two, offset layers. The tubes will be 0.01 " thick walled stainless, with an outer diameter of $3 / 8$ ". We will have enough front-end boards to read out half (160) of these channels.

Our Run Plan is:

1. One week to install planks, trigger scintillators and DAQ. The planks will have been 
tested in a cosmic ray test stand at Vanderbilt prior to installation. The DAQ software will have been tested with partial electronics prior to installation.

2. One week of running with Beam \#1 (see below) to bring the system up and adjust timing in electronics, etc.

3. One to two weeks of running with Beam \#1 to take main data sample. We require several 1-3 hour runs with access time between, to rotate a station or to move other portion of a plank into the beam.

4. Two days of data taking with Beam \#2 and Beam \#3.

5. After taking a few days to analyze the data and reach conclusions, we may go back and repeat some or all of the above. A third iteration could be necessary. Up to 4 weeks of data taking has been requested.

Beam Requirements:

1. A $1 \mathrm{kHz}$ pion beam with energy of $100 \mathrm{GeV}$ or higher and with a beam spot diameter of $4-8 \mathrm{~cm}$.

2. A beam consisting primarily of muons. Provided that the rate is at least $100 \mathrm{~Hz}$ with a beam spot area of $15 \times 15 \mathrm{~cm}^{2}$, either a beam derived from hadrons hitting a dump or a halo muon beam will be adequate. Muon purity around $99 \%$ is desirable. It would be ideal if this could be achieved by moving the stations downstream of the Mtest dump during this part of the test.

3. A $10-100 \mathrm{kHz}$ pion beam for rate studies. This might require a somewhat higher allocation of protons to Mtest area than normal for a small period of time. Depending on the situation, we may require a special high intensity run of $10^{12}$ protons per pulse for 1 day. 


\section{Bibliography}

[1] J.M. Butler et al., "Study Of Fast Gases, Resolutions And Contaminants In The D0 Muon System," Nucl. Instrum. Meth. A290, 122 (1990).

[2] S. Bhadra, S. Errede, L. Fishback, H. Keutelian and P. Schlabach, "The Design And Construction Of The CDF Central Drift Tube Array," Nucl. Instr. Meth. A268, 92 (1988).

[3] "A Fast, Low Power, Amplifier-Shaper-Discriminator for High Rate Straw Tracking Systems," F.M. Newcomer et al., Trans. Nuclear Science, Vol 40, N4, P630-636, August, 1993.

[4] F. Sauli in EXPERIMENTAL TECHNIQUES IN HIGH-ENERGY NUCLEAR AND PARTICLE PHYSICS (2nd ed.), edited by T. Ferbel, World Scientific, Singapore 1991, p. 79.

[5] ATLAS Collaboration, "ATLAS inner detector: Technical design report. Vol. 2," CERNLHCC-97-17.

[6] R. Blair et al. [CDF-II Collaboration], "The CDF-II detector: Technical design report," FERMILAB-PUB-96-390-E.

[7] M. Newcomer, Private Communication. 


\section{Chapter 8}

\section{The BTeV Trigger Performance}

\subsection{Introduction}

The proposed broad charm and beauty physics program of $\mathrm{BTeV} / \mathrm{C} 0$ calls for a trigger that is very efficient for heavy-quark decay events which can be found off-line and is relatively independent of decay mode.

The baseline trigger is a multi-level trigger with several "branches" that can be independently prescaled and then combined into a global trigger. It is described in detail in chapter 4. The main $B$-physics trigger focuses on the identification of detached vertices at Level 1 . The trigger must be capable of reducing the event rate by at least a factor of 100 at Level 1 , and another factor of 25-50 at Levels 2 and 3.

Much work has been done on the Level 1 trigger since the EOI. We present in this chapter the results of studies of the baseline algorithm under more realistic experimental conditions including such effects as electronic noise, additional hits due to secondary hadronic interactions and photon conversions, and multiple interactions per crossing. We have also increased the thickness of the pixel detectors in our simulations to account for the material in support and cooling structures.

We present a status report on Level 1 trigger R\&D including an overview of our hardware prototype and timing results for Level 1 trigger software running on digital signal processors. We also present alternative Level 1 designs in which the pixel detector is modified to reduce the material budget.

Preliminary studies of the Level 2 trigger indicate that the event rate can be reduced by a further factor of 10 through the improved measurement of track parameters and rejection of spurious tracks.

\subsection{Advanced Level 1 Trigger Studies}

The goal of the trigger is to reconstruct tracks and find vertices in every interaction, up to a luminosity of $2 \times 10^{32} \mathrm{~cm}^{-2} \mathrm{sec}^{-1}$. It must be capable of reducing the trigger rate by a 
factor of about one hundred. We employ parallelism both at the subevent level and at the event level. The detector layout has "stations" consisting of three planes of pixel detectors. Processors organize the hits in each triplet of planes into minivectors or "station hits". Still at the subevent level, individual processors work on the station hits from a " $\phi$-slice" ${ }^{1}$ of the detector to form tracks. At the event level, tracks from individual slices are brought together in a vertex finder node. The corresponding algorithms for these various stages are described in detail elsewhere [1],[2]. Here we report on:

- Trigger diagnostics and 'software instrumentation';

- Performance studies of this baseline algorithm, in presence of noise backgrounds, both random and correlated;

- Performance studies on the baseline trigger in the presence of multiple interactions per crossing.

These studies are performed using a full hit-level simulation and a full pattern recognition algorithm. Although the final criteria for triggering will depend on the luminosity, the algorithm that we have implemented runs well on crossings which have as many as 3 interactions in them.

\subsubsection{Trigger Diagnostics and 'Software Instrumentation'}

The Level 1 trigger simulation uses MCFast output "hits" and the MCFast geometry as input data. This is a genuine reconstruction package including pattern recognition (i.e., MonteCarlo generator information is never used to assemble tracks or vertices). However, backpointers to this Monte-Carlo information are extensively used to monitor the performance of this pattern recognition. In addition, the Kalman filter from MCFast is also used to compare the crude errors on the track parameters to the nominal tracking resolution one could achieve with the detector. Finally, a preliminary version of the $B^{o} \rightarrow \pi^{+} \pi^{-}$analysis presented in chapter 11, section 4, has been run in conjunction with the trigger simulation, allowing us to study and refine the trigger efficiency and rejection. We have measured the trigger efficiency on a variety of other states presented in chapter 11 .

\subsubsection{Uncorrelated Noise Study}

Here we investigate the effects on the trigger algorithm of adding random noise to the pixel detector. This study was performed by throwing random hits from a Poisson distribution of average value $\langle\mathrm{N}>$, uniformly distributed over the area of each plane. Electronic noise or neutrons could cause this kind of distribution.

Fig. 8.1 shows an event with no random noise. Fig. 8.2 shows the same event, but with an average of 25 noise hits distributed randomly over each of the 93 pixel planes.

\footnotetext{
${ }^{1}$ See Chapter 4 for definition of this particular event parallelism
} 
Z vs. Y (PlaneHits Coordinates)

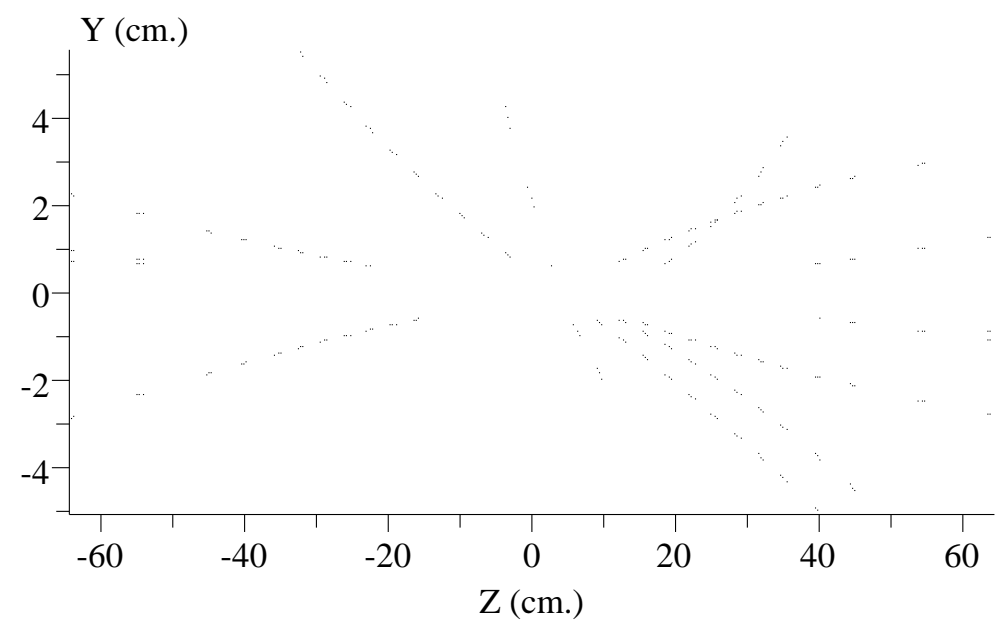

Figure 8.1: Pixel hits in Z-Y projection with no random noise from an MCFAST simulation

Fig. 8.3 shows the efficiency for minimum bias events, which we want the trigger to reject, and signal events (in this case events with a decay $B^{o} \rightarrow \pi^{+} \pi^{-}$), which we want the trigger to accept, plotted against the average number of random noise hits per plane. Three tracks with $3 \sigma$ detachment were required in this example.

We believe that it should be possible, and the experience with existing detectors bears this out, to achieve random noise levels below $10^{-5}$ if hot channel suppression is included in the pixel readout system. The result of our study is that the efficiency and rejection of the baseline trigger are preserved up to $\langle\mathrm{N}\rangle \approx 100$, which corresponds to a contribution of random background to the occupancy of $10^{-4}$. We expect the detector to have a much lower noise level than this. However, it was observed that processing time did get somewhat longer when the number of hits rose above a mean of 25 . A real estimate of the processing time in the DSP used to reconstruct the minivectors has been made and is described in section 8.6.

\subsubsection{Correlated Noise Study}

In this study, we used GEANT to simulate the events and turned on all its physics processes. This led to additional hits from hadronic interactions, photon conversions, decays in flight, and curlers (a.k.a loopers). Fig. 8.4 shows that average number of hits per plane rises by about $20 \%$ from $\approx 8$ hits/plane to $\approx 10$ hits/plane. A degradation of 1.5 is observed in the overall rejection capability of the trigger.

There are other sources of correlated noise. For example, beam-gas interactions or beam halo particles might leave long tracks in the detector. Although the time between crossings is long, there may still be background hits from previous crossings whose source and distribution we are not currently modeling. None of these is expected to be a serious problem but more work does need to be done to prove this definitively. 


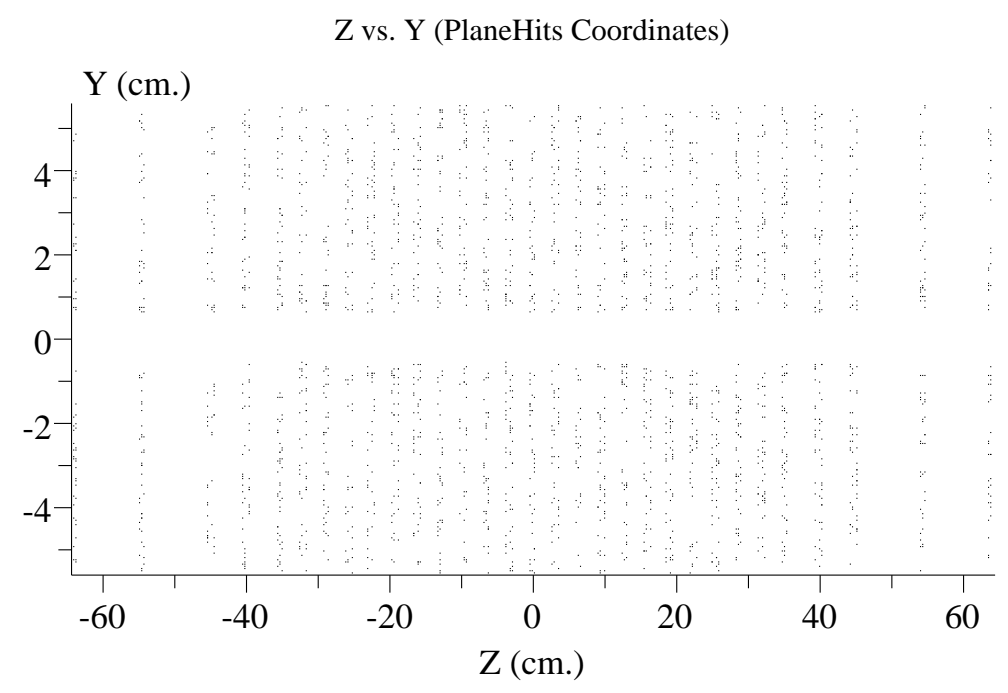

Figure 8.2: Pixel hits in Z-Y projection with an average of 25 random noise hits from an MCFAST simulation

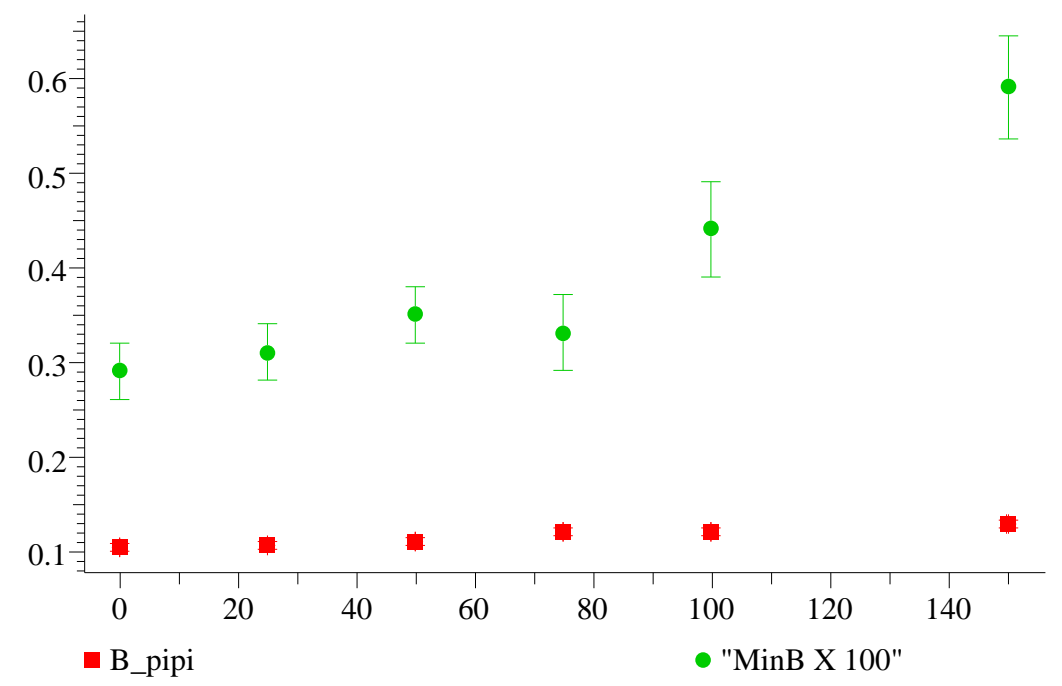

Figure 8.3: Efficiency vs average number of random noise hits for minimum bias and $B$ events. Three detached tracks were required. The acceptance for $B$ events includes all events, even those not falling into the geometrical acceptance. 


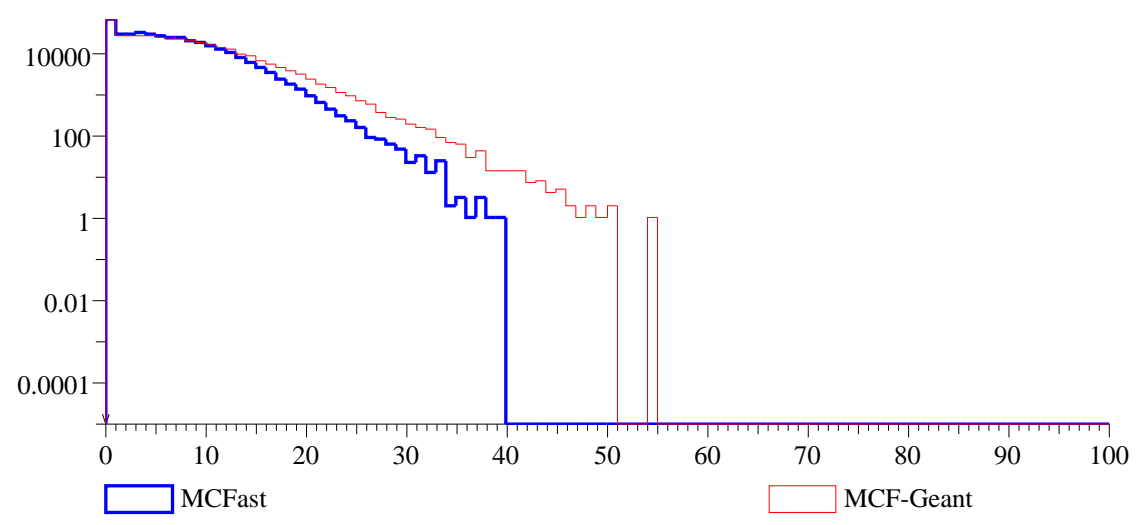

Figure 8.4: Hit multiplicity for minimum bias events in GEANT simulation and MCFAST simulation

\subsubsection{Multiple Interactions per Crossing Study}

While BTeV will begin running at a luminosity of $5 \times 10^{31} \mathrm{~cm}^{-2} \mathrm{~s}^{-1}$, in order to achieve the desired statistical precision, we will eventually want to run at a luminosity of at least $2 \times 10^{32}$. Because the Tevatron will have only 108 bunches, this means that the experiment, and in particular the trigger, must be able to handle multiple interactions per crossing. The lower luminosity creates an average of 0.5 inelastic interactions per crossing, while the higher luminosity generates an average of 2.0.

As shown below, even in the presence of multiple interactions, the trigger continues to give good efficiency for states with $B$ decays while providing good rejection against minimum bias events because the occupancy of the pixel vertex detector is very low and the luminous region of the Tevatron is long.

In order to achieve good results when there are multiple primary vertices, we had to make only a small refinement to the trigger algorithm: we put an upper limit on the impact parameter (with respect to the primary vertex) which is used to define secondary vertex track candidates. This prevented tracks not used in estimating a given primary vertex (but associated to this interaction) from automatically appearing as 'detached' when compared to another primary vertex. Here we present only a brief summary of the results of an extensive study [3].

\section{Performance on Single Interaction Crossings}

The average number of tracks per interaction (primary and secondary) which pass through at least three stations of the pixel detector is 30 for minimum bias events and 38 for $B^{\circ} \rightarrow$ $\pi^{+} \pi^{-}$events (which will be called $B$ events from now on). After a set of cleanup cuts on the tracks, the average multiplicity of tracks that the trigger uses in the primary vertex search is $\approx 12$ for minimum bias events and $\approx 20$ for $B$ events. Two additional cuts are placed on tracks which are candidates for use in the primary vertex: they must have a transverse 


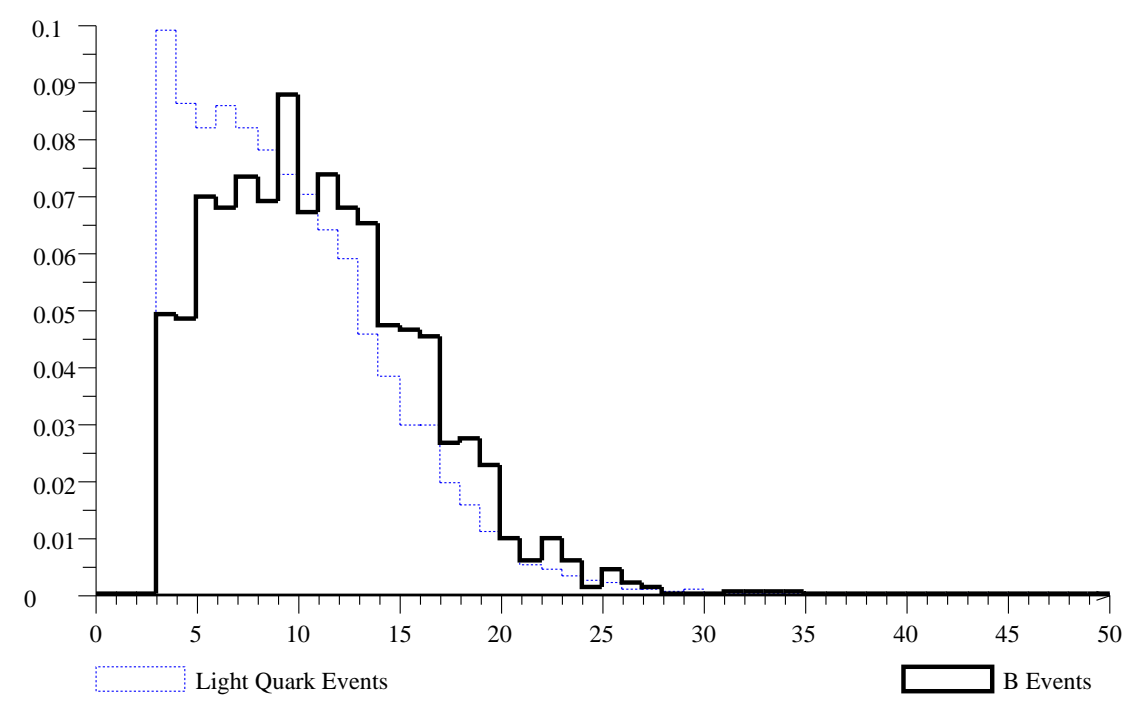

Figure 8.5: The track multiplicity distribution in the primary vertex, for single interaction crossings. The average is 9.6 for minimum bias events, and 11.1 for $B \rightarrow \pi \pi$.

momentum of less than $1.0 \mathrm{GeV} / \mathrm{c}$; and they must intersect the nominal beam line within the luminous region. With these requirements, the resultant multiplicity of primary vertex tracks found by the trigger algorithm in a crossing with exactly one interaction is given in Fig. 8.5 for minimum bias and $B$ events. The $Z$ resolution (i.e. along the beam axis) of the primary vertex is given in Fig. 8.6. Note that this distribution has typically more tails for the $B$ sample because of pattern recognition failures in events with multiple vertices: if many tracks from a $B$ decay are mistakenly taken as primary tracks, the $B$ decay vertex could be wrongly taken as the primary. Nevertheless, since some tracks are still likely to be detached, these events could pass the trigger.

To form a primary vertex requires at least 3 tracks. As a consequence of this cut and the transverse momentum cut, the probability to find a second, false, primary vertex is very small, only $0.05 \%$, in minimum bias events. The 'leftover' track multiplicity after primary vertex formation is very different for minimum bias and $B$ events. If one applies an additional detachment cut on the normalized impact parameter $\frac{\sigma_{b}}{b}$ of 3 and a transverse momentum cut $P_{t}>0.5 \mathrm{GeV} / \mathrm{c}$ one has reconstituted the analysis we presented in 1997 [1]. To deal with multiple interactions, we added the requirement that the 'detached track candidate' not be too far from the primary vertex - the impact parameter $b$ must be less than 0.2 $\mathrm{cm}$ from the primary. This cut also allows us to reject tracks from $K_{s}$ decays. In addition, further cleanup cuts on these detached tracks are now applied. For instance 4 station hits (or minivectors) on a track are required. Then, one obtains the distribution in Fig. 8.7.

Table 8.1 shows the trigger acceptance for minimum bias events and $B$ events for single interaction crossings. Our goal for Level 1 was to accept fewer than $\approx 1 \%$ of minimum bias events, which is achieved in all conditions. Other triggers based on global properties of the 


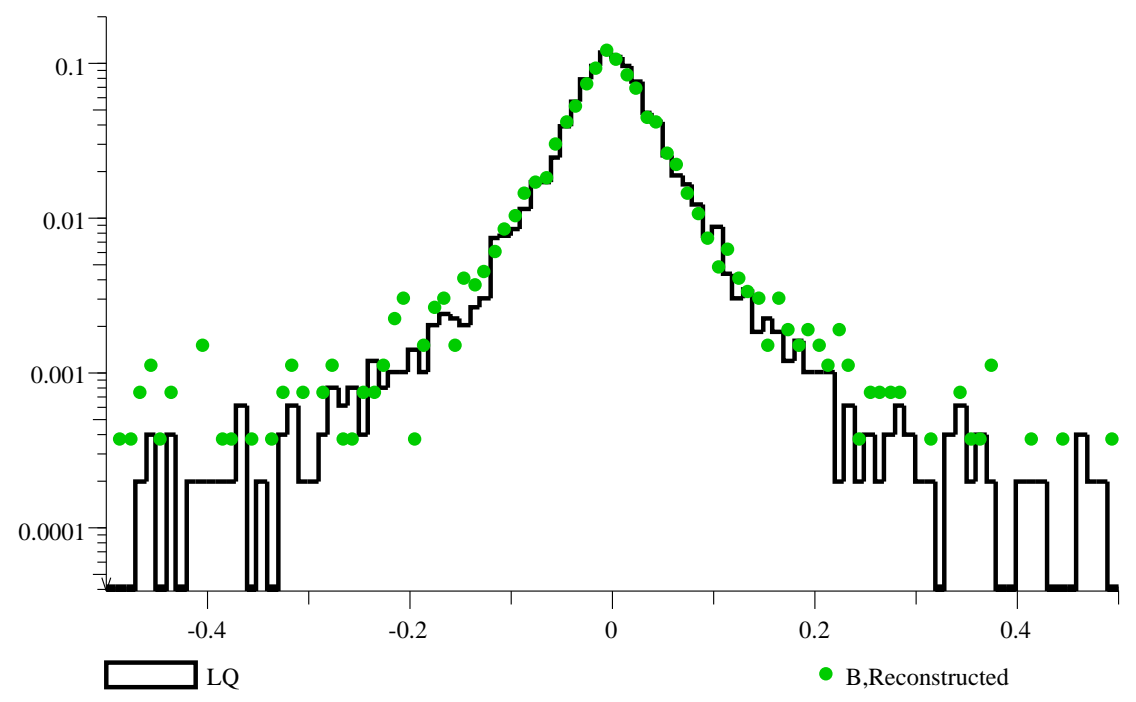

Figure 8.6: The primary vertex $Z$ resolution, for single primary vertex events. The width $(\sigma)$ of the core of these distributions is approximately $270 \mu \mathrm{m}$, while the r.m.s is about 760 microns, reflecting the presence of tails in the distributions.

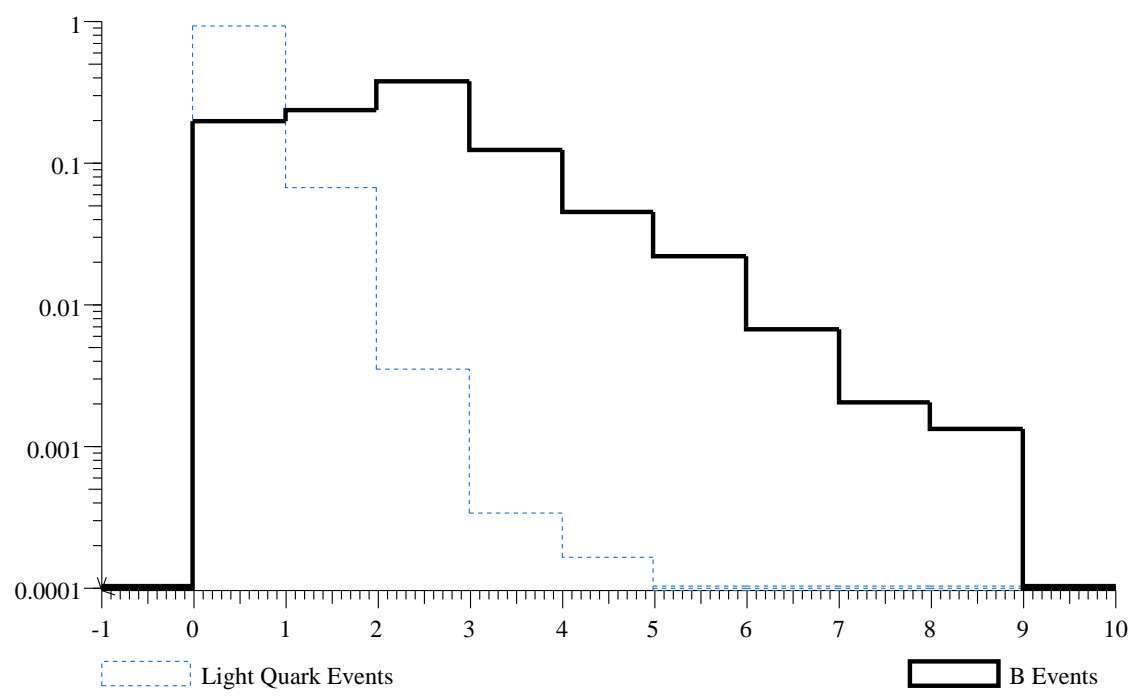

Figure 8.7: The detached, $P_{t}>0.5 \mathrm{GeV} / \mathrm{c}$ track multiplicity for both samples. The average track multiplicity for minimum bias $(B)$ is $0.6(1.5)$. 
Table 8.1: The trigger acceptance (\%) for single interaction crossings, for the minimum bias sample and the $B \rightarrow \pi \pi$ sample, versus selection criteria (number of tracks detached from the primary, detachment cut, $P_{t}$ on each detached track, and impact parameter upper limit in $\mathrm{cm})$. The errors are statistical only.

\begin{tabular}{||l|c|c||}
\hline \hline Criteria & Minimum Bias & $B \rightarrow \pi \pi$ \\
\hline 2 detached tracks $\left(4 \sigma ; P_{t}>0.5 \mathrm{GeV} / \mathrm{c} ; b_{t}<0.2\right)$ & $0.9 \pm 0.1$ & $54.9 \pm 0.9$ \\
3 det. $\operatorname{tr} .\left(3 \sigma ; P_{t}>0.5 \mathrm{GeV} / \mathrm{c} ; b_{t}<0.2\right)$ & $0.05 \pm 0.02$ & $18.5 \pm 1.2$ \\
3 det. $\operatorname{tr} .\left(3 \sigma ; b_{t}<0.2 ; S d_{P t}>1.0\right)$ & $0.17 \pm 0.04$ & $27.0 \pm 1.5$ \\
\hline \hline
\end{tabular}

vertex and its ensemble of detached tracks have been studied. For instance, the total scalar transverse momentum $S d_{P t}$ summed over all these detached tracks could be used, as in the third line of Table 8.1.

\section{Performance on Multiple Interaction Crossings}

Fig. 8.8 shows the number of primary vertices reconstructed by the trigger algorithm for an average of 0.5 and 2.5 interactions per crossing, corresponding roughly to luminosities of $0.5 \times 10^{32}$ and $2.5 \times 10^{32} \mathrm{~cm}^{-2} \mathrm{~s}^{-1}$. These plots suggest that the number of reconstructed vertices is well correlated to the number of generated vertices even in these busy events, which can be seen as follows: The distribution can be qualitatively understood as a Poisson distributed number of interactions with a fixed vertex finding efficiency of about $62 \%$. The efficiency is low because the trigger is deliberately made insensitive to very soft collisions such as quasi-elastic and low multiplicity interactions which are unlikely to contain $B$ 's.

Fig. 8.9 shows that the primary vertex $Z$ resolution is independent of the number of primary vertices per crossing. No degradation of this resolution is observed as the luminosity increases. Each interaction can be studied independently because the average distance between primary vertices is much larger than the resolution.

Fig. 8.10 shows the 'multiplicity distribution' of detached tracks for different numbers of reconstructed vertices when the average number of interactions per crossing is $\approx 0.5$ (corresponding to a luminosity of $\approx 5 \times 10^{31} \mathrm{~cm}^{-2} \mathrm{~s}^{-1}$ ), and a luminosity 5 times this amount. The main point is that there is not a large tail of events in which the number of detached tracks begins suddenly to become troublesome as the number of reconstructed vertices increases. We observe that the efficiency for detecting $B$ events does not degrade as the number of actual or reconstructed vertices increases. Note also that the detached tracks must be associated to the same primary vertex in order to satisfy the trigger.

The total yield of $B$ events per unit time does not degrade as additional detached tracks are found in other primary vertices, provided of course one does not explicitly reject these multiple primary vertex crossings. If one does, the yield follows what one would expect from the simple Poisson law. The probability of triggering on a crossing with at least one inelastic interaction is shown vs luminosity (in units of $10^{32} \mathrm{~cm}^{-2} \mathrm{~s}^{-1}$ ) in Fig. 8.11. (Here, we have used a trigger requirement of at least 3 tracks each missing the primary by $\geq 3 \sigma$.) This 

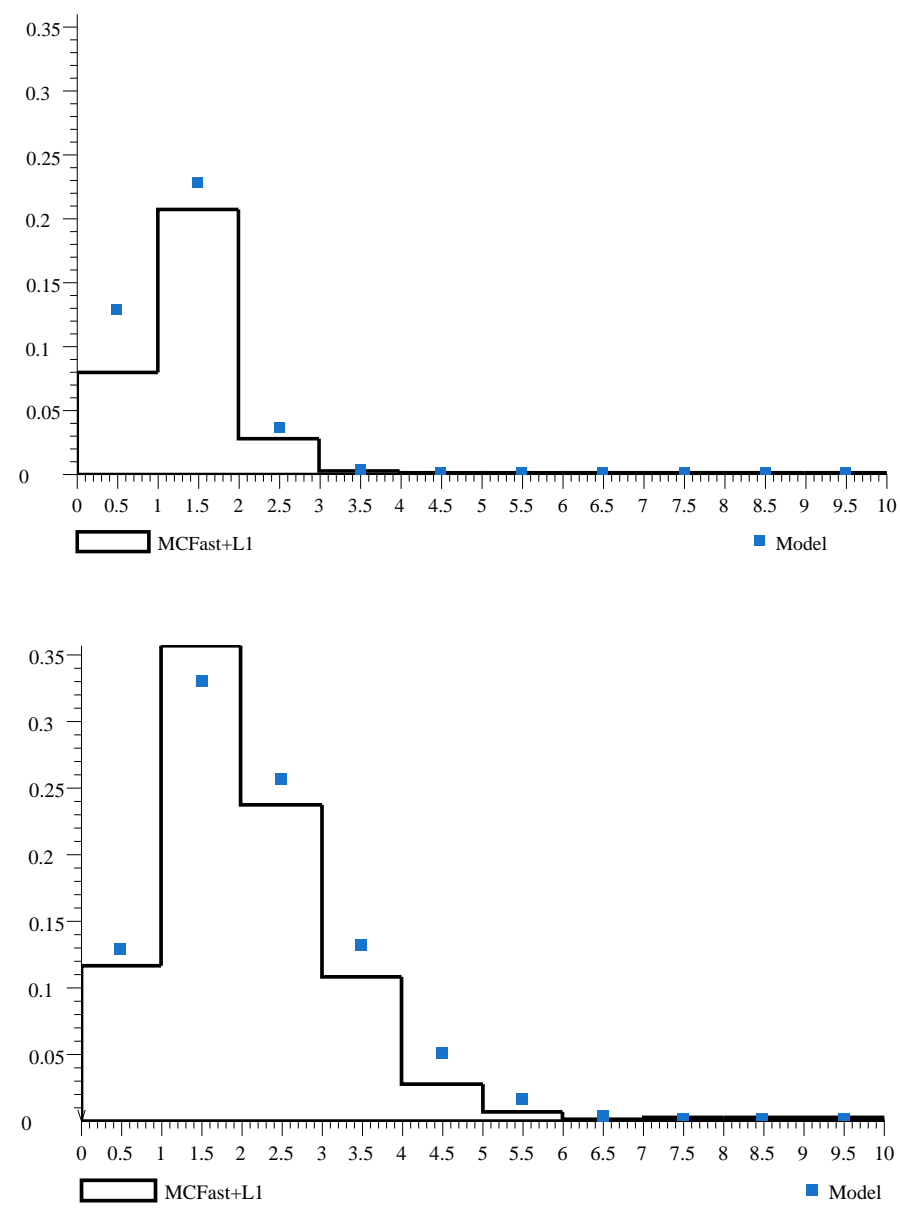

Figure 8.8: The Level 1 primary vertex multiplicity distribution running at luminosities of $5 \times 10^{31}$ (top plot) and $2.5 \times 10^{32}$ (bottom plot). These histograms are normalized to the total number of $132 \mathrm{~ns}$ crossings in the run. For instance running at $2.5 \times 10^{32}$, the probability to reconstruct one and only one primary vertex is about $35 \%$. Also shown is a simple model assuming a fixed vertex geometrical acceptance and reconstruction efficiency of about $62 \%$. 


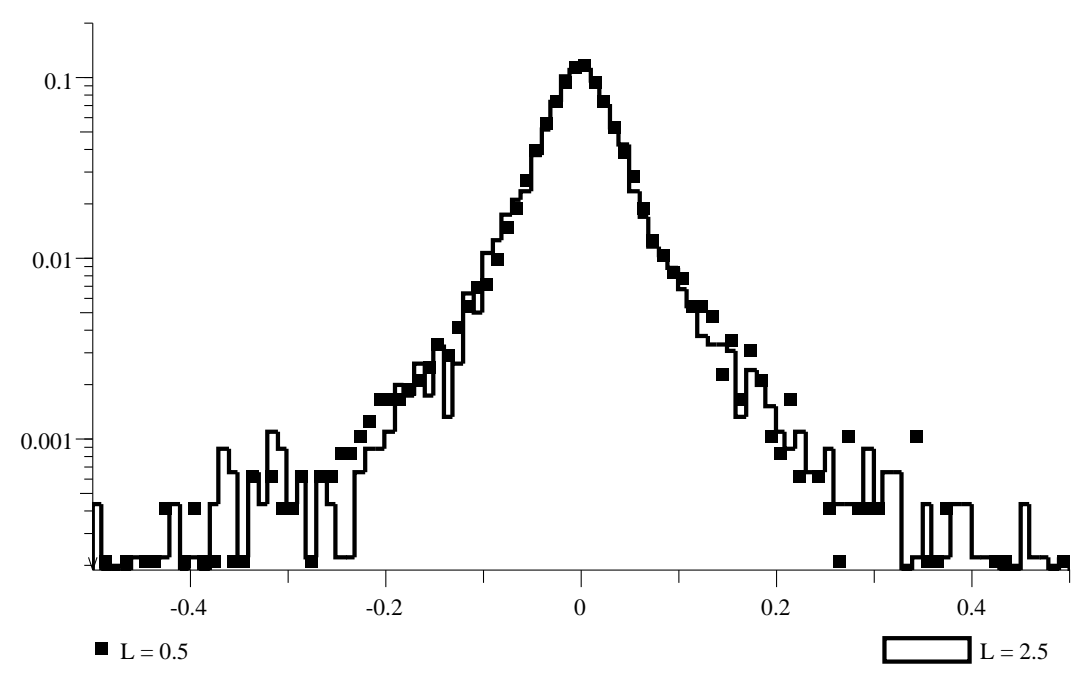

Figure 8.9: The $\mathrm{Z}$ position primary vertex resolution plot, running at luminosities of $5 \times 10^{31}$ and $2.5 \times 10^{32}$.

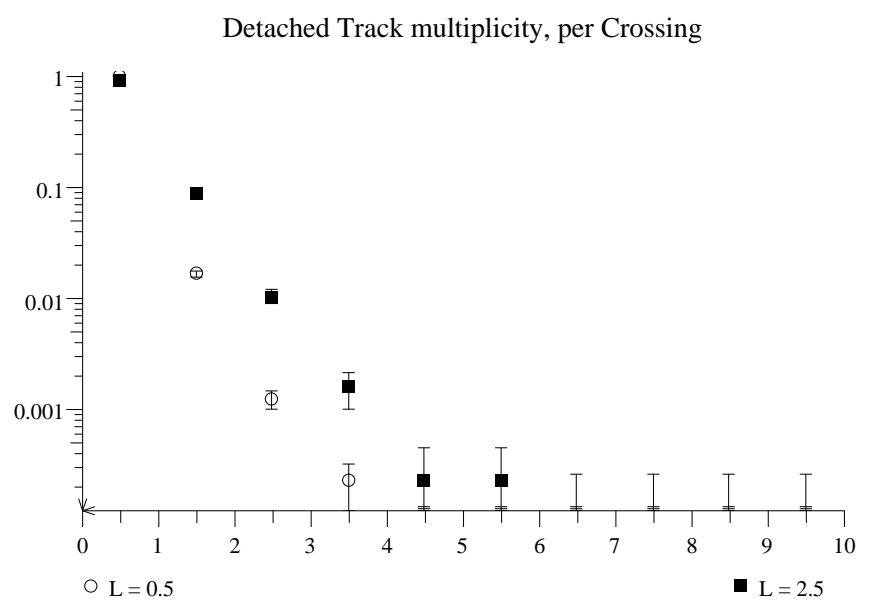

Figure 8.10: The detached $\left(3 \sigma, P_{t}>0.5\right)$ track multiplicity distributions for light quark events running at a luminosities of $5 \times 10^{31}$ and $2.5 \times 10^{32}$. These are normalized to the total number of crossings in the run: the probability to observe 1 detached track at $L=5 \times 10^{31}$ is $\approx 1.6 \%$, and at $L=2.5 \times 10^{32}$, it is $\approx 8.5 \%$ 
probability is about twice as large if the two detached track at $4 \sigma$ trigger is used. Ideally, one would expect the yield per unit time to increase linearly and it does increase roughly linearly as long as one does not restrict the number of interactions too severely. The probability for triggering on crossings with inelastic interactions does increase but stays below $1 \%$ up to a luminosity of $2.5 \times 10^{32}$ if we allow at most three reconstructed vertices. For the two tracks at $4 \sigma$, in the worst case, the probability is slightly above $1 \%$. There are many additional requirements (such as the presence of a true secondary vertex rather than tracks missing the primary) that can be imposed at Level 1 to reduce the trigger rate even more.

Our results on the performance of the baseline trigger in the presence of multiple interactions per crossing are quite encouraging. In essence,

- The trigger rate is not dominated by unresolved multiple vertices up to at least $2 \times 10^{32}$

- A Level 1 rejection factor of about one in a hundred is possible with a Level 2 bandwith that can be achieved (see chapter 9).

- One gains sensitivity by running above an average of 1 interaction per crossing provided we accept crossings with up to three interaction vertices.

Thus, we believe that it is possible to achieve a rejection against minimum bias events better than 1/100 at Level 1 up to a luminosity of $2 \times 10^{32}$ with good efficiency for $B$ events we feel confident to reconstruct offline. One can work above $2 \times 10^{32}$ provided one can handle the data rate of falsely accepted minimum bias events into the Level 2 trigger or one can reduce the rate into Level 2 by adding more requirements on Level 1 . However, there is more work to be done in making the simulation more realistic and in refining the algorithm. Also, we need to estimate the required CPU power at each stage of the reconstruction, to balance the data flow in the pipeline. We have started looking into the queuing problems at the front end of the triggering system. We look forward to reporting further on this in the future.

\subsection{Efficiency and Rejection of the BTeV Level 1 Vertex Trigger for Selected Physics States of Interest}

The results are documented in the above section and in part 3. Here we summarize our results. The rejection factor on light quark events for the two detached track trigger is about $1 / 100$ for single interaction crossing and is proportional to the luminosity if multiple interactions per crossing are accepted. We summarize the efficiency for this trigger in table 8.2. In all cases, the opposite b-hadron decays following the standard QQ decay table (defined in part3). 
Probability to trigger/crossing (\%)

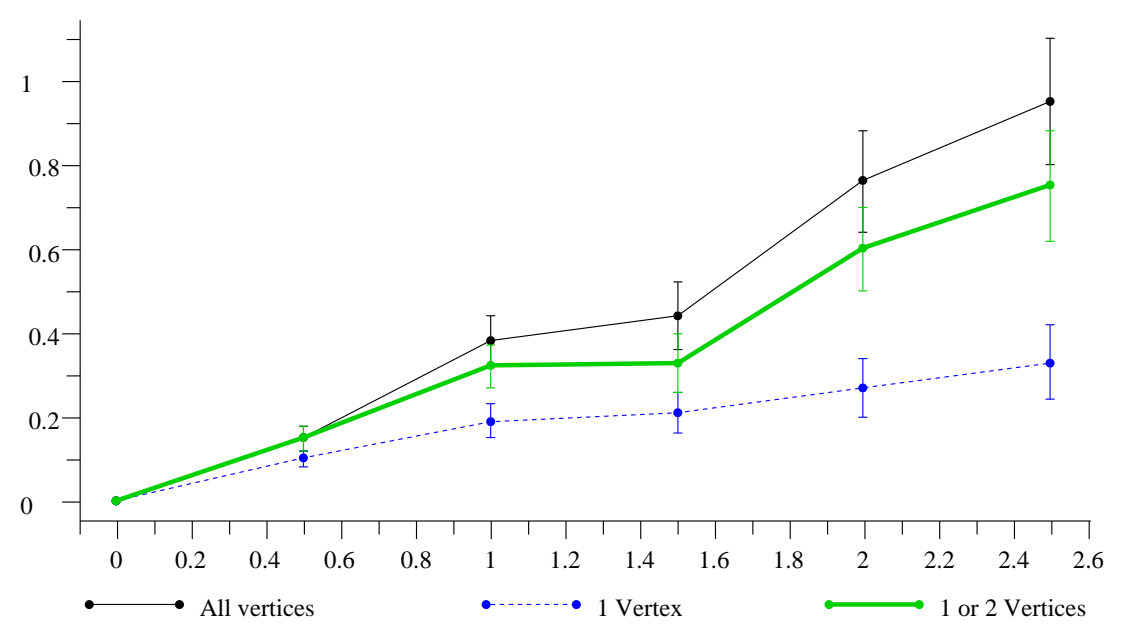

Figure 8.11: The absolute probability for triggering per $132 \mathrm{~ns}$ beam crossing. The trigger is based on three detached tracks pointing to the same longitudinal hemisphere, with $S d_{P t}>$ 1.0. For both plots, the abscissa axis is the luminosity in units of $10^{32} \mathrm{~cm}^{-2} \mathrm{~s}^{-1}$. The solid line is for events with any number of primary vertices; the dotted line, for events with one and only one reconstructed vertex; and the thick gray line for events with 1 or 2 reconstructed vertices.

Table 8.2: The two detached track at $4 \sigma$ trigger efficiency for various $B$ decays.

\begin{tabular}{||l|c||}
\hline \hline Final State & Efficiency (\%) \\
\hline$B \rightarrow \pi^{+} \pi^{-}$ & 55 \\
$B_{s} \rightarrow D_{s} K$ & 70 \\
$B^{-} \rightarrow D^{0} K^{-}$ & 60 \\
$B^{-} \rightarrow K_{s} \pi^{-}$ & 40 \\
$B^{0} \rightarrow K^{+} \pi^{-}$ & 54 \\
$B^{0} \rightarrow J / \psi K_{s}$ & 50 \\
$B_{s} \rightarrow J / \psi K^{*}$ & 69 \\
$B^{0} \rightarrow K^{*} \gamma$ & 40 \\
\hline \hline
\end{tabular}




\subsection{Other Level 1 triggers}

While we have emphasized the "vertex/impact parameter" trigger because it is our most crucial and most ambitious trigger, we will also have other triggers at Level 1 . These will include muon, minimum bias, and (possibly) electron triggers and perhaps some triggers derived from the same hardware that is used for the vertex trigger but optimized for different physics.

\subsection{Status Report on Level 1 trigger R\&D and trigger prototype}

The Level 1 vertex trigger is a key feature of the $\mathrm{BTeV}$ detector design. A proposed scheme for the vertex trigger has been worked out by the Penn group in collaboration with D. Husby at Fermilab. The hardware design for this system is described in detail in references [4] and [5]. Although the design can be implemented with DSPs (digital signal processors) and FPGAs (field-programmable gate arrays) that are available today, an intensive R\&D program is needed to develop the complete design, test the viability of the system, and perhaps modify some of the hardware components as new hardware becomes available. The $\mathrm{R} \& \mathrm{D}$ program has three main components:

- Simulations;

- Hardware prototyping;

- Software development.

Simulations of the trigger are an important aspect of the trigger $R \& D$ program. We generate pixel hits using the MCFast simulation program, and process the hits using a software implementation of our Level 1 trigger algorithm. This procedure allows us to test the trigger algorithm, and to refine the algorithm as we include more realistic running conditions for the experiment. We also use trigger simulations to learn more about the performance of the Level 1 trigger under "normal" and "extreme" running conditions, and to develop the software that is needed for our trigger prototype (see Fig. 8.12).

Although much of the testing of the trigger algorithm can be done with simulations, there are tests that are best done with a hardware trigger prototype. The trigger prototype is a single-board module that implements most of the data paths and processing steps used in the trigger system. It has FIFOs to load data generated by the MCFast simulation program, and it includes four hit processors, four farm (track or vertex) processors, and the merging and switching circuitry that connects them.

The prototype is used to test data flow through the trigger system. There are three primary data paths that are tested: 
1. Single station-quadrant to track finder.

In this test, the prototype is used to test the path from one quadrant of a pixel station to the farm processors that reconstruct tracks. Pixel hits from the three planes of a station-quadrant (see Fig. 8.13) are divided into eight $\phi$ slices, and fed to an array of eight hit processors. The prototype is used to test paths through four of the eight processors. These hit processors execute the segment-finding algorithm that finds minivectors in the vertex trigger. The minivectors are sent through the sorting network to the farm processors. Each farm processor runs the standard track-finding code to produce track records that are written to a FIFO, which is used to read out and verify test results. The results are used to investigate hit-processor throughput, study the effects of extreme event sizes, and test the ability of the switching network to sort hits.

2. 31 stations to track finder.

This tests the flow of data for all 31 pixel stations that make up a single $\phi$ slice. The four hit processors execute code that mimics the data generated by 31 hit processors. We use the results from this test to study the performance of the switching network under a full load.

3. 32 track finders to vertex finder.

This tests the flow of data from 32 track processors to the vertex processing farm. The four hit processors execute code that mimics data generated by the track processors for all $\phi$ slices. We use the results to study the switching network, and the vertex finding algorithm.

For each of the three tests, a performance monitor keeps track of statistics on the data paths that are used during each cycle. These statistics are sent to a host computer for graphical display and analysis. The host computer is also used to configure, monitor, and control each of the processors and FPGAs in the prototype.

The design of the trigger prototype entails both hardware and software development. The design of the hardware has been completed. The software that is being developed for the prototype consists of code that is used to implement test scenarios, and the three programs that implement the Level 1 vertex trigger algorithm. The first of these three programs runs on hit processors (each hit processor is a Texas Instruments DSP) and finds track minivectors. The second and third programs run on farm processors (each farm processor is an Analog Devices DSP) and perform track and vertex reconstruction, respectively. The Texas Instruments and Analog Devices DSPs differ in their hardware architecture. Each DSP requires software for data flow and buffer management, and the software to implement the Level 1 trigger algorithm.

The software development for the DSPs encompasses three different aspects:

- Data flow and buffer management; 
- Trigger algorithm;

- Optimization.

The code that handles the data flow and buffer management is specific to the hardware architecture of each DSP, and depends on the design details of the trigger prototype. The code that implements the vertex trigger algorithm is based on the trigger code used in our MCFast simulations, but significant modifications are required to produce code for the DSPs. Compared to the trigger code in MCFast, the software for the DSPs is optimized to take advantage of the DSP architecture. This optimization requires assembly language programming to achieve the performance (see timing results in the following section) that we require for the Level 1 vertex trigger.

\subsection{Results on Baseline Level 1 Algorithm Timing on Digital Signal Processors}

With 100 gigabytes of pixel data coming into the Level 1 trigger every second, a beam crossing every $132 \mathrm{~ns}$, and an average of 2 interactions per crossing, the time required for each pipeline step in the trigger is critical. Timing issues are studied by performing a variety of trigger simulations. These include Monte Carlo studies of the pattern recognition and reconstruction algorithms, and simulations of the trigger hardware. Furthermore, the timing of both the trigger hardware and software algorithms will be extensively tested with the trigger prototype described in the previous section. In this section, we describe timing studies for algorithms on DSPs (digital signal processors).

The BTeV trigger utilizes two types of DSPs: a fixed-point DSP, and a floating-point DSP. The fixed-point DSP is made by Texas Instruments and belongs to the TMS320C6X family of DSPs. The floating-point DSP is an Analog Devices ADSP-2106x SHARC. The trigger code for both the Texas Instruments DSP and the Analog Devices DSP is developed with the aid of simulation software provided by each company. We also use this software to obtain the timing results presented in this section.

Simulations of the Level 1 design show that the first stage of the trigger, the segment finder, requires the most computational power compared to other parts of the trigger. A large number of operations are performed by the segment finder, due to the number of combinations of pixel hits that must be sampled to find the hits that define a mini-vector. To address this problem, we introduce considerable parallelism in the trigger architecture. This is a key feature of the Level 1 trigger design. The parallelism begins with the organization of the data readout from the pixel detectors. Pixel hits are read out in parallel for each quadrant of a pixel plane. The hits from the three planes that belong to a pixel station are brought together in a quadrant processor, which represents one of 124 identical circuit boards that make up the first stage of the trigger. Additional parallelism is introduced by subdividing the pixel hits in a quadrant into $8 \phi$ slices, for a total of $32 \phi$ slices per pixel station. The hits in each $\phi$ slice are processed in parallel by one of the 992 TMS320C6X DSPs 


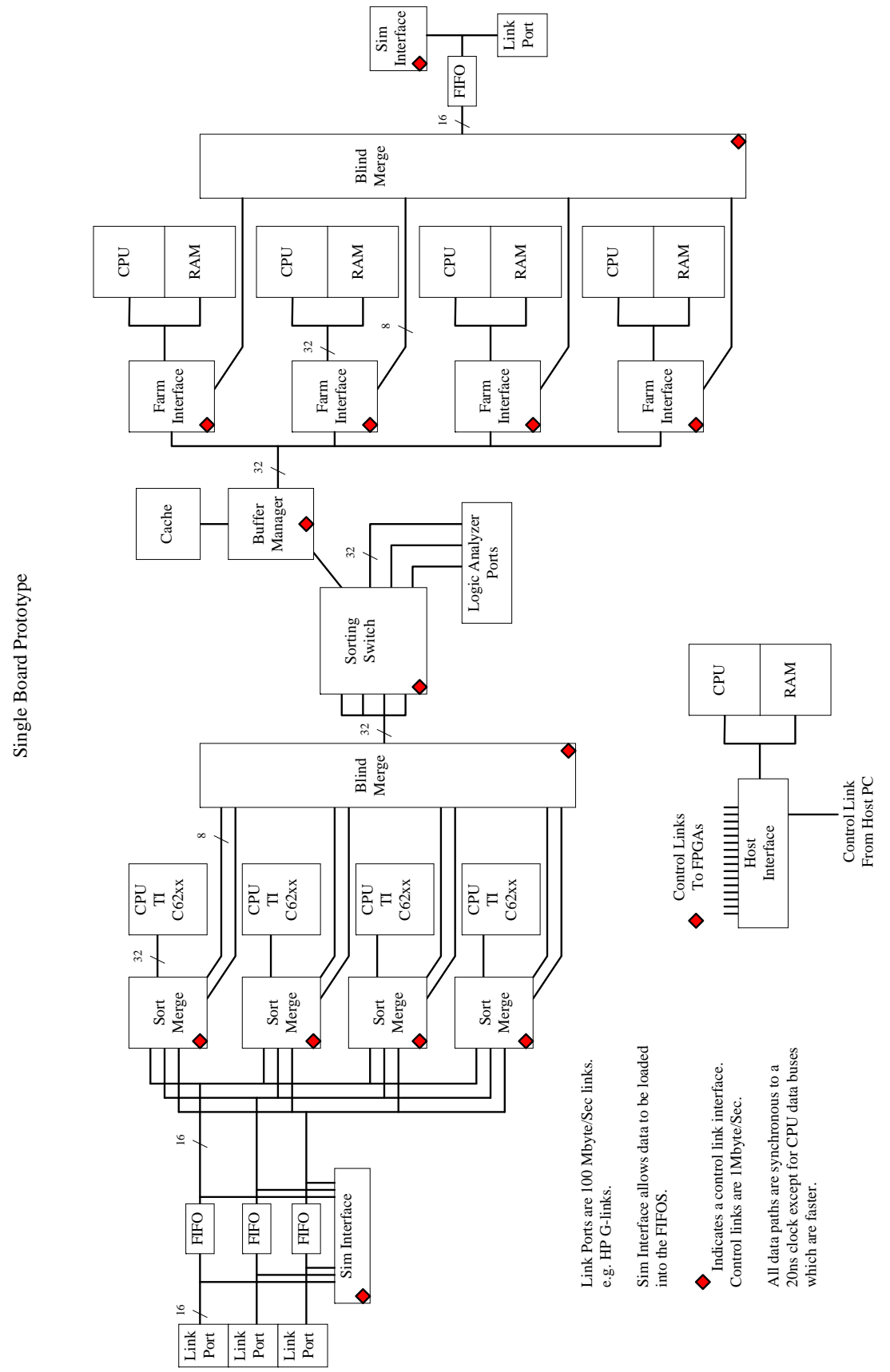

Figure 8.12: Schematic of Single Board Trigger Prototype 


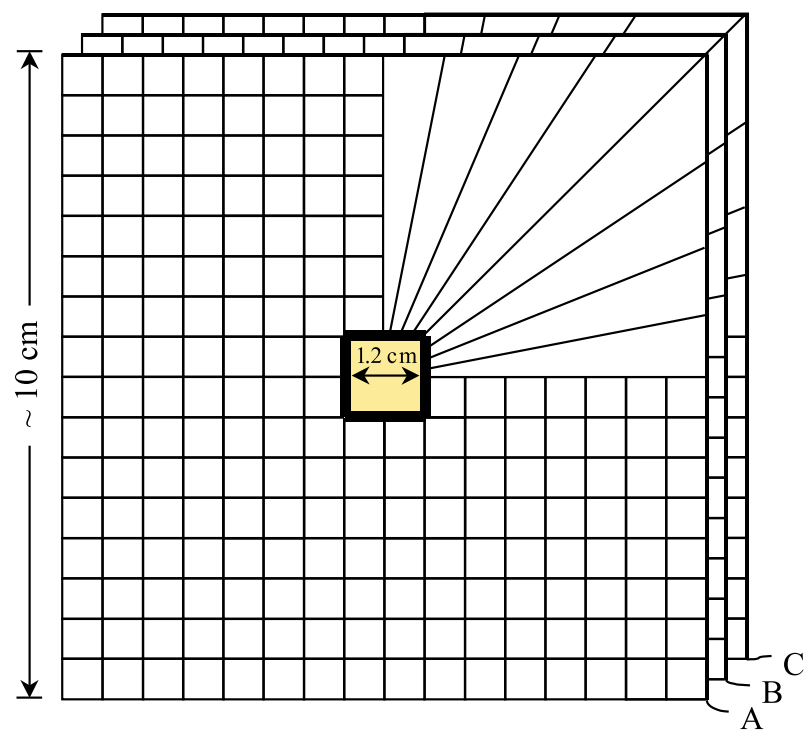

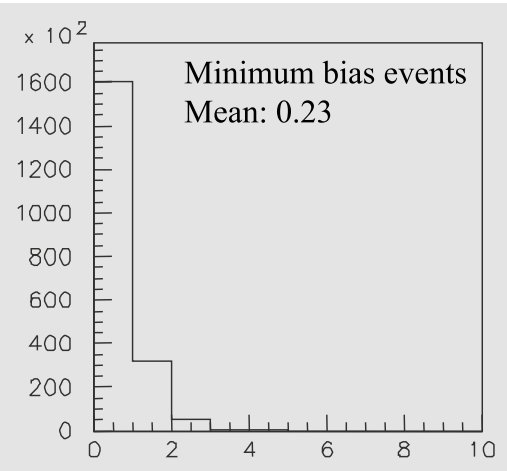

Number of hits per $\phi_{32}$ slice per pixel plane per interaction

Figure 8.13: Trigger view of one quadrant in a pixel station with planes A, B, and C. The histogram on the right shows the number of hits for each plane per $\phi$ slice for minimum bias events.

(a total of 992 DSPs for 31 pixel stations, each with $32 \phi$-slice DSPs). Fig. 8.13 shows the "trigger view" of one quadrant, and shows that the subdivision of hits into $\phi$ slices reduces the mean number of hits per pixel plane to a value of 0.23 hits per $\phi$ slice for minimum bias events.

Even with the small number of hits per $\phi$ slice, the timing for the segment finder is critical. Table 8.3 shows timing results for the segment finder algorithm developed for the TMS320C6X DSP. In the table we choose four cases to present our results: (i) For a given $\phi$ slice and with three planes (A, B, and C) in a pixel station (as shown in Fig. 8.13), we have a single hit in each plane for one track passing through a pixel station. This is the $(1,1,1)$ case in Table 8.3. (ii) For two tracks passing through the same $\phi$ slice we have two hits per plane, or $(2,2,2)$. (iii,iv) With three and four tracks in the same $\phi$ slice we get the $(3,3,3)$ and $(4,4,4)$ cases, respectively.

The timing results for these four cases are divided into three categories that represent different levels of optimization for the segment finder algorithm. The first category involves $C$ code that has not been optimized (the code is compiled without any optimization), and we use this to establish a reference measurement for subsequent measurements involving different levels of optimization. For the second category we allow the $C$ compiler for the TMS320C6X DSP to optimize the algorithm, and for the third case the programming is done directly in assembly language. Although programming in assembly language is more cumbersome, we can optimize the code so that all hardware components in the DSP are performing useful operations most of the time. Our timing results in Table 8.3 show that 


\begin{tabular}{|c|c|c|c|}
\hline \hline $\begin{array}{c}\text { Number of hits } \\
\text { per } \phi \text { slice in } \\
\text { planes }(\mathrm{A}, \mathrm{B}, \mathrm{C})\end{array}$ & $\begin{array}{c}\text { Time for } \\
\text { non-optimized } \\
\text { segment finder(ns) }\end{array}$ & $\begin{array}{c}\text { Time for compiler } \\
\text { optimized } \\
\text { segment finder(ns) }\end{array}$ & $\begin{array}{c}\text { Time for assembler } \\
\text { optimized } \\
\text { segment finder(ns) }\end{array}$ \\
\hline$(1,1,1)$ & 1915 & 1630 & 135 \\
$(2,2,2)$ & 5705 & 3670 & 315 \\
$(3,3,3)$ & 14015 & 7560 & 1340 \\
$(4,4,4)$ & 28915 & 13840 & 2885 \\
\hline weighted average & 530 & 395 & 37 \\
\hline \hline
\end{tabular}

Table 8.3: Timing results in nanoseconds for the segment finder algorithm running on the TMS320C6X DSP.

the $C$ compiler for the TMS320C6X DSP is not very effective at optimizing the segment finder algorithm; the compiler achieves only a minor performance gain for the $(1,1,1)$ case, and at best a factor of two improvement for cases with more hits in a $\phi$ slice. Table 8.3 also shows that we are able to achieve almost an order of magnitude improvement in the timing by optimizing the algorithm in assembly language. This does not include additional improvements that can be achieved for the $(3,3,3)$ and $(4,4,4)$ cases, which have not yet been fully optimized.

To obtain an estimate of the average time required to find mini-vectors in minimum bias events, we compute a weighted average by using the results in Table 8.3 weighted by the distribution of hits in Fig. 8.13. We use a value of zero for the $(0,0,0)$ case, since the DSP does not perform any operations when there are no hits in a $\phi$ slice. The average time for each level of optimization is shown in the last row of Table 8.3. Although these results are somewhat oversimplified, they do indicate that an assembly language implementation of the segment finder algorithm is necessary so that the first stage of the trigger stays within a budget of $132 \mathrm{~ns}$ per beam crossing. The average time of $37 \mathrm{~ns}$ for the segment finder is encouraging, since it suggests that we are close to having a viable implementation for the segment finder in our Level 1 design.

The track and vertex processor timing are also under study. We hope to present results soon.

\subsection{Options to the Baseline Level 1 Vertex Trigger}

\subsubsection{An Alternative Using Two Plane Stations}

The choice of three-plane pixel stations in the baseline detector is dictated by the baseline trigger design. That trigger uses the three planes to make minivectors that can then be projected to a small portion of the next station, which substantially reduces the combinatorics. Any attempt to use fewer planes per station will have to cope with larger combinatorics when starting the search for tracks. 

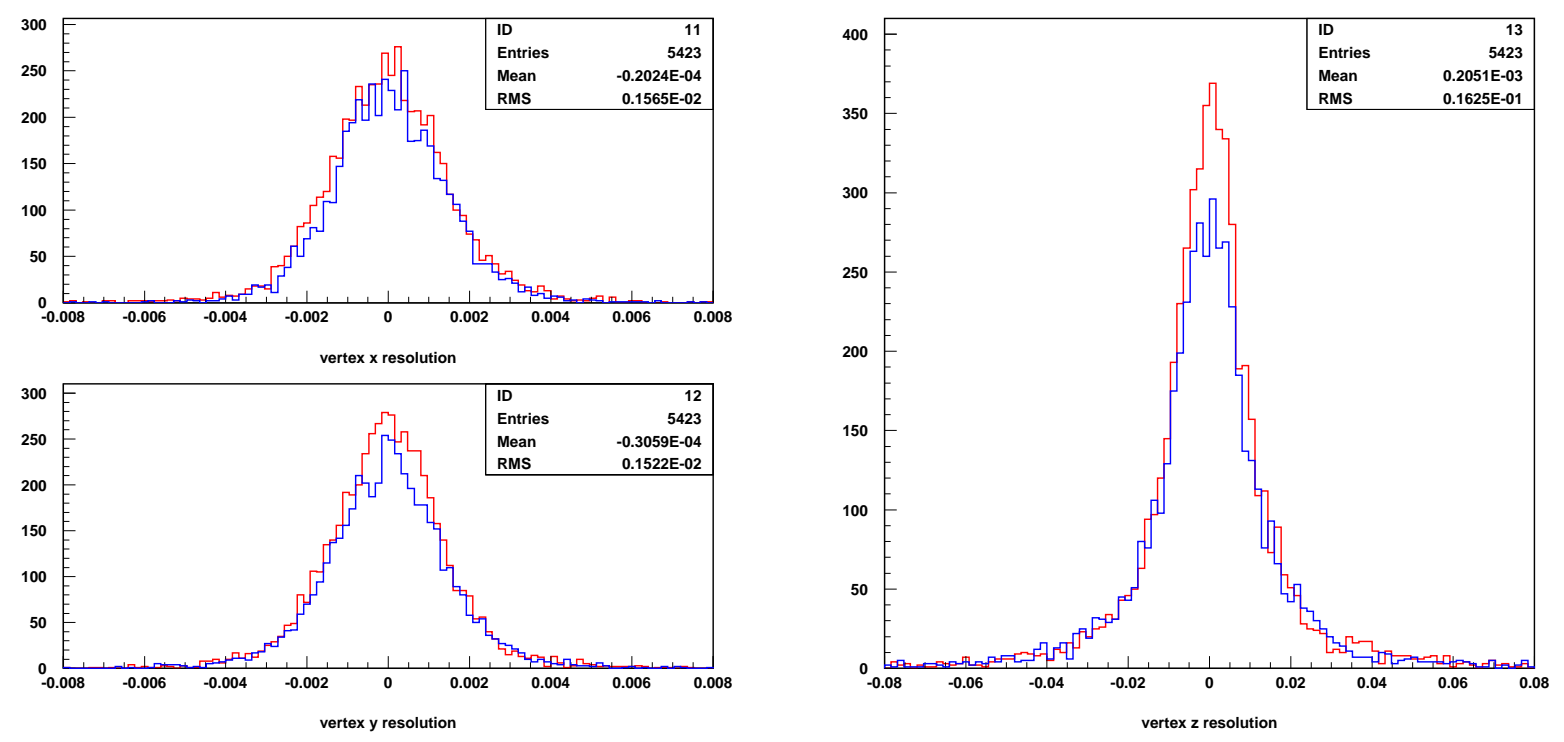

Figure 8.14: Comparison of the vertex resolution for $B^{0} \rightarrow \pi^{+} \pi^{-}$for two-plane pixel stations and three-plane pixel stations-left top $x$, left bottom $y$, right $z$.

The removal of one plane per station has advantages of lower cost, a simpler detector, and less material with no loss of physics capability. Simulations using the identical pixel detectors arranged in 31 stations of three planes and 34 stations of two planes showed almost no difference in the effective resolution and efficiency of the two detectors on charged particle states. A full offline analysis was done for several interesting physics channels, $B^{0} \rightarrow \pi^{+} \pi^{-}$, $B_{s}^{0} \rightarrow \Psi K^{*}$ and $B_{s}^{0} \rightarrow D_{s}^{+} \pi^{-}$. In the case of $B^{0} \rightarrow \pi^{+} \pi^{-}$the secondary vertex resolution is a good figure of merit and is shown in figure 8.14. The vertex resolution is almost identical in the two detector configurations. The $5 \%$ difference in efficiency may be recoverable with optimization of station placement.

The time resolution for the $B_{s}$ decays $B_{s}^{0} \rightarrow \psi K^{*}$ and $B_{s}^{0} \rightarrow D_{s}^{+} \pi^{-}$(used to study $B_{s}$ mixing) is also unaffected by the choice of two-plane or three-plane stations. Simulations show that for the first decay mode the time resolution is $44 \mathrm{fs}$ for both two-plane and threeplane geometries, and for the second decay mode the two-plane is slightly better at $54 \mathrm{fs}$ versus 56 fs for the three-plane geometry. These results can be understood by the fact that multiple scattering is an important contributor to the resolution, and the reduction in the material compensates for the lost measurements.

\section{i. Algorithm Design and Simulation}

The basic pattern recognition procedure is still finding straight lines. However they are found across stations instead of within a single station. Because we cannot point into the next station we will have to search over a large section of the next station. It is efficient to restrict the search to quadrants of each station and we do so. 


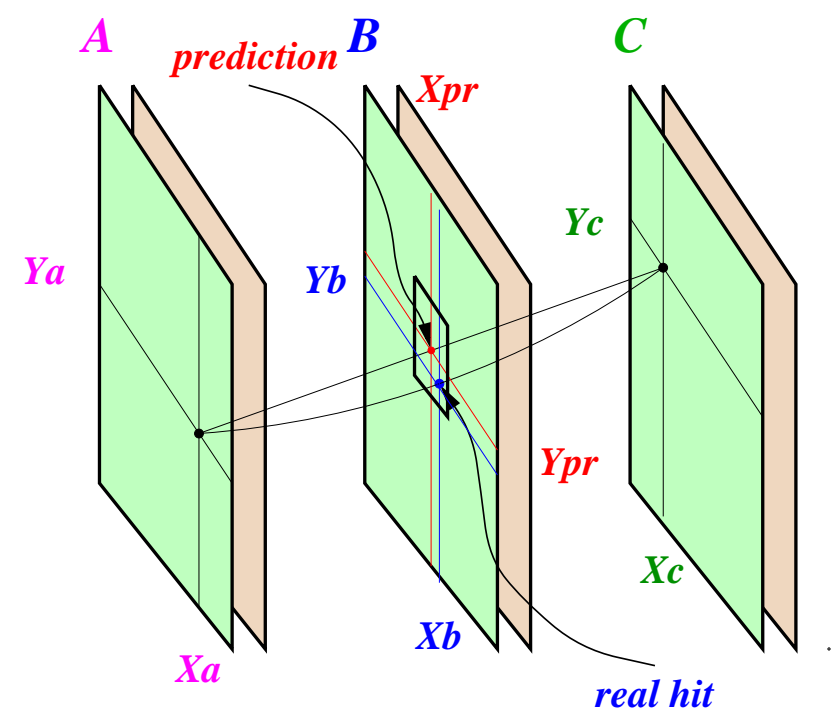

Figure 8.15: Sketch of the basic interpolation algorithm for the planes with fine pitch in $x$.

We choose three adjacent stations and try to find straight lines by using the hits in the two outer stations called $\mathrm{A}$ and $\mathrm{C}$ to predict the hit in the middle station $\mathrm{B}$. The prediction is made in both the $x$ and $y$ coordinates since the pixels have $2 \mathrm{D}$ information. The algorithm is pictured in Figure 8.15:

$$
\begin{gathered}
\left|\frac{x_{A}+x_{C}}{2}-x_{B}\right|<\Delta_{x} \\
\left|\frac{y_{A}+y_{C}}{2}-y_{B}\right|<\Delta_{y}
\end{gathered}
$$

The size of $\Delta_{x}$ and $\Delta_{y}$ is dictated by the resolution of the pixel detectors, the multiple scattering, and for the $y$ view the bending due to the magnet. In terms of pixel widths, the windows are $\Delta_{x}=3, \Delta_{y}=2$ for $x$ planes and $\Delta_{x}=2, \Delta_{y}=10$ for $y$ planes. The windows are 6 pixels in $x$ planes and 20 pixels in $y$ planes out of 125000 pixels in a quadrant, so the background suppression is excellent. Since the window sizes are larger than the pixels we do not need the full analog information at this stage but only the address of the cluster peak or similar information.

The three stations used to find line segments are overlapped, with each station sending its data to three different line segment finders. At this stage the ensemble of hits is in fact a collection of linked lists. These lists can easily be matched to tracks without combinatorial searches. Track segment fitting is currently less developed, but the momentum and intercept estimators used in the baseline trigger is a fall back option.

\section{ii. Feasibility of Implementing the Design}


The basic pattern recognition ability of the pixel detector is more than adequate to find line segments due to real tracks. The main concern is whether the hardware will be fast enough to keep up with the data rate. At a mean of two interactions per crossing we expect to see an average of 8 hits per quadrant and $95 \%$ of all quadrants in all events have fewer than 16 hits. If we implement the algorithm using a microprocessor performing loops then the time to complete the search will go like $N^{3}$, so we must introduce some parallelism into the problem.

The basic calculation consists of an addition, a multiplication (which can be done as a bit shift), a subtraction, and a test. All of these calculations can be done as integers. Programmable Logic Devices (PLDs, also called FPGAs) contain a large number of simple logic units that can perform hundreds of these calculations in parallel. This is significantly more parallelism than in the baseline trigger design allowing this design to handle the larger combinatorics.

The design can be broken into three sections: an input section that receives and buffers the hits; an interpolation section that does pattern recognition; and an output section that collects line segments to pass on to the next level of processing. The times to complete the various sections scale differently with event complexity. The timing in the input and buffering section scales as the number of hits divided by the number of input paths. If the time for this section is large due to many hits, the hits can be brought in in parallel as long as there are sufficient I/O pins on the chip. The timing of the interpolation section scales with the number of bits used in the interpolation, so it is independent of the size of the event. However, there will be a loss of efficiency should the event exceed the maximum capability of the processor. The timing of the output section scales with the number of line segments found, which makes it stable against random noise and low energy backgrounds.

A design of the line segment finder has been done for a Xilinx PLD. The basic idea is to read all of the hits from the $\mathrm{A}$ and $\mathrm{C}$ stations into one PLD. These are then used to feed a matrix of interpolation units which will do each hit from the B plane sequentially (see Figure 8.16). We use bit-serial arithmetic to shrink the data bus width and better utilize routing and logic resources. The time do this comparison goes as 1 clock cycle per bit of hit coordinate.

A single chip can check four B plane hits against the interpolations from all A and C plane hits in a quadrant in parallel. Simulation predicts this will take about 120 ns to handle 12 bits of hit information at a clock period of $10 \mathrm{~ns}$. In order to keep up with the data rate 2 chips per station quadrant per view would be required.

The size of a PLD is measured in either gates or configurable logic blocks (CLBs). The design of the interpolation nodes requires 5 CLBs per node so a PLD that can handle a maximum of 16 hits at a time would require $16 \times 16 \times 5=1280$. Currently we target a Xilinx PLD that has $8464=92 \times 92$ CLBs, so 4 arrays of interpolation nodes can be placed with $40 \%$ of the chip left for control logic, input buffers, etc. The new Virtex family chips from Xilinx are more capable and available now.

The I/O capabilities of these PLDs are more than adequate. Seventy-two I/O pins are needed to read in the 12 bits of each coordinate for a hit from each of three planes. The 


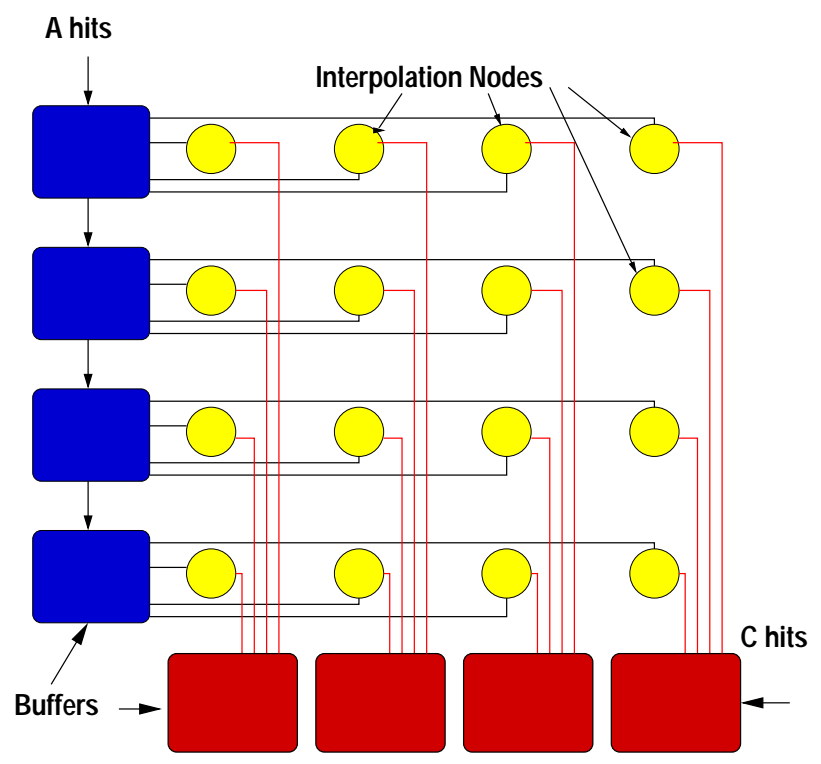

Figure 8.16: Block diagram showing the matrix of interpolation nodes and input buffering for the outer planes.

PLD under consideration has $448 \mathrm{I} / \mathrm{O}$ pins. There are more than enough I/O pins to bring in two or three times more hits in parallel. Hits from $\mathrm{A}$ and $\mathrm{C}$ planes are buffered on the PLD from one event while the previous event is analyzed.

A simple cross check using a Lucent PLD confirms the basic performance. The interpolation portion of the line segment finder was run through the Lucent device simulation and equivalent results were found. The design should work in a variety of devices so while present devices are adequate in both speed and I/O capabilities, we can delay the final choice to take advantage of better and/or cheaper devices as they become available.

\subsubsection{The 'Megapixel Trigger'}

The Megapixel Trigger is an alternative Level 1 trigger scheme. It benefits from concepts that have been explored in the context of the BTeV baseline vertex trigger and the 2-plane vertex trigger described in the previous section. Like the 2-plane trigger, the Megapixel Trigger is designed for a pixel system with two planes per tracking station. Moreover, the track reconstruction is initiated in a similar manner by finding track segments that span three adjacent pixel stations. Unlike the 2-plane trigger, the Megapixel Trigger limits the track reconstruction to track segments that are found as inner/outer tracks, which involve inner and outer pixel hits (defined below). The inner/outer tracks are used to "seed" the track-finding algorithm. Seed tracks are extended to adjacent pixel planes to find all hits belonging to a trajectory. An important feature of the Megapixel Trigger is that seed tracks are found relatively close to the primary-interaction vertex. This feature can be exploited 
to extend parallelism in the hardware architecture throughout the Level 1 trigger.

One of the central concepts of the Megapixel Trigger is the distinction between inner and outer pixel hits, where a single hit corresponds to an $(\mathrm{x}, \mathrm{y})$ position measurement made by a clustering algorithm, which combines data from adjacent pixels. Inner hits are defined as hits located in a region close to the Tevatron beams. For hits in a pixel plane, this region is defined as a square with a side of length $D$ centered on the beams and having the same orientation as the square beam hole in the pixel plane. In practice, the magnitude of $D$ is determined by the size of individual pixel sensors, so that the innermost sensors are all within the square region that surrounds the beam hole. The pixel hits from these innermost sensors are the inner hits, and about one third of all hits are inner hits. All of the remaining hits, outside the square region, are outer hits.

The Megapixel Trigger combines hits from three adjacent pixel stations to reconstruct track segments. The track finding is constrained to combinations of three hits, in which the first hit is an inner hit, and the second and third hits are outer hits. By imposing this constraint, the Megapixel Trigger finds only those trajectories that pass through at least three pixel stations, and pass from the inner to the outer pixel region. The 3-station track segments that are found in this manner are referred to as inner/outer tracks. The advantages of this approach are that (1) only a limited number of hit combinations need to be considered for pattern recognition, and (2) each trajectory is found once by the trigger hardware. By finding tracks only once, the Megapixel Trigger differs significantly from the $\mathrm{BTeV}$ baseline trigger, where track segments belonging to a single trajectory are found for each pixel station that recorded hits for the trajectory.

The percentage of tracks found as inner/outer tracks is quite high, and is shown in Table 8.4. The table shows the percentage of inner/outer tracks relative to all tracks that pass through at least three pixel stations and have a momentum greater than $1 \mathrm{GeV} / \mathrm{c}$. Results are shown for different types of tracks in minimum bias, $B$, and charm events for several values of $d$, where $d$ (which is equal to $D / 2$ ) is the distance from beam center to the border that defines the inner and outer pixel regions. Table 8.4 shows that for most types of tracks the percentage of inner/outer tracks does not vary with $d$, for the range of $d$ values we have considered. For tracks coming from $B$ decays there is a slight increase in inner/outer tracks for larger values of $d$, due to the larger $p_{t}$ for these tracks. From these results we conclude that a large percentage (about $90 \%$ ) of tracks coming from primary interaction vertices can be found as inner/outer tracks, and used to reconstruct primary vertices in the Megapixel Trigger. Furthermore, we conclude that a large percentage of $B$-decay tracks (more than $90 \%$ for values of $d$ greater than $1.1 \mathrm{~cm}$ ) can be found, and used to trigger on $B$ events.

After the inner/outer tracks are found, the Megapixel Trigger identifies the innermost pixel hits for each trajectory found as an inner/outer track. The goal is to reconstruct the track segments closest to the point of origin. These are the track segments that are least likely to suffer from multiple Coulomb scattering errors, and are required to reconstruct vertices and identify $B$-decay tracks. For example, Fig. 8.17 shows pixel hits for a simulated $B \rightarrow \psi K_{s}$ event in the $z-y$ projection. In Fig. 8.17a inner/outer tracks are shown as line 


\begin{tabular}{|c|c|c|c|c|}
\hline $\begin{array}{l}\text { Distance of } \\
\text { inner/outer } \\
\text { border from } \\
\text { beam center }\end{array}$ & $\begin{array}{c}\text { Minimum Bias } \\
\text { Tracks } \\
\text { from } \\
\text { primary vtx. }\end{array}$ & $\begin{array}{c}\text { Generic } B \\
\text { Tracks } \\
\text { from } \\
\text { primary vtx. }\end{array}$ & $\begin{array}{c}\text { Generic } B \\
\text { Tracks } \\
\text { from } \\
\text { secondary vtx. }\end{array}$ & $\begin{array}{c}\text { Generic charm } \\
\text { Tracks } \\
\text { from } \\
\text { secondary vtx. }\end{array}$ \\
\hline $\mathrm{d}=1.0 \mathrm{~cm}$ & $91 \%$ & $91 \%$ & $87 \%$ & $89 \%$ \\
\hline $\mathrm{d}=1.1 \mathrm{~cm}$ & $91 \%$ & $91 \%$ & $91 \%$ & $90 \%$ \\
\hline $\mathrm{d}=1.2 \mathrm{~cm}$ & $91 \%$ & $92 \%$ & $93 \%$ & $90 \%$ \\
\hline $\mathrm{d}=1.32 \mathrm{~cm}$ & $90 \%$ & $91 \%$ & $94 \%$ & $90 \%$ \\
\hline $\mathrm{d}=1.5 \mathrm{~cm}$ & $89 \%$ & $89 \%$ & $95 \%$ & $89 \%$ \\
\hline
\end{tabular}

Table 8.4: Percentage of tracks found as inner/outer tracks, where tracks are required to have momentum greater than $1 \mathrm{GeV} / \mathrm{c}$ and are required to pass through at least three pixel stations.

segments. There are two segments per track: one from the first hit to the second hit on the track and one from the second hit to the third hit. Fig. 8.17b shows the corresponding track segments for the innermost pixel hits associated with each track that was found as an inner/outer track. In many cases, the inner/outer tracks already include the innermost pixel hits, and no additional step is required to find these hits. For the remaining tracks, we are considering two algorithms to find the innermost hits. In the first algorithm, we extend tracks to adjacent pixel stations to locate the innermost hits by using an approach that is based on the linking of minivectors in the BTeV baseline trigger. In the second algorithm, we reconstruct inner/inner tracks in the first stage of the Megapixel Trigger, and then eliminate track candidates that do not include the innermost hits. For both algorithms, the track segments that are found are subjected to a fitting procedure to determine track parameters.

The hardware architecture of the Megapixel Trigger is organized as a pipeline with parallelism in $z$ (the beam direction), and individual track and vertex processors (digital signal processors, for example) are assigned to a range of $z$ positions in the pixel vertex detector. Processors assigned to a particular range in $z$ receive the data for tracks that originate at a vertex within that range. This can be accomplished with a data-driven architecture that evaluates the apparent origin for each track based on the slope of the track. The purpose is to "funnel" all of the relevant data for an interaction to the same processor, where a trigger decision can be made.

The details of the parallel-pipeline architecture have not been worked out. However, the advantage of this architecture is that the majority of tracks associated with an interaction are funneled to a processor based on the $z$ position of the interaction vertex. This has two consequences. The first is that tracks associated with a primary vertex (call it vertex $A$ ), and tracks from $B$-decay vertices are brought together in a single processor for vertex reconstruction, impact-parameter calculations, and a Level 1 vertex-trigger decision. The second consequence of this architecture is that tracks that occur during the same beam crossing, but are unrelated to vertex $A$, tend to be funneled to other processors. Unrelated tracks 


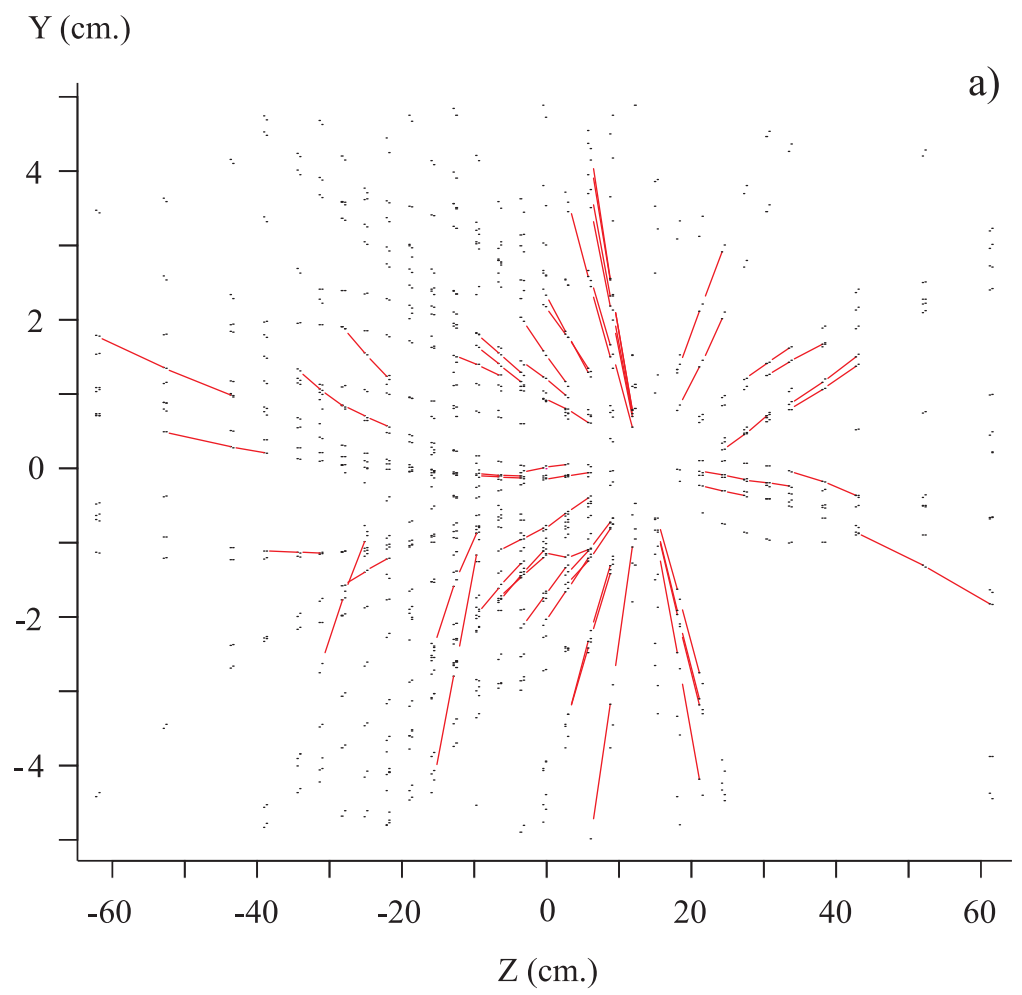

$\mathrm{Y}(\mathrm{cm}$.

b)

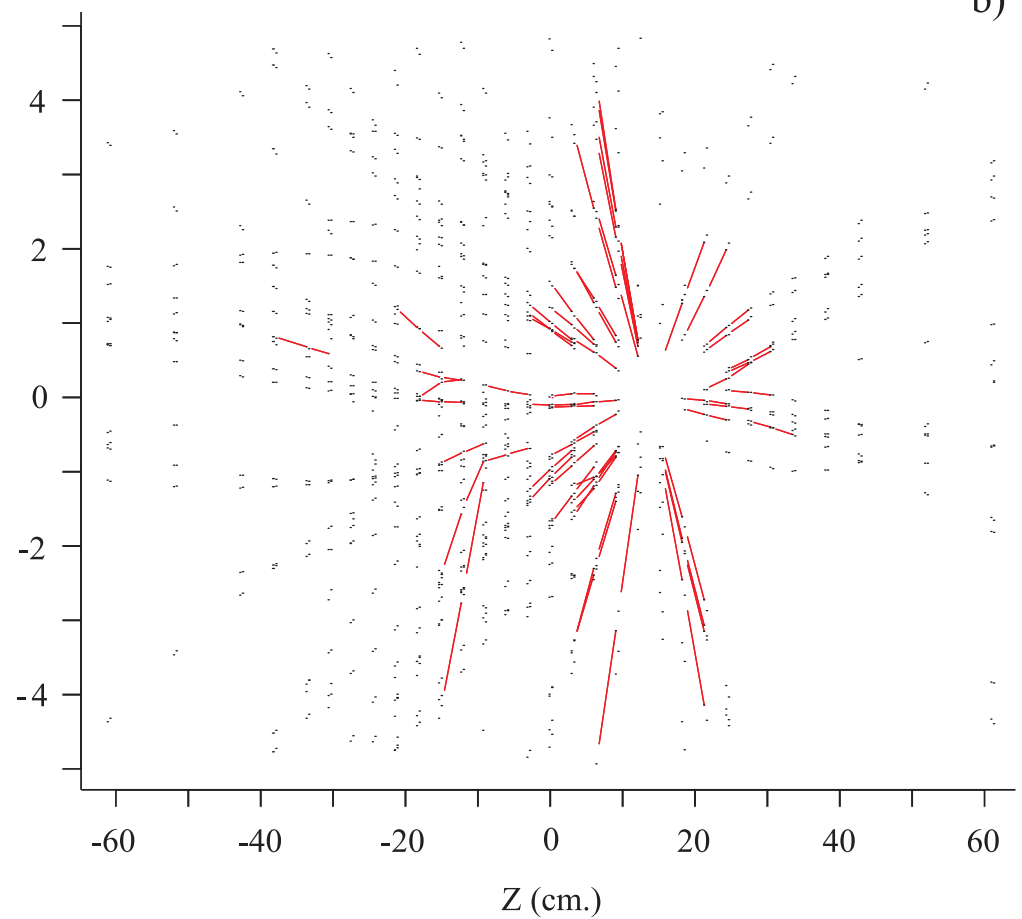

Figure 8.17: Pixel hits (shown as dots) for a $B \rightarrow \psi K_{s}$ event in the $z-y$ projection. The figure shows a) inner/outer tracks (two line segments per track), and b) track segments made up of the three innermost pixel hits for each inner/outer track found in the event. 
include those tracks associated with other vertices, and tracks produced by reinteractions in the material of the vertex detector. These tracks are processed independently of tracks associated with vertex $A$, and do not contribute to the computational load for that vertex.

To conclude, the primary goal of the Megapixel Trigger is to generate Level 1 trigger decisions with high efficiency for $B$ and charm events. A large percentage (more than 90\%) of tracks are found once as inner/outer tracks. The inner/outer tracks "seed" the track reconstruction, and are used to find the track segments that are made up of the innermost pixel hits. These track segments are close to their point of origin. They are funneled to track and vertex processors by a data-driven architecture. This means that the processing load for a particular vertex is reduced by excluding unrelated tracks from that vertex, and the load for multiple interaction vertices in a single beam crossing is distributed over multiple processors.

\subsection{The Global Level 1 Trigger Framework}

At the output of the first level trigger there will be a 'global Level 1 trigger' subsystem that will allow us to combine the various individual trigger elements into final triggers (for example, an event with only weak evidence for a secondary vertex but with an indication of a high $p_{T}$ single lepton might be accepted). It will also have the ability to apply a programmable prescale to the various triggers before they are 'or'ed together to form the final trigger. We will also include the ability to dynamically change the prescales during the course of the store. This device plays a central role in the trigger and data acquisition architecture and is discussed in great detail in chapter 9 .

\subsection{The Level 2 and 3 Triggers}

We have shown that the Level 1 trigger can reduce the trigger rate by a factor of about one hundred. The event rate is then reduced to $\approx 100 \mathrm{kHz}$ in the worst case during the highest luminosity running. While it is technically feasible to move all this data, amounting to a maximum rate of $10 \mathrm{~GB} / \mathrm{s}$, to the Level 2 trigger, we discuss below an architecture that allows the Level 2 decision to be made on a subset of the event data, thereby reducing the total bandwidth needed. Only for events passing Level 2 is the complete event assembled. We define further processing done on the complete event to be Level 3. If necessary, the Level 3 software can be quite comparable to the offline analysis in previous-generation experiments.

We anticipate that the data from Level 1 will be dominated by light quark events: the $b-\bar{b}$ production rate is about $20 \mathrm{kHz}$ and not all of these are within the detector or trigger acceptance. The charm cross section is higher, but the triggering efficiency will be poorer by design. Hence, the primary task of the Level 2 trigger will be to reject the remaining light quark events from real collisions at $\sqrt{(s)}=2$. TeV. In addition, Level 2 will provide enough kinematic information about the event so that a meaningful decision on the event decay properties can be quickly taken by Level 3 . 
Level 1 treats each sub-detector completely independently from the others: muon and pixel detectors (Level 1 vertex) for a given crossing are not treated sequentially in the same processor. They are handled asynchronously in different processing units. Events passing the global Level 1 trigger do not come in sequential order. Level 2 decisions are based on partially reconstructed events. By this, we mean that not all information from all subdetectors is available in the memory of a given level 2 processor. Possibly, the information on a given sub-detector element (i.e., pixel detector or the forward silicon tracker (FSI)) is partial: only a few planes could be used.

While the Level 1 vertex trigger broadly classifies the events in terms of their transverse detachment, looking for a few detached track with moderately high $P_{t}$, the task of Level 2 will be to confirm that the event surviving Level 1 cuts do indeed contain a heavy quark decay. Level 3 will classify these heavy Quark candidates in terms of final states, and make a decision according to an exhaustive trigger table.

In this section, we propose a few algorithms for the Level 2 trigger based on the pixel detector, the two most upstream FSI chambers, and the two most upstream straw chambers

(STRAW). The basic idea is to firm up the measurement of the transverse momentum of the tracks, and improve the vertex position resolution using this momentum information. The main software tool for this task is the MCFast Kalman filter (or fitter) to (i) test the hypothesis that a track is real and self-consistent and (ii) improve the track parameters. No attempts at doing particle i.d., search for neutral kaons, $\pi^{0}$, etc. are currently proposed at Level 2. Cuts based on the invariant mass of detached tracks could also be successful, but are obviously final-state specific.

\subsubsection{Preliminary studies of the Level 2 algorithm}

We have simulated a possible Level 2 algorithm. Note that the vertex pattern recognition, currently done as the last stage, could be done at any time after the first stage, as the Level 2 tracks are genuine, complete 3D tracks with a covariance matrix. Level 1 tracks with at least 3 minivectors are used as seeds for the Level 2 pattern recognition. The pion particle identification is always assumed. The Level 2 algorithm works in several sequential phases:

1. Adding pixel hits. The Level 1 pixel pattern recognition has known deficiencies. For instance, a track may cross a $\phi$ slice boundary and be partially reconstructed. Level 1 algorithms tend to limit the number of hits found on a track to a minimum, in order to gain time in assembling the track. Despite the very low cell occupancy in the pixel detector, there are still a few confused or fake tracks. Adding new hits and performing a Kalman filter allows us to:

- Reject spurious tracks. About $10 \%$ of the Level 1 detached tracks do not pass the Kalman filter confidence level requirement.

- Improve the parameters of the track. 
- Compute the best possible estimation of the error on these parameters, such that displacement errors at the vertex are correctly estimated. Errors due to multiple scattering are properly taken into account.

2. Adding hits from the straw chamber or the forward silicon tracker. The momentum resolution $\triangle P / P$ using the pixel information only for forward tracks is about $6 \%$. These tracks are extrapolated to the location of the most upstream tracking station (about $\pm 1.0 \mathrm{~m}$ from the interaction point). Confirming hits are searched for within a window typically $1.0 \mathrm{~cm}$ wide. The left-right ambiguities for straw hits are resolved. The second tracking station, located $\pm 1.5 \mathrm{~m}$ from the interaction point, is also used. The momentum resolution, shown in Fig. 8.18, is around $2 \%$ for tracks reaching the first or second station. Note that these two detectors do not cover the same rapidity range, tracks reaching the second station tend to have higher momentum and therefore have smaller sagitta and bigger $\Delta P / P$. They are however measured over a longer lever arm. These two effects partially compensate. The transverse momentum resolution also improves, from $\approx 20 \%$ at Level 1 down to a few percent.

3. All Level 2 tracks with a good Kalman fit confidence level are considered for the vertex pattern recognition phase. They are required to be "forward", i.e., have straw or silicon strip hits. However, if they point to these detectors, and no hits are found for a few consecutive planes, they will be rejected. The level 2 primary vertex resolution is shown in Fig 8.19.

The detachment criterion at level 2 is based on an exact calculation of the distance of closest approach of the track to the primary vertex and the error on this quantity. The detached track multiplicity, requiring a $5 \sigma$ detachment, is shown in Fig 8.20, for events passing the Level 1 trigger (based on 2 detached tracks). Note the level 2 detached tracks are not necessarily the same tracks that were called detached at Level 1. A combined Level 1 and Level 2 rejection factor on light quark events of about 1 in 1,000 is achievable, with an acceptance for $B \rightarrow \pi^{+} \pi^{-}$of about $40 \%$. Finally, at this stage, the $B \rightarrow \pi^{+} \pi^{-}$mass resolution is about $98 \mathrm{MeV}$, assuming pion particle identification. The mass resolution is, of course, significantly improved when information is used from all the forward tracking stations.

These Level 1 and Level 2 studies are based on a full pattern recognition code. The Monte-Carlo generator information is used solely to measure the performance of the code, not to select hits on tracks. Future efforts will be concentrated on improving the CPU performance and tuning the algorithm to minimize the computing needed in the Level 2 processors.

\subsubsection{Level 3}

The final trigger level will combine information from different sub-detectors to further suppress background events. Particle identification will be available at this stage and could be 


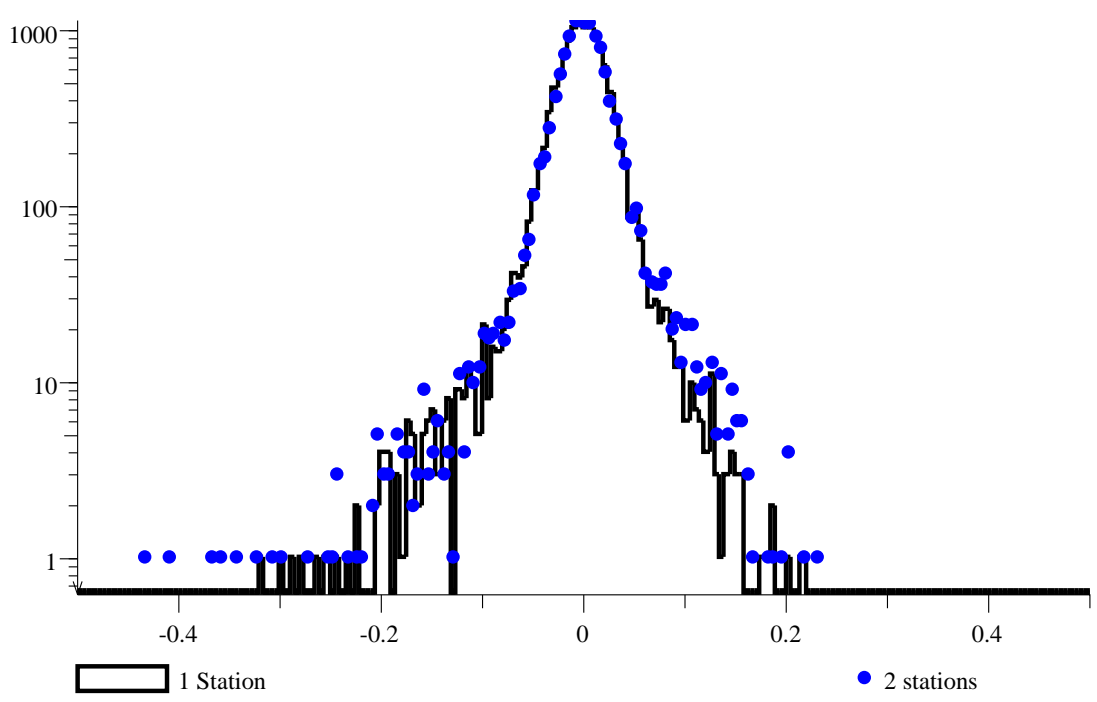

Figure 8.18: The momentum resolution for the light quark sample, at level 2, using the additional pixel hits and the forward silicon strip tracking system. The plot labeled " 1 station" refers to track with silicon hits from the most upstream station, the plot "2 stations" uses the two most upstream stations, with at least one hit in either station. The $\sigma$ of the core distribution is about $2.15 \%$ for " 1 station" plot and $2.05 \%$ for the "2 stations" plot. 

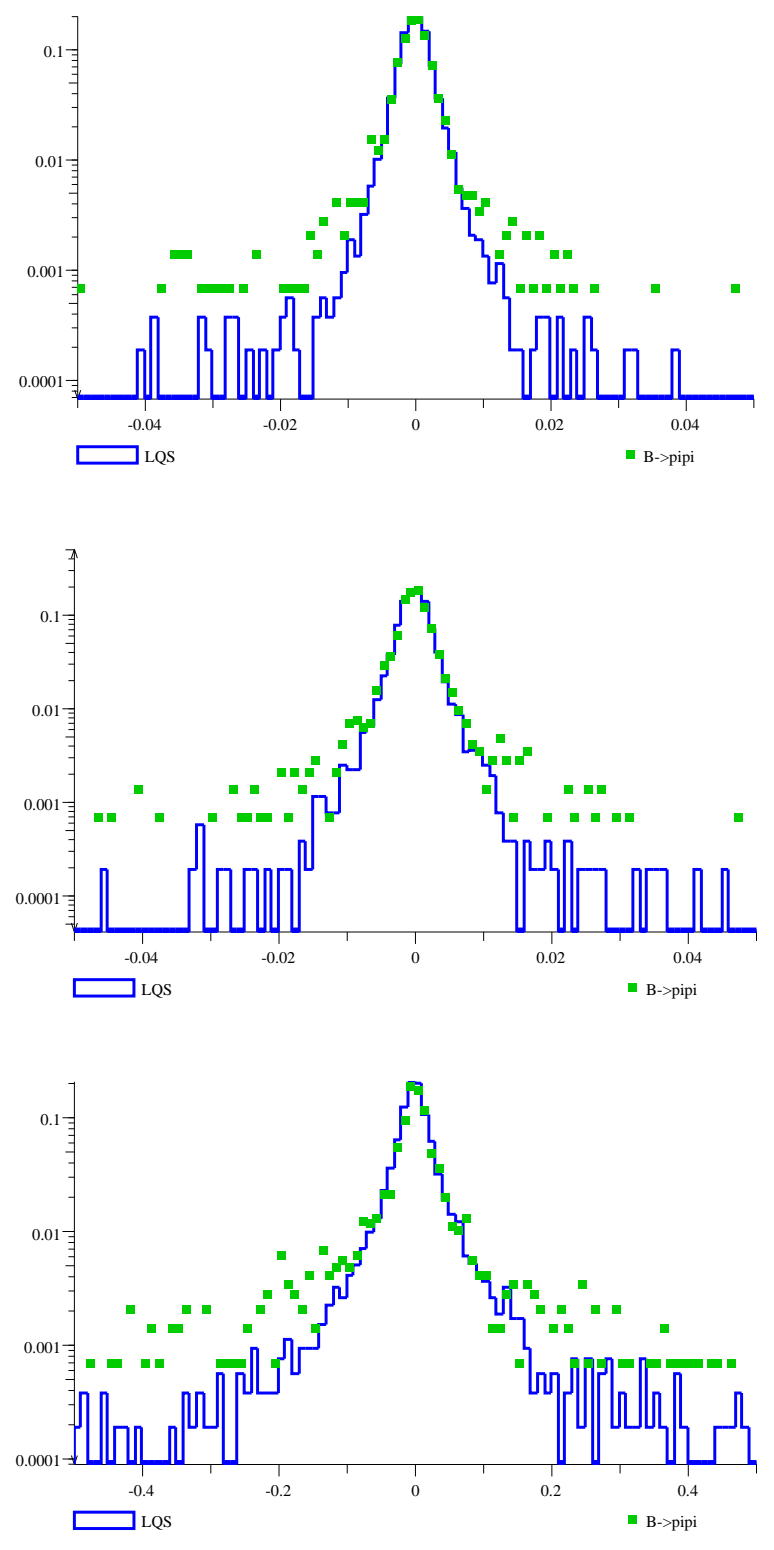

Figure 8.19: The primary vertex resolution along the $\mathrm{X}$ (top), $\mathrm{Y}$ (middle) and $\mathrm{Z}$ (bottom) direction at Level 2, for the light quark sample and for the $B \rightarrow \pi \pi$ sample. 


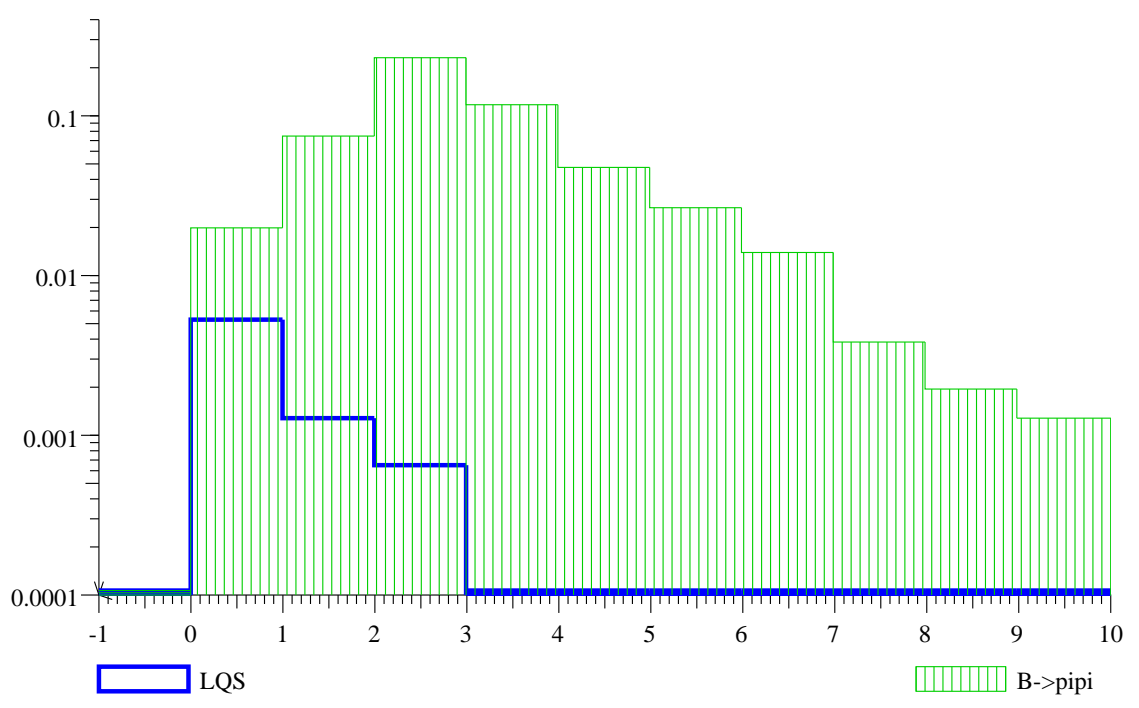

Figure 8.20: The Level 2 detached $(5 . \sigma)$ track multiplicity for the light quark event sample and for the $B \rightarrow \pi \pi$ sample selected by the offline reconstruction program. In addition, these Level 2 tracks must have a transverse momentum greater than $0.5 \mathrm{GeV} / \mathrm{c}$. The Level 1 trigger criteria has been applied. The vertical axis is the probability of accepting a beam crossing given the number of detached tracks on the horizontal axis. 
used to obtain a very clean direct charm signal for specific final states. In addition, the Level 3 trigger is likely to go through a long list of heavy quark decays to final states of interest. We intend to fully reconstruct the events at this level before the data are sent to a storage system and made available for off-line processing.

Because the event rate surviving this last level may still be a few $\mathrm{kHz}$, the software will probably have to reduce the amount of data per event to archival storage by writing out an event summary which eliminates much of the raw data. The event summary would be around $20 \mathrm{~KB}$ so that the output rate could still be as high as $80 \mathrm{MB} / \mathrm{s}$. This results in a dataset size that could be as high as $800 \mathrm{~TB} / \mathrm{yr}$. However, initial phases of the experiment will run at much lower rates and will be comparable in dataset size to a current fixed target experiment. 


\section{Bibliography}

[1] BTeV EOI (May 1997).

[2] These results are based on the work of R. Isik, W. Selove, and K. Sterner, "Monte Carlo Results for a Secondary-vertex Trigger with On-line Tracking," Univ. of Penn. preprint UPR-234E (1996).

[3] P. Lebrun, "On the Vertex Pattern Recognition in the BTeV Trigger," BTeV internal note 97/17 (1997). See also BTeV internal note 99/6 and 99/7. (1999)

[4] D. Husby, P. Chew, W. Selove, and K. Sterner, Nucl. Instr. and Meth. A 383 (1996) 193.

[5] For updated vertex trigger documentation, see http://wwwese.fnal.gov/eseproj/trigger/default.htm and E. Gottschalk, BTeV-pub-99/1, presented at DPF99 


\section{Chapter 9}

\section{Trigger and Data Acquisition Architecture and Front End Electronics}

\subsection{Data Acquisition}

The BTeV system will digitize, sparsify, and transmit data at the beam crossing rate of 7.6 MHz into off-detector buffer memories. This approach is taken because a large subset of the data is used in the first level trigger and must be digitized in any case and a very sophisticated first level trigger is planned. With this approach applied to all subdetectors, the first level buffers can hold many more beam crossings of data than those found in typical front-end integrated circuits. This means that the decision time of the Level 1 trigger can be extended by as much as two orders of magnitude, if desired, allowing for much more sophisticated trigger processing than would be possible given a fixed Level 1 latency.

Three distinct logical trigger levels are described in chapter 8 . From an engineering perspective, there is little difference between Level 2 and Level 3: these levels are both executed in the general purpose processors. The only distinction between these levels is that the start of the Level 3 processing presupposes that ALL data have been transferred to the processor, whereas the Level 2 processing may operate on a subset of the total data. Thus, triggers in the BTeV system are separated into two physical levels, referred as L1 and L2/3. This is typical of most proposed large scale data acquisition systems and acknowledges the greatly increased performance and decreased cost of general purpose processors. In effect, much of the early and intermediate processing in previous trigger architectures has been moved into the first level hardware, Level 1 in our case, while the remaining processing occurs in a greatly expanded array of general purpose processors.

Following the Level 1 trigger, the remainder of the system is sized for a minimum L1 rejection of $75: 1$, which represents an L1 accept rate of $\approx 100 \mathrm{KHz}$. This rate must be absorbed by the L2/3 processors though a switch. The baseline design assumes that this can be achieved with $\approx 4000$ general purpose processors of about 1000 MIPS each in the L2/3 array. The use of general purpose processors does not preclude the use of dedicated L2 trigger logic in the form of attached coprocessors. 


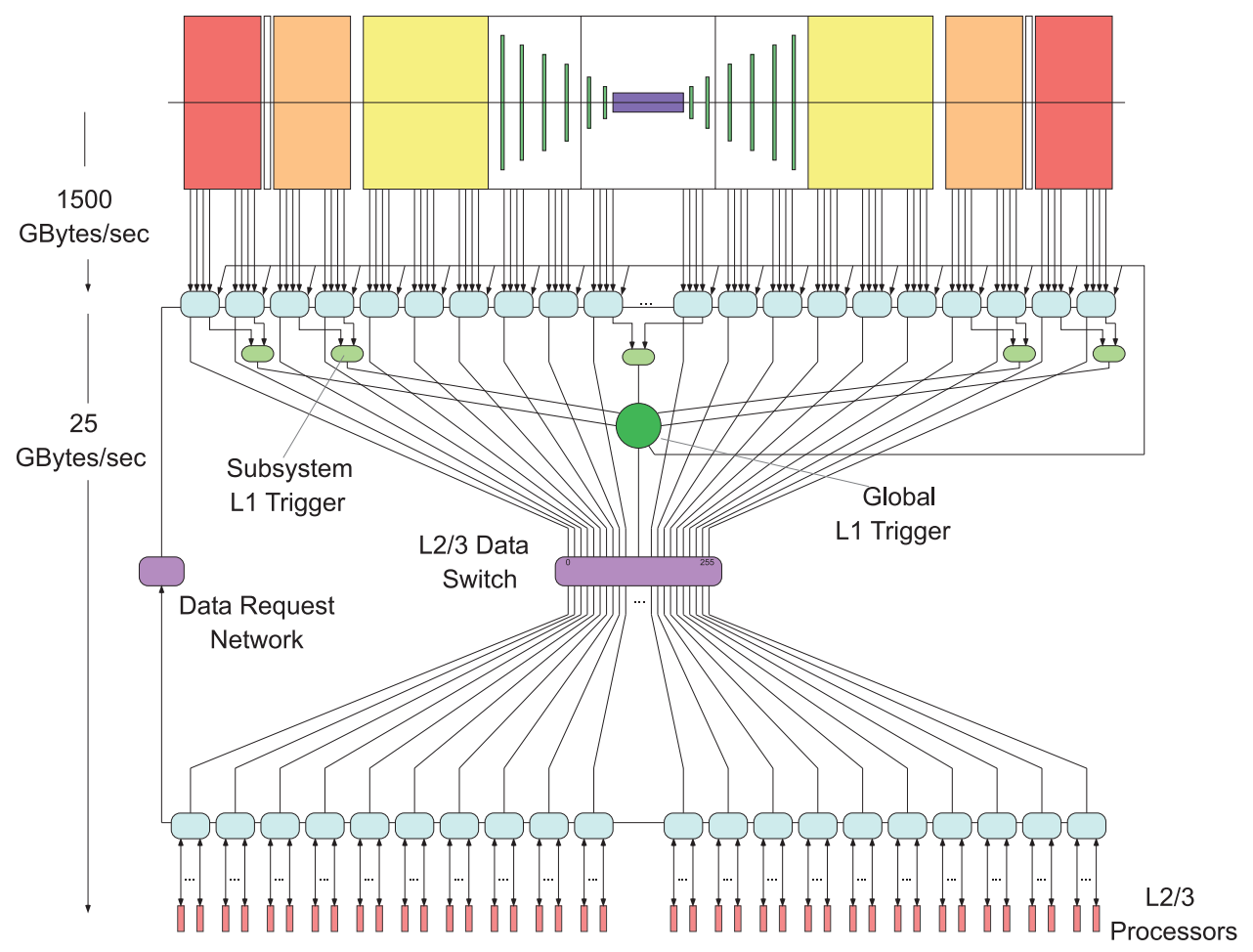

Figure 9.1: Block Diagram of BTeV Trigger and Data Acquisition Hardware

The specifications shown in Table 9.1are used as the baseline for the BTeV data acquisition. These numbers represent both arms of the detector.

\begin{tabular}{|l|l|}
\hline \hline event size & 200 KBytes \\
number of detector data links & 2048 \\
number of L1 data buffers & 2048 \\
number of L2/3 data links & 256 \\
number of L2/3 processors & 4096 \\
\hline \hline
\end{tabular}

Table 9.1: Estimates of Hardware for BTeV Trigger and Data Acquisition System

The total system buffer memory, assuming 2048 L1/switch input buffers and 256 switch output buffers, is almost 600 Gigabytes. Buffers in the L1/2/3 processors will push total system memory to approximately 1 Terabyte. A block diagram of the trigger and data acquisition system is shown in Fig. 9.1.

\subsubsection{Detector Multiplexing}

To efficiently balance data rates on the detector data links, a relatively large number of detector channels will be multiplexed into each high speed link. In the case of the RICH 
subsystem, for example, it is expected that approximately 3000 single bit signals will be multiplexed and encoded to drive one link. The input to this module will depend on the detector subsystem, but the use of programmable logic will allow for some variation in signal widths and sparsification algorithms with common hardware. The detector multiplexer drives the detector data link and receives the coded clock signal for fanout to the front-end components.

\subsubsection{Detector Data Links}

Each detector data link must transfer approximately $800 \mathrm{MBytes} / \mathrm{sec}$. This rate can be accommodated by a single 10 Gbps fiber, but more likely will be implemented with eight 1 Gbps fibers or thirty-two LVDS copper connections per link. The choice of technology and link speed depends on the physical placement of the first level buffers. The total bandwidth of the detector data links is 1.6 Terabytes/sec.

\subsubsection{L1 and Switch Input Buffers}

Each Level 1 Buffer must accept data from a subsection of the detector at the 800 MBytes/sec average rate and the data must be held in this buffer pending a Level 1 trigger decision. The BTeV L1 trigger is based in large part on track reconstruction in the vertex detector and decision times may vary over a wide range depending on event complexity. For this reason, it is desirable that the L1 buffer controller support receipt of both asynchronous and out-of-order L1 accepts and rejects. Following an L1 accept, the data are transferred to a switch input buffer. The function of the switch input buffer is to hold data until an L2/3 processor assignment is made, the data have been requested by the processor, and the switch rotation allows transmission of the data. Data requests to the switch input buffer also arrive asynchronously and in arbitrary order.

As a practical matter, the L1 buffer and switch input buffer will likely use the same physical memory, with the data "transferred" between buffers by reassignment of pointers. The size of an individual data buffer is expected to be approximately 256 MBytes and may be implemented using direct Rambus protocol compatible with next generation PC memory architectures.

Data from some detector data links will also be used in the L1 trigger. The L1 buffer module provides a direct datapath from the link receivers to an attached module which may contain local L1 trigger logic. The output of this module connects to the subsystem L1 trigger and finally to the Global L1 trigger.

\subsubsection{Ring Buffer Interconnects}

Our design approach is sufficiently flexible to accommodate different options for data transmission to the $\mathrm{L} 2 / 3$ processors. If all data is transmitted to the $\mathrm{L} 2 / 3$ processors following an L1 accept, the most natural arrangement of L1 buffers is to place an equal number on 
each switch input link. If the data is transmitted in steps (staged readout), placement of the L1 buffers should be optimized for the frequency of readout. Using a ring architecture achieves the same multiplexing functionality as a fixed backplane bus, while also allowing reconfiguration of the L1 buffers to match changes in trigger rate, switch size, and access frequency of the buffers.

The ring and switch interconnects utilize 1 Gbps serial links based on the same physical layer standard as Gigabit Ethernet, Fiber Channel, and NGIO. All data are transferred in fixed length packets, similar in size to an ATM cell.

\subsubsection{Staged Readout}

To reduce the size of the data switch, the Level 2 trigger may operate in stages. In each stage, it would then request only enough information from the switch input buffers to make a decision to continue to the next stage of processing. Events may be then rejected at any stage in the Level 2 analysis, with the remaining data cleared from the input buffers without being transmitted through the data switch.

The reduction in data switch bandwidth requirement is offset by the increase in bandwidth required for the data request messages flowing in the reverse direction. In addition, the input buffer depth must be increased significantly to provide greater latency for event data used in later stages of the L2 algorithm. Buffer management logic also becomes more complicated. The cost reduction comes from the potential decrease in the number of required output buffers. An analysis of the minimum number of required output buffers and the expected fraction of data needed in the average L2 calculation will be performed to determine if the staged readout approach is cost-effective.

\subsubsection{Data Switch}

A simple, unidirectional, packet synchronous, TDM switch has been shown to be the most efficient for event building applications. The switch configuration follows a standard "barrel shift" rotation and can be expanded to any size in NlogN. The BTeV data switch is expected to require a maximum of 256 channels, which can be implemented using two stages of $16 \mathrm{X}$ 16 switch modules.

The advantage of this switch is that it requires no internal control. Source and destination addresses contained in the data packets are used only to specify buffers on a common ring and are not needed by the switch for data routing. The switch is unidirectional, but packets are recirculated by connecting the last output buffer to the first input buffer in each ring. This provides a mechanism for input to input message transfers (throttle requests to the global trigger), output to output message transfers (L3 accepted events to logging processors) and output to input messages (event requests to the global trigger/event manager).

Because the switch is easily expanded, full implementation can be delayed until the accept rates of the L1 and L2/3 triggers are determined, and the switch sized accordingly. The cost of the switch is not a large factor in the overall system cost. 


\subsubsection{Data Request Router}

If a staged readout is not required, the L2 accept/reject messages can be broadcast to all switch input buffers. For a staged readout, a broadcast router is needed to selectively distribute the accept/reject messages to the switch input buffers. The bandwidth required for these messages can be accommodated by Fast Ethernet (100 Mbps) if multiple requests are buffered in each packet.

\subsubsection{Switch Output Buffers}

The switch output buffers are similar in function to the switch input buffers. This is the point where all the requested data from a single event is received from the switch and forwarded to a processor. Connection to processors is by way of Fast Ethernet links, with 16 links per switch output buffer. Up to 4096 processors can be connected to 256 switch output buffers, and additional buffers can be added to each ring if the processor array is expanded.

\subsubsection{Data Logging}

Events accepted by the Level 3 trigger are sent to data logging processors. These processors are attached to switch output buffers in the same way as any other L $2 / 3$ processor. Accepted events are placed back on the ring and recirculate through the data switch to the appropriate output port. Given the high trigger rejection, This utilizes approximately $1 \%$ of the data switch bandwidth while avoiding the need for a separate data logging network.

\subsubsection{Detector Control Links}

All detector subsystems transmit data at the crossing rate. This simplifies the delivery of clock and control signals to the detector front-end components because L1 and L2 accept/reject signals are not required at this level. It is assumed that a $53 \mathrm{MHz}$ coded clock will be sufficient to provide crossing and synchronization information. Clock and data recovery should be provided by the detector multiplexing module and then delivered independently to the front-end.

\subsubsection{Diagnostics}

All system buffers have an Ethernet link for the purpose of sending or receiving data request messages. This provides a convenient path for system diagnostics at little additional cost. We expect to make widespread use of embedded processing and embedded servers in these modules to allow remote test and status monitoring. 


\subsubsection{General Operation}

At startup, buffers can be initialized through the standard Ethernet link. The data switch is initialized by filling the rings with empty data packets, after which it operates in a packet synchronous mode. The front-end systems are synchronized through the clock distribution system.

Event fragments are timestamped with the crossing number which is used for all Level 1 trigger accept/reject messages. Events accepted at L1 are tracked through the remainder of the system by the L1 event number. An L1 accept causes the buffer control logic to move a memory pointer from the crossing number list to the L1 event number list. At the same time, the L1 event number is added to the list of events available for L2/3 processing.

An event request from an L2/3 processor is sent to the Global L1 trigger (or event manager logic attached to the Global trigger). The Global trigger returns a packet containing an L1 event number, along with any trigger information considered useful to the processor. At this point the processor is responsible for disposition of the event. It may request some or all of the data from switch input buffers for use in the L2 decision. Following an L2 accept or reject the processor makes a final data request to all remaining switch input buffers. Buffers then delete or transmit/delete the event data. Each switch input buffer receives exactly one data request message per event.

\subsubsection{Component Placement}

The detector data links and L1 buffers represent a large part of the overall data acquisition system cost. Placement of the buffers influences the technology and cost of the links. The buffers will occupy the equivalent of $1286 \mathrm{U}$ subracks. The L1 subsystem triggers should be located in the same area as the L1 buffers. Ideally, these components would be placed outside of the collision hall to facilitate maintenance and to allow reconfiguration of rings based on changes in trigger rate and buffer access frequency. This requires the use of optical links. Placement of all other data acquisition components (switch, output buffers and processors) is not critical, since there are a relatively small number of interconnects.

\subsection{Global Level 1 Trigger System}

The picture of the Level 1 trigger that we have presented focuses on the Level 1 Vertex Trigger which is based on the pixel detector. The actual Level 1 trigger will be more complex. This complexity is managed by the Global Level 1 Trigger, GLV1.

First, there will be several different vertex related triggers. We have described a final selection based a minimum number of tracks, N, each of whose normalized impact parameter with respect to the primary vertex exceeds a value M. In fact, there will be a variety of triggers, some accepting events with a few tracks with large detachments and others accepting events with more tracks that have smaller detachments. We also want to record a sample of 
events that would otherwise have failed in various ways to understand how the efficiency of the trigger "turns on". This last group of triggers may be prescaled.

Second, there will be triggers that involve other detectors. We plan to have a "standalone dimuon trigger". In addition to improving the overall efficiency for triggering on states containing $J / \psi$ 's, this trigger will permit an independent crosscheck on the efficiency of the vertex triggers.

Third, there will probably be triggers which combine information from more than one detector at GLV1. For example, a single high $p_{t}$ lepton trigger would be interesting but may go at too high a rate to be acceptable. However, the global Level 1 trigger should permit one to accept high $p_{t}$ single muon triggers if the event also satisfies a relaxed vertex requirement - perhaps one track with a large impact parameter. (At Level 1, one would not know that the high $p_{t}$ muon corresponded to the single high impact parameter track).

Fourth, there will be special triggers. One example will be a variety of minimum or low bias triggers which will be heavily prescaled. Another example will be special alignment and calibration triggers. We will certainly collect special triggers or use minimum bias triggers to do the quasi real time alignment of the pixel detector.

Fifth, we will probably record a prescaled sample of various 'failures' and 'timed out' events to make sure they are not introducing some kind of physics bias.

There will be one or more primary physics triggers which are not prescaled. Global Level 1 must have the ability to prescale other less important physics triggers or calibrationtype triggers. We also need the ability at Level 1 to adjust the prescales dynamically. If the luminosity is not 'leveled', then we want the ability to reduce the prescales on some of the triggers as the luminosity falls. We also want the ability to increase the number of alignment triggers taken at the beginning of a store and to reduce it once enough events have been collected to establish initial alignment constants for the store.

Given these requirements, we have the following picture of the operation of the Global Level 1 trigger. The GLV1 receives 'trigger packets' from each trigger processor. The packet contains a header which has 'trigger primitives' whose format is known to the GLV1. These packets arrive asynchronously. The GLV1 buffers these packets until it receives all the packets it is supposed to receive for a crossing or until a timeout occurs for that crossing. In normal operation, as soon as all packets are received the GLV1 inspects the headers and forms all the various triggers from truth tables that have been downloaded to it. It then applies the appropriate prescale to each trigger and takes the OR of the result. If any of the triggers is true, it issues a Level 1 accept. This results in the event data being transferred to the so-called "switch buffer" for transfer to the Level 2 trigger. If no trigger is satisfied, the GLV1 issues a Level 1 reject. This eventually results in the Level 1 event buffers being freed to be used for other events. In general, the trigger processors which are talking to the GLV1 will have timeouts which are less than the GLV1 timeout. If the maximum allowed time for the Level 1 trigger primitive arrival should expire before a trigger packet from a processor arrives, this generally indicates a problem. A prescaled sample of these events can be recorded for further analysis and diagnostics. Then the rest of the GLV1 decision proceeds as normal. 
The fact that the GLV1 receives data packets from the trigger processors allows the processors to send additional data with the trigger primitive information. This could include diagnostic information. It could even include 'event information.' It is quite likely that we will want to write some of the results of the Level 1 trigger calculations out as part of the event going to the Level 2 trigger. The data may be used for diagnostic purposes or may even be used to 'prompt' the Level 2 calculations by directing it to process particular tracks, for example. There are several ways within this architecture to write such data to Level 2. Sending it as part of the trigger processor packet to GLV1 is only one method.

As we have developed the trigger and data acquisition architecture, we have begun to merge functions that are really 'event supervisor' functions into the GLV1. At present, the plan is for the GLV1 to maintain a list of events which have satisfied the Level 1 trigger. When a L2/3 processor becomes available to process a new event, it sends a request for an event to the GLV1. The GLV1 then sends a data packet to the processor which contains the event number to analyze. It also includes the status of all the triggers for that event so that the processor can execute different code depending on how the event triggered. It may also send more extensive event data from the trigger processors. Once the GLV1 has sent an event number and associated data to a Level $2 / 3$ processor, it is done with that event.

The GLV1 is the natural location for accumulating statistics on the performance of the Level 1 trigger. It will periodically send this data up to online analysis computers.

The GLV1 will also play a role in data throttling - that is trying to reduce the rate of data sent to the upper trigger levels if they begin to fall behind and the switch buffer memory approaches its capacity. The 'switch buffer memory' which holds the event data while waiting for a L2/3 processor to become free will be able to issue a 'full warning' to GLV1 when it becomes $90 \%$ full. The GLV1 can attempt to undertake some action to alleviate the problem. For example, it could begin to adjust prescales to reduce the total trigger rate. It could also stop sending events. When the 'switch buffer memory' is completely full, it will issue a 'full' indication. The GLV1 will stop issuing Level 1 accepts until this condition is cleared. How exactly the throttling will propagate back to the Level 1 buffers is not yet clear. What is clear is that this will result in deadtime and GLV1 will have to keep track of how much deadtime there is for purposes of normalization and for diagnostic purposes.

We are also discussing 'partitioning.' Partitioning refers to the ability to divide the Trigger/DA system into more than one semi-independent piece, each of which approximates a completely independent trigger/DA system. This capability is especially useful in the debugging stage of an experiment. In this stage, some subsystems may not be installed, some may not yet be operational, some may be down for maintenance, and some may be hanging or crashing. It is important to be able to work on one or a collection of subsystems independently of the state of the others. The BTeV architecture permits a reasonable level of partitioning. The ability of Level 2 processors to request subevents rather than whole events lends itself to partitioning. On the other hand, the requirement that an event be given to one and only one processor creates some difficulties for partitioning.

In our current plan, the GLV1 will maintain separate trigger lists for different 'event' types. It will also maintain separate lists of event numbers for each triggering event type. 
It will have an algorithm for assigning events satisfying triggers in more than one list. The algorithm will proably be based on the desired event rates and priorities for each event type. Level 2 processors will request an event of a specific type. The GLV1 will return an event number from the list for that type. The Level 2 processor can then request the subevents that it wants to analyze. If the 'switch buffer' is configured so that it waits a certain minimum interval before it will send an event so that it is sure that it has the whole event, and if it can send a message saying that it doesn't have a requested subevent, then the fact that some (presumably) unneeded subevents are missing does not cause a problem. With this scheme, subgroups can have their own triggers and select the subset of the data they want to read quite independently of each other. However, since each event will go to only one processor, some intelligence in GLV1 will have to decide how events satisfying the requirements of multiple trigger lists should be apportioned.

\subsection{Level $2 / 3$ Trigger}

The Level 2/3 Trigger is a processor farm. The baseline design of the farm calls for 2000-4000 general purpose processors, such as INTEL/Pentium PCs, running Linux. Each processor runs the same analysis code, and performs the necessary analysis for a single event before it requests data for a new event. Requests for new data are sent to the Global Level 1 Trigger, GLV1, which coordinates the initial data requests for each processor in the Level $2 / 3$ Trigger. The Level 2/3 analysis software for BTeV performs data analysis that is usually part of the "offline analysis" in other experiments. Consequently, the Level $2 / 3$ Trigger can select events using cuts that are usually reserved for offline analyses, and can transform event data into a condensed format (usually referred to as a DST format) before sending the data to event-logging processors.

An important aspect of the Level 2/3 Trigger architecture is that each processor is capable of making trigger decisions for both Level 2 and Level 3. The distinction between Level 2 and Level 3 depends on the memory buffers in which the data for an event reside, and will be described below. A second aspect of the trigger architecture is that each event is assigned to a single processor in the $\mathrm{PC}$ farm, so that a single processor selects or rejects an event at Level 2 and, if it passes Level 2, at Level 3.

The Level 2 analysis for an event begins when an idle processor sends a request to the Global Level 1 Trigger (GLV1) for a new event. The GLV1, which maintains a list of events that have satisfied the Level 1 Trigger, responds by sending a data packet to the processor. The data packet contains the event number, and Level 1 trigger information. The processor begins the Level 2 analysis of the event based on the Level 1 trigger information, which summarizes all of the trigger conditions that were satisfied at Level 1. In addition to the trigger information, the processor usually requires data from various detectors to complete the analysis and arrive at a trigger decision. The data reside in switch buffers. The processor requests data from these switch buffers directly. For example, the processor may send a request for data recorded by the forward tracking chambers that are closest to the dipole magnet. The requested data are sent from the switch buffer to the processor's memory and 
the memory it occupied in the switch buffer is freed. At this point, part of the event is in the processor memory and the remainder is in the switch buffer. In this example, these data are used to improve the momentum determination of tracks found by the vertex detector, so that the processor can calculate more precise impact parameters to select tracks coming from $B$ decays. After the data are received and processed, the processor may request additional data (such as from the other forward trackers or the RICH detectors), or complete the Level 2 analysis by sending a trigger decision to the switch buffer.

A processor that has completed the Level 2 analysis for an event sends one of two trigger decisions to the switch buffer. If the event is rejected, the data for that event are removed from the switch buffers, and the processor is ready to process a new event. If the event is accepted, then the switch buffer sends all of the data for that event to the processor for subsequent Level 3 analysis, and removes the data from the switch buffers. This marks the beginning of the Level 3 trigger. At this stage, the data for an event reside entirely in the memory of one processor (the same processor that performed the Level 2 analysis for the event), and nowhere else.

A processor that performs the Level 3 analysis for an event can perform a complete analysis using all of the data that are recorded for that event. The data analysis is comparable to "offline" analyses in other experiments, and includes track reconstruction, vertex reconstruction, muon identification, and possibly charged hadron identification. Events that are rejected by a processor at Level 3 are simply terminated by that processor. Events that are accepted are sent to data-logging processors.

\subsection{Data Logging and Online Event Analysis}

Each event is handled by only one Level $2 / 3$ processor. (i.e., an event is not scattered across processors nor is it copied to more than one processor). All accepted events need to be moved out of the L2/3 processors and onto tape for long term data storage, and a fraction of these needs to be made available for online monitoring (by "consumer processes").

Events coming out of the Level 3 processors will not be raw data, but already processed data (except possibly for a highly prescaled sample used for monitoring the data reduction algorithm itself). This should shrink the event size from 200 kbytes to 50 kbytes. Assuming an event size of $50 \mathrm{kbytes}$, and a $4 \mathrm{kHz}$ event rate to the loggers, the data rate to tape is on the order of $200 \mathrm{Mbytes} / \mathrm{sec}$. The consumers themselves should require an additional 5-10\% of throughput.

Since the data logging rate out of each Level 3 processor is small, it is less cost effective to attach logging media to these individual nodes than to provide a small number of separate logger nodes. The necessary bandwidth to the logger nodes is also small (1\%) compared to the raw data coming up the Level $2 / 3$ farms, so the same switch could be used to pass the event to the logger nodes as well. The switch buffers are small $(<100$ bytes) relative to the DST event, so the L2/3 processors can send single events to the logger nodes without incurring any additional penalty. A single event is routed to the next free logging node. 
The number of logging nodes themselves is clearly a function of the data rate. It is assumed that data will first be buffered to disk before being written to tape. This serves a dual purpose; bufferring enough data before a transfer to keep the tape drives streaming, and protecting against tape media errors and tape drive failures. With current market technology, a single processor node can handle 10 Mbytes/sec to tape including the initial disk write. We will therefore require 20 data logging nodes.

Consumer events should not interfere with data logging rates and additionally may contain rejected events. The consumer processes will therefore run on separate nodes from the logger processes.

\subsection{Front End Electronics}

In this section, we discuss various aspects of our front end electronics. We start with basic infrastructure such as racks and then describe our thinking about the front end electronics for the forward tracker, the ring imaging Cherenkov counter, the electromagnetic calorimeter and the muon detector. Electronics for the pixel detector is completely covered in chapter 5. We conclude with a brief discussion of software support for electronics development.

\subsubsection{Infrastructure and Other Support Systems}

A goal of the BTeV experiment is to minimize hardware and software development. This goal can be accomplished by:

- Choosing cost-effective, commercially-available hardware and software whenever possible;

- Using hardware and software developed elsewhere whenever possible; and

- When hardware and software needs to be developed for BTeV, develop them in such a way that they can be used in the maximum number of $\mathrm{BTeV}$ front-end, trigger, and data acquisition systems.

Commonality in hardware and/or software when possible in BTeV's front-end, trigger, and data acquisition electronics will save not only substantial amounts of money but valuable personnel resources. A BTeV workshop was held recently to understand

- what already-developed electronics and software can be used in BTeV and

- where hardware and/or software commonality is possible in BTeV's front-end, trigger, and data acquisition systems.

The following subsections describe our choices for some infrastructure items and other support systems. 


\subsubsection{Electronics Packaging - Subracks and Related Infrastructure}

Using a mechanical packaging and bus protocol standard that is commonly used internationally offers several advantages over the lifetime of an experiment. Cost and personnel time savings are the two most important advantages. Standards are well-documented and stable. Various electronics and mechanical components supporting the standard are commerciallyavailable from industry. For example, subracks, processor modules, (module size) adapters, extenders, subrack power supplies and interface ICs are commercially available items not requiring development. This leaves us free to concentrate only on the development of specific front-end, trigger, data acquisition, controls and monitoring electronics. By choosing a commonly-used packaging and bus protocol standard, implementers, as well as people who maintain the electronics over the lifetime of the experiment, have a mature development and testing hardware and software environment. If the standard has been used in previous experiments at Fermilab, we can realize considerable cost and personnel time savings because local expertise is already available and infrastructure is in place.

We have chosen the VME64 Extensions (VME64x) subrack mechanical packaging and bus protocol standard as the baseline implementation for the $\mathrm{BTeV}$ front-end, trigger and data acquisition electronics. This standard is an American National Standards Institute (ANSI) standard, ANSI/VITA 1.1-1997. The size of VME64x module chosen is $9 \mathrm{U} \times 400 \mathrm{~mm}$. This standard, its physics community VME64xP extension, and the $9 \mathrm{U} \times 400 \mathrm{~mm}$ module size are being used for all of CDF's and a majority of D0's Run II electronics. Over 250 VME64xP subracks have been purchased by CDF and D0. Other standards used in conjunction with this standard are:

1. IEEE1101.10 - the subrack module mechanical standard for VME64x;

2. IEEE1101.11 - the subrack rear I/O board (transition module) mechanical standard for VME64x;

3. ANSI/VITA 1.3-1997 - the VME64x 9U x 400mm module format standard;

4. ANSI/VITA 25 - VISION, a software VME API (subroutine calls) standard; and

5. ANSI/VITA 23 - VME64 Extensions for Physics (VME64xP), a VME64x-compatible standard which extends VME64x by, for example, specifying $7 \mathrm{U}$ and $10 \mathrm{U}$ subrack implementations and specifying mandatory control and status registers.

Advantages of these standards include:

1. Substantial increase in subrack user-defined I/O pins - a must in physics applications; low-noise, high-density $2 \mathrm{~mm}$ metric connectors;

2. Subracks with built-in bussing of six standard voltages and four user-defined voltages - no kludged wiring for bringing power supplies for analog or ECL electronics; 
3. Inter-module and subrack EMC and EMI protection - protects sensitive analog electronics from electrical interference from other modules in a subrack or outside noise sources; protects electronics near the subracks from noise generated by subrack modules;

4. Module electrostatic discharge (ESD) protection - protects modules from electrostatic damage during subrack insertion or extraction;

5. Injector, extractor and locking handles - provide a simple method for inserting modules into and extracting modules from subracks; needed because of the increased forces due to the substantial increase in I/O and other module connector pins;

6. Board and subrack keying - protects modules and transition modules from being inserted into incorrect slots potentially damaging the modules; and

7. Optional subrack 48 volt power bussing allowing the use of on-board DC to DC converters to power very sensitive analog electronics.

\subsubsection{Electronics Packaging - An Alternative}

An alternative method of packaging BTeV electronics has been proposed. This method draws on products developed in the world-wide, very price-competitive, PC packaging industry. No communications busses would be used. Slow controls for component initialization, downloading and monitoring would be done by interconnecting Ethernet between PC packages. Electronics would thus not be modularized but, for example, each set of front-end electronics would be housed in its own PC package. Power to that package would be via a 110 VAC line cord and power supplies within the package. This option potentially offers cost savings over bussed systems but would require the development of a completely new infrastructure for hardware testing and software development.

\subsubsection{Data and Clock/Timing Links and Link Interfaces}

Possible and probable areas of hardware commonality in data and control/timing links and other components include:

- Data links from front-end electronics to Level 1 buffers;

- Data links from the Level 1 buffers to the event building switch;

- Data links from the event building switch to event buffers preceding online processors;

- High-speed front-end data links from on-detector electronics, possibly rad-hard, to local electronics;

- Low-noise data links from on-detector electronics, possibly rad-hard, to local electronics; 
- Data links to Level 1 trigger subsystems and from those subsystems to the Global Level 1 trigger;

- Control/timing links to front-end electronics and trigger subsystems; and

- Level 1 buffer electronics.

Two ongoing developments and one existing Hewlett Packard (HP) data link series of products should satisfy most if not all of the data and control/timing link applications given above. HP's G-links are transmitters and receivers which operate up to 1.5 Gigabits per second over fiber or copper. CERN is developing a G-link compatible radiation-hard driver for use in its LHC CMS (HCal and ECal) and ATLAS experiments. Fermilab is assisting that development, and we plan to use it for pixel data readout links, and possibly other subsystem data readout links. CDF SVX Run II electronics required the development of a radiationhard, low-noise transceiver. This transceiver will go into production soon. It is compatible with the IEEE-1596 LVDS (Low-Voltage Differential Signalling) standard developed for the Scalable Coherent Interface standard, IEEE Standard 1596-1992. This standard is actually a differential current standard intended to provide low-noise transmission of digital signals at very high speeds for a few tens of meters maximum.

We will adopt a minimum set of "BTeV data link" standards and, hopefully, a single "BTeV control/timing link" standard. The types of "BTeV links" will include:

- Serial optical G-link 1.3 Gigabit per second data link transmitting up to 20 bits every $1 / 53 \mathrm{MHz}$ (radiation-hard and non radiation-hard); and

- Parallel copper LVDS-like to LVDS 53MHz data link (radiation-hard and non radiationhard).

The parallel copper LVDS-like to LVDS data link would be used to move data a short distance from the detector to an area near the detector where electronics is more accessible. It provides for relatively low-power, low-noise data transmission off the detector. An alternative for this application is an array of Vertical Cavity Surface Emitting Lasers (VCSELs) and an optical ribbon cable link. VCSELs are inherently radiation hard. If this approach is cost effective and does not add more mass than desired, only minimal electronics need be on or near the detector. All the other electronics (e.g., data multiplexers, Level 1 buffers, trigger electronics, etc.) can be in the counting room and thus very accessible.

Our front-end and trigger subsystem implementers will easily be able to interface their electronics to BTeV standard readout and data acquisition electronics and control/timing signals. This will be accomplished by providing them with data and control/timing link daughterboards. Along with the daughterboards, the implementers will be provided with mechanical and electrical specifications for the daughterboards and component and artwork specifications for circuitry on their boards used to interface to the daughterboards. The following daughterboards are under consideration:

- Serial optical G-link or G-link compatible data link transmitter daughter-board; 
- Parallel copper LVDS-like data link transmitter daughter-board; and

- Serial optical G-link data receiver control/timing link daughter-board

\subsubsection{Slow Controls and Monitoring}

In an experiment the size of BTeV, several hundred devices need to be controlled (e.g., high-voltage systems, laser pulsers, interlock systems, etc.). Several thousand 'points' (e.g., power supply voltages, temperatures, gas mixes, interlocks) need to be monitored at regular intervals. Alarm or caution situations need to be established via hard-wiring or in software for equipment and/or personnel safety. Past experiments at Fermilab have chosen to use commercial or in-house-designed hardware and in-house- developed software for slow controls and monitoring systems despite the fact that total process automation solutions (hardware, software and user interfaces) are provided by a multitude of companies worldwide. The beamline cryogenics controls and monitoring system was the first system at Fermilab to use an industry total process automation system. Their hardware controllers and input devices were purchased from Moore Products and compatible software and graphical user interfaces from Intellution. The success of this system was remarkable. Mechanical engineers bought it and implemented it with no Fermilab professional software or electronics engineering help. Significantly fewer people were required to operate the system. As a result CDF will use an identical system for its Run II slow controls and monitoring system. BTeV's baseline slow controls and monitoring system is based on this system.

\subsubsection{Clock and Timing Distribution}

The BTeV experiment is different from all other current and past Fermilab experiments in that a) tracking (pixels) will be used in the lowest level trigger and b) all front-end subsystems will be read out into Level 1 buffers at the 132 nanosecond bunch crossing rate. Most frontend subsystems will require only a bunch crossing clock and a $53 \mathrm{MHz}$ accelerator clock. Some will operate asynchronously not needing a bunch crossing clock. For Run II, CDF has used a modular subsystem for distributing clock and timing information to its front-end and trigger subsystems. Differential ECL signals over high-quality cables are used for longdistance runs from the clock/timing sources to the collision hall. Low-current differential signals (LVDS drivers and receivers) are used to transport clock and timing signals to ondetector front-end electronics and counting room electronics. We will try to use as much of the work done by CDF as practical for its clock and timing distribution subsystem.

An alternative approach to sending clock and timing information to front-end and trigger subsystems is used in the Run II CDF SVX system. A conditioned accelerator clock (with jitter essentially removed) is used to clock timing data (e.g., bunch crossing) into HP Glinks via optical cables. Optical splitters can be used to distribute the clock and timing information. At the output of the G-link receiver, the clock is rederived from the output data strobes of the G-link receiver. The bunch crossing timing signal is received as a G-link receiver data bit. 


\subsubsection{Electronics Cooling and Rack Protection}

Several systems for cooling and protecting electronics racks exist at Fermilab. Assuming 9U $\mathrm{x} 400 \mathrm{~mm}$ VME subracks, our cooling system will be designed so that the exhaust air from a VME subrack that is dissipating 2500 watts will not exceed 95 degrees Fahrenheit. The fans will minimally provide 400 linear feet per minute of air flow. The air/water heat exchangers will reduce the temperature of 95 degrees Fahrenheit input air to 85 degrees Fahrenheit at the above air flow. There is no reason we can't use either CDF's or D0's Run II electronics cooling and rack protection system. This system will be connected to the slow controls and monitoring system described above.

\subsubsection{Forward Tracker}

\subsubsection{Straw Tracking}

The baseline design has seven stations of straw tubes in each arm. The three stations closest to the collision point on each side of the IR have inner regions instrumented with silicon strip detectors. Each straw tracking chamber at a station consists of six independent modules comprising the three views, for a total of 84 modules. As described earlier, we expect to use one of the U. Penn. ASD chips together with a new TDC to process straw hits. The TDC's may be located on the same printed circuit boards which hold the ASD chips, or on separate boards separated by short copper ribbon cables. Zero suppressed data from a group of straws will be read out to the Level 1 buffers on gigabit optical fiber links.

The straw tracker data output will consist of wire number and TDC data with a resolution of approximately one ns. Preliminary occupancy studies indicate an average of 70 hits per station per event or about 1000 system hits per event. The peak should be 5000 hits per event. The data for each hit include station ID, layer and view ID, Wire ID, and the TDC data for the leading edge; this should fit into three bytes (24 bits). For this estimate, a 4-byte data word will be used. Therefore, the system data will be 4 Kbytes per event, typical, and 20 Kbytes maximum. With an average of two events per crossing, there are 8 Kbytes per 132 ns or 61 Gbytes/sec. If the maximum event size is used to specify the system bandwidth, each of the 84 modules will require $30 \mathrm{Gbit} / \mathrm{sec}$ fiber links, for a total requirement of 2520 Fiber Optic links for the straw system.

\subsubsection{Silicon Strip Tracking}

The first three forward tracking stations in each arm will contain silicon strip detectors covering the inner $24 \mathrm{~cm}$ by $24 \mathrm{~cm}$ area. The silicon strip detectors will be 100 micron pitch sensors in two double sided planes comprising four views, $\mathrm{X}, \mathrm{Y}, \mathrm{U}$, and V. There will be 57,600 channels of data from six stations of four views. The readout electronics for this system has radiation tolerance requirements. The circuitry design is simple except for the fast readout time necessary.

The silicon detector data will consist of only hit location information, with no analog 
information. Strips with hits over threshold are read out to the Level 1 buffers. With occupancy of $1 \%$, there would be an average of 600 system hits per event. The peak occupancy is unknown at this time but will be estimated for now at 4\%. The data for each hit would be an address containing station ID, view ID, and strip ID. This should fit into two bytes (16 bits). The system data would be $1.2 \mathrm{~K}$ bytes per event, typical, $4.8 \mathrm{~K}$ bytes maximum. With an average of two events per crossing, there are $2.4 \mathrm{~K}$ bytes per $132 \mathrm{~ns}$, or an average of 18 Gbytes/sec. The maximum estimated rate is 72 Gbytes/sec. Each silicon station will produce 3 Gbytes/sec average, 12 Gbytes/sec maximum. Since there are four views per station, each view will require $24 \mathrm{Gbit} / \mathrm{sec}$ fiber optic links. For mechanical reasons, each view will be split at least in half and possibly in quarters. Providing each quarter with eight fiber optic links will allow for peak readout rates. This results in a silicon system total of 576 fiber optic links. Using the option above to move the data off the detector with copper LVDS links requires about four more subracks away from the detector in the collision hall.

\subsubsection{Ring Imaging Cherenkov Counter}

Simulations of the RICH detector indicate that $\sim 3.1 \mathrm{~m}^{2}$ should be covered with photodetectors per arm. The baseline design assumes use of multi-anode PMTs. With close packing of the PMTs, we expect to deploy 4,600 multi-anode PMTs in each arm. The resolution of the RICH detector is based partly on the segmentation density of the PMTs. Hamamatsu currently offers multi-anode PMTs with several choices of segmentation while the physical size of the tube remains the same $\left(26 \times 26 \mathrm{~mm}^{2}\right)$ :

- a 4 channel tube $(2 \times 2)$;

- a 16 channel tube $(4 \times 4)$; and

- a 64 channel tube $(8 \times 8)$.

The mix of these tubes is physics and budget driven, and is under study. The photo-detection area populated densely with $C_{4} F_{10}$ photons $\left(\sim 1.22 \mathrm{~m}^{2}\right.$ per arm) will need to be covered with the finest segmentation $(1,8008 \times 8$ tubes per arm). The rest of the photo-detection area, populated mostly by aerogel photons, will be covered with the coarser segmentation, at present assumed to be $4 \times 4$ tubes (2,800 per arm). With this segmentation the channel count is 160,000 per arm, 320,000 total.

Simulations also indicate that for $\beta=1$ tracks the number of detected photons per ring will be about 50 for gas rings and about 9 for aerogel rings. For a generic $b \bar{b}$ event, we expect each arm to produce $\sim 1000$ hits from the gas radiator and $\sim 220$ hits from the aerogel radiator. These are underestimates, since the simulation does not include secondary interactions. The probability of 2 photons striking the same the same anode cell is rather low, so it is valid to say that the number of channels fired is approximately equal to the number of photons produced by each radiator.

The PMTs need high voltage of the order of $1 \mathrm{kV}$. Because of the large number of tubes, it is likely that several tubes will share the same high voltage line. 
Read-out of multi-anode PMTs will be binary (i.e. we will detect only which anode cells have been fired). The scheme for setting discriminator thresholds needs to be studied. It will depend on gain uniformity of the individual channels, the signal to noise ratio, and on the design of the HV distribution system.

Initiation of a new design project to design 3 styles of front-end boards (a 4, 16, and 64 channel version) is attractive. However, the BTeV Muon readout system is already deploying a PMT readout system based on the ASDx series of ICs developed by Mitch Newcomer's team at the University of Pennsylvania. Each ASDx IC incorporates 8 channels of amplifier, shaper and discriminator in a single rad-hardened ASIC. The RICH system will use a read out system similar to the Muon readout system. The common system components can only simplify design, testing, sparing, installation and support. The Muon system deploys a 4 and 16 channel front-end card, a 64 channel card is needed (incorporating eight 8-channel ASDx ICs). See the Muon system description for a details.

For planning purposes, the 320,000 readout channels routed through 8 channels/IC ASDx ICs implies 40,000 ASDx ICs in total (two arms). They would be installed on 3 types of front-end boards corresponding to the tube segmentation. At one front-end board per PMT, there will be $\sim 9,200$ front-end boards, mounted directly behind the PMT bases.

The ASDx ICs require no clocking, and their output signals are low-voltage differential signals (LVDS) which are synchronized as they are latched into Doubler/Serializer boards.

The baseline muon read-out design does not sparsify the data either at the front-end electronics or at the Level 1 buffers. For common electronics to be used between the Muon system and the RICH system, provision for data sparsification will be added to the system.

The figure in the Muon section illustrates the proposed block diagram of the Muon front-end electronics subsystem. The Doubler/Serializer boards will be located outside the detector, but as close as possible to minimize the length of the cable connections from the front-end PMTs. Approximately 3,330 Doubler/Serializer boards are required. Since the Muon system uses 720 such cards, the 4,000 card total is dominated by RICH detector needs.

The output of the Doubler/Serializer boards will be sent to the data acquisition system's Level 1 buffers. The electrical protocol of the serial link to the Level 1 buffers can be differential LVDS or a higher-speed serial optical link. The data can be multiplexed into a single data stream to a high speed serial optical link ( $>1$ Gbit/s) with headers containing time stamp and channel address, sent to the switched data stream.

\subsubsection{Electromagnetic Calorimeter}

Our $\mathrm{PbWO}_{4}$ crystal calorimeter is very similar to the KTeV CsI calorimeter. The momentum range of photons that need to be detected is from about $1 \mathrm{GeV} / \mathrm{c}$ to $70 \mathrm{GeV} / \mathrm{c}$ in both $\mathrm{KTeV}$ and BTeV. For both experiments, energy resolution is of prime importance. The light output of the $\mathrm{PbWO}_{4}$ crystals is expected to be only 2.5 times lower than that for CsI crystals. Furthermore, the decay times of both scintillators are comparable, less than 100 nanoseconds for $\mathrm{PbWO}_{4}$ crystals and less than 76 nanoseconds for CsI crystals. The 
similarity of the two systems and the excellent performance reported by KTeV makes a good argument why we should use the technology developed at $\mathrm{KTeV}$ in the $\mathrm{BTeV}$ experiment.

The KTeV readout uses special Hamamatsu phototubes, with low gain $(\sim 4000)$ and good linearity. The tubes (YA2117s) act like a current source which is linear to better than $0.02 \%$ for a tube output up to 30 milliamps. Even this small non-linearity can be corrected. The phototube output goes to a Fermilab custom ASIC called the QIE5 (The entire system is called QIE) This IC is used in conjunction with an 8-bit FADC to digitize the output pulse at a fast rate with variable resolution over a 16-bit dynamic range. It accomplishes this by using eight nonoverlapping binary scaled ranges. The device is pipelined so it is deadtimeless.

The output of the phototubes will be connected to a PMT digitizer chassis or subrack (containing the QIE chip or similar device) via time-matched ribbon cable coax. The decision was made to mount the QIE electronics in a separate chassis rather than being mounted on the phototubes due to the radiation levels in the area of the PMTs and the limited space available behind the crystal wall (20,000 - PbWO4 crystal scintillators). Assuming the $\mathrm{PbWO}_{4}$ crystals are $2.47 \mathrm{~cm} \times 2.47 \mathrm{~cm} \times 22.5 \mathrm{~cm}$, a 20480-channel crystal wall approximately $12 \mathrm{ft}$ by $12 \mathrm{ft}$ will be formed in the area near the beam line and requires floor space nearby for either $406 \mathrm{U}$ subracks or 80 chassis of readout electronics mounted in 10-15 racks. Data from a number of phototubes will be multiplexed and zero suppressed by concentrator modules located in the same racks. These modules will send data to Level 1 bufferers using BTeV standard optical fiber links.

\subsubsection{Muon Detector}

In order to read out 69120 channels we will use 8640 ASD chips. Each front-end board will host two of ASD chips. Hence the total number of front-end boards is 4320 . The output signals are differential LVDS. The ASD chips are amplifiers and discriminators. They provide an asynchronous LVDS output. No clock is used in this chip. The data must be synchronized when it is latched into the "Doubler and Serializer" board. The Doubler and Serializer boards will be located off the detector, as close as possible to minimize the cable connections from the front-end. The function of this module is to multiplex the signals coming from 12 ASD chips. 720 Doubler and Serializer modules are required.

The output of the Doubler-Serializer boards will be sent to the Muon Trigger and DAQ system. The Muon Trigger will receive only one third of the total data, since the trigger is based on these data. The electrical protocol of the serial link to the Trigger and DAQ can be LVDS differential or RS485 over twisted pair cable.

For compatibility reasons the muon trigger and DAQ memory buffers to store the information can be designed on the same kind of boards selected for other BTeV subsystems. VME64 is a potential candidate. The number of boards will depend on the selected standard. 


\subsubsection{Software for Module and Subsystem Development and the Online Data Acquisition and Readout System}

$\mathrm{BTeV}$ front-end and trigger subsystem developers can use existing software for developing modules and subsystems. An infrastructure for testing VME-based boards has been developed for CDF, and is the foundation for testing CDF's Run II SVX, Muon and Calorimetry system hardware. This framework, called CDFVME, is Java-based code that communicates to the test subrack(s) via CORBA, a remote procedure-like software tool. The CORBA implementation is ROBIN, and requires a VME processor in the subrack running the VxWorks operating system. VxWorks is the real-time operating system that will be supported at Fermilab throughout Run II for both CDF and D0.

The CDFVME software consists of a series of Java classes to interface to generic VME boards (e.g., read and write register commands), plus templates that users can customize for their specific board. CDFVME also contains a framework in which to run a series of tests in batch mode, and cycle the readout code. Error logging is also provided.

CDFVME uses FISION, the Fermilab supported implementation of the ANSI standard software (ANSI/VITA 25) called VISION to communicate across the VME backplane. FISION is currently supported on Motorola MV16x and PowerPC microprocessor VME CPU modules. CDFVME was jointly developed by Yale, the Online and Database Support Department of the Computing Division and CDF.

The choice of downstream L2/3 processors in $\mathrm{BTeV}$ will be driven by price/performance issues. Current market trends indicate that the processors will be some type of $\mathrm{PC}$ processor running Linux. Linux PCs will be used for both CDF and D0 Level 3 online processor farms.

Licensing costs and various support issues are generating interest for software implementers to evaluate real time operating system alternatives to VxWorks. Real-time Linux is a possible contender for post Collider Run II data acquisition systems.

Development code for data acquisition should move in the direction of modern programming standards. Run II software has already been designed for object oriented languages such as $\mathrm{C}++$ and Java. CORBA is the standard protocol to communicate between objects on remote nodes. 


\section{Part III}

\section{Simulations Results: BTeV Physics Reach}




\section{Chapter 10}

\section{Status of the BTeV Simulation Effort}

\subsection{Update on MCFast}

The physics simulations for BTeV have been carried out using MCFast v4_2 [1], a fast Monte Carlo package developed by the Fermilab Computing Division for detector design studies. MCFast provides a general framework for the comparison of differing detector geometries and is interfaced to a variety of generators that simulate the production and decay of $\mathrm{B}$ hadrons in a collider environment or fixed target environment.

The primary goal of MCFast is speed and flexibility which is achieved through parameterization. The package was designed to serve as an analysis tool that could be used to compare the physics reach of a variety of detector options. The program emphasizes fast tracing of particle trajectories through simple geometrical shapes. In the original version used for the EOI (MCFast v2_6), the particles were traced through the detector following a straight line or a helical trajectory. The effects of multiple scattering were added by calculating the track covariance matrix using a Kalman filter technique[2][3]. Smeared particle trajectories were supplied to the user for further analysis. Parameterized showering was implemented making it possible to simulate electromagnetic and hadronic showers and energy deposition inside calorimeters and absorbers. The simulation of showers in calorimeters and absorbers in MCFast is described in detail below. An interface between the MCFast geometry framework and GEANT was developed at this time and has been used for more detailed simulation of detector response.

In preparation for this document, we updated the package and have implemented multiple scattering, $\mathrm{dE} / \mathrm{dx}$, and bremsstrahlung during the tracing step. Decays in flight, pair conversions and secondary hadronic interactions are also included. Hits are now generated during the tracing step and are passed to a new track fitting package which is based on the Kalman filter technique. Realistic hit generation in the pixel detectors has been studied and a parameterized resolution as a function of track angle is included in the physics simulations presented here. The generated hits are also fed to the L1 trigger simulation package which does complete pattern recognition and has its own track fitter and vertex finder.

The current version of the MCFast package is written in Fortran and $\mathrm{C}$ and $\mathrm{C}^{++}$. It is 
interfaced to the standard HEP event generators Pythia[6], Herwig[7] and Isajet[8] through the StdHep interface. The decay of charm and bottom hadrons is done by QQ[9], a Monte Carlo package developed for CLEO. A 3-D Graphics package based on OpenInventor has been developed to display the detector geometry, tracks and hits.

MCFast v4_2 contains the new Kalman filter track fitter, and an optional $\mathrm{C}^{++}$tracing and showering algorithm coupled to a more efficient hit generation package for the calorimeters. The new Kalman filter was used in the preparation of the results presented in this document and will be used extensively in future tracking studies. The $\mathrm{C}^{++}$tracing and showering options, while complete, are still under test and were not used in the simulations presented in this document. The tracing and showering improvements will give us more efficient code and the ability to add radial detector elements such as a beampipes into the $\mathrm{BTeV}$ detector description.

Pythia 6.125 was used to generate physics processes for the BTeV simulation studies described below. Heavy quark decays were modeled through the CLEO decay Monte Carlo QQ 9.2b.

The size of the interaction region was taken as $\sigma_{x}=\sigma_{y}=50 \mu \mathrm{m}$ and $\sigma_{z}=30 \mathrm{~cm}$ corresponding to our current understanding of the running conditions in the $\mathrm{C} 0$ interaction region. The number of interactions per crossing is expected to be on average 2 at a luminosity of $2 \times 10^{32} \mathrm{~cm}^{-2} \mathrm{~s}^{-1}$ which means that there will often be more than one interaction per crossing. The effects of multiple interactions per crossing have been accounted for in these studies. The length of the interaction region and the station repetition in the vertex detector design allow us to separate tracks from two different primary interactions in each event as long as the interactions are more than a few centimeters apart.

\subsection{Kalman Filter}

In High Energy Physics today the method of choice for track fitting is Kalman Filtering [3, 5, 4], a method which produces an unbiased, optimal, linear estimator of the track parameters. By optimal it is meant that the estimator has the minimum variance of any unbiased, linear estimator. While these properties are only true in the limit of Gaussian multiple scattering, and Gaussian measurement errors, these conditions are sufficiently well approximated by the $\mathrm{BTeV}$ detector. The utility of the method is well established with operational experience in many HEP experiments. An important property of Kalman filtering is that it does not involve the inversion of large matrices and, therefore, that the execution time is linear in the number of hits. The method is also known in the HEP literature as Billoir fitting[2].

Since the last BTeV report, a Kalman Filter has been incorporated into MCFast for use a as stand-alone fitter. By stand-alone it is meant that the Kalman filter is not incorporated into the pattern recognition.

The present operation of MCFast is a two step process. In the first step, tracks are followed from their production point along their trajectory and, at each detector surface that is encountered, one or more operations takes place. These operations include multiple scattering, energy loss, elastic and inelastic hadronic scattering, bremsstrahlung and pair 
conversion. If the surface represents a sensitive element then a hit may also be created. In addition, decay in flight may occur at any point along the track and a shower may start when a particle passes through dense material such as magnet yokes, calorimeters and hadron filters. The collection of all points on one track which are recorded in this step is referred to as the trace list of the track. In the second step, the trace list of each track is used as the input to a Kalman filter. The output of the Kalman filter has been validated over the full range of momentum and angles which are important for $\mathrm{BTeV}$.

With the advent of a working Kalman filter, several sorts of studies have become possible. These include the study of non-Gaussian resolution functions, validating the approximations used in the Level 1 trigger code, implementing the first Level 2 trigger algorithm and studying defects in pattern recognition.

The first of these has been carried out and is discussed in sections 10.3 and 5.2.2.4. The conclusion is that using realistic descriptions for the spatial resolution of the pixels grows only small non-Gaussian tails on all of the important physics quantities. All of these tails are small enough that they have no significant detrimental effect on the physics.

Because of stringent requirements on computing speed, the Level 1 trigger code must often make approximations for the properties of the tracks and vertices which it finds. For example, it may decide that the resolution on some quantity is sufficiently dominated by multiple scattering that other contributions to the resolution may be ignored. In order to verify these approximations the Kalman filter has been used to fit the tracks found by the Level 1 trigger. We have compared quantities computed by the trigger code to the corresponding quantities computed from the output of the Kalman filter; that is we are comparing the approximation against the best possible computation of the same quantity. This allows us to make quantitative judgments about the cost/benefit of replacing one approximation with another. This program has already resulted in improvements to the Level 1 trigger code and work is continuing.

A candidate Level 2 trigger algorithm, which is discussed in section 8.9, relies heavily on the Kalman filter code.

No results are yet available from the study of pattern recognition defects; they will be reported as they become available. The plan of this work is as follows. Before Kalman filtering a track we inspect the trace list and look for hits which do not belong to the track but which are close to it. If a hit is sufficiently close we add this hit to the trace list, either augmenting or replacing the nearby correct hit. This procedure grows tails in the physics distributions and, using this procedure, we will be able to estimate our sensitivity to errors in pattern recognition before a complete pattern recognition program is available.

Finally, the Kalman filter is already available for use as a final fitter once true pattern recognition algorithms are developed.

\subsection{Towards Realistic Pixel Resolution Functions}

The physics which governs the spatial resolution of the pixel detectors has been discussed in section 5.2.2. Using the results of that study, four different models of the spatial resolution 
of the pixel detectors have been incorporated into our simulations, the constant $\sigma$ model, the variable $\sigma$ model, fast clustering and slow clustering. The default model, the one used for most calculations presented in this document, is the variable $\sigma$ model.

The constant $\sigma$ model is the model used in the EOI. In this model the spatial resolution is a Gaussian with a constant width which is equal to the pitch divided by $\sqrt{12}$. This is true for both the short and the long measurement directions of the pixel.

In the variable $\sigma$ model, the spatial resolution remains Gaussian but the width of the Gaussian for the short dimension of the pixel is a function of the angle of incidence of the track on the pixel plane. The width of the Gaussian for the long dimension of the pixel remains at the pitch divided by $\sqrt{12}$. For reference, the distribution of the $x$ and $y$ angles of incidence are shown in Fig. 10.1 parts a) and b). These figures were made using typical $b \bar{b}$ events and they only include hits from tracks with at least 20 hits. The width of the distribution for $\tan \theta_{y}$, is greater than that for $\tan \theta_{x}$ because of the bending of the tracks in the magnetic field of the analyzing dipole. Fig. 10.1 parts c) and d) show the value of $\sigma$, as a function of angle of incidence, for, respectively, pixels which measure $x$ and pixels which measure $y$. The minimum of the $y$ resolution is offset from $\theta_{y}=$ because of Lorentz force effects. These functions were made by making a piecewise linear approximation to the 2-bit data shown in Fig. 5.9, in section 5.2.2.4.

In the fast clustering model a Monte Carlo simulation is run for each intersection of a track with a pixel plane. Using the physics discussed in section 5.2.2, this simulation explicitly generates the digitized pulse heights recorded on nearby pixels. A 3-bit linear $\mathrm{ADC}$ and a threshold of $2000 e^{-}$were assumed. Once each cluster has been generated, the pulse heights are used to obtain an estimator of the position of the hit; this estimator uses the knowledge of the angle of incidence.

In addition to returning an estimator of the $(x, y)$ position of the hit, the fast clustering model also returns an estimate for the error on that position. This error is used by the Kalman filter and is similar to the error used in the variable $\sigma$ model. Parts a) and b) of Fig. 10.2 show values of these errors for the short pixel dimension for hits on tracks, in typical $b \bar{b}$ events, with more than 20 hits. These functions are double valued because, at a given angle of incidence, not all tracks trigger the same number of pixels. A comparison with the corresponding plots for the variable $\sigma$ model will show that, in significant parts of the cell, the resolution in the fast clustering model is slightly poorer. This, at first, seems surprising given that the fast clustering model uses more ADC bits. However, at the time that the fast clustering model was created, the Monte Carlo code which simulates the pulse heights had been improved by the addition of more processes which degrade the resolution. Fig. 10.2c) shows the quantity $\left(x_{\text {measured }}-x_{\text {generated }}\right) / \sigma_{x}$ using the fast clustering model. In this figure there are two things to notice, one of which is the size of the non-gaussian tails; they are small enough to be manageable. The second is that the main part of the distribution is very box-like, which can be understood by seeing in part e) that, about half of the time, only a single pixel is hit. Because the resolution function of a single pixel is box-like, not Gaussian, the box-like shape makes an important contribution to part c). Parts d) and f) show similar information for pixels which measure $y$. In this case, however, the spreading due to the 
Lorentz force ensures that, only rarely, is a single pixel hit; therefore the distribution in part $\mathrm{d}$ ) is not box like. It also has quite small tails. The data in part $\mathrm{d}$ ) which lie between -2 and 2 were fitted to a Gaussian and the width was measured to be $0.924 \pm 0.005$, which suggests that the fast clustering model returns values of $\sigma_{y}$ which are usually a little too large.

The above discussion concerned only the resolution in the short dimension of the pixels. In the long dimension the nominal resolution is the pitch divided by $\sqrt{12}$ and, because almost all clusters are only one pixel wide, the shape of the resolution function is box-like.

The important improvement which is present in the fast clustering method is that its resolution functions are closer to reality, having a box-like component and noticeable tails. Using the fast clustering model, however, adds about $70 \%$ to the execution time of a typical physics analysis job. This is a sufficient degradation that this model is not routinely used for physics analyses and has only been used for a few specific studies. One such study, discussed in section 5.2.2.4, shows that the resolution functions discussed above do produce tails in several important physics quantities but that these tails are manageable.

The principal weakness of the fast clustering model is that the overlapping of two or more clusters is not considered. This weakness is addressed in the slow clustering model, in which the generation of pulse heights on pixels proceeds as in the fast clustering model. However the information about pulse heights on individual pixels is retained for a full event. Once an event has been fully generated, cluster finding code examines the wafers to identify clusters of hits and to estimate positions from the clusters. This code executes the most slowly of all models and has only been used for a few studies of occupancy. There are several obvious speed optimizations which can be applied to the implementation of this model and these optimizations are in the upgrade plan. The implementation of a fast algorithm which simulates overlapping clusters will be important for the simulation of the trigger.

A final improvement which is in the upgrade plan is to simulate clusters including delta ray production.

\subsection{Multiple interactions per crossing}

In $\mathrm{BTeV}$, b events and c events represent, respectively, of order one part in 500 and one part in 100 of the total cross section. At design luminosity, $2 \times 10^{32} \mathrm{~cm}^{-2} \mathrm{~s}^{-1}$, we expect an average of 2 interactions per beam crossing. Therefore, to a good approximation, we can neglect the contribution of multiple $\mathrm{b}$ and $\mathrm{c}$ interactions in a single beam crossing. These considerations lead to the following simple model of multiple interactions which is implemented in MCFast.

MCFast requires the mean number of interactions per beam crossing and two input files, one containing signal events and the other containing background events. Typical background samples include events containing primary uds quarks, or generic QCD events, both as generated by Pythia. To generate one beam crossing, MCFast draws a number of interactions, $n$, from a Poisson distribution with the specified mean. If $n$ is zero, then MCFast will redraw it. No record is kept of the frequency of redraws since that information 
is not needed to compute event yields. Once $n$ is determined, MCFast will read one event from the signal file and $n-1$ events from the background file.

An upgrade is planned in which we deal with the fully general problem of many input streams, allowing zero or more interactions from all streams, as demanded by Poisson and multi-nomial statistics.

\subsection{Simulation of Secondary Hadronic Interactions}

Since the BTeV EOI, a new package has been added to MCFast to simulate secondary hadronic interactions. The approach is purely phenomenological, no attempt is made to model the underlying hard scattering process. The probability of an interaction occurring is determined by the collision length and absorption length of the material. Energy dependent cross-sections are not used.

\subsubsection{Inelastic Secondary Interactions}

The number of charged particles produced in a secondary interaction of a charged pion on a proton target is a function of $\mathrm{s}$. The mean is given by

$$
a+b \log s+c(\log s)^{2} .
$$

where $a=-0.35, b=1.21$ and $c=0.03$ as given by Dumarchez et al. for pion-proton interactions [10]. The distribution is characterized by KNO scaling which is modeled as a truncated Gaussian. Fig 10.3 can be compared with Fig 5 in Firestone et al. [14]. For a heavier target the mean increases as a function of the atomic number A [11]. The average number of neutrals is assumed to be $50 \%$ of the charged multiplicity. The number of secondary particles from an interaction is limited to a maximum of 25 to avoid overflowing arrays.

The momentum distribution of secondary particles is approximately flat in rapidity and Gaussian in $p_{T}$ with respect to the direction of the interacting particle [12]. The simulated distributions for a particle with momentum $50 \mathrm{GeV} / \mathrm{c}$ are shown in Fig 10.4. These can be compared with Fig 30 in Brenner et al.[12] and Fig 69 in [11]. The momentum of each outgoing particle is selected from these distributions and then energy is conserved by rescaling the momenta. The momenta are then rotated into the lab frame and the new particles are added to the StdHep list.

\subsection{Elastic Interactions}

Elastic scattering is characterized by the distribution of four-momentum transfer $\operatorname{squared}\left(-t=p_{T}^{2}\right)$. The distribution is given by

$$
d \sigma / d t=e^{(b t)}
$$

where $b \approx 8$ [13]. The simulated distribution is shown in Fig 10.5. 


\subsection{Calorimeter Simulation}

\subsubsection{Simulation Techniques}

We use three levels of simulation:

- Studies using the momentum vector of the generated photon, smeared according to simple resolution functions. This level of simulation can give insights into many issues and is fast (requires few computer resources). However, many details are beyond its scope. In particular, it does not distribute energy over calorimeter elements so one cannot use it to study or develop shower reconstruction. We have used it to study mass resolution in states containing photons and charged particles, to take a first look at combinatoric backgrounds, and to search for useful kinematic variables to cut on.

- Studies using a GEANT simulation. This is a full simulation of the shower development and involves tracking all the products of the cascade of pair conversions and bremsstrahlung. It contains detailed knowledge of all relevant physics processes. However, it takes a large amount of computer time and the generation of large numbers of events, especially for background rejection studies, is beyond our capabilities.

- Studies using the MCFast showering/calorimetry subpackage. This deposits (smeared) amounts of energy in detector elements based not on tracking of individual particles but based on parametrized shower shape distributions. This procedure does produce showers distributed over detector elements which can be processed by realistic shower reconstruction programs. The program executes much faster than a GEANT simulation but represents the energy deposition in detectors quite well and has been shown to be consistent with GEANT over the range of energies relevant to BTeV. We have used this program extensively in our background studies since the computer time is manageable.

All three methods have been used in designing the electromagnetic calorimeter and results obtained from each method appear in this document. Below we describe the MCFast program because it was used to produce the physics results presented in chapter 11.

\subsubsection{The MCFast showering/calorimetry subpackage}

The MCFast showering/calorimetry subpackage is completely integrated into the MCFast Monte-Carlo package: the user defines tracking systems and calorimeters and absorbers through the detector description file, and MCFast takes care of converting particles to showers automatically, through ad-hoc interruption of the tracing processes. In addition, energy losses and multiple scattering in the bulk material of the calorimeters and absorbers are taken into account.

As with other features of MCFast, the showering simulation is based on an extensive parametrization scheme. That is, there is no detailed, particle by particle, description of an 
electromagnetic or hadronic shower. Instead, the energy densities due to the high energy showering processes within volumes are parametrized, and shower fluctuations are simulated globably rather than locally. Then in calorimeters, these energy densities are mapped onto a user-controlled readout grid, and calorimetry hits are generated.

Unlike particle tracing in MCFast, the simulation of the shower propagation is intrinsically 3-dimensional. To keep the program simple, only three volume types are recognized: BOX, TUBE and CONE. These shapes must be oriented in such a way that the symmetry axis is along the Z-axis.

At the initialization stage, MCFast computes surface boundaries for these volumes. At the tracing stage, once such a surface is reached, the calorimetry subpackage returns an estimate of the mean free path length for the particle. For electrons/positrons and gammas it is simulated according the formula $f(x)=e^{-x / X_{0}}$, where $X_{0}$ is related to the radiation length. For hadrons $f(x)=e^{-x / \lambda}$, where $\lambda$ is absorption length (the mean free path for inelastic interactions). Based on this information and a random number(s), the location of the interaction inside the calorimeter is computed, if need be. Should a primary interaction occur, the trace identity switches from "elementary particle" to "shower" and the core of that shower is propagated across the geometry by the MCFast tracing routine. This allows shower propagation from one calorimeter to the next.

Upstream of this conversion point, the particle behaves as a minimum ionizing particle (MIP), sensitive to magnetic field, if any, and undergoing energy loss and multiple scattering. The corresponding energy deposition is recorded, e.g., calorimetry hits are created.

Showering switches on at the shower conversion point. However, there is currently no provision for shower sensitivity to magnetic field inside a calorimeter. Unlike normal charged particles, the core of the shower propagates in a straight line.

Downstream of the conversion point, the deposited energy is calculated on a step by step basis, according to the shower longitudinal profile formulae. After each step, the shower core position and energy/momentum are estimated and the control is given to the hit generation if necessary. The package can optionally simulate energy deposition to the cells surrounding the shower core.

Note that a shower can spread over multiple volumes both longitudinally and transversely. Thus, even if a shower develops in an absorber, it still may produce hits in nearby calorimeters (if any).

The electromagnetic shower longitudinal profile is described as

$$
d E / d x=E_{0} b \frac{(b t)^{a-1} e^{-b t}}{\Gamma(a)}
$$

where

$$
\begin{gathered}
t=x / X_{0}, \quad b \simeq 0.5, \quad a=1+b t_{\text {max }}, \\
t_{\text {max }}=1.0 \times\left(\ln (y)+C_{i}\right), \quad i=e, \gamma, \quad\left(C_{\gamma}=+0.5, \quad C_{e}=-0.5\right) \\
y=E / E_{c}, \quad E_{c}=\frac{800 M e V}{Z+1.2},
\end{gathered}
$$


( $Z$ is atomic number, $E_{0}$ is energy of incident particle).

For hadronic showers we use

$$
d E / d x=E_{0}\left(w b \frac{(b t)^{a-1} e^{-b t}}{\Gamma(a)}+(1-w) d \frac{(d u)^{c-1} e^{-d u}}{\Gamma(c)}\right)
$$

where

$$
\begin{aligned}
t & =x / X_{0} \\
u & =x / \lambda \\
a & =0.6165+0.3183 \ln E_{0} \\
b & =0.2198 \\
c & =a \\
d & =0.9099-0.0237 \ln E_{0} \\
w & =0.4634
\end{aligned}
$$

When the shower transverse profile is turned on, the energy deposited at each step is distributed around the shower axis according to the shower transverse profile (which becomes wider as the shower moves deeper into a calorimeter).

The transverse profile for both electromagnetic and hadronic showers is described as

$$
\frac{d E}{d R}=e^{\left(-R / A_{1}\right)}+A_{2} e^{\left(-R / A_{3}\right)}
$$

In this formula, parameters $A_{1}, A_{2}, A_{3}$ depend on the material properties and on the current shower depth. The dependencies are different for electromagnetic and hadronic showers.

The distribution of deposited energy occurs in a plane perpendicular to the direction of motion of the shower core. Since showers may have crossed particular calorimeter boundaries, both longitudinally and transversely, energy deposition is also computed in the corresponding cells of those neighboring calorimeters.

The total deposited energy is smeared during the process of showering simulation according to the energy resolution formula

$$
\frac{\sigma_{E}}{E}=\frac{a}{\sqrt{ } E} \oplus b
$$

where $a$ and $b$ are input parameters for the code. For a sampling calorimeter, a visible fraction of energy may be calculated (depending on the densities and proportion by volume of material from which a calorimeter is built).

In the version of MCFast (V4.2) used for the studies described below, energy deposition in calorimetric cells is organized in a list of "hits" in each calorimeter in the system, and the whole container of calorimetric hits in the system is organized as a "map": the calorimeter 
ID that refers to the geometry database vs. a list of hits in the calorimeter. A "Hit" refers to a set of variables describing the cell address, energy deposition via minimum ionization, electromagnetic, and hadronic processes, and information on particles/showers contributing to a given hit, i.e. track number referring to the HEP event list, along with the percentage of the hit total energy caused by the particle/shower.

As pointed out above, showers may spread over multiple volumes both longitudinally and transversely, and thus hits are produced in as many calorimeters as affected. And finally, calorimetric hits are available for analysis via a set of application user interface routines.

\subsection{Simulation of the BTeV Trigger}

Since the last BTeV report a great deal of work has gone into development of the trigger. This work includes improvements to the baseline Level 1 algorithm, an improved characterization and understanding of the baseline Level 1 algorithm, consideration of alternate Level 1 algorithms and a first look at details of a candidate Level 2 algorithm. Most of this work was done within the framework of the $\mathrm{BTeV}$ simulation package. The above material is discussed in chapter 8. Additional simulation of the queueing aspect of the Level 1 frontend has been done using the CNCL package [15]. Additionally, work on the DAQ system, which is intimately coupled to the details of the trigger implementation, is in progress. This material is discussed in chapter 9 .

\subsection{BTeV Geometry Description for MCFast}

The geometry description for our MCFast simulations is more complete and more realistic than the one used in the EoI. The detector elements are better defined and the simulations reflect the more accurate estimates of material thickness and detector resolution. We have also taken into account multiple interactions and secondary interactions. Particles traced through the detector elements suffer the effects of multiple scattering, $\mathrm{dE} / \mathrm{dx}$ and bremsstrahlung. Decays in flight and pair conversions are also included in the simulations. The trigger simulations use full pattern recognition in the tracking detectors. The physics simulation do not yet employ pattern recognition to build tracks from hits; the effects of the material thickness and realistic detector resolutions have been taken into account.

The standard geometry description of the baseline BTeV detector for MCFast physics studies includes:

- a dipole field described by 7 regions of constant field extending to $\pm 264 \mathrm{~cm}$ including the fringe and a yoke and coils which act as absorbers. The central $90 \mathrm{~cm}$ around the vertes detector is in a horizontal field of $1.6 \mathrm{~T}$.

- a silicon pixel vertex detector with 31 stations with 3 planes each (Y-X-Y). The silicon and electronics is assumed to be $1 \mathrm{~mm}$ thickness and the outer layers of each have an extra 100 microns in thickness added to simulate the station shielding. The pixel 
dimensions are $50 \times 400$ micron. The resolution of the pixel detectors is parameterized as a function of angle.

- forward silicon detectors with 3 stations of strip detectors in each arm. Each station has 3 planes (i.e. 3 views $\mathrm{X}, \mathrm{Y}, \mathrm{U}$ ). For these simulations, the strip pitch is 300 microns.

- a set of forward tracking chambers with 7 stations in each arm. The inner three devices in each arm have larger holes to accommodate the forward silicon tracker. There are 9 planes of straws with 3 views in each chamber $(\mathrm{Y}, \mathrm{U}, \mathrm{V})$. The straw chamber resolution is assumed to be 150 microns.

- a RICH detector with $\mathrm{C}_{4} \mathrm{~F}_{10}$ gas and a Lucite mirror.

- an electromagnetic calorimeter with $2.47 \mathrm{~cm} \mathrm{PbWO}_{4}$ crystals which are $22.25 \mathrm{~cm}$ in length.

- muon system with muon detectors and absorbers. The muon detector and toroid simulations are described in Chapter 4, Section 4.2.7.

We have also generated a standard geometry description with a more detailed description of the vertex detector. Here each pixel chip is represented as an individual wafer and the wafers are in a tiled geometry. We have done studies of the trigger efficiency and resolution using this detailed description. The results of the resolution study are shown in Section 10.3.

The Level 1 vertex trigger algorithm including hit finding, pattern recognition, track finding and vertex finding is applied to each event during analysis in order to study the efficiency of vertex trigger. Level 2 trigger studies and muon trigger studies are currently underway and are described in detail in Chapters 8 and 4.

The physics simulations presented in this chapter use the standard baseline geometry description. The vertex detector is planned to be inside the beam pipe. The effects of the beam pipe on the outer tracker is currently under investigation. 

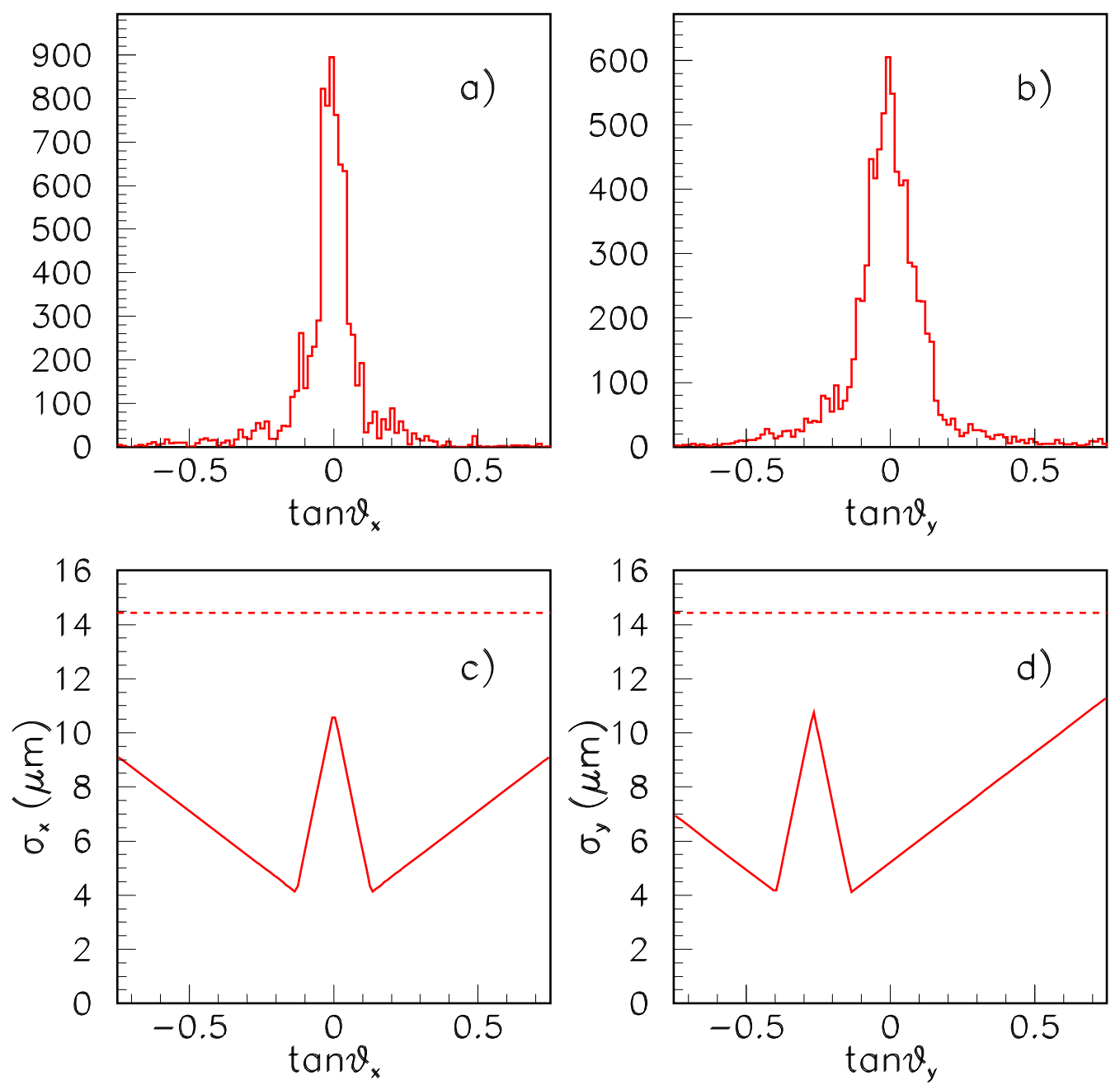

Figure 10.1: The distributions of a) $\tan \theta_{x}$ and b) $\tan \theta_{y}$ for hits on tracks, in $b \bar{b}$ events, with at least 20 hits. Here $\theta_{x}$ is the angle of incidence, in the $x z$ plane, of a track which intersects a pixel detector. Similarly for $\theta_{y}$. For most studies reported in this document, the pixel detectors are modeled as having a spatial resolution function which is Gaussian. The dependence of the $\sigma$ of the Gaussian on angle of incidence is shown in c) for pixels which measure $x$ and in d) for pixels which measure $y$. In both cases the solid line shows the value of $\sigma$ for the short dimension while the dashed line shows the value for the long dimension. 

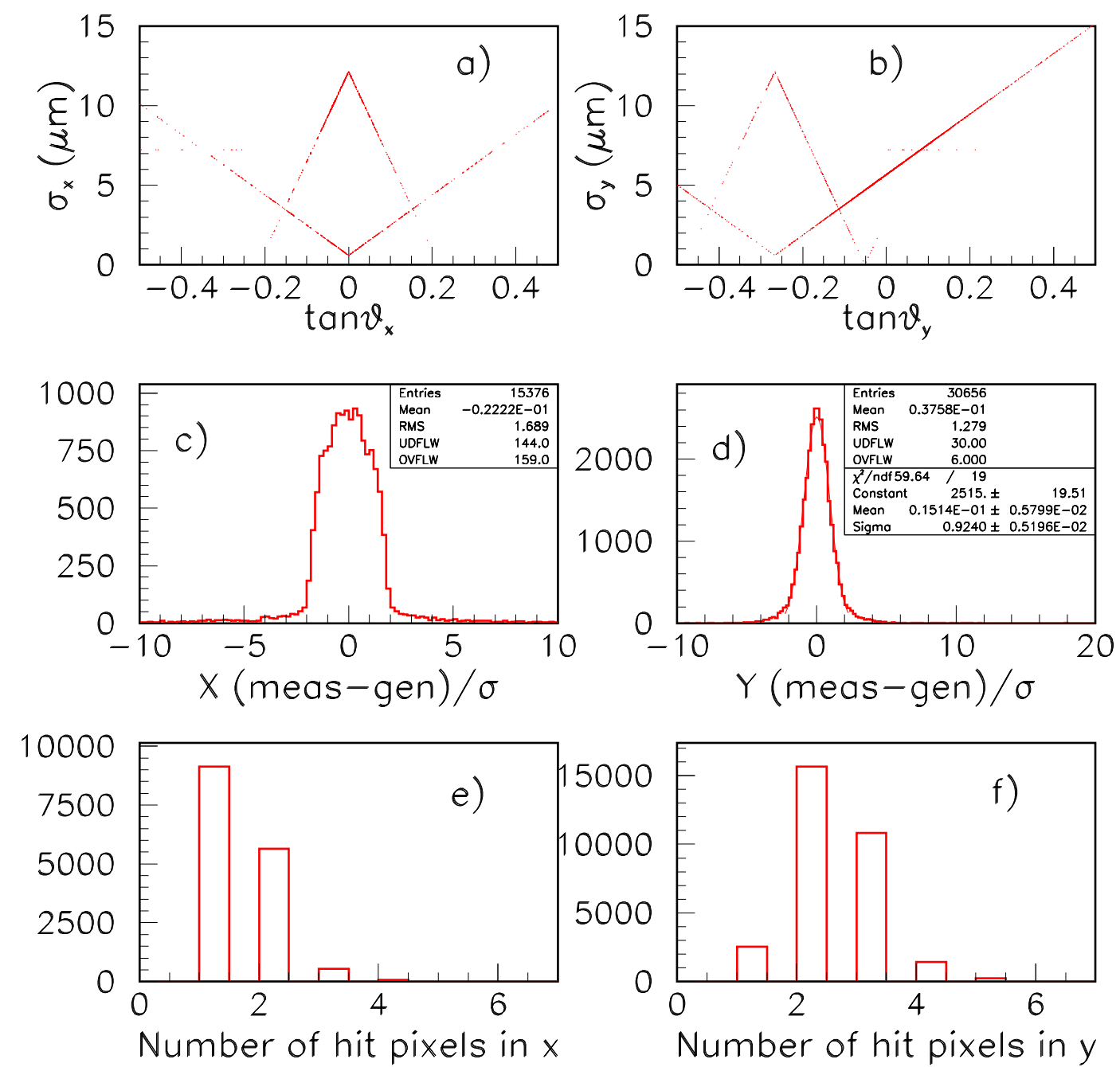

Figure 10.2: The values of the position errors in the fast clustering model for a) pixels measuring $x$ and b) pixels measuring $y$. The angles $\theta_{x}$ and $\theta_{y}$ are defined in the caption of the previous figure. The functions are double valued because two tracks at the same angle of incidence may trigger a different number of pixels. The information in these figures is only for the short dimension of the pixel. Part c) shows the distribution of $\left(x_{\text {measured }}-x_{\text {generated }}\right) / \sigma_{x}$ while part $\mathrm{d}$ ) shows the corresponding distribution for $y$. The shapes of these distributions are discussed in the text. Parts e) and f) show the distributions of the number of pixels in a cluster. 


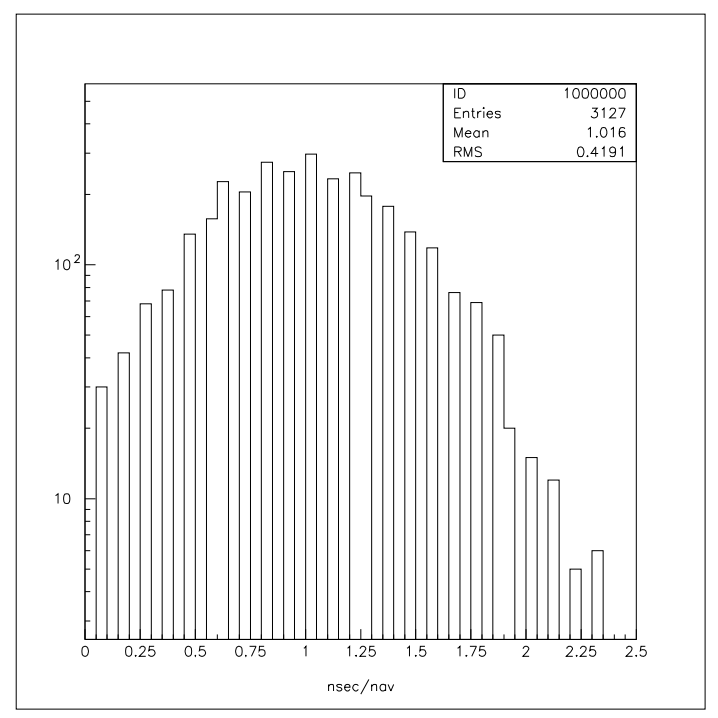

Figure 10.3: KNO scaling: $n_{\text {sec }} /<n>$ is a universal distribution
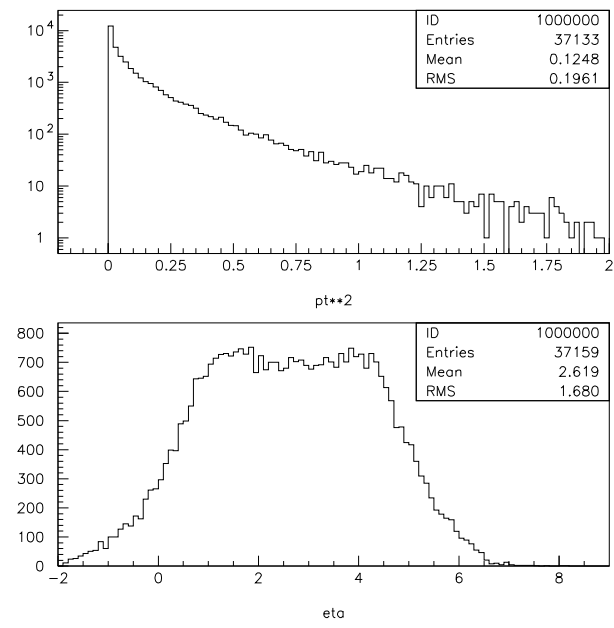

Figure 10.4: Simulated Kinematic distributions of secondary particles for an incident particle of $50 \mathrm{GeV} / \mathrm{c}$ (a) $p_{T}$, (b) $\eta$ 


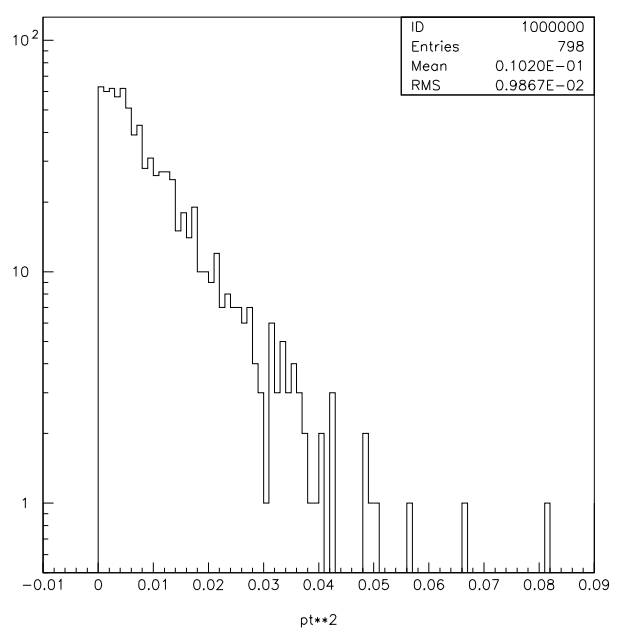

Figure 10.5: Simulated distribution of $p_{t}^{2}$ for elastic scattering 


\section{Bibliography}

[1] P. Avery et al, "MCFast: A Fast Simulation Package for Detector Design Studies". in the Proceedings of the International Conference on Computing in High Energy Physics, Berlin, (1997) and http://fnpspa.fnal.gov/mcfast/mcfast_docs.html

[2] P. Billoir, NIM 225, 352-366 (1984).

[3] Paul Avery http://www.phys.ufl.edu/ avery/fitting.html, Fitting Theory V.

[4] R. Bock et. al., "Data Analysis Techniques for High-Energy Physics Experiments", Cambridge University Press (1990).

[5] M.Regler and R. Fruwirth, "Reconstruction of Charged Particle Trajectories", Plenum Publishing Company (1989).

[6] H. U. Bengtsson and T. Sjostrand, Comput. Phys. Comun. 46,43 (1987).

[7] G. Marchesini and B.R. Webber, Nucl. Phys. B310, 461 (1988); Nucl. Phys. B330, 261 (1988);

I. G. Knowles, Nucl. Phys. B310, 571 (1988);

G. Abbiendi, et al., Comp. Phys. Comm. 67, 465 (1992).

[8] F. Paige and S. Protopopescu, Brookhaven National Laboratory Report BNL37066(1985)(unpublished).

[9] QQ is developed and maintained by the CLEO Collaboration. See http://www.lns.cornell.edu/public/CLEO/soft/QQ/ For the studies reported here, it is used for its model of the decays of hadrons containing heavy flavors.

[10] "Average Multiplicity and KNO Scaling in Hadron-Hadron Interactions", J. Dumarchez et al, Il Nuovo Cimento 66A, 114 (1981)

[11] "GHEISHA: The simulation of Hadronic Showers - Physics and Applications", H. Fesefeldt, PITHA 85/02

[12] "Experimental Study of single-particle inclusive hadron scattering and associated multiplicities", A. Brenner et al, Phys. Rev. D 26 1497(1982) 
[13] "Hadron-proton elastic scattering at 50, 100, and $200 \mathrm{GeV} / \mathrm{c}$ momentum", C. W. Akerlof et al, Phys. Rev. D 142864 (1976)

[14] " $\pi^{-} p$ interactions at $360 \mathrm{GeV} / \mathrm{c}$ : Measurement of the total and elastic cross sections and the charged-particle multiplicity distribution", A. Firestone et al., Phys. Rev. D 142902 (1976)

[15] See http://www.comnets.rwth-aachen.de/doc/cncl.html. 


\section{Chapter 11}

\section{The Physics reach of $\mathrm{BTeV}$}

In this chapter, we present results of physics simulations of some of the key measurements discussed in chapters 1 and 2. We begin with three techniques for measuring the CKM angle $\gamma$. We also discuss two channels useful for getting information on the CKM angle $\alpha, B^{o} \rightarrow \rho \pi$ and $B^{o} \rightarrow \pi^{+} \pi^{-}$. We also present one result on a typical rare decay, $B^{+} \rightarrow K^{+} \mu^{+} \mu^{-}$and one involving a photon, $B^{o} \rightarrow K^{* o} \gamma$. We include a discussion of the measurement of $B^{o} \rightarrow \psi K_{s}$ and present our sensitivity for the measurement of $B_{s}$ mixing. We also present studies on several methods of flavor tagging.

In calculating the number of events per year that we expect to reconstruct we assume a luminosity of $\mathcal{L}=2 \times 10^{32} \mathrm{~cm}^{-2} \mathrm{~s}^{-1}$. In this work MCfast was used to determine the "reconstruction efficiency". MCfast provides the user with a list of smeared tracks that are within the geometrical acceptance, each characterized by a list of hits for each subdetector. Cuts on these hit multiplicites are typically applied on each of the tracks and other topological cuts may be applied. The reconstruction efficiency is based on these cuts and does not reflect possible losses due to pattern recognition defects. 


\subsection{Measurement of the CKM angle $\gamma$}

There have been several suggestions on how to measure the CKM angle $\gamma$ (see chapter 1 , section 9). There are discrete ambiguities inherent in all of these methods so using several methods will help remove some of these ambiguities as well help control systematic errors. We give a status report here of our simulations of three methods.

\subsection{1 $\quad B_{s} \rightarrow D_{s} K$}

This method measures the CP violation resulting from the interference of direct and mixing induced decays.

When a final state $f$ can come from both a pure $B^{0}$ and a pure $\bar{B}^{0}$, the amplitudes for the direct decay $B^{0} \rightarrow f$ and the mixing induced sequence $B \rightarrow \bar{B}^{0} \rightarrow f$ can interfere. A time dependent $\mathrm{CP}$-violating effect can thus appear. Consider the following decay widths $[1]:$

$$
\begin{aligned}
& \Gamma_{1}=\Gamma(B \rightarrow f)=|M|^{2} e^{-t}\left\{\cos ^{2}(x t / 2)+\rho^{2} \sin ^{2}(x t / 2)-\rho \sin (\phi+\delta) \sin (x t)\right\} \\
& \Gamma_{2}=\Gamma(\bar{B} \rightarrow \bar{f})=|M|^{2} e^{-t}\left\{\cos ^{2}(x t / 2)+\rho^{2} \sin ^{2}(x t / 2)+\rho \sin (\phi-\delta) \sin (x t)\right\} \\
& \Gamma_{3}=\Gamma(B \rightarrow \bar{f})=|M|^{2} e^{-t}\left\{\rho^{2} \cos ^{2}(x t / 2)+\sin ^{2}(x t / 2)-\rho \sin (\phi-\delta) \sin (x t)\right\} \\
& \Gamma_{4}=\Gamma(\bar{B} \rightarrow f)=|M|^{2} e^{-t}\left\{\rho^{2} \cos ^{2}(x t / 2)+\sin ^{2}(x t / 2)+\rho \sin (\phi+\delta) \sin (x t)\right\},
\end{aligned}
$$

where $M=\langle f \mid B\rangle, M^{\prime}=\langle f \mid \bar{B}\rangle, \rho=\left|M^{\prime} / M\right|, x=\Delta m / \Gamma, \delta$ is the strong phase difference between the two decays $B \rightarrow f$ and $B \rightarrow \bar{f}$, and $\phi$ is the relative CKM phase of the two decay amplitudes.

The time-dependent CP asymmetry is given by:

$$
\begin{aligned}
A_{C P}(t) & =\frac{[N(B \rightarrow f)+N(B \rightarrow \bar{f})]-[N(\bar{B} \rightarrow f)+N(\bar{B} \rightarrow \bar{f})]}{[N(B \rightarrow f)+N(B \rightarrow \bar{f})]+[N(\bar{B} \rightarrow f)+N(\bar{B} \rightarrow \bar{f})]} \\
& =\frac{\Gamma_{1}+\Gamma_{3}-\Gamma_{2}-\Gamma_{4}}{\Gamma_{1}+\Gamma_{3}+\Gamma_{2}+\Gamma_{4}} \\
& =\frac{2 \rho}{\rho^{2}+1} \cos (\delta) \sin (\phi) \sin (x t) .
\end{aligned}
$$

The CP asymmetry is optimized if $2 \rho /\left(\rho^{2}+1\right) \approx 1$, ie. the branching fractions of the decays into $\mathrm{f}$ and $\bar{f}$ are of the same order of magnitude.

A particularly useful case is where the $B_{s}$ decays into the final states $f \equiv D_{s}^{+} K^{-}$and $\bar{f} \equiv D_{s}^{-} K^{+}$. The branching fractions are expected to both be $\sim 10^{-4}$ and in this case the weak angle $\phi=\gamma$. Note that the final state is almost identical to the more prolific mode $D_{s}^{+} \pi^{-}$. Clearly, the particle identification is crucial here. 

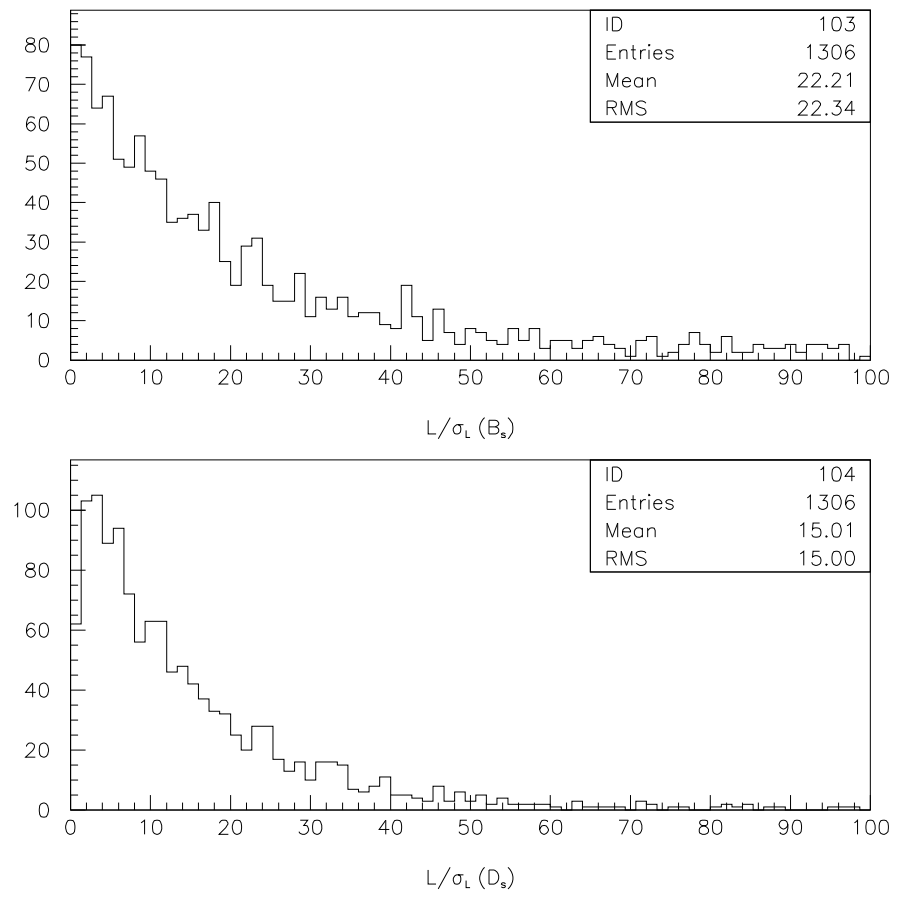

Figure 11.1: (top) $L / \sigma_{L}\left(B_{s}\right)$, (bottom) $L / \sigma_{L}\left(D_{s}\right)$

From a time-dependent measurement of the 4 decay rates $B_{s} \rightarrow D_{s}^{+} K^{-}, B_{s} \rightarrow D_{s}^{-} K^{+}$, $\bar{B}_{s} \rightarrow D_{s}^{-} K^{+}$and $\bar{B}_{s} \rightarrow D_{s}^{+} K^{-}$one can extract the quantities $\sin (\gamma+\delta), \sin (\gamma-\delta)$ and $\rho$, assuming that the value of $x_{s}$ will have been determined in a separate measurement. The quantity $\sin \gamma$ then can be extracted up to a 2-fold ambiguity.

The main handle we have at triggering on and reconstructing these decays is the detachment of the secondary $B_{s}$ and tertiary $D_{s}$ decay vertices. The distributions of detachment length divided by the resolution in the detachment $L / \sigma_{L}$ are shown in Fig 11.1. We require $L / \sigma_{L}\left(D_{s}\right)>4.0$ and $L / \sigma_{L}\left(B_{s}\right)>4.0$.

We have studied the reconstruction and trigger efficiencies of $B_{s} \rightarrow D_{s} K$ using both the $\phi \pi$ and $K^{*} K$ decay modes of the $D_{s}$. The combined geometric acceptance and reconstruction efficiency of these modes is $2.0 \%$ for the $\phi \pi$ mode and $1.7 \%$ for the $K^{*} K$, with a signal to background of about 10:1. The signal is shown in Fig. 11.2. The Level 1 trigger efficiency is $70 \%$ after all other cuts have been applied. Using branching ratios given by Aleksan et al. [31] and a tagging efficiency $\epsilon=40 \%$ we expect a total of 2680 tagged events (sum of 4 decay modes) in $10^{7} \mathrm{sec}$.

The CP asymmetry is diluted by time smearing, mistagging and background. The proper time resolution must be small to resolve the $B_{s}$ oscillations. Fig. 11.3 is a plot of the generated proper time minus the reconstructed proper time for events passing the cuts described above. The proper time is calculated from the decay length where the decay point of the $B_{s}$ comes 


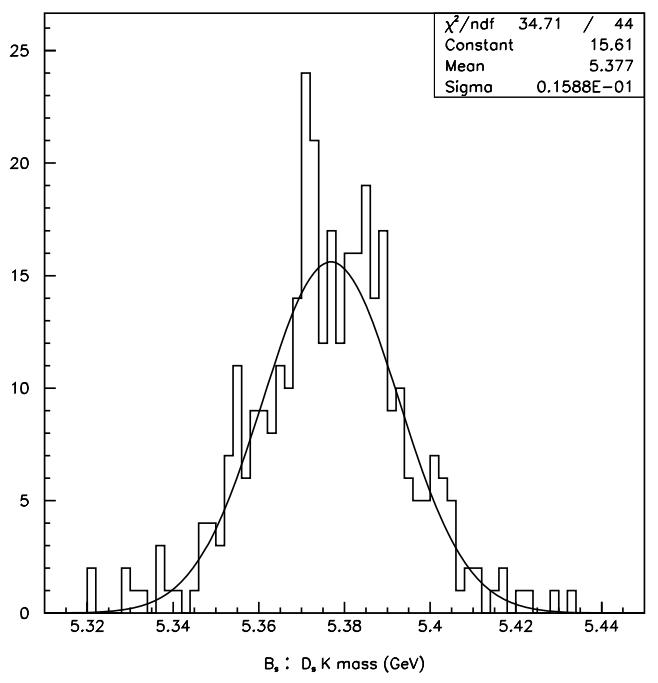

Figure 11.2: Mass Resolution for $B_{s}$

from the vertex fit of the $D_{s}$ with the $K$. A Gaussian fit to the $t_{g e n}-t_{\text {res }}$ distribution gives $\sigma_{t}=0.045$. Given that $\tau_{B_{s}}=1.54 \mathrm{psec}$, then $\sigma_{t} / t=0.030$.

We estimated the accuracy in determining $\gamma$ as follows. First, a set of "events" (ie. proper times) was generated, split into the 4 decay modes with correct time distributions. The values of the parameters were chosen to be $x_{s}=20.0, \rho=0.5, \gamma=45^{\circ}$ and $\delta=23^{\circ}$ ). Theoretically, the strong phase $\delta$ is expected to be small [2]. The proper times were smeared with a Gaussian of width $\sigma_{t}=0.030 \tau$. and a cut-off at low t simulated an $L / \sigma_{L}$ cut: $t_{\text {min }}=0.25 \tau$. A fraction of the events were then assigned to come from the "wrong flavor" parent. A mistag fraction of $30 \%$ is used. This includes mistagging due to mixing of the other B. Background events with a pure exponential time distribution are added to the "signal" events. The background is assumed to have the same lifetime as the signal.

In the absence of dilution factors the probability function is of the form:

$$
P_{1}(t)=e^{-t}\left\{\cos ^{2}(x t / 2)+\rho^{2} \sin ^{2}(x t / 2)-\rho \sin (\gamma+\delta) \sin (x t)\right\}
$$

In the presence of dilution factors the following changes are made to the probability function:

(a) to correct for time smearing:

$$
\begin{aligned}
P_{1}^{\prime}(t)= & \int_{-\infty}^{\infty} d t^{\prime} \frac{e^{-\left(t-t^{\prime}\right)^{2} / 2 \sigma^{2}}}{\sqrt{2 \pi} \sigma^{2}} P_{1}(t) \\
= & e^{\sigma^{2} / 2} e^{-t}\left[\cos ^{2}\left(x\left(t-\sigma^{2}\right)\right)\left(1+e^{-x^{2} \sigma^{2} / 2}+\rho^{2}\left(1-e^{-x^{2} \sigma^{2} / 2}\right)\right)+\right. \\
& \left.\sin ^{2}\left(x\left(t-\sigma^{2}\right)\right)\left(1-e^{-x^{2} \sigma^{2} / 2}+\rho^{2}\left(1+e^{-x^{2} \sigma^{2} / 2}\right)\right)+\sin \left(x\left(t-\sigma^{2}\right)\right) \sin (\gamma+\delta)\right],
\end{aligned}
$$

(b) to correct for background: 


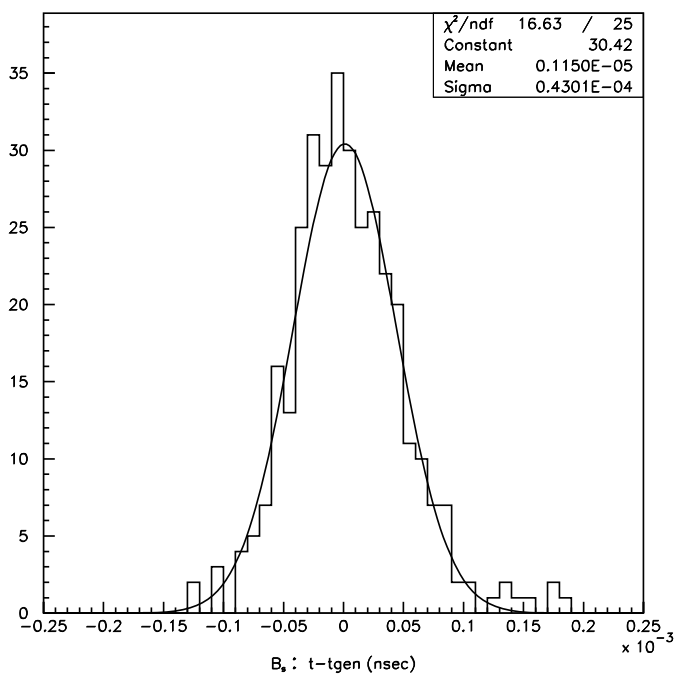

Figure 11.3: Proper Time Resolution for $B_{s}: t_{g e n}-t_{\text {rec }}(\mathrm{nsec})$

$$
P_{1}^{\prime \prime}(t)=P_{1}^{\prime}(t)+e^{-t} B
$$

(c) to correct for mistagging:

$$
P_{1}^{\prime \prime \prime}(t)=(1-w) P_{1}^{\prime \prime}(t)+w P_{4}^{\prime \prime}(t),
$$

The value of $\sin \gamma$ can be extracted from $\sin (\gamma+\delta)$ and $\sin (\gamma-\delta)$ with a 4 -fold ambiguity which reduces to a 2 -fold ambiguity if it is assumed that $\sin \gamma>0$. This still leads to a 4 -fold ambiguity in the value of $\gamma$. If the true value of $\sin (\gamma+\delta)$ or $\sin (\gamma-\delta)$ is close to 1.0 then the fitted values may be outside the physically allowed region. In particular, this is likely when the number of events is small and hence the errors are large.

In order to study the obtainable accuracy on $\gamma$ we ran 1000 "experiments," each with a signal of 2680 events where we fitted $\sin (\gamma+\delta)$ and $\sin (\gamma-\delta)$ and then calculated $\sin \gamma$ and $\cos \delta$, setting $\sin (\gamma+\delta)$ and $\sin (\gamma-\delta)$ to be equal to 1.0 if the fitted value was greater than 1.0. The results are shown in Fig. 11.4.

If $x_{s}=20$ and $\rho=0.5$ then it will be possible to measure $\sin (\gamma+\delta)$ and $\sin (\gamma-\delta)$ with a statistical error of \pm 0.15 in one year of running at $\mathcal{L}=2 \times 10^{32} \mathrm{~cm}^{-2} \mathrm{~s}^{-1}$. This will give an error on $\gamma$ of about $11^{\circ}$. The largest potential experimental problem with this method occurs when $x_{s}$ is near 60 . Then the time smearing dominates the error and the measurement error increases substantially. 

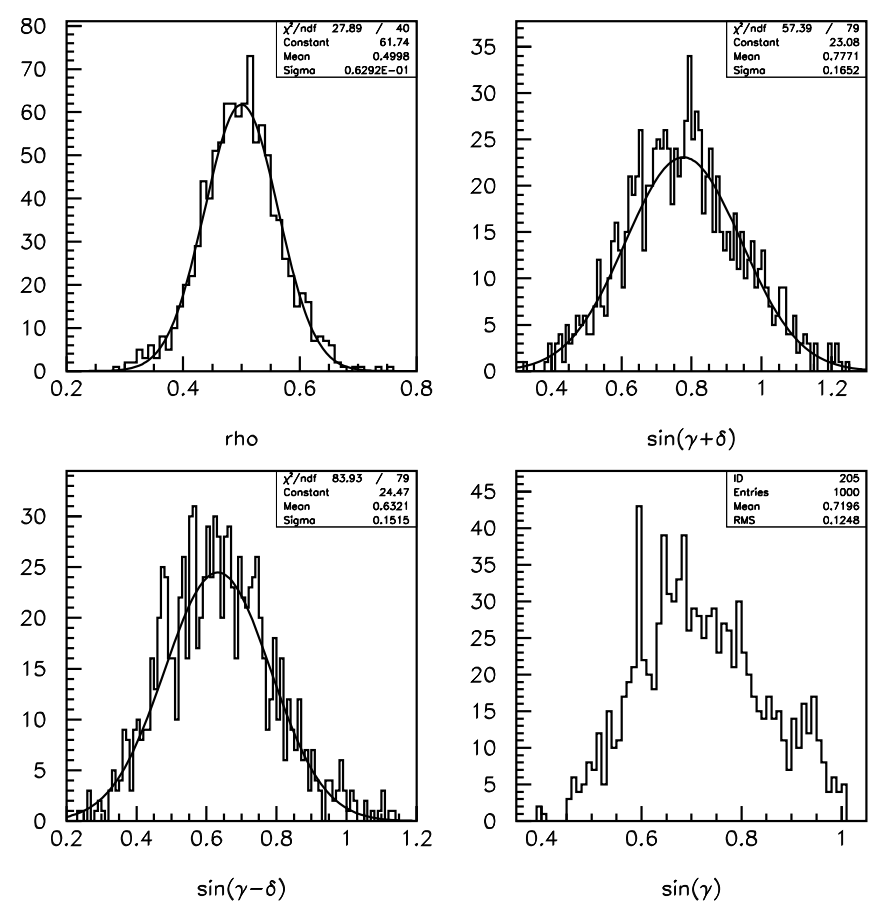

Figure 11.4: Fitted values of $\rho, \sin (\gamma+\delta), \sin (\gamma-\delta)$, and $\sin \gamma$. Input parameters $\rho=0.5$, $\sin (\gamma+\delta)=0.771, \sin (\gamma-\delta)=0.629, \sin \gamma=0.703\left(\gamma=45^{\circ}\right)$. Results of fit: $\gamma=46_{-9^{\circ}}^{+11^{\circ}}$

\subsection{2 $\quad B^{-} \rightarrow K^{-} D^{0}$}

Another method for extracting $\gamma$ has been proposed by Atwood, Dunietz and Soni [3], who refined a suggestion by Gronau and Wyler [4]. In the decay $B^{-} \rightarrow K^{-} D^{0}$ a CP asymmetry can result from the interference of the decays $B^{-} \rightarrow K^{-} D^{0}, D^{0} \rightarrow f$ and $B^{-} \rightarrow K^{-} \bar{D}^{0}, \bar{D}^{0} \rightarrow$ $f$. In particular if $f$ is a doubly Cabibbo suppressed decay of the $D^{0}$ then this asymmetry may be large. The weak phase difference between $B^{-} \rightarrow K^{-} D^{0}$ and $B^{-} \rightarrow K^{-} \bar{D}^{0}$ is $\gamma$. To observe a CP asymmetry there must also be a non-zero strong phase between the two amplitudes. It is necessary to measure the branching ratio $\mathcal{B}\left(B^{-} \rightarrow K^{-} f_{i}\right)$ for at least 2 different states $f$ in order to determine $\gamma$ up to discrete ambiguities. This method measures direct $\mathrm{CP}$ violation and requires neither tagging nor time-dependent measurements.

Define the following quantities :

$$
\begin{gathered}
a=B r\left(B^{-} \rightarrow K^{-} D^{0}\right) \\
b=B r\left(B^{-} \rightarrow K^{-} \bar{D}^{0}\right) \\
c(f 1)=B r\left(D^{0} \rightarrow f 1\right), \quad c(f 2)=\operatorname{Br}\left(D^{0} \rightarrow f 2\right)
\end{gathered}
$$




$$
\begin{array}{cc}
c(\overline{f 1})=\operatorname{Br}\left(D^{0} \rightarrow \overline{f 1}\right), & c(\overline{f 2})=\operatorname{Br}\left(D^{0} \rightarrow \overline{f 2}\right) \\
d(f 1)=\operatorname{Br}\left(B^{-} \rightarrow K^{-} f 1\right), & d(f 2)=\operatorname{Br}\left(B^{-} \rightarrow K^{-} f 2\right) \\
\bar{d}(f 1)=B r\left(B^{+} \rightarrow K^{+} f 1\right), & \bar{d}(f 2)=\operatorname{Br}\left(B^{+} \rightarrow K^{+} f 2\right)
\end{array}
$$

It is assumed that we will measure the quantities a, $c(f 1), c(f 2), c(\overline{f 1}), c(\overline{f 2}) d(f 1)$, $d(f 2), \bar{d}(f 1)$ and $\bar{d}(f 2)$ but not b.

We can express $d(f 1)$ in terms of a, b, $c(f 1), c(\overline{f 1})$, the strong phase $\xi_{1}$ and the weak phase $\gamma$.

$$
\begin{aligned}
& d(f 1)=a * c(f 1)+b * c(\overline{f 1})+2 \sqrt{a * b * c(f 1) * c(\overline{f 1})} \cos \left(\xi_{1}+\gamma\right) \\
& \bar{d}(f 1)=a * c(f 1)+b * c(\overline{f 1})+2 \sqrt{a * b * c(f 1) * c(\overline{f 1})} \cos \left(\xi_{1}-\gamma\right) \\
& d(f 2)=a * c(f 2)+b * c(\overline{f 2})+2 \sqrt{a * b * c(f 2) * c(\overline{f 2})} \cos \left(\xi_{2}+\gamma\right) \\
& \bar{d}(f 2)=a * c(f 2)+b * c(\overline{f 2})+2 \sqrt{a * b * c(f 2) * c(\overline{f 2})} \cos \left(\xi_{2}-\gamma\right)
\end{aligned}
$$

These four equations contain the 4 unknowns $\xi_{1}, \xi_{2}, b, \gamma$ which can be determined up to discrete ambiguities.

We have studied the reconstruction and trigger efficiencies of decay modes $B^{-} \rightarrow$ $K^{-}\left[K^{+} \pi^{-}\right]$and $B^{-} \rightarrow K^{-}\left[K^{+} K^{-}\right]$. The combined geometric acceptance and reconstruction efficiency was found to be $3.8 \%$ for the $K \pi$ mode and $2.5 \%$ for $K K$. We expect that the RICH will provide excellent particle ID, so we believe that most of the background in the $B^{-} \rightarrow K^{-}\left[K^{+} \pi^{-}\right]$mode will come from events where a real $D^{0}$ decays via the Cabibbo favored mode and combines with a kaon from the other $B$. We have studied this type of background and expect to achieve $\mathrm{S} / \mathrm{B} \sim 1$.

The trigger efficiency is approximately $60 \%$ for both modes. The expected number of $B^{ \pm}$ events in $10^{7} \mathrm{~s}$ is 320 in the $K \pi$ mode and 1600 in the $K^{+} K^{-}$mode (see Table 11.1). The reconstructed signal is shown in Fig. 11.5.

In order to estimate our ability to measure $\gamma$, several sets of input parameters (b, $\gamma$, $\left.\xi_{1}, x i_{2}\right)$ were chosen and for each set the expected number of events in each channel was calculated. Then 1000 trials were done for each set, smearing the number of events by $\sqrt{N+B}$. The results of one set of trials showing the distribution in fitted values of $\mathrm{b}$ and $\gamma$ are shown in Fig. 11.6.

With this number of events we expect to be able to measure $\gamma$ (up to discrete ambiguities) with a statistical error of about $13^{\circ}$ in one year of running at $\mathcal{L}=2 \times 10^{32} \mathrm{~cm}^{-2} \mathrm{~s}^{-1}$. The overall sensitivity depends on the actual values of $\gamma$ and the strong phases. 
Table 11.1: Expected Number of $\left(B^{-} \rightarrow K^{-} D^{0}\right)$ Events

\begin{tabular}{|c|cc|}
\hline & $B^{-} \rightarrow K^{-}\left(K^{+} \pi^{-}\right)$ & $B^{-} \rightarrow K^{-}\left(K^{+} K^{-}\right)$ \\
\hline$(\mathrm{a})=\mathrm{BR}\left(B^{-} \rightarrow K^{-} D^{0}\right)$ & \multicolumn{2}{|c|}{$2.5 \times 10^{-4}$} \\
$(\mathrm{~b})=\mathrm{BR}\left(B^{-} \rightarrow K^{-} \overline{D^{0}}\right)$ & .04 & \\
$(\overline{c 1})=\mathrm{BR}\left(D^{0} \rightarrow K^{-} \pi^{+}\right)$ & $3.1 \times 10^{-4}$ & \\
$(\mathrm{c} 1)=\mathrm{BR}\left(D^{0} \rightarrow K^{+} \pi^{-}\right)$ & & \\
$(\mathrm{c} 2)=\mathrm{BR}\left(D^{0} \rightarrow K^{+} K^{-}\right)$ & $1.7 \times 10^{-7}$ & $1.1 \times 10^{-3}$ \\
$(a) \times(c 1)+(b) \times(\overline{c 1})$ & & 0.025 \\
$(a) \times(c 2)$ & 0.038 & 0.60 \\
Reconstruction efficiency & 0.60 & 1600 \\
Trigger efficiency & 320 & \\
N events/year $B^{ \pm}$ & &
\end{tabular}

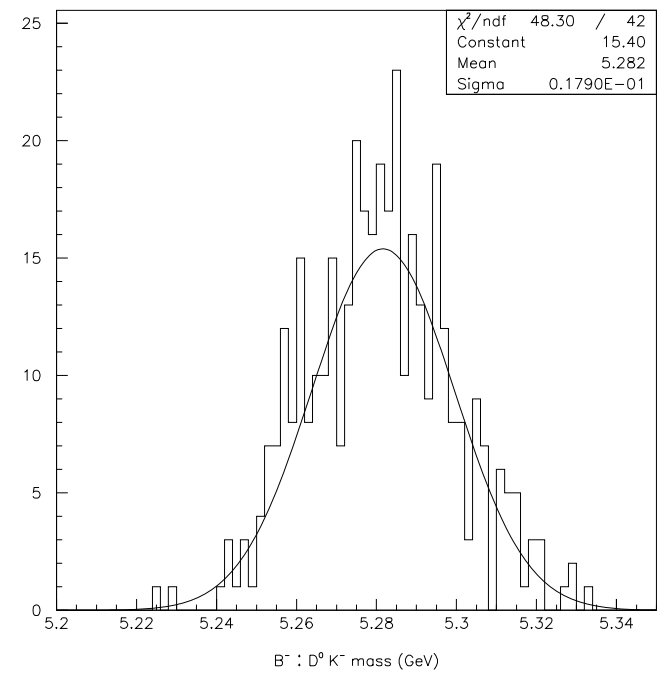

Figure 11.5: $D^{0} K^{-}$mass 

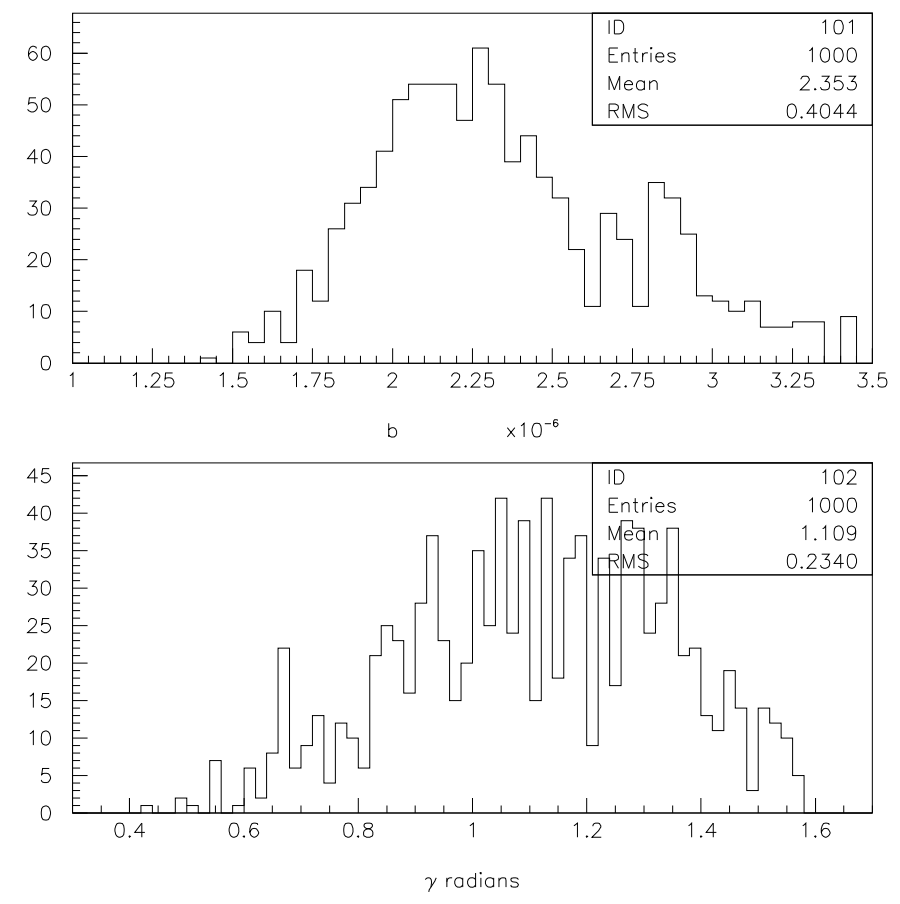

Figure 11.6: Calculated values of $\gamma$ and $b$ for 1000 trials with input parameters $\gamma=65^{\circ}$, $(1.13 \mathrm{rad}), b=2.2 \times 10^{-6}$ 


\subsection{3 $B \rightarrow K \pi$}

This method uses the branching ratios of the decays $B^{+} \rightarrow \pi^{+} K^{0}$ and $B^{0} \rightarrow \pi^{-} K^{+}$and their complex conjugates as explained in a paper by Gronau and Rosner [5]. The decay $B^{+} \rightarrow$ $\pi^{+} K^{0}$ proceeds only through a penguin amplitude and the decay $B^{0} \rightarrow \pi^{-} K^{+}$proceeds through both penguin and tree amplitudes. Ignoring electroweak penguin contributions, the penguin amplitudes in the charged and neutral $B$ decays to $K \pi$ are equal.

It is useful to define

$$
\begin{aligned}
R & \equiv \frac{\Gamma\left(B^{0} \rightarrow K^{+} \pi^{-}\right)+\Gamma\left(\bar{B}^{0} \rightarrow K^{-} \pi^{+}\right)}{\Gamma\left(B^{+} \rightarrow K^{0} \pi^{+}\right)+\Gamma\left(B^{-} \rightarrow \bar{K}^{0} \pi^{-}\right)} \\
A^{\prime} & \equiv \frac{\Gamma\left(B^{0} \rightarrow K^{+} \pi^{-}\right)-\Gamma\left(\bar{B}^{0} \rightarrow K^{-} \pi^{+}\right)}{\Gamma\left(B^{+} \rightarrow K^{0} \pi^{+}\right)+\Gamma\left(B^{-} \rightarrow \bar{K}^{0} \pi^{-}\right)} .
\end{aligned}
$$

An expression for $\gamma$ can be given in terms of $R, A^{\prime}$ and the ratio of tree to penguin amplitudes $r \equiv|T| /|P|$.

If we define $\delta$ to be the phase difference between the penguin and tree amplitudes, the ratios $R$ and $A^{\prime}$ can be rewritten :

$$
\begin{gathered}
R=1-2 r \cos \gamma \cos \delta+R^{2} \\
A^{\prime}=2 r \sin \delta \sin \gamma
\end{gathered}
$$

Combining these equations to eliminate $\delta$ we get

$$
R=1+r^{2} \pm \sqrt{4 r^{2} \cos ^{2} \gamma-A^{\prime 2} \cot ^{2} \gamma}
$$

Fleischer and Mannel[6] have claimed that if $R<1$ a useful bound can be obtained regardless of the value of $r$ or $\delta$ :

$$
\sin ^{2} \gamma \leq R
$$

If we have information on $r$ we can get a more precise estimate of $\gamma$.

CLEO has determined $R=0.65 \pm 0.40$, which suggests that the ratio could be less than 1.0 [7]. If this result holds with improved statistics then this method compliments other methods in that it will begin to exclude some of the region around $\gamma=\pi / 2$.

There has been much discussion about the effect of final state interactions on the measured branching ratios. Falk et al. [8] have shown that contributions to $B^{+} \rightarrow \pi^{+} K^{0}$ from rescattering of channels such as $B^{+} \rightarrow \pi^{0} K^{+}$lead to a modification of the Fleischer-Mannel bound:

$$
\sin ^{2} \gamma \leq R(1+2 \epsilon \sqrt{1-R})
$$


where $\epsilon$ is the ratio of the rescattered amplitude to the penguin amplitude. They estimate $\epsilon=O(0.1)$. This rescattering contribution can be determined by measuring the $K^{+} K^{-}$final state branching ratio, which $\mathrm{BTeV}$ is well equipped to do.

To study the reconstruction efficiency of $B^{+} \rightarrow \pi^{+} K_{s}$ Monte Carlo events were generated using PYTHIA with the decays of charm and bottom particles being handled by QQ. First the primary vertex was found then $K_{s}$ candidates were selected by combining all pairs of oppositely charged tracks not identified as kaons. These tracks were required to have an impact parameter greater than $5 \sigma$ with respect to the primary vertex, to form a vertex with $\chi^{2}<5$ and to be within $6 \mathrm{MeV}$ of the nominal $K_{s}$ mass. The $K_{s}$ vertex was required to have $l / \sigma>6$ with respect to the primary vertex. B candidates were selected by combining the $K_{s}$ candidates with all other charged tracks not identified as kaons and having an impact parameter greater than $3.5 \sigma$ with respect to the primary vertex. The reconstructed $\mathrm{B}$ candidate was required to have $l / \sigma>4$, and point back to the primary with an impact parameter less than $2.5 \sigma$. The combined geometric acceptance and reconstruction efficiency of the $B^{+} \rightarrow \pi^{+} K_{s}$ mode is $2.8 \%$ and the trigger efficiency is $40 \%$. The reconstructed signal is shown in Fig. 11.7.

The background has been studied using $500 \mathrm{~K}$ generic $b \bar{b}$ events with minimum bias events added to give an average of two interactions per beam crossing. Only one event with a mass above $5 \mathrm{GeV} / \mathrm{c}^{2}$ survived the cuts as described above. From these limited statistics we estimate we can achieve a signal to background ratio of about 1:1 .

The decay mode $B^{0} \rightarrow \pi^{-} K^{+}$has also been simulated. The two prong vertex should easily be distinguished from background with an $l / \sigma$ cut and the requirement that the reconstructed $B$ point back to the primary vertex. The reconstruction efficiency is found to be $9.8 \%$ and the trigger efficiency for events which pass the analysis cuts is $54 \%$. The signal is shown if Fig 11.8. The background has not yet been studied but the signal to background ratio is expected to be better than that for $B^{0} \rightarrow \pi^{+} \pi^{-}(S / B=0.6)$ because $B^{0} \rightarrow \pi^{-} K^{+}$has a higher branching ratio and there are fewer kaons than pions so the combinatoric background is lower.

We expect to reconstruct $11,000 B^{ \pm}$and $110,000 B^{0} / \bar{B}^{0}$ in $10^{7} \mathrm{~s}$ of running at $\mathcal{L}=$ $2 \times 10^{32} \mathrm{~cm}^{-2} \mathrm{~s}^{-1}$. Gronau and Rosner [5] have estimated that a $5^{\circ}$ precision in $\gamma$ can be achieved with 2400 events in each channel. This of course, doesn't take into account the above mentioned theoretical criticisms of the technique. 


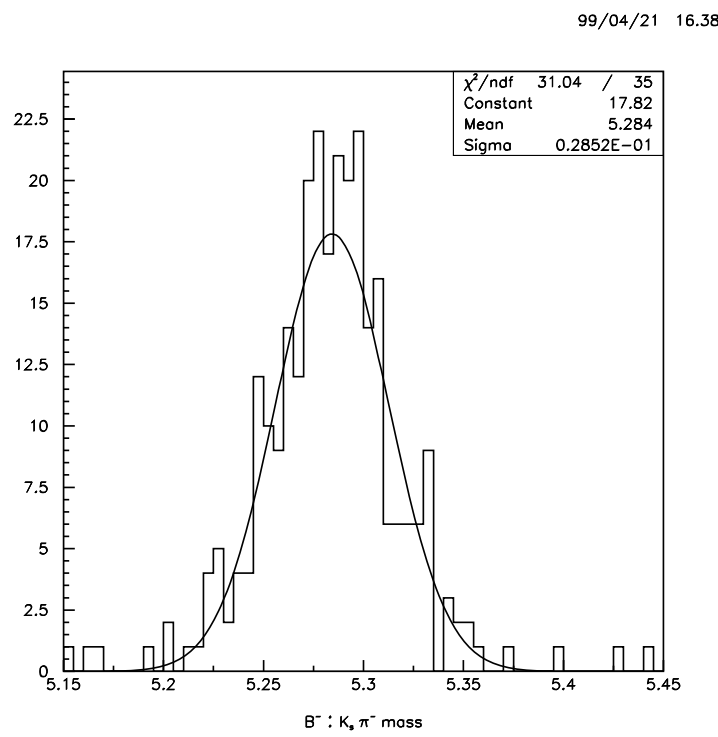

Figure 11.7: $K_{s} \pi$ mass

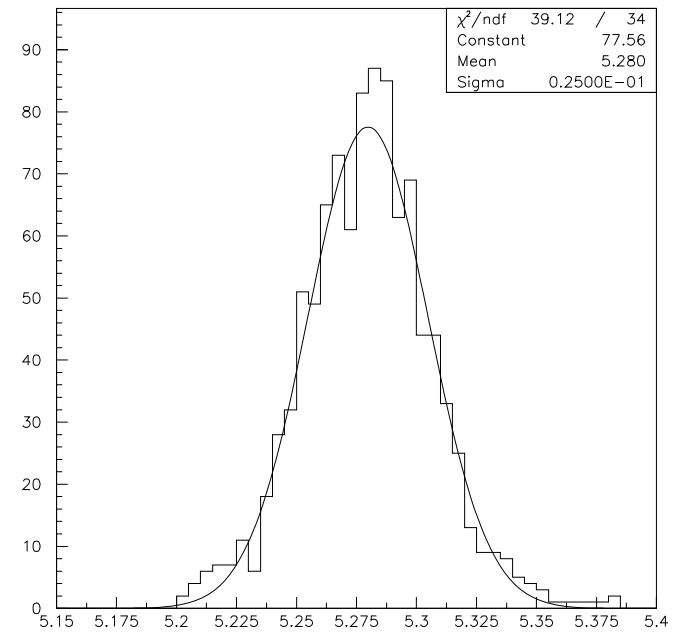

Figure 11.8: $K^{+} \pi^{-}$mass 


\section{$11.2 \quad B_{d}^{0} \rightarrow J / \psi K_{s}$}

The decay $B_{d}^{0} \rightarrow J / \psi K_{s}$ is the golden mode for measuring the CP violating angle $\beta$. A study has been made of the reconstruction efficiency and trigger efficiency of the proposed BTEV/C0 detector for the decay $B_{d}^{0} \rightarrow J / \psi K_{s} \rightarrow \mu^{+} \mu^{-} \pi^{+} \pi^{-}$

The dominant backgrounds to the decay channel $B^{0} \rightarrow \psi K_{s}$ are expected to arise from $J / \psi$ 's from B-decays combining with real $K_{s}$ 's from fragmentation or $J / \psi$ 's coming directly from hadronization combining with any $K_{s}$. CDF found that prompt $J / \psi$ 's are a large fraction of total $J / \psi$ production [10] and that this fraction increases as $p_{t}$ decreases. In the forward geometry of the BTEV/C0 detector the $p_{t}$ of the $J / \psi$ 's accepted peaks at about $2 \mathrm{GeV} / \mathrm{c}$ (see Fig 11.9). Extrapolating from the CDF results we expect that $J / \psi$ 's from B decays only comprise about $5 \%$ of the total $J / \psi$ production. Background from fake or prompt $J / \psi$ 's can be reduced by a cut on the distance between the primary vertex and decay vertex. Monte Carlo events of the type $B \rightarrow J / \psi X$ were generated to study the background arising from $J / \psi$ 's from B-decays.

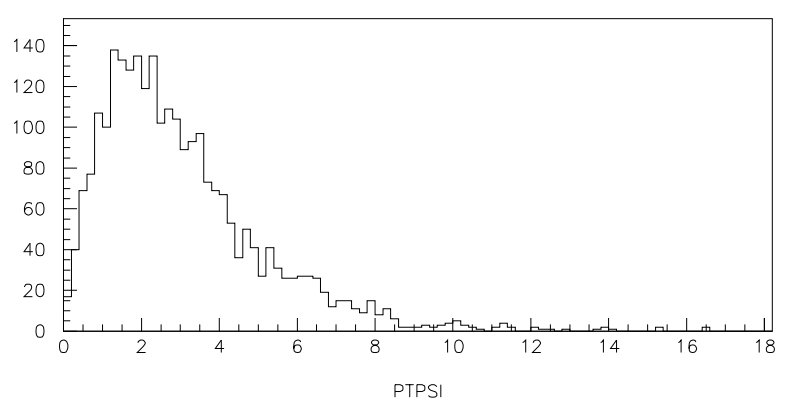

Figure 11.9: $p_{t}$ distribution of $J / \psi$ 's

To study the reconstruction efficiency Monte Carlo events were generated using PYTHIA with the decays of charm and bottom particles being handled by QQ. The following requirements were put on all events:

- Each event must pass a primary vertex fit.

- $J / \psi$ candidates were selected by combining pairs of oppositely charged tracks identified as muons. The muons were required to have a momentum greater than $5.0 \mathrm{GeV}$, and have a least one hit in the muon detector. These tracks were then required to pass a vertex fit. The normalized distance between the primary vertex and $J / \psi$ vertex $(l / \sigma)$ was required to be greater than 4.0 . This cut is $80 \%$ efficient for the signal and rejects $99.95 \%$ of the background from prompt $J / \psi$ 's. (see Fig 11.10)

- $K_{s}$ candidates were selected by combining pairs of oppositely charged tracks with a minimum momentum of $0.5 \mathrm{GeV}$ and fitting them to a vertex. Each track was required 
to miss the primary vertex by at least $3 \sigma$ and the reconstructed $K_{s}$ was required to point back to the $J / \psi$ vertex to within $3 \sigma$. (Figs 11.11 and 11.12).

- The $J / \psi$ and $K_{s}$ candidates within a $3 \sigma$ mass window of the nominal values were combined to form $B_{d}^{0}$ candidates. To reduce the background from $K_{s}$ 's from the primary vertex combining with a $J / \psi$ from a $\mathrm{B}$ decay we require the $K_{s}$ impact parameter to the secondary vertex divided by the impact parameter to the primary vertex to be less than 2.0. We also require that the reconstructed $B^{0}$ point back to the primary vertex.

The reconstructed signal and background are shown in Fig 11.13. The reconstruction efficiency of the signal is $4.0 \%$. This signal is from $10 \mathrm{~K}$ generated events.
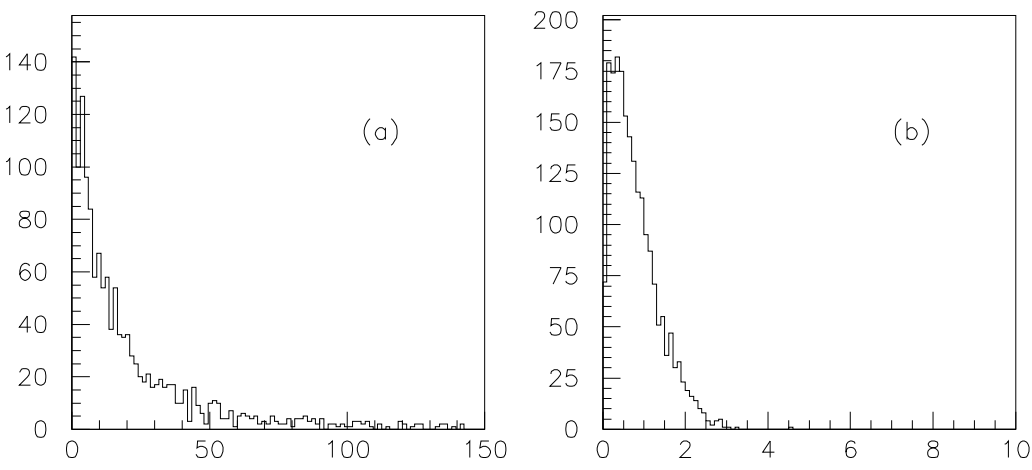

Figure 11.10: L/ $\sigma$ (a) $J / \psi$ from B decays, (b) $J / \psi$ from primary vertex
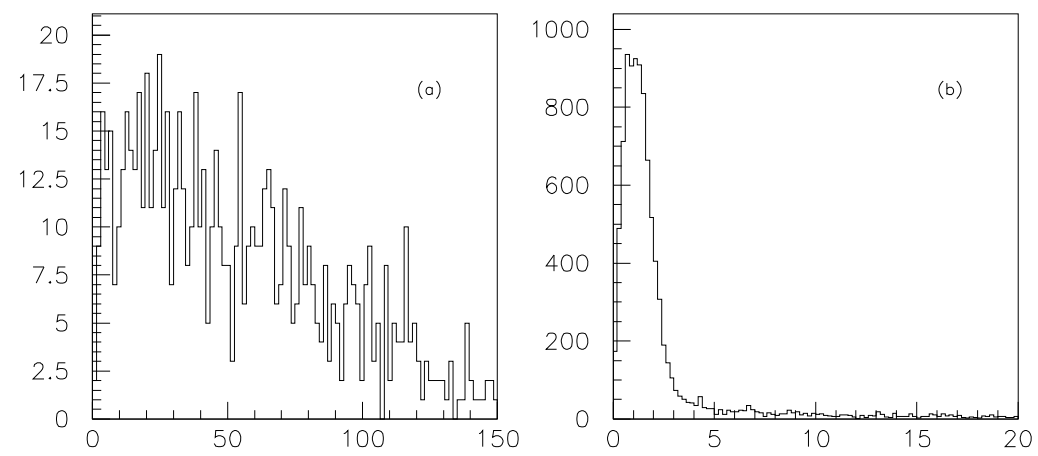

Figure 11.11: Normalized Impact parameter with respect to the primary vertex of (a) $\pi$ 's from real $K_{s}$ 's , (b) $\pi$ 's from fake $K_{s}$

The number of signal and background events expected in $10^{7} \mathrm{sec}$ of running time is shown in Table 11.2. We estimate that the efficiency of a dimuon trigger with a threshold of 10 $\mathrm{GeV} / \mathrm{c}$ is $70 \%$ for events which pass the analysis cuts. The efficiency of the Level 1 vertex 

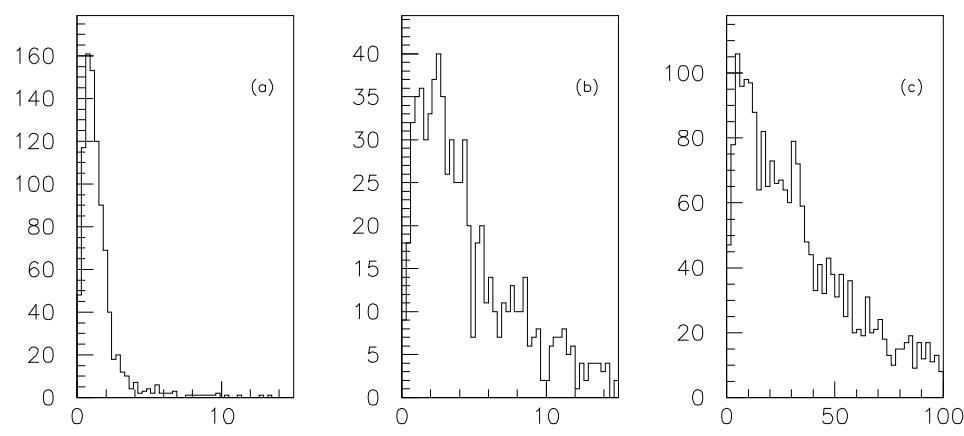

Figure 11.12: Normalized Impact parameter with respect to $J / \psi$ vertex (a) real $K_{s}$ from $B \rightarrow J / \psi K_{s}$ decay, (b) other real $K_{s}$ 's, (c) Fake $K_{s}$ 's

trigger is approximately $50 \%$ for these events so we estimate a total trigger efficiency of $85 \%$. For a time integrated asymmetry measurement the error on $\sin 2 \beta$ is given by

$$
\delta \sin (2 \beta)=\frac{1+x_{d}^{2}}{x_{d}} \frac{1}{\sqrt{\epsilon D^{2} N}} \sqrt{\frac{S+B}{S}}
$$

where $x_{d} /\left(1+x_{d}^{2}\right)=0.47$ accounts for the dilution due to the time evolution of the $B_{d}^{0}$. We estimate the effective tagging efficiency $\epsilon D^{2}$ to be $10 \%$ (see section 11.3) and assume $S / B=10$ which results in a value of $\delta \sin (2 \beta)$ of 0.021 . A time dependent analysis of the asymmetry will result in a more accurate determination of $\sin (2 \beta)$.

Table 11.2:

\begin{tabular}{c|c}
\hline Luminosity & $2 \times 10^{32}$ \\
Running time & $10^{7} \mathrm{sec}$ \\
$\sigma_{b \bar{b}} \bar{B}$ events & $100 \mu \mathrm{b}$ \\
Number of $B \bar{B}$. & $2 \times 10^{11}$ \\
Number of $B_{d}^{0} / \overline{B_{d}^{0}}, \mathrm{~s}$ & $1.6 \times 10^{11}$ \\
$\mathcal{B}\left(B_{d}^{0} \rightarrow J / \psi K_{s}^{0}\right)$ & $5 \times 10^{-4}$ \\
$\mathcal{B}\left(J / \psi \rightarrow \mu^{+} \mu^{-}\right) \times \mathrm{B}\left(K_{s}^{0} \rightarrow \pi^{+} \pi^{-}\right)$ & 0.04 \\
Reconstruction efficiency & 0.04 \\
Trigger efficiency & 0.85 \\
Number of reconstructed $B_{d}^{0} \rightarrow J / \psi K_{s}^{0}$ & 109,000 \\
Tagging efficiency $\epsilon D^{2}$ & $10 \%$ \\
$\delta \sin (2 \beta)$ & 0.021 \\
\hline
\end{tabular}




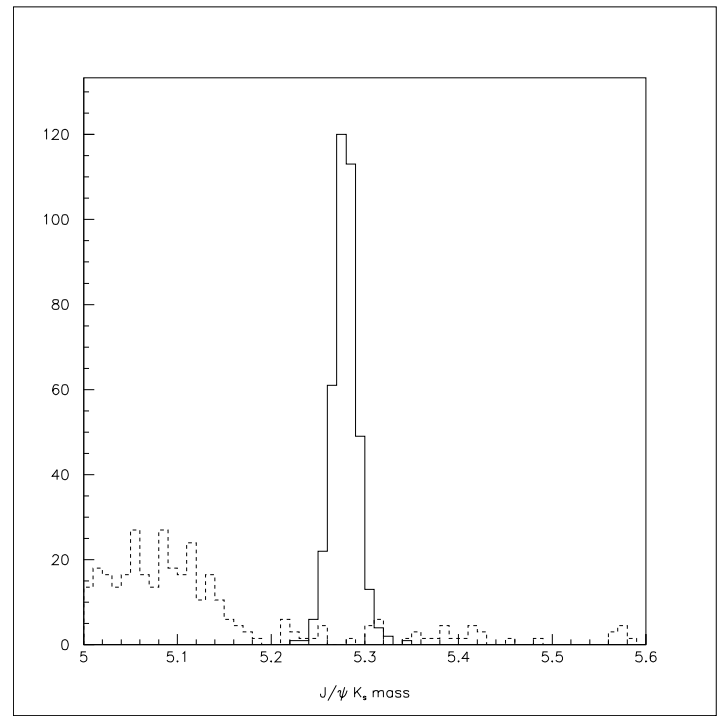

Figure 11.13: $J / \psi K_{s}$ mass - solid line: signal, dashed line: background from B decays to $J / \psi X$. Most of the background below $5.15 \mathrm{GeV} / \mathrm{c}^{2}$ is from $B \rightarrow J / \psi K^{*}$ decays 


\subsection{Flavor Tagging}

For charged $B$ mesons, the flavor of the heavy quark ( $b$ or $\bar{b}$ ) is determined by the charge of the $B$ mesons. For neutral $B$ mesons $\left(B_{d}\right.$ and $\left.B_{s}\right)$ the quark flavor can usually be determined from the flavor of the decay products, for example by the charge of the kaon in $B_{s} \rightarrow \psi \bar{K}^{*}$, $\bar{K}^{*} \rightarrow K^{-} \pi^{+}$decay. These modes can be used to measure $B_{s} \leftrightarrow \bar{B}_{s}$ and $B_{d} \leftrightarrow \bar{B}_{d}$ oscillations (sensitive to $V_{t s}$ and $V_{t d}$ respectively), if the flavor of the $b$ quark at the production point is determined independently. Decays to CP-eigenstates do not identify the $b$ quark flavor. In fact, amplitudes for these decays interfere with the mixing amplitude producing $\mathrm{CP}$ violating effects. Measurements of CP-asymmetry, resulting e.g. in determination of $\sin 2 \beta$ via $B_{d} \rightarrow \psi K_{S}^{0}$, again require determination of the $b$ quark flavor at the production point - so called "flavor tagging". Since every tagging method sometimes produces false identification, the effectiveness of flavor tagging is characterized by an effective tagging efficiency $\epsilon D^{2}$ where $\epsilon=\left(N_{R}+N_{W}\right) / N$, and $D$ is a "dilution" factor, $D=\left(N_{R}-N_{W}\right) /\left(N_{R}+N_{W}\right)$, where $N$-number of reconstructed signal events, $N_{R}$-number of right flavor tags in this sample, $N_{W}$-number of wrong flavor tags. Since the measurements mentioned above are among the most important goals of the BTeV program, large tagging efficiency is a crucial design criterion for the experiment. The forward detector geometry offers unique advantages for flavor tagging over an experiment operating in the central region.

The Away Side Tagging methods rely on determination of the flavor of the other $b$ quark in the event, since $b$ quarks are always produced in $b \bar{b}$ pairs. The flavor of the other $b$ can be determined from the charge of the lepton emitted in its semileptonic decay, the overall charge of the $b$ jet, or the charge of the kaon produced in the $b \rightarrow c \rightarrow s$ cascade.

A study had been made of the efficiency of Jet Charge tagging in the BTeV detector. PYTHIA was used to generate $b \bar{b}$ events where the $\bar{b}$ was fragmented into a $B^{0}$ which was then forced to decay via the decay chain $B^{0} \rightarrow J / \psi K_{s}, J / \psi \rightarrow \mu^{+} \mu^{-}, K_{s} \rightarrow \pi^{+} \pi^{-}$. No restrictions were placed on the $b$ fragmentation and QQ was modified to include incoherent mixing of neutral $B$ mesons [12].

An "opposite jet" is a combination of tracks consistent with being decay products of the other $B$. Candidate tracks for the opposite jet must have a transverse momentum greater than $100 \mathrm{MeV} / \mathrm{c}$ and are required to miss the primary vertex by more than $3 \sigma$. Due to the fact that the $B$ 's are produced close in pseudorapidity we require that the difference in pseudorapidity between the opposite jet candidates and the $B^{0}$ be less than 4.0 units or that both $B$ 's be in the same arm of the detector. The opposite jet is identified as a combination of tracks with a common secondary vertex which is separated from the primary by at least $1.0 \sigma$. If more than one jet is found, the one with the highest transverse momentum is selected, and if there is no secondary vertex passes the cuts as described then the highest $p_{t}$ track is selected, provided that the $p_{t}>1.0 \mathrm{GeV} / \mathrm{c}$.

The jet charge is calculated according to the formula:

$$
Q_{j e t}=\frac{\sum_{i} q_{i} \cdot\left(\vec{p}_{i} \cdot \hat{a}\right)^{k}}{\sum_{i}\left(\vec{p}_{i} \cdot \hat{a}\right)^{k}}
$$


where $q_{i}$ and $p_{i}$ are the charge and momentum of the $i^{\text {th }}$ track, $\hat{a}$ is the jet axis and $k$ is a weighting factor that we choose to be zero. When the $b$ hadronizes into a $\bar{B}$, the opposite jet typically has a neutral charge and useful tagging information is not obtained. However this has the advantage that this method is not affected by mixing. The results of this study indicate that we can achieve a tagging efficiency $\epsilon=0.39$ and a dilution $D=0.41$ which results in an effective efficiency $\epsilon D^{2}=0.065$.

Lepton tagging is limited by the low branching ratio for semi-leptonic decays and wrongsign background that results from semileptonic charm decays in $b \rightarrow c$ cascades. A study of muon tagging has been made using the same $B \rightarrow J / \psi$ events as the Jet Charge tagging study. The muons from the decays of $B$ and $D$ mesons have quite different $p_{t}$ distributions so some separation can be achieved by this method. Our simulations indicate that we can achieve $\epsilon D^{2} \approx 0.016$ for muon tagging.

Kaon tagging is the most potent method at $e^{+} e^{-} B$ factories. Potentially large backgrounds from the underlying event call for excellence in both particle identification and vertex resolution. Both are strong points of our forward detector geometry. As described in the detector design section we are aiming at efficient kaon identification in the 3-70 GeV/c momentum range. Even with a perfect kaon identification there is a large kaon background from the underlying event which may dilute tagging efficiency. Figure 11.14 illustrates the importance of a good resolution on the kaon impact parameter at the primary vertex for suppression of this background. In $\mathrm{BTeV}$ the tagging efficiency is improved by a large factor when cutting on significance of the impact parameter of the kaon. This figures also illustrates that the impact parameter resolution achievable in a central detector is not sufficient for a large improvement in tagging based on kaon identification. In $\mathrm{BTeV}$, we expect kaon tagging efficiency $\epsilon D^{2}$ in a range of 4.5-6\% depending on the exact performance of the particle identification system.

The Same Side Tagging method uses the correlations which arise between the $B$ meson and the charge of nearby tracks produced in the fragmentation chain or from $B^{* *}$ decays. For $B_{s}$ mesons the correlation is with a charged kaon. Preliminary studies indicate that this method is particularly useful for $B_{s}$ tagging where we expect to achieve $\epsilon D^{2}>5.0 \%$.

We have not yet studied the correlations between different tagging methods but estimate we can achieve an overall effective tagging efficiency $\epsilon D^{2}$ of about $10 \%$. 

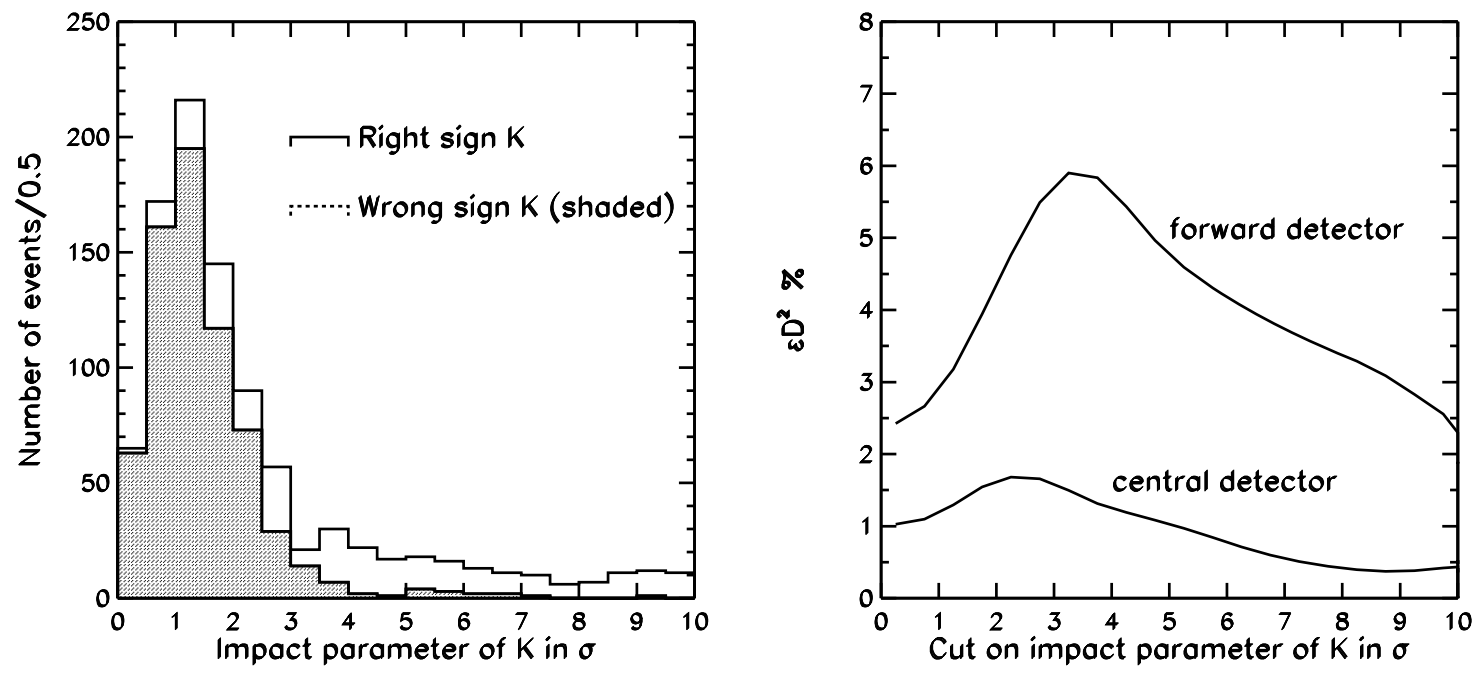

Figure 11.14: Left plot: $\mathrm{L} / \sigma$ distribution in $\mathrm{BTeV}$ for $K$ impact parameter for right sign (unshaded) and wrong sign (shaded) tags. Right plot: Kaon tagging efficiency $\left(\epsilon D^{2}\right)$ as a function of kaon impact parameter cut in units of $\mathrm{L} / \sigma$. Particle misidentification is neglected. The upper curve represents BTeV simulation. The lower curve represents a central detector with a ToF system and vertex detector with SVX-II performance. 


\section{$11.4 \quad B_{d} \rightarrow \pi^{+} \pi^{-}$}

The decay $B_{d} \rightarrow \pi^{+} \pi^{-}$is the traditional choice for measuring $\sin (2 \alpha)$, but the evidence for large penguin amplitudes in the observation of $B_{d} \rightarrow K^{+} \pi^{-}$by the CLEO collaboration means that a simple extraction of $\sin (2 \alpha)$ from this mode is no longer likely. However, this mode has been used to benchmark so many experiments it is still worthwhile to understand. In addition, it may be useful for the extraction of $\gamma$ when combined with a measurement of $B_{s} \rightarrow K^{+} K^{-}$as explained in section 1.9.4.

The signature for this decay is very simple: two oppositely charged tracks with a displaced vertex and the invariant mass of a $B^{0}$. Most of the background rejection against random combinations must come from the secondary vertex. While particle identification is vital to reject backgrounds from decays like $B^{0} \rightarrow K^{+} \pi^{-}$and $B_{s} \rightarrow K^{+} K^{-}$, it has a small effect on random combinations since most particles are pions.

It has been shown by the BCD group [13] that the dominant background to $B^{0} \rightarrow \pi^{+} \pi^{-}$ comes from random combinations of tracks in events containing $B$ 's. Tracks from real $B$ 's are already displaced from the primary vertex and have a higher probability of faking a secondary vertex. We have chosen to optimize our selection to minimize the background from real $B$ decays. Further studies will be needed to confirm that this is also sufficient to reject charm and light quark backgrounds.

We generated all our events samples using PYTHIA and QQ to decay the charm and bottom particles. One signal interaction and $n$ background interactions were used where $n$ was selected to simulate a poisson ditribution of mean 2.0. This is equivalent to a luminosity of $2 \times 10^{32} \mathrm{~cm}^{-2} \mathrm{~s}^{-1}$.

For this analysis we fit the primary vertex from tracks that are known to come from the primary. This underestimates the error on the primary but not significantly and the error on the decay distance is still dominated by the secondary decay. We loop over all opposite sign tracks with nine or more hits in the silicon vertex detector and try to fit a secondary vertex. Those with $\chi^{2}<2.0$ are kept for further analysis. After these fits we need to make four selections to reduce the random backgrounds:

- the cosine of the opening angle of the $\pi^{+} \pi^{-}$pair must be greater than zero. Random tracks that are in opposite hemispheres of the detector produce masses near the $B$, while real $B$ 's in our acceptance never have opening angles this large.

- require that the distance between the primary and secondary decay divided by its error, $L / \sigma$, be greater than 15 . This cut strongly rejects random combinations where tracks come from the primary vertex.

- require that each pion miss the primary vertex by more than 5 times its error on the impact parameter with respect to the primary vertex. This is correlated with the previous cut, but it can reject situations where a primary vertex track crosses another track making a false secondary vertex. 
- require that the $B^{0}$ point back to the primary vertex within $2 \sigma$ of its point back error. This requirement rejects false vertices that consist of two tracks from different secondary decays.

Pattern recognition in the silicon sets the requirement that each of the two $\pi$ track candidates have hits in 9 planes (3 stations) of the vertex detector. The geometric acceptance for $B^{0} \rightarrow \pi^{+} \pi^{-}$where both tracks are required to hit 3 stations is about $23 \%$. The reconstruction of the primary and secondary vertices and the background rejection cuts have an efficiency of $8.4 \%$ which means that over one third of the events in the acceptance remain after the cuts are applied.

There are several decay modes of $B$ mesons that can mimic a $B_{d} \rightarrow \pi^{+} \pi^{-}$decay. The decay $B_{s} \rightarrow K^{+} K^{-}$, which is due to a hadronic penguin decay mechanism, is the most important with other contributions from $B_{d} \rightarrow K^{+} \pi^{-}$and $B_{s} \rightarrow K^{-} \pi^{+}$. Recent results from CLEO show a confirmed signal for the $B_{d} \rightarrow K^{+} \pi^{-}$decay and an upper limit on the decay of interest, $B_{d} \rightarrow \pi^{+} \pi^{-}[7]$. While many people have previously made assumptions that the $\pi \pi$ mode was larger than or equal to the $K \pi$ mode, it now appears that the $K \pi$ mode is larger. Based on the CLEO yields of each we will make a conservative assumption that $\mathcal{B}\left(B_{d} \rightarrow K^{+} \pi^{-}\right)$is twice a large as $\mathcal{B}\left(B_{d} \rightarrow \pi^{+} \pi^{-}\right)$.

$B_{s} \rightarrow \pi^{+} K^{-}$is a $b \rightarrow u$ transition of the $B_{s}$ similar to the decay $B_{d} \rightarrow \pi^{+} \pi^{-}$, and $B_{s} \rightarrow K^{+} K^{-}$is a hadronic penguin. The modes of $B_{s}$ decays are normalized by having the total $B_{s}$ production be $34 \%$ of $B_{d}$ production [14]. In addition, we expect that the penguin and $b \rightarrow u$ decays of the $B_{s}$ will have the same pattern of branching ratios as the $B_{d}$.

Using the above results as input, we simulate the two pion mass plot without particle identification in Fig. 11.15. The plots make it clear that kinematic separation is inadequate to discriminate between these decays. The width and separations for the signals in the mass plot are almost identical to CDF's prediction in their TDR [16], although they used a more favorable ratio of $K \pi$ to $\pi \pi$ which the new CLEO data puts in doubt.

MCFast does not simulate the RICH response, but it can model the geometric acceptance, momentum threshold, decays in flight and secondary interactions. The efficiency after accounting for these effects is $6.5 \%$. Misidentification in the RICH at the $1-2 \%$ level will produce a background that is quite small compared to the background due to random combinations and it can be ignored.

There is a strong correlation between triggered events and reconstructed events. For a trigger requirement of two tracks with more than $4 \sigma$ significant miss distance from the primary vertex, the simulation shows that more than $50 \%$ of our reconstructable events pass this trigger. Such a trigger requirement rejects the light quark background at a rate of 100 to 1. This leaves us with 34,000 events per year of running before flavor tagging of the opposite $b$ has been attempted.

Our signal to background estimate is based on 280,000 generic $B$ events, which were selected at the generator level to have two tracks with a mass within $500 \mathrm{MeV} / \mathrm{c}^{2}$ (about 18 times our mass resolution) of the $B^{0}$ mass. The two tracks also had to have an opening angle less than $\pi / 2$. These cuts reduce the sample that we need to examine by a factor of 13 , so our effective background sample is $3,700,000$ events. 


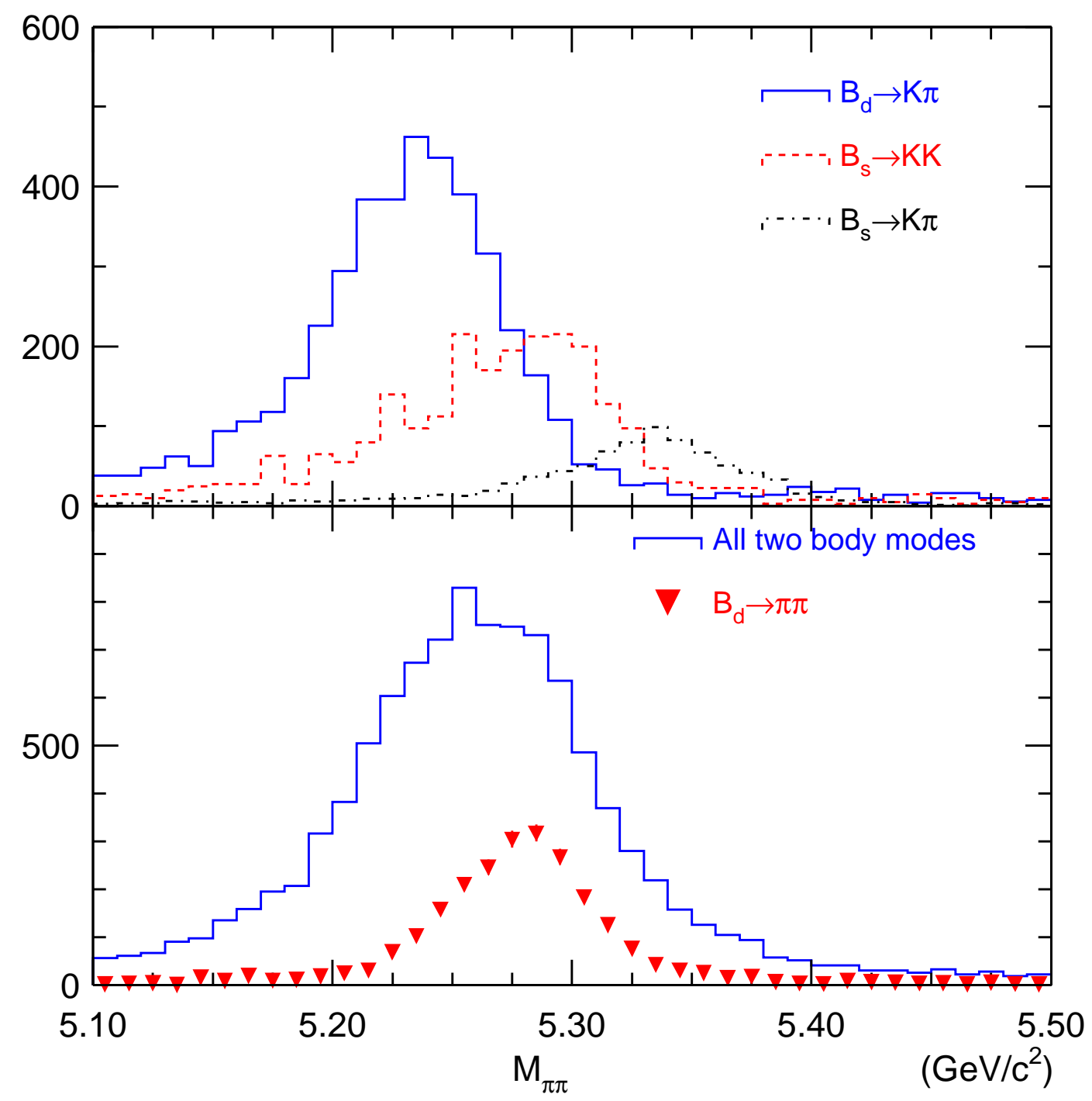

Figure 11.15: Two body mass plots without particle identification a) $B_{d} \rightarrow K^{+} \pi^{-}, B_{s} \rightarrow$ $\pi^{+} K^{-}, B_{s} \rightarrow K^{+} K^{-}$, b) $B_{d} \rightarrow \pi^{+} \pi^{-}$and a sum of all two body decay modes. All particles are assumed to be pions. 
Table 11.3: Projected yield of $B^{0} \rightarrow \pi^{+} \pi^{-}$and the uncertainty on $a_{C P}$

\begin{tabular}{|l|c|}
\hline Luminosity & $2 \times 10^{32} \mathrm{~cm}^{-2} \mathrm{~s}^{-1}$ \\
Running time & $10^{7} \mathrm{sec}$ \\
Integrated Luminosity & $2000 \mathrm{pb}^{-1}$ \\
$\sigma_{b \bar{b}}$ & $100 \mu \mathrm{b}$ \\
Number of $B \bar{B}$ events & $2 \times 10^{11}$ \\
Number of $B_{d}^{0}$ 's & $1.4 \times 10^{11}$ \\
$\mathcal{B}\left(B_{d}^{0} \rightarrow \pi^{+} \pi^{-}\right)$ & $0.75 \times 10^{-5}$ \\
Reconstruction efficiency & 0.06 \\
Trigger efficiency & 0.50 \\
Number of reconstructed $B_{d}^{0} \rightarrow \pi^{+} \pi^{-}$ & $3.4 \times 10^{4}$ \\
Tagging efficiency $\epsilon D^{2}$ & 0.10 \\
$\mathrm{~S} / \mathrm{B}$ & 0.6 \\
$\delta a_{C P}$ & 0.023 \\
\hline
\end{tabular}

From this sample only 1 event has a $\pi \pi$ mass that lies between 5.1 and $5.5 \mathrm{GeV}$, and if we scale to a $B$ signal region which is $150 \mathrm{MeV}$ wide $(\sigma=25 \mathrm{MeV})$ we expect less than one event under the peak. We will assume that the background has 1 event due to the limited statistics. If we assume a branching ratio $\mathcal{B}\left(B_{d} \rightarrow \pi^{+} \pi^{-}\right)=0.75 \times 10^{-5}$, then our $S / B=0.6$ but with large errors. Study of larger samples of backgrounds may allow us to understand how to improve our signal to background ratio.

The tagging efficiency, which is discussed in Section 11.3, is estimated to be $10 \%$. We can combine these results to find an uncertainty on the CP asymmetry. Our results are shown in Table 11.3. 


\section{5 $\quad B^{o} \rightarrow K^{* o} \gamma$}

The decay $B^{o} \rightarrow K^{* o} \gamma$ is an example of a relatively rare decay involving a single photon. This decay has already been observed by CLEO, with branching fraction $(4 \pm 1.7 \pm 0.8) \times 10^{-5}$. Decays such as $B^{o} \rightarrow K^{* o} \gamma, B^{o} \rightarrow \rho^{o} \gamma, B_{s} \rightarrow \phi^{o} \gamma$, and $B_{s} \rightarrow K^{* o} \gamma$ provide insight into $\frac{V_{t d}}{V_{t s}}$. We have chosen to study $B^{o} \rightarrow K^{* o} \gamma$ because its branching fraction is measured so a definite predictions of the reconstructed event yield is possible. Here the $K^{* o}$ decays into $K^{+} \pi^{-}$. This state can benefit from the kinematic cuts and the $p_{t}$ requirements described below to suppress the combinatoric background. Decays such as $B^{o} \rightarrow \rho^{o} \gamma$ are expected to have much smaller branching fractions and backgrounds which are comparable so more work will have to done on background rejection to prove that their detection is possible.

In order to calculate the effective signal, one has to design analysis cuts that suppress the backgrounds. A major source of background is from $B^{o, \pm} \rightarrow K^{* o}+X$ in combination with a photon from either the underlying event or the other $B$. Since the expected signal is so small compared to potential sources of background, a very large number of events must be simulated to demonstrate that the signal will not be overwhelmed. The simulation of an adequate number of events requires more computing resources than we have so we have had to make some assumptions. Within the context of these assumptions, we have obtained a fairly good estimate of the background to our signal.

The basic assumptions that we have made in estimating the background are:

- Non- $B$ events can be adequately suppressed by selecting events that have a well-defined primary vertex and a downstream decay vertex;

- The $B$ events that will cause backgrounds to this process will contain a $K^{* o}(890)$ (we do not require this to come from downstream of the primary vertex in our initial selection); and

- The events that satisfy the above requirements will only create background if they contain at least one photon which makes a high invariant mass- greater than 4.0 $\mathrm{GeV} / \mathrm{c}^{2}$ - when combined with the $K^{* o}$.

With these assumptions, we can reject $98 \%$ of all generic $B-\bar{B}$ events at the 'event generator' level. We only have to perform the full detector simulation, reconstruction, and analysis on the surviving $2 \%$. We have used 400,000 events which survived from 20,000,000 at the event generator level to compute the background. Even this large number of events is still marginal for the background estimation since each background event surviving the analysis and appearing in the $K^{* o} \gamma$ mass plot represents 10,000 events per year.

In the analysis presented below, we have assumed a calorimeter resolution with a stochastic term of $2.5 \%$ and a constant term of $0.55 \%$. If, as expected we can do better than this, the mass resolution will improve. 


\subsubsection{An Initial Shower Reconstruction Program}

The use of the showers generated by MCFast (or GEANT) in a realistic analysis requires a shower reconstruction program. Such a program looks for $\mathrm{PbWO}_{4}$ blocks with locally high maxima of energy deposition and uses them to 'seed' showers. Nearby blocks are then aggregated around the seed blocks to form the complete showers. The number of blocks to include around the seed is usually determined by GEANT simulations or from beam tests. Various techniques are used to partition energy (on a statistical basis) between showers which share cells.

We do not yet have a shower reconstruction program so we are using a 'prompted' reconstruction program. In this program, we trace the generated photons to the calorimeter to get the seed block. After that, we compute the shower energy as the sum of the energies of the seed block, the nearest neighbor blocks, and the next-to nearest neighbor blocks. A total of twenty-five blocks are summed which is consistent with our GEANT studies. The position is computed from the energy-weighted first moment of the blocks in $\mathrm{X}$ and separately in $\mathrm{Y}$. The correction to the position described in chapter 4 is not applied yet. $\pi^{o}$ 's are reconstructed simply by looping over all showers, computing the invariant mass of each pair, and cutting on the mass. No asymmetry cuts or other cuts intended to improve signal to background are made at this point.

Features of the current approach which cause it to depart from reality are:

- The current program does not permit one to find 'false' showers because it starts from the generated photons. This may result in underestimation of the combinatoric background. In a complete analysis, charged hadrons and electrons can be rejected by observing tracks pointing near the shower centroid so they in general are not a source of false photon candidates. bremsstrahlung photons, neutral hadrons, and artifacts of the reconstruction program can, however, create extraneous neutral showers.

- Since all photons, electrons, and hadrons deposit energy in the calorimeter, realistic problems with overlapping showers exist. The simple algorithms we are using do not guard against such overlaps so that some showers will have their energy and/or position

distorted. This causes the efficiency of shower reconstruction to be lower than what we ultimately expect to achieve.

This simple approach will be replaced as soon as possible by a full reconstruction program which will remedy all of these defects. However, the techniques we have used to reject combinatoric backgrounds, described in the next section, should work very well to suppress the additional backgrounds from the extraneous showers described above so we expect that the background will not be too much worse than what we see now. Moreover, there are real opportunities for improved efficiencies with an improved reconstruction program.

\subsubsection{Suppression of Combinatoric Backgrounds}

Before the advent of precision vertex detectors, the study of heavy quark states produced in hadronic interactions and decaying into all charged particles was nearly impossible due 
to combinatoric backgrounds. That is, mass combinations with the same particle content as the signal, but coming from the non-heavy quark events or the underlying part of a genuine heavy quark event, populated the mass region of interest with many more entries than did the real signal. The mass peak was then lost or obscured in the fluctuations of this background. Silicon vertex detectors permitted one to cluster the charged tracks into vertices and to include in signals only the tracks that came from a well-defined decay vertex observed downstream of the main interaction. This effectively eliminated the combinatoric background in many heavy quark decays and made possible successful programs of heavy quark studies in photoproduction and hadroproduction.

For final states containing both charged particles and neutral objects such as photons and $\pi^{\circ} \mathrm{s}$, one must again face the threat of being overwhelmed by combinatoric backgrounds. The vertex detector can be used to reconstruct the primary vertex and the $B$ vertex from the charged particles and to isolate a reasonably pure sample of $B$ events. Then the charged tracks in these vertices can be combined with photons or $\pi^{\circ}$ candidates to form the final state of interest. The problem is that the calorimeter must be at the downstream end of the system far from the interaction vertex and we know of no technology that will determine the position and direction of the neutral objects striking it well enough to tell whether they come from the primary vertex, the secondary vertex of interest, or other secondary vertices in the event. It seems then that we have to form mass combinations of every photon or $\pi^{o}$ candidate with the charged tracks and that this could produce large combinatoric backgrounds that would obscure or highly pollute any signals.

There are two ways to combat combinatoric background by eliminating many of the photons or $\pi^{o}$ s from consideration as objects in the $B$ decay of interest. The first is by taking advantage of some kinematic features of the photons from the decays of interest which differentiate them from other photons. The second is by exploiting the fact that in $\mathrm{BTeV}$ we have a rather good knowledge of the direction of the $B$ and we can insist that only photons or $\pi^{o}$ 's that balance the transverse momentum of the charged tracks relative to the $B$ direction be considered as daughters of the $B$ decay.

Kinematic cuts are effective when the photons from the $B$ final state under study have very different properties from other photons in the event. This is true, for example, for quasi-two-body decays onto a single photon and a hadronic resonance. Prime examples are

- $B^{o} \rightarrow K^{* o} \gamma$ and

- $B^{o} \rightarrow \rho^{o} \gamma$.

Figure 11.16 shows the $p_{t}$ distribution (relative to the beam) of the single photons in generic $B-\bar{B}$ events. This can be compared with the $p_{t}$ distribution of photons from $B^{o} \rightarrow K^{* o} \gamma$ shown in chapter $4.85 \%$ of the signal photons have $p_{t}$ greater than $1.0 \mathrm{GeV} / \mathrm{c}$ while $97 \%$ of the remaining photons are below $1 \mathrm{GeV} / \mathrm{c}$.

This kind of cut works to some extent, but less well, in the study of decays with one $\pi^{o}$ and a quasi two body hadronic resonance. An example of this is $B^{o} \rightarrow K^{* o} \pi^{o}$ or $B^{o} \rightarrow \rho^{o} \pi^{o}$. Here the $p_{t}$ of the $\pi^{o}$ is the same as the $p_{t}$ of the single $\gamma$ in $K^{* o} \gamma$ so the $p_{t}$ of the individual 


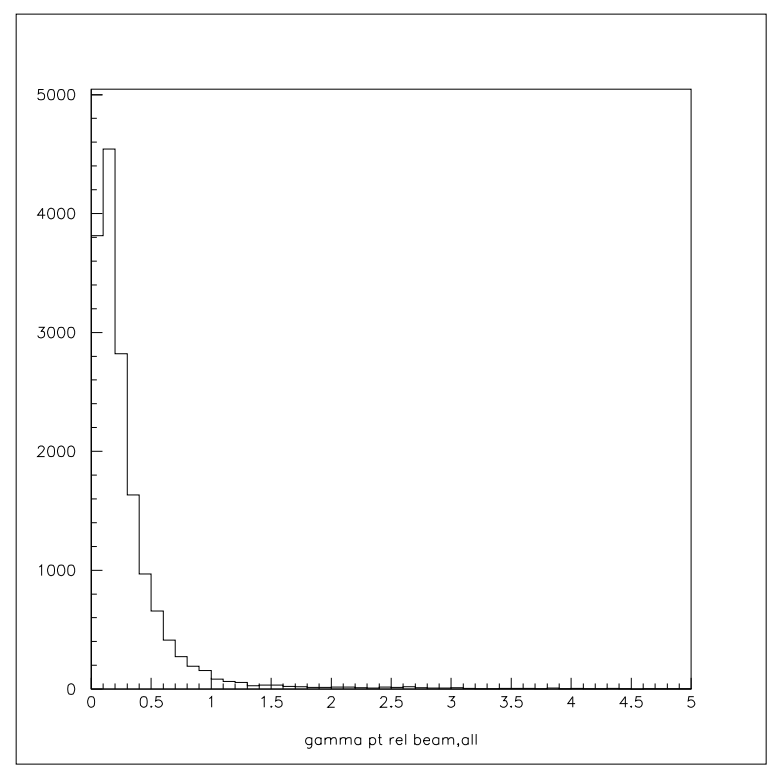

Figure 11.16: The $p_{t}$ distribution (relative to the beam) of the single photons from all photons in $B$ events.

photons will be lower. Cuts on the $p_{t}$ of the individual photons or of the $\pi^{o}$ relative to the beam will still help to reject background but the effect is not as large as for $K^{* o} \gamma$.

When the $\pi^{o}$ is a daughter of the hadronic resonance in a quasi two body decay, such as $B^{o} \rightarrow \rho^{+} \pi^{-}$, the $\pi^{o}$ and its daughter photons are even closer kinematically to the photons in the underlying event so kinematic cuts are even less effective in this case. This can be seen by comparing the $p_{t}$ of photons from $B^{o} \rightarrow \rho^{+} \pi^{-}$in Fig. 4.26 with Fig. 11.16.

A second approach uses our excellent measurement of the $B$ direction. The primary vertex is measured by fitting an average of about 10 charged tracks. In $B$ decays which contain at least two charged tracks, the secondary vertex is reconstructed with high efficiency and accuracy. The line connecting the primary and secondary vertex measures the $B$ direction with accuracies of 10-20 mr. The total momentum vector of the charged daughters and the $B$ direction define a plane in which the missing neutral must lie and the $B$ direction defines a line with respect to which the neutral particle's $p_{t}$ must be equal in magnitude and opposite in direction to the total charged particle $p_{t}$. Photons which are not daughters of the $B$ of interest but come from the underlying event or another $B$ or $D$ decay in the event will generally not satisfy this $p_{t}$ balance requirement. Similarly, if the $B$ decay is really an example of a decay mode with more than one missing neutral or a missing charged track (due to being out of the acceptance or a reconstruction failure), it will generally not satisfy the $p_{t}$ balance requirement. We have shown that, for all choices of requirements on the calorimeter which satisfy our other requirements, the calorimeter's contribution to the $p_{t^{-}}$ balance resolution is small compared to the contribution due to the finite position resolution of the vertex detector.

A final defense against the remaining combinatoric background is mass resolution. We 
have discussed this in chapter 4 . Widths of mass distributions are predicted to be 40$50 \mathrm{MeV} / \mathrm{c}^{2}$. They are dominated by the uncertainty on the measurement of the photon's momentum and are generally factors of 2.5 to 3 worse than for all charged states.

\subsection{3 $\quad K^{* o} \gamma$ Analysis Cuts}

We used the standard MCFast analysis and vertexing packages for the charged tracks and the MCFast parametrized calorimeter simulation described above. We also used the full $\mathrm{BTeV}$ Level 1 trigger simulation. The requirements we placed on events were:

- that it pass the experiment trigger;

- A good primary vertex with at least three tracks and $\chi^{2}$ per degree of freedom of less than 1.5;

- A neutral combination of a charged $\mathrm{K}$ and a charged $\pi$ which are not included in the primary vertex and which make a good fit to a common vertex with $\chi^{2}$ less than 3 ;

- $\mathrm{K}-\pi$ invariant mass between $0.840 \mathrm{GeV} / \mathrm{c}^{2}$ and $0.940 \mathrm{GeV} / \mathrm{c}^{2}$;

- $\frac{L}{\sigma_{L}}$ greater than 3 ;

- A photon with $p_{t}$ relative to the beam of greater than $1 \mathrm{GeV} / \mathrm{c}$

- That the photon satisfy a momentum balance requirement with respect to the direction of the $B$, as determined from the line connecting the primary vertex with the K- $\pi$ vertex; and

- No other charged track which is unassigned to the primary can form a vertex with the $\mathrm{K}-\pi$ with $\chi^{2}$ of less than 12.0 .

\subsubsection{Signal Properties}

The invariant mass distribution of the $K-\pi-\gamma$ combinations satisfying all these requirements is shown in Fig. 11.17. The photon $p_{t}$ is shown in Fig. 11.18. Table 11.4 shows the average values of some quantities of interest to the analysis.

Table 11.4: Average Properties of Signal Events Surviving All Analysis Cuts

\begin{tabular}{|l|c|}
\hline \hline Quantity & Value \\
\hline Mean $B$ Energy & $65.5 \mathrm{GeV}$ \\
Mean Photon Energy & $24.8 \mathrm{GeV}$ \\
Mean Photon Transverse Momentum & $3.0 \mathrm{GeV} / \mathrm{c}$ \\
Mean $\frac{L}{\sigma_{L}}$ & 11.4 \\
\hline \hline
\end{tabular}




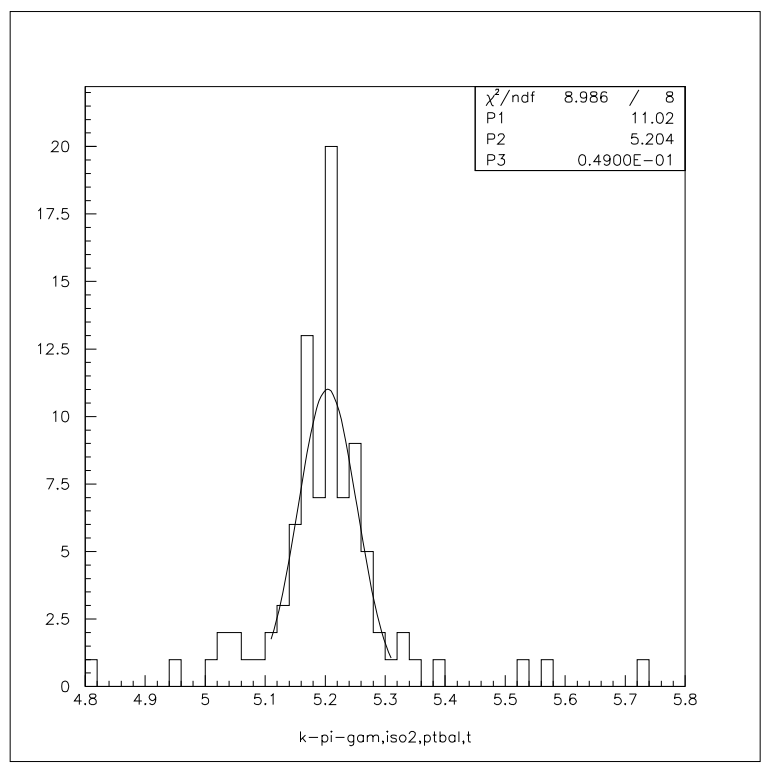

Figure 11.17: Invariant mass spectrum of $K^{-} \pi^{+} \gamma$ for events passing the trigger and all analysis cuts. The fit is to a Gaussian and has a $\sigma$ of $49 \mathrm{MeV} / \mathrm{c}^{2}$.

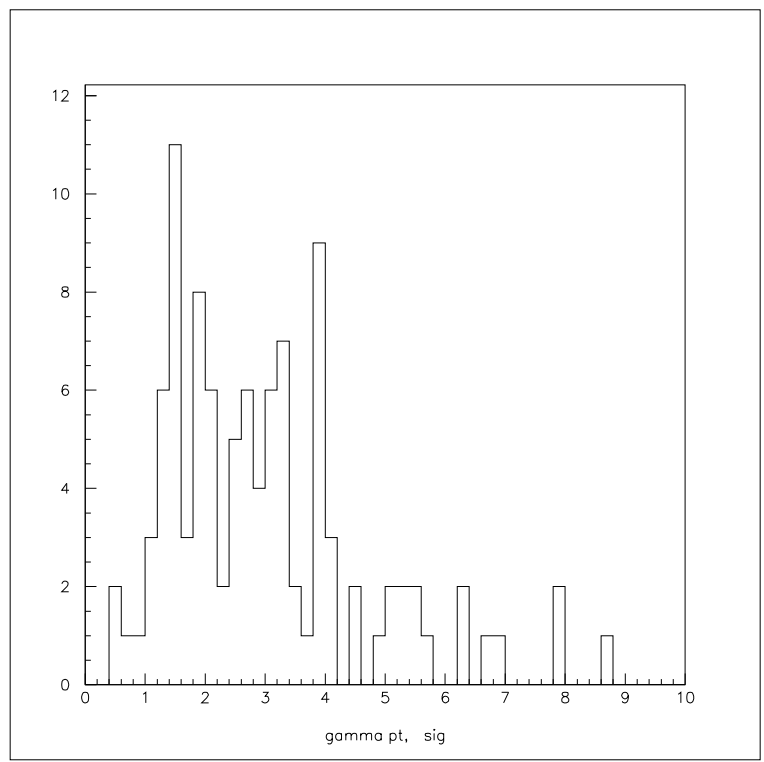

Figure 11.18: $p_{t}$ spectrum of photons from $K^{-} \pi^{+} \gamma$ combinations which pass the trigger and all analysis cuts. 


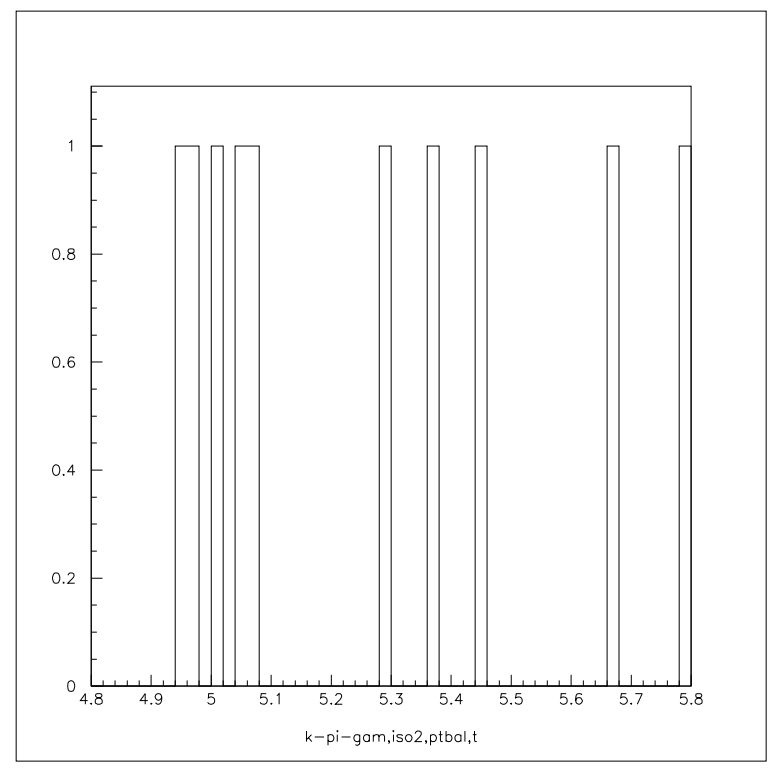

Figure 11.19: Invariant mass spectrum of $K^{-} \pi^{+} \gamma$ combinations from background events which pass the trigger and all analysis cuts.

\subsubsection{Backgrounds}

\subsubsection{Background from generic $B$ events}

The background is calculated as described above. The 400,000 events (out of 20,000,000) surviving at the event generator level are run through MCFast and the identical analysis used for obtaining the signal, described above. The result for the $K^{* o} \gamma$ invariant mass distribution is shown in Fig 11.19 .

\subsubsection{Background from $B^{o} \rightarrow K^{* o} \pi^{o}$}

There is one additional background that we have studied. In the decay $B^{o} \rightarrow K^{* o} \pi^{o}$, occasionally one photon from the $\pi^{o}$ decay will take almost all the energy. Given the finite resolution of the calorimeter, the combination of the real $K^{* o}$ and the higher energy photon from the $\pi^{o}$ could have an invariant mass that was very close to the $B^{o}$ mass. The other photon will have very small energy. If we could detect the soft photon, we could in principle pair it with the candidate single high energy photon, see that it was consistent with the $\pi^{o}$ mass, and reject the combination. However, in the complex environment of the collider and with only partial angular coverage, this is not an efficient approach.

We have studied this background by running a sample of generated $B^{o} \rightarrow K^{* o} \pi^{o}$ decays through the analysis for $B^{o} \rightarrow K^{* o} \gamma$. The result is shown in Fig. 11.20. The solid line is the $K^{* o} \gamma$ signal. The dashed line is the false signal from $K^{* o} \pi^{o}$. The relative normalization is based on the PDG branching fraction for $K^{* o} \gamma$ and the PDG upper limit for $K^{* o} \pi^{o}$. The background is not negligible but is not so large as to threaten our ability to see the signal. 


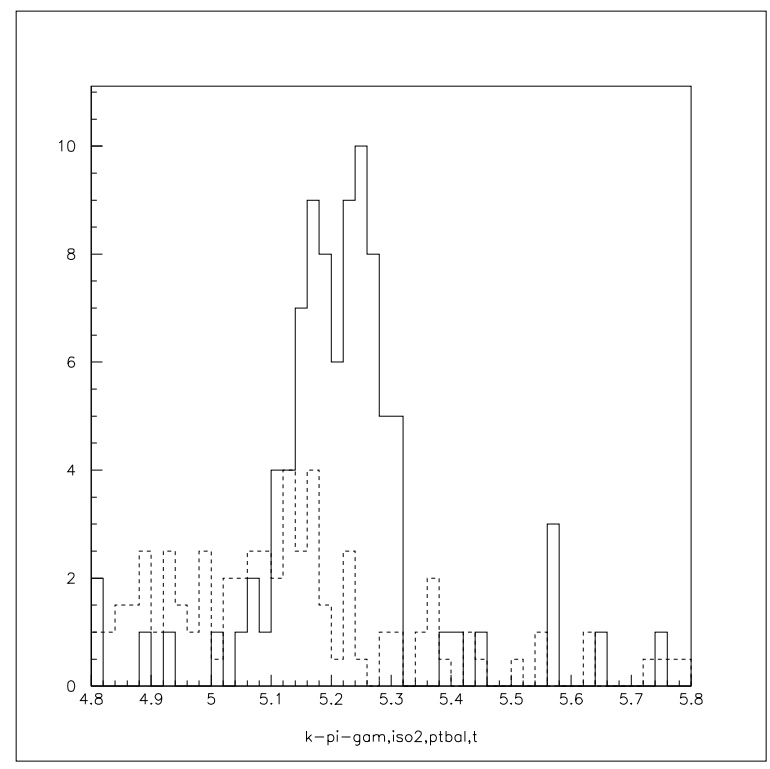

Figure 11.20: Invariant mass spectrum of $K^{-} \pi^{+} \gamma$ (solid line) compared to apparent $K^{-} \pi^{+} \gamma$ signal (dashed line) from $K^{* o} \pi^{o}$ events. The relative normalization is explained in the text.

We have not made any attempt to try to suppress it. Some of it could also be from coalesced photons rather than very asymmetric decays and for that we have rejection power that we have not yet used in this analysis. The implications for the study of $B^{o} \rightarrow \rho^{o} \gamma$, which has a much smaller branching fraction than $B^{o} \rightarrow K^{* o} \gamma$, of the background from $B^{o} \rightarrow \rho^{o} \pi^{o}$ will be studied.

\subsubsection{Projections of Signal Size and Background}

The signal size for one year of running at the design luminosity is shown in table 11.5.

Table 11.5: Number of Reconstructed $B^{o} \rightarrow K^{* o} \gamma$ per year at design luminosity

\begin{tabular}{|l|c|}
\hline \hline Total number of $B^{\prime} \mathrm{s}$ & $2.0 \times 10^{11}$ \\
Total number of $B^{o} \mathrm{~S}$ & $7.0 \times 10^{10}$ \\
Total number of $B^{o} \rightarrow K^{* o} \gamma$ & $28.0 \times 10^{5}$ \\
per $4 \times 10^{-5}$ branching fraction & \\
Total number of $B^{o} \rightarrow K^{-} \pi^{+} \gamma$ & $18.7 \times 10^{5}$ \\
$K^{* o} \rightarrow K^{-} \pi^{+}$branching fraction & \\
Total Number Reconstructed & $2.4 \times 10^{4}$ \\
$(1.3 \%$ efficiency, including trigger $)$ & \\
\hline \hline
\end{tabular}

The background can be estimated from the 10 events that survived the analysis described above. The estimate for one year of running at the design luminosity is shown in table 11.6 
Table 11.6: Number of Reconstructed Background events per year at design luminosity

\begin{tabular}{|l|r|}
\hline \hline Total number of $B$ pairs/year & $2.0 \times 10^{11}$ \\
Total number of background events & 10 \\
in $20,000,000 B$ pairs, mass between & \\
4.8 and $5.8 \mathrm{GeV} / \mathrm{c}^{2}$ & \\
Projected background events/year & $1.0 \times 10^{5}$ \\
Projected background events/year & $2.5 \times 10^{4}$ \\
under $B$ mass peak & \\
\hline \hline
\end{tabular}

While this calculation needs a number of refinements, it looks possible to achieve very large yields of reconstructed $B^{o} \rightarrow K^{* o} \gamma$ with a signal to background of no worse than a 'few' to one. The background from $K^{* o} \pi^{o}$ also looks manageable.

We have written out all events that contribute to the background as well as a larger sample of 'near misses', that is events that survived until the last few cuts. By analyzing these and running larger samples of background events, we hope to improve the background rejection and eventually to apply what we learn to the much harder problem of $B^{o} \rightarrow \rho \gamma$. We will also study $B_{s} \rightarrow \phi \gamma$ and $B_{s} \rightarrow K^{* o} \gamma$. 


\section{6 $\quad B^{o} \rightarrow \rho \pi$}

The measurement of the CKM angle $\alpha$ is beset with difficulties. It now seems that a rather complicated amplitude analysis of the decays

$$
\begin{aligned}
& B^{o} \rightarrow \rho^{+} \pi^{-} \\
& B^{o} \rightarrow \rho^{-} \pi^{+} \\
& B^{o} \rightarrow \rho^{o} \pi^{o}
\end{aligned}
$$

and their charge conjugates may be the best approach. Since the branching fractions for these decays are small, a tagged time-dependent analysis is required and a detailed multiparameter fit must be performed, a large number of events are needed.

We have begun to study these states in BTeV as part of our investigation of the capabilities of our proposed $\mathrm{PbWO}_{4}$ calorimeter. Below we present some early results on the rates of reconstructed events per year that we might achieve. We employ the same approach that we used in the analysis of the decay $B^{o} \rightarrow K^{* o} \gamma$, which was able to reject the background well enough to cleanly isolate the decay. Here we have not begun the study of the background. Moreover, because one wants to be able to carry out an amplitude analysis rather than just measure a rate, it is desirable to reduce the background to a low level. We do not yet know that the analysis cuts employed here will produce a clean enough signal to extract $\alpha$. The event yields presented here should be taken as an initial indication of what might be achieved.

Analysis cuts are similar to $B^{o} \rightarrow K^{* o} \gamma$ except that there is no cut on the $p_{t}$ relative to the beam since it would not be very effective in this case. The planarity and momentum balance requirements are now applied to the $\pi^{o}$ in this analysis.

Figure 11.21 shows the invariant mass plot of $\pi^{+} \pi^{-} \pi^{o}$ combinations which satisfy the analysis cuts. We expect the peak to narrow when we employ a more sophisticated shower reconstruction. Table 11.7 gives the estimated rate for 1 year of running for tagged events. We use $4 \times 10^{-5}$ for the branching fraction since it is consistent with theoretical predictions and used by BaBar [15].

Table 11.7: Number of Reconstructed $B^{o} \rightarrow \rho^{+} \pi^{-}$per year at design luminosity

\begin{tabular}{|l|c|}
\hline \hline Total number of $B^{\prime} \mathrm{s}$ & $2.0 \times 10^{11}$ \\
Total number of $B^{\circ} \mathrm{s}$ & $7.0 \times 10^{10}$ \\
Total number of $B^{\circ} s \rightarrow \rho^{+} \pi^{-}$ & $28.0 \times 10^{5}$ \\
per $4 \times 10^{-5}$ branching fraction & $2.8 \times 10^{4}$ \\
Total Number Reconstructed & \\
$(1.0 \pm 0.2) \%$ efficiency, including trigger) & \\
Total number reconstructed and tagged & $2.8 \times 10^{3}$ \\
(includes dilution factor) & \\
\hline \hline
\end{tabular}




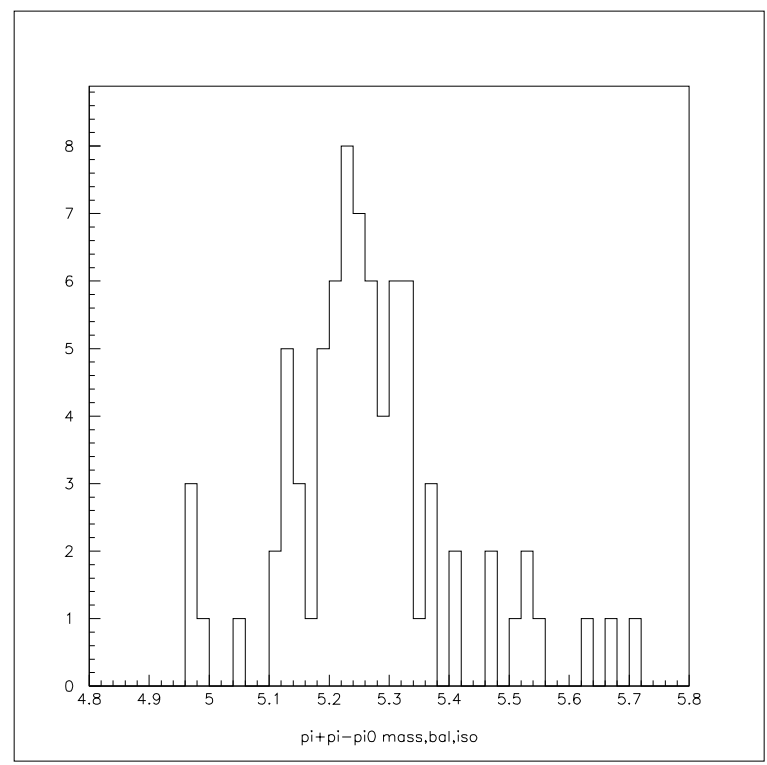

Figure 11.21: The $\pi^{+} \pi^{-} \pi^{o}$ Invariant Mass Distribution for Events Surviving All Analysis Cuts 


\subsection{Flavor-changing $B$ meson decays}

\subsubsection{The Physics Significance of Rare $B$ Meson Decays}

Within the Standard Model, flavor-changing neutral current decays of $b$ quarks may occur through loop - or penguin- diagrams or box-diagrams. Such decays, which involve small CKM matrix elements and are therefore rare, may be used to extract CKM matrix elements once various long range and perturbative QCD effects are taken into account. If, however, some of these decays occur at a much higher level than predicted by the Standard Model, this would be evidence that there were new particles whose amplitudes could contribute to the loops or boxes. This would provide a path to new physics which is at a higher mass scale than can be probed directly at existing or planned accelerators. Table 11.8 [17] gives a list of some interesting rare decays and their estimated branching fractions.

Table 11.8: Estimated Branching Fractions for Flavor-Changing Neutral Current Decays with Muons and Electrons

\begin{tabular}{||l|c|c||}
\hline Decay Mode & Est. BR(SM) & $\begin{array}{c}\text { Measurements and } \\
90 \% \text { CL upper limits }\end{array}$ \\
\hline$\left(B_{d}, B_{u}\right) \rightarrow X_{s} \mu^{+} \mu^{-}$ & $(5.7 \pm 1.2) \times 10^{-6}$ & $<3.6 \times 10^{-5}$ \\
$\left(B_{d}, B_{u}\right) \rightarrow X_{d} \mu^{+} \mu^{-}$ & $(3.3 \pm 1.9) \times 10^{-7}$ & - \\
$\left(B_{d}, B_{u}\right) \rightarrow K \mu^{+} \mu^{-}$ & $(4.0 \pm 1.5) \times 10^{-7}$ & $<0.9 \times 10^{-5}$ \\
$\left(B_{d}, B_{u}\right) \rightarrow K^{*} \mu^{+} \mu^{-}$ & $(1.5 \pm 0.6) \times 10^{-6}$ & $<2.5 \times 10^{-5}$ \\
$B_{s} \rightarrow \mu^{+} \mu^{-}$ & $(3.5 \pm 1.0) \times 10^{-9}$ & $<8.4 \times 10^{-6}$ \\
$B_{d} \rightarrow \mu^{+} \mu^{-}$ & $(1.5 \pm 0.9) \times 10^{-10}$ & $<1.6 \times 10^{-6}$ \\
\hline$\left(B_{d}, B_{u}\right) \rightarrow X_{s} e^{+} e^{-}$ & $(8.4 \pm 2.2) \times 10^{-6}$ & - \\
$\left(B_{d}, B_{u}\right) \rightarrow X_{d} e^{+} e^{-}$ & $(4.9 \pm 2.9) \times 10^{-7}$ & - \\
$\left(B_{d}, B_{u}\right) \rightarrow K e^{+} e^{-}$ & $(5.9 \pm 2.3) \times 10^{-7}$ & $<1.2 \times 10^{-5}$ \\
$\left(B_{d}, B_{u}\right) \rightarrow K^{*} e^{+} e^{-}$ & $(2.3 \pm 0.9) \times 10^{-6}$ & $<1.6 \times 10^{-5}$ \\
$B_{s} \rightarrow e^{+} e^{-}$ & $(8.0 \pm 3.5) \times 10^{-14}$ & - \\
$B_{d} \rightarrow e^{+} e^{-}$ & $(3.4 \pm 2.3) \times 10^{-15}$ & - \\
\hline \multicolumn{2}{|l}{}
\end{tabular}

\subsubsection{Rare Decays in BTeV}

Because the Tevatron produces more than $10^{11}$ b-hadrons per year, we should be able to observe some of these decays and to set stringent limits on others. However, $b$-physics at the Tevatron also has more sources of background than at $e^{+} e^{-} B$-factories and the sensitivity may be limited by the inability to reject these backgrounds. 
Below, we show the results of a study of the decay $B^{-} \rightarrow K^{-} \mu^{+} \mu^{-}$. This decay, according to Table 11.8, occurs with a branching fraction of around $\left(4.0 \pm 1.5 \times 10^{-7}\right)$. The study is based on simulation runs of 5000 signal events each containing the signal $B^{-}$decay and a generic $\bar{b}$-decay and $10^{5}$ background events each of which has both $B$-mesons decaying semileptonically.

In Fig. 11.22 we show an invariant mass distribution for $B^{-} \rightarrow K^{-} \mu^{+} \mu^{-}$which also includes background events whose origin is described below. The requirements imposed on events contributing to this plot were determined by the need to achieve very high rejection of the background while maintaining reasonable efficiency. They are:

- A primary vertex consisting of at least 2 tracks with a good $\chi^{2}$ fit;

- A secondary vertex consisting of three tracks:

1. one of which is a charged kaon and is required to pass through the RICH and have a momentum greater than $4 \mathrm{GeV} / \mathrm{c}$;

2. another is a positively charged muon which is required to hit the muon detector and have a momentum greater than $5 \mathrm{GeV} / \mathrm{c}$;

3. and another is a negatively charged muon which is required to hit the muon detector and have a momentum greater than $5 \mathrm{GeV} / \mathrm{c}$.

The secondary vertex must have a $\chi^{2}$ less than 8 . The normalized separation, $L$ between the primary vertex and the candidate secondary vertex, $L / \sigma_{L}$, must be greater than 7 and the candidate must satisfy a 'pointback' cut with respect to the primary vertex of less than 2.5 standard deviations.

It is also critical to study the background to this state. The various sources of background are:

- Minimum bias events where three particles conspire to fake a secondary vertex and two of the particles either decay downstream of the magnet or make hadronic showers which leave a signal in the muon detector (hadron punchthrough).

- Charm events with one or more real muons and kaons.

- $b$-events where portions of the two $b$-hadrons in the event appear to verticize downstream of the production point. In approximately $1 \%$ of all $b \bar{b}$ events both $B$-hadrons decay semileptonically producing two real muons. In addition, there is a charged kaon in at least one of the $b$ 's over $90 \%$ of the time.

- More generally, any variety of admixture of $B$, charm, minimum-bias events, primary interactions and secondary decays, combined with hadronic punchthrough. 


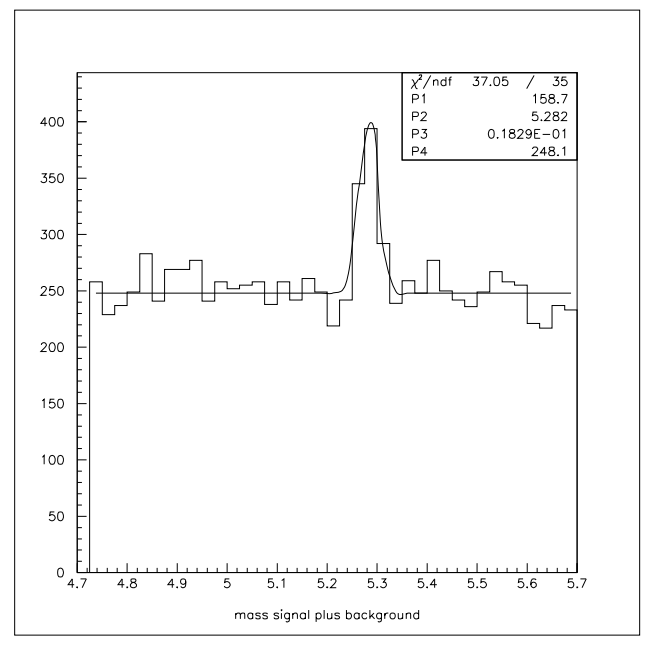

Figure 11.22: Invariant mass spectrum of $K \mu^{+} \mu^{-}$including an estimate of the background from events where both $b$ 's decay semileptonically (also obtained with MCFast).

- Fake events from single $b$ decays such as $B^{-} \rightarrow K^{-} \pi^{+} \pi^{+}$where both pions fake muon signatures by decay or punchthrough. Another possibility is the decay $B^{-} \rightarrow D^{o} \pi^{-}$ where the $D$ vertex is not seen as separated from the $B$ vertex and where both pions simulate muons. However, a mass cut on the $D$ will remove this background.

The basic weapons to combat backgrounds are:

- Excellent mass resolution on the final state.

- Excellent discrimination between the primary and secondary vertex which eliminates backgrounds from the minimum-bias events and from the underlying event within a true $b$-event.

- Excellent 'pointback' resolution between the reconstructed $b$ candidate and the primary vertex which will do much of the work to reject events that have been artificially pieced together from particles from the two separate $b$ 's in the event.

- The ability to reject events where other tracks point to the candidate vertex, where some of the tracks in the candidate vertex also point to the primary, or where some of the tracks in the candidate vertex intersect with other tracks not in the primary vertex (i.e. tracks from the associated $b$ ).

In addition, the signal-to-background depends on the quality of both the muon detector and the particle identification.

We did not include the decay $B^{-} \rightarrow \psi K^{-}$as a background. That decay is large compared to the rare decay being considered here and will interfere with the rare decay and distort the dimuon mass distribution in the vicinity of $3 \mathrm{GeV} / \mathrm{c}^{2}$. This, however, is a physics 
contribution and will certainly be observed and studied based on a mass cut on the dimuon. In fact, this state can be used to calibrate the efficiency of the analysis and can be used as a normalization for a measurement of the relative branching fraction.

We have not simulated all sources of background. Our own estimates indicate that the most serious background is from events with pairs of $B$ 's which each undergo semileptonic decays. We have analyzed $10^{5}$ simulated events of this type and have a background in the mass plot ( 4.7 to $5.7 \mathrm{GeV} / \mathrm{c}^{2}$ ) of 1 event in 40 bins (which we can make disappear with only a slight adjustment in cuts). We obviously need to run more background events. However, if we scale this result to the correct mass interval and by the correct relative normalization factor, we estimate a background of about 750 events (with a very large error) under a peak of about 300 - or signal to background of 1 to 3 and a statistical significance of 10 standard deviations. We show what this would look like by including this estimated background in Fig. 11.22. We discuss below how to reduce this background further.

The overall efficiency for this state, with cuts designed to achieve good background rejection, is about $2.5 \%$. Table 11.9 gives a calculation of the yield obtained in a one year run at a luminosity of $5 \times 10^{31} \mathrm{~cm}^{-2} \mathrm{~s}^{-1}$. We include in this calculation a triggering efficiency of $80 \%$ for those events which satisfy all the analysis cuts. This is consistent with what we expect to get from the dimuon trigger (70\%) 'or-ed' with the vertex trigger which recovers almost half of what the muon trigger failed to accept.

Table 11.9: Estimate of Yield for $B^{ \pm} \rightarrow K^{ \pm} \mu^{+} \mu^{-}$

\begin{tabular}{||l|c||}
\hline Quantity & Value \\
\hline Luminosity & $2.0 \times 10^{32} \mathrm{~cm}^{-2} \mathrm{~s}^{-1}$ \\
Running Time/year & $10^{7} \mathrm{~s}$ \\
$b \bar{b}$ cross section & $100 \mu \mathrm{b}$ \\
Total $b \bar{b}$ pairs produced & $2 \times 10^{11}$ \\
Total $B^{ \pm}$ & $1.4 \times 10^{11}$ \\
Branching fraction & $4 \times 10^{-7}$ \\
Efficiency (cuts above) & $2.5 \%$ \\
Total signal events & 1400 \\
Events satisfying trigger & 1120 \\
Estimated background & 3000 \\
Signal to background & 0.37 \\
Statistical significance & Standard deviations \\
of observation & $5 \%$ \\
Uncertainty on branching fraction & \\
\hline
\end{tabular}

There is much work left to be done, especially on understanding and rejecting the backgrounds. However, we have not exhausted all the weapons we have available to defeat these backgrounds. We have not optimized pointback cuts, $\chi^{2}$ cuts on the secondary vertex, or the $L / \sigma_{L}$ cut. Cuts that we have not yet employed include those which reject the event if there are other tracks that intersect the candidate secondary vertex and which reject the 
event if the daughter tracks of the candidate point either to the primary vertex or intersect with another track that is not in the primary (presumably from the other $b$ in the event). We have also not investigated kinematic cuts to see if any characteristic of the individual particle $p_{t}$ 's or the dimuon mass distribution, or the angular distributions of the particles in the decay rest frame can help discriminate signal from background. Nevertheless, the initial results are very encouraging.

According to the CDF II Technical Design Report [16], CDF expects a signal of 100$300 B^{+} \rightarrow \mu^{+} \mu^{-} K^{+}$events in collider RUN II. The signal-to-background is expected to be better than the 1 to 10 achieved in RUN I. However, CDF, because of its lack of particle identification for tracks above $1 \mathrm{GeV} / \mathrm{c}$, is exposed to background from all the pions in the event conspiring with the muons to create background. In $\mathrm{BTeV}$, because of the RICH, only the kaons can contribute to the background and there are fewer of them. The RICH reduces the background in this final state by about a factor of three, with a negligible loss of signal. BTeV will have intrinsically lower background than detectors without powerful particle identification on most final states. In light of the discussion above, it is reasonable to say BTeV's sensitivity for detecting rare $B$ decays is quite a bit better than CDF's. 


\section{$11.8 B_{s}$ Mixing with $B_{s} \rightarrow D_{s}^{-} \pi^{+}$and $B_{s} \rightarrow \psi \bar{K}^{* 0}$}

As discussed in section 1.10.1 the measurement of $B_{s}$ mixing allows a determination of $\left|V_{t s} / V_{t d}\right|$, which corresponds to one side of the $B$ unitarity triangle. While the theoretical discussion was given in terms of the mass splitting between the light and heavy $B_{s}$ mass eigenstates, $\Delta m_{s}$, the discussion here will be in terms of $x_{s}$, where $x_{s}=\Delta m_{s} / \Gamma$.

In this section the $x_{s}$ reach of $\mathrm{BTeV}$ will be demonstrated. This study was carried out in several steps, the first step being an MCFast based simulation of the BTeV detector, which was run on samples of both signal and background events. The output of this step was treated as real data and passed through a physics analysis program to determine the yield, the time resolution and the signal-to-background ratio in each mode. All of the preceding material is covered in section 11.8.1. This information was then passed to a separate program which computed the $x_{s}$ reach; this program is discussed in section 11.8.2.

\subsubsection{Yields, Resolutions and Signal-to-Background Ratios}

The mode for which BTeV has the most sensitivity to $x_{s}$ is $B_{s} \rightarrow D_{s}^{-} \pi^{+}$, where the $D_{s}^{-}$ decays either by $D_{s}^{-} \rightarrow \phi \pi^{-}, \phi \rightarrow K^{+} K^{-}$, or by $D_{s}^{-} \rightarrow K^{* 0} K^{-}, K^{* 0} \rightarrow K^{+} \pi^{-}$. Both of these $D_{s}^{-}$modes have narrow intermediate states and characteristic angular distributions, which can be used to improve the signal-to-background ratio. Another mode with good $x_{s}$ sensitivity is $B_{s} \rightarrow \psi \bar{K}^{* 0}, \psi \rightarrow \mu^{+} \mu^{-}, \bar{K}^{* 0} \rightarrow K^{-} \pi^{+}$. Although this mode is Cabibbo suppressed, many other factors are in its favor: the final state consists of a single detached vertex; it has a relatively low multiplicity; the state is triggerable with several independent strategies, including impact parameter triggers, secondary vertex triggers and muon or dimuon triggers [19]. While this mode does not have the reach of $D_{s}^{-} \pi^{+}$it does cover much of the range and it provides a powerful check with partly independent systematics.

In the simulation step, $p \bar{p} \rightarrow b \bar{b} X$ events were generated using Pythia 6.125. All charmed and bottom hadrons were decayed using QQ [18]. In order to simplify the analysis, all $B_{s}$ mesons were forced to decay into the signal channel and all $\bar{B}_{s}$ mesons were permitted to decay generically, but with mixing disabled and with the charge conjugate of the signal channel excluded. The effect of mixing is included in the final calculation of the $x_{s}$ reach, in section 11.8.2.

In the $D_{s}^{-} \pi^{+}$analysis, the first step was to impose track quality requirements on each reconstructed track in the event. All tracks were required to have a minimum number of hits, which is a simplified model of the fiducial requirements of the tracking system. No further requirements were placed on $\pi^{ \pm}$candidates. All $K^{ \pm}$candidates were required to lie within the fiducial acceptance of the RICH; any track which had hits in the forward tracking station which is located between the RICH and the EM Calorimeter, and which had a momentum in the range $3.0 \leq p \leq 70 \mathrm{GeV} / c$, was deemed to satisfy the fiducial requirements. It was assumed that all tracks inside the fiducial acceptance of the RICH could be correctly identified. The next step in the simulated analysis was to form all $K^{+} K^{-} \pi^{-}$combinations in an event. These combinations were fitted to a common vertex and a combination was 
retained as a $D_{s}^{-}$candidate provided the $\chi^{2}$ of the vertex fit was acceptable and provided the combination passed the mass and angle requirements of either the $\phi \pi^{-}$or the $K^{* 0} K^{-}$ hypothesis. Each $D_{s}^{-}$candidate was then vertexed with each remaining $\pi^{+}$candidate in the event and, provided the $\chi^{2}$ of the vertex fit was acceptable, the $D_{s}^{-} \pi^{+}$combination was retained as a $B_{s}$ candidate. Before computing the mass of the $B_{s}$ candidate, the mass of the $D_{s}^{-}$candidate was constrained to its PDG value.

For each event, a primary vertex was defined by looking at the generator information to identify the tracks that belonged in the primary vertex. These tracks were then fitted to a common vertex. This procedure gave the resolution of the primary vertex on an event by event basis. A separate study showed that the precision of the BTeV apparatus is sufficiently good that primary vertex pattern recognition will be highly efficient and of high purity. Once the primary vertex has been found, further requirements were placed on the $B_{s}$ candidates. They were required to have a detachment from the primary vertex of $L / \sigma_{L}>4.0$, where $L$ is the decay length of the $B_{s}$ candidate. They were required to have an impact parameter with the main vertex, $d$, of less than $3 \sigma_{d}$ and to have a $p_{T}$ balance along the line connecting the primary and secondary vertices of less than $1.0 \mathrm{GeV} / c$. The daughter $D_{s}^{-}$candidate was required to have $L / \sigma_{L}>4.0$.

Some interesting quantities for $B_{s}$ candidates are shown in Fig. 11.23. In part a) we see that the mass resolution on the $B_{s}$ is $19.2 \pm 0.8 \mathrm{MeV} / c^{2}$. Part b) shows the resolution on the proper decay time; the mean of this distribution, $50 \mathrm{fs}$, will be used, below, in the calculation of the $x_{s}$ reach. Part c) illustrates the main power of the BTeV apparatus, its excellent vertex resolution. Finally, part d) illustrates one of the important background suppression cuts; background combinations which pass the other cuts will usually not point back to the primary vertex.

In order to measure $x_{s}$, it is necessary to tag the flavor with which the $B_{s}$ meson was created. In the BTeV Expression of Interest [9] it was argued that opposite side $K^{ \pm}$tagging will have an efficiency of 0.12 with a mistag fraction of 0.25 and that opposite side muon tagging will have an efficiency of around 0.03, also with a mistag fraction of 0.25 . These tags correspond to $\epsilon D^{2}=0.075$. We expect that same side tagging of the $B_{s}$ can be a rather clean tagging method; however we have not yet investigated this tagging method in sufficient detail to use it in this analysis. As discussed in section 11.3, it is expected that the total tagging power will reach $\epsilon D^{2}=0.10$. The additional power will come from tags with much higher efficiency but with much higher mistag fractions. For purposes of this work, only the clean tags will be considered.

Finally, the BTeV Level 1 trigger simulation was run on the $B_{s} \rightarrow D_{s}^{-} \pi^{+}$sample. Using the standard requirement that there be two tracks detached by at least $4 \sigma$ and with $p_{T}>$ $0.5 \mathrm{GeV} / c^{2}$, we find that $60 \%$ of the events which pass all analysis cuts also pass the trigger.

Table 11.10 shows a breakdown of the efficiencies measured by MCFast, as well as listing all assumptions which went into the computation of the yield. In one year we expect that 16,100 events will survive all analysis cuts, have their birth flavor tagged and be triggered.

It is believed that the dominant source of backgrounds will be events of the form $B \rightarrow D_{s}^{-} X$, where $B$ may be any of $B_{u}, B_{d}$ or $B_{s}$. The background combinations arise 


\begin{tabular}{llr}
\hline Quantity & Value & $\begin{array}{c}\text { Yield } \\
\text { Events/year) }\end{array}$ \\
\hline \hline Luminosity: & $2 \times 10^{32} \mathrm{~cm}^{-2} \mathrm{~s}^{-1}$ & \\
One Year: & $10^{7} \mathrm{~s}$ & \\
$\sigma_{b \bar{b}}:$ & $100 \mu \mathrm{b}$ & \\
$\mathcal{B}\left(B_{s} \rightarrow D_{s}^{-} \pi^{+}\right):$ & $3.0 \times 10^{-3}$ & \\
$\mathcal{B}\left(D_{s}^{-} \rightarrow \phi \pi^{-}\right):$ & 0.030 & \\
$\mathcal{B}\left(D_{s}^{-} \rightarrow K^{* 0} K^{-}\right):$ & 0.036 & \\
$\mathcal{B}\left(\phi \rightarrow K^{+} K^{-}\right):$ & 0.49 & \\
$\mathcal{B}\left(K^{* 0} \rightarrow K^{+} \pi^{-}\right):$ & 0.67 & \\
$\mathcal{B}\left(\bar{b} \rightarrow B_{s}\right)$ & 0.13 & \\
$\epsilon($ Geometric $)$ & 0.08 & 108,000 \\
$\epsilon\left(\right.$ Analysis cuts $\left.: \phi \pi^{-}\right)$ & 0.40 & 16,100 \\
$\epsilon\left(\right.$ Analysis cuts $\left.: K^{* 0} K^{-}\right)$ & 0.33 & \\
$\epsilon($ Trigger $)$ & 0.60 & \\
$\epsilon($ Tag $)$ & 0.15 & \\
Mistag fraction & 0.25 & \\
$S / B$ & $3: 1$ & \\
$\sigma($ Proper Decay time $)$ & $50 \mathrm{fs}$ & \\
\hline
\end{tabular}

Table 11.10: Projected Yield for $B_{s} \rightarrow D_{s}^{-} \pi^{+}$in one year of BTeV running. The numbers in the third column give the expected yield when all of the factors down to and including that line have been considered. The branching fraction $\mathcal{B}\left(B_{s} \rightarrow D_{s}^{-} \pi^{+}\right)$was estimated to be the same as $\mathcal{B}\left(B_{d} \rightarrow D^{-} \pi^{+}\right)$. The value for $\mathcal{B}\left(\bar{b} \rightarrow B_{s}\right)$ was obtained from [14]. All efficiencies are multiplicative. The tagging efficiency and mistag fraction are quoted from the sum of clean tagging modes only.

when a true $D_{s}^{-}$combination is combined with some other track in the event. When 1 million $B \rightarrow D_{s}^{-} X$ events were passed through MCFast and the full analysis chain only 8 entries remained in a mass window 6 times larger than the mass window used to select signal $B_{s}$ candidates. From this we estimate that the signal-to-background ratio in this channel is 8.4:1. We have not yet investigated the background from direct charm production; while direct charm production is about 10 times more frequent than production of charm from $B$ decay, we believe that the required two vertex topology will greatly reduce the direct charm background. In the end we expect that background from $B \rightarrow D_{s}^{-} X$ will dominate. We have yet to do a full treatment of the impact of multiple interactions in one beam crossing. In the end we make a conservative estimate that we will achieve a signal-to-background ratio of no worse than $3: 1$.

The analysis of the $\psi \bar{K}^{* 0}$ mode proceeded similarly to that of the $D_{s}^{-} \pi^{+}$mode and only the important differences will be summarized here. In order to be considered a muon candidate, a track was required to have a momentum $p>5.0 \mathrm{GeV} / c$ and to penetrate the 
hadron filter, thereby leaving hits in the most downstream muon chambers. In this decay mode the topology is simpler, with all four tracks coming from a single vertex. A $\mu^{+} \mu^{-} K^{-} \pi^{+}$ combination was retained if the confidence level of fitting all four tracks to a single vertex was greater than 0.005 . It was also required the that resonant substructure requirements be satisfied. Combinations were considered for further analysis provided $L / \sigma_{L}>10$ and $d<3 \sigma_{d}$. Each of the four $B_{s}$ grand-daughters were required to have an impact parameter with the primary vertex, $d>2 \sigma_{d}$. Candidates with poor time resolution were rejected by demanding $\sigma_{t} \leq 0.09 \mathrm{ps}$. After constraining the mass of the $\psi$ to its PDG value, the mass resolution of the $B_{s}$ was determined to be $12.0 \pm 0.6 \mathrm{MeV} / c^{2}$. For the $\psi \bar{K}^{* 0}$ mode, the mean resolution on the proper decay time was found to be $38 \mathrm{fs}$.

The BTeV Level 1 trigger simulation was run on the $\psi \bar{K}^{* 0}$ sample, using the same trigger conditions as described above. We find that, of the candidates which pass all analysis cuts, $69 \pm 3 \%$ also pass the trigger; the error is purely statistical. However this mode can also be triggered by a single muon or a di-muon trigger and we estimate that the total trigger efficiency will be approximately $85 \%$.

By far the dominant background is expected to come from decays of the form $B \rightarrow \psi X$, $\psi \rightarrow \mu^{+} \mu^{-}$. An MCFast simulation of 500,000 such decays gives a predicted signal-tobackground level of about 2:1. Some sources of background which one might at first think to be important turn out not to be a problem. First, the more copious $B_{s} \rightarrow \psi \phi$ final state is not a significant source of background because of the excellent particle ID provided by the RICH system. Second, the mass resolution is sufficient to separate the much more copious decay $B_{d} \rightarrow \psi \bar{K}^{* 0}$.

Finally, the expected yield can be increased by a factor of at least $50 \%$ by using the mode $\psi \rightarrow e^{+} e^{-}$. This mode will have an efficiency for secondary vertex triggers which is comparable to that for $\psi \rightarrow \mu^{+} \mu^{-}$and, an as yet unspecified, electron trigger is part of the $\mathrm{BTeV}$ baseline design.

Table 11.11 shows a breakdown of the efficiencies measured by MCFast, as well as listing all assumptions which went into the computation of the expected yield. In one year we expect that 1030 events will survive all analysis cuts, have their birth flavor tagged and be triggered.

\subsubsection{Computation of the $x_{s}$ Reach}

The final step in the study was to use a mini-Monte Carlo [20] to study the $x_{s}$ reach of $\mathrm{BTeV}$. This mini-Monte Carlo generates two lifetime distributions, one for mixed events and one for unmixed events, smears the distributions and then extracts a measured value of $x_{s}$ from a simultaneous fit the two distributions. The time smearing is Gaussian, using the mean time resolutions determined above. The model includes the effects of mistagging, background under the signal, and the minimum time cut which is implied by the $L / \sigma_{L}$ cut. It is assumed that the lifetime distribution of the background is an exponential with the same mean lifetime as that of the $B_{s}$.

Figures 11.24a) and b) show the proper time distributions which result from one run of 


\begin{tabular}{llr}
\hline Quantity & Value & \multicolumn{1}{c}{$\begin{array}{c}\text { Yield } \\
\text { (Events/year) }\end{array}$} \\
\hline \hline Luminosity: & $2 \times 10^{32} \mathrm{~cm}^{-2} \mathrm{~s}^{-1}$ & \\
One Year: & $10^{7} \mathrm{~s}$ & \\
$\sigma_{b \bar{b}}:$ & $100 \mu \mathrm{b}$ & \\
$\mathcal{B}\left(B_{s} \rightarrow \psi \bar{K}^{* 0}\right):$ & $8.5 \times 10^{-5}$ & \\
$\mathcal{B}\left(\psi \rightarrow \mu^{+} \mu^{-}\right):$ & 0.061 & 180000 \\
$\mathcal{B}\left(\bar{K}^{* 0} \rightarrow K^{-} \pi^{+}\right):$ & 0.667 & \\
$\mathcal{B}\left(\bar{b} \rightarrow B_{s}\right)$ & 0.13 & 5508 \\
$\epsilon($ Geometric $)$ & 0.18 & \\
$\epsilon($ Analysis cuts $)$ & 0.17 & 7680 \\
$\epsilon($ Trigger $)$ Tracking only & 0.60 & 1030 \\
$\epsilon($ Trigger $)$ Total & 0.85 & \\
$\epsilon($ Tag $)$ & 0.15 & \\
Include $\psi \rightarrow e^{+} e^{-}$ & & \\
Mistag fraction & 0.25 & \\
$S / B$ & $2: 1$ & \\
$\sigma($ Proper Decay time $)$ & $38 \mathrm{fs}$ & \\
\hline
\end{tabular}

Table 11.11: Projected Yield for $B_{s} \rightarrow \psi \bar{K}^{* 0}$ in one year of BTeV running. The numbers in the third column give the expected yield when all of the factors down to and including that line have been considered. The estimate for $\mathcal{B}\left(B_{s} \rightarrow \psi \bar{K}^{* 0}\right)$ was obtained from [19] and that for $\mathcal{B}\left(\bar{b} \rightarrow B_{s}\right)$ was obtained from [14]. The trigger efficiency is quoted as a fraction of those events which pass the analysis cuts. The tagging efficiency and mistag fraction are quoted from the sum of clean tagging modes only. 
the mini-Monte Carlo for a generated value of $x_{s}=40$. The simulation is for the decay mode $B_{s} \rightarrow D_{s}^{-} \pi^{+}$for one year of BTeV running; that is for 16,100 signal events, with $S / B=3: 1$, a time resolution of $50 \mathrm{fs}$, and a $25 \%$ mistag rate. It was also required that the smeared decay time be greater than $200 \mathrm{fs}$, which simulates the cut of $L / \sigma_{L}>4$. Part a) shows the proper time distribution for unmixed decays while part b) shows the distribution for mixed decays. Part c) of the figure shows, as a function of $x_{s}$, the value of the unbinned negative log likelihood function computed from the simulated events. A step of 0.5 in the negative log likelihood function determines the $1 \sigma$ error bounds and a line is drawn across the figure at the level of the $5 \sigma$ error bound. A clear minimum near the generated value of $x_{s}$ is observed and the likelihood function determines the fitted value to be $x_{s}=40.0 \pm 0.04$.

The error returned by the fit was checked in two ways. First, an ensemble of miniMonte Carlo experiments was performed and the errors were found to correctly describe the dispersion of the measured values about the generated ones. Second, the errors returned by the fit were found to be approximately equal to the Cramer-Rao minimum variance bound [20].

The mini-Monte Carlo also showed how the limiting $x_{s}$ sensitivity of the experiment is approached. As the number of events in a trial is reduced, the negative log likelihood function becomes more and more ragged and the secondary minima approach the significance of the global minimum. Eventually there are secondary minima which reach depths within 12.5 units of negative log likelihood ( $5 \sigma$ ) of the global minimum. When this happens in a sufficiently large fraction of the trials, one must conclude that only a lower limit on $x_{s}$ can be established. In the region of the parameter space which was explored, the absolute error on $x_{s}$ was approximately 0.1 when this limit was reached. This was independent of the generated value of $x_{s}$.

It is awkward to map out the $x_{s}$ reach of the apparatus by running a large ensemble of mini-Monte Carlo jobs. Instead the following automated procedure was used. Following ideas from McDonald [21], the sum over events in the likelihood function was replaced with an integral over the parent distribution. Because the parent distribution does not have any statistical fluctuations, the fluctuations in the likelihood function are removed, leaving only the core information. An example of such a likelihood function is shown in Fig. 11.25.

A likelihood function computed in this way has the property that it scales linearly with the number of events being simulated. This can be stated formally as follows. Let $x_{0}$ denote the generated value of $x_{s}$ and let $\mathcal{L}\left(x ; x_{0}, N\right)$ denote the value of the likelihood function, evaluated at $x$, for a sample which has a true value of $x_{0}$ and which contains $\mathrm{N}$ events. Then,

$$
\mathcal{L}\left(x ; x_{0}, N\right)=N \mathcal{L}\left(x ; x_{0}, 1\right)
$$

Now, one can define the significance of the minimum, $n$, as,

$$
n^{2}=2.0 N\left[\mathcal{L}\left(\infty ; x_{0}, 1\right)-\mathcal{L}\left(x_{0} ; x_{0}, 1\right)\right] .
$$

For practical purposes $\infty$ was chosen to be 160 . If we did not have to worry about the missing statistical fluctuations it would be normal to define a significant signal as $5 \sigma$, or 
$n^{2}=25$. Instead, we defined significance, somewhat arbitrarily, as $n^{2}=31.25$, which allows for the usual $5 \sigma$ plus a downwards fluctuation of up to $2.5 \sigma$ anywhere else in the plot. Equation 11.18 was solved for $N$, which was then converted into the running time required to obtain a measurement of $n \sigma$ significance. This procedure was repeated for many different values of $x_{0}$ to obtain Fig. 11.26. The solid line shows, for the $D_{s}^{-} \pi^{+}$mode, the number of years needed to obtain a $5 \sigma$ significant measurement, where " $5 \sigma$ " means $n^{2}=31.25$. Had we ignored the missing fluctuations and kept $n^{2}=25$, the $x_{s}$ reach between 1 and 3 years would have been shifted higher by about 3 or 4 units of $x_{s}$. For small values of $x_{s}$ the difference is not visible. The dashed line shows the same information for the $\psi \bar{K}^{* 0}$ mode, for which the effect of setting $n^{2}=31.25$ is similar.

Inspection of Fig. 11.26 shows that, using the $D_{s}^{-} \pi^{+}$mode, $\mathrm{BTeV}$ is capable of observing all $x_{s}$ less than 60 in one year of running. Moreover there are two changes in the pipeline which we expect will improve this reach: we expect to be able to build pixel detectors which have less mass than those in the present design and we have not yet full exploited the tagging power of BTeV. 

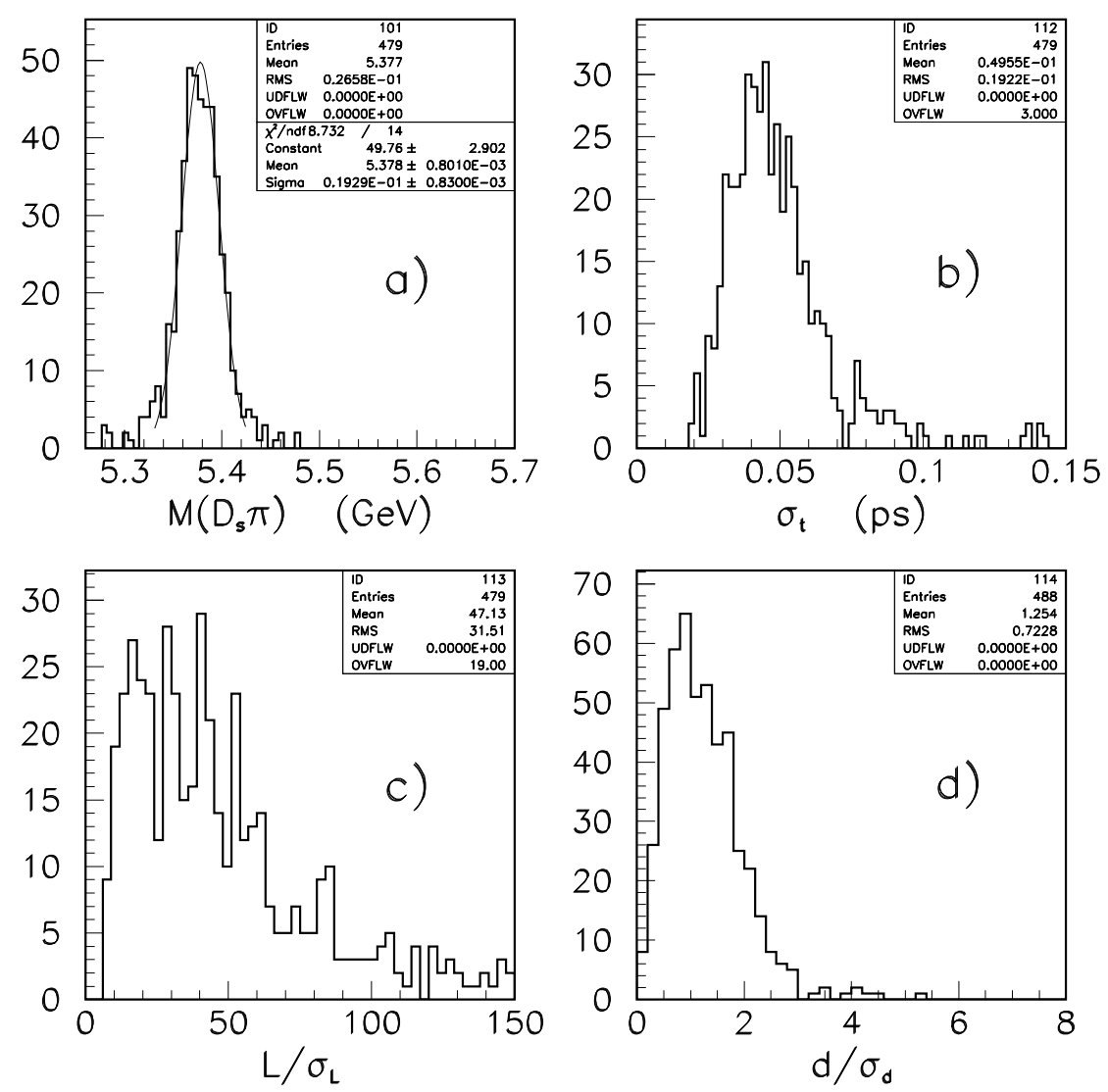

Figure 11.23: Distributions of properties of $B_{s} \rightarrow D_{s}^{-} \pi^{+}$candidates. For candidates which survive all cuts, part a) shows the invariant mass of the $B_{s}$ candidate, part b) shows the resolution on the proper decay time and part c) shows the detachment of the $B_{s}$ from the primary vertex. Part d) shows the significance of the impact parameter, $d$, of the $B_{s}$ candidate with the primary vertex; for the final selection it was required that $d / \sigma_{d} \leq 3.0$. 


\section{Simulation of $X_{s}=40$ with 50 fs Smearing}
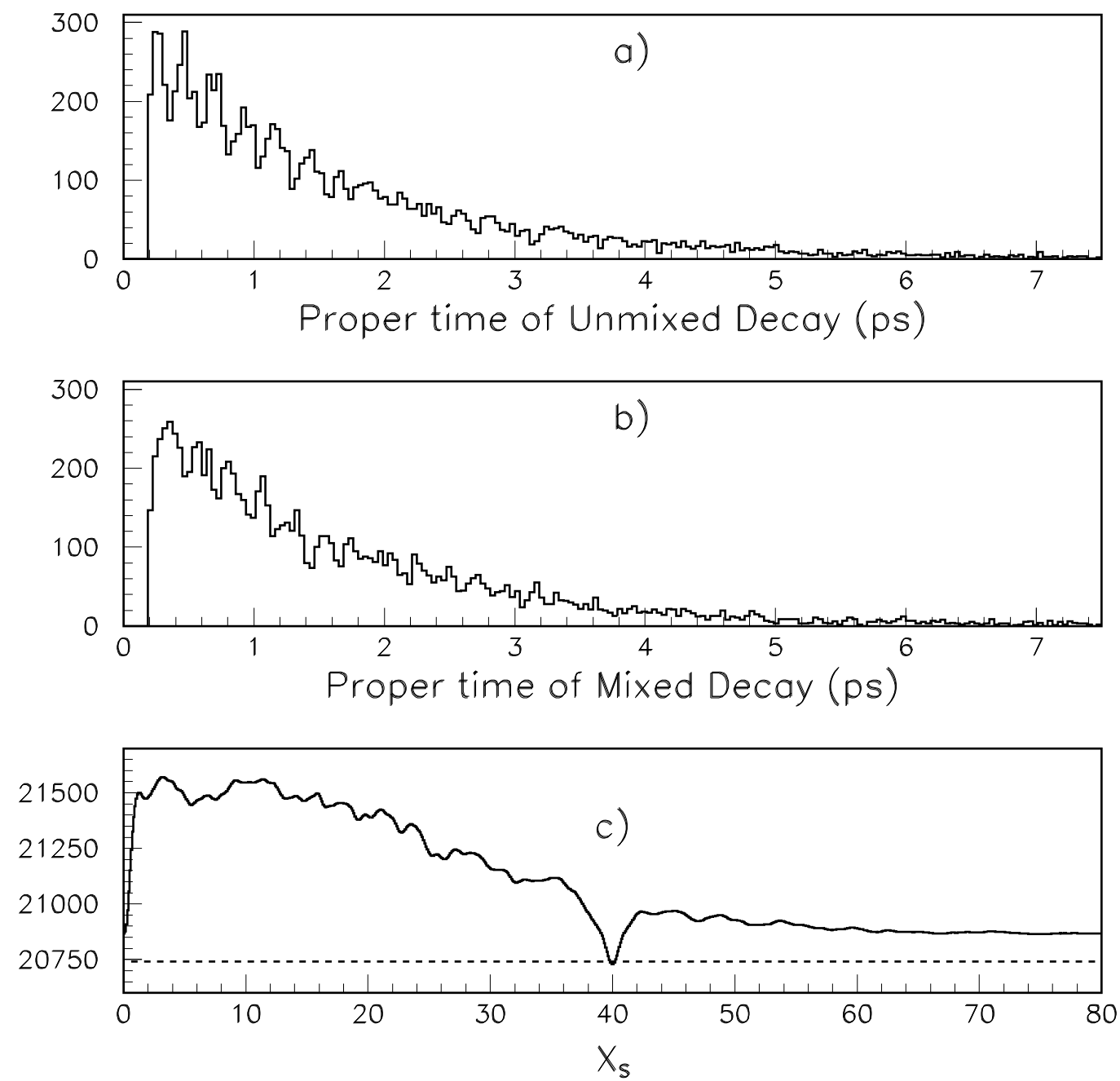

Figure 11.24: Mini-Monte Carlo Proper Lifetime plots of a) unmixed and b) mixed decays for one BTeV year of $B_{s} \rightarrow D_{s}^{-} \pi^{+}$with $x_{s}=40$, a mistag fraction of 0.25 , a time smearing of $50 \mathrm{fs}$, a signal-to-background ratio of $3: 1$ and a minimum time cut of $200 \mathrm{fs}$. The oscillations are prominent. Part c) shows the negative log likelihood as a function which was obtained from the entries in parts a) and b). A prominent minimum is seen at the generated value of $x_{s}$. The dashed line marks the level above the minimum which corresponds to $5 \sigma$ significance. 


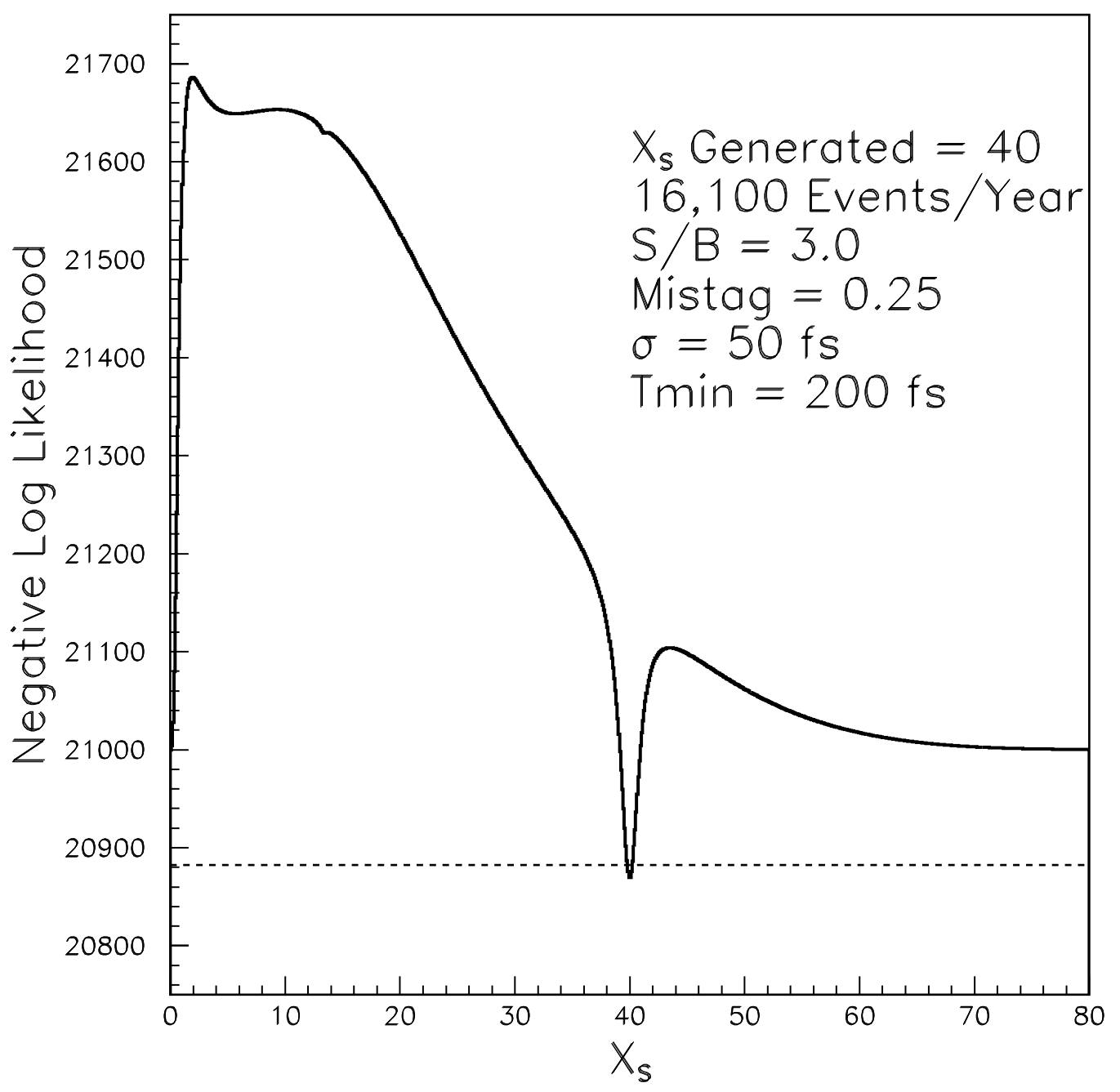

Figure 11.25: The same likelihood function as in part c) of the previous figure but using the calculation described in the text. The overall shape is the same but the statistical fluctuations have been removed. There is also an overall level shift which is related to the goodness of fit in the previous figure. 


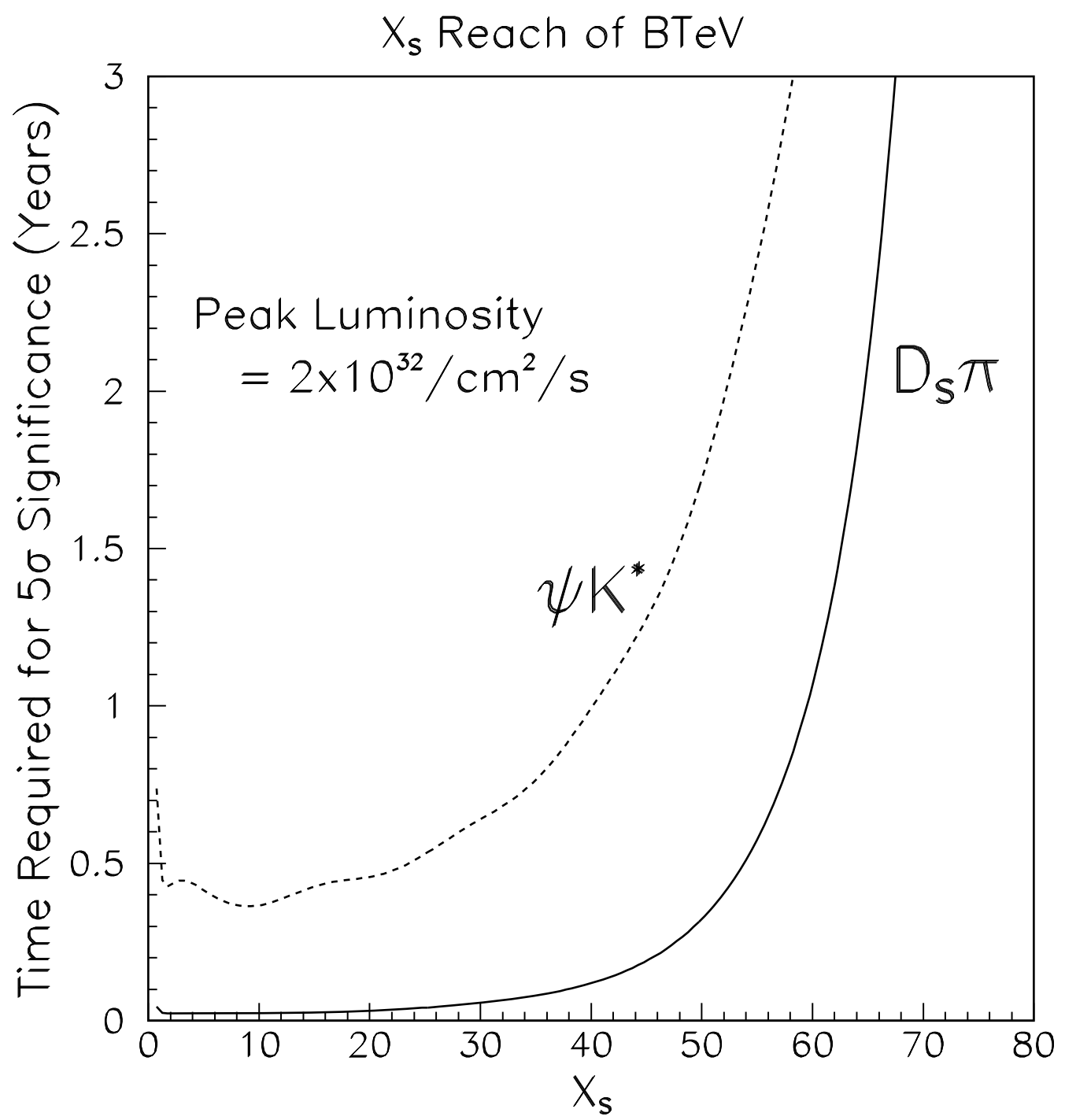

Figure 11.26: The $x_{s}$ reach of the BTeV detector. The curves indicate the number of years of running which are required to make a measurement of $x_{s}$ with a statistical significance of $5 \sigma$. The curves are shown for two different decay modes. 


\subsection{Charm Physics with the BTeV Detector}

Searches for mixing, CP violation, and rare and standard model forbidden decays in the charm sector (as discussed in chapter 2) provide a sensitive and possibly unique window to physics beyond the standard model. At a luminosity of $2 \times 10^{32} \mathrm{~cm}^{-2} \mathrm{~s}^{-1}$ the Tevatron produces on the order of $2 \times 10^{12}$ charm quark pairs $(c \bar{c})$ a year (although the $c \bar{c}$ cross section at $\sqrt{s}=2 \mathrm{TeV}$ is not well known, a relatively conservative estimate for it is $1 \mathrm{mb}$ ). Such a sample represents an opportunity unavailable anywhere except at hadron colliders, although this potential has yet to be tapped.

Charm physics simulation studies are not as advanced as the beauty studies presented previously in this report. The simulations presented here used a detector geometery with less material in the pixels and tracking stations than we used for the $b$ studies, and did not use the square hole pixel geometry that substantially improves the acceptance. We estimate that the efficiencies will not change by more than $\pm 10 \%$. In addition, no effort has yet been made to optimize triggers for charm modes. These studies are beginning now.

As a first step in studying charm physics in collider mode, we have computed the geometrical and tracking acceptances of the baseline BTeV detector (see Part II) for several charm meson decay modes using the MCFast package. We are interested in: (i) the inclusive acceptance (or "single charm"), which refers to the probability to detect all daughters of a charm or anti-charm meson, and (ii) the "associated" acceptance, defined as the probability to detect both secondary (charm and anti-charm) vertices.

At the current time we have only considered all-charged final states such as $D \rightarrow K^{-}+n \pi$ and $D^{0} \rightarrow K^{+} K^{-}$. The recoiling anti-charm (or charm) hadron decays according to PDG branching ratio values. The QQ package has been used to simulate these decays. To define our acceptance, we require that each daughter in a decay leave a minimum number of hits in the spectrometer, which directly reflects our ability to reconstruct the corresponding tracks. To be considered "accepted", a track must produce at least 4 hits in the vertex detector (which implies that it must pass through at least two stations) and 6 hits in the forward tracker.

The inclusive acceptance for $D^{0} \rightarrow K^{-} \pi^{+}, D^{+} \rightarrow K^{-} \pi^{+} \pi^{+}$and $D^{0} \rightarrow K^{-} \pi^{+} \pi^{-} \pi^{+}$is $24 \%, 15 \%$, and $11 \%$, respectively. While the acceptance does depend on decay multiplicity, it does not depend strongly on the strangeness content of the daughter tracks (e.g., kaons vs pions), so the acceptance for Cabibbo suppressed decays is similar to that for Cabibbo favored ones of equal multiplicity. The acceptance for $D^{*+} \rightarrow D^{0} \pi^{+}, D^{0} \rightarrow K^{-} \pi^{+}, K^{-} \pi^{+} \pi^{-} \pi^{+}$is noticeably lower than the acceptance for final states of the same multiplicity, due to the much softer momentum of the "bachelor" pion from the $D^{*+}$ decay. This can be mitigated by removing the requirement that this pion reach the forward tracker system. The resolution in $\Delta M=M\left(D^{*}\right)-M\left(D^{0}\right)$ deteriorates due to the diminished momentum resolution for bachelor pions not tracked in the forward tracker (see Fig. 11.27). The acceptance for these decays is approximately $17 \%$

The associated acceptance is defined to be the probability that one decay vertex is fully reconstructed and the second decay vertex is at least partially reconstructed. To calculate 
Yield

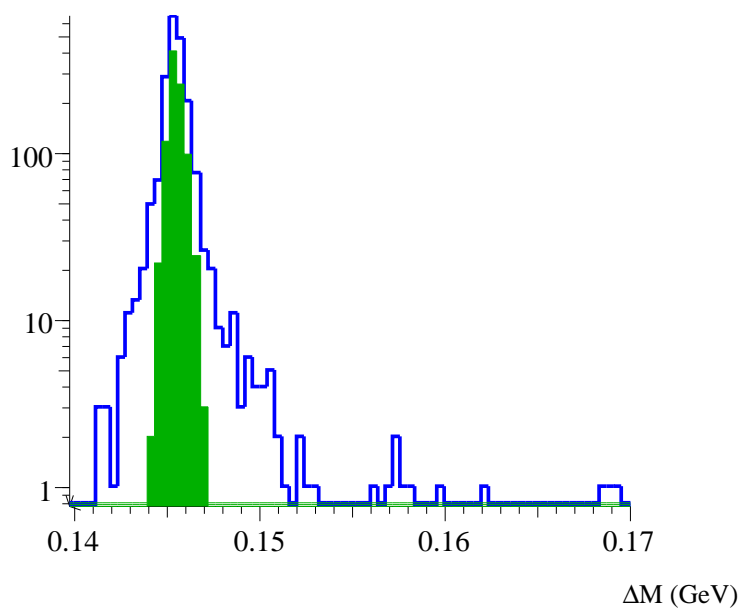

Figure 11.27: The $\Delta M=M\left(D^{*}\right)-M\left(D^{0}\right)$ mass plot, as estimated by the MCFast package, for the baseline detector, where the $D^{0}$ decays to $K^{-} \pi^{+}$and $K^{-} \pi^{+} \pi^{-} \pi^{+}$(with relative branching ratios according to PDG values). The solid shaded histogram refers to the case where the soft pion from the $D^{*}$ decays is detected by the forward spectrometer, while the solid line histogram refers to the case where this soft pion is detected only by the pixel detector.

this acceptance we again use Monte Carlo events where one daughter decays to all-charged tracks, and the other daughter decays according to PDG branching ratios. We then require that the all-charged track mode be fully reconstructed (just as in the inclusive calculation above). The other charm decay must have at least two tracks reconstructed. These tracks are required to leave at least 9 hits in the vertex and forward trackers. The acceptances for the $K^{-} \pi^{+}, K^{-} \pi^{+} \pi^{+}$and $K^{-} \pi^{+} \pi^{+} \pi^{-}$modes are $15 \%, 12 \%$ and $5 \%$, respectively. These acceptances are typically an order of magnitude higher than those observed in fixed target experiments, simply because of the strong kinematical correlation between the $c \bar{c}$ pair (if the charm meson is boosted into one of the arms of the spectrometer, it is likely that the anti-charm also gets a similar boost and is observed in the same arm). One of the benefits is cleaner primary vertices than in a fixed target configuration.

\subsubsection{Direct CP Violation in the Charm Sector}

In order to estimate the sensitivity of the $\mathrm{BTeV}$ spectrometer for a possible observation of Direct CP violation in the charm sector, we have studied in more detail the acceptance and background rejection capabilities for a specific Cabibbo-suppressed decay mode: $D^{*}(2010)^{+} \rightarrow D^{0} \pi^{+}, D^{0} \rightarrow K^{+} K^{-}$. The geometrical acceptance of the vertexing and tracking systems has already been presented in the previous subsection. Now, we concentrate on 
vertexing cuts, overall sensitivity and systematic errors.

Following the constrained vertex reconstruction algorithm, used by the experiment E687 ${ }^{1}$ [23], $K^{+} K^{-}$tracks with a relatively large invariant mass are first selected in the event. These are MCFast "offline tracks", i.e. simulated reconstruction tracks for which we have a covariance matrix allowing us to compute a vertex error and $\chi^{2}$. Note that such tracks are not the outcome of a full pattern recognition package in the forward tracker and the pixels. However, based on studies done for the pixel-based trigger, we know that the level of confusion in this vertex detector is not likely to be a limitation, thanks to its high granularity.

In addition to a minor vertex quality cut on the $D^{0}$ candidate vertex, the charged kaons are required to be positively identified as such by the Cerenkov system. We have assumed 97.5\% percent positive identification for momenta between 3 and $70 \mathrm{GeV} / \mathrm{c}$ and no identification outside this range. Under these conditions, the effective $D^{0}$ acceptance is $17.4 \%$ (the tracking acceptance for this final state is $25 \%$ ).

The soft pions from the $D^{*}$ decays are not required to be reconstructed by the forward tracker and are mostly reconstructed by the vertex detector. At least six hits are required on this soft pion track. No particle identification (P.I.D.) is required. The final tracking and P.I.D. acceptance for the fully reconstructed final state $D^{*} \rightarrow D^{0} \pi^{+}, D^{0} \rightarrow K^{+} K^{-}$is $11 \%$. The primary vertex is reconstructed with great efficiency independent of the $D^{0}$ vertex, due to the relatively large multiplicity in these events (on average, the multiplicity in this reconstructed primary vertex is about 12). The last cut applied in this simple analysis is the usual detachment between the primary and secondary vertices, $L / \sigma_{L}$. The distribution of this quantity for the generated $D^{0} \rightarrow K^{+} K^{-}$decay vertices is shown in Fig. 11.28, and gives us a final acceptance of $4.8 \%, 3.0 \%, 1.1 \%$ and $0.2 \%$ cutting at $L / \sigma_{L}>3,5,10$ and 20 respectively.

We assume that the dominant background comes from charm. The $K^{+} K^{-}$mass plot corresponding to 100,000 generated charm events is shown in Fig. 11.29, cutting at moderate values of $L / \sigma_{L}$. The observed signal to noise is acceptable at this stage, and should improve after imposing the following vertex cuts:

- A tighter $\chi^{2}$ cut on the secondary vertex

- A "point back" cut, requiring that the $D^{0}$ track points back to the primary vertex, or conversely, that the distance of closest approach (DCA) to this primary vertex vanishes within measurement errors.

- The $K^{-}$and $K^{+}$tracks should not point back to the primary.

- Isolation cut: Since the $D^{0}$ decay we are attempting to detect is fully reconstructed, no other tracks should have a small DCA with respect to this $D^{0}$ vertex.

- If another secondary vertex is found, make sure that $K^{+} K^{-}$tracks forming the $D^{0}$ vertex do not point back to that secondary vertex.

\footnotetext{
${ }^{1}$ A Charm photoproduction experiment which ran at Fermilab, 1987-1991
} 


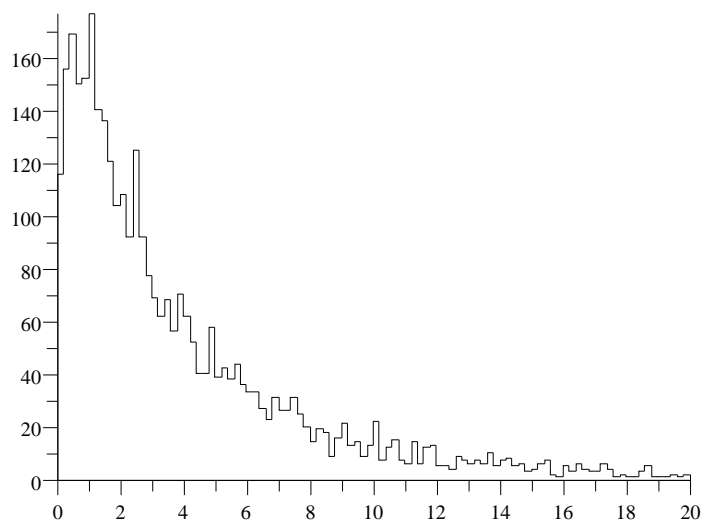

$\mathrm{L} / \sigma_{\mathrm{L}}$

Figure 11.28: The $L / \sigma_{L}$ distribution for the generated $D^{0} \rightarrow K^{+} K^{-}$

All these cuts have been used in one form or another by various fixed target experiments for this decay mode and many others.

An effective acceptance of $0.2 \%$ could be obtained with acceptable signal to noise. This includes a trigger efficiency of $10 \%$ (we hope to increase the trigger efficiency to this level with optimization for charm). Assuming a total charm $\mathrm{D}^{*}$ cross section of $1 \mathrm{mb}, \mathrm{BTeV}$ will produce and detect about 10 million such decays in $10^{7}$ seconds running at a luminosity of $2 \times 10^{32} \mathrm{~cm}^{-2} \mathrm{~s}^{-1}$. In principle, this will give us a statistical error of roughly $3 \times 10^{-4}$ on a $D^{*+} / D^{*-}$ asymmetry assuming no background. We hope to achieve sensitivities below $1 \times 10^{-3}$ even when backgrounds are present.

We believe a similar level of sensitivity is possible in searches for direct CP violation in $D^{+}$decays as well. Although there is no $D^{*}$ tag to reduce backgrounds, the longer lifetime of the $D^{+}$allows harder $L / \sigma_{L}$ cuts, and you get back the efficiency lost by requiring the bachelor pion to be found.

A proposed improvement in statistical accuracy of $1 \times 10^{-4}$ is meaningless if systematic errors are not seriously taken into account. In photoproduction, the systematic errors were as big as the statistical ones, and were primarily due to uncertainties in estimating the effective acceptance versus true lifetime and to background subtraction. Such uncertainties originate in poorly defined primary vertices, a problem we hope to resolve in a collider environment where the clean track multiplicity is higher. In photoproduction a dominant source of background is secondary interactions in the target, which is not a problem at the collider. 


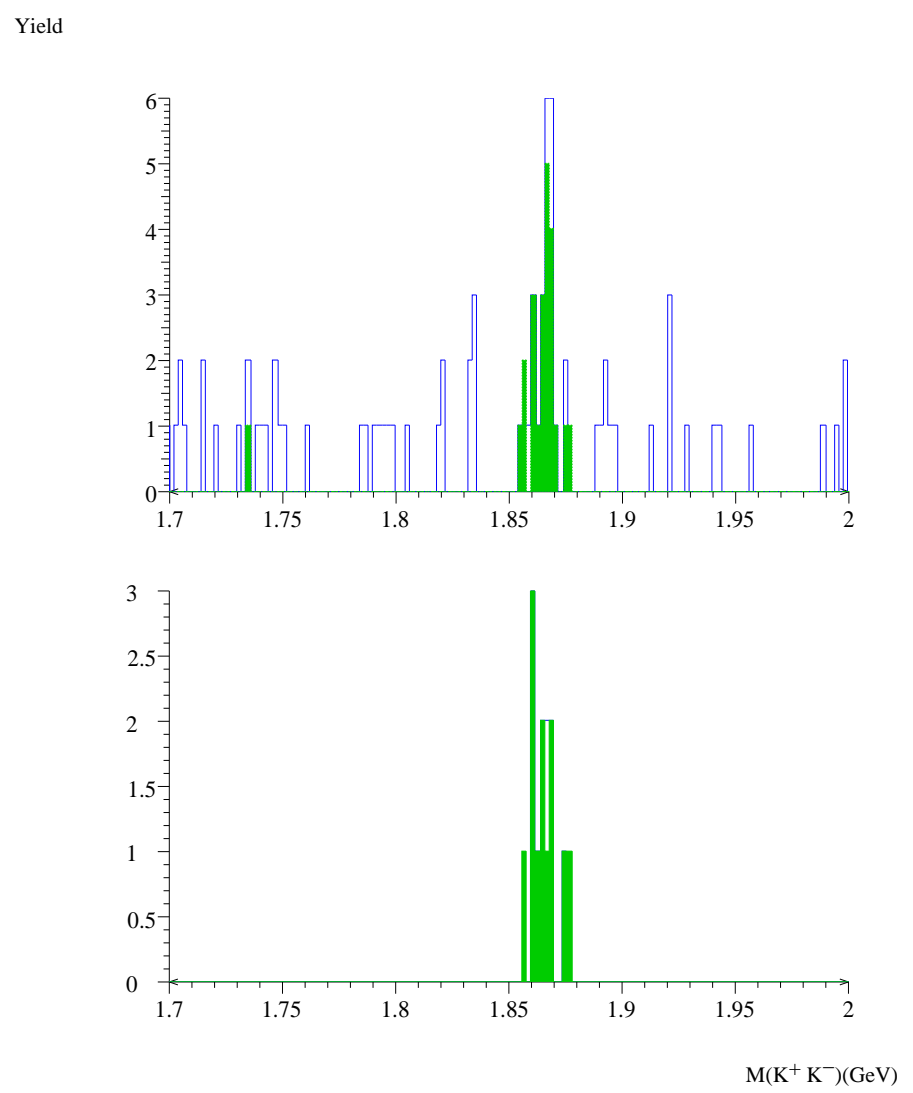

Figure 11.29: The $K^{+} K^{-}$invariant mass distribution from 100,000 generic Charm events, where the filled histogram contains the signal $D^{*} \rightarrow D^{0} \pi, D^{0} \rightarrow K^{+} K^{-}$(charge conjugation implied), while the open histogram contains all recorded events in the sample. A $\mid M\left(D^{*}\right)-$ $M\left(D^{0}\right)-0.1456 \mid<2 \mathrm{MeV}$ cut is imposed. In the top (bottom) plot a requirement of $L / \sigma_{L}>0\left(L / \sigma_{L}>3\right)$ is made. 


\subsection{Charm physics with a wire target}

We anticipate that we will be able to run with a wire target before we are able to run in collider mode at $\mathrm{C} 0$. This will allow us to take data for charm physics at an earlier date, before the full BTeV detector is ready to install. The charm physics program with the full $\mathrm{BTeV}$ detector is discussed in Section 11.9; here we discuss the physics potential of the partially instrumented detector. We consider a thin target in the halo of the proton beam and a vertex detector of some combination of pixel and microstrip planes, covering the same downstream geometrical acceptance as the final detector. Although the full BTeV trigger may not be available at this time, we discuss a possible trigger for this mode of running.

\subsubsection{Target and interaction rate}

As discussed by Marriner [24], a thin target in the beam halo will intercept beam particles that have large horizontal betatron amplitudes. Such particles will pass through the target a number of times, suffering multiple coulomb scattering until they either have a nuclear interaction in the target, or are scattered so far that they are intercepted at an aperture limit of the accelerator. This analysis suggests it is advantageous to have a low- $Z$ material for the target, e.g. beryllium or carbon. The interaction rate is then determined not by the thickness of the target, but by the rate at which particles leave the circulating beam. Marriner estimates an interaction rate on the order of $10^{5} \mathrm{~s}^{-1}$. This is likely to be a lower limit, which could be increased by a factor of 10 or more if low $\beta$ is available in $\mathrm{C} 0$ at the start of Run II. We propose further study of these considerations jointly with the Beams Division.

\subsubsection{Vertex detector and trigger}

An advantage of fixed-target mode is that the $z$-coordinate of the primary interaction is localized to the target. Thus the vertex detector only needs to cover about half the length that it needs in collider mode. The steps of the trigger which find where the interaction took place are also simplified. The algorithms of the full trigger can be tested on a reduced inventory of processors.

We have been studying a simplified trigger based on $p_{t}$ of the tracks for use in this part of the charm program. It has been established that this is a powerful signal for charm in fixed-target interactions [25], and this is confirmed in our simulations using Pythia and MCFast. It has the following advantages in the early stages of the evolution of the detector and trigger:

- The overall interaction rate may initially be lower than in collider mode, so the requirements on performance of the trigger are reduced, since a more modest rejection is acceptable at Level I.

- Charm hadron mean lifetimes are shorter than $B$ hadrons, so a $p_{t}$ trigger will be more efficient than a vertex trigger. 
- $D^{*} \rightarrow D \pi$ can be isolated without strong vertexing cuts offline, so it is advantageous not to impose these at the trigger stage.

- Tests of vertexing software can be made on relatively unbiased samples.

We have studied two versions of a $p_{t}$ trigger: (1) using the track with the highest $\left|p_{t}\right|$ in the event, and (2) using the sum of $\left|p_{t}\right|$ for all reconstructed charged tracks. Each of these can give some rejection of background, but a combination of the two measurements can give an enhanced rejection. For example, a trigger efficiency of $60 \%$ for charm can be achieved with a rejection on the order of 10 , as shown in figure 11.30 .

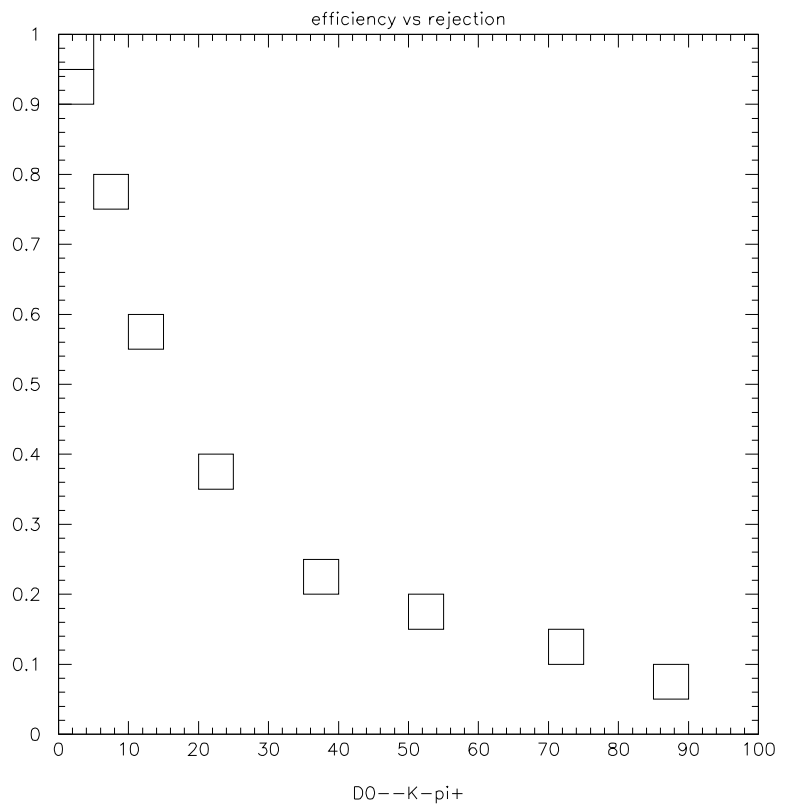

Figure 11.30: Trigger efficiency for $D^{0} \rightarrow K^{-} \pi^{+}$vs non-charm background rejection factor.

\subsubsection{Reconstruction}

Work is continuing on the definition of appropriate analysis cuts and the likely reconstruction efficiency for triggered events: preliminary results suggest this will be on the order of $20 \%$ for the more abundant modes. We compare this with the experience of E791, where similar values were achieved.

\subsubsection{Physics reach}

As an example of the potential of this mode of running, we present an estimate of the annual yield of $D^{0} \rightarrow K^{-} \pi^{+}$events which could be achieved (Table 11.12). 
Table 11.12: BTeV/C0 Summary of parameters for wire target running

\begin{tabular}{|c|c|c|}
\hline Property & Value & Comment \\
\hline Interaction rate & $10^{5}-10^{6} \mathrm{~s}^{-1}$ & thin $\mathrm{C}$ target \\
\hline Charm cross-section & $25 \mu \mathrm{b} /$ nucleon & $\begin{array}{l}2 \times 10^{-3} \text { of total } \\
\text { for } A=12\end{array}$ \\
\hline Charm rate & $200-2000 s^{-1}$ & \\
\hline Geometrical acceptances: & & \\
\hline$D^{0} \rightarrow K^{-} \pi^{+}$ & $74 \%$ & loss mostly in beam gap \\
\hline$D^{*+} \rightarrow D^{0} \pi^{+} \rightarrow K^{-} \pi^{+} \pi^{+}$ & $61 \%$ & \\
\hline Fraction of total charm which & & \\
\hline produces accepted $D^{0} \rightarrow K^{-} \pi^{+}$ & $3.5 \%$ & including $D^{0} \rightarrow K^{+} \pi^{-}$ \\
\hline $\begin{array}{l}\text { Irigger efticiency for } D \rightarrow K \pi \\
\text { Background rejection }\end{array}$ & $\begin{array}{l}60 \% \\
\times 10\end{array}$ & for accepted events \\
\hline Level I trigger rate & $10^{4}-10^{5} \mathrm{~s}^{-1}$ & \\
\hline $\begin{array}{l}\text { Number of triggered } D^{0} \rightarrow K^{-} \pi^{+} \\
\quad \text { events }\end{array}$ & $3-30 s^{-1}$ & \\
\hline Offline reconstruction efficiency & $20 \%$ & \\
\hline $\begin{array}{l}\text { Estimated number of reconstructed } \\
\qquad D^{0} \rightarrow K^{-} \pi^{+}\end{array}$ & $10^{7}-10^{8}$ & per $10^{7}$ sec \\
\hline
\end{tabular}




\section{Bibliography}

[1] "Determining the CP-violating phase $\gamma$ ",R. Aleksan, I. Dunietz, B. Kayser, Z. Phys C 54, 653-659 (1992); R. Aleksan, B. Kayser and D. London, "In Pursuit of $\gamma$," DAPNIA/SPP 93-23, hep-ph/9312338.

[2] "Determination of the CP-violating phase $\gamma$ by a sum over common decay modes to $B_{s}$ and $\bar{B}_{s}$ ",R. Aleksan, A. Le Yaouanc, L. Oliver, O. Pene, J.-C. Raynal, Z. Phys C 67, 251-260 (1995).

[3] D. Atwood, I. Dunietz and A. Soni, PRL 78, 3257(1997).

[4] M. Gronau and D. Wyler, Phys. Lett. B 265, 172 (1991).

[5] M. Gronau and J. Rosner, CALT-68-2142, hep-ph/9711246 (November 1997).

[6] R. Fleischer and T. Mannel, hep-ph/9704423.

[7] R. Godang et al, Phys. Rev. Lett. 80, 3456 (1998).

[8] A. Falk, A. Kagan, Y. Nir and A. Petrov, JHU-TIPAC-97018 (December 1997).

[9] BTev EOI (May 1997).

[10] " $J / \psi$ and $\psi(2 S)$ Production in $p \bar{p}$ Collisions at $\sqrt{s}=1.8 T e V "$, CDF Collaboration, FERMILAB-PUB-97/024-E

[11] " The CDF II Detector Technical Design Report", CDF collaboration, FERMILABPub-96/390-E

[12] http://www-pat.fnal.gov/mcfast/version_doc/v2_6_2/mcfast/mcfast_advanced.html

[13] O.R. Long, et al., "Monte Carlo Simulation of $B_{d}^{0} \rightarrow \pi^{+} \pi^{-}$from $p p$ Interactions at $\sqrt{s}=40 \mathrm{TeV} ", \mathrm{UPR} / 216 \mathrm{E}, \mathrm{PRINCETON} / \mathrm{HEP} / 92-07, \mathrm{SSCL} / \mathrm{PP} / 139$ (1992).

[14] F. Abe et al., The CDF Collaboration, Phys. Rev. D54, 6596 (1996). FERMILABPUB-96/119-E.

[15] The BaBar Physics Book, edited by P. Harrison and H. Quinn, SLAC-R-504, January 1999. 
[16] “ The CDF II Detector Technical Design Report", CDF collaboration, FERMILABPub-96/390-E (1996).

[17] A. Ali, "Rare B Decays in the Standard Model", Beauty '96, North Holland Press, 1996, the Netherlands, Table 1, page 13 and references therein.

[18] QQ is developed and maintained by the CLEO Collaboration. See http://www.lns.cornell.edu/public/CLEO/soft/QQ/ For the studies reported here, it is used for its model of the decays of hadrons containing heavy flavors.

[19] P. McBride and S. Stone, Nucl.Instrum.Meth. A368(1995) 38-40.

[20] R. Kutschke, BTeV Internal Note, 97/9, June 1997.

[21] K.T. McDonald, "Maximum Likelihood Analysis of CP Violating Asymmetries", PRINCETON-HEP-92-04, Sep 1992. 12pp, unpublished.

[22] K. Sterner and W. Selove, Univ. of Pa. Report UPR-237E, May 1997.)

[23] P.L. Frabetti et al., Nucl. Instrum. Methods. A 320, 519 (1992).

[24] J.Marriner, "Operation of the C0 experimental region with a fixed (internal) target", April 1997, unpublished.

[25] D.C.Christian, "Triggers for a high-sensitivity charm experiment", in proceedings of CHARM2000 workshop, Fermilab-conf-94/190 (1994). 


\section{Chapter 12}

\section{Comparison of $\mathrm{BTeV}$ to Other Experiments}

\subsection{Comparison with CDF, D0, CMS, and ATLAS}

Both CDF and D0 have measured the $b$ production cross section [1]. CDF has contributed to our knowledge of $b$ decay mostly by its measurements of the lifetime of $b$-flavored hadrons [2], which are competitive with those of LEP [3] and recently through its discovery of the $B_{c}$ meson [4]. CDF has also seen the first hint for CP violation in the $b$ system [5]. These detectors were designed for physics discoveries at large transverse momentum. It is remarkable that they have been able to accomplish so much in $b$ physics. They have shown that it is possible to do $b$ physics in the environment of a hadron collider.

However, these detectors, and the new central detectors ATLAS and CMS are very far from optimal for $b$ physics. BTeV has been designed with $b$ physics as its primary goal. To have an efficient trigger based on separation of $b$ decays from the primary, BTeV uses the large $\eta$ region where the $b$ 's are boosted. The detached vertex trigger allows collection of interesting purely hadronic final states such as $\pi^{+} \pi^{-}, \rho \pi, D_{s}^{+} \pi^{-}$and $D_{s}^{+} K^{-}$. It also allows us to collect enough charm to investigate mixing and $\mathrm{CP}$ violation.

The use of the forward geometry also allows for excellent charged hadron identification with a gaseous RICH detector. This is crucial for many physics issues such as separating $K \pi$ from $\pi \pi, D_{s} \pi$ from $D_{s} K$, kaon flavor tagging, etc..

Furthermore an experiment that plans on answering all the open questions in $b$ physics, requires a high quality electromagnetic calorimeter. Installation of such a calorimeter in the CLEO detector made new physics vistas possible and such a device in BTeV allows for the measurement of several crucial physics final states such as $B^{o} \rightarrow \rho \pi$, and $B_{s} \rightarrow J / \psi \eta^{\prime}$. The only central detector that is planning to have a high quality electromagnetic calorimeter is CMS.

Finally, BTeV has all the crucial elements required to study any newly suggested $b$ or charm processes or uncover new physics. The crucial elements are:

- a detached vertex trigger in the first trigger level, 
- highly efficient particle identification across the entire momentum range with good $(\approx$ 100:1) background rejection,

- an electromagnetic calorimeter with sufficiently good energy resolution and efficiency to fully reconstruct rare $B$ decay final states with single photons or neutral pions.

\subsection{Comparison with $e^{+} e^{-} B$ Factories}

Most of what is known about $b$ decays has been learned at $e^{+} e^{-}$machines [6]. Machines operating at the $\Upsilon(4 S)$ found the first fully reconstructed $B$ mesons (CLEO), $B^{\circ}-\bar{B}^{o}$ mixing (ARGUS), the first signal for the $b \rightarrow u$ transition (CLEO), and Penguin decays (CLEO). Lifetimes of $b$ hadrons were first measured by experiments at PEP, slightly later at PETRA, and extended and improved by LEP [6].

The success of the $\Upsilon(4 S)$ machines has led to the construction at KEK and SLAC of two new $\Upsilon(4 S)$ machines with luminosity goals in excess of $3 \times 10^{33} \mathrm{~cm}^{-2} \mathrm{~s}^{-1}$. These machines will have asymmetric beam energies so they can measure time dependent CP violation. They will join an upgraded CESR machine at Cornell that has symmetric beam energies. These machines will investigate only $B^{o}$ and $B^{ \pm}$decays. They will not investigate $B_{s}, B_{c}$ or $\Lambda_{b}$ decays.

Table 12.1 shows a comparison between $\mathrm{BTeV}$ and an asymmetric $e^{+} e^{-}$machine for measuring the $\mathrm{CP}$ violating asymmetry in the decay mode $B^{o} \rightarrow \pi^{+} \pi^{-}$. In Table 12.2 we show a similar comparison for the final state $B^{-} \rightarrow \bar{D}^{o} K^{-}$, a mode that could be used to determine the CKM angle $\gamma$. It is clear that the large hadronic $b$ production cross section can overwhelm the much smaller $e^{+} e^{-}$rate. Furthermore, the $e^{+} e^{-} B$ factories do not have access to the important $\mathrm{CP}$ violation measurements that need to be made in $B_{s}$ decays.

Table 12.1: Number of tagged $B^{o} \rightarrow \pi^{+} \pi^{-}\left(\mathcal{B}=0.75 \times 10^{-5}\right)$

\begin{tabular}{lrccrrr}
\hline \hline & & & & signal & tagging \\
& $\mathcal{L}\left(\mathrm{cm}^{-2} \mathrm{~s}^{-1}\right)$ & $\sigma$ & $\# B^{o} / 10^{7} \mathrm{~s}$ & efficiency & $\epsilon D^{2}$ & $\#$ tagged \\
\hline$e^{+} e^{-}$ & $3 \times 10^{33}$ & $1 \mathrm{nb}$ & $3.0 \times 10^{7}$ & 0.4 & 0.4 & 46 \\
$\mathrm{BTeV}$ & $2 \times 10^{32}$ & $100 \mu \mathrm{b}$ & $1.4 \times 10^{11}$ & 0.03 & 0.1 & 3400 \\
\hline \hline
\end{tabular}

\subsection{Comparison with LHC-B}

LHC-B is an experiment proposed for the LHC with almost the same physics goals as BTeV [7]. Here we address the issue of how BTeV can compete with LHC-B. LHC-B is being designed to run at a luminosity of $2 \times 10^{32} \mathrm{~cm}^{-2} \mathrm{~s}^{-1}$, the same as BTeV's goal. There are 
Table 12.2: Number of $B^{-} \rightarrow \bar{D}^{o} K^{-}\left(\mathcal{B}=1.5 \times 10^{-7}\right)$

\begin{tabular}{lrcrrr}
\hline \hline & & & & \multicolumn{2}{c}{ signal } \\
& $\mathcal{L}\left(\mathrm{cm}^{-2} \mathrm{~s}^{-1}\right)$ & $\sigma$ & $\# B^{-} / 10^{7} \mathrm{~s}$ & efficiency & $\#$ of events \\
\hline$e^{+} e^{-}$ & $3 \times 10^{33}$ & $1 \mathrm{nb}$ & $3.0 \times 10^{7}$ & 0.5 & 2 \\
$\mathrm{BTeV}$ & $2 \times 10^{32}$ & $100 \mu \mathrm{b}$ & $1.4 \times 10^{11}$ & 0.015 & 320 \\
\hline \hline
\end{tabular}

several inherent advantages and disadvantages that LHC-B has compared with BTeV. The issues that favor LHC-B are:

- The $b$ production cross-section is expected to be about five times larger at the LHC than at the Tevatron, while the total cross-section is only 1.6 times as large.

- The mean number of interactions per bunch is expected to be about 3 times lower at the LHC than at the Tevatron (at 132 ns bunch spacing).

The issues that favor $\mathrm{BTeV}$ are:

- BTeV is a two-arm spectrometer, which increases the signal by a factor of two compared with LHC-b.

- The short bunch spacing at the LHC, 25 ns, makes first level detached vertex triggering more difficult than at the Tevatron. It is difficult for the vertex detector electronics in LHC-b to settle in $25 \mathrm{~ns}$, while BTeV has a more relaxed $132 \mathrm{~ns}$. In fact, the current plan of LHC-b is to trigger in their first trigger level on muons, electrons or hadrons of moderate $p_{t}$, and detect detached vertices in the next trigger level.

- The seven times larger beam energy at the LHC makes the range of track momenta that need to be momentum analyzed and identified much larger and therefore more difficult. The larger energy also causes a large increase in track multiplicity per event, which makes pattern recognition and triggering more difficult.

- The interaction region at the Tevatron is three to six times longer along the beam direction than at LHC $\left(\sigma_{z}=5 \mathrm{~cm}\right)$, which allows BTeV to able to accept events with two or even three interactions per crossing, since the interactions are well separated. LHC-b currently vetos crossings with more than one interaction.

- $\mathrm{BTeV}$ is designed to have the vertex detector in the magnetic field thus allowing the rejection of low momentum tracks at the trigger level. Low momentum tracks have large multiple scattering which can cause false detached vertices leading to poor background rejection in the trigger. 
- $\mathrm{BTeV}$ is designed with a high quality $\mathrm{PbWO}_{4}$ electromagnetic calorimeter, that provides high resolution and acceptance for interesting final states with $\gamma^{\prime} \mathrm{s}, \pi^{o}$ s, and $\eta^{\left({ }^{\prime}\right)}$ 's.

- Use of a detached vertex trigger at Level 1 allows for an extensive charm physics program absent in LHC-b. It also allows for a more uniform collection of $b$ triggers.

We have more than compensated for LHC-b's initial advantages in $b$ cross-section due their higher center-of-mass energy. In fact, the high energy actually works in many ways as a disadvantage. For example, LHC-b needs two RICH counters to cover the momentum range in their one arm. Particle identification and other considerations force LHC-b to be longer than BTeV. Its single arm is as long as both BTeV arms put together. This causes the transverse size of LHC-b to be four times that of each arm of BTeV, and this allows them only to cover the same solid angle as one of BTeV's two arms. It is expensive to instrument all of this real estate with high qualtiy particle detectors. Thus, the total cost for LHC-b based only on instrumented area, (a naive assumption) would be twice the total cost for $\mathrm{BTeV}$, even though LHC-b would cover only half the solid angle. 


\section{Bibliography}

[1] K. Abe et al, (CDF), Phys. Rev. Lett. 75, 1451 (1995); S. Abachi et al, (D0), Phys. Rev. Lett. 74, 3548 (1995). See also the UA1 measurement C. Albajar et al, Phys. Lett. B186, 237 (1987); B213, 405 (1988); B256, 121 (1991).

[2] K. Abe et al, (CDF), Phys. Rev. Lett. 76, 4462 (1996); ibid 77, 1945 (1996); K. Abe et al, (CDF), Phys. Rev. D 57, 5382 (1998).

[3] T. Junk, "A Review of $B$ Hadron Lifetime Measurements from LEP, the Tevatron and SLC," in Proceedings of the 2nd Int. Conf. on B Physics and CP Violation, Univ. of Hawaii, (1997), ed. T. E. Browder et al, World Scientific, Singapore (1998).

[4] K. Abe et al, (CDF), "Observation of $B_{c}$ Mesons in $p-\bar{p}$ Collisions at $\sqrt{s}=1.8 \mathrm{TeV}$," hep-ex/9804014 (1998).

[5] M. Paulini, "B Lifetimes, Mixing and CP Violation at CDF," Review article to appear in the Int. Journal of Modern Physics A, hep-ex/9903002 (1999).

[6] See B Decays, revised 2nd Edition ed. S. Stone, World Scientific, Singapore, (1994).

[7] "LHCb Technical Proposal," CERN/LHCC 98-4, LHCC/P4 (1998). 


\section{Part IV}

\section{Costs}




\section{Chapter 13}

\section{Cost}

\subsection{Preliminary Cost Estimate}

At this time it is quite difficult to accurately predict the cost of the two-arm BTeV experiment. However, we make estimates based on comparing with other experiments. These are shown in Table 13.1.

Table 13.1: Preliminary BTeV cost estimate (\$)

\begin{tabular}{lrrl}
\hline \hline & \multicolumn{1}{c}{ Cost } & Cost & \\
Item & $(2 \mathrm{arms})$ & $(1 \mathrm{arm})$ & comment \\
\hline Pixels & $15 \mathrm{M}$ & $15 \mathrm{M}$ & based on CMS and ATLAS projections \\
Trigger Level I & $2.5 \mathrm{M}$ & $2.5 \mathrm{M}$ & based on 3200 DSP processors \\
Tracking & $10 \mathrm{M}$ & $5 \mathrm{M}$ & Based on ATLAS staw tubes, FNAL silicon \\
RICH & $8 \mathrm{M}$ & $5 \mathrm{M}$ & based on HERA-B RICH \\
EM Calorimeter & $15 \mathrm{M}$ & $7.5 \mathrm{M}$ & PbWO $_{4}$ from CMS \\
Muon & $2.5 \mathrm{M}$ & $1.5 \mathrm{M}$ & based on details in Part II \\
DAQ+Level II \& III & $4 \mathrm{M}$ & $4 \mathrm{M}$ & based on 4000 processors, 20 tape drives \\
Infrastructure & $5 \mathrm{M}$ & $5 \mathrm{M}$ & racks, crates, power supplies, etc... \\
Off-line computing & & & \\
\& data storage & $9 \mathrm{M}$ & $9 \mathrm{M}$ & based on CDF and D0 Run II estimates \\
\hline \hline
\end{tabular}

The total cost of the two-arm system is $71 \mathrm{M} \$$, compared with $54.5 \mathrm{M} \$$ for one arm. This information is useful as one arm can be fully instrumented initially, with the components of the second arm added during running. We use the baseline $\mathrm{PbWO}_{4}$ calorimeter with 10,000 crystals per arm for our cost estimate. The larger optional detector would cost twice as much. We note that costs for off-line computing and data storage are not usually included in detector cost estimates. 


\subsection{Support Required}

Simulation has been the key to developing the BTeV design. We have used simulations to establish the physics reach of the experiment. It is also useful for detailed design issues. For example, it has allowed us to see the subtle interplay between spatial resolution and material in proper time resolution. This has led to a program to reduce the pixel thickness. Continuing support of the simulation group is absolutely essential to completing the design.

Our plan for BTeV's evolution requires us continue our successful pixel, trigger, tracking and muon R\&D programs which need more funding. Test beam work this year will be most informative. R\&D on RICH and EM calorimetry, however, needs new funding in order to progress. 